Oliver Krüger

\title{
Virtualität und Unsterblichkeit
}

Gott, Evolution und die Singularität im Post- und Transhumanismus 
Oliver Krüger

Virtualität und Unsterblichkeit

Gott, Evolution und die Singularität

im Post- und Transhumanismus 


\section{ROMBACH WISSENSCHAFTEN • REIHE LITTERAE}

herausgegeben von Gerhard Neumann $(\dagger)$, Günter Schnitzler,

Maximilian Bergengruen und Thomas Klinkert

Band 123 
Oliver Krüger

\section{Virtualität und Unsterblichkeit}

Gott, Evolution und die Singularität im Post- und Transhumanismus

N"'/" rombach verlag 
Dieses Buch ist mit der Unterstützung der Philosophischen Fakultät und des Hochschulrates der Universität Freiburg (Schweiz) veröffentlicht worden.

Bibliografische Information der Deutschen Nationalbibliothek

Die Deutsche Nationalbibliothek verzeichnet diese Publikation in der Deutschen Nationalbibliografie; detaillierte bibliografische Daten sind im Internet über $<$ http:/dnb.d-nb.de $>$ abrufbar.

(C) 2019. Rombach Verlag KG, Freiburg i.Br./Berlin/Wien

2. vollständig überarbeitete und erweiterte Auflage. Alle Rechte vorbehalten Umschlag: Bärbel Engler, Rombach Verlag KG, Freiburg i.Br./Berlin/Wien Satz: rombach digitale manufaktur, Freiburg im Breisgau

Herstellung: Rombach Druck- und Verlagshaus GmbH \& Co. KG, Freiburg i.Br.

Printed in Germany

ISBN 978-3-7930-9939-0 


\section{Inhalt}

Vorwort der zweiten Auflage $\ldots \ldots \ldots \ldots \ldots \ldots \ldots \ldots$

Vorwort der ersten Auflage $\ldots \ldots \ldots \ldots \ldots \ldots \ldots \ldots \ldots \ldots$

Who is Who? des Post- und Transhumanismus ........... 13

1. Virtualität, Medien und Unsterblichkeit. Eine Einleitung $\ldots \ldots 17$

\section{Teil I Mensch und Medien}

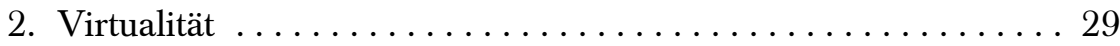

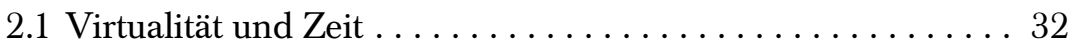

2.2 Virtualität und Raum ...................... 41

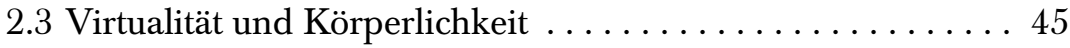

2.4 Virtualität, Realität und das Imaginäre . . . . . . . . 50

3. Die prometheische Scham . . . . . . . . . . . . . . 61

3.1 Mensch und Technik bei Günther Anders ..........6 61

3.2 Virtualität und $\operatorname{Tod} \ldots \ldots \ldots \ldots \ldots \ldots \ldots \ldots \ldots \ldots \ldots \ldots \ldots \ldots \ldots$

\section{Teil II Der technologische Posthumanismus}

4. Transhumanismus ........................ 75

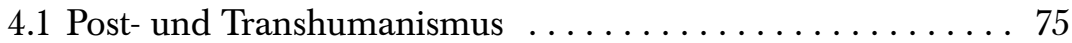

4.2 Vorläufer und Begriffsgeschichte . . . . . . . . . . 77

4.3 Der frühe Transhumanismus: Ettinger, FM-2030, Leary . . . 84

4.4 Das Extropy Institute und die (Vita-)Mores . . . . . . . . . 94

4.5 Die World Transhumanist Association / humanity+ . . . . . . . . 99

4.6 Weitere Akteure und Institutionen . . . . . . . . . . . . 103

4.7 Der religiöse und spirituelle Transhumanismus . . . . . . 110

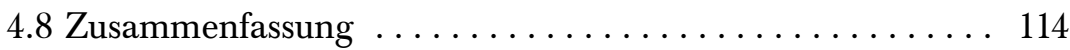


5. Der technologische Posthumanismus $\ldots \ldots \ldots \ldots \ldots \ldots 117$

5.1 Das Posthumane und der Posthumanismus . . . . . . . . 119

5.2 Das Gesicht des Posthumanismus . . . . . . . . . . . . . 122

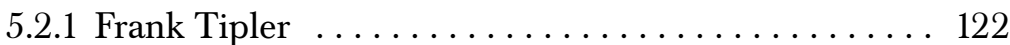

5.2 .2 Marvin Minsky . . . . . . . . . . . . . . . . . 127

5.2 .3 Hans Moravec . . . . . . . . . . . . . . . . . . . . 130

5.2 .4 Ray Kurzweil . . . . . . . . . . . . . . . 133

5.3 Posthumanismus und Kunst . . . . . . . . . . . . . . . 136

6. Ideengeschichte des technologischen Posthumanismus . . . . . 147

6.1 Methodische Vorüberlegungen zur

»Geschichte der Zukunft« . . . . . . . . . . . . . . . . 147

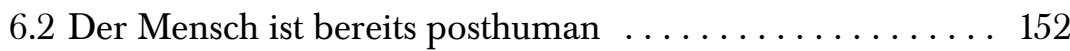

6.2.1 L'Homme Machine . . . . . . . . . . . . . . . . . . 155

6.2.2 Simulation und Identität . . . . . . . . . . . 167

6.2.3 Der Mensch im kybernetischen Paradigma .... . . . . 183

6.2.4 Der Maßstab der Vervollkommnung:

Arbeit und Wissen . . . . . . . . . . . . . . . . 201

6.3 Vernichtung oder unendlicher Fortschritt . . . . . . . . 211

6.3.1 Tod, Entropie und die drohende

Vernichtung des Lebens im Universum . . . . . . . . . 212

6.3.2 Das Opfer des Menschen ................... 220

6.3.3 Fortschritt und Vollkommenheit . . . . . . . . . 229

6.3.4 Evolution und der Aufstieg des Lebens .... . . . . . 239

6.3.5 Die Physikotheologie des Frank Tipler ......... 258

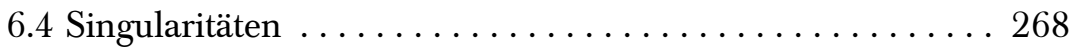

6.4.1 Die kosmologische Singularität und die

schwarzen Löcher . . . . . . . . . . . . . . . . . . . . 270

6.4.2 Die technologische Singularität . . . . . . . . . 273

6.4.3 Fortschrittsgesetze und die endless frontier . . . . . . 287

6.5 Unsterblichkeit . . . . . . . . . . . . . . . . . . . 297

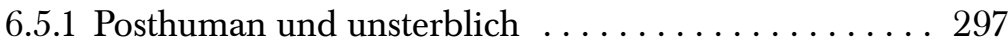

6.5.2 Von der Langlebigkeit zur computertechnischen

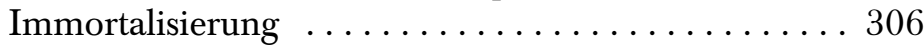

6.5.3 Die Unsterblichkeit in der Science-Fiction . . . . . . . 321

6.5 .4 Kryonik . . . . . . . . . . . . . . . . . . 334

6.5.5 Die technologische Unsterblichkeit . . . . . . . 351 
6.6 Die transzendente Superintelligenz . . . . . . . . . 355

6.6.1 Transzendenz und Übermensch . . . . . . . . . . . 355

6.6.2 Gehirn, Genie und Superintelligenz _........ 359

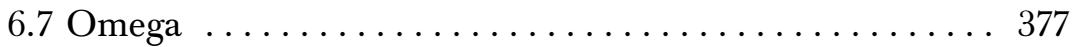

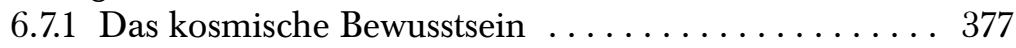

6.7.2 Teilhard de Chardin, McLuhan und die Noosphäre . . 384

7. Virtualität. Unsterblichkeit in der Mediengesellschaft ...... 393

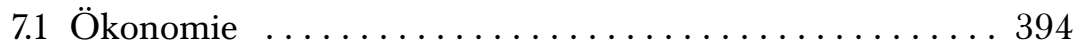

7.2 Kontrolle und Kontingenz . . . . . . . . . . . . 400

7.3 Säkularer Fortschritt und religiöse Heilserwartung . . . . . . 408

7.4 Das Ende der Kränkungen . . . . . . . . . . . . 421

Abkürzungsverzeichnis . . . . . . . . . . . . . . 427

Quellenverzeichnis ........................4429

a) Literatur . . . . . . . . . . . . . . . . . . . . . . 429

b) Spielfilme / Fernsehfilme / Dokumentationen ... . . . . . 463

c) Interviews (Videos) . . . . . . . . . . . . . . . 465

Sach- und Personenindex . . . . . . . . . . . . . 467 


\section{Vorwort der zweiten Auflage}

Die Zukunft stirbt nie. Daher erfreuen sich der Posthumanismus und der Transhumanismus anhaltender Aufmerksamkeit durch Wissenschaft, Medien und Populärkultur. Ursprünglich hatte ich den Plan gefasst, eine Aktualisierung zu den zentralen Aspekten meines Buches von 2004 zu verfassen. ${ }^{1}$ Es sind im Wesentlichen auch keine neuen Ideen und nur wenige neue Akteure auf die posthumanistische Bühne getreten. Allerdings haben sich die Themen und die Ausrichtung der Visionen deutlich verschoben.

Als ich im Jahr 2000 den Transhumanistenkongress in London besuchte, war Ray Kurzweil allen Teilnehmern, mit denen ich ins Gespräch kam, vollkommen unbekannt. Ich überlegte, ob es überhaupt legitim sei, Kurzweil in meine Analysen aufzunehmen. Seine Schriften waren im Vergleich zu denen Frank Tiplers, Hans Moravecs und Marvin Minskys nicht besonders innovativ. Vor ungefähr zehn Jahren hatte ich vermutet, dass sich der Post- und Transhumanismus als kurzlebiges Phänomen der Jahrtausendwende erweisen würde, da sich eine Reihe von Netzwerken und Organisationen auflösten. Dann aber befeuerte Ray Kurzweil mit seinem 2005 erschienenen Buch The Singularity is near und der nachfolgenden Gründung der Singularity University die Debatte. Heute steht er zweifellos im Zentrum der Aufmerksamkeit. Moravec ist jetzt vor allem als Unternehmer tätig, Minsky verstarb im Januar 2016 und Tipler fristet zwar immer noch ein Außenseiterdasein, aber seine zentrale Idee einer kosmischen Evolution wird inzwischen weitgehend akzeptiert. Stärker als in der Vergangenheit vermischen sich damit religiöse und säkulare Elemente von Fortschrittstheorien. Mit dem Fokus auf die Singularität und auf ein allumfassendes, superintelligentes Bewusstsein gerät die Thematik des Androiden und des verkörperten, künstlichen Menschen in den Hintergrund, die ich in der überarbeiteten Fassung bewusst ausklammere. Viel relevanter ist die Frage nach den kulturellen Kontexten der Idee einer Superintelligenz. Der ideengeschichtliche, zweite Teil des Buches, Der technologische Posthumanismus, wurde daher neu strukturiert und inhaltlich umfassend ergänzt. Der erste Teil, Mensch und Medien, wurde erheblich gekürzt und klarer gestaltet. Das Who is Who? des Post- und Transhumanismus soll die Orientierung in der Vielzahl von Akteuren erleichtern.

1 Vgl. Krüger 2004a. 
Diese zweite Auflage erarbeitete ich in den Jahren 2017 und 2018. DiesselbenVereinigten Staaten, in denen die Anhänger Kurzweils die Naherwartung einer Superintelligenz proklamieren, verzeichnen durch die anhaltende Drogenkrise schon im dritten Jahr in Folge einen Rückgang der Lebenserwartung. Verschwörungstheorien prägen die Realpolitik, wissenschaftliche Erkenntnisse über den Klimawandel werden von der US-Regierung geleugnet, widersprechende Wissenschaftler werden ignoriert und die Teenager-Schwangerschaften sind aufgrund christlich-fundamentalistischer Sexualerziehung auf einem Rekordhoch.

Damit ist evident, dass Fortschritt keine mathemathische Größe, sondern von sozialen Entwicklungen und der Struktur des gesellschaftlichen Wissensvorrates abhängig ist. Das Gleiche gilt für Fortschrittstheorien, wie den Post- und Transhumanismus, die Gegenstand dieser Untersuchung sind. Ihre Botschaft des exponentiellen Wachstums findet gleich einem Tanz auf dem Vulkan just in der historischen Epoche ihre radikalste Ausformung, in der die ökologischen und sozialen Folgen einer grenzenlosen Ausbeutung natürlicher und menschlicher Ressourcen mit jedem Tag sichtbarer und spürbarer werden.

Auch für diese zweite Ausgabe, deren Überarbeitung viel mehr Zeit und Lektüre benötigt hat, als ich zu Beginn meiner Arbeit erahnen konnte, bin ich vielen Freunden sowie Kolleginnen und Kollegen für ihre Hinweise, Korrekturen und Kommentare zu Dank verpflichtet. Mein besonderer Dank gilt daher Carla Hagen, Dr. Sabine Haupt, Alice Kaiser, Dr. Janina Loh, Stephanie Majerus, Adriano Montefusco, Ralf Hoffmann, Prof. Andrea Rota, Dr. Ricarda Stegmann und Prof. Helmut Zander.

Dem Hochschulrat und der Philosophischen Fakultät der Universität Freiburg i.Ü. danke ich für die finanzielle Unterstützung des Projektes sowie Herrn Dr. Torang Sinaga vom Rombach-Verlag für die gute Betreuung. Der Deutschen Vereinigung für Religionswissenschaft danke ich für die Auszeichnung mit dem 2004 erstmals verliehenen Dissertationspreis.

Das Literaturverzeichnis wurde, nachdem es in der überarbeiteten Version zunächst weit über 50 Seiten umfasste, auf die Quellen und die wichtigste Literatur beschränkt. $^{2}$

Oliver Krüger

Bern, Winter 2018

2 Alle Internetadressen im Quellenverzeichnis sowie in den Fußnoten waren - sofern nicht anders angegeben - am 15.12.2018 verfügbar. 


\section{Vorwort der ersten Auflage}

Das Problem dieses Buches und vielleicht das Problem aller Bücher ist die Tatsache, daß wir keine Tralfamadorians sind. Die Bücher dieser außerirdischen Spezies, so belehrt uns der amerikanische Schriftsteller Kurt Vonnegut (*1922), haben nämlich die Eigenart, daß man sie nicht Wort für Wort und Satz für Satz liest, sondern daß auf jeder Buchseite ein Cluster aus Zeichen das Erlebnis eines Gefühls, einer Situation oder eines Gedankens als Ganzes wiedergibt. ${ }^{1}$ In diesem Buch, das als Dissertation an der Universität Bonn eingereicht wurde, kann ich mich lediglich auf herkömmliche Weise bemühen, die überaus verzweigte Thematik von Virtualität und Unsterblichkeit sukzessiv zu entfalten, um die Wechselbeziehungen der beteiligten Diskurse auch in ihrer historischen Tiefe darzustellen.

Die Vielschichtigkeit dieser Materie habe ich nicht nur als akademischer homo viator in den verschiedensten Bibliotheken erfahren dürfen, sondern auch die große Zahl der Personen, die auf vielfältige Weise zum Gelingen dieser Arbeit beigetragen haben, spiegelt auf einer konkret sozialen Ebene die Komplexität des hier behandelten Themas wider. Ohne die fortwährende Unterstützung von Familie, Freunden und Dozenten wäre diese Arbeit so, wie sie hier vorliegt, niemals möglich gewesen.

Für die anhaltende Ermunterung zur Weiterarbeit, für die vielen anregenden Gespräche, Literaturempfehlungen, gesammelten Zeitungsartikel, für die philologische Hilfe, für die künstlerischen sowie philosophischen Reflexionen danke ich Dagmar Benner, Hatice Çiğdem, Stefanie Elbern, Stefan Guschker, Manfred Hammes, Silja Joneleit-Oesch, Elisabeth Krüger, Elias Maya, Karin Meiner, Susann Mende, Refika Sariönder, Markus Sauer, Brigitte Schön, Frank Schüller, Ulrich Vollmer, Jochen Walter, Ulrike Wittlich und Han Yan. Für die freundliche Beantwortung meiner vielen Fragen bin ich den Autoren Frank Tipler und Max More sowie Torsten Nahm von der De:Trans zu Dank verpflichtet.

Der VG Wort danke ich für die Gewährung des großzügigen Zuschusses für die Drucklegung dieses Werkes. Der Deutschen Forschungsgemeinschaft in Gestalt des Graduiertenkollegs Religion und Normativität an der Universität Heidelberg möchte ich für die Gewährung meines

1 Vgl. Vonnegut 1991, 88. 
Promotionsstipendiums danken und mich insbesondere für die Unterstützung und die pragmatischen Ratschläge bei Prof. Rudolf Wagner bedanken. Für inhaltliche Anregungen bin ich Prof. Jan Assmann, Prof. Wolfgang Gantke, Prof. David Lavery und Prof. Jan Snoek zu Dank verpflichtet.

Prof. Karl Hoheisel und Prof. Gregor Ahn möchte ich für die ungewöhnlich intensive Betreuung der Dissertation und für ihre fortwährende, in vielerlei Hinsicht ermutigende Unterstützung in den vergangenen Jahren meinen Dank aussprechen. Mein besonderer Dank gilt Ralf Hoffmann, Dardo Lessmann und Sonja Pruhs, da sie die mühevolle Arbeit des Korrekturlesens übernommen haben und den gesamten Entstehungsprozeß meiner Abhandlung mit ihren stets konstruktiven Kommentaren und Diskussionen bereichert haben. Schließlich möchte ich Frau Dr. Edelgard Spaude vom Rombach Verlag für die gelungene Zusammenarbeit danken.

Oliver Krüger

Heidelberg, Sommer 2004 
Bernal, John D. (1901-1971), Vordenker transhumanistischer Ideen. Wichtigste Publikation: The World, the Flesh, and the Devil (1929).

Bostrom, Nick (*1973), Initiator der transhumanistischen Bewegung in den 1990er Jahren, von 1998 bis 2008 Koordinator / Vorsitzender der World Transhumanist Association, seit 2005 Gründer und Direktor des Future of Humanity Institute. Wichtigste Publikation: Superintelligence: Paths, Dangers, Strategies (2014).

Brown, Bernadeane (*1937), führt seit den 1960er Jahren mit Charles Paul Brown und James Russel Strole verschiedene Vereinigungen wie die Eternal Flame oder People Unlimited Inc., die die Überwindung des Todes propagieren.

Esfandiary, Fereidoun M. / FM-2030 (1930-2000), seit den 1970er Jahren ein zentraler Vertreter transhumanistischer Ideen. Wichtigste Publikation: Are you a Transhuman?(1989).

Ettinger, Robert C. W. (1918-2011), Begründer der Kryonik. Wichtigste Publikation: The Prospect of Immortality (1964).

De Grey, Aubrey (*1963), Gerontologe mit weitreichenden Versprechungen, Leiter der SENS Research Foundation. Wichtigste Publikation: Ending Aging (2008).

Drexler, K. Eric $\left({ }^{*} 1955\right)$, Nanotechnologe, Mitbegründer des Foresight Institutes 1986. Wichtigste Publikation: Engines of Creation (1986).

Faloon, William (Bill) (*1954), Mitbegründer der Life Extension Foundation (1980) und der Church of Perpetual Life (2013), die den Vertrieb von Supplementen ermöglichen und sich für die Kryonik einsetzen.

Goertzel, Ben (*1966), IT-Unternehmer, Vorsitzender von humanity+ (2008-2010, seit 2018). Wichtigste Publikation: A Cosmist Manifesto (2010).

Haldane, John B. S. (1892-1964), Evolutionsbiologe, Vordenker transhumanistischer Ideen. Wichtigste Publikation: Daedalus; or, Science and the Future (1924).

Hughes, James J. (*1961), Soziologe, Geschäftsführer der World Transhumanist Association von 2004-2006, Begründer und Leiter des Institute for Ethics and Emerging Technologies (2004). Wichtigste Publikation: Citizen Cyborg (2004). 
Huxley, Julian (1887-1975), Evolutionsbiologe, Eugeniker, erster Direktor der UNESCO, Vordenker transhumanistischer Ideen. Wichtigste Publikation: Transhumanism (1957).

Istvan, Zoltan $\left({ }^{*} 1973\right)$, US-amerikanischer, transhumanistischer Aktivist, der mit seiner Kandidatur zu den Präsidentschaftswahlen 2016 viel Medienaufmerksamkeit erhielt.

Kent, Saul (*1939), Kryoniker und Mitbegründer der Life Extension Foundation (1980).

Kurzweil, Raymond (*1948), IT-Unternehmer, seit 2012 Director of Engineering bei Google, Mitbegründer der Singularity University (2008), Autor von futurologischen und Lebenshilfe-Büchern, bedeutender Vertreter des Trans- und Posthumanismus. Wichtigste Publikation: The Singularity is Near (2005).

Leary, Timothy (1920-1996), Psychologe, LSD-Forscher, Vordenker und Netzwerker des frühen Transhumanismus. Wichtigste Publikation: Chaos and Cyber Culture (1994).

Merkle, Ralph (*1952), Informatiker, Nanotechnologe, Kryoniker.

Minsky, Marvin (1927-2016), KI-Forscher am MIT, Lehrer zahlreicher Transhumanisten, bedeutender Vertreter des technologischen Posthumanismus.

Moravec, Hans (*1948), Robotiker, begründet mit seinem Plädoyer für die Abschaffung des Menschen zugunsten der künstlichen Intelligenzen und Roboter den technologischen Posthumanismus, popularisiert die Idee einer Immortalisierung des menschlichen Geistes mit Hilfe eines Gehirnscans. Wichtigste Publikation: Mind Children (1988).

More, Max (*1964), Transhumanist und Kryoniker, Begründer und Leiter des Extropy Institutes (1991-2006), seit 2011 Präsident und CEO des Kryonik-Anbieters Alcor. Wichtigste Publikation: Transhumanism (1990).

O'Neill, Gerard K. (1927-1992), Physiker und Vordenker der Weltraumkolonisation, der maßgeblich für die Gründung der L5 Society war, in der viele spätere Transhumanisten zusammenfanden. Wichtigste Publikation: The High Frontier (1977).

Pearce, David, Mitbegründer der World Transhumanist Association (1998).

Prisco, Giulio (*1957), Transhumanist und Kryoniker, Mitbegründer des Order of Cosmic Engineers (2008-2012).

Rothblatt, Martine $\left({ }^{*} 1954\right)$, Unternehmerin in den Biotechnologien und der Weltraumfahrt, Transhumanistin, Transgender-Aktivistin. Wichtigste Publikation: Virtually Human (2014). 
Sandberg, Anders (*1972), Neurowissenschaftler, Mitbegründer und Vorsitzender der Schwedischen Transhumanisten Vereinigung (19961998), Mitarbeiter am Future of Humanity Institute (Oxford).

Sorgner, Stefan L. ( $\left.{ }^{*} 1973\right)$, Philosoph, Begründer des Metahumanismus. Wichtigste Publikation: Transhumanismus (2016).

Stock, Gregory (*1949), Physiker und Unternehmer in den Biotechnologien. Wichtigste Publikation: Metaman (1993).

Strole, James $\left({ }^{*} 1949\right)$ führt seit den 1960er Jahren mit Charles Paul und Bernadeane Brown verschiedene Vereinigungen wie die Eternal Flame oder People Unlimited Inc., initiierte 2016 das RAAD Fest (Revolution Against Aging and Death).

Teilhard de Chardin, Pierre (1881-1955), jesuitischer Philosoph und Paläontologe, Befürworter der Eugenik, einflussreich durch seine Vorstellungen zur Zukunft des Universums im göttlichen Punkt Omega und der planetaren Noosphäre als Zusammenfluss des Denkens. Wichtigstes Buch: Der Mensch im Kosmos (1959).

Vinge, Vernor (*1944), Science-Fiction Autor, prägte maßgeblich den Begriff der technologischen Singularität.

Vita-More, Natasha $\left({ }^{*} 1950\right)$, Künstlerin und transhumanistische Aktivistin seit den 1980er Jahren. Wichtigste Publikation: The Transhumanist Reader (Hg. mit Max More, 2013).

Walford, Roy L.(1924-2004), populärer Diätologe mit dem Versprechen, die natürliche Lebensspanne enorm erweitern zu können. Wichtigste Publikation: Maximum Life Span (1983).

Warwick, Kevin (*1954), Kybernetiker mit dem Anspruch, mittels eines Chipimplantates der erste Cyborg in der Menschheitsgeschichte zu sein. Wichtigste Publikation: March of the Machines (1997).

Yudkowsky, Eliezer (*1979), KI-Forscher, Mitbegründer des Machine Intelligence Research Institute (2000). Wichtigste Publikation: Singularitarian Principles (2000). 


\title{
1. Virtualität, Medien und Unsterblichkeit. Eine Einleitung
}

\begin{abstract}
human ('hyü-men) adj. 1. of, belonging to, or typical of the extinct species Homo sapiens < the human race> 2. what consisted of or was produced by Homo sapiens < human society $>$ n. an extinct biped, Homo sapiens, characterized by carbon-based anatomy: also HUMAN BEING. ${ }^{1}$
\end{abstract}

Wired, April 2000

Gemäß der jüngsten Erhebung der seit 1964 durchgeführten Langzeitstudie Massenkommunikation verbringt der Durchschnittsdeutsche pro Tag 208 Minuten mit fernsehen, 173 Minuten mit Hörfunk, 78 Minuten mit sonstigen Medien und 107 Minuten mit dem Internet, wobei ca. $90 \%$ der Befragten das Internet auch über mobile Geräte nutzen. ${ }^{2}$ Es steht damit außer Frage, dass Medienerfahrungen ein grundlegender Bestandteil unseres sozialen Alltags geworden sind. Die Erfahrung unseres sozialen Gegenübers ist zudem zunehmend geprägt von seiner virtuellen Reproduktion - sei dies nun durch fiktive Formate, Live-Streams, Internettelefonie oder Fotoplattformen.

Jede technologische Neuerung wird natürlicherweise von praktischen Erwägungen begleitet, die Vor- und Nachteile für bestimmte Anwendungen sowie die Kosten evaluieren. Gleichzeitig beginnt aber auch eine gesellschaftliche und kulturelle Auseinandersetzung mit neuer Technologie. Dieser Prozess ist heterogen und dynamisch, das heißt, dass nicht eine einheitliche und statische Deutung vorliegt, sondern dass diese Deutungen durchaus kontrovers verlaufen und sich über die Zeit entwickeln und wieder verändern können.

Der Ansatz der Medienhermeneutik will diese Dynamiken im Rahmen der sozialwissenschaftlichen Hermeneutik und interaktionistischen Medienforschung einfangen. Schon 1962 erklärten die beiden Soziologen Elihu Katz und David Foulkes » ... the question (is) not >what do the me-

\footnotetext{
Cover von Wired 8.04.
}

2 Die Gruppe der 14 bis 29-Jährigen nutzt das Fernsehen und den Hörfunk 64 bzw. 36 Minuten weniger als der Durchschnitt, dafür beobachten wir ein Mehr von 80 Minuten bei Internetanwendungen. Vgl. Engel \& Breunig 2015, 312; Media Perspektiven 2017,85 . 
dia do to people? < but, rather, > What do people do with the media<.« ${ }^{3} \mathrm{Im}$ Gegensatz zur Annahme von deterministischen Wirkungen bestimmter Medien auf die »Masse « passiver und unaufgeklärter Medienkonsumenten müssen demnach die faktischen Rezeptionsverläufe und Deutungen von Medien und Medieninhalten sowie der soziale Gebrauch von Medien Berücksichtigung finden, also » ... welche Spielräume der Rezeption in welchen Kontexten wie genutzt werden oder ungenutzt bleiben «. ${ }^{4}$ Die Medienhermeneutik erfasst dabei neben der Medienpraxis, der Produktion und der Rezeption spezifischer Medieninhalte auch allgemeine Deutungsprozesse ausgewählter Medien. ${ }^{5}$

Im Falle der audiovisuellen Medieninnovationen, die sich von der Photographie im 19. Jahrhundert bis zu den digitalen Medien unserer Tage erstrecken, haben die virtuellen Abbilder und Simulationen des Menschen neue Fragen aufgeworfen. Wie ist das Verhältnis der Virtualität zur Wirklichkeit zu deuten? Wie verändern technische Bildmedien unser Verhältnis zum Raum, zur Zeit und zum Körper? Verändern diese Medien unsere Ideale vom Dasein?

Der technologische Posthumanismus steht für die wohl extremste Deutung von rezenten Medien. Sie ist extrem, weil sie aus dem Vergleich des biologischen Menschen mit seiner virtuellen Reproduktion die Utopie einer künftigen Unsterblichkeit entwirft, die schließlich in der Auslöschung des bisherigen Menschen mündet. Extrem ist sie ferner, weil sie aus dem Vergleich des Menschen mit fortgeschrittener Computertechnologie eine kybernetische Definition des Lebens gewinnt, die eine technozentrische Umdeutung der Evolutionsgeschichte ermöglicht. Auf dieser Grundlage formuliert der Posthumanismus den totalitären Anspruch einer kosmischen Heilsgeschichte, die unausweichlich sei. Das Eingangszitat, das in Form eines zerknitterten Lexikonartikels im April 2000 das Cover von Wired zierte, spiegelt die Reichweite dieser Ideologie wider.

Es sind nun 30 Jahre vergangen seit der amerikanische Robotiker Hans Moravec in seinem Aufsehen erregenden Werk Mind Children. The Future of Robot and Human Intelligence die Vision einer Unsterblichkeit im Computer als normatives Ziel des menschlichen Fortschrittsprozesses formulierte. Die Ideen des technologischen Posthumanismus und des mit ihm verwandten Transhumanismus sind inzwischen in Literatur,

\footnotetext{
Katz \& Foulkes 1962, 378.

Keppler 2001, 132.

Vgl. Krüger \& Rota 2015, 75-80.
} 
Kunst, Film, Fernsehen und der populären Publizistik angelangt. Filme wie Transcendence (2014) und Fernsehserien wie Altered Carbon (2018) entwerfen dramatische Szenarien um die konkrete Möglichkeit einer computertechnischen Immortalisierung. Michel Houellebecqs vollkommen trostlose Gesellschaftsanalyse Les particules élémentaires (Elementarteilchen, 1998) sowie Dan Browns Romane Inferno (2013) und Origin (2017) machen ein breites Publikum mit Logiken des eugenischen Transhumanismus bekannt. ${ }^{6}$ Derweil hat der IT-Unternehmer, Autor und jetzige Google-Chefingenieur Ray Kurzweil das posthumanistische Konzept der Singularität marktkonform für die Gründung seiner gleichnamigen Singularity University genutzt. Seine Ideen werden sogar in deutschen Klassenzimmern präsentiert. Jüngst hat Frank Schätzing das Konzept einer allmächtigen, künstlichen Superintelligenz in seinem Roman Die Tyrannei des Schmetterlings verarbeitet. ${ }^{7}$ Das Ziel meines Unterfangens ist es, zu verstehen und zu erklären, wie an der Wende zum 21. Jahrhundert die posthumanistische Idee von der Unsterblichkeit in den Medien entstehen und sich weiterentwickeln konnte. Gegenstand meiner Untersuchung ist alleinig der technologische Posthumanismus.

Von diesem ist der philosophische oder kritische Posthumanismus zu scheiden, der sich ebenfalls in den vergangenen drei Jahrzehnten ausgebildet hat. Dort werden vor allem Ansätze der poststrukturalistischen Literaturwissenschaft aufgenommen und in eine Kritik des euro- und androzentrischen Humanismus überführt. Die Initialzündung für den kritischen Posthumanismus bildete das schon 1985 veröffentliche Manifest for Cyborgs der amerikanischen Wissenschaftshistorikerin Donna Haraway. In ihrer Aufsatzsammlung Simians, Cyborgs, and Women setzt sie sich mit »dem Menschen« im Zentrum des traditionellen Humanismus auseinander und nutzt u.a. das Bild des Cyborgs, um die natürlich-essentialistischen Determinierungen des »humanistischen « Menschen aufzubrechen. ${ }^{8}$ Auch der britische Künstler Robert Pepperell erklärte bereits in seinem "post-humanen Manifest « von 1995, dass nun eine Epoche beginnt, die den arroganten Glauben an die Überlegenheit und Einzigartigkeit des Menschen überwinden werde. Den »Post-Humanismus«

6 Vgl. Pfister 2014; Kalogridis 2018; Houellebecq 1998; Brown 2013, 2017.

7 Im Jahr 2014 widmeten sich die vom Bundesministerium für Bildung und Forschung geförderten Schulkinowochen dem Thema »Digitale Gesellschaft« und stellten ausführlich die Ideen Kurzweils und der Transhumanisten vor. Vgl. Krysmanski 2014, 123f.; Schätzing 2018.

8 Vgl. Haraway 1991; Haraway 1985; Graham 2002, 200-220. 
betrachtet er als Folge des Feminismus, der Bekämpfung der Sklaverei und des Eintretens für die Rechte der Tiere. Damit will er der Ausbeutung des Menschen und seiner Umwelt Grenzen setzen: »Post-Humanism is about how we live, how we conduct our exploitation of the environment, animals and each other ... The fact that all these movements exist suggests the gradual overturning of a human-centred world is well underway. $\ll^{9}$

Karen Barad, Cary Wolfe, Pramod K. Nayar, Neil Badmington, Rosi Braidotti u.a. verstehen den philosophischen Posthumanismus als »... Abschied von einigen Grundprämissen der Aufklärung ..., nämlich vom Fortschreiten der Menschheit durch einen selbstregulierenden, teleologisch angelegten Gebrauch der Vernunft und von der säkularen wissenschaftlichen Rationalität, die auf die Perfektionierung >des< Menschen angelegt ist. « ${ }^{10}$ Der kritische Posthumanismus zielt vor allem auf eine Reform von Sprache und Wissenschaft ab und setzt sich nur peripher mit der tatsächlichen Technologisierung moderner Gesellschaften und deren sozialen Folgen auseinander. ${ }^{11}$ Stefan Herbrechter versteht unter dem kritischen Posthumanismus »das kulturelle Unbehagen oder die Euphorie, die aus dem Gefühl entstehen, wenn man die Idee des >Postanthropozentrismus< ernst zu nehmen beginnt. Den Menschen nach dem >Ende des Menschen< zu denken, ohne in apokalyptische Mystik oder neue Formen von Spiritualität und Transzendenz zu verfallen ... « ${ }^{12}$ In ihrer sehr gelungenen, systematischen Darstellung des kritischen Posthumanismus betont Janina Loh, dass dessen Vertreterinnen und Vertreter (es sind interessanterweise meist Frauen) eher durch ihre Kritik am Humanismus als durch ihre Gegenentwürfe geeint werden. ${ }^{13}$ Aus diesen wenigen Bemerkungen lässt sich schon erspüren, dass der auf rationale Vervollkommnung angelegte, technologische Posthumanismus und der kritische Posthumanismus geradezu konträre Auffassungen

9 Pepperell 1995, 176. Vgl. a.a.O., 160-177.

10 Braidotti 2014, 42.

11 Beispielhaft sei hier auf das von Ira Livingston und Judith Halberstam herausgegebene Werk Posthuman Bodies verwiesen, dessen Beiträge die posthumane Gender-Problematik in Bezug auf postmoderne Literatur, Film und Popkultur diskutieren. Vgl. Halberstam \& Livingston 1995.

12 Herbrechter 2009, 7. Herbrechter hat 2009 den Begriff des »kritischen Posthumanismus« eingeführt und betreibt ein entsprechendes Netzwerk (http://criticalposthumanism.net).

13 Einführend zu empfehlen sind hier Herbrechter 2009, 6-94; Loh 2018, 80-109; Wolfe 2010, XI-XXXIV. 
zur Überwindung des Menschen bzw. des Humanismus vertreten. Für Loh liegt der entscheidende Unterschied in der »Passivierung « des Menschen im Transhumanismus. Dieser werde zum passiven Material der Umgestaltung degradiert:

Der eigentliche Akt des Enhancements verläuft, höchstens nachdem die fragliche Person ihr Einverständnis gegeben hat, als Prozess an dem Material des zu verbessernden Menschen ab. Bei Ungeborenen (reproduktives Enhancement) oder der genetischen Verbesserung zukünftiger Generationen kann dann auch von einer im Vorhinein gegebenen Einverständniserklärung natürlich keine Rede sein; in diesen Fällen wird die im Enhancement vorgenommene Passivierung noch deutlicher. ${ }^{14}$

Von der kulturwissenschaftlichen Forschung wurden die Utopien des technologischen Posthumanismus erstmals zu Beginn der 1990er Jahre wahrgenommen - zum einen im Kunstdiskurs und zum anderen in den Berichten meist amerikanischer Publizisten und Wissenschaftler über die weitreichenden Visionen der technophilen Subkultur des Silicon Valley. Hatte Jeffrey Deitch 1992/93 die Ausstellung Post Human über jegliche Formen der Körpertransformation - von Michael Jackson bis hin zu den Inszenierungen plastischer Chirurgie der französischen Künstlerin ORLAN - organisiert, so widmete sich 1996 der von Florian Rötzer herausgegebene Doppelband des Kunstforum International mit dem Titel Die Zukunft des Körpers in zahlreichen Beiträgen explizit den Inhalten der posthumanistischen Philosophie. ${ }^{15}$ Neben Künstlern, Philosophen, Kunst- und Medienwissenschaftlern kamen hier auch einzelne Vertreter des Posthumanismus wie Hans Moravec, Luc Steels und der Aktionskünstler Stelarc selbst zu Wort. ${ }^{16}$

In derselben Periode hatten in den Vereinigten Staaten einige Journalisten über die bunten Utopien von Kryonikern, Nanotechnologen und eben Vertretern der neuen Cyberkultur berichtet, die jeweils mit verschiedenen Elementen der posthumanistischen Philosophie korrespondierten. In Great Mambo Chicken and the Transhuman Condition. Science Slightly over the Edge konnte Ed Regis 1990 einen weitläufigen Überblick über das technophile und seiner Ansicht nach von menschlicher Hybris zeugende Streben amerikanischer Forscher nach Omnipotenz geben, bevor sich 1996 Mark Dery mit Escape Velocity und der in Kalifornien lebende Pub-

14 Loh 2018, 54. Vgl. a.a.O., 51-57.

15 Vgl. Deitch 1996, 112-115.

16 Vgl. Rötzer 1996a; Moravec 1996a; Steels 1996; Stelarc 1996. 
lizist Gundolf Freyermuth mit Cyberland ausführlicher mit den zukunftsweisenden Visionen der Computerkultur beschäftigten. ${ }^{17}$ Die jüngeren Publikationen Radical Evolution (2006) des Journalisten Joel Garreau und das Werk Transcendence. The Disinformation Encyclopedia of Transhumanism and the Singularity (2015) des Cyberaktivisten R. U. Sirius ergänzen diese deskriptiven Überblickswerke um aktuelle Einsichten. ${ }^{18}$ Der von mir sehr geschätzte, aufmerksame Analytiker postmoderner Populärkultur, David Lavery (1949-2016), erörterte in Late for the Sky unterdessen die mit dem Posthumanismus verknüpften Träume des Weltraumzeitalters. Denn der Eintritt in ein posthumanes Zeitalter wurde für viele Visionäre gleichsam zur Voraussetzung für den Aufbruch des irdischen Lebens in den Weltraum. ${ }^{19}$ Als 1998 in deutscher Sprache Freyermuths Cyberland und Gero von Randows posthumanistische Streitschrift Roboter. Unsere nächsten Verwandten erschienen, öffnete sich Ende der 1990er Jahre auch die deutsche Presse der Thematik des Posthumanismus. ${ }^{20}$

Abgesehen von kulturphilosophischen und kunstgeschichtlichen Abhandlungen, die sich nur allgemein mit der vom Posthumanismus vertretenen These der Überwindung des biologischen Körpers auseinandersetzten, bleibt die kulturwissenschaftliche Beschäftigung mit dem technologischen Posthumanismus bis heute überschaubar. ${ }^{21}$ Der Publizist Franz Rottensteiner hat hier Pionierarbeit geleistet, indem er 1997 in einem Aufsatz über die Unsterblichkeit im Computer auf den Ursprung des posthumanistischen Menschenbildes in der kybernetischen Theorie verwies. ${ }^{22}$ Eine ganze Reihe von Arbeiten befasste sich in der Folgezeit mit dem Verhältnis von Science-Fiction Literatur und allgemeinen Motiven des Posthumanismus, wobei die Monographie der amerikanischen Literaturwissenschaftlerin N. Katherine Hayles How we became posthuman, die die Wechselwirkung zwischen Kybernetik und Science-Fiction Literatur analysierte, sicherlich besondere Beachtung verdient. ${ }^{23}$ Mit seiner Anthologie zur Singularität hat der Philosoph Amnon H. Eden einen wichtigen Beitrag zur kulturwissenschaftlichen Erforschung des

17 Vgl. Regis 1990; Dery 1996; Freyermuth 1996, 1997, 1998.

18 Vgl. Garreau 2006; Sirius \& Cornell 2015.

19 Vgl. Lavery 1992.

20 Vgl. Freyermuth 1998; Randow 1998.

21 Vgl. Davis 1998; Kamper 1999; Kroker \& Kroker 1996; Kroker \& Weinstein 1997; Richard 2000; Schrage 2000; Schröter 2002; Spreen 2000.

22 Vgl. Rottensteiner 1997.

23 Vgl. Flessner 2000a, 2000b; Graham 2002, 38-108; Gräfrath 2000; Hayles 1999; Innerhofer 2000; Schenkel 2000. 
technologischen Posthumanismus geleistet. Auch die Technikphilosophen Reinhard Heil und Christopher Coenen vom Karlsruher Institut für Technikfolgenabschätzung und Systemanalyse (ITAS) haben eine Reihe äußerst lesenswerter Artikel zum Transhumanismus beigesteuert. $^{24}$

Der deutsche Medienwissenschaftler und Konzeptkünstler Thomas T. Tabbert reklamiert für sich die Begründung der Artifizialanthropologie und leitete bis 2013 das gleichnamige Institut in Hamburg, das offenbar nicht mehr besteht. Mit seinem Ansatz schlägt Tabbert eine systematische Klassifikation künstlicher Menschen vor, die sowohl technische als auch kulturell-historische Aspekte umfasst. Neben seiner detailreichen Aufarbeitung der Figuren künstlicher Menschen v.a. in der deutsch- und englischsprachigen Literatur, die in der kulturwissenschaftlichen Forschung bisher auf keinen großen Widerhall stieß, legte Tabbert im Rahmen des Artislife-Verlages weitere Einzelstudien vor. ${ }^{25}$

Die umfangreichste Auseinandersetzung mit dem Posthumanismus findet inzwischen innerhalb der Philosophie und Ethik statt, die sich mit vielen praktischen Fragen des human enhancement beschäftigen. Der Literaturwissenschaftler Raimar Zons formulierte in Die Zeit des Menschen. Zur Kritik des Posthumanismus (2001) eine philosophische Antwort auf die Ziele dieser Zukunftsutopie. Im Folgejahr erschien Our Posthuman Future: Consequences of the Biotechnology Revolution des amerikanischen Philosophen Francis Fukuyama, der sich kritisch mit den sozialen Folgen der Gentechnologie befasst. ${ }^{26}$

Das Gegenstück zu Zons und Fukuyama bildet das Werk Homo Deus des israelischen Philosophen Yuval Harari von 2015, in dem er punktuell Elemente der posthumanistischen Fortschrittsidee - wie die Entwicklung eines machtvollen, unsterblichen Übermenschen - adaptiert und für seine philosophischen Spekulationen über die Zukunft der Menschheit nutzt. Seiner Ansicht nach bricht in unserer Gegenwart das Anthropozän an, in der Hunger und Seuchen überwunden sind, der Mensch die Schöpfung in die eigene Hand nimmt und Glück, Unsterblichkeit und Göttlichkeit entwickeln wird. Nicht der islamische Funda-

${ }^{24}$ Vgl. Heil 2010a, 2010b; Heil \& Coenen 2014; Coenen 2010; sowie den Sammelband Die Debatte über »Human Enhancement" (Coenen et al. 2010).

25 Vgl. Tabbert 2004, 5, 54-84.

26 Auch die kleine Streitschrift Transhumanismus - Vision und Wirklichkeit (2015) von Otto Hansmann schließt sich den Warnungen Zons' und Fukuyamas an. Vgl. Zons 2001; Fukuyama 2002; Hansmann 2015. 
mentalismus, sondern Techno-Religionen würden die Welt erobern, weil sie Heil durch Algorithmen und Gene versprächen. Zwei Ausprägungen sind für Harari wahrscheinlich: zum einen die Datenreligion, die die Ablösung des Menschen durch künstliche Intelligenz propagiert und zum anderen der Techno-Humanismus, der aus dem Homo Sapiens einen Homo Deus erschaffen will (mittels technischen Upgrades des Gehirns und des Bewusstseins). ${ }^{27}$

Eine differenzierte Analyse zu vielen konkreten Einzelaspekten der Biound Neurotechnologien bietet der neuseeländische Philosoph Nicholas Agar, der seit zwei Jahrzehnten die ethische Debatte um transhumanistische Visionen begleitet. Zeichnen sich seine frühen Studien durch einen pragmatischen Fortschrittsoptimismus aus, so ist nicht zu verkennen, dass er heute den technozentrischen Heilsversprechen zunehmend skeptisch begegnet. Agar vermisst in den glanzvollen Szenarien der Transhumanisten vor allem eine moralische Diskussion, die uns eine Entscheidung über die Zukunft des Menschen erlauben würde. ${ }^{28}$

Der Technikphilosoph Bernhard Irrgang legte 2005 mit Posthumanes Menschsein eine sehr ausdifferenzierte und philosophische "Anthropologie des homo faber « vor, die sich mit allen Formen künstlichen Menschseins - von der Robotik bis zur Gentechnik - auseinandersetzte. Der Literaturwissenschaftler und Philosoph Stefan Herbrechter präsentierte 2009 eine erste Übersicht über die poststrukturalistischen Ansätze des kritischen Posthumanismus. Der Sammelband Anthropologie 2.0? vereint kleinere Studien zum Transhumanismus und den Biowissenschaften aus Sicht der philosophischen Anthropologie. Jüngst veröffentlichte die Technikphilosophin Janina Loh eine Junius-Einführung in den Post- und Transhumanismus, die insbesondere den kritischen Posthumanismus in Anlehnung an Hannah Arendt behandelt. ${ }^{29} 2014$ edierte das Jahrbuch Pädagogik gar eine Sonderausgabe zu Menschenverbesserung und Transhumanismus mit einer Reihe analytischer Aufsätze. ${ }^{30}$ Die Soziologen Fréderic Vandenberghe und Thomas Wagner sowie der Autor und Filmemacher Philipp von Becker setzten mit ihren aktuellen Publikationen einen neuen Akzent in der Debatte, indem sie die Verbindung

\footnotetext{
27 Vgl. Harari 2017, 475-495, 536.

28 Vgl. Agar 2010.

29 Vgl. Irrgang 2005; Herbrechter 2009; Baedtke \& Brandt \& Lessing 2015; Loh 2018.

30 Vgl. Sanders 2014; Krysmanski 2014; Meyer-Drawe 2014; Kluge 2014.
} 
zwischen transhumanistischen Utopien und kapitalistischen Marktordnungen aufzeigten. ${ }^{31}$

Wie bereits in der 1. Auflage von 2004 widmet sich die vorliegende Studie in zwei Schritten der posthumanistischen Idee von der Unsterblichkeit in der Virtualität. Der erste Teil Mensch und Medien beschreibt die technologischen Rahmenbedingungen, unter denen der Posthumanismus Ende des 20. Jahrhunderts in Erscheinung treten konnte. Diese Philosophie ist nicht nur das Ergebnis ideengeschichtlicher Entwicklungen, sondern setzt Medienerfahrungen voraus, die sich erst in den vergangenen Dekaden verdichtet haben. Diese Erfahrungen mit der Virtualität verstehe ich keineswegs deterministisch, aber sie haben zweifellos einen Einfluss auf unsere Deutung von Zeit, Raum und Körperlichkeit (Kapitel 2). Die utopische, zukunftsorientierte Interpretation von Computern und Medien, wie sie der technologische Posthumanismus vornimmt, ist Teil dieser kulturellen Deutungsvielfalt. Der Philosoph Günther Anders brachte mit seinem Konzept der "prometheischen Scham« zum ersten Mal das Grundmotiv des späteren Posthumanismus zum Ausdruck: nämlich das Gefühl der Unterlegenheit und Wertlosigkeit des Menschen im Angesicht seiner vollkommenen, technologischen Schöpfungen (Kapitel 3).

Der Posthumanismus entsteht nicht ex nihilo. Die Erfahrungen mit fortgeschrittener Medien- und Computertechnik werden in etablierte kulturelle Deutungsmuster eingebettet, die vor allem auf der europäischen und amerikanischen Fortschrittstheorie, auf Elementen christlicher Heilsgeschichte und einem kybernetischen Verständnis vom Menschen beruhen. Neben kurzen Einführungen in den Transhumanismus (Kapitel 4) und technologischen Posthumanismus (Kapitel 5) widmet sich der zweite Teil dieses Buches hauptsächlich der ideengeschichtlichen Aufarbeitung der zentralen Elemente des Posthumanismus (Kapitel 6). Diese umfassen zunächst das posthumanistische Menschenbild der Kybernetik und sodann mehrere Aspekte der Fortschrittsideologie bzw. Heilsgeschichte wie das Verständnis der Evolution, der Entropie, der Singularität, der Unsterblichkeit sowie der transzendenten Superintelligenz und des Punktes Omega als letztem Ziel aller kosmischen Entwicklungen. Es ist nicht meine Absicht, mit meiner Studie eine philosophische Bewertung des Post- und Transhumanismus oder gar bestimmter Computer- und Biotechnologien vorzulegen. Allerdings kann die ideenge-

31 Vgl. Becker 2015; Wagner 2015; Vandenberghe 2006. 
schichtliche Analyse durchaus als Basis der philosophischen Debatte dienen. Diese Art von hermeneutischer »Grundlagenforschung" schärft die Sinne für Motive, Deutungsmuster und Kontexte der posthumanistischen Ideen. 
Teil I

Mensch und Medien 


\section{Virtualität}

IMAGES ARE IMMORTAL. BODIES ARE EPHEMERAL ... THE BODY NOW PERFORMS BEST AS ITS IMAGE. ${ }^{1}$

Stelarc

Es scheint, als sei der Begriff der Virtualität ebenso vieldeutig wie der Begriff der Wirklichkeit selbst. Der Germanist und Historiker Bernd Flessner, der sich seit Beginn seiner wissenschaftlichen Arbeit mit den Aspekten der Virtualität auseinandersetzt, resümiert:

Während Cybersex-Anhänger ihren umgeschnallten Koitusmaschinen digital präfabrizierte Orgasmen entlocken, führen Chirurgen mit DataGloves virtuelle Skalpelle und moderieren Redakteure Infotainment-Magazine in virtuellen Fernsehstudios. Bietet die Unterhaltungsindustrie Erlebnishungrigen virtuelle Reisen in ferne Galaxien an, so kann der Kulturinteressierte per Mausklick die Bretter nach Belieben betreten, die die Welt bedeuten ... ${ }^{2}$

Im Unterschied zum Cyberspace verkörpert die virtuelle Realität nach Auffassung der meisten Autoren jede Art einer technisch generierten, künstlichen Wirklichkeit. ${ }^{3}$ Damit begründen neben den gängigen Assoziationen von Flugsimulatoren oder Computerspielen auch Fernsehen, Radio und MP4-Player virtuelle Realitäten. Es spielt dabei vorerst keine Rolle, ob diese virtuellen Realitäten nun Fiktion (z.B. die Märchenwelt eines Computerspieles) oder ein reales Ereignis (z.B. eine Fernsehübertragung) wiedergeben.

Zwar wird gerade im englischsprachigen Diskurs der Schriftsteller William Ford Gibson als Urheber der virtuellen Realität, des Cyberspace, angeführt, ${ }^{4}$ »doch orientiert man sich an der Genese des Prinzips, so stößt man unweigerlich auf die 1964 von Stanisław Lem publizierte Summa technologiae. $~^{5}$ Der polnische Denker hatte in seinem ersten philosophischen Werk nicht nur unter dem Begriff »Phantomatik« die Möglich-

\footnotetext{
Stelarc 1998, 123.

Flessner 1997a, 7f.

Vgl. Baudrillard 1994; Flusser 1992c; Flessner 1997; Lem 1981.

4 Der Begriff cyberspace erscheint 1984 zuerst in William Gibsons Roman Neuromancer. Vgl. Gibson 2000.

5 Flessner 1996, 7.
} 
keiten einer technisch generierten, künstlichen Wirklichkeit durchdacht, sondern er hat ebenfalls die Idee der Bildschirmbrille entworfen. ${ }^{6}$ Lem weist darauf hin, dass sich bei Begegnungen mit der virtuellen Realität stets eine Divergenz zwischen dem äußeren Handeln und dem inneren Erleben auftut. Egal, ob ein Zuschauer über den virtuellen Tod eines Filmhelden in Tränen ausbricht oder ob einem Flugschüler in einem Simulator vor Aufregung Schweiß auf die Stirn tritt, stets steht diesem inneren Erleben die äußere Handlungssituation im Kino oder im Flugsimulator gegenüber: "Seine Erlebnisse würden sich aufspalten in das, was er wahrnimmt, empfindet, und das, was er tut.« ${ }^{7}$ Der Ausgangspunkt zu einer Bestimmung der Virtualität wäre damit nicht die simplifizierende Antagonie zwischen Realität und einer virtuellen Scheinwelt, sondern hätte ihren Anfang in der menschlichen Wahrnehmung - in dem von Helmuth Plessner entwickelten Gedanken einer Anthropologie der Sinne:

Für den Erlebenden ist die Welt in den Sinnen gegeben, als Farbenwelt im Auge, als Tonwelt im Ohr, als Tastwelt an der Oberfläche seines Leibes und seiner Gliedmaßen. Was wir von der Welt wissen, haben wir von den Empfindungen unserer Sinne. ${ }^{8}$

Es war Marshall McLuhan, der die Frage nach den Wirkungen von Medien mit ihrem Verhältnis zu den Wahrnehmungsbedingungen, den Sinnen des Menschen, verknüpfte. Medien waren für ihn gleichsam Extensionen des menschlichen Körpers. Über Zeiten und Räume hinweg können Medien ein Erleben des Rezipienten durch die Nachahmung (Malerei) oder technische Simulation (Computer, Fernsehen) unserer entsprechenden, meist nur visuellen und akustischen Wahrnehmungen des Ereignisses hervorrufen, womit nachhaltig die Bedeutung der körperlichen Sinne verändert wird. ${ }^{9}$

Wenn eine Technik, sei es von innen oder außen, in eine Kultur eingeführt wird und wenn sie dem einen oder anderen unserer Sinne ein neues Gewicht oder einen neuen Auftrieb gibt, dann verschiebt sie das gegenseitige Verhältnis aller

6 Vgl. Lem 1981, 319-494; Lem bezeichnet die Cyberbrille als »Gegenauge«. Vgl. a.a.O., 323ff.; Flessner 1996, 8.

7 Vgl. Lem 1981, 325.

8 Plessner 1980, 25.

9 Vgl. McLuhan 1965, 308-337; McLuhan 1995, 29-56. 
unserer Sinne. Wir fühlen uns nicht mehr als dieselben, und auch unsere Augen, Ohren und anderen Sinne bleiben nicht mehr dieselben. ${ }^{10}$

In diesem Zusammenhang möchte ich einen Begriff einführen, der vielleicht die künftigen Differenzierungen etwas erleichtern wird. Als Pendant zu dem von Alfred Schütz entwickelten Begriff der »Lebenswelt« markiert die »Erlebniswelt« jenen Wirklichkeitsbereich, den der wache und »normale « Erwachsene in der Einstellung des gesunden Menschenverstandes nicht als schlicht gegeben vorfindet und der sich seiner unmittelbaren sinnlichen Wahrnehmung entzieht. Der Begriff der Erlebniswelt beschreibt alles, was zwar auf die eine oder andere Weise objektiv vorhanden ist, was jedoch nicht grundsätzlich gegeben und selbstverständlich »wirklich « ist. ${ }^{11}$

Die knappen Beispiele haben zweierlei gezeigt: Bei den virtuellen Realitäten handelt es sich weder um Wirklichkeiten, die der blühenden Phantasie eines Einzelnen entsprungen sind, noch um Realitäten, die in der alläglichen Lebenswelt unmittelbar zugänglich sind - die somit sichtbar, greifbar oder räumlich erfahrbar wären. Virtuelle Realität simuliert demnach Sinneserfahrungen, die außerhalb unserer momentanen oder unmittelbaren Wahrnehmung liegen. Virtuelle Realität bedeutet heutzutage die visuelle, akustische und seltener die haptische Simulation von realen oder fiktiven Erfahrungen und Erlebnissen. Diese Wahrnehmungen sind dann nicht mehr primär von der physischen Konstitution des Individuums abhängig, dafür aber von Technik. Bernd Flessner redet in diesem Zusammenhang treffend von den Weltprothesen und den Prothesenwelten. ${ }^{12}$

Obwohl in der bisherigen Literatur die virtuelle Realität nicht explizit gegen die Virtualität als solche abgegrenzt wird, können wir »virtuelle Realität« eher als einzelne und meist konkret gemeinte "Wirklichkeit« oder Erlebniswelt verstehen - beispielsweise die virtuelle Realität des Grand Canyon in der Simulation einer 3D-Brille. Virtualität dagegen kann man verstehen als Gesamtheit der verfügbaren virtuellen Realitäten in einem bestimmten Medium - beispielsweise die Virtualität des Internet oder des Fernsehens.

Nach diesen allgemeineren Vorüberlegungen ist es unumgänglich, im Einzelnen die für die menschliche Wahrnehmung entscheidenden As-

10 A.a.O., 29f.

11 Vgl. Schütz \& Luckmann 1994, 25-44.

12 Vgl. Flessner 1991. 
pekte der Virtualität genauer zu betrachten. Virtualität verändert unsere Deutung und Wahrnehmung von Zeit, Raum und Körper. Sie konstituiert dadurch einen spezifischen Deutungsrahmen gegenüber der Realität und dem Imaginären, der bestimmte Interpretationen von Medien begünstigt. Diese Aspekte sind Gegenstand der nachfolgenden Erörterungen.

\subsection{Virtualität und Zeit}

Medienrealität erweist sich als ein Konstrukt in der Zeit; Medienrealität entwirft sich strikt gegenwärtig. ${ }^{13}$

Götz Großklaus

Welche Bedeutung hat die Zeit der Virtualität, die Medienzeit, für die Genese des menschlichen Zeitbewusstseins? Noch genauer gilt unser Interesse den strukturellen Bedingungen für die Darstellung von Zeitlichkeit, im Sinne von Vergänglichkeit, des Menschen in den elektronischen Medien - allen voran im Fernsehen und in computergestützten Medienanwendungen. Schrieb schon der Berliner Soziologe Dietmar Kamper, dass alle Medien »verkappte Zeitmaschinen « seien, so haben sich für die nachfolgenden Überlegungen die drei aktuellen Entwürfe einer Zeittheorie von Armin Nassehi, Klaus Beck und Götz Großklaus als richtungsweisend erwiesen. Diese sozialen Theorien der Zeit, die schließlich auch das spezifische Verhältnis von Medien und Zeit problematisieren, bauen auf einer längeren philosophischen Entwicklung auf, die an dieser Stelle kurz skizziert werden soll. ${ }^{14}$

Es ist unbestreitbar, dass jede menschliche Handlung und damit auch jede Form der menschlichen Erfahrung unauflösbar an die Existenz der Zeit geknüpft ist, wobei Zeit allgemein als die physikalisch messbare, gleichmäßig verlaufende Zeit (Newton) verstanden wird. Der Lebensphilosoph Henri Bergson führt die für alle folgenden Zeittheorien gültige Differenzierung zwischen äußerer und innerer Zeit vor dem Hintergrund von quantitativ-naturwissenschaftlichen und qualitativ-hermeneutischen Erkenntnisweisen ein. Dauer (durée) ist in seinem Ver-

13 Großklaus 1997, 45.

14 Vgl. Kamper 1995; Nassehi 1993; Beck 1994; Großklaus 1997. 
ständnis nichts den Gegenständen Anhaftendes, sondern ein Produkt des menschlichen Bewusstseinsprozesses, der nicht auf den quantitativen Aspekt der verräumlichten Zeit reduziert werden kann. Bergsons Zeittheorie behauptet, dass die zeitliche Struktur des Bewusstseins nicht nur erfahrungsgenerierend, sondern auch durch die Erfahrung generiert ist. Für eine soziologische Theorie der Zeit war Bergsons Dichotomie zwischen einer qualitativen und einer quantitativen Zeit von Bedeutung, während seine Bewusstseinsphilosophie der Zeit, die eine freie und für das Erleben der Person konstitutive, natürliche und damit »wirkliche« Zeit konzeptualisiert, ansonsten nur innerhalb der Lebensphilosophie Relevanz hat. ${ }^{15}$

Helmuth Plessner hatte diese von Bergson postulierte durée der naturgegebenen Zeit in ihrem kontinuierlichen Werden und Entwerden im Erlebnisstrom als das vorchristliche Zeitbewusstsein interpretiert, das eine zyklische Einheit zwischen Vergehen, Wiederkehr und Fortleben behauptet. Erst das jüdisch-christliche Zeitbewusstsein verleihe mit seiner individuellen und kollektiven Eschatologie der Vorstellung der endgültigen Vergänglichkeit der Gegenwart eine Form. Die lineare Zeitauffassung des Christentums konstituiere durch die Idee der Auferstehung noch vor dem Glauben an die Unsterblichkeit im Jenseits die Gewissheit der Unwiederbringlichkeit des gegenwärtigen Lebens. Nach Plessners Verständnis bedeutet Zeit in ihrer christlichen Linearität damit vor allem Vergänglichkeit des Gegenwärtigen. ${ }^{16}$

In seiner Phänomenologie des inneren Zeitbewusstseins orientiert sich Edmund Husserl dann einzig an der Rolle des menschlichen Subjektes bei der Wahrnehmung und Erfahrung der zeitlichen Erscheinungsweisen:

So wie das wirkliche Ding, die wirkliche Welt kein phänomenologisches Datum ist, so ist es auch nicht die Weltzeit, die reale Zeit, die Zeit der Natur im Sinne der Naturwissenschaft und auch der Psychologie als Naturwissenschaft des Seelischen. ${ }^{17}$

Husserl lenkt die erkenntnistheoretische Frage nach der Möglichkeit und dem Wesen der Erfahrung von Zeit auf die sich im psychischen Subjekt manifestierenden Zeiterlebnisse. Die subjektive Zeit sei daher

15 Vgl. Nassehi 1993, 35-58; Schütz 1993, 62-64.

16 Vgl. Plessner 1952, 355-362.

17 Husserl 1928, 369. Vgl. a.a.O., 368-374. 
nicht als messbare Größe zu erfassen, die gleichmäßig verstreicht, sondern sie ist ein Phänomen, das unserem Bewusstsein entspringt und das selbst wieder eine vielschichtige Struktur besitzt. ${ }^{18}$

Alfred Schütz nun verbindet mit explizitem Verweis auf Edmund Husserl und Henri Bergson die Frage nach dem Sinn sozialen Handelns mit dem Zeitproblem und kommt zu dem Schluss, dass erst die Konstituierung von einer Einheit des Erlebnisstromes in der Zeit Sinn produziert. Der Sinn eines Erlebnisses besteht also in der Einordnung dieses Erlebnisses in den vorgegebenen Gesamtzusammenhang der Erfahrungen, der als Deutungsschema für neue Erfahrungen fungiert. Das Sinnproblem ist damit ein Zeitproblem, da das Zeitproblem als Möglichkeitsbedingung der Herstellung innerer Dauer eingeführt wird. Schütz unterscheidet wie Bergson zwischen einer inneren und einer äußeren Zeit. Er verweist auf die Verbindung dieses Zeitbegriffes zur soziologischen Handlungstheorie:

Die innere Zeit und die Weltzeit sind beide unumkehrbar. Aber Denken, ein Handeln ausschließlich in der inneren Zeit, ist in einer Weise widerrufbar, die für das Wirken nicht gilt. Wirken greift in die Außenwelt ein und findet in der Weltzeit statt: was geschehen ist, ist geschehen. ${ }^{19}$

Die in Handlungen sichtbar werdende zeitliche Struktur unserer intersubjektiven Erfahrungen, die »soziale Zeit«, wird für Schütz besonders durch den nahenden Tod evident:

Auch die Erwartung meines Todes, als einer endgültigen Abkehr (von der Lebenswelt), entspringt meiner Existenz in der intersubjektiven Welt. Andere werden älter, sterben, die Welt besteht weiter (und ich in ihr). Nun ist es aber eine meiner Grunderfahrungen, daß ich älter werde. Ich werde älter, also weiß ich, daß ich sterben werde, und ich weiß, daß die Welt fortdauern wird. ${ }^{20}$

Selbst wenn Alfred Schütz den Gegenstand einer soziologischen Zeittheorie nicht explizit definiert hat, bilden seine zeittheoretischen Ausführungen einen wichtigen Beitrag zu einer handlungstheoretischen und damit soziologischen Reflexion über die Zeit. ${ }^{21}$

Mit einer ähnlichen Intention hat der Soziologe Norbert Elias in seinen Reflexionen Über die Zeit offengelegt, dass sich das wachsende Bewusst-

18 Vgl. a.a.O., 368-374.

19 Schütz \& Luckmann 1990, 32.

20 Schütz \& Luckmann 1994, 75.

21 Vgl. a.a.O., 73-86; Schütz \& Luckmann 1990, 27-32; Nassehi 1993, 84-98. 
sein der Zeit, das Zeit-Erleben, die Zeit-Erfahrung und die jeweiligen Akte der Repräsentation von Zeit in den allgemeinen Zivilisationsprozess der Menschheit einbetten. ${ }^{22}$ Zeit ist für Elias eine gesellschaftliche Konstruktion, die dem Menschen »als gemeinsame Bezugsabfolge ... für die Bestimmung von Positionen im Nacheinander einer Vielfalt von oft recht verschiedenen Geschehnisabläufen « dient. ${ }^{23}$

Durchaus mit Bezug auf Schütz aber gleichsam in der Fortführung der Handlungstheorie von George Herbert Mead und der Prozesstheorie von Alfred North Whitehead entwickelt der Soziologe Armin Nassehi einen Begriff sozialer Zeit, der weder dem Mangel erliegen will, einseitig der so genannten objektiven oder kosmischen Zeit nachempfunden zu sein, noch auf die Zeitlichkeit intentionaler Ich-Subjekte zurückzugehen. Dieser operative Zeitbegriff setzt als temporale Letzteinheiten gegenwärtige Handlungen, die sich emergierend aneinander anschließen. So stellen in der streng handlungstheoretischen Orientierung Nassehis nicht Bewusstseinserlebnisse (Bergson, Husserl), sondern Handlungen die "primordialen Urgegenwarten « des entstehenden Zeitstroms dar. ${ }^{24}$ In diesem Zusammenhang scheint es von Bedeutung zu sein, dass der Soziologe Niklas Luhmann unsere Aufmerksamkeit auf die weithin vergessene Theorie der Zeit lenkt, die der französische Philosoph Luc de Clapiers Marquis de Vauvenargues im 18. Jahrhundert entwickelt hat und die in unserem medienorientierten Zusammenhang von fundamentaler Wichtigkeit ist. ${ }^{25}$ Im Kontrast zu aktuellen Handlungstheorien, die Handeln über den vom Handelnden gemeinten Sinn und die Motive des Subjektes verstehen wollen, findet sich bei Vauvenargues der Vorschlag, Handlung grundsätzlich als Gegenbewegung gegen den Zeitstrom zu definieren:

On ne peut condamner l'activité sans accuser l'ordre de la nature. Il est faux que ce soit notre inquiétude qui nous dérobe au présent; le présent nous échappe de lui-même, et s'anéantit malgré nous. Toutes nos pensées sont mortelles, nous ne les saurions retenir; et si notre âme n'était secourue par cette activité infatigable qui répare écoulements perpétuels de notre esprit, nous ne durerions qu'un instant ... Nous ne pouvons retenir le présent que par une action qui sort du

22 Vgl. Elias 1994, XXXIII.

23 Vgl. a.a.O., 79.

24 Vgl. Nassehi 1993, 126-130.

25 Vgl. Luhmann 1979. 
présent ... cette activité qui détruit le présent, le rappelle, le reproduit, et charme les maux de la vie. ${ }^{26}$

Wenn Vauvenargues Handeln als »Gegenbewegung zur Selbstannihilation der Zeit ${ }^{27}$ identifiziert, dann weist dies schon auf die Bedeutung hin, die insbesondere das so genannte »Medienhandeln« für die soziale Konstruktion von Zeitlichkeit und Vergänglichkeit des Menschen hat. So betont der Medienwissenschaftler Klaus Beck die Rolle des »Medienhandelns «ei der Vermittlung von sozialer Zeit als Element des gesellschaftlichen Wissensvorrates:

Mediennutzung und Rezeption kann handlungstheoretisch als Medienhandeln aufgefaßt werden, das - wie alle Handlungen - eine Zeit- und Sinnstruktur besitzt ... Medienhandeln kann als Teil des Vermittlungsprozesses sozialer Zeit verstanden werden, wobei sich das Medienhandeln der Kommunikatoren und der Nutzer/Rezipienten miteinander verschränkt, weil beide Akteure ihre Partner und deren Handlungen wechselseitig in stark typisierter und anonymisierter Form wahrnehmen. ${ }^{28}$

Menschliche Kommunikation, so Klaus Beck, ist damit untrennbar mit der Konstruktion von Bedeutung und der wechselseitigen Konstruktion des Anderen verknüpft, gleich ob dies nun direkte oder medial vermittelte Kommunikation ist. Aus der konstruktivistischen Perspektive Becks wird eine objektive, physikalische Zeit nicht durch unsere Wahrnehmung reproduziert und abgebildet, sondern konstruktiv erzeugt. Die Zeitmodi Gegenwart, Zukunft und Vergangenheit sowie die Zeitqualitäten Dauer, Wiederholung und Reihenfolge sind kognitive Konstruktionen, weil sie durch die Struktur unserer Wahrnehmung und nicht durch die Beschaffenheit der Außenwelt determiniert werden. ${ }^{29}$

Die Signifikanz der Zeiterfahrung in Bezug auf die Virtualität gewinnt ihre Evidenz, wenn man der Vermutung von Hans Michael Baumgartner Berücksichtigung schenkt, dass der Ursprung der menschlichen Zeiterfahrung eng mit der Erfahrung eines existentiellen Verlustes verbunden ist: »Die Erfahrung eines Verlustes reißt aus einer Kontinuität heraus, stellt das Verlorene gleichsam als nichtwiederbringlich ins Vergange-

\footnotetext{
26 Vauvenargues, Luc de Clapiers Marquis de: Oeuvres, hg. von D. L. Gilbert. Genf 1970, 94f. Zitiert nach Luhmann 1979, 65.

27 Ebd.; Vgl. a.a.O., 65f.

28 Beck 1994, 114.

29 Vgl. a.a.O., 43f., 71.
} 
ne ...« ${ }^{30}$ Medien jedoch negieren die lebensweltlichen Bedingungen der menschlichen Zeiterfahrung und eröffnen damit neben einer zyklischen und einer absolut fortschrittsorientierten Zeitdeutung noch einen dritten Ausweg aus dem Dilemma der unwiederbringlichen Gegenwart.

Schon die Erfindung der Schrift ermöglicht ein neues Zeiterleben von vergangenen Ereignissen, das über den Akt eines rein mentalen Erinnerungsprozesses hinausgeht. Jan Assmann und Peter Gendolla betrachten die Erfindung der Schrift explizit im Kontext einer Überwindung des Todes - als Versuch, das rühmliche Leben des Einzelnen festzuhalten und ihm damit Unsterblichkeit zu verleihen. ${ }^{31}$ Ein schriftliches Dokument wird zwar mit dem Wissen gelesen, dass es in der Vergangenheit produziert wurde, aber es kann so gelesen werden, als ob es sich um gegenwärtige Äußerungen handeln würde. Leser und Schreiber stellen eine mediale (in diesem Falle: literale) Gegenwart her, indem sie in Bezug auf Schreiber und Leser so tun, als ob beide tatsächlich gegenwärtig wären. ${ }^{32}$ So stellt Thomas Luckmann fest, dass in schriftlosen Gesellschaften die Grenzlinie zwischen den abstrakten, stark objektivierten Kategorien der Zeit und der alltäglichen in »reiner Intersubjektivität« jeweils aktuell ausgehandelten und gelebten, sozialen Zeit noch nicht so stark gezogen ist. In Schriftkulturen dagegen haben sich die abstrakten Zeitkategorien einerseits vollständig von der unmittelbaren Alltagserfahrung gelöst und sind andererseits zum (natur-)wissenschaftlichen bzw. zum religiösen Sonderwissen geworden. Diese abstrakten Zeitkategorien durchdringen nun über Alltagstechniken (Kalender, Uhr, Smartphone, Smartwatch) alle Ebenen des sozialen Lebens bis hin zur sozialen Interaktion und Kontrolle. ${ }^{33}$

Die Photographie nun als erstes technisches Bildmedium, das vergangene Ereignisse visuell simuliert, koppelt uns entscheidend vom natürlichen Zeittakt unserer neurophysiologischen Wahrnehmungswirklichkeit ab und das in zwei Richtungen: durch den zeitlich beschleunigten $\mathrm{Zu}$ griff und durch die zeitliche Arretierung des Zeitsplitters. Die Photographie impliziert eine neue Art von Gegenwärtigkeit, was die technische Erfassung des Augenblickes und seine Bewahrung betrifft: »angehalten, reicht die vergangene Gegenwart bis in unsere augenblicklich gelebte

\footnotetext{
30 Baumgartner 1994, 210.

31 Vgl. Assmann 1993, 64ff.; Gendolla 1992b, 22-26.

32 Vgl. Beck 1994, 171.

33 Vgl. a.a.O., 113; Luckmann 1986.
} 
Gegenwart hinein: als Bild. ${ }^{34}$ Die Photographie gewinnt ihre Relevanz daher durch die Simulation vergangener (visueller) Erfahrungskontexte:

Die Betrachtung der Fotografie hat den Vorteil, die Vergegenwärtigung des vergangenen intensiven Augenblicks beliebig wiederholen und in der Sekundär-Wahrnehmung des Abbildes die intensive Erfahrung einer möglicherweise belanglosen Primär-Erfahrung simulieren zu können. ${ }^{35}$

Die Photographie setzt auf diese Weise eine neue Kodierung von Raum und Zeit durch, denn das photographische Bild bestätigt uns über die materiale Spur, die das »Wirkliche« hinterlassen hat, die Authentizität dessen, was "wirklich gewesen ist«, und zeigt uns die technische Simulation von wirklichen Personen und Räumen »in vergangenem Zustand « als Erscheinung unmittelbarer Präsenz. ${ }^{36}$

Photos sind gemäß Roland Barthes daher »Emanationen des vergangenen Wirklichen«, was durch das Verschwinden einer historischen Zwischenzeit im Akt der vergegenwärtigten Betrachtung als Verdichtung, als Akkumulation von Zwischenzeit beschrieben werden kann. ${ }^{37}$ Niemand anderes hat so pointiert wie Barthes in seinem Werk La Chambre Claire den subtilen Zusammenhang von Tod und Photographie herausgestellt. ${ }^{38}$ Das Photo ermöglicht uns einen augenblicklichen Zugang zu dem, was gewesen ist, so dass die individuelle Geschichte des Bürgertums zum ersten Mal visuell erfassbar wurde: Das Familienalbum galt als privates »Miniatur-Museum ${ }^{39}$ Günther Anders sprach in diesem Zusammenhang bereits von der »Unsterblichkeit zu Lebzeiten«, die der Normalbürger durch Photos inszeniert, der Filmstar jedoch - wie die immortal Garbo - schon perfektioniert habe. ${ }^{40}$

Im Gegensatz zur Fixierung des Momentes in einem Photo geben der Film und im Anschluss daran das Fernsehen, Video und digitale Filmmedien eine Abfolge von einzelnen Bildern und damit den Ablauf eines Ereignisses wieder. Zeit wird in ihrer filmischen Darstellung (Dehnung, Verdichtung, Zeitsprünge) anschaulich. ${ }^{41}$ Erst der Film beginnt,

34 Großklaus 1997, 17.

35 A.a.O., 19.

36 Vgl. a.a.O., 33; Barthes 1985, 93.

37 Vgl. a.a.O., 95-99, $126 \mathrm{ff}$.

38 Vgl. a.a.O., $102 \mathrm{f}$.

39 Vgl. a.a.O., 11-70; Großklaus 1997, 18.

40 Vgl. Anders 1983, 57f.

41 Vgl. Großklaus 1997, 22-30. 
den Zeitverlauf zu simulieren: "So setzt mit dem Film das ein, was man die Mimesis der Zeit nennen könnte. Zeit und Zeitlichkeit werden - im Gegensatz zum fotografisch fixierten Augenblick - erstmalig ansichtig in ihrem Verlauf. ${ }^{42}$ Nichtsdestotrotz wird auch hier Zeitlichkeit konserviert - Thomas Macho verweist treffend auf die Konservierung unserer Toten im Medium Fernsehen. ${ }^{43}$ Götz Großklaus präzisiert dies im Hinblick auf Fernseh- und Computermedien: »Überführen wir ständig Vergangenheit in die Gegenwart der Medienrealität - und Zukunft in die Gegenwart der Computer-Simulation, so leben wir zunehmend im künstlichen Präsens abstandsloser Augenblicke ... « ${ }^{44}$

Computersimulationen und virtuelle Realitäten können als programmierte Modelle von Objekten, Ereignissen und Abläufen drei verschiedene Bezüge zur realen Zeit herstellen: Sie simulieren, was nicht mehr ist (aber »real« war), was noch nicht ist (aber »real« möglich ist) und was nicht ist (aber »simulatorisch « möglich ist). ${ }^{45}$ Großklaus ist der Auffassung, dass schließlich die Computer das Projekt der Moderne in ihrem Ziel der Vernichtung zeitlicher und räumlicher Distanzen verwirklichen werden. In aktuellen Szenarien vergegenwärtigen die Simulationen vergangene und zukünftige Ereignisabläufe und überführen sie in die Sichtbarkeit des Gegenwärtigen. ${ }^{46}$ Ähnlich resümiert Klaus Beck Anfang der 1990er Jahre noch vor der Verbreitung des Internet, dass sich unter dem Eindruck der neuen elektronischen Massenmedien kultursoziologische Indizien finden, die eine Tendenz zur Gegenwartsausdehnung und demzufolge zur »Vergangenheitsschrumpfung « erkennen lassen. ${ }^{47}$

Was aber bedeutet die mediale Konstruktion von Zeit für das menschliche Zeitbewusstsein? Historisieren wir unsere Gegenwart durch den geschichtlichen Bezug in realen und fiktiven Welten der Virtualität? In dieser Frage möchte ich mich dem Urteil von Götz Großklaus anschließen:

Ich denke, dass wir es in der Tendenz des gesamten Modernisierungsprozesses, zeitliche und räumliche Ferne zu vernichten, mit einer Erweiterung und Verdichtung des Gegenwarts- und Jetzt-Feldes zu tun haben. Alles tendiert dazu, Gegenwart zu sein, hier zu sein, jetzt zu geschehen ... Mit der Fotografie stehen wir am Anfang dieser medialen Entwicklung; die Fotografie duldet nicht das Entstehen

\footnotetext{
42 A.a.O., 22.

43 Vgl. Macho 1994, 433.

44 Großklaus 1997, 45.

45 Vgl. a.a.O., $53 f$.

46 Vgl. a.a.O., 53-57.

47 Vgl. Beck 1994, 344.
} 
von Vergangenheit, sie duldet nicht, daß Ferne entsteht, daß sich etwas entfernt. Das Bild fordert immer wieder zur Vergegenwärtigung des Vergangenen auf ... ${ }^{48}$

In der Virtualität wird vergangene, zukünftige und fiktive Zeit vergegenwärtigt und bildet damit eine aus der Realzeit heraustretende Eigenzeit unabhängig von ihren Inhalten. Man könnte hier daher zwischen einer Lebenszeit der sozialen Lebenswelt und einer durch die jeweiligen $\mathrm{Me}$ dienereignisse induzierten Erlebniszeit differenzieren, die desto intensiver wahrgenommen wird, je stärker man in seiner Aufmerksamkeit in das Medienhandeln eingebunden ist. Computerspiele oder das endlose Surfen in YouTube-Videos lassen schnell die soziale Zeit der Lebenswelt vergessen.

Für Vauvenargues war Handeln eine notwendige Bewegung gegen die Unwiederbringlichkeit der Gegenwart. Die Vergegenwärtigung von realem, real-möglichem und fiktivem, rationalem Handeln und erst recht vom Gefühlserleben in der Virtualität bietet eine weitere Chance, die Flüchtigkeit der Gegenwart in einem von Linearität geprägten Zeitverständnis zu verdrängen. Für den Medienwissenschaftler Götz Großklaus spiegeln sich in den spezifischen Eigenschaften der virtuellen MedienZeit die Auflösungstendenzen des abendländischen linearen Zeitverständnisses wider. ${ }^{49}$

Im Hinblick auf die Sterblichkeit des Menschen ermöglicht das mediale Zeiterleben die Verdrängung der subjektiv empfundenen Zeitlichkeit, der Endlichkeit des Menschen. Wirklich »sein« bedeutet dann, jederzeit gegenwärtig sein zu können. In diesem Kontext müssen wir Günther Anders Idee von der Verleugnung der »Einmaligkeit« des Subjektes auch im Sinne von Vauvenargues als zeitliche Einmaligkeit lesen: »Sie [die mediale Reproduktion: O.K.] ist eine im größten Stile von ihm durchgeführte Gegenmaßnahme gegen sein >mich gibt's nur einmal.« ${ }^{50}$ »Realität wird durch Reproduktion produziert; erst im Plural, erst als Serie, ist >Sein < - und in der Kehrform: Einmal ist keinmal; das nur Einmalige >ist $<$ nicht; der Singular gehört noch zum Nichtsein. ${ }^{51}$

Die elektronischen Medien, die den wirklichen Menschen nicht mehr mimetisch nachahmen, sondern gemäß unserer audio-visuellen Wahr-

48 Großklaus 1997, 21. Ähnliche Gedanken finden wir bei Günther Anders, der Photographien ganz im Sinne von Vauvenargues Zeittheorie deutet. Vgl. Anders 1983, $179 \mathrm{ff}$.

49 Vgl. Großklaus 1997, 40-42; McLuhan 1965, 334 f.

50 Anders 1983, 57.

51 A.a.O., 180. 
nehmung technisch simulieren, vermitteln unserem gesellschaftlichen Wissensvorrat auf diese Weise ein Bild vom Menschen, der seiner zeitlichen Bedingtheit - seiner Zeitlichkeit - enthoben ist. Götz Großklaus hat diese Entwicklung treffend charakterisiert: »Die Geschichte der Medien ist eine Geschichte wachsender symbolischer Verfügung über nicht-anwesende ferne Zeiten und ferne Räume. ${ }^{52}$ Das impliziert jedoch gleichermaßen die symbolische Verfügung über nicht anwesende Menschen aus fernen Zeiten und Räumen. Den nicht anwesenden Räumen ist das nächste Kapitel gewidmet.

\subsection{Virtualität und Raum}

Es machte mir wieder den ungemeinsten Eindruck, wie sie den Radioapparat anstellten und von London nach Rom, von Rom nach Moskau usw. übersprangen. Zeit- und Raumbegriff sind vernichtet. Man muß zum Mystiker werden. ${ }^{53}$

Victor Klemperer

Um das Phänomen der Virtualität zu verstehen, ist es unumgänglich, auch seine räumlichen Aspekte zu thematisieren. Für den Menschen existiert der Raum nicht an sich als ein mathematisch-euklidisches, ungegenständlich Abstraktes, sondern als eine soziale Erfahrung von Grenzen und Bewegungen unseres Körpers im Raum - als ein erlebter Raum, den wir als homo viator im eigentlichen Wortsinn erfahren. »Wir sprechen «, so der Philosoph Otto Friedrich Bollnow, »um diese Verhältnisse begrifflich zu fixieren, von einem erlebten Raum und meinen damit den Raum, wie er sich dem konkreten menschlichen Leben erschließt. «" Die Wahrnehmung vom eigenen Körper im Raum ist dabei konstituierend sowohl für die motorische Steuerung der Körperteile wie auch in einem höheren Sinne für menschliche Identität:

Es handelt sich ... um das Verhältnis, das zwischen dem Menschen und seinem Raum besteht, und darin also zugleich um die Struktur des menschlichen Da-

52 Großklaus 1997, 33.

53 Klemperer 1997, 228.

54 Bollnow 1997, 18. 
seins selber, sofern dieses durch sein Verhältnis zum Raum bestimmt ist. Wir sprechen in diesem Sinn von der Räumlichkeit des menschlichen Daseins. ${ }^{55}$

Raum wird vom Menschen visuell, akustisch, haptisch und auch olfaktorisch wahrgenommen. Nähe und Ferne werden durch komplexe rezeptive Mechanismen und aus dem Schatz bisheriger Erfahrungswerte konstruiert. Die neuen Bewegungstechniken seit dem 19. Jahrhundert haben nun eine fundamentale Veränderung unserer Raumwahrnehmung bewirkt, denn der Erschließung des Raumes durch Straßen und Wege und den mittelalterlichen Fortbewegungsmöglichkeiten per Pferd oder Kutsche folgte die zunehmend schneller werdende Eisenbahn. Sie hatte als Mittel der »Raumschrumpfung" schon bei Heinrich Heine einen besonderen Eindruck hinterlassen. ${ }^{56}$ Götz Großklaus weist darauf hin, dass die Erfindung dieser Bewegungstechniken mit der Entdeckung neuer, technischer Medien zusammenfällt:

Historisch gleichzeitig seit den 30er und 40er Jahren des 19. Jahrhunderts bewirken die neuen Bewegungsmaschinen (Eisenbahn, Dampfschiff) und die neuen Bild- und Kommunikationsmedien (Fotografie, Telegrafie, Phonografie) die Tilgung räumlicher Distanzen und Zwischenräume und die Auslöschung zeitlich-geschichtlicher Intervalle - mit dem doppelten Effekt der zeiträumlichen Zusammenziehung zu prägnanten Punkten - oder der Erweiterung oder Vernetzung dieser Punkte zu Zeit- und Raumfeldern. Die alten Intervalle sind in diesen Feldern oder Netzen zu kurzen Schaltwegen, superschnellen Signallaufzeiten, instantanen Zugriff- und Abrufvorgängen, zu live- und real-time-Prozessen in der Tat geschrumpft, bei gleichzeitiger Expansion der Netze. ${ }^{57}$

Es ist offensichtlich, dass spätestens mit der Erfindung der Telegraphie und der Eisenbahn die traditionellen Abgrenzungen zwischen verschiedenen Kulturräumen, zwischen Nah- und Fernraum und zwischen öffentlichem und privatem Raum »in Bewegung « geraten sind. ${ }^{58}$ Noch gravierender als die Beschleunigung der menschlichen Bewegung durch den Raum sollte sich die Erfindung der neuen Übertragungs- und Reproduktionsmedien auswirken, denn erstens bedeutete Raumerfahrung immer weniger, dass ein Erleben von Raum an eine tatsächliche Bewegung im Raum gebunden ist und zweitens wurde es möglich, Bilder

55 A.a.O., 22. Vgl. a.a.O., 13-25.

56 Vgl. a.a.O., 96-101; Großklaus 1997, 72-79; Schivelbusch 1977, 37.

57 Großklaus 1997, 37.

58 Vgl. a.a.O., 72-102. Natürlich müssten hier auch das Teleskop und das Bild im allgemeinen hinzugefügt werden, die räumliche Distanzen negieren. 
von Räumen - also die visuelle Erfassung von Räumlichkeit - technisch zu simulieren. Zwar gibt es seit der Antike in der Malerei und Mosaikkunst teils überraschend gelungene Darstellungen des dreidimensionalen Raumes, ${ }^{59}$ jedoch begann erst in der Mitte des 19. Jahrhunderts mit der Erfindung der Daguerreotypie (1837) die technische Reproduktion von visuell erfasster Räumlichkeit. Die Natur wird nicht mehr durch die subjektive Begabung eines Künstlers in einem mimetischen Prozess "nachgeahmt", sondern ihre physikalischen Eigenschaften werden objektiv, durch das Objektiv einer Kamera, technisch erfasst und mit Hilfe geeigneter Speicher- und Reproduktionsmedien (Photopapier, Film, Magnetbänder, Fernseh- und Computerbildschirme, Smartphone-Displays) mit immer besseren Methoden sichtbar gemacht. Räumlichkeit wird nun technisch simuliert. Im selben Jahr, als Louis Daguerre seine große Entdeckung machte, entwickelte Samuel Morse den Schreibtelegraphen und machte damit erstmals die Übermittlung von Nachrichten unabhängig von einem materiellen Träger und einem Überbringer, der sich tatsächlich durch den Raum bewegen musste. Informationen wurden durch Engergiedifferenzen übermittelt. So lösten sich Stück für Stück die Bilder der Räume vom tatsächlichen Raum. ${ }^{60}$

Mit dem Auftreten der neueren und neuesten Medien: mit Fotografie, Film, Radio und Fernsehen kann jedes reale Geschehen, wo immer und wann immer es sich ereignet, wie entfernt auch immer der Ort des Geschehens sein mag, zum Nahereignis werden über sein mediales Abbild. ${ }^{61}$

Durch Film und später durch Fernsehen, das Internet und Mobiltelefonie konnten nicht nur einzelne Bilder, sondern ganze Bildsequenzen menschliches Handeln und Bewegung im Raum simulieren. Ohne eine einzige Bewegung des Beobachters im realen Raum erforderlich zu machen, bringt das Fernsehen oder der Live-Stream im Internet die Welt zu uns ins Wohn- oder Arbeitszimmer, überwindet unbeschränkt räumliche Distanzen und schafft in der Anwesenheit von Abwesenden die Ablösung der Präsenz durch die Präsentation. Das Prinzip hatte schon Günther Anders erkannt. ${ }^{62}$ Im Fernseh- und Filmbild selbst kann über

59 Das Panoptikum bildet vermutlich den Höhepunkt dieser vortechnischen Darstellung von Räumlichkeit.

60 Vgl. Hammel 1994, 62-70.

61 Großklaus 1997, 97.

62 Vgl. Anders 1983, 114-134. 
die Veränderung der Kameradistanz die räumliche Distanz zwischen $\mathrm{Zu}$ schauer und Objekt künstlich noch weiter verringert werden.

Analog zu den vorhergehenden Differenzierungen ist die Wahrnehmung einer virtuellen Realität gekennzeichnet durch das Nebeneinander eines Lebensraumes in der Lebenswelt und eines Erlebnisraumes im virtuellen Medium. Beide Räume können wirklich existieren - wie beispielsweise bei der Übertragung eines Fußballspieles. Aber für den exemplarischen Live-Stream-Zuschauer ist nur der Raum, in dem er sich befindet, real erfahrbar, während der Erlebnisraum medial vermittelt wird und die zeitliche und örtliche Anwesenheit des Beobachters dort nicht erforderlich ist. Die visuelle und akustische Wahrnehmung des Ereignisses im Erlebnisraum ist damit virtuell verfügbar, ohne dass hier etwa soziale Voraussetzungen zur Teilnahme an einem Ereignis verlangt würden.

In größeren kulturgeschichtlichen Zusammenhängen betrachtet der britische Soziologe Anthony Giddens den Übergang von der traditionalen Welt in die Moderne als eine zunehmende Trennung von Raum und Ort, die er als »Entbettung « (disembedment) bezeichnet. Gewohnheiten und Praktiken werden somit von ihren ortsgebundenen Zwängen befreit und vielfältige Möglichkeiten des Wandels ergeben sich mit dem Beginn der Moderne: »Unter Entbettung verstehe ich das >Herausheben< sozialer Beziehungen aus ortsgebundenen Interaktionszusammenhängen und ihre unbegrenzte Raum-Zeit-Spannen übergreifende Umstrukturierung. « ${ }^{63}$ Unter diesem Gesichtspunkt sind Medienerfahrungen generell von den Anwesenheitszusammenhängen ihres originären sozialen Raumes unabhängig, bloß die Befähigung zur Wahrnehmung räumlicher und akustischer Signale ermöglicht die telepräsente Teilnahme an fernen und sozial vielleicht nicht zugänglichen Ereignissen. Man denke beispielsweise an Fernsehübertragungen von Bundestagssitzungen. Kommunikation und Interaktion - und in ihrer Folge Sozialität und Identität - lösen sich von ihren ortsgebundenen, sozialen Zwängen, aber auch von einer identitätsstiftenden räumlichen Gemeinschaft. ${ }^{64}$

63 Giddens 1997, 33. Vgl. a.a.O., 28-43.

64 Vgl. a.a.O., 30-33. Auch Giddens problematisiert in diesem Zusammenhang die Frage, ob die moderne Auflösung ortsgebundener Identitäten als Gegenreaktion in manchen Staaten ein Aufkommen des lokalen Nationalismus begünstigt, wie das bekanntlich Samuel Huntington nahelegte. Vgl. a.a.O., 86. 


\subsection{Virtualität und Körperlichkeit}

The central event of the $20^{\text {th }}$ Century is the overthrow of matter. In technology, economics, and the politics of nations, wealth - in the form of physical resources - has been losing value and significance. The powers of mind are everywhere ascendant over the brute force of things. ${ }^{65}$

The Magna Charta of the Cyberspace

Die vorhergehenden Überlegungen zur veränderten Zeit- und Raumerfahrung durch die elektronischen Medien werden deutlich gemacht haben, dass sich hieraus auch Veränderungen für die Wahrnehmung der Materialität und Körperlichkeit von Um- und Mitwelt ergeben. Schon kurz nach der Entdeckung der Telegraphie und Photographie gelangte der amerikanische Arzt und Lyriker Oliver Wendell Holmes zu einer geradezu postmodernen Erkenntnis:

Form is henceforth divorced from matter. In fact, matter as a visible object is of no great use any longer, except as the mould on which form is shaped. Give us a few negatives of a thing worth seeing, taken from different points of view, and that is all we want of it. Pull it down or burn it up, if you please. We must, perhaps, sacrifice some luxury in the loss of color; but form and light and shade are the great things, and even color can be added, and perhaps by and by may be got direct from Nature ... There is only one Coliseum or Pantheon; but how many millions of potential negatives have they shed, representatives of billions of pictures, since they were erected! Matter in large masses must always be fixed and dear; form is cheap and transportable. We have got the fruit of creation now, and need not trouble ourselves with the core. ${ }^{66}$

Relevanz gewinnen diese Gedanken Holmes, wenn Erfahrungen von Materialität und konkreter Körperlichkeit in zunehmendem Maße durch elektronische Medien geprägt werden, denn der menschliche Körper selbst ist Produkt unserer Wahrnehmungen und gleichzeitig unser Erkenntnisapparat. Der Psychologe Jean Piaget betonte den körperlichen Anteil bei der Herausbildung der Intelligenz und der Philosoph Arnold Gehlen bestimmte die Höherentwicklung des Menschen als Folge seiner körperlichen Bedingtheiten, während Wilhelm Dilthey unseren Glauben an die Realität der Außenwelt in der Erfahrung des auch ma-

65 Dyson et al. 1994, 1.

66 Holmes 1859, 747. 
teriellen Widerstandes der Welt begründet sah. Menschliche Erkenntnis und Identität sind in einem beträchtlichen Ausmaß von der materiellen Form des menschlichen Körpers, seinen Wahrnehmungsmöglichkeiten und auch unserer Wahrnehmung des Körpers abhängig. Die Wahrnehmung von Körpern im Fernsehen, Internet und in Computerspielen unterscheidet sich laut Karlheinz Lüdeking jedoch erheblich von der des lebensweltlichen Alltages. ${ }^{67}$

Die durch unseren Körper, durch dessen sensorische und motorische Beschränkungen verursachte Verankerung im räumlichen Hier wird immer weiter aufgeweicht. Auch ohne Roboter-Fernlinge oder virtuelle Puppen überschreiten wir unsere sensorischen und motorischen Beschränkungen durch jede neue Form der Technologie, durch jedes Mensch-Maschine-System. Unsere Erfahrung, die stets von unserem Körper abhängig ist, verändert sich durch diese Erweiterungen oder Prothesen. Unser Körper hört nicht mehr an unserer Haut auf, wenn wir ein Fahrzeug oder einen Roboter in der Ferne steuern ... Unser Körperbild wird dadurch bestimmt, was wir durch ihn erleben und empfinden, was wir mit ihm machen können (und müssen). ${ }^{68}$

Der französische Philosoph Jean-François Lyotard hat diese durch die Verbreitung von elektronischen Medien evozierte Immaterialität der Wirklichkeit als eine der wesentlichen Eigenarten der Postmoderne identifiziert. Schon 1985 schreibt er in Immaterialität und Postmoderne:

Forschung und Entwicklung in Technowissenschaft, Kunst und Technik, ja selbst die Politik erwecken heute den Eindruck, daß die Wirklichkeit, was auch immer sie sei, ständig ungreifbarer wird, daß sie unmittelbar niemals beherrscht werden kann ... Zudem greifen wir fast nie in die Wirklichkeit ein, die wir umgestalten wollen. Die Arbeit im Beruf oder Haushalt benötigt immer mehr Apparate (matériels). Der manuelle, visuelle und geruchsmäßige Kontakt mit dem Material geht verloren ... Es ist, als hätte man zwischen uns und den Dingen einen Filter gesetzt, einen Schirm von Zahlen ... Mit den Codier- und Dekodiersystemen erfahren wir, daß es Realitäten gibt, die auf eine neue Weise ungreifbar sind ... Die Wirklichkeit besteht aus Elementen, die von Strukturgesetzen (Matrizes) in nicht mehr menschlichen Raum- und Zeitmaßstäben organisiert werden. ${ }^{69}$

Im Vergleich zur heutigen Situation beschreibt Lyotard allerdings nur eine Übergangsphase. Während die Philosophie in den 1980er Jahren zur Einsicht gelangte, dass die Wahrnehmung und Veränderung in der

67 Vgl. Hayles 1999, 220f.; Gebauer 1984, $234 f$.

68 Lüdeking 1996, 66.

69 Lyotard et al. 1985, 10f. 
Wirklichkeit immer stärker durch immaterielle Medien wie Fernsehen oder Computer geprägt sind, die sich zwischen Mensch und Wirklichkeit schalten, stehen wir seit den 1990ern am Beginn einer Entwicklung, in der Menschen einen immer größeren Teil ihrer Erfahrungen nicht mehr durch, sondern mit immateriellen, sprich virtuellen Wirklichkeiten selber machen. Handlungen zielen heute in größerem Maße als je zuvor darauf ab, Veränderungen in virtuellen Erlebnisräumen vorzunehmen - und nicht mehr nur Veränderungen in der materiellen Welt durch immaterielle Medien.

Der Medienphilosoph Vilém Flusser versteht daher die Postmoderne in Verbindung mit einem neuen Verständnis der Wirklichkeit, die die Begegnung des Menschen mit der Virtualität provozieren wird: »Irgend etwas ist dabei, sich ziemlich grundsätzlich zu ändern. Wenn wir dies etwas eleganter ausdrücken wollten, könnten wir sagen, dass wir in einem Paradigmenwechsel stehen. ${ }^{70}$

Konstituierend für dieses neue Verständnis von Wirklichkeit ist vor allem die durch Medien veränderte Wahrnehmung des menschlichen Körpers, wie Lüdeking bereits betonte. Das Körpererleben bezieht sich hierbei in den meisten Fällen jedoch weniger auf das Erleben des eigenen Körpers als vielmehr auf die Wahrnehmung und bei Computerspielen auf die interaktive Steuerung von anderen realen bzw. fiktiven Körpern. Auch wenn zur Zeit haptische und bewegungstolerante Simulationen entwickelt werden und andererseits schon mit der Erfindung des Phonographen die heute allgegenwärtige, akustische Virtualisierung einsetzte, sind die heute bedeutendsten Formen von Virtualität im Fernsehen, Computer und Internet bilddominiert. ${ }^{71}$ Einhergehend mit der geistigen Aufklärung Europas übernahm das Auge in der Nachahmung des barocken Gottesauges immer mehr Kontroll- und Selbstkontrollfunktionen und führte damit zu einer gravierenden Reduktion der Weltwahrnehmung auf den Sehsinn. Die Welt als Ganzheit der verschiedenen Sinneserfahrungen zu erleben, weicht einer visuell dominierten Welt- und Selbstwahrnehmung, die das Empfinden der eigenen Gegenwärtigkeit in der sinnlichen Reaktion des Körpers auf die Welt der Objekte verändert. Die Kontrolle des Auges versagt aber gerade in der Differenzierung zwischen Realität und Phantasmen. ${ }^{72}$ Auch Vilém Flusser

\footnotetext{
70 Flusser 1992b, 15.

71 Vgl. McLuhan 1965, 275-283.

72 Vgl. Wulf 1984, $22 f$.
} 
betont an dieser Stelle die Wichtigkeit der Herrschaft der Bilder in der europäischen Kulturgeschichte:

Was uns nicht mehr frei steht, ist, die Dominanz der technischen Bilder über die künftige Gesellschaft in Frage zu stellen. Jenes Universum, das seit einigen Jahrzehnten daran ist, in Form von Fotos, Filmen, Videos, Fernsehschirmen und Computerterminals eine Funktion zu übernehmen, welche bislang von Texten eingenommen wurde, die Funktion nämlich, die für die Gesellschaft und den Einzelnen lebenswichtigen Informationen zu tragen. Es geht um eine Kulturrevolution, deren Reichweite und Konsequenzen wir erst zu ahnen beginnen. ${ }^{73}$

Ein technisches Bild simuliert eine visuell wahrnehmbare Realität. Die künstlerischen Bilder sind nach Flusser subjektive Abstraktionen von Phänomenen, die technischen Bilder sind Konkretisierungen von objektiven Abstraktionen. ${ }^{74}$ Das technische Bild entspricht damit der Realität, wie wir sie mit unseren Augen wahrnehmen könnten und bedarf keiner Imagination des Subjekts, das in einem Denkprozess aus abstrakten, sprachlichen Symbolen wieder sinnlich erfahrbare Bilder konstruieren müsste. Technische Bilder lassen - im Gegensatz zu künstlerischen Bildern - keine Leerstellen als Raum für die subjektive Imagination. Das technisch erzeugte Bild vom Körper vollzieht nun die Dekontextualisierung der bloß visuellen Wahrnehmung des Körpers ohne seinen materiellen Kontext. Im Zuge von Bildtelegraphie und der nachfolgenden Digitalisierung visueller Darstellungen im 20. Jahrhundert wird das Bild des Körpers zur Summe mathematisch definierter Zustände. Das Bild des Körpers wird immateriell, es wird Information.

Durch diese veränderte Wahrnehmung des Körpers entwirft sein technisches Bild jedoch ein neues Verhältnis zu Zeit und Raum, weil diese Bilder räumlich und zeitlich beliebig reproduzierbar sind: »Die Bilder verselbstständigen sich und schaffen senträumlichte Räume und sentzeitlichte Zeitlichkeit<, in die sie den Betrachter hineinziehen. ${ }^{75}$

Der Soziologe Dietmar Kamper begreift diese Entwicklung technischer Bilder und die damit korrelierende Entstehung eines neuen Körperverständnisses in einem größeren kulturgeschichtlichen Zusammenhang. Die Geschichte der Zivilisation versteht er einerseits als planvolle An-

73 Flusser 1992c, 8f. Vgl. zum Begriff des technischen Bildes a.a.O., 20-27.

74 Mit technischem Bild ist im Sinne Flussers das Foto, das Bild im Fernseher und Computer gemeint. Nicht gemeint ist das künstlerische Bild (auch nicht, wenn es am PC entsteht). Vgl. Flusser 1992c, 7ff.; Flusser 1997, 75ff.

75 Wulf 1984, 42. 
eignung der Natur - als (geistige) Verallgemeinerung des (körperlichen) Besonderen - andererseits als unaufhaltsame Abstraktion und Formalisierung jeglichen Inhaltes: ein geistlos Körperliches werde unter ein körperlos Geistiges subsumiert. ${ }^{76}$ Posthumanistische wie auch gentechnische Körperutopien erscheinen damit als Fortsetzung des modernen Körperdiskurses, wie ihn vor allem Norbert Elias und Michel Foucault in verschiedenen Aspekten nachgezeichnet haben. Die zunehmende Kontrolle des Körpers ist demnach ein Kennzeichen gerade der bürgerlichen Gesellschaft des 19. Jahrhunderts, wobei in der Gegenwart die älteren, moralischen Perspektiven weitgehend den Kriterien der Effizienz und der Erlebnisrationalität gewichen sind. ${ }^{77}$ Die Entfernung des menschlichen Körpers mittels Sprachabstraktionen, durch Unterdrückung, Gesetz und Verbot oder neuerdings durch normative Bildabstraktion dient der Orientierung in der Welt. Körper werden hier von ihrer ursprünglichen Mannigfaltigkeit bis auf die Nulldimensionalität von der Präsenz zur Absenz - reduziert. ${ }^{78}$ Die Stufen dieser Abstraktion sind gekennzeichnet durch erstens die körperlose Sprache, zweitens das sprachlose Bild und schließlich durch das bilderlose Zeichen in seiner digitalen Reproduzierbarkeit. ${ }^{79}$

Technische Bilder sind nun augenscheinlich frei von materiellen Trägern, gleichzeitig aber mathematisch vollkommen determinier- und kontrollierbar. Im Prinzip werden damit Körperbilder ebenfalls technisch vollkommen determinierbar, kontrollierbar und beliebig manipulierbar. Die freie Wählbarkeit und Gestaltung von virtuellen Körpern, den Avataren, in Computerspielen und in bestimmten Kommunikations- und Spielanwendungen des Internet wecken die Hoffnung, im virtuellen Raum neue Entwürfe der eigenen, körperlichen Identität konstruieren zu können.

\footnotetext{
76 Vgl. Kamper 1976, 8.

77 Vgl. Elias 1997, 323-465; Foucault 1983; Schröter 2002, 88f.

78 Vgl. Kamper 1999, 7.

79 Vgl. a.a.O., $145 f$.
} 


\subsection{Virtualität, Realität und das Imaginäre}

Wir leben in einer Gesellschaft mit Kaffee ohne Koffein, Schokolade ohne Zucker und Virtualität als Realität ohne Realität. $^{80}$

Slavoj Žižek

Der mit den elektronischen Medien einhergehende Bedeutungswandel von Zeit und Raum kulminiert in der Auflösung des herkömmlichen Wirklichkeitsbegriffes. Die gegenseitige Durchdringung zwischen virtueller Erlebniswelt und realer Lebenswelt erschwert es, klar zwischen Virtualität und Realität zu differenzieren. Niemand hat die damit einhergehende Entleerung des traditionellen Begriffes von Wirklichkeit durch die neuen Medien hellsichtiger vorausgeahnt als Günther Anders in seinem 1956 publizierten Essay Die Welt als Phantom und Matrize:

So wie, wo das Leben als Traum gilt, Träume als Leben gelten, so wirkt nun, da jede Realität als Phantom auftritt, jedes Phantom real. Wo jedem wirklichen Vorgang durch dessen Übertragung etwas Scheinhaftes verliehen wird, muß das Scheingeschehen ... in der Übertragung seinen spezifisch ästhetischen Scheincharakter einbüßen. ${ }^{81}$

Stanisław Lem hatte für dieses Phänomen schon sehr früh den Begriff Realyse als Bezeichnung für den allgegenwärtigen Wirklichkeitsschwund eingeführt. ${ }^{82}$ Seit Günther Anders und Stanisław Lem wurde die Auflösung der Wirklichkeit unter dem Eindruck virtueller Welten beinahe inflationär oft heraufbeschworen. ${ }^{83}$ Das Resümee des Schriftstellers und Computerkünstlers Herbert W. Franke zielt in eine ähnliche Richtung, impliziert jedoch bereits eine nützliche Differenzierung:

Unabhängig von positiven oder negativen Erwartungen stößt uns diese Technik auf Fragen ganz anderer Art, beispielsweise die Unterscheidung zwischen Sein und Schein ... Bisher kannte man nur die virtuellen Räume unserer Vorstellungen, und die Aussagen, die zu diesen Problemen gemacht wurden, beruhen auf psychologischen Erfahrungen, die wir in unseren Wahrnehmungen einerseits

80 Funkhausgespräche (WDR5) mit Oskar Negt und Slavoj Žižek vom 20.09.2001.

81 Anders 1983, 143.

82 Vgl. Lem 1996c, 129. Dieser Roman, Der Futurologische Kongreß, stammt schon von 1974 !

83 Vgl. Lyotard 1987, 22; Kamper 1995; Flusser 1992c, 7f.; Baudrillard 1994, 9 f. 
und den Gedankenbildern andererseits machen. Doch jetzt wird uns in Form der virtuellen Räume eine dritte Möglichkeit geboten..${ }^{84}$

Franke spricht hier von unseren Wahrnehmungen der Welt, unseren Aussagen darüber und schließlich von der Virtualität. Auf diesem Grundgedanken aufbauend, möchte ich ein heuristisches Modell vorschlagen, das zwischen der Realität, der Virtualität und dem Imaginären unterscheidet. Es soll hier keine neue Ontologie entworfen werden das würde eine Menge weiterer philosophischer und psychologischer Fragen provozieren. Das Modell soll lediglich dazu dienen, über das Verhältnis zur Realität und dem Imaginären zu einer genaueren Bestimmung der Virtualität zu gelangen. In verschiedenen Annäherungen sollen diese drei Aspekte nun genauer charakterisiert werden - zugespitzt auf die Frage: Welche Bedeutung hat die Erfahrung von und mit Virtualität? Der Fokus dieser Frage orientiert sich an der Qualität der menschlich-sinnlichen Wahrnehmung. ${ }^{85}$

Unter Realität soll die mit menschlichen Sinnen unmittelbar erfahrbare Welt verstanden werden. ${ }^{86}$ Dass unter der Berücksichtigung konstruktivistischer oder soziolinguistischer Theorien gar keine objektive Welt unabhängig von der Leistung eines Beobachters bestehen kann, ist für dieses heuristische Modell nicht relevant. Wenn alle Welt- und Medienerfahrung als sprachliche, psychische oder medientechnische Konstruktion betrachtet wird, dann gibt es zwischen dem Imaginären, der Realität und der Virtualität weder eine anwendbare Differenzierungsmöglichkeit noch einen daraus hervorgehenden Erkenntnisgewinn. Diese objektive Realität wird von unseren visuellen, akustischen, olfaktorischen, haptischen (in jedem Fall aber) körperlich gegebenen Sinnen wahrgenommen. Realität setzt also in diesem Zusammenhang einen Betrachter voraus. Realität steht in einem zeitlichen, räumlichen und materiellen Kontext der Lebenswelt. Beim Beispiel einer Photographie besteht die lebensweltliche Realität dieses Bildes aus dem Photopapier,

84 Franke 1997, 366.

85 Die Wahrnehmung der Welt durch Tiere oder physikalische Messungen ist für die Frage nach der Virtualität zunächst unerheblich, da Virtualität Teil von Medienerfahrungen ist, die speziell für die sinnlichen Wahrnehmungsfähigkeiten des Menschen produziert werden.

86 Die Alternative, unter Realität alles zu verstehen, was unabhängig von menschlicher Wahrnehmung verstanden wird, würde nur suggerieren, dass es Aussagen über die Welt geben würde, die nach objektiven Kriterien getroffen werden könnten. Das ist aber nie der Fall, denn alle Aussagen über die Welt sind menschlich. 
den aufgetragenen Farben, dem spezifischen Geruch etc. zu einer lebensweltlich bestimmbaren Zeit und an einem bestimmten Ort, den dieses Photo einnimmt.

Das Imaginäre soll als die vom menschlichen Subjekt produzierte Imagination der Wahrnehmung, Erinnerung und Vorstellung verstanden werden. Hierbei können die Überlegungen des Germanisten Wolfgang Iser von Nutzen sein, denn er plädiert in der Literaturwissenschaft für die Aufhebung der bipolaren Opposition von Wirklichkeit und Fiktion. Iser unterscheidet zwischen dem Realen, dem Imaginären und der Fiktion. Unter dem Imaginären versteht er psychische Operationsformen, die sich spontan und unwillkürlich einstellen und umfasst damit Manifestationen des Imaginären als Phantasie, Einbildungskraft und Imagination. Durch den intentionalen Akt des Fingierens, den das Imaginäre in einem literarischen Werk durchläuft, wird es zur Fiktion. ${ }^{87}$ Lebensweltliche Realität und das bloß vorgestellte Imaginäre vereinigen sich in der Fiktion, die im Falle der Literatur schriftlich fixiert wird. ${ }^{88}$ Das Imaginäre ist damit das Produkt der rein subjektiven Imagination - also das visuelle, akustische oder sonst wie sinnliche Vorstellungsvermögen des Menschen. Handlungen, Erlebnisse, Phantasien oder Gedanken über zukünftige und vergangene Ereignisse werden in meist bildlichen Vorstellungen imaginiert und teils schon sprachlich abstrahiert. Das Imaginäre setzt in diesem Zusammenhang die subjektive Imagination voraus. Es steht in einem zeitlichen, räumlichen und materiellen Kontext des psychischen Erlebens des Subjektes. Beim Beispiel der Photographie besteht das Imaginäre dieses Bildes aus den Gedanken, Emotionen und imaginierten Erinnerungen und Phantasien, die ein Betrachter innerlich entwickelt.

Virtualität soll nun heißen: die Simulation von menschlich-sinnlicher Wahrnehmung der Realität oder des Imaginären. Die virtuelle Realität simuliert visuelle, akustische und seltener olfaktorische oder haptische Sinneserfahrungen des Körpers. Virtualität setzt daher stets eine Simulationstechnik voraus. Virtualität steht in einem zeitlichen, räumlichen und materiellen Kontext der Erlebniswelt. Beim Beispiel der Photographie besteht das Virtuelle des Bildes in der technischen Simulation einer abgelichteten Szene (z.B. eine Landschaft).

87 Vgl. Iser 1990, 23-30 und Iser 1991, 24-59.

88 Vgl. Sill 1997, 141-145. 
Einen bedeutenden Beitrag zur Frage nach dem Verhältnis zwischen Realität und Medien bzw. Virtualität hat der französische Soziologe und Philosoph Jean Baudrillard geleistet. Er gliedert die abendländische Geschichte nach der Art der Beziehung zwischen Zeichen und Bezeichnetem. Unter Zeichen versteht Baudrillard zunächst alles, was Realität kodifiziert wiedergibt: Sprache, Zahlen, Bilder, Kleidung und ähnliches. Während in der mittelalterlichen Ständegesellschaft das Zeichen als symbolische Ordnung der Gesellschaft stets seine Referenz auf das Reale beibehält, beginnt mit der Renaissance das Ende des aufgezwungenen Zeichens: ${ }^{89}$

Auf die Endogamie der Zeichen, die der Rangfolge des Status entsprachen, folgt die Demokratie der Konkurrenz. Mit der Übertragung der Prestigewerte und -zeichen von einer Klasse auf die andere geht man notwendigerweise zugleich auch zur Imitation über. ${ }^{90}$

Die Imitation von Zeichen, die als bloßes Simulakrum nur noch die Referenz auf das Reale vortäuschen, erhält mit der industriellen Revolution und der seriellen Produktion von Zeichen eine neue Qualität. Da nun alle Zeichen als industrielles Simulakrum äquivalent wiederholbar sind, verliert die Imitation von Zeichen mit ihrer ursprünglichen Referenz ihre Bedeutung an die Produktion neuer Zeichen. Die Referenz auf das Modell ersetzt damit die Referenz auf die Realität. Das Zeichen generiert selbst Bedeutung und konkurriert nicht mehr primär mit anderen Zeichen bzw. mit den Referenzbezügen dieser Zeichen.

Das bestimmende Schema unserer Gegenwart ist nach Baudrillard die Simulation, die spätestens in ihrer digitalen Kodierung von Zeichen ohne Realitätsreferenz, eine Hyperrealität ins Leben ruft: "Am Ende dieses Entwicklungsprozesses der Reproduzierbarkeit ist das Reale nicht nur das, was reproduziert werden kann, sondern das, was immer schon reproduziert worden ist. « ${ }^{91}$ Zeichen konstituieren ihre eigene Realität und lösen damit den Widerspruch zwischen Realem und Imaginärem auf. »Die Irrealität ist nicht mehr die eines Traumes oder Phantasmas, eines Diesseits oder Jenseits, es ist die Irrealität einer halluzinierenden

${ }^{89}$ Jeder Stand hatte im Mittelalter seine ihm zugewiesenen Zeichen (Kleidung, Sprache, Verhaltenskodex). Vgl. Baudrillard 1991, 79-83.

90 A.a.O., 80.

91 Vgl. Baudrillard 1991, 116. 
Ähnlichkeit des Realen mit sich selbst. ${ }^{92}$ Zeichen repräsentieren damit nicht mehr Realität - mit der Simulation erschaffen sie eine Hyperrealität. ${ }^{93}$ In seinen medienphilosophischen Analysen stellt Baudrillard die Virtualität auch in ein Verhältnis zum Imaginären, das er der Bedeutung nach als äquivalent zur Illusion versteht. Er verurteilt die Simulation der Welt in der Virtualität als das perfekte Verbrechen. Das Verbrechen sei die Ermordung der Welt durch ihre Simulation, die einhergehe mit der Vernichtung der radikalen Illusion der Welt. Illusion ist für Baudrillard nicht trügerischer Schein, sondern der lebensnotwendige, utopische Geist, die autonome Kreativität des Menschen - das Imaginäre in dem oben ausgeführten Sinne (nach Iser): ${ }^{94}$

Der Gedanke wird zur Rechenoperation, dabei wird jeder Zweifel, jede Distanz, jede unberechenbare Eventualität, jedes Spiel und jede Illusion des Gedankens unterdrückt - gerade wie der Körper als Illusion in seiner genetischen Einschrift verschwindet, wie die Welt als Illusion in ihrem technischen Artefakt verschwindet ... All dies, Künstliche Intelligenz, Echtzeit, Telesensorialität, bedeutet die endgültige Verdrängung der Illusion. Die wilde Illusion des Gedankens, der Szene, der Leidenschaft, der Intelligenz, der Illusion der Welt, die Vision der Welt (und nicht ihre Darstellung und analytische Erkenntnis) ..., die Anschauung von Gut und Böse ..., von Wahr und Falsch, die wilde Illusion des Todes, oder aber des Lebens um jeden Preis - das alles verflüchtigt sich in der Tele-Wirklichkeit, in allen Technologien, die uns im Virtuellen einfangen, das heißt in das Gegenteil der Illusion, in die totale Desillusion. ${ }^{95}$

Wenn man Baudrillards Überlegungen aufgreift, präsentiert sich die Virtualität prinzipiell als Simulation sowohl von Realität als auch von Illusion bzw. dem Imaginären. Die Auffassung, dass die Virtualität selbst keinerlei Referenz mehr auf die Realität aufweist, Zeichen also nur noch sich selbst bezeichnen, ist nicht ohne weiteres nachvollziehbar. Ähnlich wie Herbert W. Franke und Wolfgang Iser entwirft damit auch Baudrillard ein dreiteiliges Modell - in diesem Fall mit den Begriffen der realen Welt, der Illusion der Welt und der Virtualität. Alle drei Denker differenzieren damit zwischen einer Ebene der (theoretisch) objektiv vorhandenen Realität, des subjektiv konstruierten Imaginären und der medialen Simulation der Realität wie auch des Imaginären in der Virtualität. An dieser Stelle der Überführung des subjektiv Imaginären in die

\footnotetext{
92 A.a.O., 114.

93 Vgl. a.a.O., 112-123.

94 Vgl. Baudrillard 1996, 9-38.

95 Baudrillard 1994a, 19ff.
} 
sprachliche oder bildliche Simulation wäre Isers Begriff des Fiktiven einzubringen. ${ }^{96}$

Unter diesen Voraussetzungen ist evident, dass Virtualität nicht einfach Schein oder das Gegenteil von Realität bedeutet, was schon Günther Anders durch das Paradox der Phantomatisierung - real und doch nicht wirklich - ausdrücken wollte. ${ }^{97}$ Die Szene eines Flugzeugabsturzes im Fernsehen kann eine Live-Übertragung sein oder aber eine dramaturgische Inszenierung in einem entsprechenden Spielfilm. Die eine Sendung stellt ein reales Ereignis dar, die andere ein fiktives (also eine Virtualisierung des Imaginären) - beide sind für den Fernsehzuschauer jedoch nur virtuell vorhanden.

Virtualität kann daher nur vom jeweils wahrnehmenden Subjekt aus gedeutet werden. Ereignisse sind nicht an sich virtuell, sondern ihre Wahrnehmung machen sie aus der Perspektive eines bestimmten Betrachters virtuell. Eine erhellende Erfahrung war für mich persönlich eine Wahlkampfrede Helmut Kohls 1998 auf dem Bonner Marktplatz. Als nur zufälliger Besucher am Rande der Zuschauermenge musste ich in Kauf nehmen, dass der Redner vor dem Rathaus sehr weit entfernt von mir stand, aber ein japanischer Tourist filmte mit aufgeklapptem Sucherbildschirm die Szene mit seiner Videokamera direkt vor mir. Auf dem kleinen Bildschirm war Kohl viel besser zu erkennen, als wenn ich direkt auf die Tribüne geblickt hätte. Kohl war genauso wie ich real und zur selben Zeit auf dem Bonner Marktplatz anwesend. Der Kamerabildschirm vor mir erzeugte also keinen Schein, sondern simulierte technisch die sinnlichen Erfahrungen (hier diejenigen der visuellen Nähe), die mir nicht zugänglich waren. Aber auf dieselbe Weise - allerdings ohne Elektronik - produzierte auch schon meine Brille ein besseres Bild des Redners. Meine reale Lebenswelt und die virtuelle Erlebniswelt des Kamerabildschirmes waren fast identisch - aber eben nur fast.

Umso deutlicher zeigt sich an diesem »fast«, dass Virtualität stets eine technikunabhängige Wirklichkeitsebene der realen Lebenswelt voraussetzt, von der sich die virtuelle Erlebniswelt abgrenzt. Beim Beispiel des Kohl-Auftrittes waren lediglich meine akustische Wahrnehmung (Laut-

\footnotetext{
96 Während das Imaginäre einen psychischen Prozess des Subjektes darstellt, verbindet die Fiktion das rein geistig Imaginäre mit dem Realen als Text, als Film, als Bild, als Plastik, als Computerprogramm, womit das Imaginäre in materialisierter Fiktion auch außenstehende Betrachter zulässt.

97 Vgl. Anders 1983, 129ff.; Heim 1993, 109 f.
} 
sprecher) und ein Teil meiner visuellen Wahrnehmungen virtuell - also simuliert.

Über die Differenzierung zwischen realer Lebenswelt und virtueller Erlebniswelt hinaus, die sich an der konkreten Situation des Medienkonsums orientiert, gibt es keine grundsätzliche Abgrenzung von Phänomenen der Virtualität und der Realität. Alle weiteren Unterscheidungen sind gradueller Natur und abhängig von dem Maß, in dem eine technische Simulation von unverfügbaren Wahrnehmungen stattfindet. Eine Brille und ein Teleskop erzeugen genauso wie ein Live-Stream auf dem Smartphone eine virtuelle räumliche Nähe, indem sie die sinnlich (hier: visuell) unverfügbare Realität visuell verfügbar machen. ${ }^{98}$ Welches Medium nun stärkere Aspekte von Virtualität impliziert, kann nicht pauschal beantwortet werden. Dies hängt von den heterogenen Inhalten des Mediums ab, die den Einbezug und die Konzentration des Betrachters auf das virtuell wahrgenommene Geschehen beeinflussen.

Klar ist aber: Je totaler die technische Simulation von körperlich-sinnlichen Erfahrungen ist, je stärker sie sich damit löst von den realen Erfahrungsmöglichkeiten unseres Körpers und unserer Person in der realen Lebenswelt, im Hier und Jetzt, desto größer ist das Maß von technisch erzeugter Virtualität in ihren räumlichen, zeitlichen und realweltlichen Aspekten.

Der absoluten Realität, wie sie Vladimir Cherniavsky definiert, ${ }^{99}$ muss daher die absolute Virtualität gegenüber gestellt werden. In der absoluten Virtualität ist die Simulation des Realen so perfekt und total, dass wir die Simulation nicht als solche erkennen können, da uns der Bezugspunkt einer höheren Realitätsebene fehlt. Die absolute Virtualität ist von der absoluten Realität nicht unterscheidbar. Der Posthumanist Frank Tipler verklärt dies als Vision für den Fortbestand der Menschheit, Rainer Werner Fassbinder in seinem Film Welt am Draht und die

98 Vgl. Lem 1981, 365.

99 Absolute Realität ist für Cherniavsky eine Wirklichkeit, deren Existenz nicht von uns abhängt und die uns unmittelbar durch unsere Sinne gegeben ist. Darüber hinaus bleibt Cherniavskys philosophisch-mathematischer Virtualitätsbegriff kaum anwendbar für die Virtualität der Medientheorie, da er Virtualität mit Kategorien von logischer Aussagenwahrheit verknüpft und damit die so wichtigen Aspekte des Fiktiven als »Phantasiewelten« aus seinen Betrachtungen ausklammert. Vgl. Cherniavsky 1994, $4,383$. 
Matrix-Trilogie problematisieren die Auflösung des Realen unter dem Eindruck dieser perfekten Simulation. ${ }^{100}$

Je perfekter und totaler aber die Simulation ist, desto geringer fällt die geistig-kreative - also imaginäre - Leistung des Menschen aus, da alle Gefühlsdispositionen und eventuelle Handlungsoptionen vorprogrammiert und geplant sind. Christoph Wulf und Nicolas Negroponte betonen diesen Verlust von subjektiver Imagination, der mit der verwobenen Einheit von Ton und Bild im Film, Fernsehen und Computerspiel einhergeht: ${ }^{101}$

Interactive multimedia leaves very little to the imagination. Like a Hollywood film, multimedia narrative includes such a specific representation that less and less is left to the mind's eye. By contrast, the written word sparks images and evokes metaphors that get much of their meaning from the reader's imagination and experiences. When you read a novel, much of the color, sound, and motion come from you. ${ }^{102}$

Nach diesen Überlegungen über das Verhältnis zwischen der Realität, dem Imaginären und der Virtualität und über die entsprechenden zeitlichen, räumlichen und körperlichen Aspekte stellt sich nun erneut und abschließend die Frage: Was ist Virtualität?

Bereits im theologischen Werk des mittelalterlichen Logikers John Duns Scotus (1265/66-1308) galt das lateinische virtualiter als ein Begriff, der anzeigte, dass ein Objekt nicht nur empirisch beobachtbare Eigenschaften besitzt, sondern dass es auch über nur virtuell vorhandene Qualitäten verfügt, die nicht alle gleichzeitig aktuell sind. ${ }^{103}$ Und nehmen wir Bezug auf die französische und englische Wortgeschichte der Begriffe Virtualität (virtualité bzw. virtuality) und virtuell (virtuel,-elle bzw. virtual), so fällt auf, dass sich die erörterten Eigenschaften der Virtualität teils schon in den verschiedenen historischen Bedeutungsgehalten dieses Wortes wiederfinden. Dabei zeichnen sich drei Bedeutungslinien ab. Das mittelenglische vertualyte bezeichnet Macht und Fähigkeit in einem ursprünglich religiösen Kontext. Das neuenglische virtuality spiegelt ab dem 17. Jahrhundert das wahre Wesen einer Sache oder eines Menschen

\footnotetext{
${ }^{100}$ In Matrix und Welt am Draht sind bereits alle unsere sinnlichen Erfahrungen Teil eines gigantischen Computerprogrammes. Vgl. Fassbinder 1973; Lem 1981, 345f.; Wachowsky \& Wachowsky 1999.

${ }^{101}$ Vgl. dazu Hammel 1994, 73-78; Wulf 1984, 42.

${ }^{102}$ Negroponte 1996, 8.

${ }^{103}$ Vgl. Heim 1993, 132f.; Williams 2001.
} 
wider, das unabhängig von seiner materiellen Verkörperung existiert. In der französischen Sprache bereits im 15. Jahrhundert, im Englischen erst im 19. Jahrhundert, benennen virtualité bzw. virtuality und die entsprechenden Adjektive einen möglichen Zustand, eine potentielle Fähigkeit, die aber nicht tatsächlich realisiert wird. Heute treffen die verschiedenen Bedeutungszusammenhänge von Virtualität aus der Philosophie, Literatur, Religion, der Optik und der Informatik im Brennpunkt der virtuellen Realität wieder aufeinander. ${ }^{104}$

Dass der Begriff Virtualität nicht auf computergestützte Simulationstechniken sowie den von Jaron Lanier geprägten Kontext der virtual reality und die Metapher des cyberspace beschränkt werden kann, sondern dass hierbei weitergehende zeitliche, räumliche und materielle Aspekte zu berücksichtigen sind, die auch nicht-elektronische Medien implizieren, ist nach der bisherigen Erörterung der Frage offensichtlich. Frühere Annäherungen an die begriffliche Fassung der Virtualität heben meist nur bestimmte Aspekte hervor. So betont N. Katherine Hayles vor dem Hintergrund ihrer Analyse von Kybernetik und Science-Fiction Literatur die Rolle der Information:

Virtuality is the cultural perception that material objects are interpenetrated by information patterns ... Virtual reality technologies are fascinating because they make visually immediate the perception that a world of information exists parallel to the »real« world. ${ }^{105}$

Dagegen nehmen Dietmar Kamper und Vilém Flusser mit nicht weniger Plausibilität eine eher pragmatische Interpretation vor und deuten die Virtualität als Möglichkeitsraum. Der virtuelle Raum, den das Universalmedium Computer dem Menschen eröffnet, führt nach Ansicht Flussers zu einer Vielzahl alternativer Welten. Er schafft neue Möglichkeitsfelder, die so plural und singulär sind, dass sie ins Wirkliche umschlagen und konkret werden. Für Kamper jedoch schließt diese Eröffnung neuer Möglichkeiten die Eliminierung der realen Welt mit ein. ${ }^{106}$

\footnotetext{
${ }^{104}$ Der lateinische Wortstamm virtus und virtualis als Stärke, Tugend und Vermögen verweist nur indirekt auf den späteren Bedeutungskontext. Als Ausdruck für eine nicht tatsächlich realisierte Möglichkeit leiten sich die neuzeitlichen Verwendungen im foyer virtuelle (1757) bzw. dem virtual focus (1704) in der Optik und im virtual memory (1959) in der Informatik ab. Vgl. Trésor 1994, 1186f.; Simpson \& Weiner 1989, Bd. 19, 674f.

${ }^{105}$ Hayles 1999, 14.

${ }^{106}$ Vgl. Flusser 1993, 70.
} 
Das Virtuelle ist das Mögliche, das jederzeit und überall auch anders Mögliche. Es besteht in einem Abschied vom Körperlichen, indem es die Bedingungen der Zeit und des Raumes negiert und damit seine eigene Genese verleugnet. ${ }^{107}$

Wir können Virtualität im medientheoretischen Kontext so verstehen, dass etwas nach Möglichkeit vorhanden, aber nicht wirklich da ist. Es ist insofern vorhanden, als dass unsere visuellen, akustischen und ggf. weiteren Sinneswahrnehmungen eines Phänomens simuliert werden. Virtualität ist damit nicht einfach das Gegenteil von Realität und auch nicht identisch mit dem Schein oder gar dem subjektiv Imaginären. Virtualität kann die Simulation der Realität wie auch des Imaginären bedeuten. Elektronische Medien im Prozess der technischen Simulation implizieren die Idee eines Informationsmusters, das hinter der Realität steht. Meiner Überzeugung nach führen diese Überlegungen zu der folgenden, sicherlich auch nur annähernden, medientheoretischen Definition des Virtualitätsbegriffes:

Virtualität ist die Verfügbarkeit des Unverfügbaren. Unverfügbar meint räumliche, zeitliche und materiell-körperliche Unverfügbarkeit von realen oder imaginären Ereignissen. Verfügbarkeit meint die Simulation der sinnlichen Wahrnehmung dieser Ereignisse. Je unabhängiger die Erfahrungen der virtuellen Erlebniswelt von Zeit und Raum sind, desto größer ist ihre Abhängigkeit hingegen von Technik. Im medientheoretischen Kontext ermöglicht Virtualität demnach die visuelle, akustische oder sogar haptische und olfaktorische Vergegenwärtigung von räumlich, zeitlich und real nicht zugänglichen Ereignissen aus der realen Lebenswelt oder aus imaginären bzw. fiktiven Welten.

Virtualität simuliert nicht Raum, nicht Zeit und nicht Körper: Virtualität simuliert unsere Wahrnehmungen von Raum, Zeit und Körper. Denn wie Stanisław Lem in der Summa technologiae demonstrierte, bleibt uns, solange wir Menschen und nicht Teile eines Computers sind, die Fähigkeit erhalten, die Virtualität durch unsere Leiblichkeit - durch unseren nur beobachtenden Körper - zu entlarven. ${ }^{108}$ Wenn Wilhelm Dilthey feststellt, dass erst die Widerstände der materiellen Welt uns Identität und Orientierung geben können, so resümiert Dietmar Kamper sinngemäß, dass im Computerzeitalter unsere Rückenschmerzen die einzige, aber deutlich vernehmbare Erinnerung an die Realität der Welt und des Körpers bilden.

107 Kamper 1999, 22.

${ }^{108}$ Vgl. a.a.O., 58f.; Lem 1981, $335 f$. 
Die Medienerfahrungen mit der Virtualität sind Erfahrungen der Verfügbarkeit von Zeit, Raum und Körper. Interaktionen oder Beziehungen zu Medienakteuren bzw. Computerspielfiguren eröffnen uns in dem spezifischen Kontext ihrer virtuellen Erlebniszeit und ihres virtuellen Erlebnisraumes das partielle Erleben einer Unabhängigkeit von Zeit, Raum und unseren physischen Grenzen. Virtualität ist hier in ihrer Fähigeit zur Vergegenwärtigung von Zeiten gerade auch Kontrolle über die Vergänglichkeit des Lebens. Im Kontext der gesamten lebensweltlichen Wirklichkeit - und eben nicht nur der medialen - sieht sich der reale Mensch jedoch den scheinbar aller Zeitlichkeit enthobenen Simulationen des Menschen in den elektronischen Medien gegenüber. 


\section{Die prometheische Scham}

Ich nenne es vorerst für mich »Prometheische Scham«; und verstehe darunter die »Scham vor der >beschämend hohen Qualität der selbstgemachten Dinge. ${ }^{1}$

Günther Anders

\subsection{Mensch und Technik bei Günther Anders}

Der Philosoph und Publizist Günther Anders entdeckte 1942 bei dem Besuch einer Technikausstellung ein neues Scham-Motiv, das es seiner Ansicht nach in der Vergangenheit noch nicht gegeben hatte. In seinem philosophischen Hauptwerk Die Antiquiertheit des Menschen (1956) führt Anders diesen Gedanken der prometheischen Scham aus und schildert zunächst, wie der homo faber im Prozess zunehmender Industrialisierung und Entfremdung von den Produkten der Arbeit seinen Stolz auf die von ihm nicht mehr persönlich produzierten Geräte verliert. Er illustriert dies am Beispiel der komplexesten Maschine seiner Zeit, der kybernetischen computing machine. Wenn ich nachfolgend so häufigen Gebrauch von Zitaten aus Anders Schriften machen werde, so spiegelt sich hier mein Erstaunen über sein unglaubliches Gespür für die im rezenten Posthumanismus artikulierten Deutungen von Mensch und Technik wider. Diese Tatsache offenbart sich vollends nach der Lektüre der entsprechenden Zitate der posthumanistischen Autoren. Günther Anders entfaltet den Gedanken, dass sich der Mensch gegenüber den Geräten, die ihre Qualität als Geschaffenes eingebüßt haben - also einfach »da« sind - aufgrund seiner eigenen »Fehlkonstruktion« unterlegen fühlen wird und Scham über die eigene Minderwertigkeit empfindet:

Daß, was Kraft, Tempo, Präzision betrifft, der Mensch seinen Apparaten unterlegen ist; daß auch seine Denkleistungen, verglichen mit denen seiner >computing machines<, schlecht abschneiden, ist ja unbestreitbar. ${ }^{2}$

Während der Mensch sich aus der Perspektive dieser Maschinen folglich als schlecht konstruiertes Gerät neben anderen, in vielerlei Hinsicht überlegenen Geräten wahrnehmen muss und sich in einem fehlerhaften

1 Vgl. Anders 1983, 23.

2 Anders 1983, 32. 
Körper seiner Unvollkommenheit ausgesetzt sieht, würden Maschinen im täglichen Wechsel immer weiter vervollkommnet:

Und wir? Und unser Leib? Nichts von täglichem Wechsel ... Er ist morphologisch konstant; moralisch gesprochen: unfrei, widerspenstig und stur; aus der Perspektive der Geräte gesehen: konservativ, unprogressiv, antiquiert, unrevidierbar, ein Totgewicht im Aufstieg der Geräte. Kurz: die Subjekte von Freiheit und Unfreiheit sind ausgetauscht. Frei sind die Dinge: unfrei ist der Mensch. ${ }^{3}$

In der Präsenz eines sich beschleunigenden technischen Fortschrittes ist der den physischen Beschränkungen seines Körpers ausgelieferte Mensch unter dem Paradigma der Maschine antiquiert, obsolet. Er ist der Saboteur seiner eigenen Leistungen. Um sich nicht mit all seiner Inferiorität und Zurückgebliebenheit abfinden und die "Sturheit des Leibes« akzeptieren zu müssen, begibt sich der Mensch auf den vielversprechenden Weg des Human Engineering und wechselt damit in das Reich des Hybriden und Artifiziellen hinüber:

Absicht der Experimente ist es, die Physis, die (außer für Magie und Medizin) stets als »fatum « gegolten hatte, einer Metamorphose zu unterwerfen; sie ihrer Fatalität zu »entkleiden« - und das bedeutet zugleich ..., ihr alles Fatale, alles Beschämende zu nehmen. ${ }^{4}$

Damit hat Günther Anders über 30 Jahre vor dem Erscheinen der ersten normativ posthumanistischen Schriften bereits eine Theorie über den Posthumanismus skizziert, an die ich anknüpfen möchte. Die Relevanz und Aktualität von Anders brillanten Überlegungen ist heute wohl so groß wie nie zuvor, da sich uns erst jetzt tagtäglich die Bezüge zu den neueren Entwicklungen der Gentechnologie und den posthumanistischen Visionen einer Verschmelzung zwischen Mensch und Computer aufdrängen. ${ }^{5}$

Im Anschluss an Günther Anders und Hannah Arendt fügt der Philosoph Johannes Rohbeck neuerdings den drei Kränkungen des Menschen durch die Wissenschaft, die seinerzeit Sigmund Freud beschrieben hatte, eine vierte hinzu. Freud sprach von der kosmologischen Kränkung (Kopernikus), der biologischen Kränkung (Darwin) und der psychologischen Kränkung (Psychoanalyse), die jeweils das menschliche Selbstbewusstsein erschüttern sollten:

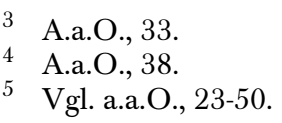


Ich meine, man könnte diesen Kränkungen noch eine vierte hinzufügen: die technologische Kränkung der Menschheit. In ihr erfahren die Menschen, daß sie nicht mehr Herr ihrer eigenen Schöpfungen sind, sondern von den selbst geschaffenen Machwerken beherrscht werden. Wie der Zauberlehrling haben sie etwas hergestellt, das nun eine eigene Dynamik entwickelt. ${ }^{6}$

Der Mensch ist laut Anders den Maschinen jedoch nicht nur aufgrund seiner »morphologischen Präformiertheit« unterlegen, denn »obwohl sturer als seine Produkte, ist der Mensch nämlich auch kurzlebiger, sterblicher als diese. ${ }^{7}$ Der einzelne Mensch sieht sich mit der quasi unsterblichen Serienexistenz der industriell gefertigten Produkte und ihren Modellen konfrontiert: »Ist es nicht >ewig« geworden durch seine Ersetzbarkeit, also durch die Reproduktionstechnik? Tod, wo ist dein Stachel? $\ll^{8}$ Dieser zweiten Inferiorität versucht der Mensch nun durch die von Anders bezeichnete Ikonomanie zu entgehen, indem er sich in Fotos, Filmen, »Fernsehphantomen« und Plakaten vervielfacht:

Unter den Gründen, die für diese zutreffend beschriebene hypertrophische Bildproduktion verantwortlich zu machen sind, ist eine der wichtigsten, daß sich der Mensch durch Bilder die Chance erobern konnte, "spare-pieces" von sich selbst zu schaffen; also seine unerträgliche Einmaligkeit Lügen zu strafen ... Nicht nur gleichverbreitet sind sie [Filmstars und Serienprodukte: O.K.], sie haben auch auf gleiche Weise ihre Sterblichkeit überwunden: Beide können sich ja nach ihrem Tode in ihren Reproduktionen weiterbewähren. ${ }^{9}$

Die prometheische Scham bestimmt Günther Anders als die Einsicht in die Desorientiertheit und Verzweiflung der sich als begrenzt erkennenden Freiheit und Individuation des Menschen, der sich nun seiner Hilflosigkeit und seines Versagens bewusst wird: " 'Sich schämen< bedeutet also: nichts dagegen tun können, daß man nichts dafür kann. ${ }^{10}$ Damit begreift er die prometheische Scham nicht als Metapher, sondern als tatsächliches Schamerlebnis in unserer Welt der Maschinen:

Wem es niemals zugestoßen ist, daß er den fälligen Griff an der Maschine verfehlte und dem wortlos weiterwandernden Fließbande ungläubig nachblickte ... wessen Blick niemals befremdet auf seine Hände fiel, auf diese tölpelhaften, deren Obsoletheit und unverbesserliche Inkompetenz seinen Fall verschuldet hatte

\footnotetext{
Rohbeck 1993, 10.

Anders 1983, 50.

A.a.O., 51.

A.a.O., 57. Vgl. hierzu a.a.O., 50-64.

10 A.a.O., 70. Vgl. hierzu a.a.O., 64-95.
} 
- der weiß nicht, welche Scham die Scham von heute ist, welche Scham heute täglich tausende Male ausbricht. ${ }^{11}$

In der Vergangenheit gab es einige kulturtheoretische Versuche, die zunehmende rationale Disziplinierung und Verhaltenssteuerung des Menschen im Zivilisationsprozess mit der Idee der Maschine in Verbindung zu setzen. Im Anschluss an Norbert Elias sehen Peter Gendolla und Wolfgang Schivelbusch zur Zeit der Aufklärung hierin ein Ideal der Rationalisierung gesellschaftlichen Verhaltens, die Elias so eindrücklich beschrieben hatte. In einer sich immer weiter ausdifferenzierenden Gesellschaft verkörpert die Maschine das Ideal eines mit steigender Komplexität notwendig immer exakter werdenden Ineinandergreifens aller Einzelteile und veranschaulicht damit die Notwendigkeit der Rationalität von individuellen und kollektiven Verhaltensregeln im gesellschaftlichen Leben. ${ }^{12}$

In ihrem Buch Die Achse Avantgarde - Faschismus lenkt Eva Hesse unsere Aufmerksamkeit auf die Frage nach dem Zusammenhang zwischen der Entstehung der Eugenik, also der Optimierung des menschlichen Körpers, Ende des 19. Jahrhunderts und der zunehmenden Industrialisierung. Inwieweit provozierte die Begegnung mit der »perfekten Maschine« die Idee einer rassischen bzw. genetischen Optimierung des Menschen? Wieso war in den rassistischen und faschistischen Ideologien kein Platz mehr für das Schwache, Unvollkommene - oder einfach Menschliche? ${ }^{13}$

So wurde die Idee von der eugenischen Vervollkommnung des Menschen und dem technischen Fortschritt vereinzelt schon um die Jahrhundertwende zueinander in Beziehung gesetzt. Der Arzt und spätere Rassenfanatiker Ludwig Woltmann sah die menschlichen Versuche einer biologischen Vervollkommnung im Schatten der ungeheuer raschen Perfektionierung der Maschinen.

11 A.a.O., 95.

12 Vgl. Elias 1997, 323-465; Gendolla 1992a, 16-29, 52-59; Schivelbusch 1977, 149-173. Hochinteressant wäre an dieser Stelle ein Vergleich von Elias' Ausführungen über die Entdeckung von Scham und Peinlichkeit und den entsprechenden Erörterungen von Anders zum Wesen der Scham. Vgl. Elias 1997, 408-419; Anders 1983, 65-82; Spreen 2000, 190f.

13 Obwohl Faschismus und avantgardistische Kunst in den dreißiger Jahren teilweise entgegengesetzte Ideologien vertraten und sich viele Futuristen, so auch Marinetti, entschieden gegen den Antisemitismus aussprachen, bestanden gerade zu Beginn beider Bewegungen in den 1920er Jahren enge ideologische Verbindungen zueinander. Vgl. Hesse 1992, 227-233, 281-336. 
Aber es gibt hier keine Überlebenden und sich fortpflanzenden besten Menschen. Nur eines vervollkommnet sich, und das ist die Maschine, welche die Menschenkraft überflüssig macht, und nicht der einzelne persönliche Mensch ... Und darum erzeugt der kapitalistische Konkurrenzkampf dieses außermenschliche und technische Resultat, weil die wirtschaftlichen Kräfte in der Maschine von individuellen Menschen losgelöst werden und eine gesellschaftliche Arbeitskraft darstellen. ${ }^{14}$

Der »neue Mensch« ist das Projekt sozialtechnischer und psychotechnischer Utopien des 19. und 20. Jahrhunderts, die sich von den »angewandten Humanwissenschaften « bis hin zu Rassentheorien der Steuerung und leistungsorientierten Optimierung menschlichen Handelns verschrieben haben. ${ }^{15}$ Diese hier nur angeschnittenen Seitenblicke veranschaulichen die Reichweite von Günther Anders Konzept der prometheischen Scham in seiner historischen Dimension, das auch Anknüpfungspunkte für die aktuelle Auseinandersetzung mit dem Transhumanismus bietet. ${ }^{16}$ Denn es war Anders, der Eugenik, Human Engineering bzw. Gentechnik als ersten Schritt einer, modern gesprochen, »Cyborgisierung« des Menschen entlarvte:

Die Experimente des »Human Engineering « sind wirklich die Initiationsriten des Roboterzeitalters; und die Versuchspersonen sind die Kandidaten und schließlich die Neophyten, die nun stolz darauf sind, ihre »Kindheit« hinter sich gebracht und die heute fällige »Erziehung des Menschengeschlechts« hinter sich gebracht zu haben. Aber wenn als »erwachsen« die Geräte gelten, dann bedeutet »die Kindheit hinter sich bringen« und »Erziehung des Menschengeschlechts« soviel wie: »das Menschsein hinter sich bringen«. ${ }^{17}$

Obwohl Günther Anders neben dem auf Maschinen und Geräte bezogenen Konzept der prometheischen Scham eine Medientheorie, eine »Phänomenologie des Fernsehens« entwickelte, bleiben die Verbindungen beider Gegenstandsbereiche in seinem Werk marginal und werden nicht entfaltet. Anders' Beispiele von der Unsterblichkeit des Filmstars in seiner unendlichen seriellen Reproduzierbarkeit im Photo und Film und der kosmetischen Selbstverdinglichung künden jedoch von dem

14 Ludwig Woltmann: Die Darwinsche Theorie und der Sozialismus. Düsseldorf 1899, 30. Zitiert nach Bolle 1962, 154. Vgl. a.a.O., 144-157.

15 Vgl. Schrage 2000, 43-52.

16 Vgl. Kluge 2014; Meyer-Drawe 2014.

17 Anders 1983, 41. 
kulturtheoretischen Potential, das die Kombination von Medien- und Maschinentheorie noch bereithält. ${ }^{18}$

\subsection{Virtualität und Tod}

All die jungen Photographen, die durch die Welt hasten, weil sie sich dem Aktualitätenfang verschrieben haben, wissen nicht, daß sie Agenten des Todes sind. ${ }^{19}$

Roland Barthes

Seit der europäischen Aufklärung ist Religion nicht mehr die alleinige Quelle von Sinn im Angesicht der menschlichen Sterblichkeit. Medizinische Utopien von einer nahezu unbegrenzten Lebensspanne wurden in den vergangenen drei Jahrhunderten immer wieder von progressiven Geistern artikuliert. Technische und mediale Immortalisierungsvisionen treten ab Ende des 19. Jahrhunderts in Erscheinung. ${ }^{20}$ Das Verhältnis von Tod, Medien und der Sehnsucht nach Unsterblichkeit unterliegt dabei einer erstaunlich persistenten Deutung in der menschlichen Kulturgeschichte. Der Heidelberger Ägyptologe Jan Assmann gelangte ausgehend von seinen Überlegungen zu altägyptischen Unsterblichkeitsvorstellungen zu der Überzeugung, dass neben Steinbauten, Plastik und Mumifizierung besonders die Verwendung der Schrift in den ägyptischen Gräbern als Ausdruck eines Unsterblichkeitswunsches zu verstehen ist. In den zugänglichen Kapellen ägyptischer Gräber erzählt die Grabinschrift die Lebensgeschichte des Verstorbenen und kann so durch den Akt des Erinnerns die Begrenztheit der Lebenszeit überwinden. Das Medium der Schrift als erste systematische Abstraktion der Realität mache die Sterblichen unsterblich: ${ }^{21}$

Der Mensch, durch sein Zuviel an Wissen aus den Ordnungen der Natur herausgefallen, muß sich eine künstliche Welt erschaffen, in der er leben kann. Das ist die Kultur. Die Kultur entspringt dem Wissen um den Tod und die Sterblichkeit. Sie stellt den Versuch dar, einen Raum und eine Zeit zu schaffen, in der der Mensch über seinen begrenzten Lebenshorizont hinausdenken und die Linien

18 Vgl. Anders 1983, 30f., 57ff.

19 Barthes 1985, 102.

20 Vgl. Kapitel 6.5.

21 Vgl. Assmann 1993, 64ff. 
seines Handelns, Erfahrens und Planens ausziehen kann in weitere Horizonte und Dimensionen der Erfüllung, in denen erst sein Sinnbedürfnis Befriedigung findet und das schmerzliche, ja unerträgliche Bewußtsein seiner existentiellen Begrenzung und Fragmentierung zur Ruhe kommt. Ohne Fantasmen der Unsterblichkeit ... kann der Mensch nicht leben. ${ }^{22}$

5000 Jahre später sehen Denker wie Boris Groys und Florian Rötzer erneut ägyptische Verhältnisse im Zentrum unserer Kultur. Auch Timothy Leary stilisiert die altägyptischen Könige gar als Vorbilder der technischen Immortalisierung: »The pharaos made it. ${ }^{23}$ Wie einstmals die Zweifel an der Unsterblichkeit der Seele in der Einbalsamierung von Körpern und in den Grabinschriften ägyptischer Pyramiden ihren Ausdruck gefunden hätten, so münde diese seelische Ungewissheit heute in einer ausufernden Medienkultur. Das Problem der menschlichen Sterblichkeit ist in der Gegenwart damit keineswegs in die Peripherie der Lebenswelt abgedriftet, sondern steht im Mittelpunkt unserer kulturellen Entwicklung:

Seit der ägyptischen Kultur mit ihren Mumien und Monumenten, seit der Einschreibung von Schrift und Bild in Stein und Papier fürchten die Menschen die Endlichkeit der Körper, Materialien und Informationen, kamen Vorstellungen auf vom unendlichen Leben ... Der Drang zur Virtualisierung fällt, parallel zu den Fortschritten der Biotechnologie, mit dem alten Begehren nach Ewigkeit zusammen. $^{24}$

Diese Diagnosen gegenwärtiger Kulturtheorie verweisen bereits auf die Signifikanz von Medien für das anthropologisch universale Todesproblem, vom Alten Ägypten bis in die Moderne. Niemand jedoch hat pointierter als der Berliner Kulturwissenschaftler Thomas Macho formuliert, was die Darstellung des Menschen in den elektronischen Medien für unsere Wahrnehmung der menschlichen Sterblichkeit bedeuten kann:

Unsere Fernsehgeräte sind heute schon Bildsärge, elektromagnetische Totenprojektionen. Haben wir jemals überlegt, wie viele von den Menschen, die allabendlich über unseren Fernsehschirm geistern, noch am Leben sind? [ ...] Es stimmt schon, die Toten sind unter uns. Wie heimatlose Nomaden bevölkern sie unsere Wohnzimmer. ${ }^{25}$

\footnotetext{
22 Assmann 2000, $13 f$.

23 Leary \& Sirius 1997, 159.

24 Rötzer 1996b, 232.

25 Macho 1994, 433.
} 
Damit ist der Bogen gespannt von den äußerst realistischen Totenportraits besonders im Ägypten römischer Zeit, über die populären Totenphotographien des 19. Jahrhunderts - nicht zuletzt von toten Kindern, dem in Mittelmeerländern und Südamerika heute verbreiteten Brauch, Grabsteine mit Photographien der Toten zu verzieren, bis hin zu den virtuellen Konservierungen vergangener Menschen in Film, Fernsehen und Computersimulationen in unserer Gegenwart - ganz zu schweigen von den expliziten Internetfriedhöfen, den virtual memorials.

Diese Deutung des Verhältnisses von Mensch, Technik und Medien, wie sie Günther Anders und später die Posthumanisten und einige Medienphilosophen formulierten, ist keine zwangsläufige Interpretation. Was ich hier beschreibe, sind keine »Eigenschaften«, die den Medien innewohnen. Diese »Sicht der Dinge« teilen in unserer heutigen Gesellschaft nur sehr wenige Menschen - auch die transhumanistische Bewegung ist trotz ihres revolutionären Anspruches nur eine marginale soziale Erscheinung. Aber die neuen sinnlichen Erfahrungen von simulierten Menschen in virtuellen Räumen und Zeiten bilden einen Rahmen, der bestimmte Deutungen, wie sie der technologische Posthumanismus hervorbringt, erst ermöglicht. Der Fernseher als »Bildsarg« steht der Sterblichkeit, der Unwiederbringlichkeit, des verstorbenen Menschen entgegen. Die virtuelle Simulation des Menschen geht über den im Subjekt verankerten Akt des Erinnerns hinaus, da sie den Menschen - zumindest visuell und akustisch - so darstellt, wie wir ihn wahrnehmen würden. Das gilt insbesondere für Menschen, die wir ohnedies nur aus den Medien kennen. Der reale Lebensstatus der Medienakteure bleibt uns verborgen.

Natürlich sind Tod und Sterblichkeit auch Thema in den Medien, was uns im Kontext von Katastrophenmeldungen aufgrund der direkten Erfahrbarkeit des Leidens sehr betroffen machen kann, sich jedoch meines Erachtens vor allem auf das Leiden der lebenden Menschen bezieht. Die unmittelbare Erfahrung eines sterbenden Menschen, der dann plötzlich nur noch eine Leiche ist, hat jedoch eine völlig andere, tiefergehende Beschaffenheit als das Erlebnis des Sterbens im Film, Fernsehen und Computerspiel, an das man heute durch die tausendfachen Tode von klein auf gewöhnt ist.

Die Virtualität der Medien führt uns einen Menschen vor, der nicht mehr den zeitlichen und räumlichen Bedingungen des Menschen entspricht. Durch die Unabhängigkeit von Raum und Zeitlichkeit konstituiert 
Virtualität eine übersinnliche Erfahrungsdimension der Wirklichkeit. Die Anwesenheit von sinnlich Unverfügbarem bietet schließlich den Schlüssel zum Verständnis der Virtualität in ihrer sozialen Relevanz und entfaltet die Signifikanz innerhalb einer thanatologischen Fragestellung. Das Unbestimmbare des Todes wird durch die Möglichkeit der beliebigen, technischen Vergegenwärtigung des Zeitlichen bestimmbar: Der kalkulierte Tod im Film und im Computerspiel ist revidierbar, wiederholbar und unterliegt damit nicht den Bedingungen der Einmaligkeit des realen Lebens. Die stets verfügbare Vergegenwärtigung von Erfahrungen, die über die natürlichen Möglichkeiten unserer Sinne und unseres Daseins hinausgehen, bleibt nicht ohne Folgen für die Vorstellungen des Menschen von seinem Tod und seiner Sterblichkeit. Es lassen sich hier vor allem zwei Konsequenzen benennen, die zunächst paradox anmuten.

Zum einen bildet die von der Unwiederbringlichkeit der Zeit enthobene Darstellung des Menschen in den elektronischen Medien einen weiteren Aspekt der Todesverdrängung, da sich die soziale Sichtbarkeit des Todes allein aufgrund der entsprechenden medialen Dispositive verringert. Wenn der Tod die soziale Dimension des Erlebens, also Wahrnehmens, eines Menschen durch seine Mitmenschen meint, dann verschleiern die aus unendlichen Wiederholungen bestehenden Medienereignisse den realen Tod, die Vergänglichkeit und die Einmaligkeit des Lebens.

Erst dort, wo das Sterben erlebt und über den Tod reflektiert wird, entsteht so etwas wie das Todesproblem, das nach Lösungen und Erklärungen verlangt. Zum anderen aber erfahren wir, dass Menschen wirklich sterben und wirklich nicht mehr unter uns weilen. Nur ihre medialen Abbilder »überleben«. Vor diesem Horizont der realen Erfahrung des Todes unserer Mitmenschen und der Abwesenheit des Todes in der Virtualität wird die technische Utopie einer medialen Immortalisierung des Menschen erklärbar.

Denn was passiert, wenn der Mensch beginnt, sich mit den technisch simulierten Menschen der elektronischen Medien zu vergleichen? Was passiert, wenn er sich entsprechend unserem historischen Ausgangspunkt im Alten Ägypten nicht mehr nur durch niedergeschriebene Lebensfragmente oder Photographien der Erinnerung seiner Nachkommen vergewissern will, sondern wenn er selbst ganz real medial unsterblich werden will? Wenn er seine virtuelle Repräsentation, seine Existenz als reines Zeichen, der körperlich-vergänglichen Realität vorziehen möchte? Wenn der Mensch, wie Dietmar Kamper es ausdrückt, in seinem "postmodernen Unsterblichkeitswahn« in die "Schöpfung aus 
Sprach- und Bildermüll« hinüberwechseln will, in der es vollkommen unmöglich geworden sei zu sterben $?^{26}$ Schlicht: Was passiert, wenn der Mensch so unsterblich werden möchte, wie die von ihm wahrgenommenen Menschen in der medialen Simulation?

Ich vertrete die These, dass die Begegnung mit der Virtualität, das heißt vor allem das Erleben von nur virtuell vorhandenen, medialen Abbildern des Menschen, eine Art prometheischer Scham evozieren kann. Nicht also die Konfrontation mit der seriellen Reproduzierbarkeit industrieller Produkte bildet die Ursache des menschlichen Minderwertigkeitsgefühls in der Mediengesellschaft, sondern die Gegenüberstellung mit den photographischen, filmischen und diversen digitalen Reproduktionen des Menschen. Die Ikonomanie als letztlich vergeblicher Versuch, der »Kalamität des Menschen ${ }^{27}$ zu entkommen, führt damit in eine neue Qualität menschlicher Inferiorität im Verhältnis zu seinen medientechnischen Produkten.

Der Mensch sieht sich Simulationen von Menschen gegenüber, die unabhängig von Zeit, Raum und Körperlichkeit virtuell existieren. Je näher diese Simulationen der Realität kommen, desto näher rückt der Vergleich des Menschen mit seinem virtuellen Gegenüber. Die Wahrnehmung von Film, Fernsehen und digitalen Medien in ihrer zeitlichen Dynamik entspricht eher unserer Wahrnehmung realer Menschen, als dies eine Photographie tut. Ein Computerspiel mit virtuellen Interaktionspartnern oder die so genannten persönlichen Assistenten wie Siri oder Alexa simulieren darüber hinaus mit wachsendem Erfolg das interaktive Moment menschlicher Kommunikation.

Das Vergleichsmoment zwischen dem Menschen und der virtuellen Simulation des Menschen ist zunächst seine Sterblichkeit und die vermeintliche Unsterblichkeit seiner medialen Simulation. Der technologische Posthumanismus ist die Antwort des Menschen auf die prometheische Scham, die der Mensch gegenüber seiner medialen Reproduktion empfindet. Er propagiert die Überwindung des Menschen durch einen bloß in der Virtualität existenten - posthumanen - unsterblichen Menschen.

Der künftige Mensch soll jedoch nicht nur die Sterblichkeit überwinden, sondern auch das biologische Dasein in all seinen Qualitäten übertreffen. Ein zweiter Aspekt des Vergleiches ist daher der kybernetischen

\footnotetext{
26 Vgl. Kamper 1999, 50f.

27 Vgl. Anders 1983, 35ff.
} 
Theorie geschuldet, die das Leben insgesamt am Grad seiner informationsverarbeitenden Leistung misst und den Menschen den Computern gegenüberstellt. Die Unsterblichkeitsfrage in Verbindung mit der Vision unermesslicher Intelligenz und Macht bilden die zwei zentralen analytischen Achsen der nachfolgenden Ideengeschichte des technologischen Posthumanismus. 


\section{Teil II \\ Der technologische Posthumanismus}




\section{Transhumanismus}

trans-húman, a. [Rare] superhuman. - trans-húmanize, vt. [Rare] To make superhuman. ${ }^{1}$

New Standard Dictionary of the English Language (1949)

\subsection{Post- und Transhumanismus}

Wenn auch häufig die verschiedensten Denker, die die Überwindung des bisherigen Menschen mit Hilfe neuer Technologien propagieren, allesamt als Transhumanisten bezeichnet werden, möchte ich nachdrücklich dafür plädieren, zwischen dem technologischen Posthumanismus und dem Transhumanismus zu differenzieren. Zu einer Klärung nötigt nicht nur die in der kunst- und kulturwissenschaftlichen Forschung gebräuchliche Rede vom Posthumanismus, sondern es bestehen spürbare Unterschiede zwischen dem Anliegen, den Inhalten und der Herkunft des Transhumanismus und des technologischen Posthumanismus.

Das erste Argument für eine Unterscheidung zwischen den beiden Futurologien ist die Genealogie. Der Transhumanismus wurde in seinen Anfängen in den 1960er Jahren, die vorwiegend in Kalifornien lokalisiert werden können, maßgeblich von den Visionen des Futuristen Fereidoun M. Esfandiary (FM-2030), dem Engagement des Vordenkers der psychodelic movement, Timothy Leary, und dem Kryoniker Robert Ettinger geprägt. Aus dieser Bewegung gingen in den späten 1980er Jahren die Extropianer um Max More und mit stärkerem europäischen Engagement die von Nick Bostrom, David Pearce und Anders Sandberg 1998 begründete World Transhumanist Association hervor.

Der technologische Posthumanismus dagegen vereinigt eine Reihe von Autoren, die seit Mitte der 1980er Jahre die Ablösung des Menschen durch ihre künstlichen Nachkommen propagieren. Seine Hauptvertreter wie Marvin Minsky, Frank Tipler, Hans Moravec und Ray Kurzweil argumentieren auf der Grundlage der kybernetischen Theorie. Diese

1 Funk 1949, 2250. 
Autoren beziehen sich bis in die frühen 2000er in keiner Weise auf die Bewegung der Transhumanisten und deren Themen.

Das zweite Argument für die Trennung zwischen Post- und Transhumanismus stützt sich auf die unterschiedlichen inhaltlichen Schwerpunkte. Transhumanisten befassen sich praktisch mit den Fragen der Lebensverlängerung und der mentalen Leistungssteigerung wie den smart drugs, lebensverlängernden Diäten, Fortschritten der Prothesentechnik, den Potentialen einer erneuerten Eugenik oder gar den Aussichten der Kryonik, während diese Anwendungen in den posthumanistischen Schriften kaum Erwähnung finden. Gerade die enge Verbindung von Kryonik und Transhumanismus veranschaulicht die Hoffnung, in ferner Zukunft aus den gekühlten Stickstofftanks wieder ins Leben zurückgerufen zu werden und dann die erhofften Möglichkeiten einer technischen Immortalisierung zu nutzen.

Diese Vernachlässigung der für die Menschen der Gegenwart drängenden, praktischen Fragen im Posthumanismus oder Postbiologismus verweist bereits auf die entscheidende Differenz gegenüber dem Transhumanismus. Während im Transhumanismus der Mensch und das, was aus ihm mit Hilfe technologischer Aufrüstungen und Erweiterungen wird, das Subjekt der Entwicklung ist, sind im Posthumanismus Roboter und künstliche Intelligenzen die künftigen Träger von Evolution und Fortschritt. Die unsterbliche Existenz des Menschen in einem virtuellen Habitat ist hier nur Begleiterscheinung des autonomen Fortschrittsprozesses der künstlich-intelligenten, posthumanen Wesen. Eine von Menschen geschaffene, posthumanistische Philosophie, die für die totale Annihilation des Menschen zugunsten von Maschinenwesen eintritt, ohne dafür die Erlangung der Unsterblichkeit zu garantieren, ist schlicht unvorstellbar. Für den Posthumanismus ist die Idee der technischen Immortalisierung daher von zentraler und konstitutiver Bedeutung. Gut sichtbar ist diese Differenz zwischen Post- und Transhumanismus beim Thema der Eugenik bzw. gentechnologischen Optimierung des Menschen. Während diese für viele Transhumanisten wesentlich ist, spielt die biologische Verbesserung des Menschen im Posthumanismus keine Rolle. Dies mag auch dem reflektierteren Umgang mit der dunklen Geschichte der Eugenik und dem familiären Hintergrund einiger Posthumanisten geschuldet sein.

Eine Sonderstellung nimmt Ray Kurzweil ein, da er neben seinen posthumanistischen Werken The Age of Spiritual Machines (1999) und The Singularity is near (2005) seit den 1990er Jahren auch Lebenshilfe-Bücher 
veröffentlicht. Kurzweil ist bis vor ca. zehn Jahren kein zentraler Akteur innerhalb der etablierten transhumanistischen Netzwerke gewesen. Durch seinen publizistischen Erfolg und die Gründung der Singularity University 2008 ist er allerdings inzwischen zum prominentesten Repräsentanten des Post- und Transhumanismus avanciert. Er selbst bezeichnet sich jedoch als Singularitarian, den wir aufgrund seiner technozentrischen Anlage dem Posthumanismus zurechnen müssen.

Der Begriff posthuman ist demnach auf verschiedene Art konnotiert: Im Transhumanismus, der in letzter Konsequenz anthropozentrisch bleibt, bezeichnet posthuman in den meisten Fällen den Zustand des weitgehend perfektionierten Menschen - des Transhumanen, der viele seiner Ziele verwirklicht hat und deshalb nicht mehr human ist. Im technologischen Posthumanismus dagegen wird derselbe Terminus mit den vollkommen synthetischen, künstlichen Intelligenzen gleichgesetzt.

\subsection{Vorläufer und Begriffsgeschichte}

Der bedeutende Historiker Eric Hobsbawm entwickelte während seiner Analyse der britischen Geschichte das Konzept der invented tradition der erfundenen oder konstruierten Tradition. Demnach wird der status quo mit der behaupteten Kontinuität einer historischen Tradition von Praktiken und Werten legitimiert. ${ }^{2}$

Der Transhumanismus präsentiert sich als Musterbeispiel eines solchen Prozesses. Der Legitimationsbedarf ist enorm, da die Bewegung auch durch ihre starke Verbindung zur Kryonik in den journalistischen Darstellungen als äußerst exotisch wahrgenommen wird. Der frühe Transhumanismus formiert sich in den späten 1960er Jahren um Timothy Leary, Robert Ettinger und Fereidoun Esfandiary (FM-2030). Bis Ende der 1990er Jahre ist dies auch innerhalb des Transhumanismus die vorherrschende Lesart der eigenen Ursprünge - die Selbstbezeichnung wird noch im Jahr 2000 auf Esfandiary zurückgeführt, der angeblich um 1970 das Wort transhuman kreiert haben soll. ${ }^{3}$

Erst mit zwei Veröffentlichungen der Transhumanisten James Hughes und Nick Bostrom aus den Jahren 2004 bzw. 2005 ändert sich dies. Eine

Vgl. Hobsbawm 2000.

3 So die aus dem Jahr 2000 stammende Homepage von Anders Sandberg http://www. aleph.se/Trans/Words/ (noch verfügbar am 19.08.2018). 
lange Reihe fortschrittsorientierter Philosophen wird in die Ahnengalerie des Transhumanismus eingeordnet: u.a. Francis Bacon, Condorcet, Benjamin Franklin, La Mettrie, Kant, (mit Einschränkungen) Nietzsche, der Biochemiker J. B. S. Haldane, der Physiker John D. Bernal sowie die ungleichen Brüder Aldous und Julian Huxley - der Schriftsteller und der Biologe. Letzterem wird auch die Kreation des Wortes transhumanism zugeschrieben. ${ }^{4}$ Vereinzelt berufen sich heutige Transhumanisten auch auf die wiederentdeckten, russischen Kosmisten des frühen 20. Jahrhunderts. $^{5}$

Ganz im Sinne einer Legitimation durch die Konstruktion von Tradition fügt Bostrom den Transhumanismus mit Verweis auf Haldane, Bernal und Julian Huxley in die akademische Kontinuität englischer Eliteuniversitäten ein und gründet exakt im Jahr seiner oben genannten Aufsatzpublikation das Future of Humanity Institute in Oxford. In der wissenschaftlichen Literatur über den Transhumanismus wird diese retrospektive Traditionsbildung teilweise unkritisch übernommen. ${ }^{6}$ Die Trias von Haldane, Bernal und Julian Huxley ist dabei nicht zufällig, denn alle drei waren ab 1930 über einen politischen Diskussionsclub in Cambridge verbunden. ${ }^{7}$

Wenn wir unter »Vorläufern des Transhumanismus« aber nicht nur die Ideengeber für einzelne Aspekte verstehen, die später im Transhumanismus in Erscheinung treten, dann sind es nur Julian Huxley und der Philosoph und Paläontologe Pierre Teilhard de Chardin, die jeweils über viele Jahrzehnte einen konsistenten philosophischen Entwurf zur Zukunft des Menschen ausgearbeitet haben. Die ideengeschichtliche Analyse in Kapitel 6 wird im Detail eine Vielzahl von Elementen der trans- und posthumanistischen Visionen beleuchten, die auf älteren Deutungen von Geschichte, Evolution und Technik in der europäischen und amerikanischen Geistesgeschichte beruhen. ${ }^{8}$

4 Vgl. Hughes 2004, 156-159; Bostrom 2005, 2-6. More und der deutsche Transhumanist Stefan Sorgner betonen entgegen vieler anderer Transhumanisten die positive Rolle Nietzsches.

5 Vgl. More 2013b, 10f.; Goertzel 2010; Sorgner 2016, 34-40.

6 Vgl. Heil 2010a, 128-131; Heil 2010b, 26-28; Heil 2010c; Heil 2018, 55-64.

7 Vgl. Brown 2005, 104.

8 Die häufig angeführten Wissenschaftler Haldane und Bernal formulierten zwar radikale Futurologien, entwickelten daraus jedoch kein philosophisches oder gesellschaftliches Programm. Auch hat Bernal seine Ideen im späteren Werk nicht weitergeführt. Vgl. Kapitel 6.5.2. 
Julian Huxleys Prägung der Begriffe transhuman und transhumanism kann bereits auf eine lange Vorgeschichte im Englischen zurückblicken. Als Verb ist trasumanar zunächst eine italienische Wortschöpfung des Dichters Dante (1256-1321) im ersten Gesang des Paradiso in der Divina Commedia (der Göttlichen Komödie). Der Poet beschreibt damit den gemeinsamen Aufstieg mit seiner Führerin Beatrice in die Sphären des Paradieses. In diesem Punkt fügt Dante nun zwei Referenzen ein: Explizit verweist er auf die Vergöttlichung des einfachen Fischers Glaucus in einen Meeresgott aus den Metamorphosen des Ovid. Indirekt - jedoch vollkommen unzweideutig - spielt der Dichter durch weitere Anmerkungen auch auf die neutestamentliche Überlieferung an: den Aufstieg des Apostel Paulus in den dritten Himmel und seine Gottesschau (2 Kor 12:1-10). Bei Dante ist das Verb trasumanar also bereits bedeutungsvoll mit zwei Konnotationen geladen: der (griechisch-mythologischen) Vergöttlichung und der (christlichen) Gottesschau. ${ }^{9}$

Als Henry Francis Cary 1814 die erste englische Übersetzung der Göttlichen Komödie anfertigt, wird aus dem italienischen Verb das englische Adjektiv transhuman - aus Dantes Trasumanar significar per verba non si poria wird bei Cary Words may not tell of that transhuman change. ${ }^{10}$ Der Begriff transhuman ist dann in der Folgezeit im Kontext der englischen Dante-Rezeption mehrfach nachgewiesen und wird schon 1949 bzw. 1959 im New Standard Dictionary of the English Language und in Webster's New International Dictionary of the English Language mit der heute von den Transhumanisten verwendeten Wortbedeutung aufgeführt. ${ }^{11}$ Als Webster's diesen letztgenannten Vorgang als »transcending human limits« beschrieb, hatte der Terminus transhuman augenscheinlich schon Eingang in den Alltagsdiskurs gefunden, wie ein Beitrag in der Londoner Wirtschaftszeitung The Economist von 1957 belegt. $^{12}$

Die Entstehung des Begriffes transhumanism nun geht auf den kanadischen Philosophen William Douw Lighthall (1857-1954) zurück. ${ }^{13}$ Wie

9 Vgl. Harrison \& Wolyniak 2015, $265 f$.

10 Vgl. Simpson \& Weiner 1989, Bd. 18, 403; Harrison \& Wolyniak 2015, 265f.; Heil 2010a, 128.

11 Vgl. Funk 1949, 2250.

12 Vgl. Neilson 1959, 2691; Simpson \& Weiner 1989, Bd. 18, 403; Gove 1993, Bd. 3, 2428.

13 Entgegen der seit James Hughes Buch Citizen Cyborg (2004) verbreiteten Annahme, dass Julian Huxley 1927 den Begriff in seinem Buch Religion without Revelation erfunden habe, konnten Peter Harrison und Joseph Wolyniak belegen, dass die Wortschöpfung von Lighthall stammt. Dieser Irrtum wird bis dato auch in der gesamten wissen- 
viele seiner Zeitgenossen - zu nennen sind hier vor allem Henri Bergson, Alfred North Whitehead und Pierre Teilhard de Chardin - war Lighthall darum bemüht, Metaphysik und Naturwissenschaft im Zeitalter nach Darwin miteinander zu versöhnen. ${ }^{14}$ Als liberaler Protestant und Freimaurer entwirft er eine kosmische Evolutionsgeschichte, die als ersten und außerhalb des Universums stehenden Grund ein personales Bewusstsein identifiziert: the Outer Consciousness, the Directive Power, the Thinker of Evolution. Aus der Beobachtung des biologischen Lebens meint Lighthall einen vitalen Drang der Evolution nach Tugend und dem Guten ableiten zu können, der als »Teleologie der Freude« den Rang eines Naturgesetzes einnimmt: »It is marked by marvellous, beneficent, and progressive devices, notably illustrated by the instincts and cerebral cortex of Man; and now so hugely numerous that they form an unanswerable argument for purposiveness. ${ }^{15}$ Das heutige, proteinbasierte Leben auf der Erde wird sich gemäß Lighthall in ferner Zukunft, wenn es die irdischen und solaren Grenzen verlässt, vielleicht in gänzlich anderer Form verwirklichen, » ... shall that spirit not take on more glorious forms of body, mind and heart, ever advancing in oneness with the Person of Evolution. $\ll^{16}$

Nicht als philosophisches System, sondern als Zustand des Apostels Paulus, führt Lighthall sodann den Transhumanismus ein:

Over all, we should remember Dante's supernal principle:

Trasumanar significar per verba

Non si porria.

and Paul's Transhumanism: »Eye has not seen, nor ear heard, neither has it entered into the conception of man. ${ }^{17}$

schaftlichen Literatur wiederholt oder gar auf Huxleys Werk New Bottles for New Wine (1957) datiert. Hughes und mit ihm Bostrom verwenden fälschlicherweise als Beleg ein Zitat, das aus ebendieser späten Aufsatzsammlung Huxleys stammt. Vgl. Harrison \& Wolyniak 2015, 465-467; Hughes 2004, 158; Bostrom 2005, 6; Huxley 1957b, 17.

14 Explizit bezieht sich Lighthall auch auf Bergson, Schopenhauer, Whitehead, Haeckel und den deutschen Lebensphilosophen Hans Driesch, mit dem er sich brieflich austauschte. Dass sein Ansatz, der demjenigen Teilhards sehr ähnelt, so wenig Resonanz erfuhr, mag vermutlich an der schlechten Vernetzung Lighthalls liegen. Vgl. Lighthall 1930, 14-21, $201 \mathrm{ff}$.

15 Lighthall 1940, 135. Vgl. Lighthall 1930, 125-140.

16 Lighthall 1930, 200.

17 Lighthall 1940, 139. Lighthall zitiert hier ungenau 1 Kor 2:9 »But as it is written, eye hath not seen, nor ear heard, neither have entered into the heart of man, the things which God hath prepared for them that love him.« (nach der King James Bible). 
Aus dem weiteren Kontext geht hervor, dass hier der stetige Aufstieg des Menschen gemeint ist. Die Nutzung des Begriffes bleibt für Lighthall allerdings singulär. ${ }^{18}$ Es ist unklar, ob Julian Huxley den Aufsatz des kanadischen Philosophen kannte. Belege für diese Vermutung bestehen nicht. Wichtig ist an dieser Stelle, dass Lighthall seine kosmische und evolutionäre Metaphysik mit der bekannten Passage aus Dantes Paradiso verbindet, die Vergöttlichung und christliche Gottesschau einschließt. Dieser literarische Kontext muss auch Julian Huxley (1887-1975) bekannt gewesen sein, als er erstmals in den William Alanson White Memorial Lectures von 1951 den von ihm begründeten, evolutionären Humanismus als transhumanism bezeichnet: »It is the idea of humanity attempting to overcome its limitations and to arrive at fuller fruition. $"{ }^{19}$ Konzeptionell entwirft Huxley die Idee einer durch den vernunftbegabten Menschen gesteuerten Evolution schon in seinen frühen Schriften, die stark von der zeitgenössischen Eugenik geprägt sind:

... life in his person has become self-conscious, and evolution is handed over to him as trustee and director. »Nature « will no longer do the work unaided. Nature - if by that we mean blind and non-conscious forces - has, marvelously, produced man and consciousness; they must carry on the task to new results which she alone can never reach. ${ }^{20}$

Huxley will mit Hilfe der Wissenschaft die Qualität (und nicht die Quantität) des menschlichen Lebens fördern. Biologisch soll der Mensch mit Hilfe der Eugenik verbessert werden, gesellschaftlich durch Bildung, eine gute Gesundheitsversorgung und Wohlfahrt für die ganze Menschheit. Diese Agenda verfolgte Huxley auch, als er 1946 zum ersten Direktor der UNESCO ernannt wurde. Der Fortschritt einer geeinten Menschheit werde Religionen und Nationalismus überwinden. ${ }^{21}$

Ab den 1950er Jahren und besonders in seiner Aufsatzsammlung New Bottles for New Wine (1957) fasst Huxley sein Programm des evolutionären Humanismus unter der griffigeren Formel des Transhumanismus zusammen, den er einerseits als streng wissenschaftliche Weltanschauung begreift, andererseits als glaubensmäßiges Bekenntnis zum Aufstieg des Menschen.

18 Vgl. Harrison \& Wolyniak 2015, 465-467.

19 Huxley 1951, 139. Die Vorlesung wurde im selben Jahr im Journal Psychiatry veröffentlicht. Vgl. Harrison \& Wolyniak 2015, 465.

20 Huxley 1923, XIII.

21 Vgl. Huxley 1957b, 16f.; Huxley 1957c, 21, 39f.; Huxley 1957c, 39f.; Huxley 1957d. 
The human species can, if it wishes, transcend itself - not just sporadically, an individual here in one way, an individual there in another way - but in its entirety, as humanity. We need a name for this new belief. Perhaps transhumanism will serve: man remaining man, but transcending himself, by realizing new possibilities of and for his human nature. "I believe in transhumanism «: once there are enough people who can truly say that, the human species will be on the threshold of a new kind of existence, as different from ours as ours is from that of Pekin [sic!] man. It will at last be consciously fulfilling its real destiny. ${ }^{22}$

Seit Beginn der Konstruktion einer transhumanistischen Tradition durch James Hughes, Nick Bostrom und Max More wird Huxley zwar die Wortschöpfung zugeschrieben, aber eine reflektierte Würdigung seines Beitrages - gar eine positive Rezeption seines programmatischen Ansatzes - bleibt aus. Max More spielt Huxleys Rolle sogar dezidiert herunter, um dann zu betonen, dass der Transhumanismus als philosophisches System erst durch seinen eigenen Aufsatz »Transhumanism: Toward a Futurist Philosophy« von 1990 begründet wurde. ${ }^{23}$ Wenn diese Selbsteinschätzung Mores anfechtbar ist, so spiegelt sich hier auch der Umstand wider, dass Huxley sich seinerzeit mit dem Eintreten für die Eugenik zusehends ins Abseits manövrierte. Die gesellschaftliche Kritik, Aufarbeitung und Einstellung von eugenischen Praktiken in Europa und den Vereinigten Staaten war nach dem 2. Weltkrieg nicht mehr vereinbar mit Huxleys teils eugenischen Zukunftsvisionen. ${ }^{24}$

Intensiver gestaltet sich im Transhumanismus die Auseinandersetzung mit dem literarischen Werk von Julians älterem Bruder, Aldous Huxley (1894-1963), dem dystopischen Roman Brave New World von 1932. Transhumanisten sehen sich in der Pflicht, die von Aldous geschilderten Gefahren einer totalitären, eugenischen Klassengesellschaft zu entkräften und betonen den freiheitlichen Charakter ihrer Vision. ${ }^{25}$

Erwähnt Julian Huxley in seiner obenstehenden Begründung des Transhumanismus den vorzeitlichen Peking-Menschen, so ist dies ein überdeutlicher Verweis auf den französischen Paläontologen und jesuitischen Philosophen Pierre Teilhard de Chardin (1881-1955), der 1929 nämlich maßgeblich an der Entdeckung des Sinanthropus Pekinensis beteiligt war. Obwohl der direkte Einfluss von Teilhards Ideen auf den Post- und

22 Huxley 1957b, 17.

23 Vgl. Hughes 2004, 158; Bostrom 2005, 6; More 2013b, 8f.; Sorgner 2016, $23 f$.

24 So erwähnt z.B. der Physiker Gerald Feinberg, der mit seinem Project Prometheus (und dem gleichnamigen Buchtitel von 1969) für eine radikale Überwindung des bisherigen Menschen eintritt, Huxley mit keiner Silbe.

25 Vgl. Bostrom 2005, 5; Hughes 2005, 49, 56f.; Ettinger 1989, 80f. 
Transhumanismus ungleich größer ist als derjenige Huxleys, wird dieser in der transhumanistischen Geschichtskonstruktion ganz bewusst ausgeblendet. ${ }^{26}$ Hughes und More sowie der deutsche Transhumanist Stefan L. Sorgner führen ihn gar nicht auf und Bostrom stellt lakonisch fest: »However, while these ideas might appeal to those who fancy a marriage between mysticism and science, they have not caught on either among transhumanists or the larger scientific community. « ${ }^{27}$

Pierre Teilhard de Chardin fokussierte nach seinem Eintritt in den Jesuitenorden, der Priesterweihe und seinem ausgedehnten Studium der Theologie, Philosophie und verschiedener Naturwissenschaften sein wissenschaftliches Interesse immer stärker auf die Paläontologie, insbesondere die Vorgeschichte des Menschen. Schon seine frühen, teils mystisch anmutenden Schriften während und nach dem Ersten Weltkrieg, den er als Sanitäter erlebte, sind geprägt von dem Gedanken einer göttlich gelenkten Evolution des Kosmos, den er in den folgenden 40 Jahren seines wissenschaftlichen und theologischen Schaffens weiterentwickelte. Trotz zahlreicher weltlicher Ehrungen wie der Ernennung zum Ritter der französischen Ehrenlegion (1947) und zum nichtresidierenden Mitglied des Institut de France (1950) untersagte der Vatikan weitgehend die Veröffentlichung von Teilhards philosophischem und theologischem Werk. Die Anerkennung und Weiterführung der darwinistischen Evolutionstheorie erschien der römischen Zensur und seinem Orden damals zu progressiv.

Trotz des Publikationsverbotes waren Teilhards Ideen durch seine Vortragstätigkeit und umfassende Korrespondenzen insbesondere in philosophischen und naturwissenschaftlichen Kreisen soweit bekannt, dass noch im Todesjahr die Veröffentlichung einer Werkausgabe unter dem Patronat vieler prominenter Wissenschaftler (allen voran Julian Huxley, Arnold Toynbee und Adolf Portmann) begonnen wurde. Parallel zum zweiten Vatikanischen Konzil (1962-1965) setzte die weit über die katholische Kirche hinausgehende Rezeption von Teilhards Lebenswerk ein. Schon Ende der 1970er Jahre wiesen verschiedene Bibliographien mehr als 10.000 Titel an Sekundärliteratur über Teilhard auf und innerhalb des New Age war er die wichtigste Referenz für die Autorinnen und

26 Auch in der wissenschaftlichen Darstellung des Trans- und Posthumanismus würdigen nur Heil (2018, 68-77) und Krüger (2004a, 335-347) die Rolle Teilhards.

27 Bostrom 2005, 8. 
Autoren dieser Bewegung. Sein Eintreten für die Eugenik wird in dieser breiten Rezeption seines Werkes verschwiegen. ${ }^{28}$

Was Teilhards Ideen so besonders macht, ist die Verbindung zwischen christlicher Heilsgeschichte, dem Evolutionsgedanken und einer kosmischen Perspektive. Als gottgewollten Plan betrachtet er den Aufstieg des Lebens aus der toten Materie und die Entstehung von Bewusstsein und Denken seit den ersten Hominiden. In diesem dynamischen Gottesbild entfaltet sich Gott selbst mit seiner Schöpfung bis diese am Ende der Zeit wieder mit ihm im Punkt Omega konvergiert. Die Relativierungen, die die katholische Lehre durch Kopernikus und Darwin erfahren musste, sind damit aufgehoben. Innerhalb des Posthumanismus baut der Ansatz von Frank Tipler vollständig auf Teilhards Kosmologie auf, die auch Hans Moravec teilt. Ray Kurzweil übernimmt in seinem Verständnis der Singularität ebenfalls alle wesentlichen Elemente von Teilhards universalem Entwurf der Heilsgeschichte. Die theologischen Anteile werden dabei mal mehr und mal weniger betont. ${ }^{29}$

Der kurze Einblick in die Vorgeschichte des Transhumanismus hat vor allem die normativen Mechanismen der transhumanistischen Traditionsbildung offengelegt. Während die Rolle von religionskritischen Denkern wie J. B. S. Haldane, John D. Bernal und Julian Huxley hervorgehoben wird, wird der Einfluss theologischer Autoren wie Teilhard de Chardin systematisch ausgeblendet, obwohl sie für Frank Tipler und alle Formen des religiösen Transhumanismus zentral sind. Diese religiösen Anteile, die auch in der Dante-Rezeption des Begriffes transhuman präsent sind, werden von Hughes, Bostrom und More in ihrem Bemühen um Seriösität und Glaubwürdigkeit offenbar als Störfaktor ihrer säkularen und wissenschaftlichen Ausrichtung des Transhumanismus wahrgenommen.

\subsection{Der frühe Transhumanismus: Ettinger, FM-2030, Leary}

Während sich andere Denker noch mit Einzelaspekten des wissenschaftlichen und technischen Fortschrittes befassten, formulierten seit Beginn

${ }^{28}$ Die bedeutende New Age-Autorin Marylin Ferguson präsentiert in ihrem Buch The Aquarian Conspiracy (1980) eine Untersuchung, die aufzeigt, dass Teilhard de Chardin der am häufigsten zitierte Denker unter allen New Age-Anhängern ist. Vgl. Ferguson 1980, 50-51, 93, 420; Daecke 2000; Leary \& Sirius 1997, 47.

29 Vgl. Kapitel 6.7. 
der 1970er Jahre vor allem zwei amerikanische Autoren eine ausgefeilte Futurologie, die bereits das umfasste, was später einmal als Transhumanismus bezeichnet werden würde. Die Rede ist vom Kryonik-Begründer Robert C. W. Ettinger (1918-2011) und vom kalifornischen Futuristen Fereidoun M. Esfandiary (1930-2000).

Ettingers Bedeutung für den Transhumanismus lag nicht allein in seinem Engagement für die Kryonik, sondern ebenso in der Ausarbeitung einer allgemeinen transhumanistischen Zukunftsvision. 1964 veröffentlichte er sein prophetisches Buch The Prospect of Immortality, das die Überwindung des Todes durch Einfrieren der Verstorbenen verspricht. ${ }^{30}$ Aber schon 1972 band er die Kryonik in seinem Werk Man into Superman in eine umfassendere Utopie ein. Hier und an anderer Stelle forderte und prophezeite er den Anbruch eines transhumanen Zeitalters. Seit dem ersten Erscheinen von Kultur sieht Ettinger den Menschen auf dem Weg der Befreiung aus der unintelligenten, natürlichen Evolution und auf dem Weg zum superhuman und zur transhumanity. ${ }^{31}$

On the level of repair work and prostheses there has indeed been notable success, mostly in recent times. With our eyeglasses, gold inlays and birth control pills we are substantially superhuman; we have transcended the apparent limitations of our design, without even taking into account our vehicles and other machinery. But the basic design has not been noticeably improved. ${ }^{32}$

Ettinger betrachtet es als wissenschaftliche Notwendigkeit, den Menschen neu zu erschaffen:

Thus humanity itself is a disease, of which we must now proceed to cure ourselves ... To be born human is an affliction ... To do this, it must first be shown that homo sapiens is only a botched beginning; when he clearly sees himself as an error, he may not only be motivated to sculpt himself, but to make at least a few swift and confident strokes. ${ }^{33}$

Denn die Evolution habe den Menschen und all ihre biologischen Schöpfungen mit vielerlei Fehlern ausgestattet - nicht aus Bosheit oder Unvermögen, sondern weil das Entwicklungsprinzip der Evolution einfach nicht an der Hervorbringung der optimalen Lebensformen inter-

${ }^{30}$ Vgl. Ettinger 1964. Die Geschichte und der kulturelle Kontext der Kryonik werden gesondert in Kapitel 6.5.4 behandelt.

31 Vgl. Ettinger 1989, 29, 35, 101, 110f., 116, 127, 129, 162.

32 A.a.O., 10.

33 A.a.O., 11. 
essiert sei. Vielmehr steuerten Zufälle und weitreichende Kompromisse mit den jeweiligen Umweltbedingungen das Überleben und Aussterben der Arten - das Leben entstammt in den Worten Ettingers daher einer »Mülltonne«. ${ }^{34}$

The purely physical shortcomings of the human animal are legion, and we need only tick off a few, most of them well known. The worst weakness of all, of course, we share with every other large animal: the susceptibility to degenerative disease, senile debility, and death from old age. A less critical, but still serious, defect is our manner of gestating and bearing children, which represents not only inconvenience but danger to the mothers. If some of us temporarily choose to regard these as »natural, « there are plenty of others that are clearly pathological. ${ }^{35}$

Den Ausweg aus dieser Katastrophe der natürlichen Evolution, deren unrühmliches Ergebnis der Mensch sei, erblickt Ettinger zunächst in der Formulierung einer neuen Philosophie, die den Weg zur Verwirklichung eines unsterblichen Übermenschen ebne.

It should be amply clear by now that the immortal superman represents not just a goal, but a way of life, a world-view only partly compatible with today's dominant ideologies. We might call this fresh outlook the new meliorism, of which the cryonics or people-freezing program is an important current element. ${ }^{36}$

Unter Berücksichtigung einer möglichst weiten anthropologischen Basis entwickelt Ettinger das Bild eines Übermenschen, der die maskulin-heroischen Eigenschaften des sumerischen Helden Gilgamesch, des fiktiven Detektivs Sherlock Holmes, von Nietzsches philosophischem Übermenschen und den Superhelden diverser Science-Fiction Geschichten in sich vereint. Der künftige Mensch soll dabei bloß die schon vorhandenen Eigenschaften biologischer Lebewesen kombinieren und erweitern: Er soll gegen Kälte und Hitze unempfindlich sein wie Angehörige einiger Indianerstämme; er soll beliebig seine Hautfarbe wechseln können; er soll in der Lage sein, so viel zu essen und zu genießen, wie er will, da Übergewicht »repariert« werden könne. Der gesamte Körper soll mit einer stabilen, aber flexiblen Hautrüstung ausgestattet werden, während gleichzeitig einige aktive Verteidigungsorgane (elektrische Organe oder eine Art Flammenwerfer) die Sicherheit dieses transhumanen Lebewesens gewährleisten sollen. Die Form des Körpers könne dabei vom flie-

\footnotetext{
${ }^{34}$ Vgl. a.a.O., $13 \mathrm{ff}$.

35 A.a.O., 17.

36 A.a.O., 174.
} 
genden Batman bis hin zum schwimmenden Aquaman, vom Zwerg bis zum Riesen und Cyborg variieren. Im Laufe der Jahrtausende werde das angesammelte Wissen jedoch ein enormes Wachstum unserer Gehirne mit sich bringen. ${ }^{37}$

Tons of brain tissue? Of course: doubtlessly, some irreducible minimum amount of matter, in mass and volume, is required to store a unit of information, and if we jettison no memories, we must become gigantic. Even storing »our« memories in a separate mechanical store or computer, plugged in at will, cannot avoid giantism for several reasons. In any case, we should not want to avoid giantism - it is our salvation with respect to the accidental death bogey. ${ }^{38}$

Vollkommen neu gestalten will Ettinger auch das ihm erbärmlich erscheinende, menschliche Sexualleben - es sollen sowohl neue Geschlechter als auch neue Genitalien konstruiert werden, die zudem nach Belieben austauschbar wären. Frauen will Ettinger von der »Krankheit des Gebärens« befreien, die den weiblichen Körper fast neun Monate lang in einen Sklavenstatus degradiere und danach in der Zeit des Stillens zur biologischen Maschine herabsetze. Sexualität werde von der Last der Fortpflanzung befreit und diene nur noch dem immerwährenden Superorgasmus. ${ }^{39}$

The sexual superwoman may be riddled with cleverly designed orifices of various kinds, something like a wriggly Swiss cheese, but shapelier and more fragrant; and her supermate may sprout assorted protuberances, so that they intertwine and roll all over each other in a million permutations of The Act, tireless as hydraulic pumps. (We may have hydraulic pumps, if we are cyborgs.) A perpetual grapple, no holes barred, could produce a continuous state of multiple orgasm. ${ }^{40}$

Die für die heutigen Extropianer typische Verbindung von wirtschaftlichem Liberalismus und Transhumanismus formulierte auch bereits Ettinger, denn er konstatiert zu Beginn der 1970er Jahre, dass die Umweltverschmutzung nicht so gravierend sei, wie es scheine, und dass das Wohlergehen des Menschen schließlich wichtiger sei als das Überleben einiger unwesentlicher Tierarten und Blumen - zudem könne man wohl

\footnotetext{
37 Vgl. a.a.O., 22-66.

38 A.a.O., 61.

39 Vgl. a.a.O., 66-89.

40 A.a.O., 68.
} 
schon bald ausgestorbene Tiere selbst aus Fossilienfunden wieder vollständig rekonstruieren und reanimieren.

Immortality costs money: to make it as individuals, we must earn and save substantial amounts; to make it as a society, we must increase the GNP [Gross National Product: OK], and rapidly. The notion that we can enjoy the fruits of labor without first laboring is a pollution of the mind, and it is this pollution which is the greater threat. ${ }^{41}$

Dieses Verständnis vom immerwährenden Fortschritt als Aufgabe des Menschen betrachtet Ettinger in voller Übereinstimmung mit der christlichen Heilsgeschichte:

The Christians among us are not rebelling against God nor aspiring to equality with him (if such a thing were conceivable); they seek rather to become his more effective tools, his worthier stewards. Neither do we seek endless change just for the sake of change; we pursue intermediate goals on what we hope will be an ascending road, a road perhaps some day leading to the Celestial City - wherever and whatever that may be. Does not Christianity need supermen? Can any but a superman be a complete Christian ${ }^{42}$

Parallel zu Ettinger, jedoch mit stärkerem politischen Akzent, ersann Fereidoun M. Esfandiary die Zukunft des Menschen. Der in Belgien geborene Sohn eines iranischen Diplomaten hatte schon als Elfjähriger in mehr als einem Dutzend Ländern gelebt, durfte sein Heimatland sogar 1948 bei den olympischen Spielen in London vertreten und arbeitete später als Schriftsteller, als Mitglied der UN-Beratungskommission für Palästina und als Dozent an der University of New York. Waren seine frühen Romane zwar mitunter fiktionalen Charakters (The Day of Sacrifice, The Beggar, Identity Card), ${ }^{43}$ so entwarf er in den nachfolgenden Monographien Optimism One. The Emerging Radicalism (1970) und Up-Wingers (1973) eine politisch-technizistische Utopie, die dermaßen futuristisch war, dass Esfandiary 1989 seinen Namen in FM-2030 ändern ließ: $:^{44}$ »The name 2030 reflects my conviction that the years around 2030 will be a magical time. The solar system will be alive with people linking in

41 A.a.O., 133.

42 A.a.O., 155.

43 Vgl. Esfandiary 1959, 1965, 1966. The Day of Sacrifice wurde auch ins Deutsche übersetzt: Der Opfertag. München 1962.

44 Vgl. zur Biographie Esfandiarys: West 2000. Einer breiteren Öffentlichkeit wurde dieses Namensspiel von Transhumanisten durch Dan Browns Roman Inferno bekannt. Vgl. Brown 2013. 
and out of planets and moons and orbital communities. In 2030 we will be ageless and everyone will have an excellent chance to live forever. 2030 is a dream and a goal. ${ }^{45}$

In seinem späteren Buch Are you a transhuman? von 1989 stellt Esfandiary den Kerngedanken seiner radikalen Fortschrittsutopie noch einmal deutlich heraus. Erst hier rückt der Begriff transhuman ins Zentrum einer seiner Texte. ${ }^{46}$

The most urgent problem facing us is not social - economic - political. The most pressing problem facing us *all* everywhere is death. All other human constraints are derivative. So long as there is death no one is free. So long as there is death we cannot upgrade the basic quality of life. The elimination of death has never been on anyone's agenda because throughout the ages we were never able to do anything about it ... Immortality is now a question of when - not if. The elimination of death will not do away with problems. It will take away the tragedy in human life. Once we attain immortality everything will be possible. ${ }^{47}$

Die Grabenkämpfe zwischen dem linken und rechten politischen Flügel sollten beendet werden, so dass sich alle politischen Kräfte als Up-Wingers gemeinsam einer viel größeren Aufgabe widmen können: »Even more profound evolutionary changes are now evident. We are striving to deanimalize our species - debiologize intelligence - deplanetize. ${ }^{48}$ So müsse nun der Körper des Menschen vollkommen neu erschaffen werden (redesign), indem die zufälligen Werke der natürlichen Evolution durch die von Vernunft geleitete Schöpfung des Menschen ersetzt werden. Denn bisher sind wir laut Esfandiary nur prehuman, aber das transhumane Zeitalter werde dem Wassermann-Zeitalter folgen. ${ }^{49}$

Eine Vielzahl technischer und sozialer Aspekte vereinigt Esfandiary in seiner transhumanen Utopie, die inzwischen an Aktualität gewonnen haben. So erwartet er die beginnende Kolonisierung des Weltraums, prophezeit die Entwicklung eines interaktiven, allgegenwärtigen Kommunikationssystems (telespheres) ${ }^{50}$ und fordert, dass mit Hilfe gentech-

45 Aus einem Interview von Bircan Unver mit Flora Schnall, einer langjährigen Kollegin und Freundin von Esfandiary vom 18.10.2000. Verfügbar unter: https://www.lightmillennium.org/fall/fm_interviewpart1.html. Vgl. auch Jordan \& Frewer 2010a, $155 f$.

46 Nur an einer peripheren Stelle spricht Esfandiary in Up-Wingers von künftigen transhumans $(1973,79)$. Ettingers Werk ist Esfandiary seit 1970 bekannt, vgl. Esfandiary $1970,72$.

47 FM-2030 1989, 116.

48 Ebd.

49 Vgl. Esfandiary 1973; Lavery 1992, 76ff.

50 Vgl. Esfandiary 1977. 
nologischer Methoden nur noch ausgewählte Samen- und Eizellen mit besten Erbanlagen zur Fortpflanzung freigegeben werden (collaborative procreation) ${ }^{51}$

Die Lösung wirklich aller Probleme sieht Esfandiary in der Verbreitung und Weiterentwicklung von Technik:

We no longer only strive for better schools, more teachers, better textbooks. Schools, colleges, and textbooks are becoming anachronisms. We need more and more communication satellites, lasers, and magnetic tapes to transmit knowledge and information to every individual anywhere on the planet. Literacy itself is no longer a prerequisite for social progress. What will literacy mean in a world where instantaneous global communication has replaced the written word? ${ }^{52}$

Das transhumane Zeitalter sei vor allem gekennzeichnet durch einen unermesslichen Zuwachs an menschlicher Macht. So präsentieren sich die frühen Schriften des Transhumanismus hauptsächlich als menschliches Sehnen nach Omnipotenz:

We are no longer content with simply diverting the course of rivers, reclaiming seas and deserts, creating islands, producing rain, harnessing solar energy. All this is now increasingly commonplace. We want to make alterations in the universe. We want to reclaim more planets, create new moons, nudge old ones to more suitable orbits, harness the life-bestowing energies of more giant suns. ${ }^{53}$

Den Vorwurf, in seinen transhumanistischen Werken eine Selbstvergötterung des Menschen zu propagieren, übertrifft Esfandiary jedoch noch mit seiner Forderung: »We humans do not want to play god or to be god. We aspire to much more. $\ll^{54}$

Fereidoun Esfandiary und Robert Ettinger formulierten bereits in den 1970er Jahren Ziele und Wege des heutigen Transhumanismus. So unterschiedlich ihre Ansätze im Detail auch sind, sie fokussierten doch beide quasi als Brennpunkt und Motivation ihres Fortschrittsgedankens die menschliche Sterblichkeit und strebten die damit verbundene Überwindung des biologischen Menschen an. Auch kommt der bei Julian Huxley schon präsente Gedanke zum Vorschein, dass der wissende Mensch der Gegenwart sich aus der zufälligen, natürlichen Evolutionsgeschichte befreien müsse.

51 Vgl. FM-2030 1989, 118.

52 Esfandiary 1970, 83.

53 Ebd.

54 Esfandiary 1973, 143. 
Beide Denker haben mit ihren Büchern und Aufsätzen sicherlich großen Anteil an der Verbreitung transhumanistischer Ideen gehabt, jedoch war Esfandiary darüber hinaus maßgeblich an der Institutionalisierung der transhumanistischen Bewegung in den Vereinigten Staaten beteiligt, wo er die sich formierenden Extropianer mit Beiträgen auf Konferenzen und in Zeitschriften unterstützte und bis heute als der große Vordenker des Transhumanismus verehrt wird. Die Rolle Ettingers als Begründer der Kryonik wird in der transhumanistischen Literatur durchaus gewürdigt. Seine Prägung des Begriffes transhuman jedoch, die derjenigen Esfandiarys fast zwei Jahrzehnte vorausgeht, wird kaum zur Kenntnis genommen. Man kann darüber spekulieren, ob nicht viele der comicgleichen Schilderungen von Ettingers superman und superwoman selbst den heutigen Transhumanisten zu bizarr anmuten und damit das Bild einer wissenschaftlich fundierten Gesellschaftsutopie gefährden würden. ${ }^{55}$

Auch wenn er sich nie als Vertreter einer rückhaltlosen Technikeuphorie betrachtet hat, darf der Psychologe Timothy Leary (1920-1996) als herausragende Gestalt der Hippie-Bewegung nicht unerwähnt bleiben. Er trat gegen die Unterdrückung des Menschen durch den technischen wie auch politischen Apparat während der sozialen Perspektiv- und Sinnlosigkeit ein, die Präsident Dwight D. Eisenhower und der Vietnamkrieg hinterlassen hatten. Seine psychologischen Experimente mit LSD und anderen bewusstseinserweiternden Substanzen sollten erste Schritte auf dem Weg zu einer größeren Freiheit des Menschen darstellen, während er in seinen späteren Lebensjahren ein sich auch publizistisch niederschlagendes, reges Interesse für die Möglichkeiten der »digitalen Unsterblichkeit«, der Nanotechnologie und Kryonik an den Tag legte. Entschied er sich persönlich für die Kremation seines Leichnams und nicht etwa für dessen kryonische Präservation, so war er doch in Kalifornien ein prominenter Mittelpunkt innerhalb der transhumanistischen Avantgarde. ${ }^{56}$ Spätestens mit seinem Buch Chaos and Cyber Culture von 1994 verschrieb sich Leary einer posthumanistischen Agenda, die enthusiastisch das kommende Zeitalter feiert:

We are mutating into another species - from Aquaria to the Terrarium, and now we're moving into Cyberia. We are creatures crawling to the center of the cyber-

55 Vgl. More 2013b, 11; Bostrom 2005, 10f.; Hughes 2004, 30, 160.

56 Vgl. Leary \& Sirius 1997, 7-10, 143-174; Lavery 1992, 30f.; Stephenson 2014, 281-289. 
netic world. But cybernetics are the stuff of which the world is made. Matter is simply frozen information. ${ }^{57}$

Im Austausch mit seinem Freund Marshall McLuhan entwickelte Leary die Vorstellung, dass das Gehirn wie die neuen Medien letztlich durch elektrischen Strom die Sinneseindrücke der Umwelt verarbeitet. Wie Moravec platziert Leary zu Beginn seines Buches eine Graphik zur Acceleration of Brain Power mit Hilfe von Fernsehen, PCs, virtueller Realität etc., die schließlich eine neue Spezies (new breed) hervorbringen werde. Der homo sapiens electronicus werde kein höriger Arbeiter mehr sein, sondern der Computer werde ihm die Freiheit zu Selbstverwirklichung und unermesslichem Vergnügen vermitteln:

Owning it defines you as member of a new breed postindustrial, postbiological, post-human because your humble VM (Volks-Mac) permits you to think and act in terms of clusters of electrons. It allows you to cruise around in the chaotic post-Newtonian information ocean, to think and communicate in the lingua franca of the universe, the binary dialect of galaxies and atoms. Light. ${ }^{58}$

Leary war auch ein wichtiges Bindeglied zu Vertretern des Space Age, die für die baldige Kolonisierung des Weltraums eintraten. Ein Argument für die Überwindung des heutigen Menschen ist aus Sicht des Posthumanismus diese kosmische Expansion, für die der biologische Mensch ungeeignet sei. David Lavery hat in seinem Buch Late for the Sky eindrücklich demonstriert, wie sich aus dem vom NASA-Ingenieur Krafft Ehricke formulierten »extraterrestrischen Imperativ« die Obsoletheit des Menschen ableiten ließ. Die Weltraumfuturistin Barbara Marx Hubbard verkündete daraufhin, dass es nun an der Zeit sei, den menschlichen Körper vollkommen, wunderschön und weltraumtauglich zu konstruieren. ${ }^{59}$

Als Katalysator dieser Weltraumvision bildete sich 1975 die so genannte L5 Society und konnte große Aufmerksamkeit in der amerikanischen

57 Leary 1994, VII.

58 A.a.O., 45. Vgl. a.a.O., 45-50; Stephenson 2014, 289-291.

59 Vgl. Lavery 1992, 13f., 48, 80f. Manfred Clynes und Nathan Kline hatten 1960 den Begriff Cyborg aus cybernetic organism gebildet - als Bezeichnung für ein »artificially extended homeostatic control system functioning unconsciously « - also ein sich selbst regulierendes Mensch-Maschine-System. Sie spekulierten auch darüber, den Menschen mit zusätzlichen Implantaten und Drogen auszurüsten, so dass er sich ohne Raumanzug im Weltraum bewegen könne. Vgl. Clynes \& Kline 1960, 27; Gray 2002, $18 f$. 
Öffentlichkeit erlangen - ebenso wie das Buch The High Frontier des Princeton Physikers Gerard K. O'Neill. Er entwickelte hier die Idee einer orbitalen Kolonie der Menschheit, die an einem stabilen Punkt zwischen Erde und Mond errichtet werden sollte. ${ }^{60}$ Frühe Mitglieder dieser Gesellschaft, die in ihren besten Zeiten an die 10.000 Anhänger hatte, waren der Nanowissenschaftler Eric K. Drexler, die Science-Fiction Schriftsteller Isaac Asimov und Robert Heinlein und neben Timothy Leary zwei zentrale Figuren des technologischen Posthumanismus: Hans Moravec und Marvin Minsky. ${ }^{61}$

Anders als die späteren Posthumanisten traten aber Ettinger, Esfandiary und Leary weder für die Ablösung des Menschen durch künstliche Intelligenzen als Träger der künftigen Evolution ein, noch entwarfen sie eine technische Lösung des menschlichen Mortalitätsproblemes, wie sie in den 1980er Jahren von Hans Moravec und anderen entwickelt wurde. In der heutigen transhumanistischen Bewegung fügen sich die Ideen Ettingers, Esfandiarys, Learys und die der posthumanistischen Denker zu einer neuen Synthese.

Wichtige Foren für den frühen Transhumanismus waren ab den 1980er Jahren auch die beiden technophilen Zeitschriften bOING bOING (1988 bis 1997) und Mondo 2000 (auch unter den Titeln High Frontiers / Reality Hackers, 1984-1998), wobei letztere von R.U. Sirius und zeitweise zusammen mit Timothy Leary und der Hackerin St. Jude (d.i. Judith Milhon) herausgegeben wurde. Die Zeitschrift mit dem frühen Untertitel A Space Age Newspaper of Psychedelics, Science, Human Potential, Irrevence and Modern Art verband die einstige Hippie-Rebellion, Anarcho-Liberalismus, New Age-Begeisterung, Antiintellektualismus und zukunftseuphorischen Cyberpunkutopien mit Themen aus der Popkultur, Kryonik, Kunst und der transhumanen Lebensart. ${ }^{62}$

Die Inhalte dieser Zeitschriften verweisen bereits auf die fließenden Übergänge der verschiedenen, technikorientierten Diskurse. So finden sich viele Visionen der Posthumanisten und Transhumanisten bereits in den Ideen der stark von der Cyberpunk-Literatur beeinflussten Subkul-

60 Dieser Punkt, an dem sich die Gravitationskräfte zwischen Erde und Mond aufheben, wird nach dem französischen Astronomen Joseph-Louis Lagrange (1736-1813) als Lagrange Point 5 bezeichnet - daher der Name der vom Kryoniker Keith Henson begründeten L5 Society. Vgl. O’Neill 1977. Deutscher Titel: Unsere Zukunft im Raum. Stuttgart 1978.

61 Vgl. Dery 1996, 36; Regis 1992, 62-68.

62 Vgl. Dery 1996, 31-41; Freyermuth 1998, 41-44; Graham 2002, 157f.; Rutsky 1999, 1. 
tur der Hacker wieder, als deren Vertreterin beispielsweise St. Jude einer breiteren Öffentlichkeit bekannt wurde. Jenseits des naiven Zukunftsoptimismus vieler Transhumanisten werden hier meist mit kritischem Blick Modifikationen des Körpers (body hack), des Gehirns (brain hack) und der Sinne (sensory hack) diskutiert. ${ }^{63}$

\subsection{Das Extropy Institute und die (Vita-)Mores}

Die Brücke vom frühen Transhumanismus zur Bewegung der Jahrtausendwende bildet die Künstlerin Natasha Vita-More, d.i. Nancie Clark (*1950), die eng mit Esfandiary verbunden war. Zusammen mit dem »High-Priest« der amerikanischen psychodelic movement, Timothy Leary, begründeten die drei 1982 die transhumanistische Kunst (Transart) in Los Angeles. Vita-More formuliert im Folgejahr das Transhuman Manifesto, um Perspektiven des künftigen Menschseins aufzuzeigen. ${ }^{64}$

We are transhumans.

Transhumans integrate the most eminent progression of creativity and sensibility merged by discovery.

Transhumans want to elevate and extend life.

Transhumans seek to expand life.

As our tools and ideas continue to evolve, so too shall we.

We are designing the technologies to enhance our senses and increase our understanding.

The transhumanist ecology and freedom exercises self-awareness and self-responsibility.

Let us choose to be transhumanist not only in our bodies, but also in our values.

Toward diversity, multiplicity.

Toward non-partisan ideology (transpolitics, transpartisan, transmodernity).

Toward transhuman rights of morphological freedom, existence safety, personhood preservation.

Toward a more humane transhumanity. ${ }^{65}$

Auf einer Party von Timothy Leary lernte Vita-More ihren späteren Ehemann, Max More, kennen, mit dem sie bis heute den kalifornischen Transhumanismus prägt. Von 2010 bis 2018 fungierte sie als geschäftsführende Direktorin von Humanity+, der Nachfolgeorganisation der

63 Vgl. Dery 1996, 198.

64 Vgl. Vita-More 2000a.

65 Vita-More 1983. Zur Kunst vgl. Kapitel 5.3. 
World Transhumanist Association. Zusammen mit Max More edierte sie 2013 den Transhumanist Reader, eine Sammlung historischer und programmatischer Beiträge. $^{66}$

Max More (*1964), vormals Max Terrence O’Connor, studierte Philosophie in Oxford und traf am College mit anderen Zukunftseuphorikern zusammen, darunter auch Garret Smyth. Begeistert von der amerikanischen Zeitschrift Cryonics gründeten die Freunde 1986 die englische Kryonik-Organisation Mizar Limited, den Vorläufer der späteren Alcor $U K L t d{ }^{67}$ Im Folgejahr siedelt Max O'Connor nach Kalifornien über, nennt sich fortan Max More und entwickelt »seine Philosophie«:

I wanted to develop a way of thinking that would wake up the world to the possibilities of the future and help to stretch the minds of humanity ... Frankly I was pissed off at death and people who were aging and dying and no one seemed to do anything about it. I was frustrated that we still stuck on this planet ... My ideas have merged into a clear vision, I now set out to bring together the finest minds to join me in shouting out to the world »Wake up! The future can be better than you ever imagined. ${ }^{68}$

Zusammen mit einem Anwalt mit dem Künstlernamen T. O. Morrow (d.i. Tom W. Bell) gründete Max More 1988 das Magazin Extropy. Vaccine for Future Shock, das seinen Untertitel nach den ersten Ausgaben in Journal for Transhumanist Thought änderte. Der Begriff Extropy geht dabei auf T. O. Morrow zurück und soll das Gegenteil von Entropie, dem unvermeidlichen Wärmetod des Universums, ausdrücken. Extropie symbolisiere daher den unerschütterlichen Lebenswillen der Extropianer. ${ }^{69}$ Ohne Bezug auf den früheren Wortgebrauch durch Julian Huxley verfasste More 1990 den knappen Aufsatz Transhumanism. Towards a Futurist Philosophy, der dennoch viele Ähnlichkeiten mit Huxleys Ansatz erkennen lässt. ${ }^{70}$ More kritisierte darin mit Verweis auf Karl Marx, Ludwig Feuerbach und Friedrich Nietzsche die Religion als Kraft der Entropie, die ihre Macht auf Angst (vor dem Tod) und sozialer Kontrolle aufbaut.

66 Vgl. More \& Vita-More 2013.

67 Vgl. zur Geschichte des Extropy Institute: Freyermuth 1996; Freyermuth 1998, 195-258; More 2013b, 12.

68 More 2000a, 2.

69 Vgl. Kapitel 6.3.1.

70 Die Unabhängigkeitserklärung von der Natur sowie die Überwindung von Religion mit einem planbaren Fortschritt teilen Huxley und More miteinander. Vgl. More 2013b, 8f.; Huxley 1923, XIII; Huxley 1929. 
Dem setzte er den Extropianismus als höchste Form des Transhumanismus entgegen:

The goal of religion is communion with, or merely serving, God, a being superior to us. The Extropian goal is our own expansion and progress without end ... God was a primitive notion invented by primitive people, people just beginning to step out of ignorance and unconsciousness ... Our own process of endless expansion into higher forms should and will replace this religious idea ... No more gods, no more faith, no more timid holding back. Let us blast off our old forms, our ignorance, our weakness, and our mortality. The future is ours. ${ }^{71}$

Max More wurde 1995 an der University of Southern California mit einer Dissertation im Fach Philosophie bei Janet Levin promoviert, wobei seine Doktorarbeit The Diachronic Self: Identity, Continuity, Transformation im Grunde das Fundament einer posthumanistischen und kryonischen Identitätstheorie entwirft. ${ }^{72}$ Seine Lebensaufgabe sieht More vor allem in der philosophischen Zusammenführung der disparaten technischen Innovationen und naturwissenschaftlichen Denkansätze in einer ganzheitlichen Futurologie:

Extropianismus ist die erste neue Philosophie nach dem Scheitern der traditionellen Denksysteme am Ende dieses Jahrhunderts ... Wir entwickeln die erste systematische Philosophie für das nächste Millennium. Wir sind die neue Aufklärung. ${ }^{73}$

1999 gründete More einen kurzlebigen Nachrichtensender, der ausschließlich positive Nachrichten über neue Psychotechniken und Erfindungen verkündete, die von der Weiterentwicklung der menschlichen Spezies zeugen. ${ }^{74}$ Als impulsiver Anhänger von Fitnesstraining und Bodybuilding warnte Max More jedoch davor, sich ausschließlich auf die technischen Möglichkeiten der Körper- und Geistverbesserungen zu verlassen, denn bis in einigen Jahren die Implantate und Medikamente zur Verfügung stünden, müsse man seinen Körper auf konventionelle Weise fit halten. Der Transhumanismus sei daher keineswegs eine körperverachtende Philosophie. ${ }^{75}$

More 1996b, 4f.

Vgl. More 1995a, 24ff.

Max More im Gespräch mit Gundolf Freyermuth: Freyermuth 1998, 253.

74 Vgl. Freyermuth 1998, 214.

75 Vgl. a.a.O., 198-203. 
Kennzeichnend für einen Extropianer sei primär sein »dynamischer Optimismus«, der Erfahrungen positiv bewertet und so positive Ergebnisse herbeiführt, wobei More hier offensichtlich die Philosophie des Positive Thinking von Joseph Murphy und Norman Vincent Peale rezipiert, zumal auch die Gesundheit, das Lebensgefühl und beruflicher Erfolg von diesem Optimismus positiv beeinflusst werden könnten: »DYNAMIC OPTIMISM is an active, empowering, constructive attitude that creates conditions for success by focusing and acting on possibilities and opportunities. ${ }^{76}$ Die extropianischen Prinzipien sollten die stets zu verbessernde und zu erweiternde Grundlage der Philosophie des neuen Jahrtausends bilden:

\section{Immerwährender Fortschritt (Perpetual Progress)}

Nur ein anhaltender Fortschrittsprozess garantiere, dass die Evolution unter der Verwendung von Wissenschaft und Technik nicht stagnieren, sondern auch den Menschen zu immer höheren Formen führen werde, die seine biologischen und geistigen Fähigkeiten unaufhörlich erweitern.

\section{Selbsttransformation (Self-Transformation)}

Unter Verwendung von Technik und Drogen solle auf individueller Ebene eine stetige Verbesserung von psychischen, physischen, intellektuellen und ethischen Qualitäten erreicht werden.

\section{Praktischer Optimismus (Practical Optimism)}

Statt entropische Haltungen wie Kleinmut, Zukunftsangst und Resignation an den Tag zu legen, erfordere der praktische oder dynamische Optimismus einen ungebremsten Tatendrang als Fortsetzung des Evolutionsprozesses und zur Verwirklichung der extropianischen Ziele im Diesseits: »Where others say enough is enough, we say Forward! Upward! Outward! «77

\section{Intelligente Technik (Intelligent Technology)}

Technik als Ausdruck des menschlichen Intellektes und Willens solle als Fortführung der natürlichen Evolution den Menschen zu mehr Freiheit führen.

\section{Offene Gesellschaft (Open Society)}

Mit dem Modell einer offenen Gesellschaft, das auf Karl Poppers in den USA stark rezipiertes Werk Die offene Gesellschaft und ihre Feinde zurückzugreifen scheint, zumal More auf den kritischen Rationalismus anspielt, tritt der Extropianismus für eine pluralistische, dezentrale und stets für

76 More 1998b, 1.

77 More 1998a, 3. 
Verbesserungen offene Gesellschaft ein. Auf diese Weise könne ein liberaler Staat seiner Hauptaufgabe, der Förderung von Glück und Fortschritt, am ehesten entsprechen.

\section{Selbstbestimmung (Self-Direction)}

Das Recht auf unabhängiges Denken und individuelle Freiheit soll fest verankert werden.

\section{Rationales Denken (Rational Thinking)}

Die Überlegenheit des rationalen Denkens und der Vernunft über den blinden Glauben und Dogmatismus werde anerkannt. ${ }^{78}$

Nicht nur durch seine programmatischen Ideen, sondern vor allem durch die Gründung des Extropy Institute im kalifornischen Marina del Rey (1991) war More bedeutend für die Institutionalisierung des Transhumanismus. Von hier aus organisierte er von 1994 bis 2001 fünf Konferenzen namens Extro und 2004 noch den Vital Progress Summit. Bekannte Post- und Transhumanisten präsentierten hier ihre philosophischen und technischen Beiträge: Hans Moravec (1994), Roy L. Walford (1995, 1999, 2004), Natasha Vita-More (1995, 1999, 2001, 2004), FM2030 (1995), Eric K. Drexler (1996), Marvin Minsky (1995, 1997, 2004), Vernor Vinge (1999), Ray Kurzweil (2001, 2004) sowie Aubrey de Grey (2004). Mit fünf Extropianern und Transhumanisten leitete More das Extropy Institute im Direktorium, darunter Anders Sandberg und Natasha Vita-More, während sich als wissenschaftliche Berater auch prominentere Vertreter wie Roy L. Walford, Marvin Minsky, Gregory Stock und Raymond Kurzweil betätigten. ${ }^{79}$

2006 wurden die Aktivitäten des Extropy Institutes eingestellt. Die Extropianer sind offenbar im allgemeinen Transhumanismus oder unter den Anhängern der Singularität aufgegangen. Die meisten Extropianer stammten aus den High-Tech-Zentren im Westen der USA, vor allem aus dem Silicon-Valley und fast alle Anhänger waren weiße Männer. Ökonomisch und politisch waren sie überwiegend Verfechter eines uneingeschränkten Liberalismus und vertrauten auf die fortschrittsbegünstigende Wirkung einer freien Konkurrenzwirtschaft - wodurch sie sich von anderen transhumanistischen Bewegungen unterschieden. ${ }^{80}$

\footnotetext{
78 Vgl. More 1998a.

79 Vgl. www.extropy.org.

${ }^{80}$ Vgl. Freyermuth 1998, 214; More 2000c, 1.
} 


\subsection{Die World Transhumanist Association / humanity+}

Etwas später als in den USA formierten sich auch die europäischen Transhumanisten in einem festen Gefüge von Organisationen. Das Zusammenfinden der Gleichgesinnten wurde durch verschiedene Diskussionsforen im Internet erst ermöglicht. Auf der ersten europäischen Transhumanismus-Konferenz Transvision98 im Juni 1998 in Weesp (Niederlande) fanden sich die nationalen Organisationen aus den Niederlanden (Transcedo) und Schweden (Transhumanististka Föreningen, ALEPH) zusammen und begründeten durch die Initiative von Nick Bostrom und David Pearce die World Transhumanist Association (WTA), die die Aktionen der nationalen Verbände in Zukunft koordinieren und forcieren sollte. Pearce und Bostrom waren zunächst die beiden Koordinatoren der WTA, von 2002 bis 2008 hatte dann Bostrom den Vorsitz im Board of Directors inne. ${ }^{81}$ Es folgte 1999 auch in Deutschland die Gründung von De:Trans als eingetragener Verein und 2004 die UK Transhumanist Association, die beide nur bis 2008 aktiv waren. ${ }^{82}$ In diesem Jahr änderte die WTA auch ihre Bezeichnung in humanity+, um den Menschen stärker ins Zentrum zu rücken. Nach Ben Goertzel führte Natasha Vita-More von 2010 bis 2018 die Organisation, deren Vorsitz jüngst wiederum Ben Goertzel übernommen hat. ${ }^{83}$ Goertzel (*1966) ist ein amerikanischer IT-Unternehmer, KI-Forscher und Buchautor, der den Transhumanismus insbesondere an die Tradition der russischen Kosmisten anbinden möchte. Unter den weiteren Transhumanisten, die prägend für WTA / humanity + sind oder waren, finden sich der amerikanische Soziologe James Hughes (*1961), der schwedische Bioinformatiker Anders Sandberg (*1972), die vielseitige Unternehmerin Martine Rothblatt (*1954), der Gerontologe Aubrey de Grey $\left({ }^{*} 1963\right)$ sowie der KI-Forscher Eliezer Yudkowsky $\left({ }^{*} 1979\right)$.

Die Transhumanist Declaration von 1998 beruht wesentlich auf Sandbergs, Pearce' und Bostroms Initiative. Die zentralen Sätze beinhalten die bekannten Forderungen:

81 Da Bostrom seine Leitungsfunktion in der WTA nicht auf seiner Webseite aufführt, wurden die Daten über die archivierten Webseiten der WTA (transhumanism.org) recherchiert.

82 Vgl. hpluspedia.org/wiki/UK_Transhumanist_Association. Laut dem Internetarchiv (archive.org) ist die letzte Präsenz der deutschen Homepage auf den 28.12.2008 datiert; die letzte Aktualisierung der niederländischen Webseite wurde 2013 vorgenommen (http://www.transcedo.org).

83 Vgl. humanityplus.org (und entsprechende Archivseiten). 
(1) Humanity will be radically changed by technology in the future. We foresee the feasibility of redesigning the human condition, including such parameters as the inevitability of aging, limitations on human and artificial intellects, unchosen psychology, suffering, and our confinement to the planet earth ...

(4) Transhumanists advocate the moral right for those who wish to use technology to extend their mental and physical capacities and to improve their control over their own lives. We seek personal growth beyond our current biological limitations. ${ }^{84}$

Die eingängige Definition des Transhumanismus, die Anders Sandberg 2004 formulierte, spiegelt diese wachstumsorientierten Werthaltungen exakt wider:

TRANSHUMANISM: Philosophies of life ... that seek the continuation and acceleration of the evolution of intelligent life beyond its currently human form and human limitations by means of science and technology, guided by life-promoting principles and values. ${ }^{85}$

Die Online-Zeitschrift der WTA / humanity+, das Journal of Evolution and Technology (bis 2001 unter dem Titel: Journal of Transhumanism), wird z.Z. vom australischen Schriftsteller und Philosophen Russel Blackford und von James Hughes herausgegeben. Im umfangreichen Herausgebergremium sind auch der Religionswissenschaftler William S. Bainbridge, Aubrey de Grey, der Ökonom Robin Hanson, David Pearce, Anders Sandberg, der Bio-Tech-Unternehmer Gregory Stock sowie der deutsche Transhumanist Stefan L. Sorgner aktiv. Kreisten die meisten Artikel zu Beginn um die Fragen, wann welche Zukunftstechnik zur Verfügung stehen werde, erhielten in den vergangenen Jahren ethische und soziale Fragen nach den potentiellen Folgen von Robotik, künstlicher Intelligenz und Human Enhancement mehr Aufmerksamkeit. ${ }^{86}$

Sorgner ist meines Wissens auch der einzige deutschsprachige Transhumanist bzw. Metahumanist, der publizistisch tätig ist. Zusammen mit dem Medien- und Performancekünstler Jaime de $\mathrm{Val}^{87}$ hat Sorgner den Metahumanismus begründet, der eine Vermittlerposition zwischen einem technologisch verstandenen Transhumanismus und dem philosophi-

84 The Transhumanist Declaration, 1998. Auch alle späteren Versionen finden sich unter https://hpluspedia.org/wiki/Transhumanist_Declaration.

85 http://www.aleph.se/Trans/Words/, 1.6.2004.

${ }^{86} \mathrm{Zu}$ Beginn gehörte auch Frank Tipler zum Herausgeberkreis. Vgl. jetpress.org.

87 Aktuell kreisen Jaime de Vals Projekte um den Metabody und sollen die bisherige Erfahrungen von Körper und Körperlichkeit überwinden. Vgl. metabody.eu. 
schen Posthumanismus einnehmen soll. Sorgner $\left({ }^{*} 1973\right)$ ist promovierter Philosoph und lehrt derzeit an der amerikanischen John Cabot University in Rom zu Themen des Posthumanismus und der Bioethik. Weitgehend unabhängig vom etablierten Transhumanismus hat er sein Beyond $\mathrm{Hu}$ manism-Netzwerk vor allem unter Beteiligung von süd- und osteuropäischen Philosophinnen und Philosophen aufgebaut. Seit 2014 erscheint die inzwischen 9 Bände umfassende Buchreihe Beyond Humanism: Transand Posthumanism / Jenseits des Humanismus: Trans- und Posthumanismus (im Peter Lang Verlag). ${ }^{88}$ Inwieweit es ihm gelingen wird, den Metahumanismus als philosophische Schule zu etablieren, ist eine offene Frage. ${ }^{89}$

Eine besondere Stellung unter den Transhumanisten nimmt die amerikanische Unternehmerin Martine Rothblatt ein, die im Bereich der Biotechnologie und elektrischen Flugtechnik aktiv ist. Mit ihrer zu Beginn der 2000er Jahre gegründeten Terasem-Bewegung (abgeleitet von Erde \& Samen) will sie mit Hilfe von Nanotechnik und digitalen Klonen (mind clones) die menschliche Unsterblichkeit verwirklichen. Von 2006 bis 2014 edierte sie die beiden Journals Geoethical Nanotechnology und Personal Cyberconsciousness mit den bekannten Beiträgen namhafter Transhumanisten. ${ }^{90}$ Ihre technologischen Visionen verbindet Rothblatt mit dem Eintreten für Transgenderrechte und Diversität - im Alter von 40 Jahren hat sie selbst das Geschlecht gewechselt. ${ }^{91}$

Trotz der mitunter verwirrenden und im Gesamten kaum überschaubaren Vielfalt von Ideen im Umfeld des Transhumanismus bestehen auch gewisse Abgrenzungsmechanismen der transhumanistischen Bewegung gegenüber anderen Diskursen. Dies betrifft zunächst die Abgrenzung gegenüber religiösen Traditionen und Ideen. So unterstreichen Bostrom und Yudkowsky, dass der Transhumanismus nur auf Vernunft und wissenschaftlichen Erkenntnissen beruhen dürfe. Die Versuche Tiplers und anderer, Mystik und technologische Prophetie miteinander zu verschmelzen, weist Bostrom entschieden zurück. ${ }^{92}$

${ }^{88}$ Vgl. http://beyondhumanism.org.

89 Vgl. Loh 2018, 173-175. Sorgners Buch Transhumanismus (2016) eignet sich kaum als Überblick oder Einführung in den Transhumanismus. Der Autor verfolgt offenbar vorrangig das Ziel, sein eigenes Verständnis vom Transhumanismus darzulegen sowie sich selbst und den von ihm begründeten Metahumanismus im Verhältnis zum Transhumanismus zu verorten. Dadurch entstehen ideengeschichtliche und normativ geprägte Unschärfen. Vgl. z.B. Sorgner 2016, 17-33.

90 Vgl. http://www.terasemjournals.org.

91 Vgl. https://www.terasemcentral.org; Rothblatt 2014.

92 Vgl. Bostrom 2005, 8; Bostrom 2014, 2; Yudkowsky 2000a, 2000 b. 
Wie die Soziolinguistik gezeigt hat, ist auch die Ausbildung einer gruppenspezifischen Sprache ein entscheidender Faktor zur Formierung eines gemeinschaftlichen Handelns und Denkens. Sie schafft damit die Voraussetzung für die Genese kollektiver wie individueller Identität. ${ }^{93}$ Den Bemühungen des schwedischen Transhumanisten Anders Sandberg ist es zu verdanken, dass wir über eine ausführliche Zusammenstellung von Termini und Neologismen verfügen, die kennzeichnend für den internen transhumanistischen Diskurs sind. Einige Beispiele hieraus sollen den normativen Bezugsrahmen der Transhumanisten veranschaulichen.

AMORTALIST: A person who opposes death.

ARCH-ANARCHY: The view that we should seek to void all limits on our freedom, including those imposed by the laws of nature. [T. O. Morrow, 1990]

ASEX: A person who has been physically and mentally altered so that it is no longer male or female. [Greg Egan, Distress]

ATHANASIA: The act of preventing death.

ATHANOPHY: A philosophical system that offers a possible means of overcoming death scientifically.

AUTOEVOLUTION: Evolution directed by intelligent beings instead of natural selection.

BIOCHAUVINISM: The prejudice that biological systems have an intrinsic superiority that will always give them a monopoly on self-reproduction and intelligence.

BIOLOGICAL FUNDAMENTALISM: A new conservatism that resists asexual reproduction, genetic engineering, altering the human anatomy, overcoming death. A resistance to the evolution from the human to the posthuman. [FM-2030]

BIOPHILIAC: someone who values life of all kinds for its own sake.

CRYONAUT: A cryonically suspended person.

DEANIMALIZE: Replace our animal organs and body parts with durable, pain-free non-flesh prostheses. [FM-2030]

DEATHISM: The set of beliefs and attitudes which glorifies or accepts death and rejects or despises immortality.

DOWNLOAD: To transfer a mind from one computational matrix to another, especially a slower one.

EXTROPY: A measure of intelligence, information, energy, life, experience, diversity, opportunity, and growth. The collection of forces which oppose entropy. [T.O. Morrow, 1988]

IMMORTALIST: A person who believes in the possibility of, and who seeks to attain, physical immortality.

93 Vgl. Lavery 1992, 69f. 
JUPITER-BRAIN: A posthuman being of extremely high computational power and size. This is the archetypal concentrated intelligence.

LONGEVIST: A person who seeks to extend their life beyond current norms (but who may not wish to live forever).

NEOLOGOMANIA: The transhuman enthusiasm for neologisms, especially the creation of words with many prefixes.

SINGULARITY: The postulated point or short period in our future when our self-guided evolutionary development accelerates enormously (powered by nanotechnology, neuroscience, AI, and perhaps uploading) so that nothing beyond that time can reliably be conceived.

TIPLERITE: A person with religious faith in Tipler's Omega Point Theory (So far very rare, if any).

TRANSCEND: v. To become vastly superhuman and incomprehensible for unaugmented beings.

UPLOAD: (a) To transfer the consciousness and mental structure of a person from a biological matrix to an electronic or informational matrix (this assumes that the strong AI postulate holds). The term »Downloading « is also sometimes used, mainly to denote the mind to a slower or less spacious matrix. (b) The resulting infomorph person.

UNIVERSAL IMMORTALISM: The view that the problem of death can be solved in its entirety (including bringing back those »dead« who were not placed into biostasis) through a rational, scientific approach.

WETWARE: Similar to hardware, but denotes a biological system, most commonly the human nervous system (see also dryware).

XOX: (from Xerox) An (atomically) identical copy of a person. ${ }^{94}$

Gerade die Kontrastierung der negativen, den Tod implizierenden Begriffe zur Kennzeichnung von Kritikern des Transhumanismus (biochauvinism, biophiliac, deathism) mit den positiven, auf die Unsterblichkeit verweisenden Selbstbezeichnungen (amortalist, athanasia, athanophy, immortalist, longevist) verstärkt die Konstruktion einer elitären Identität, die die quantitativ marginale Bewegung der Transhumanisten gegenüber der massiven humanistischen Kritik stabilisiert.

\subsection{Weitere Akteure und Institutionen}

$\mathrm{Zu}$ Beginn der 2000er Jahre konnte der Transhumanismus zunächst einen beachtenswerten politischen Erfolg verbuchen, der sich allerdings

${ }^{94}$ Vgl. hierzu http://www.aleph.se/Trans/Words/. 
als Pyrrhussieg erweisen sollte. Unter der Leitung des Nanowissenschaftlers Mihail Roco und des Religionssoziologen William S. Bainbridge, der große Sympathien für den Transhumanismus hegt, ${ }^{95}$ wurde 2003 ein erster Bericht zur Konvergenz der NBIC-Technologien erstellt (Nanotechnology, Biotechnology, Information technology und Cognitive science). Der Bericht ging auf einen Workshop der amerikanischen National Science Foundation aus dem Jahr 2001 zurück, der hochrangige Vertreter der US-Regierung, Wissenschaftspolitik und Forschung miteinander ins Gespräch brachte. Der von Roco und Bainbridge edierte Bericht unter dem Titel Converging technologies for improving human performance folgte dabei stark einer unkritischen, transhumanistischen Agenda, die von Eric Drexlers Nanofuturologie geprägt war. Ein goldenes Zeitalter des Friedens und des Wohlstandes mit deutlich verlängerten Lebensperspektiven versprachen die Autoren.

The twenty-first century could end in world peace, universal prosperity, and evolution to a higher level of compassion and accomplishment. It is hard to find the right metaphor to see a century into the future, but it may be that humanity would become like a single, distributed and interconnected »brain « based in new core pathways of society. ${ }^{96}$

Verwunderung und das Bemühen um Schadensbegrenzung war die Reaktion der etablierten Wissenschaftsgemeinde auf den Bericht, die nun aufmerksamer und skeptischer die transhumanistischen Aktivitäten registrierte. ${ }^{97}$ Auf Seite der Transhumanisten setzte nachfolgend eine Gründungswelle von akademischen Instituten ein, die ihre Aufgabe darin sehen, die eigenen futuristischen Szenarien zu evaluieren und bekannt zu machen.

Zu den Transhumanisten der ersten Stunde zählt sicherlich der Nanowissenschaftler Kim Eric Drexler (*1955), der in den 1970er Jahren eng mit Gerard O'Neill zusammenarbeitete und in der L5 Society eine herausragende Rolle spielte. In seinem populären Buch Engines of Creation von 1986 präsentierte er die Nanotechnologie als universalen Heilsbringer. Mit Hilfe von künftigen Nanorobotern, die in den menschlichen Körper eingeflößt werden, könnten die zellulären Schäden von eingefrorenen Kryonikern sowie von alternden Menschen behoben werden. Diese

\footnotetext{
95 Vgl. Bainbridge 2005, 2011, 2014.

96 Vgl. Roco \& Bainbridge 2003, 6.

97 Vgl. Coenen 2010, 71-78; Heil \& Coenen 2014, $153 f$.
} 
Idee hatte 1972 zwar schon Robert Ettinger, aber Drexler fundiert die Vision mit den Einsichten und den weitreichenden Verheißungen der damals noch jungen Nanowissenschaft. Molekülgroße Materieumwandler (universal assembler) sollen in naher Zukunft für medizinische Probleme aber auch zur ökonomischen Ressourcenproduktion eingesetzt werden. Drexler und sein Kollege Ralph Merkle sind vollkommen davon überzeugt, mit Hilfe der Nanotechnologie einem goldenen Zeitalter ohne Krankheit und Alter entgegen zu steuern. ${ }^{98}$

If we succeed (and if you survive) then you may be honoured with endless questions from pesky great-grandchildren: »What was it like when you were a kid, back before the Breakthrough?« and »What was it like growing old?« ... By your answers you will tell once more the tale of how the future was won. ${ }^{99}$

Gemeinsam mit der futuristischen Autorin Christine Peterson und dem Unternehmer James C. Bennet - alle kennen sich aus der L5 Society gründete Drexler 1986 das Foresight Institute im kalifornischen Palo Alto, das sich der Verbreitung der nanotechnischen Zukunftsvision verschrieben hat. ${ }^{100}$ Kaum ein transhumanistischer Autor verzichtet heute auf die Verheißungen der Nanotechnologie.

Ein weiteres zentrales Element transhumanistischer Futurologie ist die Verschmelzung von Mensch und Maschine zum Cyborg, insbesondere die Idee einer neuronalen Schnittstelle zwischen dem menschlichen Gehirn und einem Computer bzw. dem Internet. Kevin Warwick (*1954), Professor für Kybernetik an der Coventry University (England), erregte zu Beginn der 2000er Jahre Aufsehen mit seinen Selbstexperimenten, die ihn seiner Ansicht nach zum ersten Cyborg der Menschheitsgeschichte machten. Tatsächlich war er der erste Mensch, der sich einen Computerchip implantieren ließ. Dieser sandte ein Lokalisierungssignal aus und vereinfachte die Licht- und Türsteuerung in seiner Universität. ${ }^{101}$ Neben der Erweiterung der körperlichen Sinne sieht Warwick in der Anwendung von Neuroimplantaten eine Chance auf die Heilung von Krebs und Depressionen und allgemeiner die Möglichkeit einer künst-

98 Vgl. Ettinger 1989, 193-196; Drexler 1996, 138f.; Regis 1990, 108-143; Heil 2010b, 34-40.

99 Drexler 1996, 239.

${ }^{100}$ Vgl. https://foresight.org.

${ }^{101}$ Heute verwenden wenige Firmen bereits Chip-Implantate zur Lokalisierung ihrer Mitarbeiter. Einige Bundesstaaten in den USA haben daher gesetzliche Regulierungen zum Einsatz dieser Chips erlassen. https://en.wikipedia.org/wiki/Microchip_ implant_(human). 
lichen Kontrolle unserer Stimmungen und Gefühle. Fasziniert zeigt er sich von der Idee einer Schnittstelle, eines implantierten Interface, zwischen dem menschlichen Gehirn und dem Computer, das uns erlauben würde, Informationen direkt ins Gehirn einspeisen zu können und mit anderen Gehirnen in einer höheren Kommunikationsform ohne den Umweg über das Medium Sprache Informationen auszutauschen. Diese Möglichkeiten mittels Gehirnimplantaten Superhumans zu schaffen, sei gemäß Warwick die einzige Chance, noch einige Zeit mit den intelligenten Maschinen der Zukunft mitzuhalten und als Mensch nicht zum unterdrückten Wesen zweiter Klasse degradiert zu werden. »I believe humans will become cyborgs and no longer stand-alone entities. ${ }^{102}$ Ähnlich bewertet der Biologe und Direktor des Institutes für künstliche Intelligenz der Freien Universität Brüssel, Luc Steels (*1954), die vielfältigen Verbindungen von Leben und Computern durchweg positiv. Sowohl die von Menschen geplante Erschaffung der künstlichen Lebensformen als auch die Verknüpfung von menschlichem Gehirn und dem Internet bedeute einen großen Fortschritt in der Evolutionsgeschichte. ${ }^{103}$

Falls irgendwie Verbindungen zwischen dem Gehirn und der elektronischen Datenautobahn eingerichtet werden könnten, dann gäbe es die fesselnde Möglichkeit, daß das Gehirn zu riesigen Informationsmengen Zugang hat und im Gegenzug Handlungen über die Entfernung hinweg durch die Vermittlung von elektronischen Geräten ausführen kann. ${ }^{104}$

Hatte bereits der Science-Fiction Schriftsteller Bruce Sterling eine dichotome Evolution des Lebens in der Zukunft prophezeit, so sieht Luc Steels am fernen Horizont der Entwicklung den Homo Cyber Sapiens, der schrittweise unabhängiger von seiner biologischen Wetware würde, und daneben das aufsteigende, künstliche Leben des Robot Hominidus Intelligens. ${ }^{105}$

Eine gegenüber Steels und Warwick differenziertere Abwägung einer Cyborgisierung des Menschen liefert Chris Hables Gray in seinem Buch Cyborg Citizen. Politics in the Posthuman Age von 2002. Zwar sieht er auch die Gefahr einer zunehmenden Kontrolle des Menschen durch die teil-

\footnotetext{
${ }^{102}$ Warwick 2000, 151. Vgl. auch Warwick 2004.

${ }^{103}$ Vgl. Steels 1994; Brooks \& Steels 1995; Flessner 1997b, 104.

${ }^{104}$ Steels $1996,86$.

${ }^{105}$ Vgl. a.a.O., 85-89. Ähnlich wie Steels und Warwick, jedoch mit einer Konzentration auf die Gentechnik argumentiert der Biologe Gregory Stock in seinem Buch Redesigning Humans. Vgl. Stock 2002.
} 
weise Maschinisierung und technischen Erweiterungen des Körpers, jedoch überwiegen seiner Einschätzung nach die Vorteile:

Cyborgization will not make us like gods, thank goodness, but we may be able to live better and longer than any human or protohuman has before. We may be able not only to live long and prosper, but to push the species into new, enlightening adventures in inner and outer spaces ... It is thrive or die. ${ }^{106}$

Im Vergleich zu den Verheißungen von Aubrey de Grey klingen diese Cyborg-Visionen allerdings bescheiden. Der britische Gerontologe prophezeit nicht nur die Verwirklichung einer Lebensspanne von über 1000 oder gar 5000 Jahren, sondern er behauptet im gleichen Atemzug, dass wahrscheinlich sogar seine Altersgenossen diese Lebensfülle noch werden realisieren können. De Grey war Software-Ingenieur und gelangte über seine spätere Gattin, die Genetikerin Adelaide Carpenter, in die Altersforschung. Im Jahr 2000 verlieh ihm die Universität Cambridge für seine Forschungen zu den Mitochondrien einen Doktortitel in Biologie. Zusammen mit dem Unternehmer David Gobel gründete De Grey 2003 die Methuselah Foundation, die seither verschiedene Wettbewerbe zu Langlebigkeitsexperimenten ausgerufen hat und auch von Silicon Valley-Magnaten wie Peter Thiel finanziell unterstützt wird.

Im Jahr 2009 rief De Grey die SENS Research Foundation ins Leben (bis 2013 firmierte sie unter dem Namen SENS Foundation). Hinter dem Kürzel verbirgt sich die Zielmarke von De Greys Forschungen: Strategies for Engineered Negligible Senescence - Strategien zur technischen Vermeidung von Alterserscheinungen. Anders als Walford empfiehlt De Grey nämlich keine Diät oder disziplinierte Lebensführung. Das Altern an sich bewertet der ambitionierte Forscher als Krankheit, die wahrscheinlich auf sieben differenten Zellprozessen basiert und sich durch gentechnische Eingriffe behandeln lassen werde. Seine Stiftung finanziert sich groBenteils über das Erbe seiner Mutter in Höhe von 11 Millionen Pfund. Die Dokumentation The Immortalist von Jason Sussberg und unzählige Portraits in internationalen Medien haben zum Bild des exzentrischen Genies beigetragen. Durch seine phantastisch anmutenden Visionen und seinen messianischen Führungsanspruch steht ihm die biogerontologische Forschungsgemeinschaft jedoch weitgehend kritisch gegenüber. Obwohl de Grey die US-amerikanische Partei der Transhumanisten im Bereich anti-aging berät und auch Anhänger der Kryonik ist, lehnt er

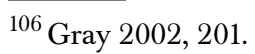


das Label des Transhumanisten für sich selbst ab: Er sieht sich eher als Immortalisten. ${ }^{107}$

Im Gegensatz zu diesen forschungsorientierten Institutionen haben sich drei Einrichtungen zum Ziel gesetzt, den realen und erwarteten technischen Fortschritt zu bewerten. Die von Ray Kurzweil 2008 gestartete Singularity University zähle ich ausdrücklich nicht dazu. ${ }^{108}$ Die Initialzündung für die Etablierung eines akademischen Netzwerkes von Transhumanisten ging von dem KI-Forscher Eliezer Yudkowsky aus, der im Jahr 2000 mit finanzieller Unterstützung der Internet-Unternehmer Brian und Sabine Atkins das Singularity Institute for Artificial Intelligence in Berkeley ins Leben rief. Das Institut, das sich für die Erforschung von menschenfreundlicher KI einsetzen wollte, erfreute sich 2006 einer größeren Spende seitens des Pay-Pal-Gründers Peter Thiel. Mit dieser Finanzierung konnte der erste Singularity Summit im selben Jahr in Stanford organisiert werden (mit Max More, Nick Bostrom, Eric Drexler, Ray Kurzweil, Peter Thiel u.a. als Gäste). Von 2007 bis 2010 war Kurzweil hier als Co-Direktor engagiert, Aubrey de Grey und Bostrom dienten dem Beratergremium des Instituts, letzterer bis heute. Im Jahr 2013 wurde der Name in Machine Intelligence Research Institute (MIRI) umgewandelt. Das Institut widmet sich seither vor allem mathematischer und theoretischer Computerwissenschaft. ${ }^{109}$ Mehrere Mitarbeiter des MIRI gehörten im Januar 2015 zu den Erstunterzeichnern des offenen Briefes Research Priorities for Robust and Beneficial Artificial Intelligence, der sich für eine kontrollierte und nutzbringende KI-Forschung aussprach. ${ }^{110}$ 2004 gründeten James Hughes und Nick Bostrom das amerikanische Institute for Ethics and Emerging Technologies (IEET) als transhumanistischen Think Tank mit einer »technoprogressiven Agenda«. Als LobbyOrganisation hat das Institut die Aufgabe, die Vorteile der erhofften Zukunftstechnologien zu betonen und das Gespräch mit seinen Kritikern zu suchen. Es ist vor allem Hughes zu verdanken, dass gesellschaftspolitische Fragen in die Diskussion eingebracht wurden. Seine Zielsetzung

\footnotetext{
${ }^{107}$ Vgl. De Grey 2008 (dt.: Aubrey De Grey \& Michael Rae: Niemals alt! So lässt sich das Altern umkehren. Fortschritte der Verjüngungsforschung. Bielefeld 2010); Agar 2010, 83-132; Hooper 2005; Sussberg 2014.

${ }^{108}$ Vgl. Kapitel 6.4.2.

${ }^{109}$ Vgl. Wagner 2015, 63-69, 77-124; https://intelligence.org.

${ }^{110}$ Initiator war das 2014 gegründete Future of Life Institute (Cambridge). Weitere Unterzeichner waren Stephen Hawking, Elon Musk, Nick Bostrom, Anders Sandberg und Vernor Vinge. https://futureoflife.org/ai-open-letter.
} 
ist ein demokratisch fundierter Transhumanismus, dessen künftige Errungenschaften allen Menschen gleichermaßen zugutekommen sollen. ${ }^{111}$ Die Liste der Fellows des Institutes liest sich wie das Who is Who? des Transhumanismus: Aubrey de Grey, Ben Goertzel, William S. Bainbridge, David Pearce, Martine Rothblatt, Stefan L. Sorgner und Natasha Vita-More waren hier aktiv; Giulio Prisco ist Teil des Vorstandes. Von 2005 bis 2014 fanden eine Reihe von Tagungen zur Biopolitik, Ethik und künstlicher Intelligenz statt (die ersten beiden aufgeführten Tagungen sind dabei identisch mit den letzten internationalen Tagungen der World Transhumanist Association). Das transhumanistische Journal of Evolution and Technology wird vom IEET herausgegeben. ${ }^{112}$

Das erstaunlichste Phänomen innerhalb des transhumanistischen Institutionalisierungsprozesses ist jedoch der schwedische Philosoph Nick Bostrom (d.i. Nicholas Boström) und sein 2005 realisiertes Future of $\mathrm{Hu}$ manity Institute $(F H I)$ an der rennomierten Universität Oxford. Während der ersten Jahre, als Bostrom gleichzeitig auch noch Vorsitzender der World Transhumanist Association war, verfolgte die Einrichtung ähnlich wie das IEET und das Foresight Institute eine sichtbare transhumanistische Agenda. Drei Forschungsbereiche wurden zunächst anvisiert: (1) Die Transformation des Menschen, (2) Risiken für globale Katastrophen und (3) die Zukunft des intelligenten Lebens. Der erste Aspekt sollte das Potential für eine Erweiterung menschlicher Fähigkeiten sondieren, Technologien des enhancement bewerten und Strategien entwickeln, um die vorhandenen Vor- und Fehlurteile über diese Technologien zu reduzieren. ${ }^{113}$ Anders Sandberg ist seit der ersten Stunde des Institutes Bostroms enger Mitarbeiter und Eric Drexler sowie Ralph Merkle gehörten zu den frühen Vortragsgästen.

Seit Bostroms erfolgreichem Buch Superintelligence. Paths, Dangers, Strategies von 2014 akzentuiert das FHI neuerdings auch die Risiken von Zukunftstechnologien. Im Fokus stehen zwei Bereiche: zum einen das Bemühen um eine sichere Nutzung von künstlicher Intelligenz, v.a. der erwarteten Superintelligenz (governance of $A I$ ). Zum anderen berät das FHI politische Gremien zu den Chancen und Risiken von Biotechnologien, so den US-amerikanischen President's Council on Bioethics, den UK

${ }^{111}$ Hughes' Utopie einer harmonischen Zukunftsgesellschaft wird aber auch innerhalb des Transhumanismus kritisiert und als unrealistisch eingeschätzt. Vgl. Hughes 2004, 187-220; Agar 2010, 151-177.

${ }^{112} \mathrm{Vgl}$. https://ieet.org.

${ }^{113}$ Vgl. https://www.fhi.ox.ac.uk (13.10.2005). 
Synthetic Biology Leadership Council und das US-Verteidigungsministerium (DARPA). Dieser neue Akzent ist dabei nicht als Paradigmenwechsel zu bewerten, sondern entspricht exakt den 2005 veröffentlichten Zielsetzungen (Vorurteile auszuräumen und das Potential des enhancement zu betonen). Es kann kein Zweifel daran bestehen, dass Bostrom der Entstehung einer Superintelligenz mit Euphorie entgegenblickt, verbindet er doch damit die Hoffnung, dass sie alle Probleme der Menschheit lösen könne. ${ }^{114}$ Die geäußerten, theoretischen Bedenken und die Rhetorik einer sicheren künstlichen Intelligenz erhöhen allerdings die gesellschaftliche Akzeptanz dieser Innovation. Im Bereich der Biotechnologie herrscht das bereits von Warwick artikulierte Narrativ vor, dass die Risiken künftiger Technologien durch menschliche Anpassung wieder ausbalanciert werden könnten: "Arms races or proliferation with advanced bioweapons could pose existential risks to humanity, while advanced medical countermeasures could dramatically reduce these risks. Human enhancement technologies could radically change the human condition. ${ }^{115}$

Die Fokussierung auf diese erwartete Superintelligenz spiegelt die transhumanistische Selbstbezogenheit und Technozentrik wider. Die aktuelle Destabilisierung politischer Systeme und die Verbreitung von Verschwörungstheorien mit Hilfe recht simpler Algorithmen, Social Bots und menschlicher Agenten / »Trollen« in ökonomisch determinierten, sozialen Medien bei einem gleichzeitigen Verfall öffentlicher Bildungssysteme, befindet sich aufgrund der weitgehenden Ignoranz gegenüber gesellschaftlichen Prozessen nicht im Fokus des »Institutes der Zukunft der Menschheit«.

\subsection{Der religiöse und spirituelle Transhumanismus}

Nach Frank Tiplers stark christlich geprägter Futurologie war es zu erwarten, dass der Trans- und Posthumanismus auch auf eine positive Rezeption unter einer progressiven, christlichen Minderheit hoffen durfte. Überraschenderweise waren es die Mormonen, die sich zuerst und aktiv in den Transhumanismus einbrachten. Schon 2006 wurde die Mormon

\footnotetext{
${ }^{114}$ Vgl. Kapitel 6.4.2.

${ }^{115}$ Homepage des FHI(Research / Biotechnology) https://www.fhi.ox.ac.uk.
} 
Transhumanist Association in Utah ins Leben gerufen, die seitdem auch mit humanity + affiliiert ist. Nach eigenen Angaben verfügte sie 2017 über 637 Mitglieder weltweit, von denen sich jedoch nur $62 \%$ als Mormonen und 59\% als Theisten identifizieren. Voraussetzung für die Mitgliedschaft ist die Akzeptanz der Transhumanist Declaration von humanity + und der Mormon Transhumanist Affirmation, die ein Bekenntnis zu Jesus Christus und dem von Gott vorgesehenen wissenschaftlichen Fortschritt verlangt. Dieser solle dem Menschen Unsterblichkeit, Auferstehung und die Erneuerung der Welt ermöglichen. Entsprechend dem biblischen Ereignis der Verklärung (transfiguration), verstehen sich die transhumanistischen Mormonen als transfigurationalists: In jeder Religion könne man nämlich einen Moment der Verwandlung des Menschlichen in ein Höheres, Göttliches, beobachten. Sie haben keine eigenen kultischen Praktiken entwickelt, organisieren jedoch neben drei lokalen Gesprächsgruppen in den Vereinigten Staaten jährliche Tagungen zur Diskussion technischer und theologischer Fragen. ${ }^{116}$

Die Christian Transhumanist Association wiederum hat sich erst 2014 mitten im amerikanischen bible belt, in Tennessee gegründet. Das Glaubensbekenntnis (Christian Transhumanist Affirmation) entspricht inhaltlich dem mormonischen, betont jedoch stärker die Nachfolge Christi. Neben Aktivitäten auf Facebook trifft sich eine lokale Diskussionsgruppe einmal monatlich in Nashville. Im wissenschaftlichen Beirat werden James Hughes und Frank Tipler aufgeführt, Aubrey de Grey war 2018 Gast der Jahrestagung. ${ }^{117}$

Die älteste und zugleich jüngste Spielart des religiösen Transhumanismus formiert sich um »Bernadeane « Brown und James Russel Strole. Es ist die älteste Spielart, weil die Bewegung bereits in den frühen 1960er Jahren entstand. Sie ist zugleich die jüngste, da sie sichtbar erst seit drei Jahren Kontakte zum etablierten Transhumanismus aufbaut und sich mit dessen medizinischen Ansätzen zur Lebensverlängerung identifiziert. Die Ursprünge der Bewegung gehen auf Charles Paul Brown (19352014) zurück, der während seiner Ausbildung zum Pfarrer der Assembly of God 1959 ein Erweckungserlebnis erfuhr. Er war davon überzeugt, dass Jesus seine Gene verändert habe, ihm damit körperliche Unsterblichkeit verliehen habe und dass dieser Heilsweg allen Menschen offen stünde. Zusammen mit seiner Frau Bernadeane und ab 1968 auch mit

${ }^{116}$ Vgl. https://transfigurism.org.

117 Vgl. https://www.christiantranshumanism.org. 
James Strole predigte er seine immortalistische Botschaft, die vor allem jede Art des herkömmlichen »Todeskultes« bekämpft (extreme optimism). Ähnlich wie im Extropianismus wird die Rede vom Sterben und vom Lebensende vollkommen negiert. Ab den 1980er Jahren lehrt Brown, dass er die zelluläre Unsterblichkeit auch durch Kommunikation und körperliche Nähe übertragen könne. Zunächst unter dem Namen Eternal Flame, später als Flame Foundation und People Forever, CBJ (nach den Führerinitialen) und jüngst als People Unlimited Inc. ist die Gruppierung seit 1971 in Scottsdale (Arizona) angesiedelt. Nach kritischer Berichterstattung durch die US-Medien in den 1990er Jahren, die dem Leitungstrio persönliche Bereicherung vorwarfen, trat die Gemeinschaft bis zum Tod von Brown 2014 nicht mehr besonders in Erscheinung. ${ }^{118}$

Vor allem auf Betreiben von James Strole wurde Ende 2015 die nonprofit Coalition for Radical Life Extension als Plattform verschiedener Aktivisten begründet, die ab 2016 ein jährliches RAAD Fest organisiert - die Abkürzung steht für Revolution Against Aging and Death. Ganz im Wortlaut der Emancipation Proclamation von Abraham Lincoln, die 1863 das Ende der Sklaverei erklärte, lässt der Vorstand der Coalition das Ende des Alterns verkünden:

That all persons held as slaves to aging within any Country, State or designated part of a State, the people whereof shall then be in rebellion against the disease known as "aging", shall be then, thenceforward, and forever free; and the Executive Governments of all Countries will recognize and maintain the freedom of such persons, and will do no act or acts to repress such persons, or any of them, in any efforts they may make for their actual freedom. ${ }^{119}$

Das mehrtägige RAAD Fest erscheint als Mischung zwischen transhumanistischen Vorträgen und Diskussionen, allgemeinem Lebenscoaching und marketingkonformen Businesspräsentationen vor allem der Anbieter von Ernährungssupplementen und Anti-Aging-Therapien. Mit über 1000 Besuchern ist das »Fest« als äußerst erfolgreich zu bewerten. $\mathrm{Zu}$ den Vortragenden gehören neben Bernadeane und James Strole, Aubrey de Grey, Ben Goertzel, Zoltan Istvan, Ray Kurzweil, Max More und Natasha Vita-More. ${ }^{120}$

\footnotetext{
${ }^{118}$ Vgl. Van Velzer 2014; https://peopleunlimitedinc.com; http://www.rlecoalition.com/ about.

${ }^{119}$ Zitiert nach https://hpluspedia.org/wiki/RAAD_Fest.

${ }^{120}$ Vgl. https://www.raadfest.com.
} 
Die konkretesten kultischen Formen hat die seit 2013 aktive Church of Perpetual Life in Hollywood (Florida) angenommen. Sie ist eng verbunden mit der von Saul Kent, einem Urgestein der Kryonik, und William Faloon seit 1980 geführten Life Extension Foundation, die (vermeintlich) lebensverlängernde Supplemente und Medikamente vertreibt und 2013 über ein Vermögen von 25 Millionen \$ verfügte. Im Mai 2013 verlor die Stiftung ihre Steuerbefreiung. ${ }^{121}$ Einen Monat später öffnete die von Faloon initiierte Church of Perpetual Life ihre Pforten, die wie alle Kirchen in den Vereinigten Staaten steuerbefreit agieren darf. Einmal monatlich versammelt sich die etwa 500 Personen umfassende Gemeinde, die die "Krankheit des Alterns" mit Hilfe von Supplementen und Kryonik überwinden will. Als Propheten werden der russische Kosmist Nikolai Fedorov und der britische Science-Fiction Schriftsteller Arthur C. Clarke verehrt. Innerhalb des Transhumanismus fühlt man sich dem Immortalismus zugehörig. Die auch auf YouTube verfügbaren celebrations bestehen aus philosophischen Beiträgen zur Erreichung der Unsterblichkeit, der Vermarktung von Supplement-Produkten, Songs und Vorträgen von transhumanistischen Aktivisten wie Natasha Vita-More, Aubrey de Grey, Zoltan Istvan, Max More, Martine Rothblatt sowie James Strole und Bernadeane. An Weihnachten wird der eingefrorenen Kryoniker, den ressurectables, gedacht. ${ }^{122}$ Die Life Extension Foundation ist ein wichtiger Sponsor des RAAD Festes, auf dem auch William Faloon auftritt.

Offenbar bislang nicht viel mehr als ein Internet-Phänomen ist die Turing Church. Der italienische Transhumanist und Kryoniker Giulio Prisco, der kurzzeitig auch im Vorstand von humanity+ engagiert war, gründete schon 2008 zusammen mit Philippe Van Nedervelde, Martine Rothblatt und anderen den Order of Cosmic Engineers, der allerdings nur bis 2012 aktiv war und sich für radikal lebensverlängernde Technologien (insbesondere Nanotechnologie) einsetzen wollte. Im Anschluss rief Prisco mit einigem Medienecho die Etablierung der Turing Church aus, in deren Zentrum die Gottwerdung des Menschen und die technische Immortalisierung steht. Man sieht sich in der Tradition der russischen Kosmisten. Prisco schwebt statt lokaler Zusammenkünfte eine globale, virtuelle Kir-

${ }^{121}$ Rechtliche Auseinandersetzungen über den illegalen Vertrieb von Medikamenten und den Gemeinützigkeitsstatus prägen die Geschichte der Foundation (vgl. engl. Wikipedia).

${ }^{122}$ Vgl. Volpicelli 2014 ; http://www.churchofperpetuallife.org; https://www.youtube. com/user/COPL18/featured. 
che vor, die sich in Second Life zum Austausch trifft. ${ }^{123}$ Empirisch kaum fassbar ist auch die von Anthony Levandowski (*1980) begründete Way of the Future Church. Der Ingenieur, der für seine Forschungen zu selbstfahrenden Autos bekannt ist, wirbt für einen friedlichen Übergang zu einer von künstlicher Superintelligenz beherrschten Erde. ${ }^{124}$

\subsection{Zusammenfassung}

Viele akademische und religiöse Organisationen sowie auch politische Bewegungen, die seit den 1960er Jahren mit den Themen des Transhumanismus verbunden waren, erwiesen sich im Verhältnis zu ihren Zielsetzungen als äußerst antagonistisch: Sie waren sehr kurzlebig. ${ }^{125}$ Transhumanisten sind in der Regel Einzelkämpfer. Rivalitäten um die öffentliche Aufmerksamkeit und die transhumanistische Deutungshoheit sind stark ausgeprägt und spiegeln sich in der Anzahl von proklamierten Manifesten, von Neologismen für Schulen und von Institutsgründungen wider. ${ }^{126}$ Originalität generiert Aufmerksamkeit und damit finanzielle Zuwendungen, auf die alle genannten Organisationen angewiesen sind. Eine einheitliche Charakterisierung des Transhumanismus anhand der erwarteten technischen Fortschritte ist heute noch weniger möglich als während meiner Studien vor 15 Jahren. Es ist vielmehr die gemeinsame Hoffnung auf die künftigen Technologien, die mindestens das Altern und den Tod überwinden sollen, die die verschiedenen Strömungen vereinigt. Innerhalb des transhumanistischen Spektrums ist dabei offen, ob dieser Durchbruch menschengemacht oder bereits die Folge einer wirksamen Superintelligenz ist und ob dieser Fortschritt gentechnologisch oder kybernetisch sein wird. Auch für den religiösen Transhumanismus gilt, dass diese Segnungen dem technischen Fortschritt und nicht dem direkten Eingreifen einer göttlichen Macht geschuldet sind.

\footnotetext{
${ }^{123}$ Vgl. https://turingchurch.net; https://turingchurch.com.

${ }^{124}$ Vgl. http://www.wayofthefuture.church.

${ }^{125}$ Wie z.B. die zahlreichen Kryonik- und Unsterblichkeitsvereine der 1960er Jahre. Vgl. Kapitel 6.5.4.

${ }^{126}$ Die Zukunft der jüngst gegründeten, transhumanistischen Parteien sowie des umtriebigen Kandidaten für die US-Präsidentschaftswahlen 2016, Zoltan Istvan, ist daher ungewiss. Vgl. Loh 2018, 49 f.
} 
Der Transhumanismus ist höchst dynamisch. Es liegt offenbar in der Natur seiner Anhänger, ihn stets neu zu erfinden und kontinuierlich neue Schulen, Organisationen und Neologismen hervorzubringen. Die "Zukunft« an sich existiert nur sehr abstrakt. Die transhumanistische Deutung dieser Zukunft erfolgt durch den Einsatz verschiedener Filter, die negative Aspekte ausblenden oder marginalisieren - paradigmatisch hierfür ist die Tabuisierung des Todes. Technische Fantasmen wie die Unsterblichkeit im Computer oder die genetische Umprogrammierung werden als faktische Projekte präsentiert, deren Realisierung nur noch wenige Jahre Forschung und großzügige finanzielle Unterstützung erfordern. In Ermangelung tatsächlicher Fortschritte und angesichts wachsender gesellschaftlicher Probleme muss diese Zukunft jedoch stets neu erfunden werden. Diese Einsicht gilt ebenso für den technologischen Posthumanismus. 


\title{
5. Der technologische Posthumanismus
}

\begin{abstract}
Wir leben in einer entscheidenden historischen Phase: Der Ära des Posthumanen. Dieses Zeitalter ist charakterisiert durch ein gnadenloses Bemühen seitens der virtuellen Klasse, das Verlassen des Körpers in Bausch und Bogen zu forcieren, die sinnliche Wahrnehmung auf den Müll zu kippen und sie stattdessen durch eine entkörperte Welt leerer Datenströme zu ersetzen. ${ }^{1}$
\end{abstract}

Arthur und Marilouise Kroker

Meiner Einschätzung nach übertreiben die kanadischen Medienphilosophen Arthur und Marilouise Kroker keineswegs, wenn sie heute den Beginn einer neuen historischen Ära verkünden, die auch der Medienwissenschaftler Bernd Flessner als das »Zeitalter der Posthumanität« bezeichnet hat. ${ }^{2}$ Am Ende des zweiten Milleniums trat der Posthumanismus nicht mehr als Auswuchs vereinzelter, exzentrischer Denker in Erscheinung. Vielmehr überblicken wir heute eine philosophische Strömung mit mehreren zentralen Autoren, einer etablierten transhumanistischen Bewegung und akademischen Institutionen. Eine ganze Reihe von philosophierenden Naturwissenschaftlern, die sich meist mit der Erforschung künstlicher Intelligenz und Robotik beschäftigen, vertreten heutzutage offensiv die Auffassung, dass der bisherige Mensch im Angesicht der zu erwartenden technischen Errungenschaften obsolet geworden sei. Im Gegensatz zu scharfsinnigen Beobachtern der industriellen Kultur wie Günther Anders fordern sie nun normativ die Ablösung des Menschen in der Evolutionsgeschichte durch seine vermeintlich überlegenen, technischen Produkte. Zunächst als Cyborg - als menschlich-maschineller Hybrid - und später als perfekte Simulation seiner Person im Speicher eines Computers soll der heutige Mensch an dieser Entwicklung teilhaben, die das intelligente Leben von seinen biologisch bedingten Schranken befreien will. Die natürlichen Grenzen unseres Intellektes und unseres Körpers, die altersbedingte Degeneration, Krankheiten und schließlich die menschliche Sterblichkeit sollen durch die Partizipation des Menschen an der posthumanen Evolution überwunden werden.

Kroker \& Kroker 1996, 87.

2 Vgl. Flessner 2000a, 13. 
Der technologische Posthumanismus drückt die Art und Weise aus, wie Menschen über Technik denken und welche Sinndeutung des Lebens sie mit ihr verbinden. So fügen sich in der posthumanistischen Philosophie Ideen aus der Literatur, der Populärkultur, der Wissenschaft gleichwie aus Philosophie und Theologie zusammen. ${ }^{3}$ Das Erscheinen dieser neuartigen posthumanistischen Philosophie wirft Fragen auf: Warum hier? Warum jetzt? Wo sind die Ursprünge der für den Posthumanismus konstituierenden Idee einer technischen Immortalisierung des Menschen? Gab es eine ideengeschichtliche Konstellation, die die Entstehung des Posthumanismus in den Vereinigten Staaten und Europa begünstigte? Wo sind seine philosophischen Voraussetzungen zu finden? Wie können wir also verstehen und erklären, wie Menschen an der Wende zum 21. Jahrhundert für die Überwindung ihrer eigenen Spezies plädieren und das Ende allen biologischen Lebens fordern oder zumindest billigen würden?

In den vergangenen 20 Jahren wurde viel über den Posthumanismus geschrieben. Leider zeichnet sich ein Großteil dieser Arbeiten dadurch aus, dass zwar einzelne Elemente der posthumanistischen Futurologie kommentiert, die entsprechenden Quellen jedoch kaum berücksichtigt werden. Eine ideengeschichtliche Kontextualisierung bleibt meist völlig aus. In Fortführung der ersten Auflage des vorliegenden Werkes von 2004 soll diese Perspektive hier vertieft werden. Der analytische Teil ist vollkommen neu gestaltet, da sich das Feld mit der Diskussion um die Singularität und die Superintelligenz heute ungleich komplexer gestaltet. Die detaillierte Untersuchung von sechs zentralen Aspekten des technologischen Posthumanismus ist nun klarer strukturiert: das posthumanistische Menschenbild (Kapitel 6.2 Der Mensch ist bereits posthuman), die Fortschrittstheorie (Kapitel 6.3 Vernichtung oder unendlicher Fortschritt), die Idee der Singularität (Kapitel 6.4 Singularitäten), die Unsterblichkeit (Kapitel 6.5), die transzendente Superintelligenz (Kapitel 6.6) und der Zielpunkt der Fortschrittsbewegung (Kapitel 6.7 Omega). Eingerahmt werden die Analysen durch eine Einführung (Kapitel 5.1) zu den vier wichtigsten Autoren des technologischen Posthumanismus sowie der Kunst (Kapitel 5.3) und ein abschließendes Resümee (Kapitel 7). Die aktuellen Publikationen dieser Autoren wurden in die jetzigen Analysen miteinbezogen. Zwar haben in den vergangenen Jahren auch weitere Autoren (und eine Autorin) wie Ben Goertzel oder Martine Rothblatt posthuma-

3 Vgl. Dery 1996, 15; Flessner 2000b, $259 f$. 
nistische Werke veröffentlicht - ideengeschichtlich waren diese jüngeren Beiträge jedoch nicht mehr besonders innovativ. In den Detailanalysen wurde die jüngere Literatur natürlich berücksichtigt. Gleichzeitig konnte die ideengeschichtliche Kontextualisierung dank verbesserter Recherchetechniken spürbar vertieft werden (um das Jahr 2000 war ich noch auf Zettelkataloge angewiesen, Onlineenzyklopädien bestanden nur rudimentär).

\subsection{Das Posthumane und der Posthumanismus}

Hatte bereits im 17. Jahrhundert Thomas Blount (1618-1679) das Wort posthumian in seiner Glossographia (1656) als ein zukünftig Kommendes definiert (»following or to come, that shall be«), so hat 1977 der amerikanische Kulturtheoretiker Ihab Hassan (1925-2015) in seinem Text Prometheus as Performer: Toward a Posthumanist Culture? als erster den Begriff posthumanism für philosophische Versuche der Überwindung des Menschen und damit auch des Humanismus eingeführt. ${ }^{4}$ Hassan spannt einen weiten Bogen von der griechischen Mythologie bis zur Philosophie und Kybernetik seiner Zeit. Die Figur des Prometheus ist ihm Sinnbild für die Überschreitung bisheriger Grenzen des Menschseins. Mit Bezug auf Marshall McLuhan, Teilhard de Chardin, Buckminster Fuller und vielen anderen Denkern meint Hassan, die Entstehung eines neuen kosmischen, prometheischen Bewusstseins beobachten zu können, das den seit der Renaissance vorherrschenden Humanismus ablösen werde. Daneben werde auch die Kybernetik das Verständnis des Menschen verändern: ${ }^{5}$

Will artificial intelligences supersede the human brain, rectify it, or simply extend its powers? We do not know. But this we do know: artificial intelligences, from the humblest calculator to the most transcendent computer, help to transform the image of man, the concept of the human. They are agents of a new posthumanism... ${ }^{6}$

4 Vgl. Blount 1656; Hassan 1977, 212; Raulerson 2013, 32f.; Hayles 1999, 247; Simpson \& Weiner 1989, Bd. 12, 197; Loh 2018, 94f. In diesem Sinne wird er auch bei Zons (2001, 7-27) verwendet.

5 Vgl. Hassan 1977, 834, 843-850.

6 Hassan 1977, 846. Der Aufsatz ging aus einem Vortrag von 1976 hervor. 
In ähnlicher Weise fasst auch noch der britische Künstler Robert Pepperell (*1963) in seiner Monographie The Post-Human Condition von 1995 unter dem Begriff posthumanism einerseits die Periode in der Philosophiegeschichte, die das Ende des Humanismus markiert, andererseits die beginnende Verschmelzung von biologischen Organismen und Technologie bis zu dem Punkt, an dem sie ununterscheidbar werden. ${ }^{7}$ In seiner Novelle Schismatrix von 1979 spricht der Science-Fiction Autor Bruce Sterling ebenfalls von post-human als Zustand einer nachmenschlichen Lebensform, die sich in die zwei Subspezies der gentechnisch orientierten Shaper und der auf kybernetische Prothesen fixierten Mechanics aufgliedern werde. ${ }^{8}$

Nachdem der Robotiker Hans Moravec noch in seinem für den technologischen Posthumanismus konstituierenden Werk Mind Children. The Future of Robot and Human Intelligence (1988) die Vision einer postbiologischen (postbiological) und übernatürlichen (supernatural) Zukunft des Menschen verkündet hatte, wurde während der 1990er Jahre in Publikationen über diese neue Philosophie die Bezeichnung Postbiologismus zunehmend von Posthumanismus verdrängt. ${ }^{9}$ Führende Denker der um die Umsetzung des Posthumanismus bemühten Transhumanisten versuchen die verschiedenen Aspekte des posthumanen Menschen auf folgende Weise zu systematisieren:

A posthuman is a human descendant who has been augmented to such a degree as to be no longer a human. Many transhumanists want to become posthuman. As a posthuman, your mental and physical abilities would far surpass those of any unaugmented human. You would be smarter than any human genius and be able to remember things much more easily. Your body will not be susceptible to disease and it will not deteriorate with age, giving you indefinite youth and vigor. You may have a greatly expanded capacity to feel emotions and to experience pleasure and love and artistic beauty. You would not need to feel tired, bored or irritated about petty things.

The means by which transhumanists hope to achieve posthuman status include, but are not limited to, the following: molecular nanotechnology, genetic engineering, artificial intelligence (some think artificial intelligences will be the first posthumans), mood drugs, anti-aging therapies, neurological interfaces,

Vgl. Pepperell 1995, I.

8 Vgl. Sterling 1985, dt.: Schismatrix. München 1989. Schröter 2002, 84.

9 So spricht Regis 1990 noch ausschließlich vom postbiologischen Menschen bzw. Geist (postbiological man bzw. mind), während Dery 1996 und Hayles 1999 fast durchweg nur noch den Begriff posthuman (posthuman) bzw. Posthumanismus (posthumanism) benutzen. Vgl. Dery 1996, 371; Hayles 1999, 343; Moravec 1988, 1; Regis 1990, 7, 144. 
advanced information management tools, memory enhancing drugs, wearable computers, economic inventions ... and cognitive techniques ... In general, technological or social inventions that improve overall economic efficiency tend to benefit transhumanist aims.

Posthumans could be completely synthetic (based on artificial intelligence) or they could be the result of making many partial augmentations of a biological human or a transhuman. Some posthumans may even find it advantageous to get rid of their bodies and live as information patterns on large super-fast computer networks. It is sometimes said that it is impossible for us humans to imagine what it would be like to be a posthuman. They may have activities and aspirations that we can't even begin to fathom, much as an ape could never hope to understand the complexities of a human life. ${ }^{10}$

\title{
Mit einem anderen Akzent bestimmt N. Katherine Hayles das Wesen des Posthumanismus über die zugrunde liegenden theoretischen Annah- men, die eine Überführung des biologischen Menschen in eine rein vir- tuelle Existenz im Speicher eines Computers ermöglichen sollen:
}

\begin{abstract}
First, the posthuman view privileges informational pattern over material instantiation, so that embodiment in a biological substrate is seen as an accident of history rather than an inevitability of life. Second, the posthuman view considers consciousness ... as an epiphenomenon, as an evolutionary upstart trying to claim that it is the whole show when in actuality it is only a minor side-show. Third, the posthuman view thinks of the body as the original prosthesis we all learn to manipulate, so that extending or replacing the body with other prostheses becomes a continuation of a process that began before we were born.

Fourth and most important, by these and other means, the posthuman view configures human being so that it can be seamlessly articulated with intelligent machines. In the posthuman, there are no essential differences or absolute demarcations between bodily existence and computer simulation, cybernetic mechanism and biological organism, robot teleology and human goals. ${ }^{11}$
\end{abstract}

Gemäß diesen vorläufigen Kennzeichnungen strebt der technologische Posthumanismus soweit eine unbegrenzte Erweiterung der Fähigkeiten des biologischen Menschen an, bis er kein Mensch mehr ist. Der ökonomische und utilitaristische Charakter dieser Utopie ist dabei deutlich geworden (»overall economic efficiency«). N. Katherine Hayles hat den Kern der posthumanistischen Logik herausgearbeitet: Der Mensch wird

10 http://www.transhumanism.org/resources/faq.html (30.10.2002). Jens Schröter spiegelt diese Elemente in seiner Definition wider. Vgl. Schröter 2002, 84f.

11 Hayles 1999, 2 f. 
zunächst als intelligente Maschine definiert und ist folglich durch diese ersetzbar.

Was in bisherigen Studien allerdings vernachlässigt wurde, ist erstens die Rolle der Unsterblichkeit in der posthumanistischen Vision und zweitens die Rezeption religiöser Elemente im Kontext eines kosmischen Heilsentwurfes.

\subsection{Das Gesicht des Posthumanismus}

I consider these future machines our progeny, "mind children « built in our image and likeness, ourselves in more potent form. ${ }^{12}$

Hans Moravec

Zu den wichtigsten Vertretern des Posthumanismus zählen Frank Tipler, Marvin Minsky, Hans Moravec und Ray Kurzweil. Die Reihenfolge der nun folgenden Darstellung ergibt sich aus der Tatsache, dass Tipler bereits 1986 das Grundprinzip seiner späteren Physik der Unsterblichkeit entwickelt und publiziert hat und Minsky wiederum als »Vater der künstlichen Intelligenz« die Ideen von Moravec und Kurzweil in hohem Maße beeinflusste.

\subsubsection{Frank Tipler}

$\mathrm{Zu}$ Beginn seines wohl bekanntesten Werkes The Physics of Immortality (1994) konstatiert Frank Tipler, dass er nicht weniger über die Schlussfolgerungen seines Buches überrascht sei als seine Leser: ${ }^{13}$

When I began my career as a cosmologist some twenty years ago, I was a convinced atheist. I never in my wildest dreams imagined that one day I would be writing a book purporting to show that the central claims of Judeo-Christian theology are in fact true, that these claims are straight-forward deductions of the laws of physics as we now understand them. I have been forced into these conclusions by the inexorable logic of my own special branch of physics. ${ }^{14}$

Moravec 1999, 13.

13 Vgl. Tipler 1995. Deutscher Titel: Die Physik der Unsterblichkeit. Moderne Kosmologie, Gott und die Auferstehung der Toten. München 1995. Die vierte deutsche Auflage erschien 2007.

14 Ebd., IX. 
Frank Jennings Tipler $\left({ }^{*} 1947\right)$ absolvierte seine akademische Ausbildung als Physiker am Massachusetts Institute of Technology und der Universität Maryland (Ph.D. 1976), bevor er nach mehreren Universitätsstellen (Berkeley, Oxford, Austin) schließlich 1981 einen Lehrstuhl für mathematische Physik an der Tulane University in New Orleans erhielt. Hauptsächlich konzentrieren sich seine Forschungen auf Fragen der allgemeinen Relativitätstheorie, Quantentheorie und Kosmologie, die er im Laufe seiner wissenschaftlichen Tätigkeit immer stärker miteinander verbindet. Auch seine frühen und zahlreichen Publikationen, die die Nichtexistenz von außerirdischer Intelligenz im Universum beweisen sollen, ${ }^{15}$ stehen in Zusammenhang mit seinem Interesse für die Genese und die zukünftige Entwicklung des Kosmos. ${ }^{16}$ Schon bevor 1986 sein wissenschaftliches Hauptwerk The Anthropic Cosmological Principle ${ }^{17}$ erschien, das er gemeinsam mit dem englischen Kosmophysiker John David Barrow verfasst hat, veröffentlichte das Autorenteam seit Ende der 1970er Jahre zahlreiche Artikel über kosmologische Fragen. Das Verhältnis vom Menschen zur kosmischen Evolution stand bereits 1984 im Zentrum ihrer Monographie L'homme et le cosmos. Le principe anthropique en astrophysique moderne, die sie zusammen mit der französischen Wissenschaftsjournalistin Marie-Odile Monchicourt publizierten. ${ }^{18}$

Spätestens 1988 endete die zehnjährige wissenschaftliche Kooperation zwischen Tipler und Barrow, ${ }^{19}$ der inzwischen einen Lehrstuhl für angewandte Mathematik und theoretische Physik an der Universität Cambridge innehatte und mit seinen über 500 Veröffentlichungen als einer der führenden Kosmologen unserer Zeit gilt. Barrow betont anders als Tipler auch die Grenzen der physikalischen Kosmologie. ${ }^{20}$

Während The Anthropic Cosmological Principle in der fachfremden Öffentlichkeit weitgehend unbeachtet geblieben war, erlangte Frank Tipler gewissermaßen über Nacht Berühmtheit mit seinem 1994 erschienenen Buch The Physics of Immortality. Modern Cosmology, God and the Resurrection of the Dead. Obwohl Tipler hier lediglich seine Omegapunkt-Theorie,

15 Vgl. Tipler 1981, 1991 und Tiplers bibliographische Angaben in: Barrow \& Tipler 1986.

16 Schon 1980 verfasste er den Aufsatz General Relativity and the Eternal Return zu Fragen nach Anfang und Ende des Universums. Vgl. Tipler 1980.

17 Barrow \& Tipler 1986.

18 Vgl. Barrow \& Tipler 1978, 1979, 1981, 1985b; Barrow \& Tipler \& Monchicourt 1984.

19 Die letzte gemeinsame Publikation diskutiert Wirkungsprinzipien im geschlossenen Universum. Vgl. Barrow \& Tipler 1988.

20 Vgl. Barrow 1997. 
die er bereits 1988 auf einem theologischen Kongress vorgestellt und als Aufsatz publiziert hatte, ${ }^{21}$ weiter ausführte und in radikalen Formulierungen zuspitzte, erregte das Werk großes Aufsehen. Es wurde noch im selben Jahr ins Deutsche übersetzt und stand mehrere Wochen auf der Sachbuch-Bestsellerliste von Spiegel und Focus. Der provokante Versuch, die religiösen Fragen der Menschheit mit mathematischer Genauigkeit zu beantworten, stieß in den Rezensionen weitgehend auf staunendes Unverständnis und schroffe Zurückweisung. Immerhin fühlten sich sogar der anglikanische Theologe John Polkinghorne und der protestantische Theologe Wolfhart Pannenberg zu einem kritischen Kommentar herausgefordert. ${ }^{22}$ Tipler selbst beschreibt seine religiöse Herkunft als einen Weg vom christlichen Fundamentalismus der Kindheit und Jugend bei den Southern Baptists, über eine Zeit des skeptischen Agnostizismus und Atheismus hin zum »fundamentalistischen Physiker «, der die Wahrheit des Christentums beweisen kann. Der fünfzehnjährige Austausch mit Pannenberg sei jedoch der Grund für seine Konversion vom Physiker zum gläubigen Christen gewesen. ${ }^{23}$

In seiner letzten Monographie, The Physics of Christianity von 2007, wiederholt Tipler einerseits seine physikalischen Thesen zum Auferstehungsglauben für ein populäres Lesepublikum. Andererseits beschäftigt er sich mit naturwissenschaftlichen Erklärungen von biblischen Mythen und Wundern wie dem Stern von Bethlehem (Supernova), der jungfräulichen Geburt (Parthenogenese), Erbsünde (genetische Vererbung) und Jesus' Gang über das Wasser (Neutrinostrahl). ${ }^{24}$

In vielen Punkten unterscheidet sich Tiplers Position von der anderer Posthumanisten - seien dies nun der kosmologische Akzent, seine euphorischen Bilder vom virtuellen Paradies oder sein naturwissenschaftlicher Inklusivismus, der Religion nicht überwinden, sondern integrieren will. In seiner kosmologisch fundierten Perspektive geht Tipler davon aus, dass das Universum geschlossen ist und in ferner Zukunft im Punkt

21 Tipler nahm im November 1988 am 2. Pannenberg Symposium am Chicago Center for Religion and Science (Lutheran School of Theology) teil. Vgl. Tipler 1989.

22 Pannenberg lobt ausdrücklich die möglichen Konvergenzen zwischen Physik und Theologie, während Polkinghorne Tipler als »Reduktionisten « und "physikalischen Imperialisten« scharf attackiert. Vgl. Pannenberg 1995; Pannenberg 1995, 3f.; Polkinghorne 1995.

23 Vgl. Tipler 2007, 217; Tipler 2013 (Interview).

24 Vgl. Tipler 2007, 101-242; Tipler 2013 (Interview). Deutscher Titel: Die Physik des Christentums. Ein naturwissenschaftliches Experiment. München 2008. Die Kritiken dieses Buches fielen ebenfalls harsch aus. 
Omega enden wird. Der zeiträumliche Beginn und das Ende des Universums werden durch die initiale und die finale Singularität markiert. ${ }^{25}$ Bis zum Moment dieser finalen Singularität muss intelligentes Leben - das ist die Menschheit mit ihren maschinellen Nachfahren - die völlige Kontrolle über das Universum erlangt haben, während parallel die Menge an Information, die das Leben verarbeitet, mit Annäherung an den Punkt Omega gegen unendlich divergieren wird. Wenn die Sonne in vielen Milliarden Jahren ihre Brennstoffe verbraucht haben wird, besteht Tipler zufolge die einzige Überlebenschance der Menschen in einer virtuellen Existenz in gigantischen Computern. Den Zielpunkt kosmologischer Entwicklungen, den Punkt Omega, identifiziert Tipler mit Gott.

Die Bezüge zu anderen posthumanistischen Autoren sind nur peripher. Tipler verweist in Zusammenhang mit der Idee einer virtuellen Unsterblichkeit und den technischen Möglichkeiten von Computern auf Hans Moravec, der 1990 an seinem Omega Point Colloquium teilnahm. ${ }^{26}$ Marvin Minskys Society of Mind erwähnt Tipler noch in seinem Werk, während er Ray Kurzweils Werke weitgehend ignoriert. ${ }^{27}$ Frank Tipler war zeitweise Mitherausgeber der hauseigenen Zeitschrift der World Transhumanist Association, des Journal of Evolution and Technology. Stoßen die religiösen Implikationen seiner Theorie innerhalb der transhumanistischen Bewegung teils auf starke Ablehnung, so findet der grundsätzliche Gedanke einer kosmischen Teleologie auch viele Befürworter. Moravec gibt Tiplers Omega-Theorie wieder, ohne selbst Stellung zu beziehen. Der Transhumanist Anders Sandberg sieht Tipler zusammen mit Freeman Dyson als Begründer einer physikalischen Eschatologie, hält die Thesen Tiplers jedoch für wissenschaftlich kaum haltbar. ${ }^{28}$ Einen gewissen Einfluss auf die mormonischen und die christlichen Transhumanisten kann man Tipler jedoch nicht absprechen. Der letztgenannten Vereinigung dient Tipler im Academic Advisory Council. ${ }^{29}$

25 Während sich ein offenes Universum beginnend mit dem Urknall immer weiter ausdehnen würde, bremsen im Konzept des geschlossenen Universums die Gravitationskräfte der Materie die Ausdehnung so weit, dass sich der Kosmos schließlich wieder zusammenzieht.

26 Vgl. Tipler 1995, XXIII, 17, 23f., $225 f$.

27 Vgl. a.a.O., 201. Lediglich einen kurzen Verweis auf die Beschleunigung der Rechengeschwindigkeit von Computern laut »Ray Kurtzweil« (sic!) fügt Tipler ein. Vgl. Tipler 2007, 250, 290.

28 Vgl. Moravec 1999, 201f.; Sandberg 1998.

29 Vgl. www.christiantranshumanism.org; https://transfigurism.org. 
Über den biographischen Kontext seines Werkes erhalten Tiplers Leser nur wenige Anhaltspunkte. Auf der ersten Seite der Physics of Immortality widmet er sein Buch den Großeltern seiner Frau, die während der deutschen Besetzung Polens im 2. Weltkrieg ermordet wurden: »Who died in the hope of the Universal Resurrection, and whose hope, as I shall show in this book, will be fulfilled near the End of Time. $"$ Ein weiteres Mal gewährt Tipler uns einen Blick hinter den Vorhang der physikalischen Formeln auf seine persönlichen Motive: "I once visited a Nazi death camp; there I was reinforced in my conviction, that there is nothing uglier than extermination. ${ }^{31}$ Ebenso erläutert er das Theodizeeproblem und den hieraus entstehenden Atheismus am Beispiel des Holocaust. ${ }^{32}$ Und trotz seiner Annahme, dass der Punkt Omega am Ende der Zeiten notwendigerweise alle Sünden eines Hitler oder Stalin vergeben wird, betrachtet Tipler es als gerechtfertigt, Hitler von dieser universalen Vergebung auszuschließen. ${ }^{33}$ Die persönlich bedeutsame Thematisierung des Todes im Kontext des Holocaust führt Tipler direkt zum Ausgangspunkt seiner physikalischen Unsterblichkeitssuche: »We physicists know that a beautiful postulate is more likely to be correct than an ugly one. Why not adopt this Postulate of Eternal Life, at least as a working hypothesis? « 34

Die naturwissenschaftliche Heilsbotschaft ist daher auch in ihrer Wortwahl religiös geprägt: »If any reader has lost a loved one, or is afraid of death, modern physics says: >Be comforted, you and they shall live again. « ${ }^{35} \mathrm{Da}$ es zweifellos nicht die moderne Physik ist, die das behauptet, sondern allein Frank Tipler, so können die persönlichen Anteile in der Physik der Unsterblichkeit nicht unterschätzt werden, die dem Autoren helfen, das rein innerweltliche Sinndefizit, das der Tod ihm gerade in seiner Familiengeschichte offenbart hat, zu bewältigen. ${ }^{36}$

30 Tipler 1995, V.

31 A.a.O., 11.

32 Tipler 1995, 260.

33 Vgl. a.a.O., 253.

34 A.a.O., 11.

35 A.a.O., 1.

36 Daneben bietet die Omega-Theorie Tipler auch ganz triviale Vereinfachungen seines Alltages. Bei einem opulenten Dinner mit einem Journalisten rechtfertigt er seinen übermäßigen Kaloriengenuss mit Verweis auf seine kommende Unsterblichkeit. Vgl. Liversidge 1994, 89. 


\subsubsection{Marvin Minsky}

Weniger umstritten sind - jedenfalls innerhalb des transhumanistischen Diskurses - die Ansichten des amerikanischen Informatikers Marvin Minsky. Obwohl er nie eine Monographie verfasste, die explizit das Ziel verfolgte, den Ersatz des Menschen durch seine posthumanen Nachfolger zu propagieren, kann Minskys Einfluss auf die posthumanistische Philosophie kaum überschätzt werden. Der Mitbegründer des Media Lab am Massachusetts Institute of Technology war Lehrer und Mentor einer ganzen Reihe von heutigen Vertretern des Posthumanismus und Transhumanismus: Ray Kurzweil, Luc Steels, Eric Drexler und Sasha Chislenko studierten bei ihm; Hans Moravec und seinen Kollegen, den Mathematiker und Psychologen Seymour Papert, prägte er mit seinen Forschungen zur künstlichen Intelligenz und zusammen mit Nicholas Negroponte rief er das MIT Media Lab ins Leben. Mit einigen Science-Fiction Autoren - wie Arthur C. Clarke, Robert Heinlein und Frank Herbert - verband ihn ein freundschaftlicher Ideenaustausch. ${ }^{37}$

Marvin Lee Minsky (1927-2016) absolvierte Studium und Promotion an den Universitäten Harvard und Princeton. Er befasste sich von Beginn seiner wissenschaftlichen Karriere an mit der Entwicklung künstlicher Intelligenz. ${ }^{38}$ Seine hochangesehenen Mentoren Norbert Wiener, John von Neumann, Warren McCulloch und Claude Shannon führten sein Erkenntnisinteresse zu der ihn auf Jahrzehnte bestimmenden Frage: Wie funktioniert das menschliche Denken? Ein besseres Verständnis dieser mentalen Prozesse war für Minsky die Voraussetzung für die Entwicklung einer künstlich-intelligenten Software. Immer wieder beschäftigte er sich mit dem Verhältnis zwischen sinnlicher Wahrnehmung und gedanklicher Konstruktion der objektiven Umwelt und der psychischen Innenwelt, Gefühlen und Ideen. ${ }^{39}$

1977 gelang ihm in Kooperation mit seinem Kollegen Seymour Papert der theoretische Durchbruch mit der Idee der gedanklichen Agenten: Der menschliche Verstand entspricht demnach keinem zentralistischen Modell, das durch eine übergeordnete, leitende Stelle im Gehirn gesteuert wird, sondern unsere gedanklichen Leistungen beruhen auf der komplexen Verknüpfung mentaler Agenten (agents), die interagieren

37 Vgl. Artikel Marvin Minsky (engl. Wikipedia).

38 Die Dissertation verfasste er zum Thema Neural Nets and the Brain Model Problem. Vgl. Minsky 1954; Minsky 1967, 32-66.

39 Vgl. Minsky 1965. 
und auch miteinander konkurrieren. ${ }^{40}$ Agenten bilden Bedeutungseinheiten wie »essen«, »heben« oder »Vogel«, die sich erst in semantischen Kontextualisierungen mit anderen Agenten sinnvoll auflösen lassen und menschliches Handeln steuern. Das Zusammenspiel dieser symbolischen Repräsentationen bezeichnet Minsky als Society of Mind - dies ist auch der Titel seines Hauptwerkes von $1985 .{ }^{41}$

Man könnte fast zu der Überzeugung gelangen, Minsky würde eine Replik auf Tiplers physikalischen Reduktionismus verfassen, wenn er eindrücklich und wiederholt davor warnte, das Geschehen in der Welt auf einfache mathematische Regeln zurückzuführen. ${ }^{42}$ Auch wenn Minsky Verständnis hat für die Sehnsucht nach Sicherheit als menschliches Grundbedürfnis, werden seiner Ansicht nach Mathematik und Theologie mit ihrer eindimensionalen Betrachtungsweise der Vieldeutigkeit der objektiven Welt nicht gerecht. ${ }^{43}$ Auch menschliches Denken beruhe daher nicht allein auf den Gesetzen der Logik - ja reine Logik ohne Ziel würde nach Meinung Minskys sogar direkt in den Wahnsinn führen. Vielmehr vermag Denken unlogische Erfahrungen in logische Sätze zu überführen, um damit wenigstens den Regeln der Vernunft zu genügen. Vernunft könne deshalb nicht allein auf Logik reduziert werden. Sein 2006 erschienenes Spätwerk The Emotion Machine vertieft diese Überlegungen zur Komplexität des menschlichen Denkens, das auch das Fühlen - wie Liebe, Schmerz und Leiden - mit einschließt. Den Begriff der »Agenten« gab Minsky hier zugunsten des Konzeptes der »Ressourcen « auf, da zu viele Leserinnen und Leser die Agenten mit Einheiten einer persönlichen oder individuellen Motivation gleichsetzen würden. Das Buch schließt überraschenderweise mit einem Ausdruck der Hochachtung vor der Komplexität des menschlichen Verstandes (the dignity of complexity): Die Vielfalt der Ressourcen des Geistes beruhen laut Minskys Einsicht nämlich auf der genetischen Vererbung, der kulturellen Sozialisation sowie den individuellen Erfahrungen. ${ }^{44}$

Wie ich in späteren Kapiteln zeigen werde, liegt Minskys Bedeutung für den Posthumanismus vor allen Dingen in der Formulierung der informationstechnischen Grundlagen des posthumanistischen Menschenbildes. Sogar in einem unauffälligen Lehrbuch für Informatik setzte er die Evo-

\footnotetext{
40 Vgl. Minsky 1977.

41 Vgl. Minsky 1988.

42 Vgl. a.a.O., 96; Minsky 1992b, 2.

43 Vgl. Minsky 1988, 127, $301 \mathrm{f}$.

44 Vgl. a.a.O., 184-189; Minsky 2006, 8, 341-346.
} 
lution des Menschen in Beziehung zu derjenigen der Maschinen: »Man has within a single generation found himself sharing the world with a strange new species: the computers and computer-like machines. $\ll^{45}$ Darüber hinaus bekannte sich Minsky zum Materialismus und ging davon aus, dass Religion in erster Linie soziale Funktionen erfülle. Da die Religionen vor allem empirisch und logisch unlösbare (zirkuläre) Fragen beantworten und diese eindeutigen Antworten durch Indoktrination verbreiten, würden sie zur Stabilisierung der gesellschaftlichen Ordnung beitragen. ${ }^{46}$ In ihrer Bedeutung für die Wissenschaft und Aufklärung verurteilte Minsky - und mit ihm Hans Moravec - die Religion als rückständig und repressiv, da sie über Jahrtausende verhindert habe, dass sich der Mensch um die technische Realisierung der Unsterblichkeit bemühte:

They have always assumed that personal death was in the very nature of things. Most of their recorded history describes how their leaders were always inventing imaginary superbeings. Then, instead of trying to solve the hard technical problems, those leaders convinced their followers that simply believing those marvelous tales would endow them with everlasting life - whereas disbelief would be punished by death. ${ }^{47}$

Die heilsversprechenden Visionen einer Optimierung des Menschen durch maschinelle Substitute erwähnte er zunächst bloß am Rande und konkretisierte erst seit den 1990er Jahren seine posthumanistischen Ideen in Aufsätzen. Sein Ansehen als weltweit prominentester Vertreter der KI-Forschung verband er in seinen letzten Jahrzehnten mit dem Engagement für die transhumanistischen Organisationen. 1997 bekannte er sich auf der dritten Konferenz des amerikanischen Extropy Institute zur Kryonik, gehörte seitdem zu den wissenschaftlichen Beratern der größten Kryonik-Stiftung Alcor in Kalifornien und hat auch den Scientist's open Letter on Cryonics (2004-2009) unterzeichnet. ${ }^{48}$

45 Minsky 1967, VII.

46 Vgl. Minsky 1988, 49, 247, 283-286, 306f.; Minsky 1992a, $24 f$.

47 Minsky 1992a, 24. Vgl. auch Minsky 1988, 41; Moravec 1988, 4.

48 Mit Rücksichtnahme auf die privacy policy will Alcor das Einfrieren von Minskys Leichnam weder bestätigen noch dementieren. Alcors Fallbeschreibung von »Patient 144, gest. am 24.01.2016 « legt jedoch nahe, dass es sich hierbei um Marvin Minsky handelt, der gegen den Willen seiner Familie drei Tage nach seinem Tod eingefroren wurde (http://www.alcor.org/blog/a-1700-case-summary-patient-144/). Vgl. auch Kurzweil 2016 (Video). 


\subsubsection{Hans Moravec}

Seit der frühesten Kindheit war Hans Moravec $\left({ }^{*} 1948\right)$, der als Heranwachsender fast nur Science-Fiction Literatur las, von Robotern fasziniert. Mit 10 Jahren gelang ihm erstmals die Konstruktion eines Roboters aus Metallschrott mit einem motorisierten Arm. Mit seinen Eltern immigrierte er 1953 von Österreich nach Kanada. ${ }^{49}$ Angeregt durch seinen Vater, einen Elektroingenieur, bastelte er als Jugendlicher an einer lichtgesteuerten Roboterschildkröte und auch an einem ersten Computer. Seit Beginn seines Studiums der Mathematik und Computerwissenschaften in verschiedenen Roboterlaboratorien der Vereinigten Staaten und Kanadas arbeitete er als Programmierer und Forschungsassistent. Nach seiner Promotion über Robotik 1980 an der Stanford University erhielt er eine Forschungsprofessur an der Carnegie-Mellon University, wo er bis 2005 das Mobile Robot Laboratory leitete. 2003 gründete er in Pittsburgh die Seegrid Corporation, die autonome Transport- und Steuerungssysteme für Industrie- und Lagertechnik entwickelt und produziert. 1988 erschien sein zuvor schon in Manuskripten verteiltes Werk Mind Children. The Future of Robot and Human Intelligence, ${ }^{50}$ das ihn für viele heutige Anhänger zum eigentlichen Begründer einer posthumanistischen Philosophie machte. Denn während in den frühen Werken von Tipler und Minsky posthumanistische Entwicklungsmöglichkeiten des Lebens lediglich am Rande Erwähnung finden, ${ }^{51}$ rückt der Robotiker Moravec seine hoffnungsvollen Visionen ins Zentrum. Schon das Vorwort wirkt wie eine Präambel des Posthumanismus:

Engaged for billions of years in a relentless, spiraling arms race with one another, our genes have finally outsmarted themselves ... What awaits us is not oblivion but rather a future which, from our present vantage point, is best described by the words "postbiological« or even "supernatural«. It is a world in which the human race has been swept away by the tide of cultural change, usurped by its own artificial progeny ... within the next century they [the machines: O. K.] will mature into entities as complex as ourselves, and eventually into something transcending everything we know - in whom we can take pride when they refer to themselves as our descendants. ${ }^{52}$

${ }^{49}$ Vgl. Moravec 1999, VII.

50 Vgl. Moravec 1988. Deutscher Titel: Mind Children. Der Wettlauf zwischen menschlicher und künstlicher Intelligenz. Hamburg 1990.

51 Vgl. Barrow \& Tipler 1986; Minsky 1988.

52 Moravec 1988, 1. 
Moravec ist der Ansicht, dass diese posthumanen, künstlichen Intelligenzen zum Menschen in einem ähnlichen Verhältnis wie Kinder zu ihren Eltern stehen. ${ }^{53}$ Und im Rückgriff auf eine Metapher des Evolutionstheoretikers Richard Dawkins, der Gott als blinden Uhrmacher charakterisierte, übernähmen die Menschheit und ihre posthumanen Erben in der Deutung Moravecs soeben die Führung dieser Hand des Uhrmachers. ${ }^{54}$

Diese Botschaft wiederholt Moravec seit mehr als drei Dekaden. In zahlreichen Publikationen kündigt er das Erscheinen künstlicher Intelligenz an, die vom Zeitpunkt der Prognosen aus jeweils in ca. 30 Jahren so leistungsfähig sein würden wie der menschliche Verstand. ${ }^{55}$ Seine zweite geplante Monographie The Age of Mind. Transcending the Human Condition through Robots (1994) blieb wegen Schwierigkeiten mit dem Verleger unveröffentlicht, ${ }^{56}$ während sein letztes Werk Robot. Mere Machines to Transcendent Mind (1999) auf großes Interesse stieß. ${ }^{57}$ Der publizistische Erfolg dieses Buches, das zeitgleich mit Ray Kurzweils The Age of Spiritual Machines ${ }^{58}$ erschien, hat sein starkes Echo wohl der technikeuphorischen Stimmung in den ausgehenden 1990er Jahren zu verdanken.

Moravecs futuristische Prophezeiungen werden jedoch von der Fachwelt der KI-Forschung vor allen Dingen deshalb als haltlose Spekulationen zurückgewiesen, weil Moravec als Robotiker argumentiert: Er befasst sich nur peripher mit der Entwicklung komplexer KI, hauptsächlich jedoch mit der autonomen Steuerung von Maschinen im Raum. Als Kriterium der Intelligenz beachtet Moravec nur die Speicherkapazität und Rechengeschwindigkeit eines Systems und vernachlässigt weitestgehend die »Software« des Denkens, deren Entwicklung der KI-Forschung so viele Probleme bereitet. ${ }^{59}$

In seiner Weitsicht behandelt Moravec darüber hinaus auch soziologische Fragestellungen der zukünftigen Gesellschaft, wenn menschliche

53 Vgl. Moravec 1999, 13.

54 Vgl. a.a.O., 158f. Dawkins wiederum greift die Metapher des Theologen William Paley in seiner Natural Theology von Gott als dem kosmischen Uhrmacher auf. Vgl. Dawkins 1996.

55 Vgl. a.a.O., 68-74; Moravec 1996b, 1996c, 1998, 1999, 72ff; Moravec \& Shieber 1997.

56 Moravec hat nach der gescheiterten Veröffentlichung offenbar einzelne Kapitel publiziert und diese schließlich in Robot. Mere Machines to Transcendent Mind. (1999) neu zusammenfasst. Vgl. Moravec 1996b, 1996c, 1998.

57 Vgl. Moravec 1994, 1999; McGinn 1999.

58 Vgl. Kurzweil 1999.

59 Vgl. Moravec \& Shieber 1997; Minsky 1988. 
Arbeit überflüssig geworden sein und die Steuerung der Wirtschaft in den Händen von Roboterkonzernen liegen wird. ${ }^{60}$ Der Mensch werde dann jedoch nicht zum Wesen zweiter Klasse degradiert, sondern erhalte in der Symbiose mit den Computern die Chance, seine biologische Bedingtheit zu überwinden. ${ }^{61}$

Moravecs herausragende Bedeutung für die posthumanistische Philosophie rührt vor allem daher, dass er 1988 als erster Wissenschaftler die technischen Möglichkeiten der Unsterblichkeit in der Virtualität formuliert hat. Nicht als Science-Fiction Autor, sondern als wissenschaftlicher Visionär stellt Moravec die normativen Leitgedanken der zukünftigen Entwicklung der Menschheit dar. Präzise schildert er den technischen Vorgang dieser transmigration (Seelenwanderung). ${ }^{62}$

You've just been wheeled into the operating room. A robot brain surgeon is in attendance. By your side is a computer waiting to become a human equivalent, lacking only a program to run ... The robot surgeon opens your brain case and places a hand on the brain's surface ... Instruments in the hand scan the first few millimeters of brain surface ... These measurements, added to a comprehensive understanding of human neural architecture, allow the surgeon to write a program that models the behavior of the uppermost layer of scanned brain tissue. This program is installed in a small portion of the waiting computer and activated ... The process is repeated for the next layer... In a final disorientating step the surgeon lifts out his hand. Your suddenly abandoned body goes into spasms and dies. For a moment you experience only quite and dark. Then, once again, you can open your eyes... Your metamorphosis is complete. ${ }^{63}$

In einem frühen Beitrag in einem Science-Fiction Magazin von 1979 prophezeite Moravec, dass die computertechnischen Voraussetzungen also Speicher, Rechenleistung und künstlich intelligente Computerprogramme auf menschlichem Niveau bereits 10 Jahre später zur Verfügung stehen könnten. ${ }^{64}$ Auf diese Weise wird der Mensch in der Vision Moravecs als virtuelle Simulation im Speicher eines Computers seine unendliche Fortexistenz sichern, während die biologische Menschheit langsam ausstirbt. Nach eigenem Bekunden hatte Moravec die Idee des uploading

60 Vgl. Moravec 1999, 127-162.

61 Vgl. Moravec 1988, 100ff.

62 Vgl. a.a.O., 108f. In einem Aufsatz von 1993 spricht Moravec allerdings von der religiöse Implikationen vermeidenden Geisttransplantation (mind transplant). Vgl. Moravec \& Pohl 1993, 66. Moravec geht davon aus, dass auch schon Alan Turing diese Option einer technischen Seelenwanderung vorschwebte. Vgl. Moravec 1999, $77 f$.

63 A.a.O., 109f. Eine frühe Version findet sich in Moravec 1979, 78-82.

64 Vgl. Moravec 1979, 76f. 
schon während seiner Highschool-Zeit entwickelt. ${ }^{65}$ Diese Stelle aus Moravecs Mind Children zeichnet für alle späteren posthumanistischen Autoren den konkreten technischen Vorgang einer Immortalisierung vor: Das materielle Gehirn dient in einem Scan-Prozess als Vorlage für die weitere, unbegrenzte Existenz in der Virtualität. Transhumanisten wie auch deren Kritiker referieren stets diese technische Deskription des uploads. ${ }^{66}$ Dabei hatte Moravec noch drei weitere Möglichkeiten einer Aufzeichnung der Inhalte unseres Gehirns in Betracht gezogen: erstens durch einen hochauflösenden Gehirn-Scan, der das originale Gehirn nicht zerstört, jedoch das philosophische Problem eines virtuellen Doppelgängers mit sich bringen würde; zweitens durch eine Art tragbaren Computer, der alle Aktivitäten des Gehirns und interaktive Erfahrungen mit der Umwelt aufzeichnet. Drittens stellt sich Moravec vor, dass die größte Verbindung zwischen den beiden Hemisphären unseres Gehirns, das corpus callosum, von einem Computer angezapft werden könnte:

Suppose in the future, when the function of the brain is sufficiently understood, your corpus callosum is served and cables leading to an external computer are connected to the severed ends ... In time, as your original brain faded away with age, the computer would smoothly assume the loss of functions. Ultimately your brain would die, and your mind would find itself entirely in the computer. ${ }^{67}$

Hans Moravec scheint in keiner der transhumanistischen Organisationen offizielle Funktionen auszuüben, hat jedoch einen Beitrag im Journal of Evolution and Technology veröffentlicht und auf der ersten Tagung des Extropy Instituts 1994 als Eröffnungsredner mitgewirkt. Seit seiner Firmengründung 2003 ist er kaum noch publizistisch aktiv. ${ }^{68}$

\subsubsection{Ray Kurzweil}

Raymond C. Kurzweil (*1948) ist inzwischen sicherlich der bekannteste ehemalige Student Marvin Minskys, der seit seinem Universitätsabschluss am MIT 1970 nicht weniger als sechs Firmen in der Informa-

65 Vgl. Regis 1990, 156.

66 Vgl. Dery 1996, 299ff.; Leary \& Sirius 1997, 171; Regis 1990, 4.

67 Moravec 1988, 112. Ähnliche Positionen wie Moravec vertrat im deutschsprachigen Raum der Wissenschaftsjournalist Gero von Randow in seinem Buch Roboter. Unsere nächsten Verwandten (1998).

68 Vgl. Moravec 1992, Moravec 1998; Moravec 1999, 219 und http://www.extropy.org/ events. 
tionstechnologie-Branche gegründet hat. ${ }^{69} \mathrm{Ihm}$ gelangen Innovationen wie die Lesemaschine Xerox für Blinde (1976), die gelesene Texte in gesprochene Sprache verwandelt, Spracherkennungsprogramme, spezielle Computerprogramme für Kompositionen sowie Software für Keyboards. Den Höhepunkt seiner beruflichen Karriere markiert gewiss die Berufung zum Director of Engineering des Google-Konzerns 2012. Mehrere Dutzend nationale und internationale Auszeichnungen - darunter 20 Ehrendoktorwürden - belohnten bisher die Bemühungen des Bostoner Erfinders. In verschiedenen Interviews unterstreicht Kurzweil stets, dass er alles daransetzt, selbst die so genannte Singularität zu erreichen, den heilsgeschichtlichen Punkt, an dem die technische Unsterblichkeit verfügbar sein soll. Er hält deshalb eine strenge Diät ein und nimmt bis zu 90 Vitaminpillen täglich zu sich. ${ }^{70}$

Sein Buch The Age of Spiritual Machines. When Computers exceed Human Intelligence $(1999)^{71}$ vermarktete der Unternehmer mit einer professionellen Werbekampagne gleichzeitig in mehreren Ländern und genoss in Deutschland die Aufmerksamkeit von renommierten Zeitungen wie der Frankfurter Allgemeinen Zeitung und der ZEIT - ja sogar die deutsche Technikkritik wählte sich Kurzweil als den führenden Vertreter einer posthumanistischen Techno-Kultur. Meinem Eindruck nach ist Kurzweil damit der einzige Protagonist posthumanistischer Ideen, der bisher von einer breiteren Öffentlichkeit wahrgenommen wird. 2009 wurde sogar ein Dokumentarfilm über Kurzweil unter dem Titel Transcendent Man. The Life and Ideas of Ray Kurzweil präsentiert. ${ }^{72}$

Dabei handelt es sich bei seinem frühen, 1990 erschienenen Werk The Age of Intelligent Machines, ${ }^{73}$ das damals auch als meistverkauftes Buch der Computerwissenschaften überzeugte, noch um eine rein technikgeschichtliche Betrachtung der Entwicklung künstlicher Intelligenz. Das kurze Zukunftsszenario innerhalb des Buches beinhaltet geläufige Folgen des zunehmenden Einsatzes von Maschinen in der Arbeitswelt und

69 Das sind Kurzweil Computer Products, Kurzweil Music Systems, Kurzweil Applied Intelligence, Waltham, Kurzweil Educational Systems und Kurzweil Technologien. Vgl. Kurzweil 1999a, $367 f$.

70 Vgl. Keller 2003, 14-17; Agar 2010, 35-81.

71 Vgl. Kurzweil 1999a. Deutscher Titel: Homo s@piens. Leben im 21. Jahrhundert. Was bleibt vom Menschen? Köln 1999. Zwei Jahre später erschien bereits die dritte deutsche Auflage des Buches.

72 Vgl. Wagner 2015, 100.

73 Vgl. Kurzweil 1990. Deutscher Titel: KI - das Zeitalter der künstlichen Intelligenz. München 1993. 
einige Aussichten auf die künftige Freizeitgestaltung. ${ }^{74}$ Während 1990 seine weitreichendste Prophezeiung ein Computer war, der zwischen 2020 und 2070 ein eigenes Bewusstsein entwickelt haben wird, ${ }^{75}$ will Kurzweil im Jahr 1999 den Anfang vom Ende der Menschheit einleiten: Demnach würden im Jahr 2099 Mensch und Maschine miteinander verschmolzen sein und der Mensch seine biologische Bedingtheit überwunden haben. ${ }^{76}$ In seinem radikalsten Werk, The Singularity is near von 2005, werden die heilsgeschichtlichen Aussichten um ein halbes Jahrhundert auf das Jahr 2045 vorverlegt und mit der universalen Lösung aller Menschheitsprobleme verknüpft. ${ }^{77}$

Ray Kurzweil kombiniert in seinen späteren Werken die posthumanistischen Visionen und Argumentationsmuster von Marvin Minsky, Hans Moravec, Frank Tipler und anderen Futuristen wie insbesondere Vernor Vinge, der ab den 1980er Jahren das Konzept der technologischen Singularität prägte. Obwohl seine Ideen kaum Neuerungen innerhalb des posthumanistischen Diskurses enthalten, erklärt sich sein publizistischer Erfolg aus der Fähigkeit, bestimmte Ideen des Posthumanismus sehr pointiert und leicht verständlich zu präsentieren. Kurzweil stand zwar nie im Zentrum der transhumanistischen Organisationen und Einrichtungen, ist aber dennoch - wie wir im vorangegangenen Kapitel sehen konnten - äußerst gut vernetzt und als Vortragsgast gern gesehen.

Seit den 1990ern schreibt Kurzweil auch Lebenshilfebücher wie zunächst The 10\% Solution for a Healthy Life (1993), in dem er für eine fettarme Ernährung eintritt. Diesen Ratgeber setzte er 2004 mit Fantastic Voyage: Live Long Enough to Live Forever fort (mit Co-Autor Terry Grossman). Die Notwendigkeit zur gesunden Lebensführung besteht darin, dass nach Meinung der Autoren ab ca. 2024 Therapien mittels Gentechnik und Nanobots zur Verfügung stehen werden, die den Alterungsprozess stoppen und umkehren können. Den eigenen Tod könne man daher so lange aufschieben, bis auch diese letzte Grenze des Lebens technisch übersprungen werde. ${ }^{78} \mathrm{Im}$ etwas modifizierten Nachfolgeband, Transcend: Nine Steps to Living Well Forever (2009), propagieren beide Autoren nun die Transzendierung der »menschlichen Steinzeitgene«.

\footnotetext{
74 Vgl. a.a.O., 401-416.

75 Vgl. a.a.O., 483.

76 Vgl. Kurzweil 1999a, 277-280.

77 Vgl. Kurzweil 2005. Deutscher Titel: Menschheit 2.0. Berlin 2013.

78 Kurzweil \& Grossman 2004, 4. Aubrey de Grey gilt ihnen als Garant für diese Utopie.
} 
Insgesamt zeigt sich bei einem Großteil der wichtigsten Post- und Transhumanisten eine bemerkenswerte biographische Parallele: Sie sind amerikanische Einwanderer der ersten oder zweiten Generation: Hans Moravec immigrierte aus Österreich bzw. Kanada in die Vereinigten Staaten, Ray Kurzweil ist Sohn jüdischer Emigranten aus Wien. Der im Iran geborene Fereidoun M. Esfandiary, und der in England aufgewachsene Führer der Extropianer, Maximilian O'Connor änderten in ihrer amerikanischen Wahlheimat sogar die ihre ethnische Herkunft offenbarenden Namen in FM-2030 bzw. Max More, wobei letzterer seine philosophische Doktorarbeit (The Diachronic Self) an der University of Southern California just über eine Theorie der vom Körper unabhängigen Identitätskonstruktion verfasste.

An dieser Stelle kann nur die Vermutung geäußert werden, dass der biographische Kontext einiger Posthumanisten als Emigranten bzw. als Kinder von Emigranten in den Vereinigten Staaten ein besonderes Verhältnis zu ihrer subjektiven Konstruktion von Identität bedingt hat. Die angesprochenen Autoren sind offensichtlich keine Vertreter einer kulturellen Getthoisierung, sondern haben sich äußerst erfolgreich in der amerikanischen Gesellschaft etabliert. Diesem Anpassungsprozess liegt eine Auffassung zugrunde, die Identität nicht als ethnisches Erbe, also als Produkt einer körperlichen Genealogie, versteht, sondern als Ergebnis der Enkulturation: Nicht der Körper bestimmt die persönliche Identität, sondern der Geist. Diese Annahme ist insofern bemerkenswert, als dass genau hier der Ausgangspunkt der für den Posthumanismus fundamentalen Mustertheorie der Identität zu finden ist.

\subsection{Posthumanismus und Kunst}

In fact it is now time to REDESIGN HUMANS, TO MAKE THEM MORE COMPATIBLE WITH THEIR MACHINES. ${ }^{79}$

Stelarc

Seit der Begründung des Futurismus durch den italienischen Poeten Emilio Filippo Thomaso Marinetti ist die Zukunft Gegenstand des

79 Stelarc 1998, 121. 
künstlerischen Schaffens. Parallel zu den philosophisch-technischen Entwürfen des Posthumanismus und den technologischen Debatten im Transhumanismus wird der künftige Mensch in der Gegenwartskunst verhandelt. Die gesamte wissenschaftliche Auseinandersetzung mit dem Posthumanismus begann sogar im Kunstforum International. ${ }^{80}$ Das Verhältnis von Mensch und Technologie bewegte schon 1909 Marinetti zur Veröffentlichung des Manifeste Initial du Futurisme, das wie im Rausch die Welt der Maschinen verherrlichte und den menschlichen Körper veraltet erscheinen ließ:

\begin{abstract}
Allons, dis-je, mes amis! Partons! Enfin la Mythologie et l'Idéal mystique sont surpassés. Nous allons assister à la naissance du Centaure et nous verrons bientôt voler les premiers Anges! ... Nous nous approchâmes des trois machines renâclantes pour flatter leur poitrail. Je m'allongeai sur la mienne comme un cadavre dans sa bière ... Nous sommes sur le promontoire extrême des siècles! ... A quoi bon regarder derrière nous, du moment qu'il nous faut défoncer les vantaux mystérieux de l'Impossible? Le Temps et l'Espace sont morts hier. Nous vivons déjà dans l'absolu, puisque nous avons déjà créé l'éternelle vitesse omniprésente. ${ }^{81}$
\end{abstract}

In Marinettis Konzept des Futurismus wurde die Natur förmlich von der Geschwindigkeit, Kraft und Schönheit der Automobile und Aeroplane überholt und zurück blieb der »kotige und übelriechende Körper des Menschen ${ }^{82}$ Voller Überschwang forderte Marinetti den mechanischen, nicht-menschlichen Typ, der mit der allgegenwärtigen Geschwindigkeit Schritt halten könne und dem Verwandlungs- und Optimierungspotential der Maschinen entsprechen werde. ${ }^{83}$

Ganz in der Tradition Marinettis veröffentlichte der englische Künstler Robert Christian Pepperell, der an der Cardiff School of Art and Design lehrt, 1995 sein Post-Human Manifesto. Das 16-seitige Bekenntnis berührt neben allgemeinen Feststellungen die Aspekte des Bewusstseins, der Wissenschaft, der Ordnung und Kontinuität unserer Erfahrungswelten, Kunst, Kreativität und künstliche Wesenheiten:

Post-Humanists are people who understand how the world is changing. By understanding this they are changing the world ...

${ }^{80}$ Vgl. Rötzer 1989, 1996.

81 F. T. Marinetti: Manifeste Initial du Futurisme. In: Figaro, 20.02.1909. Zitiert nach: Andreoli-de-Villers 1986, 43-49.

82 Vgl. a.a.O., 45.

83 Vgl. Rötzer 1997, 74. 
It is now clear that humans are no longer the most important things in the universe. This is something the humanists have yet to accept ...

In the Post-Human era machines will be gods ...

Complex machines are an emergent life form ...

Currently the output of computers is predictable. The Post-Human era begins in full when the output of computers is unpredictable $\ldots{ }^{84}$

In anderen Punkten unterscheidet sich Pepperell grundsätzlich von Vertretern des technologischen Posthumanismus. Bewusstsein ist für ihn eine Leistung, die der gesamte menschliche Organismus hervorbringt und nicht nur das Gehirn. Alles Sein ist gemäß Pepperell durch Energie bestimmt, so dass folglich keine ontologische Differenz zwischen Lebewesen und Dingen besteht. Die Stellung des Menschen in seiner Welt gestaltet sich daher als offene Frage: »The humanist era was characterized by certainty about the operation of the universe and the place of humans within it. The Post-Human era is characterized by uncertainty about the operation of the universe and about what it is to be human. ${ }^{85}$ Viele dieser frühen Gedanken Pepperells treten später im kritischen Posthumanismus in Erscheinung, ohne dass jedoch auf den Künstler Bezug genommen wird.

Während Marinetti in seiner Begeisterung für das "moderne Leben« den Menschen als Maschine definierte und für die Verbesserung dieser Menschmaschine eintrat, knüpft in unseren Tagen der australische Aktionskünstler Stelarc (d.i. Stelios Arcadiou) (*1946) an futuristische Ideen an, indem er die Verschmelzung zwischen Mensch und Technik zelebriert. Seine Botschaft, die er seit 50 Jahren bewusst antiphilosophisch in Aufsehen erregenden "physiologischen Performances" inszeniert, wiederholt stets die Erkenntnis, dass der menschliche Körper obsolet sei. Stelarc ist damit einer der frühesten Vertreter der gegenwärtigen transhumanistischen Visionen. ${ }^{86} \mathrm{Er}$ ist davon überzeugt, dass in einer postmodernen Gesellschaft, die sich anschickt, in den Weltraum aufzubrechen, ein weicher und wässriger Körper nur hinderlich sei: ${ }^{87}$

\footnotetext{
${ }^{84}$ Pepperell 1995, 180-195.

85 Pepperell 1995, 191. Vgl. a.a.O., 181-191.

86 Vgl. Koplos 1993, 104. Texte von Stelarc, besonders zu den technischen Einzelheiten seiner Visionen, und Beschreibungen seiner Projekte finden sich auf Stelarcs Homepage: http://stelarc.org.

87 Vgl. Stelarc 1996, 73ff.; Stelarc 1998, 118.
} 
It is time to question whether a bipedal, breathing body with binocular vision and a $1400 \mathrm{ccm}$ brain is an adequate biological form. It cannot cope with the quantity, complexity, and quality of information it has accumulated; it is intimidated by the precision, speed, and power of technology and it is biologically ill-equipped to cope with its new extraterrestrial environment. ${ }^{88}$

1981 präsentierte Stelarc in der Performance The Third Hand in Tokyo erstmals eine künstliche Roboterhand, die an seinem rechten Arm befestigt war. Über ein komplexes System von Sensoren bewegte sich diese Hand in späteren Performances teils unabhängig von Stelarcs Vorgaben wie auch die künstlichen Laseraugen, die Strahlen aussandten. Während diese technischen Erweiterungen des Körpers bis Ende der 1980er Jahre als Prothesen des Körpers vorgeführt wurden, bildete Stelarcs Körper in den neueren Vorstellungen eine Symbiose mit den maschinellen Supplementen. Diese Inszenierung des paradigmatischen Erdbebens, wie es die Kunstkritik empfand, gelang Stelarc in Structure/Substance: Amplified Body, Laser Eyes, and Third Hand, Re-Wired/Re-Mixed, Propel (1990, 2015, 2016), Host Body/Couple Gestures: Event for Virtual Arm, Robot Manipulatore, and Third Hand (1992) und Remote Gestures/Obsolete Desires (1993), indem die Bewegungen und Reaktionen der mit seinem Körper gekoppelten Technik unwillkürlich vom Herzschlag, den Gehirnströmen und den Bauchmuskeln des Künstlers gesteuert wurden. Erfassten Sensoren auf diese Weise einerseits Stelarcs Sinnesreizungen, so waren andererseits seine Körpermuskeln mit elektronischen Stimulatoren verbunden, die auf seine Bewegungen reagierten und ihn wie eine Marionette erzittern ließen. Die künftige Harmonie des Menschen mit den Maschinen erlebte ihren bisherigen Höhepunkt in Stelarcs Performance Fractal Flesh: An Internet Body Upload Performance (1995), in der die Muskelstimulatoren an Signale aus dem Internet gekoppelt wurden und der Künstler einen archaisch anmutenden Tanz synchron mit einem stählernen Industrieroboter vollführte. ${ }^{89}$ Einen weiteren Schritt ging Stelarc 2008, als er sich ein drittes $\mathrm{Ohr}$ als Silikon-Implantat operativ in den Unterarm einpflanzen ließ (Ear on Arm). ${ }^{90}$

Die physiologischen Inszenierungen Stelarcs geraten zum Gesamtkunstwerk, das nicht nur die geistige Überwindung des Körpers jenseits von Fleisch und Blut propagiert - in seinen frühen Performances und jüngst

\footnotetext{
88 A.a.O., 117.

89 Vgl. Koplos 1993; Caygill 1995; Dery 1996, 153-169.

90 Vgl. http://stelarc.org.
} 
wieder 2012 (Ear on Arm Suspension) ließ er sich an durch die Haut gezogenen Stahlhaken aufhängen. Stelarc will damit den schwächlichen biologischen Körper auf die künftige Vereinigung mit technischen Körpererweiterungen vorbereiten. ${ }^{91}$

Mit der bewussten Planung kommender Lebensformen durch die Gentechnik und durch den Einsatz von technischen Implantaten ist für Stelarc das Zeitalter der Postevolution angebrochen: Der Körper wird seine biologische Konstitution überwinden und die Form von der Materie befreien, um einen von der irdischen Biosphäre unabhängigen Planeten-Menschen zu entwerfen. Große Hoffnungen setzt Stelarc dabei in die Nanotechnologie, die von innen heraus den Menschen zu einer Maschine umformen soll, deren fehlerhafte Einzelteile man dann problemlos austauschen könnte: ${ }^{92}$

THERE WILL TECHNICALLY BE NO BIRTH. And if the body can be redesigned in a modular fashion to facilitate the replacement of malfunctioning parts, then TECHNICALLY THERE WOULD BE NO REASON FOR DEATH ... Death does not authenticate existence. It is an outmoded evolutionary strategy ... In the extended space - time of extraterrestrial environments, THE BODY MUST BECOME IMMORTAL TO ADAPT. ${ }^{93}$

Die weitreichendsten Körper-Visionen enden auch bei Stelarc im virtuellen Raum, da ein materieller Körper stets fehleranfällig bleibe und sich nur mühsam durch den realen Raum bewegen könne. Die Möglichkeiten als Phantomkörper, als Bild (Image), in der virtuellen Realität offenbare die »Impotenz « des physischen Körpers. ${ }^{94}$

Die gleichzeitige Verschmelzung mit künstlicher Intelligenz, die im virtuellen Raum vollzogen werden soll, eröffne dem Menschen das höhere Bewusstsein eines neuen Zeitalters: »The significance of interfacing with it is that they culminate in an ALTERNATE AWARENESS THAT IS PAN-HISTORIC AND POST-HUMAN. « ${ }^{95}$ Trotz seines Engagements für die Überwindung des gegenwärtigen Menschen ist Stelarc skeptisch gegenüber den Unsterblichkeitsvisionen Hans Moravecs und will sich mit praktischen Fragen der Erweiterung des Körpers befassen - nicht mit dessen metaphysischer Überwindung:

\footnotetext{
91 Vgl. Neesham \& Smith 1995; Rötzer 1997, S. 74ff.

92 Vgl. Virilio 1994, 120-123.

93 Stelarc 1998, 120.

94 Vgl. a.a.O., 123.

95 Ebd.
} 
Ohne Körper kein Bewusstsein. Bewusstsein ist doch das Resultat einer Interaktion mit der Welt ... Die Vorstellung eines vom Körper losgelösten Bewusstseins ist Blödsinn ... Meine Ideen, die ich in den Performances umsetze, sind keine Science-Fiction Ideen. Ich bin nicht interessiert an Dingen und Vorstellungen, die man nicht über den Körper, über die Operation mit Maschinen, über andere Körper oder andere soziale Systeme erfahren kann. ${ }^{96}$

Ist Stelarc innerhalb der internationalen Performance-Kunstszene sicherlich einer der bedeutendsten Gegenwartskünstler, so bleibt Natasha Vita-More außerhalb der transhumanistischen Bewegung weitgehend unbekannt und nimmt auch keinen Bezug auf rezente Künstlerinnen und Künstler. ${ }^{97}$ Ihr skizzenhaftes Manifest der transhumanistischen Kunst von 1983 blieb lange Zeit unveröffentlicht, dennoch erhebt sie den Anspruch, als Wegbereiterin einer transhumanen Kunstepoche das Ende der modernen Kunst einzuläuten. ${ }^{98}$ Und sind es bei Marinetti die rasenden Automobile, die den stürmischen Fortschrittsoptimismus legitimieren, so werden bei Vita-More die Raumschiffe des 21. Jahrhunderts zur Metapher der unbegrenzten technischen Möglichkeiten:

How will the $21^{\text {st }}$ Century look like? It will look like the $21^{\text {st }}$ Century technology space craft that will steam across the solar system and beyond; like the new and versatile durable immortal bodies that we will inhabit ... like the vision of those imaginative to transcend the limitations of their conditions with the fusion of biology and intelligent machines. ${ }^{99}$

Die transhumanistische Kunst habe vor allem die Aufgabe, die futuristischen Visionen künstlerisch zu illustrieren. Vita-More, die hauptsächlich Videokunst und Bilder produziert, verfasste 1997 das Extropic Art Manifesto:

We are transhumans. I am the architect of my existence. My art reflects my vision und represents my values. It conveys the very essence of my being - coalescing imagination and insight, challenging all limits ... We are neo-cyberneticists utilizing high-end creativity ... Extropic Art emphasizes the infinite possibilities of self-transformation ... We are active participants in our own evolution from human to posthuman. ${ }^{100}$

\footnotetext{
96 Stelarc 2000, 123. Zu Stelarc allgemein vgl. auch Tabbert 2004, 414-423.

97 Vgl. Vita-More 2013.

98 Vgl. Vita-More 1997b, 3f; Vita-More 2000a, 2.

99 Vita-More 1995, 2.

${ }^{100}$ Vita-More 1997b.
} 
Vorrangige Themen sind für Vita-More die Befreiung von der natürlichen Evolution, die Bewahrung ewiger Jugend und Schönheit und die Aussichten auf den vollkommenen Körper der Zukunft. Kunst soll sich nämlich nicht nur deskriptiv zu den technischen Visionen verhalten, sondern das künftige Design des posthumanen Menschen maßgeblich mitgestalten. Sie soll dabei eine besondere Aufmerksamkeit auf die Entwicklung einer automorphen Sexualität legen - das heißt auf den Entwurf neuer und besserer Genitalien. ${ }^{101}$ So wirbt eine fiktionale Anzeige transhumanistischer Künstler für das Körpermodell Primo $3 M+$, das gegenüber unserem jetzigen Leib alterslos, umweltfreundlich und turbo-optimistisch sein werde. Es soll über 100 Quadrillionen Synapsen und eine automatische Fehlerkorrektur verfügen sowie den willkürlichen Geschlechterwechsel ermöglichen. ${ }^{102}$ Kunst wird in der Vorstellung Vita-Mores in dem Prozess einer Neuschöpfung des Menschen zur zentralen Vermittlungs- und Verwirklichungsinstanz zwischen Wissenschaft, Technik und Kultur. ${ }^{103}$

Die ersten Schritte auf diesem Weg der Autotransformation ist die französische Aktionskünstlerin ORLAN bereits gegangen, als sie in den 1970er Jahren die Art Charnel ins Leben rief. Zahlreiche Performances und Ausstellungen in renommierten Museen und Kunsteinrichtungen spiegeln das weltweite Interesse am Werk dieser bis heute höchst aktiven Künstlerin wider. ${ }^{104}$ Mit Stelarc ist sie der festen Überzeugung, dass der menschliche Körper obsolet sei - er könne mit den beschleunigten technischen Fortschritten nicht mehr mithalten: »We are at the junction of a world for which we are no longer mentally or physically prepared.« ${ }^{105}$ Sie tritt vehement für die Befreiung von den Beschränkungen der Natur ein:

Are we still convinced that we should bend to the determinations of nature? This lottery of arbitrarily distributed genes ... My work is a fight against, the innate, the inexorable, the programmed, nature, DNA (which is our direct rival as artists of representation) and God! One can therefore say that my work is blasphe-

\footnotetext{
${ }^{101}$ Vgl. a.a.O., 4; Vita-More 1997a, 2 f.

${ }^{102}$ Vgl. Primo 3M+ unter: http://www.natasha.cc.

${ }^{103}$ Vgl. Vita-More 2000b.

${ }^{104}$ Zur Einführung in das Werk ORLANs vgl. O'Bryan 2005 sowie ihre Homepage http://www.orlan.eu.

${ }^{105}$ Zitiert nach Reitmeier 1996, 12.
} 
mous. It is an attempt to move the bars of the cage, a radical and uncomfortable attempt. It is only an attempt. ${ }^{106}$

Die biologische Fortpflanzung und Geburt erachtet ORLAN als anachronistisch und lächerlich in einer hochtechnisierten Welt, in der biologisch bedingte Empfindungen wie Schmerz chemisch der freien Manipulierbarkeit unterliegen: » ... désormais nous avons la péridurale et de multiples anesthésiants ainsi que les analgésiques, vive la morphine! A bas la douleur! « ${ }^{107}$ Schon in ihrer ersten viel beachteten Performance $L e$ baiser de l'artiste 1977 in Paris changierte sie als Sainte-ORLAN zwischen den Polen traditioneller Frauenbilder: Maria und Maria Magdalena der Mutterfigur und der Prostituierten. In späteren Performances soll der Körper antipodisch zum biblischen Verhältnis als Text, als wortgewordenes Fleisch, die künstlerische Botschaft repräsentieren: Der Körper selbst wird zur veränderlichen Software. ${ }^{108}$ In ihrem Manifeste de L'Art Charnelvon 1997 formulierte die provokative Künstlerin die Grundzüge ihres universalen Körperprojektes:

L'Art Charnel est un travail d'autoportrait au sens classique, mais avec des moyens technologiques qui sont ceux de son temps. Il s'inscrit dans la chair parce que notre époque commence à en donner la possibilité. Le corps devient un « ready-made modifié » car il n'est plus ce ready-made idéal qu'il suffit de signer. ${ }^{109}$

Steht auch ihre frühe Arbeit in der Tradition der Körperkunst, in der der menschliche Körper als künstlerisches Erlebnisobjekt dient, so begann ORLAN 1990 mit der Art Charnel die bisherigen Grenzen von Kunst zu überschreiten. In ihrer Performance Self-Hybridations, die als lebenslanges Gesamtkunstwerk angelegt ist, lässt sie ihren eigenen Körper sukzessiv durch kosmetische Operationen verändern. Diesen Prozess bezeichnet sie selbst als réincarnation de Sainte-ORLAN. Während der chirurgischen Eingriffe, die per Video aufgezeichnet wurden und per Satellit live an Kunstmuseen und Galerien weltweit übertragen wurden, trägt die Künstlerin philosophische Texte vor. Sie empfindet ihre körperliche Transformation als rite de passage, den sie als selbstverwirklichende Anpassung ihrer äußeren Hülle an ihre innere, geschlechtsübergreifen-

\footnotetext{
${ }^{106}$ Ebd.

${ }^{107}$ ORLAN 2000, 2.

${ }^{108}$ Vgl. ebd.; MacCorquodale 1996; Dery 1996, $239 f$.

109 ORLAN 2000, 1.
} 
de Identität versteht: »Je suis une homme et un femme! «110 Nach ihrem Tod möchte sie den vollendeten Körper ORLANs mumifizieren und als dauerhafte Installation in einem Museum ausstellen lassen. ${ }^{111}$

Da sie die plastische Chirurgie als größten Ausdruck männlicher Machtentfaltung verurteilt, widersetzt sich ORLAN bewusst den patriarchalen Schönheitsidealen der Frau und dem absoluten Jugendlichkeitskult. So ließ sie sich die Wangenknochen mit auffälligen Implantaten verstärken und 1999 wurde ihr Nasenrücken in ihrer 7. Operation deutlich vergröBert. ${ }^{112}$ Mit ihren Aktionen möchte ORLAN die Frage nach dem Status des Körpers aufwerfen. Was bedeutet es, wenn wir im anbrechenden Zeitalter der Gentechnik unsere körperliche Erscheinung nahezu beliebig verändern können? Wie kann sich die Verwirklichung persönlicher Identität gegen die konformistischen Körperideale einer allgegenwärtigen Medienindustrie behaupten? ${ }^{113}$ In ihrem jüngsten Projekt von 2018 kreierte sie ein künstlich intelligentes Roboterabbild von sich selbst, die ORLANOÏDE, die mit dem Publikum interagiert und erneut die Frage nach den Normativen unserer technischen Schöpfungen aufwirft. ${ }^{114}$ ORLAN inszeniert in ihren Performances, die in Frankreich und international auch über die engere Kunstszene hinaus viel beachtet werden, die willkürliche Veränderbarkeit des als obsolet verstandenen menschlichen Körpers. Sie will jedoch die Befreiung von der Herrschaft der Gene nicht durch neue und alte soziale Unfreiheiten gefährdet wissen.

Auch eine Reihe jüngerer Künstlerinnen und Künstler sucht die kritische Auseinandersetzung mit dem Posthumanen. Bio- und Kommunikationstechnologien beschäftigen den brasilianischen Künstler Eduardo Kac (*1962), der sich nach eigenem Bekunden als erster Mensch 1997 einen Computership unter die Haut implantieren ließ. In seiner transgenic art bzw. bioart experimentiert er mit menschlicher, pflanzlicher und tierischer DNA und präsentierte im Jahr 2000 einen fluoreszierenden Hasen (Alba). Kac will die Grenzen des Menschlichen künstlerisch erfahrbar machen und zur Debatte stellen. ${ }^{115}$

Auf den Spuren von Stelarc und Kevin Warwick befindet sich das Künstlerpaar Neil Harbisson $\left({ }^{*} 1984\right)$ und Moon Ribas $(* 1985)$. Harbisson, der

\footnotetext{
${ }^{110}$ Zitiert nach Reitmeier 1996, 3.

${ }^{111}$ Vgl. a.a.O., 13.

112 Vgl. ORLAN 1999; Reitmeier 1996, 8-11.

113 Vgl. Reitmeier 1996, 7.

${ }^{114}$ Vgl. http://www.orlan.eu/orlan-et-lorlanoide.

${ }^{115}$ Vgl. http://www.ekac.org.
} 
von Geburt an keine Farben sehen kann (Achromatopsie), ließ sich 2004 auf dem Hinterhauptbein seines Schädels eine Antenne und einen Mikroship implantieren, die Farben in hörbare Töne verwandeln. In seinen Kunstprojekten nimmt er auch externe Signale auf (z.B. über internetbasierte Kommunikation), die er dann in Farbbilder umsetzt. Moon Ribas hat sich 2013 einen seismischen Sensor in den Unterarm einpflanzen lassen, der Vibrationen abgibt, wenn auf unserem Planeten die Erde bebt (das Signal erhält der Sensor über eine globale Messstation im Internet). Die seismischen Wahrnehmungen setzt sie in Tanz und Musik um. Mit Harbisson entwickelte Ribas auch die »transdentale Kommunikation", die es beiden erlaubt, über Morsezeichen und vibrierende Zahnimplantate miteinander zu kommunizieren. ${ }^{116} 2010$ gründeten Harbisson und Ribas die Cyborg-Foundation, die Menschen dabei unterstützen will, Cyborgs zu werden und als solche ihre Rechte einzufordern. Dazu gehören das Recht auf körperliche Integrität inklusive technischer Bestandteile, das Recht auf technische Veränderungen oder Erweiterungen des Körpers, den gleichen Rechtstatus für Mutanten und Menschen sowie das Besitzrecht an allen dauerhaften Erweiterungen des Körpers. ${ }^{117} 2017$ riefen Harbisson und Ribas zusammen mit dem spanischen Künstler Manel Muñoz die Transpecies Society in Barcelona ins Leben. Gemeinsam ist den engagierten Künstlern, dass sie Technik als Teil der Natur begreifen. Als Angehörige einer nicht-menschlichen Spezies wollen sie die Freiheit in Anspruch nehmen, ihren Körper selbstbestimmt mit neuen Organen und Sinnen auszustatten. Auch Muñoz versteht sich als Cyborg-Künstler, dessen Implantat den gemessenen Luftdruck und seine Veränderungen in Vibrationen umsetzt. ${ }^{118}$

Direkten Bezug auf den technologichen Posthumanismus nehmen die zwei französischen Künstler Fabien Giraud (*1980) und Raphaël Siboni (*1981) in ihrer Videokunst. Mensch und Technik in einem historischen Bogen von der Eroberung des Silicon Valley im 16. Jahrhundert bis zum projizierten Death of Ray Kurzweil - 2045 sind das Thema ihrer meditativ-abstrakten Filme (The Unmanned) von 2014. So wird in 1997 - The Brute Force der leere Raum nach der Niederlage Garri Kasparows gegen den Computer Deep Blue mit zeitlupengleichen Kamerafahrten erfasst. ${ }^{119}$ Eher dokumentarischer Art ist das Werk des Schweizer Photo-

\footnotetext{
${ }^{116}$ Vgl. https://www.cyborgarts.com.

117 Vgl. https://www.cyborgfoundation.com.

${ }^{118}$ Vgl. https://www.transpeciessociety.com; http://www.manelmunoz.com.

${ }^{119}$ Vgl. http://www.theunmanned.com.
} 
graphen Matthieu Gafsou (*1981), der aktuell unter dem Projekttitel $H+$ (2018) Techniken und Akteure des europäischen Transhumanismus und der Robotik visuell einfängt. ${ }^{120}$

Die Art Charnel ORLANs definiert sich wie Stelarcs Technozentrik und Natasha Vita-Mores Extropian Art in jeweils verschiedenen Aspekten der posthumanen Entgrenzung des Körpers über den künstlerischen Beitrag zum Design der künftigen Lebensformen. Allen gemeinsam ist die Verachtung für die natürliche und zufällige Evolution der biologischen Spezies Mensch, deren Fehlerhaftigkeit und Impotenz im Angesicht der berauschenden Kraft der Maschinen schon Marinetti im Manifeste du Futurisme offenbarte. Die Überwindung der körperlichen Beschränkungen verspricht die Freiheit von Alter, Krankheit, von der Erde, von den Grenzen des Intellektes und nicht zuletzt die Freiheit von der Religion und Gott, die meist als gesellschaftliche Repression des menschlichen Vervollkommnungsstrebens wahrgenommen werden. ${ }^{121}$

Die künstlerische Auseinandersetzung mit dem Posthumanen gestaltet sich vielfältig. Sie reicht von konkret-spielerischen Entwürfen (Stelarc), über naiv-visionäre Illustrationen (Vita-More) bis zu problemorientierten Impulsen (ORLAN, Giraud \& Siboni). Im Zentrum steht die Frage nach Macht und Ohnmacht des heutigen Menschen angesichts der erhofften Freiheiten und der befürchteten Verluste menschlicher Lebensmöglichkeiten. Da die Zukunft nicht stirbt, ist zu erwarten, dass sich auch die nächste Künstlergeneration der Thematik kreativ annehmen wird.

${ }^{120}$ Vgl. http://www.gafsou.ch/hplus.

${ }^{121}$ Vgl. Caygill 1997, 51; Vita-More 2000b; Reitmeier 1996, 12. Von der Kunstkritik kaum beachtet, jedoch mit ihren Illustrationen von menschlich-maschinellen Hybriden, Cyborgs und Aliens haben der surrealistische Maler Hans Rudi Giger und auch der weniger bekannte, deutsche Maler und Graphikkünstler Joachim Luetke mit ihrer "posthumanen « Kunst eine gewisse Bedeutung in der transhumanistischen Subkultur. Vgl. Dery 1996, 280ff.; Luetke 2000. 


\section{Ideengeschichte des technologischen Posthumanismus}

Observed progress is mainly technical, whereas believed progress is mainly spiritual. ${ }^{1}$

John Baillie

\subsection{Methodische Vorüberlegungen zur »Geschichte der Zukunft»}

In meinen bisherigen Ausführungen habe ich mich bemüht, die Relevanz von Erfahrungen mit der Virtualität für die menschliche Selbstdeutung aufzuzeigen. Aus diesen Medienerfahrungen - jedenfalls dort, wo sie für das Handeln des Einzelnen eine Bedeutung erlangt haben - lassen sich zwei Konsequenzen ableiten. Zum einen kann die Erfahrung mit den quasi unsterblichen, medialen Abbildern des Menschen als ein weiterer kultureller Mechanismus der Todesverdrängung fungieren oder aber zum anderen den Wunsch nach einer gleichartigen, virtuellen Unsterblichkeit evozieren. Den Zusammenhang mit der medialen Erfahrung von Zeit und Raum habe ich eingehend geschildert. Die geistige Verarbeitung der primären technischen Erfahrung mit Virtualität ist jedoch keine anthropologisch konstante Größe, sondern sie ist kulturell und zeitlich gebunden. Darauf verweist schon die simple Beobachtung, dass die Vereinigten Staaten und nicht etwa technisch gleich entwickelte Länder in Europa oder Japan den Ursprung des technologischen Posthumanismus markieren. Nicht in einem kulturellen Niemandsland entstand diese technophile Strömung, sondern vor allem im Rahmen der amerikanischen Kulturgeschichte (und ihrer spezifischen Rezeption europäischer Philosophie).

Der Posthumanismus interpretiert das vergangene, gegenwärtige und künftige Verhältnis des Menschen zur Technik, wobei ontologische Grundfragen zur Beziehung zwischen Materie und Geist, Körper und

1 Baillie 1950, 156. 
Seele oder die Definition von Intelligenz und Leben berührt werden. Der Posthumanismus deutet hierbei nicht nur den Menschen und die Technik, sondern nimmt in seinem Rekurs auf ältere geistesgeschichtliche Konzepte bereits Interpretationen der Interpretationen von Mensch und Technik vor - dies jedoch in einem vorwiegend kybernetisch bzw. physikalisch determinierten Kontext. Wir haben es also mit wechselseitigen Transferleistungen von naturwissenschaftlichen, philosophischen und religiösen Ideen zu tun.

Um diese komplexen Prozesse der Umdeutungen und neuen Kontextualisierungen präzise erfassen zu können, halte ich einen rezeptionsgeschichtlichen Ansatz für besonders geeignet. Hatte Hans Blumenberg schon 1958 herausgearbeitet, dass »Mythen« wie die epochemachende kopernikanische Wende ihre geschichtliche Kraft von Anbeginn aus der Rezeption und der Anreicherung ihrer Bedeutungen durch die ständige Umerzählung erlangen, so begründete Hans-Georg Gadamer mit der Analyse des wirkungsgeschichtlichen Bewusstseins die philosophische Hermeneutik. ${ }^{2}$ Aus seinem Prinzip der Wirkungsgeschichte, demzufolge ein Werk nur unter Einbeziehung seiner Wirkung verstanden werden kann, hat die literarische Hermeneutik das korrelate Prinzip der Rezeptionsgeschichte entwickelt. Sie geht nicht vom Werk und seiner Wahrheit, sondern vom verstehenden Bewusstsein als Subjekt der Erfahrung aus. Wirkung wird dabei meist als das vom Text bedingte, Rezeption als das vom Adressaten bedingte Element der so genannten Konkretisation bestimmt. ${ }^{3}$ In Ergänzung zu einer Wirkungsgeschichte, der es vor allem um Einfluss- oder Abhängigkeitsforschung geht und die dabei einen semantisch konstanten »Text « voraussetzt, der seine »Spuren « in der Geistesgeschichte hinterlässt, sucht die Rezeptionsgeschichte ihren Ausgangspunkt in der Perspektive der Rezipienten.

Im Anschluss an den polnischen Phänomenologen Roman Ingarden entwickelte sich die »Konkretisation« zu einem Schlüsselbegriff der Literatursemiotik und Rezeptionsästhetik, was vor allem dem Prager Strukturalismus (Felix Vodicka, Jan Mukasovský) und der literaturwissenschaftlichen Konstanzer Schule um Hans Robert Jauß zu verdanken ist. Erst die Rezeption eines Werkes versieht es mit geschichtlichem Leben: In spezifischen sozialen, biographischen oder kulturellen Kontexten bringt es in fortschreitenden Interpretationen seine Struktur in der

2 Vgl. Blumenberg 1958; Gadamer 1960.

3 Vgl. Jauß 1987, $16 f$. 
offenen Reihe seiner Konkretisationen oder Rezeptionsgestalten zum Ausdruck. In einer kritischen Weiterführung der phänomenologischen Ästhetik Ingardens hat die Konstanzer Schule die Konstitution und Neubildung von Sinn bei der Aufnahme eines ästhetischen Objekts in der Geschichte seiner Rezeption untersucht. ${ }^{4}$

Ein rezeptionsgeschichtliches Paradigma bezieht daher sowohl die Geschichtlichkeit eines Werkes als auch den historischen Kontext der Interpreten ein. Es legt zugrunde, dass ein Kunstwerk weder in seiner Struktur noch die Kunst in ihrer Geschichte als eine Substanz oder Entelechie verstanden werden kann, wie es Hans Robert Jauß formulierte:

Erst wenn die Differenz und Vielfalt der Auslegbarkeit nicht länger auf den objektiven Gehalt des Schriftsinns, sondern auf die subjektiven Bedingungen des Verstehens, näherhin: auf die Leistung verschiedener Auslegungsweisen gegründet werden, tritt die Wende zur modernen Hermeneutik ein. Danach gilt der Sinn des Textes nicht mehr als autoritativ vorgegeben, sondern als einem produktiven Verstehen zur Suche aufgegeben. Erst dann öffnet sich der Horizont des Verstehens auf Möglichkeiten, den Text im späteren Kontext immer wieder anders, näherhin als Antwort auf Fragen zu verstehen, die sich in seinem primären Kontext noch nicht stellen konnten. ${ }^{5}$

In diesem Sinne möchte ich die Rezeption von philosophischen, religiösen und literarischen Motiven und Konzepten in den Schriften der Posthumanisten analysieren. In seiner umfangreichen Dissertation über die europäische Faszination Zarathushtra hat Michael Stausberg die Möglichkeiten der rezeptionsgeschichtlichen Methode erstmals für die Religionswissenschaft fruchtbar gemacht. Dabei ergeben sich aus dem hermeneutischen Ansatz der Rezeptionsgeschichte gravierende Konsequenzen für die gängige Darstellung der »Lehre« oder der »Botschaft« einer bestimmten Religion, Philosophie oder eines bestimmten Textes:

Der historisch arbeitenden Religionswissenschaft wäre somit nicht so sehr die Aufgabe gestellt, aus der Vielzahl der Quellen die Theologie oder Mythologie einer Religion zu systematisieren, sondern Religionsgeschichte als eine Verkettung der von Anhängern bestimmter Religionen selbst in bestimmten geschichtlichen Situationen erbrachten produktiven Rezeptions- und Selektionsleistungen bestimmter Motive, Themen, Konzeptionen oder Texte vorzustellen ... ${ }^{6}$

4 Vgl. Jauß 1987.

5 Jauß 1987, 9. Vgl. hierzu auch Stausberg 1998, 2.

Stausberg 1998, 3. 
Unter dieser hermeneutischen Prämisse sind von einer semantisch konstanten Struktur her konzipierte Kategorien wie »Verkanntwerden« oder "Missdeutung " nicht anwendbar. Die scheinbaren »Interpretationsfehler«, die den Posthumanisten aufgrund der Unkenntnis von Originaltexten oder philosophiegeschichtlichen Zusammenhängen unterlaufen, sind für uns nur insofern von Nutzen, als dass hier die Konkretisationen in ihren neuen, bisher unbekannten Kontextualisierungen deutlicher zu Tage treten und somit auf die Frage nach den besonderen Selektions- und Interpretationsmustern drängen. Denn nicht der von einer vermeintlich objektiveren, wissenschaftlichen Perspektive her konstruierten »Irrtumsgeschichte« der posthumanistischen Philosophierezeption gilt unser Interesse, sondern den innovativen Anwendungen philosophischer Konzepte in einem neuen semantischen Kontext. ${ }^{7}$

Das Zentrum des technologischen Posthumanismus bildet die Aussicht auf die Verwirklichung menschlicher Unsterblichkeit. Sie markiert genealogisch den Beginn der transhumanistischen Bewegung in den Werken von FM-2030 und Robert Ettinger in den 1970er Jahren. Die Unsterblichkeit ist jedoch auch das alles verbindende Glied in der posthumanistischen Argumentationskette. Ohne dieses Element bleibt das grundlegende Plädoyer der Posthumanisten für die Überwindung des Menschen sinnlos. Das Opfer des Menschen für seine künstlichen Nachkommen - seine Auslöschung - erfordert das Versprechen einer noch höheren Wiedergutmachung: das ewige Leben. Alle weiteren Aspekte wie das Verschmelzen des Menschen mit einer universalen $\mathrm{Su}_{-}$ perintelligenz, die intergalaktische Omnipräsenz oder die Rettung des Kosmos vor dem Wärmetod, befinden sich in der Peripherie des posthumanistischen Denkens und sind bei seinen Vertretern verschieden stark ausgeprägt.

Der technologische Posthumanismus verhandelt daher im Hinblick auf die Unsterblichkeit vor allem zwei miteinander verwobene, fundamentale Fragen. Zum einen: Wie kann der Mensch seine perfekte Simulation im Speicher eines Computers generieren? Und zum anderen: Wie gelangen wir in diese verheißungsvolle Zukunft?

Die Diskussion um das Menschenbild berührt ein ganzes Bündel an ontologischen und anthropologischen Grundfragen, die im Posthumanismus beantwortet werden, um die theoretischen Voraussetzungen für eine Immortalisierung des Menschen zu schaffen: Was ist Leben?

7 Vgl. hierzu auch a.a.O., 2ff. 
Was ist der Mensch? Was macht das Wesen des Individuums aus? Ein kybernetisches Welt- und Menschenbild, das den Menschen schon vor seiner technischen Transformation als Maschine deutet, bildet die Voraussetzung für alle posthumanistischen Visionen. Nur unter dieser Bedingung wird die Idee einer computertechnischen Kopie des Menschen überhaupt erst denkbar. Das Kapitel 6.2 Der Mensch ist bereits posthuman wird sich daher verschiedenen Aspekten der Analyse dieses posthumanistischen Verständnisses vom Menschen widmen. Die Rezeption von Descartes unter einem kybernetischen Paradigma nimmt hierbei eine herausragende Stellung ein.

Die zweite Frage zielt auf die Fortschrittsidee des Posthumanismus. Beginnend mit Ernst Bloch und seiner philosophiegeschichtlichen Analyse der Utopie im Prinzip Hoffnung rückt die Geschichte der Zukunft in den Mittelpunkt. In den vergangenen Jahrzehnten hat vor allem der Historiker Lucian Hölscher diesen Ansatz theoretisch ausgearbeitet. Eine historische Zukunftsforschung sei demnach entscheidend für das Verständnis einer Epoche, denn hier lassen sich Leitbilder und Zielrichtungen politischer und wirtschaftlicher Erwägungen nachzeichnen. Die Ausgestaltung dieser Möglichkeitsräume greift dabei auf verschiedenste Formen wie technische Prognosen, Belletristik, religiöse Prophetien oder auch politische Programme und Planungen zurück. ${ }^{8}$ Während die Definition des Menschen durchweg auf materialistischen Weltanschauungen fußt, ist die posthumanistische Fortschrittstheorie ein hybrides Gemisch aus naturwissenschaftlichen, philosophischen und religiösen Anteilen. Warum es Fortschritt geben muss, wie und in welchen Schritten er sich vollziehen wird und was die Ziele dieses Prozesses sind - all dies wird in den Kapiteln 6.3 bis 6.7 detailliert aus einer rezeptionsgeschichtlichen Perspektive durchleuchtet. Ein besonderes Augenmerk gilt hier dem Konzept der Singularität, der Superintelligenz und der von Teilhard de Chardin geprägten Noosphäre.

In Hinsicht auf beide Aspekte - dem Menschenbild und dem Fortschrittsprozess - werde ich zunächst die wesentlichen Positionen der post- und transhumanistischen Denker darlegen, sie vergleichen und dann versuchen, die ideengeschichtlichen Traditionen und philosophischen sowie literarischen Kontexte ihrer Entwürfe aufzuzeigen. Im Fokus stehen hier vor allem die Werke von Frank Tipler, Marvin Minsky, Hans Moravec, Ray Kurzweil und Arbeiten weiterer Autorinnen und Autoren.

8 Hölscher 2017b, 7-21. 


\subsection{Der Mensch ist bereits posthuman}

DER SINN DES BOTEN IST DIE BOTSCHAFT. Denn die

Organismen dienen der Übermittlung und nicht umgekehrt. ${ }^{9}$

GOLEM XIV

Der Technikphilosoph Bernhard Irrgang stößt uns auf die grundlegenden Fragen, die der Posthumanismus aufwirft:

Was sind wir als Menschen eigentlich, wenn wir uns als Menschen die Aufgabe stellen, uns als Menschen zu verändern? Nach wessen Bilde sollen wir Menschen aber nun den Menschen schaffen? Wenn uns unsere biowissenschaftlichen Erfolge die Möglichkeit an die Hand geben würden, Menschen nach Maß zu schaffen, dann fragt sich immer noch: nach welchem Maß? ${ }^{10}$

Allgemein berührt dieser Fragenkomplex unzählige Aspekte der gegenwärtigen Maschinisierung und Digitalisierung: die Verdrängung des Menschen durch Maschinen in der Arbeitswelt; Roboter als Haustiere, Diener und Konkurrenten; die Rolle des Erfinders bei der Erschaffung künstlicher Menschmaschinen und künstlicher Intelligenz; Automatae, Roboter und Computer in Literatur und Film. Damit werden Perspektiven der Arbeits-, Technik- und Mediensoziologie, der Medienpsychologie sowie soziologische und anthropologische Grundfragen tangiert.

Eine Analyse dieser Gesichtspunkte innerhalb des posthumanistischen Diskurses wäre sicherlich vielversprechend. Die im Zentrum unseres Erkenntnisinteresses stehende Frage aber, wie der Posthumanismus die technische Möglichkeit einer Unsterblichkeit im Computer begründet, ist gleichbedeutend mit der Frage, wie die Simulation eines realen Menschen im Computer verwirklicht werden kann. Sie leitet uns zu den philosophischen Voraussetzungen der posthumanistischen Vision von Unsterblichkeit. Was sind also die Bedingungen für die Vereinigung zwischen Mensch und Technik?

Es ist vor allem dem Philosophen Günther Anders und der amerikanischen Literaturwissenschaftlerin N. Katherine Hayles zu verdanken, dass wir heute die Argumentationsstrukturen eines normativ posthumanistischen Diskurses mit größerer Klarheit nachzeichnen und verstehen können. Die posthumanistischen Autoren fordern mit im Detail erheb-

9 Lem 1986, 49.

10 Irrgang 2010, 243. 
lich differierenden Akzentuierungen den Ersatz des Menschen durch posthumane Lebensformen und damit einhergehend die entsprechende Weiterentwicklung des biologischen Menschen. Keiner der Posthumanisten verlangt jedoch die völlige Abschaffung des Menschen - der Mensch, wie er heute ist, soll lediglich überwunden werden. Auch die weitestgehenden Vorstellungen von Hans Moravec und Frank Tipler, die sogar die baldige Auslöschung des Menschen in der Evolutionsgeschichte billigend in Kauf nehmen, retten den gegenwärtigen Menschen in ihrer Hoffnung auf eine Art virtuellen Reservates bzw. auf die zukünftige Auferstehung aller Menschen. Im Allgemeinen ergänzen sich die Visionen von der anfänglichen Aufrüstung des Menschen mit technischen Implantaten und der Kreation biologisch-maschineller Mischwesen, den Cyborgs, zur Forderung einer weitestgehenden Mechanisierung des menschlichen Körpers bis hin zum Upload des menschlichen Geistes in einen Computer. Der biologische Körper soll durch einen in allen physischen und psychischen Qualitäten leistungsfähigeren Maschinenkörper ersetzt werden - der Mensch wird zur Maschine.

Nun haben Günther Anders und N. Katherine Hayles eindrücklich demonstriert, dass die Menschmaschine nicht nur das Ziel, sondern bereits die Voraussetzung posthumanistischer Forderungen konstituiert, denn der Mensch ist nach den Deutungen der Posthumanisten längst posthuman: "Homo sapiens are so transfigured in conception and purpose that they can appropriately be called posthuman. " ${ }^{11}$ Als Selbstverdinglichung und Dehumanisierung im Roboterzeitalter bezeichnet Günther Anders die freiwillige Degradierung des Menschen zum Gerät, zur fehlerhaften Maschine. ${ }^{12}$

Der Rahmen für die Fragestellungen, die Bernhard Irrgang eingangs aufgeworfen hat, ist daher bereits abgesteckt: Nur wenn der Mensch eine Maschine ist, kann er prinzipiell von einer anderen Maschine, dem Computer, simuliert und ersetzt werden. Die materialistische Definition des Menschen als Maschine bildet damit eines der beiden zentralen Elemente der posthumanistischen Philosophie. Denn wenn der Mensch mehr sein sollte als die Summe und Anordnung seiner molekularen Bestandteile, wenn er über die physikalische Materie hinaus eine von uns bisher unverstandene Metaphysik sein Eigen nennen könnte, wenn der Mensch gar so etwas wie eine Seele besäße, dann wäre jede Utopie ei-

11 Hayles 1999, 11.

12 Vgl. Anders 1983, 30-56. 
ner technischen Simulation des Menschen und der maschinell erzeugten Unsterblichkeit zunichte gemacht. Nicht nur die technische Machbarkeit einer Simulation wäre damit ihres Fundamentes beraubt worden, sondern auch der Vergleich der »fehlerhaften Menschmaschine« mit den überlegenen künstlichen Intelligenzen. Damit der Mensch in diesem Vergleich stehen kann, muss er bereits als Maschine definiert werden.

Das nachstehende Kapitel 6.2.1 L'Homme Machine - im Titel angelehnt an La Mettries bekannte Abhandlung von 1747 - stellt zunächst die mechanistische Interpretation des menschlichen Körpers und Geistes bei den wichtigsten Posthumanisten dar und reflektiert deren geistesgeschichtliche Tradition.

Bemerkenswert bei der posthumanistischen Vision einer technischen Immortalisierung ist vor allem die Tatsache, dass beim so genannten Upload das menschliche Gehirn als Sitz des Verstandes (mind) und nicht der gesamte Körper gescannt und in einem Computer simuliert werden soll. Dieser Zusammenhang zwischen der perfekten Simulation und der implizierten Reduktion der menschlichen Identität auf den im Gehirn situierten menschlichen Geist wird im Kapitel 6.2.2 Simulation und Identität angesprochen und insbesondere im Hinblick auf die Rezeption von Descartes problematisiert.

In einem dritten Kapitel (6.2.3 Der Mensch im kybernetischen Paradigma) werde ich der Frage nachgehen, warum die Posthumanisten bei ihrer Rezeption der philosophischen Konzepte von Descartes, La Mettrie oder der englischen Physikotheologen bestimmte Aspekte selektiert und andere wiederum völlig ignoriert haben. Es wird sich hierbei zeigen, dass die philosophische Rezeptionsleistung der Posthumanisten unter einem informationstechnischen Paradigma steht, das von der kybernetischen Theorie Norbert Wieners, John von Neumanns, Alan Turings und Claude Shannons geprägt wurde.

Es war die These von N. Katherine Hayles, dass die Science-Fiction Literatur entscheidend zur Verbreitung einer informationstechnischen Deutung des Menschen beigetragen hat. In den späteren Kapiteln (unter 6.3), die die Verwirklichung des vollkommenen Menschen behandeln, werden diese Aspekte im Einzelnen erörtert. 


\subsubsection{L'Homme Machine}

And though most people still consider it degrading to be regarded as machines, I hope, this book will make them entertain, instead, the thought of how wonderful it is to be machines with such marvelous powers. $^{13}$

Marvin Minsky

Während die Mehrheit der posthumanistischen Autoren bei ihrem Vergleich zwischen Mensch und Maschine die vermeintlich mechanistische Funktionsweise des menschlichen Körpers und Geistes als Ausgangspunkt wählen, versucht Frank Tipler zu zeigen, dass auch Maschinen unter bestimmten Bedingungen als lebendig erachtet werden müssen. Frank Tipler und John D. Barrow definieren Leben zunächst ausgehend von der Fähigkeit der Selbstreproduktion der Lebewesen, deren Zellen die Information zu ihrer Wiederherstellung bzw. Vermehrung enthalten. So erkennen Tipler und Barrow nicht nur einen Virus und ein heterosexuelles Menschenpaar als lebendig an, sondern sie werten auch geistige Ideen und sogar Autos als Lebewesen, deren natürliche Selektion im freien Markt garantiert ist und die im Verbund mit den Autoherstellern reproduziert werden. ${ }^{14}$

In dem Vergleich zwischen einem biologischen Virus und der von Tipler eingeführten Von-Neumann-Sonde zeigen die beiden Kosmologen, dass auch Maschinen zu den Lebewesen gerechnet werden können, sofern sie den Anforderungen der vorhergehenden Definition genügen. Der Mathematiker und Kybernetiker John von Neumann entwickelte die Idee eines Automaten, der dazu in der Lage ist, autonom Nachbildungen von sich selber anzufertigen. Diese Maschine sollte im Idealfall aus einem universal constructor, der Materie bearbeiten kann, und einer Datenbank mit den nötigen Konstruktionsanweisungen bestehen. ${ }^{15}$ Die funktionale Analogie ist für Tipler und Barrow der Beleg dafür, dass sich selbst reproduzierende Maschinen und Viren gleichermaßen lebendig sind. ${ }^{16}$ Die entscheidende Aufgabe kommt dabei der gespeicherten Information zu, die die Reproduktion steuert, so dass Tipler und Barrow zu einer weiteren Definition von Leben gelangen:

13 Minsky 1988, 323. Vgl. auch a.a.O., 30.

14 Vgl. Barrow \& Tipler 1986, 513-522.

15 Vgl. Neumann 1966.

16 Vgl. Barrow \& Tipler 1986, 515-521; Tipler 1981; Tipler 1986, 73-78. 
... we may even say that a human being is a program designed to run on particular hardware called a human body, coding its data in very special types of data storage devices called DNA molecules and nerve cells. The essence of a human being is not the body but the program which controls the body ... In principle, the program corresponding to a human being could be stored in many different forms - in books, on computer disks, in RAM - and not just in the brain of a particular human body. ${ }^{17}$

In seinem späteren Werk führte Turing diese informationszentrierte Lebensdefinition noch weiter aus und kommt provokativ zu dem Schluss:

I therefore regard a human being as nothing but a particular type of machine, the human brain as nothing but an information processing device, the human soul as nothing but a program being run on a computer called the brain. Further, all possible types of living beings, intelligent or not, are of the same nature, and subject to the same laws of physics as constrain all information processing devices. ${ }^{18}$

Der Mensch wird unter Tiplers informationstechnischem Paradigma selbst zum Computer und ein Mensch wird mit demselben Ansatz erst dadurch zur Person, dass er den Turing-Test besteht. Der englische Mathematiker Alan Turing hatte in den 1950er Jahren einen Test vorgeschlagen, um zu überprüfen, ob ein Computer Intelligenz besitzt oder nicht: Angenommen eine Testperson kommuniziert über einen Computer mit einem menschlichen Gegenüber und einem weiteren, vermeintlich intelligenten Computer, so wird dieser Computer dann als intelligent angesehen, wenn die menschliche Testperson nicht mehr eindeutig die Maschinen- bzw. Menschenidentität seines Gegenübers bestimmen kann. ${ }^{19}$ Tipler nun dreht die Beweisführung um und der Mensch selbst wird zum Testobjekt:

Thus »life« is a form of information processing, and the human mind - and the human soul - is a very complex computer program. Specifically, a "person« is defined to be a computer program which can pass the Turing test ... ${ }^{20}$

Aus einem Test für Maschinen wurde damit ein Test für den Menschen, weil der Mensch aus der physikalischen Perspektive Tiplers nichts anderes als eine Maschine ist. Eine »Person« zu sein, macht Tipler abhängig von der Intelligenz eines informationsverarbeitenden Gerätes, so dass

\footnotetext{
17 Barrow \& Tipler 1986, 659.

18 Tipler 1995, XI; vgl. a.a.O., $124 f$.

19 Vgl. Barrow \& Tipler 1986, 523; Tipler 1995, 20f.; Turing 1950.

20 Tipler 1995, 124.
} 
Behinderte oder psychisch kranke Menschen - »unfortunately, there are such people ${ }^{21}{ }^{2}$ - den Turing-Test nicht bewältigen würden, da sie ohne sprachlich erkennbare Intelligenz auch keine »Person« wären.

Das Augenmerk von Marvin Minsky richtet sich nicht in erster Linie darauf, klare Definitionen für Begriffe wie Denken, Intelligenz und Bewusstsein zu finden, sondern unter einem funktionalistischen Paradigma möchte er zunächst besser verstehen, wie diese mentalen Prozesse ablaufen. ${ }^{22}$ Es steht für Minsky außer Zweifel, dass der Mensch ein intelligentes System ist, das heißt im allgemeinen, dass er die Fähigkeit zur Lösung komplexer Probleme besitzt. Tiere dagegen verfügten über keine Intelligenz, da sie kein Bewusstsein für die Lösung eines Problems aufzuweisen hätten. ${ }^{23}$ In prinzipieller Abgrenzung zu den nächsten Artverwandten des Menschen - dem Orang-Utan, Gorilla und Schimpansen - betrachtet Minsky den homo sapiens als Anti-Evolutionisten, der sein überlegenes Gehirn der aktiven Aneignung neuer Fähigkeiten auf einer früheren Entwicklungsstufe zu verdanken hat. ${ }^{24}$

In einem fiktiven Gespräch zweier Außerirdischer koppelt der Kybernetiker Minsky dann generelle Existenzrechte an die Intelligenz der Lebewesen. ${ }^{25}$ Jenseits einer informationstechnischen Terminologie ist der Mensch auch für Minsky ausschließlich durch die Denkfähigkeiten seines Verstandes (mind) charakterisiert; Tipler sprach an dieser Stelle von Informationsverarbeitung.

Sämtliche mentalen Prozesse, auch Gefühle und Empfindungen, werden auf bestimmte Funktionen reduziert, die zur Stabilisierung und Weiterentwicklung der Society of Mind beitragen. Insbesondere wendet sich Minsky gegen den Mythos vom unverstandenen emotionalen Denken, denn Gefühle seien als allgemeine Zweckmaschine nötig zur Entscheidungsfindung und unverzichtbar zur Entstehung von intelligentem Denken und Handeln. ${ }^{26}$ Auch musikalisches Empfinden und Humor dienen Minsky zufolge dem Erhalt unserer Verstandeskräfte und werden bei der Entfaltung künstlicher Intelligenz nicht fehlen - selbst wenn uns derzeit Visionen von witzigen Computern noch sehr fern erscheinen

21 A.a.O., 20.

22 Vgl. Minsky 1988, 39, 71.

23 Vgl. a.a.O., 71.

24 Vgl. a.a.O., 321 .

25 Vgl. Minsky 1992a, 24f.

26 Vgl. Minsky 1981, 1f.; Minsky 1988, 163f.; Minsky 2006, 9-35. 
mögen. ${ }^{27}$ Jede Mystifizierung geistiger Vorgänge weist er entschieden zurück, sei dies nun der schier übermenschliche Scharfsinn der Genies, der lediglich auf einer besseren Lern- und Wissensorganisation beruhe, ${ }^{28}$ oder sei dies ein wie auch immer gearteter religiöser Geistbegriff. Die Vorstellung einer immateriellen Seele, die den Kern des Menschen ausmachen soll, ist für Minsky gleichbedeutend mit dem Ende geistiger Entwicklung:

And that's exactly what we get with inborn souls that cannot grow: a destiny the same as death, an ending in a permanence incapable of any change and, hence, devoid of intellect ... But minds are just the opposite. We start as little embryos, which then build great and wondrous selves - whose merit lies entirely within their own coherency. The value of a human self lies not in some small, precious core, but in its vast constructed crust. ${ }^{29}$

Ein von Minsky ohne weitere Differenzierungen rezipierter, religiöser Seelenbegriff wurde hier unter einem informationstechnischen Paradigma bewertet: Welche Funktion könnte eine unwandelbare Seele für das Denken eines Systems haben? Mit dieser Voraussetzung kommt Minsky zwangsläufig zu der Schlussfolgerung, dass das Konzept der Seele keinen Sinn mache. ${ }^{30}$

Minds are simply what brains do ... There is not the slightest reason to doubt that brains are anything other than machines with enormous numbers of parts that work in perfect accord with physical laws. ${ }^{31}$

Der Verstand selber, der Bewusstsein und Identität eines Systems generiere, sei als Summe von Denkprozessen immateriell. Wenn Minsky konstatiert, dass die Erforschung künstlicher Intelligenz das Bild vom Menschen und vom Denken nachhaltig beeinflussen wird, so bekräftigt er damit sein ganz persönliches Eintreten für die grundsätzliche Wesenseinheit von Mensch und Maschine. ${ }^{32}$ Der Mensch sei dabei keine triviale Maschine, sondern mit dem wachsenden Verständnis unseres Geistes würden wir in der Hoffnung Minskys sehen, was für komplexe

27 Humor dient als Zensor zum Erlernen normativer Tabus. Vgl. Minsky 1988, 278f. (Klassische!) Musik könne zur Ordnung und Bewältigung von Alltagserlebnissen oder zur Beruhigung benutzt werden. Vgl. Minsky 1981, 5 f.

28 Vgl. Minsky 1988, 80.

29 A.a.O., 41.

30 Vgl. A.a.O., 287f.

31 Ebd.

32 Vgl. Minsky 1967, 4; Minsky 1982, 9. 
und herrliche Apparate wir Menschen doch seien. ${ }^{33}$ Die vermeintlichen Unterschiede zwischen Mensch und Maschine würden sich in der Reduktion menschlicher Fähigkeiten auf eine reine Denktätigkeit und in den Visionen zukünftiger Computerpotentiale verlieren:

When people ask, »Could a machine ever be conscious?« I'm often tempted to ask back, »Could a person ever be conscious? I I mean this as a serious reply, because we seem so ill equipped to understand ourselves ... However, we can design our new machines as we wish, and provide them with better ways to keep and examine records of their own activities - and this means that machines are potentially capable of far more consciousness than we are. ${ }^{34}$

Es wird nach den bisherigen Ausführungen kaum verwundern, dass auch der Robotiker Hans Moravec den Menschen als eine höchst komplexe Maschine definiert: "Living organisms are clearly machines when viewed at the molecular scale ... « ${ }^{35}$ Und die Natur als Ganzes wird ebenso als totale Maschine interpretiert. ${ }^{36}$

Mit weitreichenden Konsequenzen für unser Verständnis von Tod und Leben unterstreicht Moravec nachdrücklich, dass unter der Voraussetzung, dass Leben in der Biologie und Chemie als sehr besondere und komplexe Organisation von Materie begriffen wird, ein heutiger Computer ebenfalls als lebendig angesehen werden muss. ${ }^{37}$ Bewusstsein würden die künstlichen Intelligenzen über ihre Selbstwahrnehmung erzeugen können und darüber hinaus angelernte Gefühle - wie Liebe zum Besitzer, Zorn und Schmerz - zur besseren Verhaltenssteuerung nutzen. ${ }^{38}$ Zugunsten einer ausdrucksstarken Vision der Umwandlung aller Materie in Geist, das heißt in Computer, verzichtet Moravec jedoch auf tiefergehende Reflexionen über den Lebensbegriff:

Thoughtful machinery violates the equally obvious and sacred dichotomy of the living and the dead, a difference embedded in our mentality ... In the old metaphor, we are in the process of inspiriting the matter around us. It will soon be our honor to welcome some of it to the land of the living ... ${ }^{39}$

33 Vgl. Minsky 1988, 30, 323.

34 A.a.O., 160. Vgl. auch a.a.O., 63.

35 Moravec 1988, 72.

36 Vgl. Moravec 1999, 127.

37 Vgl. a.a.O., 110f.

38 Vgl. a.a.O., 114-124.

39 A.a.O., 111. 
Ray Kurzweil, Schüler von Marvin Minsky, gelangte ebenso zu einer technozentrischen Deutung des Menschen, wobei die Simulation des Gehirns für ihn keine besondere Hürde darstellt: »Ich glaube ganz einfach, dass das Gehirn eigentlich banal funktioniert, recht simpel. Es ist ein Rechner, der zwar komplexe Aufgaben bewältigen kann, der aber in seiner Struktur doch ziemlich einfach aufgebaut ist ... $\ll^{40}$ Diese Grundhaltung vertritt Kurzweil auch in seinem jüngsten Buch How to Create a Mind, in der er konkrete Ideen zur Simulation des menschlichen Gehirns diskutiert. ${ }^{41}$

Wenn Menschen und Maschinen so verschieden voneinander wären, wie es manche Humanisten behaupten - so Max More - dann müsse es wahr sein, dass Maschinen in keiner Weise dem Menschen ähneln und der Mensch nicht den Maschinen. Jedoch habe die Wissenschaft gezeigt, dass der Mensch von den kleinsten Bausteinen an exakt wie das Zusammenspiel vieler Maschinen funktioniere. Das versteht More allerdings nicht als Herabsetzung des Menschen: »To say that humans are composed of machines is not to say that we are merely machines. Humans are dignified machines. « ${ }^{42}$ In seiner Diskussion der Irreversibilität des Todes zieht More die Analogie zwischen einem defekten Auto und einem gerade verstorbenen Menschen heran, um zu verdeutlichen, dass nicht nur die Funktionen des Autos in den meisten Fällen durch eine Reparatur wiederhergestellt werden können. Daher könne man ja auch kaum den definitiven Todeszeitpunkt eines Autos bestimmen. Dies gelte ebenso für den Menschen: Solange noch Informationen über die materielle Struktur und Funktion des Verstorbenen vorhanden sind, kann die Persönlichkeit wieder zum Leben erweckt werden. ${ }^{43}$ Andererseits lehnt More den Begriff Maschine für den Menschen wieder ab, da der Mensch frei, verantwortlich, moralisch und rational handele, was geplante und programmierte Maschinen (noch) nicht täten. ${ }^{44}$

Über im Detail differierende Wege gelangen Tipler, Moravec, Minsky, Kurzweil und More zu dem Schluss, dass der Mensch als Ganzes eine Maschine sei, dass der menschliche Geist als operative Funktion eines rein materiellen Gehirns zu betrachten sei und dass komplexe Maschinen, die Bewusstsein entwickeln bzw. sich selbst reproduzieren können,

\footnotetext{
${ }^{40}$ Interview mit Christoph Keller, Keller 2003, 16. Vgl. auch Kurzweil 1999a, 5.

41 Vgl. Kurzweil 2012b; Kurzweil 2005, 212-220.

42 More 1997b, 1.

43 Vgl. More 1995a, 27f.

44 Vgl. More 1997b, 1.
} 
als lebendig betrachtet werden müssen. Alle mentalen Vorgänge unterlägen dabei strikt den Gesetzen der Physik. Hans Moravec verweist ohne Umschweife auf René Descartes (1596-1650) als den philosophischen Urheber des Vergleiches zwischen Mensch und Maschine, dessen Erfahrungswelt vor 400 Jahren durch die Erfindung neuartiger Maschinen geprägt war.

Historisch gesehen begann die mechanistische Deutung des Menschen im frühen 17. Jahrhundert. Mit Thomas Hobbes und seiner mechanistisch-materialistischen Anthropologie im Leviathan (1651) und noch deutlicher in Descartes' 1633 verfasstem, jedoch erst posthum erschienenen Traité de l'Homme, wird der Körper als Einheit von Teilstücken gedeutet. Diese seien mechanistischen Grundprinzipien unterworfen. Descartes versichert zwar, dass er nie behaupten würde, der Körper des Menschen sei wirklich eine Maschine, jedoch schildert er detailliert, wie eine hypothetische Maschine von einem göttlichen Schöpfer gestaltet werden müsste, die unserem Körper entsprechen würde:

Ich stelle mir einmal vor, daß der Körper nichts anderes sei als eine Statue oder Maschine aus Erde, die Gott gänzlich in der Absicht formt, sie uns so ähnlich wie möglich zu machen, und zwar derart, daß er ihr nicht nur äußerlich die Farbe und Gestalt aller unserer Glieder gibt, sondern auch in ihr Inneres alle jene Teile legt, die notwendig sind, um sie laufen, essen, atmen, kurz all unsere Funktionen nachahmen zu lassen, von denen man sich vorstellen könnte, daß sie aus der Materie ihren Ursprung nehmen und lediglich von den Dispositionen der Organe abhängen. Wir sehen Uhren, kunstvolle Wasserspiele, Mühlen und ähnliche Maschinen, die, obwohl sie von Menschenhand hergestellt wurden, nicht der Kraft entbehren, sich aus sich selbst auf ganz verschiedene Weisen zu bewegen. ${ }^{45}$

Am Schluss seines Traktates über den Menschen hebt Descartes noch einmal den Grundgedanken seiner hypothetischen Konstruktion eines maschinellen Körpers hervor, der im Zusammenwirken seiner einzelnen Organe so funktionieren sollte wie ein menschlicher Körper:

... ich wünsche, sage ich, daß man bedenke, daß die Funktionen in dieser Maschine alle von Natur aus allein aus der Disposition ihrer Organe hervorgehen, nicht mehr und nicht weniger, als die Bewegungen einer Uhr oder eines anderen Automaten von der Anordnung ihrer Gewichte und ihrer Räder abhängen. Daher ist es in keiner Weise erforderlich, hier für diese (die Maschine) eine

45 Descartes 1969, 44. 
vegetative oder sensitive Seele oder ein anderes Bewegungs- und Lebensprinzip anzunehmen als ihr Blut und ihre Spiritus, die durch die Hitze des Feuers bewegt werden, das dauernd in ihrem Herzen brennt und das keine andere Natur besitzt als alle Feuer, die sich in unbeseelten Körpern befinden. ${ }^{46}$

Indem Moravec konstatiert, dass der Körper für Descartes' eine komplexe Maschine sei, verschärft er Descartes' ursprünglichen Vergleich, dass die körperlichen Organe in ihrem Zusammenspiel wie eine Maschine funktionieren würden. ${ }^{47}$ Den Weg vom cartesianischen Denken bis zum Posthumanismus markiert allerdings nichts eindrücklicher als die Metapher von der Schöpfung als Uhrwerk und ihrem göttlichen Uhrmacher. Spricht Descartes hier noch von Gott als dem Schöpfer der hypothetischen Körper-Maschine, die wie ein Uhrwerk funktioniere, so redet schon 1802 der Verfasser der Natural Theology, William Paley, von Gott als dem Uhrmacher. ${ }^{48}$ In diesem Sinne charakterisiert der von vielen Posthumanisten rezipierte Evolutionstheoretiker Richard Dawkins Gott heute als den blinden Uhrmacher (blind watchmaker). Für Hans Moravec ist nun der Zeitpunkt gekommen, an dem die Menschheit und ihre posthumanen Erben die Führung der Hand dieses Uhrmachers übernähmen. ${ }^{49}$ Es war ebenfalls Paley, der bereits vor 200 Jahren dem Vorwurf der Herabwürdigung des Menschen zur simplen Maschine, ähnlich wie Minsky, den Einwand entgegensetzte, dass die großartige Schöpfung des menschlichen Körpers als »most complicated or most flexible machine that was ever contrived « bewundert werden müsse. ${ }^{50}$ Descartes rechnet zu den rein körperlichen Funktionen auch die Erinnerungsfähigkeit des Gedächtnisses, die Gemütsbewegungen und

${ }^{46}$ Descartes 1969, 135f. » ... je désire, dis-je, que vous considérez que ces fonctions suivent toutes naturellement, en cette machine, de la seule disposition de ces organes, ne plus ne moins que font les mouvements d'une horloge, ou autre automate, de celle de ses contrepoids et de ses roues; en sorte qu'il ne faut point à leur occasion en celle aucun autre principe de mouvement et de vie, que son sang et ses esprits, agités par la chaleur du feu qui brûle continuellement dans son cœur, et qui n'est point d'autre nature que tous les feux qui sont dans les corps inanimés. « Descartes 1967a, 479f.

47 Vgl. Moravec 1999, 121f.

48 Paley allerdings musste sich schon zu Lebzeiten den Vorwurf des Plagiatismus seiner berühmten Uhrenmetapher gefallen lassen, die er wohl dem Werk Regt gebruik der Werelt Beschouwingen, 1716 (engl: The Religious Philosopher, 1730) des holländischen Descartes-Schülers Bernard Nieuwentyt entnommen hatte.

49 Vgl. Moravec 1988, 158f; Dawkins 1996, 4f; Paley 1802. Zur Rezeption von Dawkins vgl. Moravec 1988, 136; Moravec 1999, 4, 213; Tipler 1994, 125f.; Barrow \& Tipler 1986, 522; auch Wiener 1963, 76ff.

50 Vgl. Paley 1802, 490f. 
Stimmungen, das Vorstellungsvermögen und den Gemeinsinn (sensus communis), die allesamt durch die feinsten, aber rein materiell gedachten Blutkörperchen - die spiritus animales (esprits animaux) - gesteuert würden. ${ }^{51}$ Sofern für Descartes das Wesen aller materiellen Körper (res extensa) in ihrer Ausdehnung besteht und sofern Vorgänge materieller Körper für ihn nichts anderes als Bewegungsvorgänge darstellen, müssten die Phänomene im Bereich der Organe als körperliche Mechanismen verstanden werden. Die cartesianische Physiologie setzt in ihrem Selbstverständnis als Teilbereich der Physik voraus, dass alles materielle Geschehen mechanischer Natur sei und im Idealfall mathematisch beschrieben werden könne. ${ }^{52}$ Die Fähigkeit des vernünftigen Denkens, des Erkennens und Wollens jedoch schreibt Descartes der unsterblichen, immateriellen Seele des Menschen zu.

Die Nichtbeachtung der Differenz zwischen den körperlichen Sinnen und dem immateriellen Erkenntnissubjekt in der Konzeption Descartes', eben jener vernunftbegabten Seele, verweist auf die materialistische Deutung, mit der Moravec an Descartes herantritt. Ferner würde Descartes nach Ansicht Moravecs seinen - für den Existenzbeweis so grundlegenden - Dualismus zwischen Körper und vernunftbegabter Seele unter dem Eindruck der heutigen, technischen Möglichkeiten zugunsten eines gänzlich materialistischen Menschenbildes aufgegeben haben: ${ }^{53}$

Lacking a mechanical model of thought, he retained part of the medieval idea ... If he were working today, Descartes might well have found, in computers, a material model for mind and become a thorough-going materialist. But, alas, there were no computers in the seventeenth century. ${ }^{54}$

Ohne dass die meisten Posthumanisten nun explizit Bezug auf die grundlegenden Zeilen in Descartes' Traité de l'Homme nehmen würden, sondern vielmehr die cartesianische Philosophie hauptsächlich über Sekundärliteratur rezipieren, ist offensichtlich, dass wir hier ein wichtiges geistesgeschichtliches Fundament der posthumanistischen Deutung des

51 Vgl. Descartes 1967a, 387ff., 437f., 448-453. Descartes reduziert hier die antike Lehre Galens von den drei Arten des Spiritus (naturalis, vitalis, animalis) auf die Idee von den flammengleichen, schnellen und bewegten spiritus animales, die rein materieller Natur seien. Vgl. a.a.O., 388 (FN 1), Descartes 1969, 52 (FN 2).

52 Vgl. Röd 1995, 130-134.

53 Vgl. Moravec 1999, 121f.

54 A.a.O., 121. 
Menschen als Maschine antreffen. ${ }^{55}$ Wenn auch die mit Descartes auf einen Körper-Geist-Dualismus angelegte Metaphysik schwierige Fragen ausblendet, so stilisieren einige der heutigen Posthumanisten den Philosophen zum Urheber der Idee einer maschinellen Simulation des Menschen, da er im Traité de l'Homme erstmals in der europäischen Neuzeit die gedankliche Möglichkeit formulierte, eine dem Menschen nachempfundene, rein materielle Maschine zu konstruieren, die alle Funktionen des menschlichen Körpers nachahmen könne. Indes muss man die $\mathrm{Zu}-$ rückhaltung anderer Posthumanisten mit expliziten Verweisen auf Descartes wohl gerade dem von Moravec ausgeblendeten, fundamentalen Dualismus zwischen Materie, res extensa, und Geist, res cogitas, im cartesianischen Denken zuschreiben.

Der materialistischen Position des Posthumanismus entspricht vielmehr das Werk des weithin vergessenen und von den Posthumanisten nach meiner Kenntnis auch nicht explizit beachteten Mediziners und Philosophen Julien Offray de La Mettrie (1709-1751), der erst im 19. Jahrhundert vor allem von einem Vertreter des deutschen Idealismus, Friedrich Albert Lange, als eigentlicher Begründer des materialistischen Monismus rezipiert wurde. La Mettrie markiert den Endpunkt einer von Descartes initiierten und bis ins 18. Jahrhundert reichenden philosophischen Debatte um das Wesen und das Verhältnis von Körper, Geist und Seele. Schon für Thomas Hobbes galten die mechanischen Gesetze auch für die geistigen Phänomene, die vollkommen vom Körper abhängen würden. ${ }^{56}$ Aber erst unter dem Einfluss von Gottfried Wilhelm Leibniz' Monadentheorie und dem rationalen Empirismus John Lockes führt die noch dualistische Annahme zweier »Automatae«, nämlich Körper und Geist, die jeder für sich mechanischen Gesetzen gehorchen, schließlich $\mathrm{zu}$ einer monistischen Interpretation. Diese kam schon in der Wende zum 18. Jahrhundert bei den Philosophen Pierre Sylvain Régis, Georges-Louis Le Sage und Claude Buffier zum Vorschein, und gipfelte schließlich in der materialistischen Position La Mettries. ${ }^{57}$ In seinem 1747 niedergelegten Werk L'Homme Machine führt La Mettrie die Essenz' seiner bisherigen materialistischen Abhandlungen zusammen, indem er zwar einerseits Descartes' mechanistische Deutung des menschlichen Körpers adaptiert, andererseits jedoch über Descartes hinausgeht, da er

\footnotetext{
55 Vgl. Barrow \& Tipler 1986, 53f.; Moravec 1988, 180; Moravec 1999, 78, 121, 191; Kurzweil 1999, 60, 262.

56 Vgl. Kirkinen 1960, 219.

57 Vgl. a.a.O., 444-449.
} 
gleichermaßen den menschlichen Geist bzw. die Seele funktionalistisch und materialistisch interpretiert: »Die Seele ist also nur ein nichtiger Ausdruck, von dem man keine rechte Vorstellung hat und dessen sich ein guter Kopf nur zur Benennung des in uns denkenden Prinzips bedienen sollte. $\ll^{58}$

Nachdem der umstrittene Philosoph unter einer schweren Fieberkrankheit gelitten hatte, erkannte er die enge Wechselwirkung zwischen Körper und Geist. Fortan wies er alle dualistischen Erklärungen dieses Verhältnisses vehement zurück, da hier die Kategorien des Immateriellen und des Geistigen (spirituelles) unzulässigerweise vermengt worden seien. Mit Aristoteles, den Epikuräern und Thomas von Aquin wendet sich La Mettrie damit ausdrücklich gegen den Dualismus von Platon, Augustinus und Descartes. In seiner Histoire Naturelle de l'âme ou Traité de l'âme von 1745 führt La Mettrie alle Funktionen des menschlichen Geistes im Sinne eines materialistischen Monismus gänzlich auf die Funktionen des Körpers zurück: Der Körper denkt! ${ }^{59}$

In der Tat, wenn das, was in meinem Gehirne denkt, nicht ein Teil desselben und folglich des ganzen Körpers ist, warum erhitzt sich ... wenn ich abstrakten Gedanken nachhänge mein Blut? ... folglich ist die Seele nur ein Bewegungsprinzip, oder ein empfindlicher materieller Teil des Gehirns ... ${ }^{60}$

Während La Mettrie die Lebenskraft als Eigenschaft der Organisation der Körperteile und inneren Organe charakterisiert, verortet er das denkende Prinzip des Menschen im Gehirn, wo es all unsere Gedanken, Gefühle und Leidenschaften hervorbringt. Aus der Tatsache, dass der menschliche Körper ein durch unübertroffene Kunst und Geschicklichkeit hergestelltes Uhrwerk sei und dass er damit ebenso wie die Tiere eine Maschine sei, leitet La Mettrie eine große Achtung für die Phänomene der Natur ab. Jede metaphyische Aussage über Gott und das Jenseits - gleich welcher Provenienz - verbannt er aus seiner dem Empirismus verpflichteten Philosophie. ${ }^{61}$

Entscheidend zur Verbreitung dieser materialistischen Philosophie hatte seit dem 19. Jahrhundert im Zuge der stürmischen Entwicklung der positivistischen Naturwissenschaft die monistische Bewegung beigetragen. Paul Carus (1852-1919) in den Vereinigten Staaten und vor allem

58 La Mettrie 1994, 78.

59 Vgl. La Mettrie 1994, 80ff.

60 A.a.O., 84, 86.

61 Vgl. a.a.O., 92-101; Irrgang 2005, 33f. 
der Evolutionstheoretiker Ernst Haeckel (1834-1919) mit seinen in 24 Sprachen übersetzten Welträthseln ${ }^{62}$ konnten zu Beginn des 20. Jahrhunderts in allen Gesellschaftsschichten die auf einem weitgehend materialistischen Monismus beruhenden Ideen popularisieren. Dies fand in der Gründung des Deutschen Monistenbundes im Jahre 1906 und unzähliger monistischer, freikirchlicher Gruppierungen weltweit seine Krönung. ${ }^{63}$

Seitdem die ersten mechanischen Uhrwerke im 15. Jahrhundert die menschliche Lebenswelt zu prägen begannen, geben unzählige Monographien Zeugnis ab von der bewegenden Diskussion um das Verhältnis zwischen Menschen und Maschinen sowie der menschlichen Selbstreflexion unter dem Einfluss der Technik. Bernhard Dotzler, Peter Gendolla und Jörgen Schäfer führen über 1700 europäische und amerikanische Titel seit dem Jahr 1420 auf, die sich philosophisch oder literarisch geleitet von warnender Polemik bis hin zu euphorischer Technikbegeisterung mit dem Thema befassen. ${ }^{64}$ Wenn hier auch nicht der Raum gegeben ist, diese Auseinandersetzung der vergangenen 500 Jahre auch nur skizzenhaft nachzuzeichnen, so möchte ich aus heuristischen Motiven dennoch nicht die kurze Kritik an der Gleichsetzung von Mensch und Maschine vorenthalten, die der englische Philosoph Colin McGinn an den Posthumanisten übt:

It is true that human minds manipulate symbols and engage in mental computations, as when doing arithmetic. But it does not follow from this that computing is the essence of mind ... The fallacy here is analogous to reasoning that if a human body is a device for taking you from A to B, and a car also does this, then the human body is the same thing as a car. ${ }^{65}$

Nach Frank Tiplers und John D. Barrows »Beweis«, dass Autos ebenfalls Lebewesen seien, und Max Mores Analogieschluss zwischen der Reparatur eines Autos und eines verstorbenen Menschen, veranschaulicht nun McGinns Kritik sehr transparent das Prinzip dieser philosophischen Gleichsetzung von Mensch und Maschine. Beziehen sich die posthumanistischen Autoren explizit nur auf Descartes, so ist deutlich geworden, dass diese rein materialistische Descartes-Rezeption - seine Metaphysik

\footnotetext{
62 Vgl. Haeckel 1960.

63 Vgl. Mehlhausen \& Dunkel 1994.

64 Vgl. Dotzler \& Gendolla \& Schäfer 1992.

65 McGinn 1999, 12.
} 
wird ja ausgeblendet - durch Interpretationsmuster des mit La Mettrie beginnenden Materialismus bedingt ist, die durch die populäre monistische Bewegung seit dem Ende des 19. Jahrhunderts gerade auch in der amerikanischen Wissenschaft weite Verbreitung gefunden haben.

\subsubsection{Simulation und Identität}

The optimist's proof of the rationality of the general constitution of things turned out to be a proof of its essential immortality.

Arthur O. Lovejoy ${ }^{66}$

Wenn Frank Tipler behauptet, dass die Essenz des Menschen nicht im Körper, sondern im »Programm« liegt, das den Körper erschafft, so vertritt er die Haltung der Mustertheorie der Identität. Nicht der Träger der Information, der menschliche Körper, sondern sein immaterielles Programm bilde prinzipiell unabhängig von seiner Verkörperung die menschliche Identität. Tipler beruft sich dabei vor allem auf Aristoteles und Thomas von Aquin. Ferner zieht er Plutarchs Bericht vom Schiff des Theseus heran, auf den schon Thomas Hobbes zurückgriff. Die Athener bewahrten demnach über mehrere Jahrhunderte das Schiff ihres großen Helden auf, erneuerten aber laufend dessen Bestandteile. Trotz uneiniger Schlüsse der antiken Philosophen und einer gegenteiligen Lösung bei Hobbes, plädiert Tipler mit der Quantentheorie für die Identität des Schiffes mit dem Original. Die Bewahrung des ursprünglichen Musters stellt für ihn das ausschlaggebende Kriterium dar. ${ }^{67}$

Ähnlich argumentiert der Physiker, wenn er auf den ständigen materiellen Wandel auf submolekularer Ebene hinweist, wo in weniger als $10^{-23}$ Sekunden die Bestandteile der Neutronen und Protonen vernichtet und

66 Zitiert nach Dobrée 1959, 29.

67 Bei Plutarch in den oi ßíor $\pi \alpha \rho \alpha \dot{\lambda} \lambda \lambda \eta \lambda$ or / Vitae parallelae. Vgl. Hobbes 1967, 111-116; Tipler 1995, 234f. Bei Hobbes gestaltet sich die Antwort auf das Identitätsproblem differenzierter und anders als in Tiplers und Minskys Rezeption. Ist für Menschen, Institutionen und Staaten die Kontinuität der Identität mit der Kontinuität der »Form«, dem Verhalten, also dem »Lebensprinzip der Bewegung « verbunden, so gilt dies nach Hobbes gerade nicht für »Dinge«, deren Identität auf der Materie beruht: »Ein Schiff, unter welchem Namen wir eine bestimmt gestaltete Materie verstehen, wird dasselbe sein, solange seine Materie dieselbe bleibt; ist kein Teil der letzteren mehr derselbe, dann ist es numerisch ein anderes Schiff geworden ... » Hobbes 1967, 115. Tipler und Minsky rezipieren Hobbes' rein materialistisch, adaptieren jedoch seine nur in einem dualistischen Konzept gültige Identitätstheorie. 
neu gebildet werden. Im gleichen Zusammenhang führt er das Kannibalenproblem in Thomas von Aquins Summa contra gentiles an, das er mit der Möglichkeit einer Wiederherstellung des immateriellen Musters einer Person lösen will. ${ }^{68} \mathrm{Da}$ die mittelalterliche Vorstellung von der Seele in aristotelischer Tradition als abstrakte Form der Aktivität des Körpers verstanden wurde und sich etymologisch der Begriff Information von Form ableiten lässt, folgert Tipler schlicht:

There is actually an astonishing similarity between the mind-as-computer-program idea and the medieval Christian idea of the »soul«. Both are fundamental immaterial ... For Aquinas, a human soul needed a body to think and feel, just as a computer program needs a physical computer to run ... Even semantically, the information theory of the soul is the same as the Aristotle-Aquinas theory. ${ }^{69}$

Dass sich gerade Thomas von Aquin kaum dazu eignet, die Identität einer Person auf ihr »immaterielles Programm« zu reduzieren, kann hier nicht weiter problematisiert werden. ${ }^{70}$ Die Mustertheorie der Identität bietet Tipler die Basis für die technisch ermöglichte Auferstehung der Toten.

Aus den Ausführungen Marvin Minskys geht klar hervor, dass wenn der Mensch unter dem Maßstab der Maschinen betrachtet wird, die Maschinen schon immer die besseren Menschen gewesen sind. Der menschliche Körper in seiner biologischen Form bildet in dieser informationszentrierten Perspektive Minskys eine zu vernachlässigende Größe. Wesen und Funktion dessen, was den Menschen als Menschen charakterisiert, sei allein das Denken. Indem Minsky konstatiert, dass der Mensch nie im direkten Kontakt zur Welt steht, sondern nur über Rezeptoren und Nervenbahnen des Körpers Informationen zum Gehirn gelangen, setzt er den Menschen mit dem Verstand (mind) gleich und löst ihn vom Körper. ${ }^{71}$ Kurz bevor Minsky die zentrale Rolle von Besitz (possession) erläutert, unterstreicht er noch einmal die Trennung von Körper und Geist. Da Minsky Besitz als die unabdingbare Voraussetzung für die Kontrolle

68 Vgl. Tipler 1995, $235 f$.

69 A.a.O., 127f; vgl. auch Barrow \& Tipler 1986, 659.

70 Bei Thomas von Aquin zerfällt der Mensch eben gerade nicht in zwei voneinander zu trennende Substanzen, Seele und Leib, sondern er ist die Einheit, die durch seine Form, die Geistseele als Lebensprinzip seines Leibes, und durch die Materie gebildet wird. Nur als diese Einheit ist der Mensch Person. Vgl. Scherer 1995, 886.

71 Vgl. Minsky 1988, 110, 286. 
und Nutzung eines Werkzeuges betrachtet, können wir folgern, dass er den Körper als Besitz und Hilfsmittel des Geistes auffasst. ${ }^{72}$

Damit aber ist der Körper als Datenträger beliebig austauschbar. Minsky argumentiert analog zu Tipler, dass ein Schiff, dessen Einzelteile ausgetauscht werden, stets die gleiche Funktion erfüllen wird. Genauso wird ein Gehirn, dessen Gehirnzellen komplett durch künstliche Computerchips ersetzt werden, die gleiche Funktion wie das originale Denkorgan übernehmen und damit identisch sein. Eine hundertprozentige Übereinstimmung, die technisch kaum zu realisieren ist, sei nicht notwendig, da der menschliche Körper ohnehin einem ständigen Wandel unterworfen sei. ${ }^{73}$

Nachdem er die Substitution des gesamten biologischen Körpers durch künstliche Einzelteile erörtert hat, macht Minsky eine bemerkenswerte Aussage: "Needless to say, in doing so, we'll be making ourselves into machines. ${ }^{74}$ In aller Dichte veranschaulicht Minsky auf diese Weise den visionären Diskurs unserer posthumanen Weiterentwicklung, die N. Katherine Hayles beschrieben hat. Unzählige Male wiederholt Minsky, dass der Mensch nichts anderes als eine (Denk-)Maschine sei, und dann schildert er den technischen Vorgang des faktischen, menschlichen Aufgehens in der Maschine. Diese doppelte Maschinisierung des Menschen impliziert bei allen Posthumanisten eine subtile Differenzierung: Zwar wird der Mensch zunächst so definiert, dass er problemlos mit den Maschinen verglichen werden kann - er ist damit in seiner Selbstdeutung schon posthuman. ${ }^{75}$ Dann erst kann er zur wirklichen Maschine aus elektronischen und mechanischen Substituten transformiert werden.

Eine bestimmte Art von Dualität weist auch Moravecs Menschenbild auf - nämlich die Differenzierung zwischen dem materiellen Körper und den geistigen Funktionen, die dieser Körper hervorzubringen imstande ist. Geist (mind) wird von Moravec nicht als im herkömmlichen Sinne metaphysische Substanz - wie etwa ein Seelenbegriff - gedeutet, sondern etwas unpräzise als Summe der geistigen Operationen dieses Körpers. Nichtsdestotrotz ist Moravec optimistisch, dass den Robotern und intelligenten Computern eine Seele zugesprochen werde, sobald diese Maschinen als Personen anerkannt worden seien. ${ }^{76}$ Diese Funktionen

72 Vgl. a.a.O., $292 \mathrm{f}$.

73 Vgl. a.a.O., 289. Minsky erwähnt nicht explizit den Bericht Plutarchs bzw. Hobbes.

74 Minsky 1994, 109f.

75 Vgl. Hayles 1999, 11.

76 Vgl. Moravec 1999, 77. 
des Geistes beruhten zwar auf der materiellen Basis des menschlichen Körpers, sind aber nach Moravec nicht abhängig von der spezifischen Form dieses Körpers. Die geistigen Funktionen - also die ausschließlich vom materiellen Körper erbrachte Informationsverarbeitung - können demnach auch von einer perfekten Simulation des Körpers, insbesondere des Gehirns, geleistet werden. Damit stellt der physische Tod des Menschen nicht das besiegelte Ende des Lebens dar: ${ }^{77}$

Death of a body should no more destroy a soul - or its history or potential - than clearing an abacus destroys a number. Nor should death destroy sensations and consciousness - those are properties of the abstraction. Only the perfect correlation between the consciousness and the physical world would be lost. ${ }^{78}$

Ebenso wie Tipler und Minsky argumentiert Moravec als Verfechter der radikalen Mustertheorie, wenn er die Identität des Menschen unabhängig von ihrer materiellen Verkörperung verankert. Der Körper wird als Nachricht begriffen, dessen Identität durch die exakte Simulation seines Musters, seiner Struktur, bewahrt bleibt. Die Kontinuität der persönlichen Identität als Fortsetzung der gedanklichen Operationen wird somit durch das funktionale Äquivalent eines nur virtuell existierenden Körpers garantiert. Trotz einer gewissen Brisanz der philosophischen Problematik seiner Idee, beharrt Moravec darauf, dass zugleich mehrere Kopien einer Person mit Recht behaupten können, das Original zu sein und es auch sind: ${ }^{79}$ "It is you. And when that demon in your belly at last makes the body you have occupied all these years useless, and the couple of pounds of wetware in your skull has to die ... you live on in the machine. $\ll^{80}$

Die Denkfähigkeit allein wird im Verständnis Ray Kurzweils in ausdrücklicher Anlehnung an Descartes zum Inbegriff des Menschseins und damit zum Existenzbeweis. Die computertechnische Simulation des Gehirns werde damit auch die Kontinuität von Bewusstsein und Identität garantieren, da das simulierte Subjekt davon überzeugt sein würde. ${ }^{81}$ Die baldige Simulation aller Gehirnteile werde es uns ersparen, dass

\footnotetext{
77 Vgl. Moravec 1988, 162-169.

78 Moravec 1999, 76.

79 Vgl. Moravec 1988, 162-166. Dieses Problem diskutierte Lem bereits 1956 ausführlich in seinen Dialogen. Vgl. Lem 1980.

80 Moravec \& Pohl 1993, 72. Vgl. auch Rothblatt 2014, 9-53.

81 Vgl. Kurzweil 1999a, 53-60.
} 
wir alle Funktionen des Gehirns physiologisch erschöpfend verstehen müssten, zumal die künstlichen Intelligenzen der kommenden Quantencomputer mit größerer Geschwindigkeit einschließlich des irrationalen Denkens alles leisten könnten, was ein menschliches Gehirn vollführen kann. ${ }^{82}$

Indem Max More in seiner Dissertation The Diachronic Self auf den psychologischen Reduktionismus Derek Parfits zurückgreift, vertritt er einen rein funktionalistischen Standpunkt, der die Genese von Bewusstsein allein auf die Prozesse komplexer physikalischer Systeme zurückführt. ${ }^{83}$ Schon auf Seite vier seiner dem Problem von Kontinuität und Überleben von Identität gewidmeten Doktorarbeit legitimiert More die Idee der Unsterblichkeit im Computer mit der Anknüpfung an eine weitest gefasste, reduktionistische Betrachtungsweise. Diese knüpft Identität nicht an einen bestimmten physischen Träger (das Gehirn), sondern an ein funktionales Äquivalent der mentalen Prozesse. ${ }^{84}$ Detailliert führt More nun den Beweis, dass die Kontinuität der Person nicht von der Kontinuität ihrer materiellen Verkörperung abhänge: »According to psychological reductionism I am nothing more than the connectedness and continuity of my psychological states. $\ll^{85}$

In einer weitergehenden Differenzierung vertritt More die Position der so genannten konservativen Interpretation der weitesten, reduktionistischen Betrachtungsweise, die die Kontinuität der Identität an die Aktivität des simulierten Menschen knüpft. Die Identität eines Menschen mit seinem Original, der sich zum Beispiel in einem Computer simulieren ließe, sei nur dann gewährleistet, wenn dieser Mensch eine kausale Beziehung zu seiner Simulation aufweise. More bestreitet aus diesem Grunde, dass Tiplers Vorschlag einer Auferstehung aller quantenphysikalisch möglichen Verstorbenen durch einen Computer die Identität der Personen garantieren würde, da diese Identitäten keine Rolle spielen würden » ... neither in the coming into existence nor the qualitative identity ... ${ }^{86}{ }^{86}$

${ }^{82}$ Vgl. a.a.O., 111f., 124f.; Kurzweil 2005, 312-320.

83 Referenzwerk bildet vor allem Derek Parfits Reasons and Persons. Oxford 1984. Vgl. auch More 2000c, 1.

84 Vgl. More 1995a, 4f. Seitenangaben entsprechen dem Seiten-Layout, Microsoft Word 97 SR-1.

85 A.a.O., 16. Vgl. hierzu a.a.O., $15 f f$.

86 Vgl. a.a.O., 5-9; Gräfrath 2000, $296 \mathrm{f}$. 
Als Anhänger der radikalen Mustertheorie der Identität sind für More die Bewahrung der strukturellen und funktionalen Muster entscheidend für die Kontinuität der Identität, da sich mit diesen Informationen die Simulation eines Bewusstseins bewerkstelligen ließe. Demnach wäre der permanente und irreversible Tod erst durch die Zerstörung dieser Informationen vollendet. More differenziert daher zwischen einem endgültigen, irreversiblen Tod und einem reversiblen Zustand, den er als Deanimation (deanimation) bezeichnet. Diese Todesdefintion stammt aus der Kryonik - nicht zufällig fungiert More als CEO des Kryonik-Anbieters Alcor. Damit ist es letztlich unmöglich, den definitiven Tod eines Menschen festzustellen. ${ }^{87}$

Überdies wehrt sich More gegen den Vorwurf, der Posthumanismus würde für die Abschaffung des Körpers eintreten, da die technische Aufrüstung des Körpers ja gerade dessen physische und psychische Wahrnehmungs- und Handlungsvielfalt erweitern solle. Denn es gehe den Extropianern nicht um die Abschaffung des Körpers, sondern allein um die Ausweitung seiner Fähigkeiten und das schließe die Überwindung des rein biologischen Körpers mit ein: ${ }^{88}$

The contribution of bodily features to personal continuity is entirely of instrumental importance. Parts of a body gain their instrumental importance from their functional roles. The particular matter constituting a body, and even the specific form of a body, have no intrinsic significance for personal identity. ${ }^{89}$

Da die menschliche Identitätsbildung in großem Maße von physischen Eigenschaften unserer gegenwärtigen Körper abhängt, ist More dennoch der Ansicht, dass wir in unserer Transformation zur posthumanen Lebensform nicht einfach unsere Körperlichkeit aufgeben können. Von einem psychologisch-reduktionistischen Standpunkt aus seien für die Bewahrung der persönlichen Identität zwar die spezifische Form und materielle Basis des Körpers entbehrlich, nicht aber die körperlichen Funktionen, die in einer virtuellen Existenz simuliert werden könnten:

Without this consideration of our physical nature, and the relationship between our physicality and our psychology, a psychological reductionist view of identity would be in danger of falling into a faulty dualism. ${ }^{90}$

\footnotetext{
87 Vgl. Ettinger 1964, 3f.; Freyermuth 1996, 154; Richard 2000, 66.

${ }^{88}$ Vgl. More 1997a, 1f.

89 More 1995a, 91.

90 A.a.O., , 95.
} 
Somit soll allein die Kontinuität der körperlichen Funktionen, die ausgehend vom materialistischen Paradigma des Posthumanismus zugleich alle geistigen Funktionen implizieren, die Kontinuität der persönlichen Identität gewährleisten, ohne an dualistische Konzepte eines dominierenden, immateriellen Geistes oder einer Seele anknüpfen zu müssen. ${ }^{91}$

Erstaunlicherweise bleibt der gesamte psychologische Diskurs - außer bei More - mit den über ein Jahrhundert währenden Auseinandersetzungen um das Verhältnis von Gehirn, Geist und Seele im Posthumanismus beinahe vollkommen unberücksichtigt. Aus den Bemerkungen von Marvin Minsky und Ray Kurzweil geht hervor, dass unter der Annahme eines vollkommen materiell determinierten Menschen die perfekte Simulation jedes Moleküls eines Körpers bzw. eines Gehirns die perfekte Simulation einer Person erlauben wird, ohne dass wir die Funktionsweise unseres Gehirns auch nur annähernd verstehen müssten! ${ }^{92}$ Der Fokus auf das menschliche Gehirn muss in Zusammenhang mit dem Genieund Gehirnkult des 19. Jahrhunderts gesehen werden. ${ }^{93}$

Dort, wo innerhalb des posthumanistischen Diskurses die Kontinuität der Identität in der Simulation problematisiert wird, erscheinen zuvorderst zwei ineinander verwobene Argumentationsstränge. Der erste setzt zur Absicherung der Identität in der Simulation die Bewahrung der Form und Struktur über die Bewahrung der konkreten Materie. Der zweite bindet die Bewahrung der persönlichen Identität an die Bewahrung des Geistes, das heißt an die mentalen Operationen allein des Gehirns. Um den Vorzug der Bewahrung der Form und Struktur zu legitimieren, beziehen sich die Posthumanisten nur vereinzelt und peripher auf Aristoteles und Thomas von Aquin (Tipler) bzw. auf den psychologischen Funktionalismus (More). ${ }^{94}$ Vielmehr entspringt die Idee, die Kontinuität eines Körpers durch die Bewahrung der Informationen über seine Form und Struktur zu garantieren, bei Minsky, Moravec und Kurzweil unbestreitbar der Mustertheorie der Identität, wie sie innerhalb der kybernetischen Informationstheorie entwickelt wurde. Auch die Herangehensweise des Physikers Tipler und des Philosophen More scheint von diesem informationstechnischen Paradigma geleitet zu sein, das ich im anschließenden Kapitel herausarbeiten werde.

91 Vgl. a.a.O., 90-95.

92 Vgl. Minsky 1988, 306f.; Kurzweil 1999a, $124 f$.

93 Vgl. Kapitel 6.6.2.

94 Vgl. Barrow \& Tipler 1986, 659; Tipler 1995, $127 f$. 
Indem sich alle Posthumanisten beim technischen Prozess des Uploads auf das Einscannen der Gehirnmuster beschränken, wird nachhaltig illustriert, wie persönliche Identität ausschließlich als Ergebnis der mentalen Funktionen des Gehirns verstanden wird. Auch More betonte ja ausdrücklich, dass die spezifische Materie unseres Körpers keine Relevanz für unsere Identitätsbildung hat. Die Dominanz des Geistes gegenüber dem Körper leiten Hans Moravec, Ray Kurzweil und offensichtlich auch Marvin Minsky aus der Erkenntnistheorie René Descartes her.

Descartes widmet sich in seinen Meditationes de prima philosophia ${ }^{95}$ der systematischen Suche nach dem unbezweifelbaren Fundament der Erkenntnis, wobei er schrittweise alles ausschließt, was einem möglichen Zweifel unterliegen könnte. Alle Objekte der Umwelt, alle Sinneswahrnehmungen und schließlich der eigene Körper fallen unter den Verdacht, Illusion zu sein:

Ich setze also voraus, daß alles was ich sehe, falsch ist, ich glaube, daß niemals etwas von dem allen existiert hat, was das trügerische Gedächtnis mir darstellt: ich habe überhaupt keine Sinne; Körper, Gestalt, Größe, Bewegung und Ort sind nichts als Chimären. Was also bleibt Wahres übrig? Vielleicht nur das Eine, daß es nichts Gewisses gibt. ${ }^{96}$

Die letzte Gewissheit sieht Descartes demzufolge in der geistigen Handlung, die den Menschen zum fundamentalen Zweifel an allen Wahrnehmungen befähigt. Was auch immer man sieht, träumt, halluziniert - all dies bestätigt nur eines mit Gewissheit: dass eine geistige Handlung stattfindet, deren Gegenstand und Zweck zwar letztlich ungewiss bleiben, die aber als geistige Handlung - und nur als diese - existent ist. Denn wenn alle körperlichen Erscheinungen nur Illusion sind, was bin ich dann?

Das Denken ist's, es allein kann von mir nicht getrennt werden: Ich bin, ich existiere, das ist gewiß. Wie lange aber bin ich? Nun, so lange, als ich denke ... Ich bin also genau nur ein denkendes Ding (res cogitas), d. h. Geist (mens), Seele (animus), Verstand (intellectus), Vernunft (ratio) - lauter Ausdrücke, deren Bedeutung mir früher unbekannt war ... Und was heißt das? Nun - ein Ding, das

95 Die französische Übersetzung der 1641 bzw. 1642 in Paris bzw. Leiden erschienenen lateinischen Originalfassung wurde erst 1647 publiziert. Vgl. Descartes 1967b, 171-176, 377-381.

96 Descartes 1972, 17. »Suppono igitur omnia quae video falsa esse; credo nihil unquam exitisse eorum quae mendax memoria repraesentat; nullos plane habeo sensus; corpus, figura, extensio, motus, locusque sunt chimerae. Quid igitur erit verum? Fortassis hoc unum, nihil esse certi.« Descartes 1967b, 182. 
zweifelt, einsieht, bejaht, verneint, will, nicht will und das auch Einbildung und Empfindung hat. ${ }^{97}$

Das Denken selbst wird damit zum Existenzbeweis: Cogito, ergo sum! Die cartesianische Existenzgewissheit, die allein in der Fähigkeit des Denkens verankert wird, entledigt sich damit des Körpers als Erkenntnissubjekt. Der radikale Erkenntniszweifel, der als einzige sichere Erkenntnis die Fähigkeit zum Zweifeln selbst anerkennt, führt auf diesem Wege zu der Idee der vollkommenen Loslösung des Denkens vom materiellen Körper. Der Körper sei damit als vermeintliche Illusion für das Denken ebenso unwichtig wie ein Traum. ${ }^{98}$

Sehr deutlich wehrt sich Descartes in seinem posthum publizierten, physiologischen Essay La description du corps humain von 1647 dagegen, die Seele des Menschen als Prinzip und Ursache aller Bewegungen und Funktionen des menschlichen Körpers zu bestimmen, da die physiologischen Mechanismen der Organe gleich dem Räderwerk einer Uhr keiner Seele bedürfen, sondern sich von sich aus bewegen würden:

Doch bei der Bemühung, unsere Natur genauer kennenzulernen, können wir statt dessen feststellen, daß unsere Seele als eine vom Körper verschiedene Substanz einzig dadurch bekannt ist, daß sie denkt ... ${ }^{99}$

Hier zeigt sich, wie tiefgreifend die Interpretationsleistung sein muss, wenn man aus der mechanistischen Erklärung des menschlichen Körpers einen materialistischen Monismus konstruiert. Denn Descartes hat den Menschen schon früh - und nicht erst in den Meditationes - als dualistische Einheit von Körper und Seele verstanden, zumal auch die »Lebensgeister", die spiritus animalis, rein körperlich definiert sind und keinen Zwischenstatus einnehmen: "Ces hommes seront composés, comme nous, d'une Ame et d'un corps. « ${ }^{100}$ Wenn Descartes auch die

97 Descartes 1972, 20f. »Cogitatio est; haec sola a me divelli nequit. Ego sum, ego existo; certum est. Quandiu autem? Nempe quandiu cogito ... sum igitur praecise tantùm res cogitans, id est, mens, sive animus, sive intellectus, sive ratio, voces mihi prìus significationis ignotae ... Quid est hoc? Nempe dubitans, intelligens, affirmans, negans, volens, nolens, imaginans quoque, sentiens.« Descartes 1967b, $184 \mathrm{ff}$.

98 Vgl. a.a.O., 404-413; Gebauer 1984, $235 f$.

99 Descartes 1969, 140; Vgl. a.a.O., 139ff.

${ }^{100}$ Descartes 1967a, 379. 
Verhältnisse von Körper, Seele und Geist nie eindeutig klärte, so bleibt unbestritten, dass er eine Trennung von Leib und Seele voraussetzt: ${ }^{101}$

Nun bemerke ich hier erstlich, daß ein großer Unterschied zwischen Geist und Körper insofern vorhanden ist, als der Körper seiner Natur nach stets teilbar, der Geist hingegen durchaus unteilbar ist ... und das allein würde hinreichen, um mich zu lehren, daß der Geist (l'esprit ou l'âme) vom Körper gänzlich verschieden ist ... 102

Die cartesianische Trennung in eine körperliche und eine immaterielle Welt, die zwar nicht aufeinander, aber doch irgendwie miteinander wirken, sollte innerhalb und außerhalb der Medizin unter Theologen, Philosophen und Ärzten heftige, jahrzehntelange Fehden um das von Descartes ungelöste Leib-Seele-Problem heraufbeschwören. Und es wird kaum jemanden überraschen, wenn schon zu Descartes' Lebzeiten viele Varianten seiner Werkinterpretation die Diskussion bereicherten. So muss Descartes bereits 1641 das noch häufig wiederholte Missverständnis des Theologen Arnauld korrigieren, der den cartesianischen Leib-Seele-Dualismus als Wiedereinführung des platonischen Seelenverständnisses interpretieren wollte. ${ }^{103}$ Descartes hat unterdessen selbst diese platonische Deutung provoziert, indem er in der sechsten Meditation seiner Gewissheit darüber Ausdruck verlieh, dass er nur als »denkendes Ding " wahrhaft vom Körper verschieden sei und auch ohne ihn existieren könne. ${ }^{104}$ Dem cartesianischen Menschenbild liegt jedoch die alles bestimmende Auffassung zugrunde, dass im Menschen die von ihrer Natur her differenten, in sich vollständigen Substanzen der res cogitans - die immaterielle, vernunftbegabte Seele - und der res extensa - der räumliche, materielle Körper - aufs engste in einer Einheit miteinander verbunden seien. Damit repräsentiert der Körper nicht bloß das »Fahrzeug« der

${ }^{101}$ Descartes erwähnt mehrfach die Absicht, analog zu der Beschreibung der körperlichen Funktionen eine Schilderung der seelischen Vorgänge anzuschließen. Vermutlich weil er die Notwendigkeit sah, zunächst seine Metaphysik zu begründen, wurde der Plan verschoben und blieb dann unausgeführt. Vgl. Descartes 1969, 43 (FN 1), 133 (FN 1).

102 Descartes 1972, 74. »Nempe imprimis hîc adverto magnam esse differentiam inter mentem \& corpus, in eo quòd corpus ex natura suâ sit semper divisible, mens autem plane indivisibilis ... quod unum sufficeret ad me docendum, mentem a corpore omnino esse diversam ... « Descartes 1967b, 232.

${ }^{103}$ Vgl. Quatrièmes Objections faites par Monsieur Arnauld, Docteur en Théologie. Descartes $1967 \mathrm{~b}, 640$.

${ }^{104}$ Vgl. a.a.O., 488. 
Seele. ${ }^{105}$ Der Descartes-Kenner Wolfgang Röd meint abschließend zum cartesianischen Körper-Seele Problem:

Nur innerhalb der Metaphysik bezeichnet »Ich« ausschließlich das denkende Subjekt der Erkenntnistheorie, dem der Körper als bloße »Gliedermaschine«, somit als Teil der Außenwelt, gegenübersteht, während dieser Ausdruck im Leben die konkrete Person bedeutet, zu der die Körperlichkeit wesentlich gehört. ${ }^{106}$

Es kann an dieser Stelle weder darum gehen, die vermeintlich »richtige« Interpretation des Leib-Seele-Problems im philosophischen Werk Descartes' zu rekonstruieren, noch darum, die äußerst komplexe und für den philosophischen Laien kaum überschaubare Rezeptionsgeschichte auch nur annähernd nachzuzeichnen. Es kann hier einzig um die hermeneutisch motivierte Frage gehen, welche Bedeutung Descartes für die Frage der Unsterblichkeit im Posthumanismus hat.

Gerade in der Kritik des Philosophen Ludwig Wittgenstein an Descartes gewinnt die posthumanistische Rezeption von Descartes' Körper-Geist-Modell noch schärfere Konturen. Wittgenstein hat in seinen posthum veröffentlichten Überlegungen Über Gewißheit die immanenten Irrtümer der cartesianischen Erkenntnistheorie zu Tage gefördert, indem er konstatierte, dass sich die Begriffe »Wissen « und »Zweifel « stets in einem System befinden müssen und voraussetzen, dass eine Prüfung des zu gewinnenden Wissens möglich ist. Ferner ist damit die Annahme impliziert, dass die Verfahren zur Gewinnung einer Erkenntnis von dem Verfahren zur sicheren Prüfung dieser Erkenntnis getrennt sind. ${ }^{107}$ Wodurch wolle Descartes garantieren, dass die prüfende Instanz, also der Geist, dem radikalen Erkenntniszweifel standhalten kann? Die prüfende Instanz sei letztlich nicht sicherer als das zu prüfende Wissen. Anders als Descartes führt Wittgenstein den Beginn des Denkens auf die Setzung von Gewissheiten zurück, die erst den Rahmen für den Zweifel manifestieren. Diese Gewissheiten sind in der materiellen Form unseres Körpers verankert: als Gewissheit über die Hände, Finger an jeder Hand, Füße, den Mund usw. Dadurch liegen die mit Hilfe unseres Körpers gewonnenen Gewissheiten tiefer als die Gewissheiten unseres Weltbildes.

Wittgenstein und der Entwicklungspsychologe Jean Piaget stimmen darin überein, dass die Gewissheit des Körpers die menschliche Erkennt-

${ }^{105}$ Vgl. a.a.O., 228; Gouhier 1962, 353-394; Röd 1995, 136-140.

${ }^{106}$ Vgl. Röd 1995, 144.

${ }^{107}$ Vgl. Gebauer 1984, 237f.; Wittgenstein 1969, § 24. 
nisbedingung fundiert. ${ }^{108}$ Die Kritik Wittgensteins veranschaulicht den posthumanistischen Standpunkt umso deutlicher, als der Posthumanismus mit Bezug auf Descartes die Rolle des Körpers bei der Gewinnung von sicherer Erkenntnis und der Genese von Identität negiert. Hier unterscheiden sich die Ansätze der Posthumanisten auch klar von der so genannten embodied cognition in der KI-Forschung, die die Körperlichkeit als Grundvoraussetzung von Denken und Bewusstsein erachtet. ${ }^{109}$

Bei keinem Vertreter des Posthumanismus kommt den Bezügen auf die Philosophie Descartes' eine so hohe Bedeutung zu wie bei dem mit philosophischen Exkursen ansonsten sehr sparsamen Autor Hans Moravec. Cogito, ergo sum! wird zum Leitmotiv von Moravecs zweiter Monographie Robot. Mere Machines to Transcendent Mind, indem Rodins berühmte Denkerplastik und als ihr Spiegel ein denkender Roboter das Titelbild der amerikanischen Ausgabe zieren. Ungeachtet der Tatsache, dass der cartesianische Existenzbeweis ad absurdum geführt werden würde, dokumentiert Moravec nun die ausschließliche Fähigkeit zum Denken als Existenzbeweis mit einem im Computer simulierten Descartes. Hierdurch sollte gleichzeitig die Existenz von künstlichen Intelligenzen oder simulierten Menschen in der Virtualität bewiesen werden. In der perfekt simulierten Welt im Innern eines Computers, einer Emulation, hätte die Simulation des denkenden Subjektes keine Prüfungsmöglichkeit über ihren ontologischen Status - weder in körperlicher noch in geistiger Hinsicht: Alle Wahrnehmungen der Umwelt und des Selbst würden also ebenso real wie in der Realität erscheinen. Die Ermessensgrundlage bilden für Moravec nicht die philosophischen Kriterien von Schein und Wahrheit, sondern ganz pragmatisch steht die Gewährleistung der Kontinuität des denkenden Subjektes durch die technische Simulation aller für das menschliche Denken notwendigen Voraussetzungen im Vordergrund. Das schließt die Simulation der mentalen Funktionen ebenso wie die Simulation der Sinneseindrücke ein. Wenn die Simulation nicht als solche entlarvt werden kann, ist sie real - das Wahrheitskriterium bleibt dabei unberücksichtigt. Entscheidend ist lediglich, dass mit der Fortführung des Denkens die persönliche Existenz gesichert wird. ${ }^{110}$

\footnotetext{
${ }^{108}$ Vgl. Gebauer 1984, 235-243; Wittgenstein 1969, §55f., 496f., 579; Piaget 1969, 34-45. ${ }^{109}$ Vgl. Schöner 2014.

${ }^{110}$ Vgl. Moravec 1988, 180. Mit der Simulation eines Descartes in einem Computer illustriert Moravec eindrücklich die ihm unbekannte Kritik Wittgensteins am cartesianischen Existenzbeweis.
} 
A simulated world hosting a simulated person can be a closed self-contained entity ... The inhabitant might, by patient experimentation and interference, deduce some representations of the simulation laws, but not the nature or even existence of the simulating computer. ${ }^{111}$

Entsprechend knüpft Ray Kurzweil an Descartes' Existenzbeweis an, wenn er das Vorhandensein von menschenähnlicher Intelligenz im Computer mit dem Entstehen eines Bewusstseins verbindet: ${ }^{112}$

Before 2030, we will have machines proclaiming Descartes's dictum [I Think, Therefore I Am: O.K.] ... The machines will convince us that they are conscious, that they have their own agenda worthy of our respect. We will come to believe that they are conscious much as we believe that of each other. ${ }^{113}$

Moravec präsentiert Descartes darüber hinaus als einen Vordenker der Idee einer virtuellen Realität, da dieser schon im 17. Jahrhundert die Möglichkeit in Betracht gezogen habe, dass unsere Wirklichkeit die Illusion eines bösen Dämonen sei, der all unsere körperlichen Sinne (sehen, hören, riechen, schmecken, fühlen) kontrolliere, während nun im 21. Jahrhundert die Wissenschaft selbst die illusorischen Möglichkeiten der Virtualitätstechniken hervorbringe. ${ }^{114}$

Wenn Marvin Minsky nun den Körper als Besitz und Werkzeug des denkenden Geistes charakterisiert, der eben nur durch diesen Körper und nie direkt im Kontakt zur Welt stehe, spiegelt er desgleichen das Menschenbild der cartesianischen Erkenntnistheorie wider. ${ }^{115}$ N. Katherine Hayles verweist in diesem Zusammenhang auf die liberal-humanistischen Implikationen des körperlichen Besitzdenkens im Posthumanismus. ${ }^{116}$

${ }^{111}$ Moravec 1999, 192.

112 Vgl. Kurzweil 1999, 60; Kurzweil 2005, 173. Obwohl Kurzweil nur oberflächlich eine Descartes-Einführung von Jonathan Rée (Descartes, New York 1974) und einen Lexikonartikel (Dictionary of Scientific Biography) rezipiert, erachtet er Descartes' Werk für wichtig genug, um auch drei der Originalwerke bibliographisch aufzuführen. Vgl. Kurzweil 1999, 325, 349.

113 A.a.O., 60, 63.

114 Vgl. Moravec 1999, 191. Dass die Idee eines Betrüger-Gottes nur eine methodische Hypothese der ersten Meditation, die Descartes überdies später wieder aufhebt, darstellt, ignoriert Moravec. Vgl. Descartes 1967b, 181.

${ }^{115}$ Vgl. Minsky 1988, 110, 286. Frank Tipler knüpft nur indirekt über den Philosophen George Berkeley an Descartes an, dessen teils vom Cartesianismus beeinflusste Erkenntnistheorie die Grundlagen des anthropischen Prinzips bildet. Vgl. Tipler 1995, $211 \mathrm{f}$.

${ }^{116}$ Vgl. Hayles 1999, 4. 
Offenbar steht darüber hinaus bei Moravec auch die Verwendung der so genannten Vielwelteninterpretation der Quantenmechanik im Zusammenhang mit der von Descartes' und Lockes Empirismus maßgeblich beeinflussten Erkenntnistheorie George Berkeleys. Dieser hatte 1710 in seinen Principles of Human Knowledge ergründet, dass kein Objekt unserer Erkenntnis außerhalb unseres Geistes existieren kann, da Materie und äußerliche Dinge nur durch unsere bewusste Wahrnehmung zur Existenz gelangen. Dieser existentiell bedeutsame Vorrang des menschlichen Geistes vor der materiellen Welt findet sich in Frank Tiplers anthropozentrischer Interpretation der Rolle intelligenter Beobachter im Universum wie auch in Moravecs solipsistischer Auslegung der Vielweltentheorie. ${ }^{117}$ Auch in der Philosophie der so genannten Physikotheologen des 19. Jahrhunderts, in deren Tradition sich Tipler ausdrücklich stellt, wird das Verhältnis zwischen Körper und Geist eindeutig als "superiority of the soul to the body, of the rational to the animal part of our constitution « bestimmt. ${ }^{118}$

Unter Berücksichtigung der posthumanistischen Rezeptionsleistung lässt sich abschließend feststellen, dass Descartes' Verhältnisbestimmung von Körper und Geist in dreierlei Hinsicht für den Posthumanismus eine gewisse Relevanz erlangt hat. Alle Posthumanisten rechtfertigen dabei mit Verweis auf Descartes die Annahme, dass das denkende Subjekt allein den Status seiner Existenz und die individuelle Identität determiniert. Daraus ergeben sich folgende drei Konsequenzen:

1. Der konkrete Körper hat keine Bedeutung für die Existenz eines Wesens, da nur das vernunftbegabt denkende und mit Gewissheit existierende Subjekt - also der im Gehirn lokalisierte menschliche Geist (mind) - die individuelle Persönlichkeit eines Menschen hervorbringt. Als austauschbares »Werkzeug" des Geistes bildet der »Datenträger Körper« eine für die menschliche Existenz zu vernachlässigende Größe.

2. Da der menschliche Geist auch ohne den Körper existieren kann, ist die Weiterexistenz des Menschen als ein nur »denkendes Ding « (res cogitans) im Computer auf ewige Zeiten gewährleistet. Die Unsterblichkeit der Person ist damit gleichbedeutend mit der Kontinuität der Denkprozesse.

3. Wenn ein künstlich intelligentes Computerprogramm sich selbst als existent bezeichnet, so kann diese Aussage von keinem Außenstehen-

${ }^{117}$ Vgl. Moravec 1988, 153f. u. 187f.; Moravec 1999, 205ff.; Barrow \& Tipler 1986, $21 \mathrm{ff}$. ${ }^{118}$ Paley 1842, 6. 
den bestritten werden und das Programm kann wie alle intelligenten Lebewesen seine Existenzrechte einfordern. Nur das Bewusstsein der intelligenten Maschine kann den Status seiner Existenz bestimmen.

Diese großenteils mit Descartes begründeten Bestandteile der posthumanistischen Definition des Menschen werfen unmittelbar die Frage auf, inwieweit diesem Menschenbild eine dualistische oder eine monistische Auffassung zugrunde liegt. Alle Posthumanisten gehen davon aus, dass der Mensch nicht mehr als eine äußerst komplexe Anordnung von Materie ist, deren Funktionen letztlich vollkommen auf physikalische Gesetze zurückzuführen sind. Der Posthumanismus ist damit eine materialistische Philosophie.

In einem zweiten Schritt aber werden die mentalen Funktionen des Körpers - und allein diese - als das Wesen des Menschen bestimmt. Der menschliche Geist als Summe und Kontinuität dieser Funktionen wird mit der individuellen Persönlichkeit gleichgesetzt, indem der Geist oder bisweilen auch die menschliche Seele als Computerprogramm bezeichnet werden, die auf einem Computer namens Gehirn ablaufen (Tipler). Marvin Minskys voll und ganz auf den menschlichen Geist (mind) ausgerichtetes Konzept muss wohl als maßgeblich für Tipler, Moravec und Kurzweil angenommen werden. Die für die menschliche Persönlichkeit entscheidende Software des Geistes wird auf diese Weise als eine von ihrer spezifischen Hardware bzw. der so genannten Wetware verschiedene Einheit etabliert, deren Leistungsfähigkeit - wie Max More hervorhebt - nicht auf die konkrete Form und Struktur des Körpers angewiesen ist. Ausschlaggebend für die Kontinuität der geistigen Prozesse und der gleichbedeutenden Kontinuität einer menschlichen Persönlichkeit ist die Fortsetzung der operativen Funktionen des Gehirns, das auf diese Weise mit jedem anderen »Datenträger« beliebig austauschbar wird, der die mentalen Funktionen simulieren könnte. Die Unsterblichkeit des Menschen ist daher als Unsterblichkeit des Geistes determiniert.

Sofern ein materialistischer Monismus die Dualismen von Form und Inhalt, Geist und Natur, Materie und Struktur ablehnt, wie es August Schleicher formulierte, liegt die Schlussfolgerung nahe, dass der Posthumanismus durch den von ihm propagierten Vorrang des Geistes über den zu vernachlässigenden Körper eine dualistische Auffassung vertritt. ${ }^{119}$ Dem materiellen Körper werden die allein als bedeutend erachteten, geistigen Funktionen des Körpers gegenübergestellt. Das dieser Betrachtungswei-

${ }^{119}$ Vgl. Mehlhausen \& Dunkel 1994, 213. 
se zugrunde liegende funktionalistische Paradigma ist damit gänzlich auf die geistige Funktion der Verarbeitung von Informationen ausgerichtet: Der Mensch wird als informationsverarbeitende Maschine definiert. Das spezifische »Programm« der informationsverarbeitenden Prozesse, der individuelle Geist, kennzeichnet als körperunabhängige Einheit die dualistische Deutung des Menschen in der posthumanistischen Philosophie. ${ }^{120}$ Im Kontext verschiedener Fragestellungen werden in der posthumanistischen Philosophie teils materialistisch-monistische Aspekte und teils dualistisch-funktionalistische Aspekte betont.

Mark Seltzer entdeckte in seiner Analyse sozialer Auswirkungen des Naturalismus im späten 19. Jahrhundert ein ähnliches Phänomen, wie es uns heute im Posthumanismus begegnet. Er machte die zunächst widersprüchlich erscheinende Entdeckung, dass der Naturalismus einerseits die materialistische oder physikalische Bedingtheit von Personen, Repräsentationen und Handlungen hervorhebt. Andererseits konnte er feststellen, dass der Naturalismus diesen Standpunkt gerade mit dem Verweis auf die Abstraktionen von Personen, Körpern und Handlungen in immateriellen Zahlen, Zeichen, Modellen und Diagrammen legitimiert. Diese Ideologie nennt Seltzer einen dematerialisierten Materialismus. ${ }^{121}$

Wenn man bisweilen die philosophische Konsistenz in der posthumanistischen Auseinandersetzung um Dualismus und Monismus des Menschenbildes vermissen mag, so muss betont werden, dass die entsprechenden Autoren nicht vordringlich als Philosophen, sondern als Pragmatiker, als Informationstechniker und Robotiker argumentieren. Das heißt, einerseits wird der Mensch gänzlich als materielle Maschine definiert, die somit prinzipiell computertechnisch simuliert werden könne. Andererseits wird die menschliche Persönlichkeit auf den menschlichen Geist - also die Funktionen des Gehirns - reduziert, da die Simulation in der Virtualität die Loslösung des für Alter und Tod verantwortlichen, materiellen Körpers einschließen solle. Seit Aristoteles bildet das Verhältnis zwischen Form und Stoff, zwischen Geist und Materie ein Hauptkapitel der abendländischen Metaphysik. In einer um Schärfe bemühten Formulierung des Berliner Soziologen Dietmar Kamper entspricht der Geist der Seite der Form und die Materie der des Stoffes, wobei man dem ersteren eine aktive, der letzteren eine passive

${ }^{120}$ Lediglich Max More warnt vor einem »falschen Dualismus«. Vgl. More 1995a, 91-95. ${ }^{121}$ Vgl. Seltzer 1994, 14. 
Rolle zuschreibt: Der Geist sei klug - die Materie dumm. Die Form, der Geist, erst befreie die Materie aus dem Chaos und führe sie in Stufen zu den Gipfeln, an denen der jeweilige Denker gerade stehe. Die Menschen seien daher heute von der Idee der reinen Form eines selbstbezüglichen Geistes so sehr fasziniert, dass sie ohne Stofflichkeit und Materialität auskommen wollten. ${ }^{122}$

\subsubsection{Der Mensch im kybernetischen Paradigma}

Es ist offensichtlich, dass posthumanistische Denker die Werke ihrer philosophischen Vorläufer durch eine besondere »Brille« rezipieren. Über diesen produktiven Rezeptionsakt gelingt es beispielsweise Frank Tipler, einen physikalischen und materialistischen Reduktionismus mit den untereinander divergierenden Philosophien eines Platon, Aristoteles, Augustinus und Thomas von Aquin zu verbinden. Hans Moravec vermag es, einerseits Descartes materialistisch und monistisch zu deuten und andererseits die in Descartes' Existenzbeweis als Dualismus angelegte, absolute Dominanz des Geistes über den Körper für seine posthumanistische Argumentation zu verwerten. Die Konsistenz dieser Unternehmungen aus einer philosophischen Perspektive zu beurteilen, liegt außerhalb meines Unterfangens. Unter der von mir verfolgten, rezeptionsgeschichtlichen Fragestellung gilt es, die spezifischen Selektions- und Interpretationsmuster der Posthumanisten herauszuarbeiten, die zu den in den beiden vorhergehenden Kapiteln aufgeführten Konkretisationen von insbesondere Descartes' Werk und der materialistischen Philosophie geführt haben. Was kennzeichnet also das Paradigma der Posthumanisten, das die Voraussetzung für diese Interpretationsleistung schafft? Im Folgenden werde ich erörtern, welchen Anteil die Grundlagen der kybernetischen Wissenschaft an der Genese eines noch genauer zu bestimmenden, kybernetischen Paradigmas hatten, das signifikant für die posthumanistische Philosophie werden sollte. Im Mittelpunkt dieser Betrachtung stehen hier drei miteinander korrespondierende Aspekte der Kybernetik: nämlich erstens die Bestimmung des organischen Nervensystems als informationsverarbeitende Maschine, zweitens die Gleichstellung einer künstlich intelligenten Maschine mit dem Menschen und abschließend die Deutung des Verhältnisses zwischen Information und

${ }^{122}$ V gl. Kamper 1999, 110ff.; Lavery 1992, $71 \mathrm{f}$. 
Materie bzw. Körper, die im posthumanistischen Diskurs einen so gewichtigen Einfluss auf das Verständnis des menschlichen Geistes haben sollte. Es wird sich hierbei zeigen, dass die Positionen der führenden Kybernetiker sich merklich von denen der Posthumanisten abheben, aber immerhin ein spezifisch kybernetisches Interpretationsmuster zur Verfügung stellen, das die Posthumanisten auf die Unsterblichkeitsfrage hin anwenden konnten.

An der Begründung der kybernetischen Wissenschaft mit ihrem interdisziplinär angelegten Forschungsprogramm waren seit den vierziger Jahren des 20. Jahrhunderts eine ganze Reihe namhafter Mathematiker, Biologen, Mediziner und Ingenieure involviert, unter denen Alan Turing, John von Neumann, Claude Shannon und vor allem Norbert Wiener aufgrund ihrer Pionierleistungen besondere Bekanntheit erlangten. Der Mathematiker Wiener lehrte seit 1919 am Massachusetts Institute of Technology (MIT) und definierte die Aufgaben der Kybernetik wie folgt:

Dieses Studium von Nachrichten und insbesondere von Regelungsnachrichten ist der Gegenstand einer Wissenschaft, für die ich in einem früheren Buche den Namen Kybernetik eingeführt habe. Der Name bedeutet die Kunst des Piloten oder Steuermanns, griechisch $\kappa \cup \beta \varepsilon \rho \nu \eta \tau \eta \sigma$, lateinisch gubernator; der Stamm dieser Wörter findet sich auch im englischen governor, das in der Ingenieurwissenschaft den Regler einer Maschine bezeichnet. ${ }^{123}$

Allgemein befasst sich die Kybernetik mit abstrakten Systemen, die als theoretische Analogiemodelle bestimmte Bereiche der Wirklichkeit simulieren und somit zu mathematisch-berechenbaren Lösungen von speziellen Problemen beitragen können. Im Mittelpunkt der Kybernetik steht die Frage, wie Information von einem System zum anderen weitergegeben, wie sie aufgenommen und gespeichert wird und welche Aktionen von Maschinen sich damit steuern lassen.

Zusammen mit Oskar Morgenstern entwickelte John von Neumann die kybernetische Spieltheorie, die als mathematische Methode zur Auswahl optimaler Verhaltensweisen aus der Menge aller möglichen Verhaltensweisen in Konfliktsituationen geschaffen wurde. ${ }^{124}$ Während und nach dem 2. Weltkrieg gewann die Spieltheorie eine besondere Bedeutung innerhalb der militärischen Strategieplanung sowie in der Programmierung der ersten elektronischen Großrechner. Der ENIAC (Electronic

\footnotetext{
${ }^{123}$ Wiener 1964, 20. Vgl. Wiener 1963.

${ }^{124}$ Vgl. Neumann \& Morgenstern 1966; Klaus 1969b, 598-600; Heims 1981, 83-93, 294 299.
} 
Numerical Integrator and Computer), der 1943-1946 an der University of Pennsylvania unter Mitarbeit von John von Neumann gebaut wurde, sollte Flugbahnen von Raketen und anderen ballistischen Körpern berechnen. ${ }^{25}$

Wiener und von Neumann waren also bemüht, eine Methode zu finden, um Vorgänge unserer Realität in einem abstrakten Modell der Realität, einem Programm, zu simulieren. Immer mehr Abläufe in unserer realen Lebenswelt mussten dann symbolisch durch Algorithmen als informationsverarbeitendes System im Computer modelliert werden. Das reale System eines Wirtschaftsunternehmens, eines Staates oder einer Rakete wurde in einem Computerprogramm nachgeahmt, so dass man beispielsweise verschiedene Szenarien eines Atomkrieges durchspielen lassen und die politische und militärische Strategie nach den berechneten Ergebnissen ausrichten konnte. ${ }^{126}$ Nach dem Willen ihrer Begründer sollte sich die Kybernetik als universale Wissenschaft von der Art der Welterkenntnis etablieren. In ihren ersten beiden Jahrzehnten gewann die kybernetische Theorie einen spürbaren Einfluss auf die neu entstandene kybernetische Psychologie, Soziologie und Pädagogik. ${ }^{127}$

1943 veröffentlichten Norbert Wiener, Julian Bigelow und Arturo Rosenblueth das so genannte Cybernetic Manifesto unter dem Titel Behaviour, Purpose and Teleology. Es folgte eine Verteidigung ihrer Thesen (Purposeful and Non-Purposeful Behaviour), in denen unter behavioristischen Vorzeichen die Äquivalenz zwischen biologischer und maschineller Verhaltenssteuerung behauptet wurde. Wiener setzte damit erstmals die Mechanismen von Kontrolle, Information und Kommunikation von organischen Lebewesen und Maschinen gleich. ${ }^{128}$ Die Autoren führen die Kategorie des zweckgerichteten (purposeful) Verhaltens in die kybernetische Debatte ein und begründen die Etablierung dieses umstrittenen teleologischen Handlungsmodells mit dem zu erwartenden Erkenntnisgewinn beim Verstehen maschineller Steuerungsprozesse. Wiener, Bigelow und Rosenblueth propagieren das Konzept des zweckgerichteten Verhaltens von Mensch und Maschine, das die Abhängigkeit von einem mit der Außenwelt des Systems verbundenen Ziel beinhaltet, ausdrücklich als methodisches Konzept und nicht als ontologische Aussage

${ }^{125}$ Vgl. a.a.O., 365 .

${ }^{126}$ Vgl. Anders 1983, 59-64; Weizenbaum 1980, 316ff.

${ }^{127}$ Vgl. Hayles 1999, 96ff.; Klaus 1969a, 324-337. Zur Geschichte der Kybernetik vgl. Wiener 1963, 25-62.

${ }^{128}$ Vgl. Wiener 1985, 187-195. 
über das Wesen des Menschen. ${ }^{129}$ Die humanwissenschaftlich geprägten Begriffe »Zweck" (purpose) und »Ziel« (goal, teleology) des Handelns verwenden die Verfasser des Cybernetic Manifesto unter der Annahme der Äquivalenz menschlichen und maschinellen Verhaltens:

We believe, that men and other animals are like machines from the scientific standpoint because we believe that the only fruitful methods for the study of human and animal behaviour are the methods applicable to the behaviour of mechanical objects as well. Thus, our main reason for selecting the terms in question was to emphasize that, as objects of scientific inquiry, humans do not differ from machines. ${ }^{130}$

Aus der wissenschaftlich dienlichen Gleichsetzung von Mensch und Maschine unter einem kybernetischen Paradigma leitet Wiener die Entstehung der zwei Teilbereiche der Biokybernetik ab: die Neurokybernetik und die medizinische Kybernetik. ${ }^{131}$

Bei einem Treffen mit dem Mathematiker Walter Pitts, der die mathematischen Eigenschaften neuronaler Netze analysierte, bezeichnete Wiener die Funktionen der modernen Vakuumröhren als operative Äquivalente zur organischen Neuronenentladung, die sich völlig analog zur Auswahl einer binären Ziffer - also Entladung oder nicht - gestalten würde. Die ultraschnelle Rechenmaschine müsste aus diesem Blickwinkel ein beinahe ideales Modell der sich aus dem Nervensystem ergebenen Prozesse darstellen. Aber nicht nur weil die organischen Nervenzellen dem binären »Alles-oder-Nichts«-Prinzip des Computers entsprechen würden, sondern auch weiterreichende Analogien zwischen dem menschlichen Gedächtnis und dem einer Rechenmaschine, veranlassen Wiener zu der Folgerung, dass das Gehirn wie eine logische Maschine arbeite: ${ }^{132}$

Wir beginnen einzusehen, daß solche wichtigen Elemente wie die Neuronen, die Atome des Nervenkomplexes unseres Körpers, ihre Arbeit unter fast den gleichen Bedingungen wie Vakuumröhren verrichten, mit ihrer relativ kleinen Energie ... und daß die Bilanz, die sehr wesentlich ist, keine Energiebilanz ist. Kurz, die neuere Untersuchung der Automaten, ob aus Metall oder aus Fleisch, ist ein Zweig der Nachrichtentechnik, und ihre Hauptbegriffe sind jene der Nachricht,

\footnotetext{
${ }^{129}$ Vgl. a.a.O., $193 \mathrm{f}$.

${ }^{130}$ A.a.O., 195. Hervorhebungen von O. K.

${ }^{131}$ Norbert Wiener: »Introduction to Neurocybernetics.« Vgl. a.a.O., 400-406.

${ }^{132}$ Vgl. Wiener 1963, $42 \mathrm{f}$.
} 
Betrag der Störung oder »Rauschen« ... Größe der Information, Kodierverfahren und so fort. ${ }^{133}$

Bedeutungsvoll für die von Wiener und der frühen Kybernetik vorgenommene Nebeneinanderstellung von menschlichem und maschinellem Denken war das neuronale Modell, das Warren McCulloch, der langjährige Leiter der wichtigen Macy Conferences, entwarf. Dieses mathematische Modell des Gehirns zeigte letztendlich, »that brains do not secrete thought as the liver secretes bile but ... they compute thought the way electronic computers calculate numbers. ${ }^{134}$ Die Neuronen des menschlichen Gehirns illustrierte McCulloch entsprechend dem Schema elektronischer Schaltungen. Damit sollte bewiesen werden, dass Menschen und Maschinen nicht nur denselben universellen physikalischen Gesetzen unterworfen waren, sondern auch dass ihre Denkprozesse nach den gleichen Mechanismen ablaufen. ${ }^{135}$

Zur selben Zeit entwarf John von Neumann eine allgemeine und systematische Theorie der Automaten, die aus heuristischen Gründen auf den Analogien zwischen organischen Lebewesen und Computern aufbaute und die er als künstliche Automaten (Computer, Radiosysteme) bzw. natürliche Automaten (Nervensystem, selbstreproduzierende Systeme) kategorisiert. Seine Theory of Self-Reproduction Automata greift die Idee einer unbeschränkten Reproduktion eines Musters durch eine Maschine auf und entwirft das theoretische Konzept eines organischen oder maschinellen Automaten, der alles berechnen, alles konstruieren und sich selbst reproduzieren kann. ${ }^{136}$ Auch Wiener nennt neben der Lernfähigkeit die Möglichkeit der eigenen Reproduktion als kennzeichnend für lebende Systeme, was von Frank Tipler dekontextualisiert zum entscheidenden Merkmal des Lebens erhoben wurde: auf diese Weise können selbst Autos als lebendig erachtet werden. ${ }^{137}$

Liegt von Neumanns Bedeutung für die Kybernetik vordringlich in seinem Vergleich von organischen und maschinellen Steuerungsmechanismen, so kommt er dennoch zu dem Schluss, dass die Zurückführung der cerebralen Verarbeitung von Informationen auf mathematische Abstraktionen keine absolute und wahre Beschreibung der hinter der

${ }^{133}$ Wiener 1963, 78f.

${ }^{134}$ Warren McCulloch: The Beginning of Cybernetics. McCulloch Papers, B/M139. Zitiert nach Hayles 1999, 58.

${ }^{135}$ Vgl. Hayles 1999, 58-61; Hagner 2004, 288-296.

${ }^{136}$ Vgl. Neumann 1966, 251-296.

${ }^{137}$ Vgl. Wiener 1963, 241-256; Barrow \& Tipler 1986, 511-523; Tipler 1995, 44-55. 
Wirklichkeit stehenden Form ist: vielmehr ist auch die mathematische Sprache wie jede andere natürliche Sprache Ergebnis historischer und kultureller Prozesse. ${ }^{138}$

Im Vertrauen auf die intellektuellen, sozialen und emotionalen Qualitäten des Menschen wendet sich Norbert Wiener von seinem entschieden humanistischen Standpunkt aus gegen Überlegungen, die Mensch und Maschine als solche direkt auf eine Stufe stellen und den Menschen durch seine mangelnde Effizienz gegenüber den Maschinen als obsolet und ersetzbar herabwürdigen:

Those who value man not in his own right but merely as an instrument for production see eye to eye with the slave merchant ... This evaluation of man is Fascism ... Those who devaluate man in the presence of the superior efficiency of the machine, are simply those who have devaluated man in their own hearts, and their works speak for them ... If we rate a man only as a factory hand, for the cost of his daily wages, then the machine will indeed displace man over much of his range of activity. But in essence, the factory hand is first a human being, and only secondarily a factory hand. ${ }^{139}$

John von Neumann und erst recht den vor der Macht der Maschinen warnenden, späteren Werken Norbert Wieners kann nicht vorgehalten werden, dass sie den Menschen grundsätzlich als Maschine betrachteten. ${ }^{140}$ Aber selbst der immanent wissenschaftliche Vergleich zwischen Mensch und Maschine wird - sobald die Thematik im Alltagsdiskurs steht - schon allein durch die sprachliche Nivellierung den Menschen und die Maschine als ein im außerwissenschaftlichen und allgemeineren Sinne wesentlich gleiches System zur Verarbeitung von Informationen konstruieren. $\mathrm{Ob}$ nun Analogien oder Differenzen festgestellt werden - wenn Menschen und Maschinen im selben Bezugssystem miteinander verglichen werden, wird mit der prinzipiellen Vergleichbarkeit gewöhnlich eine prinzipielle Wesensgleichheit impliziert. So illustriert etwa Wiener elementare Unterschiede des Wahrnehmens durch den Vergleich zwischen dem menschlichen Auge und einem Fernsehempfänger und begrüßt dabei überhaupt die Möglichkeit, die Mechanismen der Automaten aufgrund ihrer organischen Äquivalenz mit Begrif-

\footnotetext{
${ }^{138}$ Vgl. Neumann 1991, 76 f.

${ }^{139}$ Norbert Wiener: »The Future of Automatic Machinery.« Wiener 1985, 132. Vgl. auch Roszak 1994, 9ff.

${ }^{140}$ Vgl. Wiener 1963, 59-62.
} 
fen der menschlichen Physiologie belegen zu können. So redet er von den Sinnesorganen und dem Nervensystem eines Automaten. ${ }^{141}$

Um die Verschiedenheit zwischen den »natürlichen und künstlichen Automaten« zu akzentuieren, weisen Wiener und von Neumann mehrfach darauf hin, dass das menschliche Nervensystem ungleich komplexer ist als die entsprechenden Steuerungsmechanismen der Maschinen: »Thus the machine, for all the similarities that its functioning shows with that of the human organism, is at a much lower level of organization and complexity ... «. ${ }^{142}$ Damit aber sind die Kybernetiker schon in der Falle des Vergleiches gefangen, die mit der steigenden Komplexität maschineller Informationsverarbeitung zuschnappt. Noch deutlicher wird dieser Zusammenhang, wenn Wiener selbst den Begriff des Transzendierens menschlichen Verhaltens durch die differierenden Qualitäten der Maschinen einbringt: »Examples, however, are readily found of man-made machines with behaviour that transcends human behaviour. ${ }^{143}$ Indem Wiener anschließend auf die nur maschinellen Möglichkeiten, elektrischen Strom oder Radiowellen auszusenden, verweist, hat er bereits einen Vergleich zwischen Mensch und Maschine durchgeführt und im selben Augenblick das technische Potential der Transzendierung des Menschen durch die Maschine offenbart, was sich in den entsprechenden Metaphern bei Hans Moravec und Ray Kurzweil widerspiegeln wird. ${ }^{144}$ Dass just die Idee der künstlichen Intelligenz, das heißt der komplexesten informationsverarbeitenden Systeme, eine zentrale Rolle bei der Gleichsetzung von Mensch und Maschine spielt, scheint nach den bisherigen Überlegungen evident zu sein:

In the same way, whether they are understood as like or unlike, ranging human intelligence alongside an intelligent machine puts the two into a relay system that constitutes the human as a special kind of information machine and the information machine as a special kind of a human. ${ }^{145}$

Schon lange bevor der am MIT tätige Kybernetiker John McCarthy 1960 den Begriff von der künstlichen Intelligenz (artificial intelligence) prägte, beschäftigte sich der englische Computerpionier Alan Turing

\footnotetext{
${ }^{141}$ Vgl. Wiener 1985, 185; Wiener 1963, 80.

142 Wiener 1985, 665. Vgl. Neumann 1966, 42-56.

${ }^{143}$ Wiener $1985,184$.

${ }^{144}$ Vgl. a.a.O., 180-186.Vgl. Hayles 1999, 94 f.

145 A.a.O., $64 f$.
} 
mit der Frage: Kann eine Maschine denken? ${ }^{146}$ Wurden die Computerriesen ENIAC und sein britisches Pendant Colossus während des zweiten Weltkrieges für die Dechiffrierung von verschlüsselten Nachrichten und Berechnungen zum Bau der Atombombe benötigt, so entwickelten Alan Turing und John von Neumann in der Folgezeit die ersten Programme, die Probleme selbstständig durchdenken und zu Lösungen gelangen konnten. 1950 konzipierten Turing und einige andere Forscher Programme für logisches Denken und intellektuelle Spiele wie Dame und Schach, die heute bekanntlich schon so ausgefeilt und mit so gewaltigen Rechnerkapazitäten verbunden sind, dass Schachweltmeister die besten Programme nicht mehr schlagen können. ${ }^{147}$

Alan Turings wohl bedeutendster Beitrag für die Verbreitung der Idee von künstlicher Intelligenz war 1950 sein Aufsatz Computing Machinery and Intelligence, der das von allen Posthumanisten stets referierte und später als Turing-Test bekannte Imitationsspiel entfaltete. ${ }^{148}$ In den Darstellungen des Turing-Testes wird allerdings häufig vernachlässigt, dass Turing in seinem Experiment zunächst vorschlägt, einen Mann (A), eine Frau (B) und einen dritten Spieler in getrennten Räumen unterzubringen und sie nur durch einen Fernschreiber miteinander kommunizieren zu lassen. Die Aufgabe des drittens Spieler bestehe nun darin, anhand von Fragen zu erkennen, welcher der beiden Spieler A und B nun weiblich und welcher männlich sei. Mit diesem Spiel hatte Turing aber mehr im Sinn:

>What will happen when a machine takes the part of A in this game?< Will the interrogator decide wrongly as often when the game is played ...? These questions replace our original, >Can machines think? ${ }^{149}$

Auf diese Weise, so Turing, würde es gelingen, die intellektuellen Qualitäten des Menschen unabhängig von seinen physischen Fähigkeiten mit den Möglichkeiten einer intelligenten Maschine, einem digitalen Computer, zu beurteilen. ${ }^{150}$ Turing ging davon aus, dass um das Jahr 2000 Maschinen und Menschen in diesem Test mit großer Wahrscheinlichkeit nicht mehr voneinander zu unterscheiden wären. Interessanterweise fin-

\footnotetext{
${ }^{146}$ Vgl. Moravec 1988, 8; Turing 1992, 107-128.

147 Vgl. Moravec 1988, 8f.

${ }^{148}$ Vgl. Turing 1950; Barrow \& Tipler 1986, 523; Tipler 1995, 20f.; Moravec 1999, 73-88; Kurzweil 1999a, 61-65.

149 Turing 1950, 434.

${ }^{150}$ Vgl. a.a.O., $434 \mathrm{f}$.
} 
den wir in Descartes' Discours de la Méthode (1637) bereits einen Vorläufer dieses Prüfgedankens. Der Philosoph vermutet in diesem Text, dass es zwar möglich wäre, ein künstliches Tier täuschend echt zu fabrizieren. Ein künstlicher Mensch dagegen könne nach Ansicht Descartes' höchstens einige wenige menschliche Reaktionen und Worte simulieren, zu einem echten Gespräch im Austausch mit seiner Umwelt jedoch wäre ein solcher Maschinenmensch niemals in der Lage. ${ }^{151}$

Während der eigentliche Turing-Test keine große Aussagekraft besitzt, nachdem schon 1966 ein simples Sprachanalyseprogramm (ELIZA von Joseph Weizenbaum) die menschlichen Gesprächspartner über seine wahre Identität hinwegtäuschen konnte, liegt die vordringliche Bedeutung von Turings Aufsatz für den Posthumanismus in seiner Entgegnung möglicher Einwände, die einer Gleichstellung von Mensch und Maschine zuwiderlaufen. ${ }^{152}$ Diese verschiedenen Aspekte, die Hans Moravec im Übrigen in allen Einzelheiten referiert, betreffen großenteils die von Wiener und von Neumann anerkannte, überlegene Komplexität des menschlichen Organismus, die Turing im Zuge des zu erwartenden technischen Fortschrittes dahinschwinden sieht. ${ }^{153}$

Turing hält es für möglich, eine Maschine zu entwerfen, die ebenso wie ein Mensch alle universellen Regeln des Verhaltens lernen und auf jede Situation reagieren kann, wobei die Unterschiede zwischen den kontinuierlichen Signalen des organischen Nervensystems und den diskreten Signalen eines Computers letztlich unerheblich für das Hervorbringen logischer Denkprozesse seien. Computer würden auch in der Lage sein, Handlungen auszuführen, für die sie nicht konkret programmiert wurden, zumal Turing der Überzeugung ist, dass Maschinen ebenso einen Sinn für Schönheit, Humor, Liebe etc. entwickeln könnten: »The criticism that a machine cannot have much diversity of behaviour is just a way of saying that it cannot have much storage capacy.« ${ }^{154}$

Während Turing die Vorstellung einer unsterblichen Seele vehement zurückweist, hält er das seinerzeit vorgetragene Argument von den wissenschaftlich beglaubigten, übersinnlichen Fähigkeiten, wie Telepathie und Psychokinese, für schwerwiegender und schlägt vor, eventuell durch einen »telepathisch sicheren Raum« die Testbedingungen des Imitationsspieles anzupassen, während Moravec diese Phänomene als Schwindel

${ }^{151}$ Vgl. Descartes 1824, 189f.; Gunderson 1964.

${ }^{152}$ V gl. Weizenbaum 1980, 14-33.

${ }^{153}$ V gl. Moravec 1999, 72-88.

154 Turing 1950, 449. 
und wissenschaftlichen Irrtum verurteilt. Paradoxe mathematische Probleme, die nicht aus der reinen Arithmetik eines Computers deduziert werden können, würden jedoch die bisherige mathematische Überlegenheit des Menschen belegen. ${ }^{155}$

Trotz der (marginalen) verbleibenden Differenzen, die im Widerspruch zu einer vollkommenen Äquivalenz zwischen Mensch und intelligenter Maschine stehen, zeigt sich Turing zuversichtlich, dass seine Argumente zu ihrer vollen Geltung gelangen werden, wenn am Ende des 20. Jahrhunderts die Computer den Imitationstest erfolgreich bestanden haben würden. Dies werde zunächst wohl mit einer relativ einfach zu konstruierenden »Kindermaschine" gelingen, die nur den kindlichen Entwicklungsstand erreiche. ${ }^{156}$

Otto Mayr zeigt in seinem Buch Authority, Liberty, and Automatic Machinery in Early Modern Europe, wie dieses an sich ältere Ideal einer Maschine, die lernfähig ist und sich selbst reproduzieren kann, mit der aufklärerischen Idee eines selbstregulierenden Marktes und mit dezentralistischen, demokratischen Ambitionen korrespondiert. ${ }^{157}$ Diese historische Untersuchung Mayrs gewinnt eine besondere Relevanz für den Posthumanismus, wenn man bedenkt, dass amerikanische Posthumanisten, allen voran die Extropianer, ausgesprochene Verfechter eines uneingeschränkten wirtschaftlichen Liberalismus sind: Dies korreliert offenbar mit den gegenwärtigen Hoffnungen auf die künstliche Intelligenz von autonomen Maschinen.

Das posthumanistische Menschenbild ist nur zu einem Teil von einer mechanistischen und materialistischen Interpretation geprägt. Noch viel wichtiger als die Deutung des Menschen als Maschine ist für die Unsterblichkeitsfrage die dualistische Teilung des Menschen in einen Körper und einen Geist - in unserem Fall also den geistigen Funktionen dieses Körpers. Da in den Visionen der Posthumanisten nur der konkrete Geist, also der informationsverarbeitende Prozess, im Computer simuliert werden soll, während der Körper meist als beliebig austauschbar beurteilt wird, lautet unsere zentrale Frage nun: Wie konnte Information als eine Einheit konzeptualisiert werden, die verschieden ist von ihrer materiellen Verkörperung?

\footnotetext{
${ }^{155}$ Wie beispielsweise Gödels Theorem. Vgl. a.a.O., 443-454.

${ }^{156}$ Vgl. a.a.O., $455 \mathrm{ff}$.

${ }^{157}$ Otto Mayr: Authority, Liberty, and Automatic Machinery in Early Modern Europe. Baltimore 1986. Vgl. Hayles 1999, 86.
} 
Norbert Wiener macht darauf aufmerksam, dass Wissenschaft grundsätzlich auf einer operational dualistischen Basis beruht, ohne dass sich hieraus jedoch zwangsläufig ein metaphysischer Dualismus ergeben müsse. Wissenschaft bestehe aus Abstraktionen der Wirklichkeit, die sich in formalen und materiellen Modellen des Universums niederschlagen und die in Tiplers Vision einer Emulation des ganzen Universums gipfeln - damit aber nach Meinung Wieners ihren Zweck verfehlen:

The ideal formal model would be one which would cover the entire universe, which would agree with it in complexity, and which would have a one to one correspondence with it. Any one capable of elaborating and comprehending such a model in its entirety, would find the model unnecessary, because he could then grasp the universe directly as a whole. ${ }^{158}$

Auf der ersten Sitzung der Macy Conferences on Cybernetics, die von 1943 bis 1954 Wissenschaftlern verschiedener Fachdisziplinen eine Gelegenheit zum Austausch über kybernetische Forschungsprojekte bot, gelang es John von Neumann und Norbert Wiener den Teilnehmern zu demonstrieren, dass nicht - wie bisher weithin angenommen - Energie, sondern Information die wichtigste Einheit in der Mensch-MaschineGleichung ist. N. Katherine Hayles bezeichnet dies als den Triumph der Information über die Materie. ${ }^{159}$

Als dann 1948 Claude Shannon im Bell System TechnicalJournal seinen für die Informationstheorie grundlegenden Aufsatz The Mathematical Theory of Communication veröffentlichte, der im Folgejahr unter ergänzender Mitwirkung von Warren Weaver in Buchform erschien, sollte damit der vorläufige Höhepunkt einer wissenschaftlichen Tendenz zu Abstraktion und Formalisierung erreicht sein. Weaver differenziert hier zunächst drei Ebenen der Kommunikationstheorie im Allgemeinen, die sich jeweils mit unterschiedlichen Fragen beschäftigen:

1. Das technische Problem: Wie genau können Zeichen der Kommunikation übertragen werden?

2. Das semantische Problem: Wie genau entsprechen die übertragenen Zeichen der gewünschten Bedeutung?

3. Das Effektivitätsproblem: Wie effektiv beeinflusst die empfangene Nachricht das Verhalten in der gewünschten Weise?

\footnotetext{
${ }^{158}$ Norbert Wiener \& A. Rosenblueth: »The Role of the Models in Science.« Wiener $1985,450$.

${ }^{159}$ Vgl. Hayles 1999, 6f., 50ff.
} 
Obwohl Weaver anschließend betont, dass Shannons mathematische Kommunikationstheorie auch eine gewisse Bedeutung für die Semantik und Effektivität von Nachrichten hat, gilt Shannons Herangehensweise eindeutig dem erstgenannten Problem der technischen Übertragung von Nachrichten. ${ }^{160}$ Das grundlegende Problem, mit dem sich die mathematische Kommunikationstheorie beschäftigt, bestehe folglich darin, an einer Stelle entweder genau oder annähernd eine Nachricht wiederzugeben, die im Kontext gewisser physikalischer oder begrifflicher GröBen steht - dort also Bedeutung hat. Shannon unterstreicht hier:

Diese semantischen Aspekte der Kommunikation stehen nicht in Zusammenhang mit den technischen Problemen. Der technisch bedeutungsvolle Aspekt ist, daß die tatsächliche Nachricht aus einem Vorrat von möglichen Nachrichten ausgewählt worden ist. ${ }^{161}$

Die von Shannon initiierte Informationstheorie befasst sich demnach ausschließlich mit dem Problem der technischen und meist digitalen Übertragung von Informationen in der Telekommunikation, die sich in zwei separate Teilprobleme gliedert: nämlich erstens die Repräsentation der Ausgangssignale der Quelle durch binäre Symbole (Quellkodierung) und zweitens die eigentliche Übertragung von binären Zufallsfolgen über einen Kanal (Kanalkodierung). Das zentrale Anliegen galt der sicheren Übertragung von Informationen, so dass der Kanaldekodierer trotz der stets auftretenden Störungen des Kanals aus den übertragenen Signalen die ursprüngliche Nachricht zuverlässig rekonstruieren kann. Seit den 1960er Jahren wurden, basierend auf umfassender algebraischer Struktur, viele Systeme zur Fehlerkontrolle entwickelt, die durch die nachfolgende Entwicklung in der Mikroelektronik komplexe Algorithmen der Fehlerkontrolle hervorbrachten und heute die Grundlage für digitale Kommunikations- und Speichermedien bilden. So wurden beispielsweise bestimmte Kodes für die Beseitigung von Fehlern durch Kratzer und Schmutz beim Abspielen von CDs angewendet, um die ursprünglichen binären Daten durch den Kanaldekodierer wiederherstellen zu können. Ähnliches gilt natürlich auch für die Übertragung von Daten durch Glasfaserkabel oder drahtlose Netzwerke. ${ }^{162}$

\footnotetext{
${ }^{160}$ Vgl. Shannon \& Weaver 1976, 11-15; vgl. dazu auch Roszak 1994, 11 ff.

${ }^{161}$ Shannon \& Weaver 1976, 41.

${ }^{162}$ Vgl. Johannesson 1992, 11-14.
} 
Nach Shannons Auffassung repräsentiert Information die Wahl einer Botschaft unter mehreren möglichen Botschaften. Im Falle einer begrenzten Anzahl von möglichen Informationen schlägt Shannon als Maßstab der Information eine logarithmische Funktion vor, um die gleichwahrscheinliche Auswahl einer Information aus dem Vorrat möglicher Informationen mathematisch zu erfassen. Als logarithmische Basis dieses Informationsmaßes führte Shannon bekanntlich die Binärziffern 0 und 1 (binary digits: bits) ein. Das Maß der Information wird auf diese Weise als mathematische Wahrscheinlichkeitsfunktion der Wahl eines bestimmten Elementes einer Nachricht eingeführt. ${ }^{163}$

Shannons Definition der Information, die ohne Berücksichtigung des materiellen Trägers oder des semantischen Kontextes determiniert wird, setzte sich gegen einen konkurrierenden Entwurf des britischen Forschers Donald McKay durch, der die Veränderung der Bedeutung von Information vom jeweils spezifischen Kontext eines Empfängers abhängig machen wollte. Shannons technischer Vorteil bestand jedoch gerade darin, dass Information ohne hohe Kosten freifließend und unbeeinflusst von Kontexten und Medien durch eine mathematische und damit allgemeingültige Formalisierung übertragbar war. Wenn der mathematische Wert der Information an ihre Bedeutung gekoppelt wäre, müsste sie eventuell in jedem neuen Kontext ihren Betrag ändern, da die Kontexte dann die Bedeutung beeinflussen würden. ${ }^{164}$

Gegenüber den Einwänden, Information von ihrer Bedeutung zu trennen, bekräftigte Shannon stets, dass seine Theorie nur auf die spezielle technische Telekommunikation und nicht auf die Kommunikation im Allgemeinen anwendbar sei. Warren Weaver drückt dieses Verhältnis folgendermaßen aus:

Eine technische Kommunikationstheorie ist geradeso wie eine gute und diskrete Postangestellte, die Ihre Telegramme annimmt. Sie achtet nicht auf die Bedeutung, ob sie nun traurig oder fröhlich oder unangenehm ist. Aber sie muß bereit sein, sich um alles zu kümmern, was auf ihrem Schreibtisch landet. ${ }^{165}$

Carolyn Marvin hat auf die kulturellen und ideologischen Implikationen von Shannons dekontextualisiertem Informationsbegriff hingewiesen,

${ }^{163}$ Ich verzichte hier auf eine ohnehin nur unzureichende mathematische Darstellung und verweise auf: Shannon \& Weaver 1976, 42f.; Brillouin 1956, 1-27; Johannesson 1992, 19-33.

${ }^{164}$ Vgl. Hayles 1999, 19

${ }^{165}$ Shannon \& Weaver 1976, 38. 
der mit dem amerikanischen Bedürfnis der kulturellen Unabhängigkeit gegenüber Europa in Zusammenhang steht. Technisch habe dies in dem Vorzug digitaler Informationsverarbeitung vor einer stärker kontextabhängigen, analogen Informationsverarbeitung seinen Niederschlag gefunden. ${ }^{166}$ Überdies weist Norbert Wiener selbst in seinem Aufsatz Homeostasis in the Individual and Society darauf hin, wie gering die Verbindung der Menschen in der »esoteric hot house civilization of Southern California« zu ihren kulturellen und genealogischen Ursprüngen ist. ${ }^{167}$ Kalifornien ist inzwischen nicht nur zu einem der bedeutendsten Standorte der weltweiten Computerindustrie geworden, sondern ebenso das Zentrum der auf einem dekontextualisierten Informationsbegriff fundierten Bewegung der Extropianer und Singularitarians.

Information wird durch die Arbeiten Shannons und Wieners als stochastische Größe eingeführt, die ungeachtet ihrer differierenden Kontexte und unabhängig von ihrem Transport über verschiedene materielle Träger auf demselben mathematischen Weg berechnet werden kann. Damit aber wird der Mensch unter einem technischen Gesichtspunkt selbst zur »Nachricht«, zu einer komplexen Organisation von Information, die unabhängig von ihrer materiellen Verkörperung - also dem menschlichen Körper - konstruiert wird. Für Norbert Wiener ist damit unbestreitbar: »... die körperliche Identität eines Individuums beruht nicht auf der Identität der Substanz, aus der es gemacht ist. ${ }^{168}$ Dieses Verhältnis schildert er folgendermaßen:

[Es: O.K.] wird auch keine innere Unstetigkeit bei einem lebenden Individuum eintreten, das sich in zwei Individuen aufteilt oder gabelt ... Es gibt aber keinen Grund, warum es nicht auch ohne entsprechende Spaltung des Körpers mit dem, was wir Geist nennen, geschehen könnte ... Die Individualität des Körpers ist eher die einer Flamme als die eines Steines, eher die einer Form als die eines Teilchens Materie. Diese Form kann übermittelt, oder abgeändert und verdoppelt werden ... Da dies so ist, gibt es keine fundamentale absolute Grenze zwischen den Übermittlungstypen, die wir gebrauchen können, um ein Telegramm von Land zu Land zu senden und den Übermittlungstypen, die für einen lebenden Organismus wie den Menschen zum mindesten theoretisch möglich sind. ${ }^{169}$

\footnotetext{
${ }^{166}$ Vgl. Marvin 1987.

167 Vgl. Wiener 1985, 383.

${ }^{168}$ Wiener 1964, 99. Vgl. a.a.O., 94-101; Hayles 1999, 50-54. Vgl. hierzu auch die knappen aber treffenden Hinweise auf die informationstheoretischen Hintergründe bei Rottensteiner 1997, $366 f$.

${ }^{169}$ Wiener 1964, 99f.
} 
Im Anschluss an diese Überlegungen spekuliert Wiener über die technischen Probleme eines vollständigen und exakten Körper-Rasters (scanning) des Menschen und diskutiert in aller Kürze die Schwierigkeiten der Wiederherstellung dieses Körpers! ${ }^{170}$

Die Bedeutung Marvin Minskys für die Vermittlung des kybernetischen Paradigmas kann kaum überschätzt werden, denn Minsky war nicht nur ein Schüler von Wiener, McCulloch, Shannon und von Neumann, sondern er konnte seinerseits als Professor am Bostoner MITHans Moravec, Ray Kurzweil und den Transhumanisten Sasha Chislenko maßgeblich prägen. Die Reduktion menschlicher Qualitäten auf unsere gedanklichen Fähigkeiten und die Subsumierung aller vermeintlich irrationalen Eigenschaften wie Humor oder der musischen Sinne als Funktionen der Ratio propagiert vor allem Minsky und konstituiert damit die posthumanistische Ideologie einer Herrschaft des Geistes über den Körper - oder allgemeiner: die Herrschaft der informationsverarbeitenden Einheit über die Materie.

Auch innerhalb der technophilen KI-Forschung ist dieses Verständnis von Leben und Intelligenz umstritten. Selbstkritisch blickt der australische Robotiker und KI-Forscher Rodney Brooks auf die erkenntnisleitenden Paradigmen seiner Zunft:

To a large extent we have all become computational bigots, believers that any problem can be solved with enough computing power. Although I do firmly believe that the brain is a machine, whether this machine is a computer is another question.

I recall that in centuries past the brain was considered a hydrodynamic machine ... When I was a child, the prevailing view was that the brain was a kind of telephone-switching network. When I was a teenager, it became an electronic computer, and later, a massively parallel digital computer. A few years ago someone asked me at a talk I was giving, »Isn't the brain just like the World Wide Web? « We use these metaphors as the basis for our philosophical thinking and even let them pervade our understanding of what the brain truly does. None of our past metaphors for the brain has stood the test of time... ${ }^{171}$

Es ist nach diesen Ausführungen offensichtlich, dass der Posthumanismus in weiten Teilen auf einem kybernetischen Paradigma basiert. Dieses Paradigma interpretiert den Menschen unter einer wissenschaftlichen Fragestellung als Maschine und von einer kommunikationstech-

${ }^{170}$ Vgl. a.a.O., 100 f.

${ }^{171}$ Brooks 2008. 
nischen Perspektive her als Informationsmuster. Der Posthumanismus dekontextualisiert auf diese Weise den semantikverneinenden Informationsbegriff Claude Shannons aus seinem rein technisch determinierten Kontext und schöpft hieraus eine Ideologie, die die persönliche Identität des Menschen in eben diesem entkörperlichten Informationsmuster verankert. Norbert Wieners Definition des Menschen als Nachricht und seine Spekulationen über die Versendung dieser Nachricht mittels eines Körperrasters aus dem Jahre 1950 sind scheinbar nur einen kleinen Schritt entfernt von den sich offenkundig anschließenden Erwägungen eines Hans Moravec, diese »Nachricht Mensch « in einem Computer zu speichern und ihr eine ewige Weiterexistenz zu bescheren. Während jedoch die Kybernetik der 1940er und 1950er Jahre einen fundamentalen Dualismus zwischen Materie und Information vertrat, müssen wir die Position des Posthumanismus erheblich differenzierter betrachten. ${ }^{172}$ Der Mensch entwickelt sich unter dem kybernetischen Paradigma zu einer informationsverarbeitenden Maschine, deren immaterielles »Programm« mit seinen spezifischen Anweisungen zur Verarbeitung von Informationen die einmalige Persönlichkeit eines Menschen konstituiert. Die Posthumanisten wollen nämlich nicht die immaterielle Form unseres vollständigen Körpers in einem Computer simulieren, sondern lediglich das exakte Muster unseres denkenden Gehirns technisch rekonstruieren. Die für das Individuum wesentliche Identität wird als körperunabhängige, geistige Einheit bestimmt, so dass ein Mensch ohne seine konkrete körperliche Form in der Virtualität simuliert werden soll. Der rezeptionsgeschichtliche Ansatz ermöglicht es zu verstehen, wie die Posthumanisten mit einem kybernetischen Paradigma die Philosophie Descartes' interpretiert haben. Während die Dominanz der denkenden Seele über den Körper bei Descartes nur im Kontext des cartesianischen Existenzbeweises besteht, verabsolutiert der Posthumanismus das denkende Prinzip - also die informationsverarbeitenden Funktionen des Gehirns - als die Essenz unseres menschlichen Wesens schlechthin. Die posthumanistische Philosophie gehört damit jedoch nur zur Hälfte in den Bereich der Geistesgeschichte - sie ist gleichzeitig immer auch die Geschichte der Physis: Körpergeschichte. Denn wenn Frank Tipler in Begeisterung für seine Idee einer technisch ermöglichten Unsterblichkeit nun fragt »Tod, wo ist Dein Stachel?« (1 Kor 15:55), dann weiß die

${ }^{172}$ Hier liegen m. E. die Schwächen von Hayles Arbeit, da sie außer einigen kurzen Verweisen auf Moravecs Mind Children die posthumanistische Literatur völlig unberücksichtigt lässt und nicht weiter differenziert. 
Soziologin Barbara Ossege genau, wo der Stachel bei den Posthumanisten sitzt: ${ }^{173}$

Letztendlich ist der Stachel des Todes der Körper selbst. Hier wird begehrt und zwar immer sündig-schuldhaft. Das Leben ist eine sexuell übertragbare Krankheit, die sich bisher in 100 Prozent der Fälle als tödlich erweist. ${ }^{174}$

Die posthumanistischen Überlegungen zur Natur des Menschen, die die Prävalenz des Geistes vor dem Körper in einem Maße akzentuieren, dass der Körper nur noch eine zu vernachlässigende Größe abgibt, knüpfen daher an den postmodernen Diskurs über das Verschwinden des Körpers an. ${ }^{175}$ In den nachfolgenden Kapiteln werde ich genauer zeigen, wie der Ersatz des Menschen durch das Posthumane weitgehend identisch ist mit der Abschaffung des menschlichen Körpers, dessen vermeintlich überholte Erscheinungsform und funktionale Unterlegenheit dem unaufhaltsamen Fortschrittsprozess zum Opfer fallen soll.

Die Schwierigkeit einer Geschichte des Körpers besteht vor allem darin, dass der Körper offiziell keine Geschichte besitzt. Weder gilt die Körpersprache als Sprache, noch Gesten als Taten. Seine Gestaltungen zeigen ihren Niederschlag nicht nur in der Kunst, sondern Körperbilder werden in der Medizin, in der Philosophie und in religiösen Traktaten und Ikonen ebenso konstruiert wie in Modezeitschriften, Filmen und neuerdings in Computerspielen und sozialen Medien. Abseits der spektakulären Ereignisse der offiziellen Geschichte, so Dietmar Kamper, fand diese Schlacht zwischen Körper und Geist, die den Körper in einem Triumph Descartes' schließlich zum Schweigen brachte, in den vergangenen 500 Jahren der europäischen Neuzeit im Untergrund des gesellschaftlichen Selbstverständnisses statt. ${ }^{176}$ Die Geschichte der Zivilisation bestimmt Dietmar Kamper demzufolge einerseits als planvolle Aneignung der Natur - als (geistige) Verallgemeinerung des (körperlichen) Besonderen - andererseits als unaufhaltsame Abstraktion und Formalisierung jeglichen Inhaltes: Ein geistlos Körperliches wird unter ein körperlos Geistiges subsumiert. ${ }^{177}$ Durch die Idee einer vollendeten Abstraktion des Menschen in der virtuellen Existenz zielt der Posthumanismus auf die in der materiellen Welt unerreichbare Herrschaft des Geistes über einen

\footnotetext{
173 Tipler 1995, 268 (=Tipler 1989, 250).

174 Ossege 1999, 179.

${ }^{175}$ Vgl. Kamper 1976, 1999; Kamper \& Wulf 1984b.

176 Vgl. Kamper 1976, 7f.; Kamper 1999, 36ff., 49.

177 Vgl. Kamper 1976, 8.
} 
dann nur noch virtuellen, geisterhaften Körper ab. Das Modell für das in der europäischen Neuzeit zunehmende Bedürfnis der Disziplinierung und Kontrolle des Körpers findet in der Maschine seinen Abschluss wie Christoph Wulf meint:

War zunächst der Mensch das Modell für die Maschine, so wird allmählich die Maschine zum Modell für die Ausrichtung des Menschen. Mensch und Maschine gleichen sich an. Wie Gott den Menschen, vermag der Mensch die Maschine zu erschaffen. Darin sucht er Gott ähnlich zu werden. In der Herstellung der Menschenmaschine realisieren sich seine narzißtischen Allmachtsphantasien. ${ }^{178}$

Der Posthumanismus repräsentiert unter dieser Prämisse die gegenwärtige Spitze einer Entwicklung, die der Soziologe Norbert Elias als den Prozess der Zivilisation bezeichnet hat. Durch die Eigendynamik eines gesellschaftlichen Beziehungsgeflechtes steigt bei zunehmender funktionaler Differenzierung der Gesellschaft auch die Notwendigkeit, das Verhalten von immer mehr Menschen aufeinander abzustimmen. Der Einzelne ist daher gezwungen, sein Verhalten immer differenzierter, immer gleichmäßiger und stabiler zu regulieren. Elias spricht hier von einem Automatismus des Selbstzwanges, sich bewusst oder unbewusst den komplexer werdenden gesellschaftlichen Gegebenheiten anzupassen. ${ }^{179}$ Sichtbar wird das Bedürfnis nach einer zunehmenden Kontrolle des unbeherrschbaren Körpers nicht nur an jener eigentümlichen sozialen Modellierung des Triebhaushaltes, die von "Scham" und "Peinlichkeitsempfinden " charakterisiert waren, sondern auch an der zunehmenden Gestaltung des Körpers nach medizinischen und modischen Normvorstellungen, die im anbrechenden Maschinenzeitalter, nämlich in der zweiten Hälfte des 19. Jahrhunderts, einen ersten Höhepunkt erreichen sollten. ${ }^{180}$

In der posthumanistischen Philosophie kulminiert diese abendländisch-zivilisatorische Idee der Herrschaft des Geistes über den Körper in der Vision eines willkürlich veränderbaren und unendlich perfektionierbaren Körpers, der im virtuellen Raum einem technisch immortalisierten Geist nach Belieben zur Verfügung steht. Markiert bislang der Tod die Grenze der Herrschaft des Geistes über einen von ihm verschieden

\footnotetext{
178 Wulf $1984,31$.

179 Vgl. Elias 1997, 323-329.

${ }^{180}$ Vgl. a.a.O., 339ff., 408-420. Die bürgerliche Bekämpfung der Onanie beispielsweise war in der Radikalität ihrer Techniken wohl nie so einfallsreich wie im ausgehenden 19. Jahrhundert. Vgl. Foucault 1983.
} 
erachteten Körper, so konvergiert nun die posthumanistische Vision von der technischen Überwindung der Sterblichkeit mit der endgültigen Bezwingung des menschlichen Körpers.

\subsubsection{Der Maßstab der Vervollkommnung: Arbeit und Wissen}

Wenn der Mensch gemäß diesem kybernetischen Paradigma nichts anderes als eine informationsverarbeitende Maschine ist, dann stellt sich die Frage, wie eine Vervollkommnung des Menschen erreicht werden kann. Was kennzeichnet - aus dieser posthumanistischen Perspektive die Perfektionierung des menschlichen Daseins?

In der kosmologischen Konzeption Frank Tiplers ist unverkennbar, dass der Fortschritt im Universum auf das Ausmaß von Informationsverarbeitung zurückgeführt wird. Sofern Tipler, wie wir bereits gesehen haben, Leben grundsätzlich als Informationsverarbeitung definiert, so bedeutet jeder Fortschritt des Lebens eine Steigerung der Geschwindigkeit, mit der Daten verarbeitet werden, sowie eine Ausweitung des Gedächtnisses, also des Informationsspeichers, dieser Lebensformen. Tipler propagiert daher nicht nur die notwendige Überwindung der cerebralen Grenzen des biologischen Menschen, sondern auch die Kolonisierung der Galaxis durch intelligentes Leben mit dem Ziel, die Zahl der verarbeiteten Information bis ins Unendliche zu steigern. Nur eine immense Steigerung der »Rechenleistung « der heutigen intelligenten Lebensformen könne die Entfaltung verschiedener Vollkommenheiten garantieren, die Tipler zunächst nicht weiter spezifiziert. Auch die finale Vereinigung mit Gott im verwirklichten Punkt Omega und die damit einhergehende Auferstehung (oder Simulation) aller Toten sei demnach abhängig von der künftigen Leistung der Datenverarbeitung, um die gewaltigen Informationsmengen, die zu dieser Vervollkommnung nötig sind, zu berechnen. ${ }^{181}$ Kollektiver Träger des Fortschritts sind für Tipler all jene Gattungen, deren Mitglieder potentiell selbstprogrammierende Turingmaschinen sind, das heißt die Menschen und ihre maschinellen Nachfahren - eben alle intelligenten »informationsverarbeitenden Systeme ${ }^{182}$

In ähnlicher Weise fixiert Marvin Minsky Denken im Sinne von Problemlösungen als absoluten Zweck intelligenter Systeme. Er verurteilt

${ }^{181}$ Vgl. Barrow \& Tipler 1986, 675ff.; Tipler 1989, 245-249; Tipler 1995, 55-65.

${ }^{182}$ Vgl. a.a.O., 124-128. 
die trivialen Unterhaltungsstrategien des Menschen (football und Popmusik) als reine Verschwendung der Denkkapazitäten teurer Gehirne. ${ }^{183}$ Aus der fiktiven Perspektive eines religiösen Experten und nicht etwa aus der eines Ökonomen kommentiert Minsky diese offensichtliche Vergeudung menschlicher Geistesgaben:

It would be fun to ask the religious ones to consider whether it is not a sin to waste such wondrous hardware on watching adults kicking balls around? My own view is that this is less a sin than a symptom - of infection by a parasitic meme ... which has self-propagated through our culture like a software virus, a cancer of the intellect so insidious that virtually no one thinks/dares to question it. Now, in the same way we see grown people playing and working in the context of popular music ... that sets at least part of one's brain in a loop. Is it o.k. that we, with our hard earned brains, should welcome and accept this indignity - or should we resent it as an assault on an evident vulnerability? ${ }^{184}$

Dort, wo Minsky die rastlose Denkarbeit durch den parasitären Virus der seichten Unterhaltung bedroht sieht, versagen auf einmal seine sehr offenen Erklärungsversuche menschlichen Denkens. Gefühle, Humor und den Konsum klassischer Musik deutet er noch als Mechanismen des Lernens und der Problemlösung, während Beschäftigungen vermeintlicher kultureller Trivialität nur als Verschwendung menschlichen Geistes zu verurteilen sind. Aus diesem Grunde wäre auch die Fortsetzung des rein biologischen Lebens nichts anderes als die Verschwendung künftiger potentieller Denkkapazitäten der Roboter: »We owe our minds to the deaths and lives of all the creatures that were ever engaged in the struggle called Evolution. Our job is to see that all this work shall not end up in meaningless waste. ${ }^{185}$

Wenn darüber hinaus das Ziel des Fortschrittes die Erlangung von unendlichem Reichtum sein soll, wobei Reichtum für Minsky beruflichen Erfolg quantifiziert, ${ }^{186}$ und er die Verschwendung der »teuren Gehirne» in geistlosen Vergnügungen als kulturelles Krebsgeschwür anprangert, dann rezipiert der Kybernetiker an dieser Stelle die calvinistischen Ideale rastloser Berufsarbeit in ihrer spezifisch amerikanischen Ausprägung. Minsky leistet hier nichts weniger als die Formulierung einer normativen informationstechnischen Arbeitsethik, die Berufsarbeit mit Denkar-

\footnotetext{
${ }^{183}$ Vgl. Minsky 1992c, 35.

${ }^{184}$ Ebd. Vgl. entsprechende Äußerungen von Arthur C. Clarke (1960, 219).

${ }^{185}$ Minsky 1994, 113.

${ }^{186}$ Vgl. Minsky 1988, 284.
} 
beit und wiederum mit der maschinellen Informationsverarbeitung gleichsetzt. Jede Ablenkung von problemorientierter Denkarbeit weist er asketisch zurück. Erst in Verbindung mit einem effizienten Einsatz der geistigen Kapazitäten kennzeichnet die Steigerung der informationsverarbeitenden Leistungen damit für Marvin Minsky den Maßstab der Vervollkommnung intelligenten Lebens.

Hans Moravec, Ray Kurzweil und der Kybernetiker Kevin Warwick verknüpfen Fortschritt ebenfalls mit der Erhöhung der Denkleistungen, akzentuieren in diesem Zusammenhang jedoch stärker technische und quantitative Vergleiche zwischen biologischen Gehirnen und den bisherigen sowie den künftig zu erwartenden Leistungen der Computer. Anschauliche Tabellen und seitenlange Ausführungen über die beschleunigt ansteigende Zahl von Rechenschritten pro Sekunde im Laufe der Technik- oder gar Evolutionsgeschichte werden mit der Vorstellung einer gleichsam zunehmenden Intelligenz der denkenden Systeme verbunden. Denn während die Entstehung intelligenter Computerprogramme trotz der eingestandenen Schwierigkeiten als zwangsläufige Entwicklung der kommenden Jahrzehnte präsentiert wird, konzentrieren sich alle Ansätze einer Futurologie fast ausschließlich auf Spekulationen über die künftigen Rechenleistungen und Speicherkapazitäten. Die Frage nach der intelligenten Software wird von den Posthumanisten weitgehend marginalisiert. Moravec führt exemplarisch insbesondere die durch Schachpartien bekannt gewordenen Computer wie Deep Blue an und spiegelt damit die öffentliche Inszenierung von »Computerintelligenz« anhand dieses Duells. ${ }^{187}$ Ray Kurzweils Annahme, dass schon im Jahr 2029 an die 99\% der irdischen Denkleistung von Computern ausgeführt werden, illustriert plastisch den quantitativen Maßstab von cerebralen und informationstechnischen Leistungen, der dieser Fortschrittsentwicklung zugrunde gelegt wird. ${ }^{188}$

Die Basis der Vervollkommnung menschlichen und künstlichen Lebens ist für alle Posthumanisten die Maximierung der Leistung informationsverarbeitender Systeme. Eine leistungsorientierte Steigerung der Informationsverarbeitung impliziert damit bereits zwei Aspekte eines Fortschrittsprozesses: zum einen den Fortschritt der Arbeit und zum anderen den des Wissens.

\footnotetext{
${ }^{187}$ Vgl. Moravec 1988, 51-74; Moravec 1999, 51-72; Kurzweil 1999a, 9-39; Warwick 1998, 257-279.

${ }^{188}$ Vgl. Kurzweil 1999a, 189-252.
} 
Werden auch nur im Ausnahmefall - wie bei Minsky - solch deutliche Verweise auf die protestantische Arbeitsethik gemacht, so rezipieren darüber hinaus auch andere Posthumanisten protestantische und philosophische Konzeptionen von Arbeit, die hauptsächlich schon im 18. Jahrhundert entstanden sind und im Laufe der vergangenen 200 Jahre besonders die amerikanische Debatte prägen konnten. Der französische Philosoph Condorcet, die schottischen Philosophen Adam Smith, David Hume und Adam Ferguson sowie Henry Home gelangten im 18. Jahrhundert aus ihren jeweiligen Kontexten der politischen Ökonomie, der Moralphilosophie und der Theologie zu der Überzeugung, dass Arbeit nicht nur ein Mittel zur Erlangung von Gegenständen des Genusses sei, sondern dass Arbeit selbst ein anthropologisches Bedürfnis des Menschen darstelle. Der Drang zur Arbeit ist laut Adam Ferguson deshalb höher zu gewichten, weil die rein konsumtiven Bedürfnisse des Menschen, da die Befriedigung sinnlicher Triebe nur von kurzer Dauer und durch ständige Wiederholung geprägt sei. Arbeit dagegen entspreche der natürlichen Veranlagung des Menschen, fortgesetzt seine Fähigkeiten $\mathrm{zu}$ bewähren und somit eine historische Kontinuität seiner Leistungen zu ermöglichen. Die schottischen Moralphilosophen definierten daher Arbeit selbst als eine originäre Quelle des Glückes. Anders als Hume, Home und Smith verbindet Ferguson in seiner Eigenschaft als presbyterianischer Geistlicher diese Auffassung jedoch mit den protestantischen Tugenden des Triebverzichtes und der rastlosen Berufsarbeit: ${ }^{189}$

Such men do not chuse pain as preferable to pleasure, but they are incited by a restless disposition to make continued exertions of capacy and resolution; they triumph in the midst of their struggles; they droop, and they languish, when the occasion of their labor has ceased. ${ }^{190}$

Der bekannte Exponent der Physikotheologie, William Paley, führt diese Gedanken weiter aus und unterstreicht mit Nachdruck das Entwicklungsmoment der Arbeit. Er betont, dass die Freude an der Entfaltung unserer physischen und geistigen Fähigkeiten in der Arbeit uns erst dann wirklich glücklich machen werde, wenn wir sie im Bewusstsein über das zukünftige Ziel unserer Handlungen tätigen:

\footnotetext{
${ }^{189}$ Vgl. Rohbeck 1987, 88-119. Die schottischen Calvinisten werden als Presbyterianer bezeichnet.

${ }^{190}$ Adam Ferguson: An Essay on the History of Civil Society. London 1793, 67f. Zitiert nach Rohbeck 1987, 119. Vgl. a.a.O., 117-120.
} 
A man who is earnest in his endeavours after the happiness of a future state, has in this respect, an advantage over all the world: for, he has constantly before his eyes an object of supreme importance, productive of perpetual engagement and activity, and of which the pursuit ... lasts him to his life's end. ${ }^{191}$

Tiplers Teleologie der informationstechnischen Fortentwicklung und Minskys Ermahnung zur Konzentration auf die wirklichen Ziele unseres Denkens - eben die Erweiterung »unserer« intellektuellen Fähigkeiten - rezipieren das von Paley und den schottischen Philosophen zum Ausdruck gebrachte Verständnis von Arbeit: Die Quelle des Glücks sei nun Denkarbeit oder Informationsverarbeitung. Es ist ebenfalls Paley, der hervorhebt, dass der Mensch nicht am absoluten Wert einer Handlung, sondern nur an der Steigerung seiner Fähigkeiten Befriedigung gewinnt, so dass Arbeit letztlich den Maßstab des Fortschrittes determiniert. ${ }^{192}$ Im 18. Jahrhundert hatten bereits Turgot und Adam Smith eine ökonomische Stadientheorie entworfen, die die Steigerung der Arbeitsleistung und Produktivität als Kennzeichen geschichtlicher Fortentwicklung etablierte. ${ }^{193}$ In diesem Zusammenhang betonten sowohl die französischen als auch die schottischen Philosophen Turgot, Condorcet, Fontenelle, Adam Smith und John Millar die Schlüsselfunktion einer Weiterentwicklung der Arbeitsmittel und Werkzeuge innerhalb des Fortschrittsprozesses. ${ }^{194}$

Posthumanistische Theorien haben nun die Eigenschaft, dass sie die zentralen Mittel des Fortschrittes zu seinem eigentlichen Subjekt umdeuten. Unter der Voraussetzung, dass die Arbeitsleistung den Maßstab des Fortschritts markiert, gewinnen die Maschinen und Computer mit zunehmender Leistung eine größere Bedeutung für den Fortschrittsprozess als die originären Fähigkeiten des Menschen. In diesem Sinne wird der Mensch über seine Leistungsfähigkeit wahrgenommen und als Mängelwesen (Herder, Gehlen), homo faber (Henri Bergson) oder gar als tool making animal (Benjamin Franklin) definiert. Der Fortschritt der Technik wird zum eigentlichen Kennzeichen jeder Höherentwicklung des menschlichen Lebens. Die gesamte Geschichte der Zivilisation löst sich, wie der Geograph Ernst Kapp feststellte, »in die Geschichte der

\footnotetext{
191 Paley 1842, 8.

192 A.a.O., 9.

${ }^{193}$ Vgl. Rohbeck 1987, 88-119.

${ }^{194}$ Vgl. a.a.O., 158ff.
} 
Erfindung besserer Werkzeuge auf.« ${ }^{195}$ Völlig neu ist in der posthumanistischen Argumentation, dass das Werkzeug nun zum absoluten Maß des Menschen wird. Wie wir unlängst sahen, wurde der Mensch bereits als eine (schlechte) Maschine definiert.

Damit verbunden ist auch der zweite Aspekt des Fortschritts der »informationsverarbeitenden Systeme«, da vor allem die französischen Philosophen des 18. Jahrhunderts den Fortschritt des Wissens in Abhängigkeit von der Weiterentwicklung der Erkenntnismittel wie der Mathematik und Physik sahen. ${ }^{196}$

So wie die Idee eines allgemeinen geschichtlichen Fortschrittes im Sinne einer Entwicklung zum Besseren ihre Vorläufer schon in den Werken der christlichen Kirchenväter Tertullian, Origines und Lactanz findet, die das Christentum als historischen Fortschritt gegenüber der »heidnischen« Religion beurteilten, so stand auch die Idee eines Fortschrittes des Wissens zunächst in einem eigentümlich christlichen Kontext. Während der Kirchenlehrer Augustinus die Idee eines weltlichen Fortschrittes in der Zeit zugunsten der Vorstellung einer spirituellen Verbesserung durch die christliche Offenbarung ablehnte, konnte sein Zeitgenosse Vincent von Lerins in seinem Commonitorium dennoch von einer Weiterentwicklung der kirchlichen Lehre ausgehen, die im Laufe der Jahrhunderte die Weisheit der Kirche immerfort vermehren würde. In der mittelalterlichen Scholastik sind es Anselm von Canterbury und Gerhoch von Reichersberg, die sich nachdrücklich für ein Voranschreiten (proficere) in der kirchlichen Lehre und Auslegung aussprechen, um die veritatis ratio zur vollen Entfaltung zu bringen. Die Notwendigkeit eines kontinuierlichen Fortschrittes des religiösen Wissens ergibt sich für die Scholastiker aus dem kurzen Leben der einzelnen Kirchenlehrer, das nicht ausreiche, um noch vor dem Weltenende alle Erkenntnis zu erlangen. ${ }^{197}$

Im Anschluss an das Werk De Magnete (1600) des Arztes William Gilbert entwirft Francis Bacon in seinen Schriften The Advancement of Learning (1605) und Novum Organum (1620) die Idee des Fortschrittes im Bereich des empirischen, naturwissenschaftlichen Wissens, die im frühen 17. Jahrhundert mit großer Wirkung vor allem in England propagiert wurde. Hier schon findet sich die weithin anerkannte Überzeugung, dass

\footnotetext{
${ }^{195}$ Ernst Kapp: Grundlinien einer Philosophie der Technik. Zur Entstehungsgeschichte der Cultur aus neuen Gesichtspunkten. Braunschweig 1877. Zitiert nach Rapp 1992, 97. Vgl. a.a.O., 97-103.

${ }^{196}$ Vgl. Rohbeck 1987, 160-167.

${ }^{197}$ Vgl. Kosellek 1975, 363-368; Spadafora 1990, 85-90.
} 
sich der Zuwachs an Wissen parallel zur steigenden Zahl wissenschaftlicher Entdeckungen beschleunigen wird. ${ }^{198}$ War die Idee des wissenschaftlichen Fortschrittes bereits bei Bacon und Newton in einen breiten theologischen Kontext eingebunden - Wissenschaft als Huldigung an die göttliche Schöpfung - so fundierten im Laufe des 17. Jahrhunderts eine Reihe puritanischer und puritanisch beeinflusster Denker diese Verknüpfung zwischen einem Fortschritt des theologischen Wissens und der naturwissenschaftlichen Erkenntnis. David Spadafora beschreibt in seiner umfassenden Arbeit über die englische Fortschrittsidee diese Synthese des wissenschaftlichen und des religiösen Fortschrittsgedankens als das geistige Erbe, aus dem sich die englische und großenteils die amerikanische Fortschrittstheorie entwickeln konnte. ${ }^{199}$

Für die Genese des Posthumanismus ist der englische Fortschrittsdiskurs des 18. Jahrhunderts von besonderem Interesse, weil hiermit der simplifizierenden Ansicht entgegengewirkt werden kann, dass die englische (und amerikanische) Fortschrittstheorie gänzlich ein französischer Import sei. Die Implikationen dieser Theorie, nämlich die Annahme einer konsequenten Dichotomie zwischen religiöser Heilsgeschichte einerseits und deren »säkularisiertem " Pendant im rein innerweltlichen Fortschrittsglauben andererseits, würden sich als erschwerend für die Analyse der posthumanistischen Fortschrittsidee erweisen. Da das 19. Jahrhundert nach der Einschätzung Spadaforas keine wirklich neue Fortschrittstheorie hervorbrachte, sondern lediglich ältere Muster rezipierte oder fortschrittskritische Verfallstheorien entwarf, sind die Konzeptionen des 18. Jahrhunderts weiterhin von immenser Bedeutung für die englische und amerikanische Gegenwartsphilosophie. ${ }^{200}$

Im England des 18. Jahrhunderts waren es vor allem drei herausragende Gelehrte, die eine von den säkularen französischen Theorien differente Fortschrittsidee prägen konnten und immensen Einfluss auf die englische und amerikanische Geisteswelt hatten. Der anglikanische Bischof von Carlisle, Edmund Law, hatte als Mentor in Cambridge eine ganze Generation von Studenten formen können, darunter auch William Paley. Der Arianer Richard Price hatte seinerseits eine nachhaltige Wirkung auf die berühmte Reformergruppe der Chatamites in Shelburne. Der unitarische Geistliche Joseph Priestley schließlich galt als der bedeutendste Universalgelehrte seiner Zeit in der britischen Hemisphäre:

\footnotetext{
${ }^{198}$ Vgl. a.a.O., $19 f f$.

${ }^{199}$ Vgl. Dawson 1935, 159-175; Spadafora 1990, $19 \mathrm{ff}$.

${ }^{200}$ Vgl. a.a.O., 381-387.
} 
Neben seinem politischen Engagement und den philosophischen Arbeiten wirkte er als Historiker, Linguist und Chemiker und erzielte auf manchen dieser Gebiete beachtliche Ergebnisse. Durch seinen Einsatz für die Bewegung des Unitarian New Meeting, die von scharfen Attacken gegen die anglikanische Kirche geprägt war, sowie durch sein anhaltendes Bemühen um politische Reformen, sah sich Priestley 1794 gezwungen, England zu verlassen. Er wurde zum seinerzeit prominentesten Einwanderer Amerikas. Eine enge Freundschaft verband ihn mit dem amerikanischen Naturforscher und Staatsdenker Benjamin Franklin. Als führendes Mitglied des englischen Intellektuellen-Clubs, der Lunar Society, die sich für wissenschaftlichen und politischen Fortschritt einsetzte, geriet neben seinen Studenten wie dem utilitaristischen Philosophen Jeremy Bentham auch der Naturforscher Erasmus Darwin unter seinen Einfluss. ${ }^{201}$ Für Law, Price und Priestley und eine beträchtliche Anzahl weiterer Gelehrter, die sowohl ein Interesse an naturwissenschaftlichen Fragen wie auch an theologischen und (moral-)philosophischen Problemen an den Tag legten, war das Christentum letztlich der Fokus ihrer philosophischen und politischen Überzeugungen. Ihre teils nur fragmentarischen Konzeptionen einer Theorie des Fortschrittes vollzogen eine enge Verknüpfung zwischen der Idee einer göttlichen Vorsehung und dem wahrnehmbaren, weltlichen und wissenschaftlichen Fortschritt, wobei sich dieser Fortschritt des wahren Wissens nach Priestleys Ansicht zunehmend beschleunigen würde. ${ }^{202}$

Anglikanische Geistliche wie John Edwards, William Worthington und Edmund Law entwickelten zwischen 1699 und 1745 die These, dass man aufgrund des sichtbaren vergangenen Fortschrittes im religiösen Wissen darauf schließen könne, dass auch in der Zukunft das menschliche Wissen um Gott stetig vergrößert werde. Nach der Vorstellung dieses religious progress offenbart Gott dem Menschen je nach Reife und Erkenntnisfähigkeit seiner Geschöpfe neues, höheres Wissen über seine Natur und seinen Willen. Erst im Laufe des 18. Jahrhunderts setzte sich allmählich die Auffassung durch, dass die Vertiefung religiösen Wissens auch auf menschliches Bemühen zurückgehe und nicht nur Teil einer vom Menschen passiv empfangenen göttlichen Offenbarung sei. So nahmen die anglikanischen Bischöfe John Ross und William Warburton an, dass das Wissen um Gott, sich gegenseitig ergänzend, sowohl von göttlicher Of-

${ }^{201}$ Vgl. Graham 1995, 1-41; Spadafora 1990, 252.

${ }^{202}$ Vgl. a.a.O., 235, $252 \mathrm{ff}$. 
fenbarung als auch vom menschlichem Fortschritt in der Interpretation der heiligen Texte herrühren könne.

Der Arzt, Psychologe und anglikanische Laie David Hartley gelangte zu dem Schluss, dass das Verständnis der alttestamentarischen Propheten und der späteren schriftlichen Offenbarungen Gottes in der Bibel dem jeweiligen intellektuellen Stand der Gläubigen entspreche, der fortschreite und daher auch zu einer Steigerung des religiösen Wissens führe. Sein Zeitgenosse, der englische Presbyterianer John Taylor, drückte diesen Gedanken metaphorisch in Analogie zu den biologischen Lebensphasen des Menschen aus: So wie die Weisheit des Menschen in der Abfolge von Kindheit, Jugend, der Zeit als Erwachsener und im Alter beständig zunehme, so werde sich der progress of religion von Beginn der Menschheitsgeschichte bis in ihr Alter als graduelle Verbesserung ihrer geistigen und religiösen Erkenntnisfähigkeit darstellen. Fortschritt wird hier - wie bei Hartley - sowohl individuell als stufenweiser Fortschritt im spirituellen Leben einer Seele als auch kollektiv als allgemeiner Fortschritt der christlichen Religion interpretiert. Der liberale anglikanische Bischof von Llandlaff, Richard Watson, ging sogar so weit, zu behaupten, dass die Verbreitung des Christentums mit der aufklärerischen Mission von Vernunft und Wissenschaft einhergehe.

Einerseits wird in diesen Aussagen anglikanischer Vertreter des 18. Jahrhunderts die Nähe zum Deismus offensichtlich, andererseits jedoch auch, wie gerade die anglikanische Theologie eine Fortschrittstheorie im Kampf gegen den Deismus entwickelte. Denn somit stand die Idee eines stückweisen Fortschrittes im Bereich des religiösen Wissens, der sich am Geistesstand der Menschen orientierte, nicht im Gegensatz zu der Vorstellung einer göttlichen Offenbarung und Vorsehung.

Allen hier vorgestellten englischen Fortschrittsdenkern ist gemeinsam, dass sie einen vergangenen und zukünftigen Fortschritt des Wissens zugrunde legen. Auch ist es Konsens, dass sich dieser Fortschrittsprozess stetig beschleunigt. Soweit stimmen sie mit den Annahmen der französischen Philosophen überein. Jedoch war ein weltlicher Fortschritt des Wissens, anders als in der säkularen französischen Philosophie, im englischen und amerikanischen Kontext meist ein wesentlicher Bestandteil der christlichen Heilsgeschichte, die das Voranschreiten der Wissenschaften und der Vernunft mit der wachsenden Erkenntnis Gottes verbanden. Der Fortschritt der Wissenschaften wurde als Teil von Gottes Plan ausgelegt. William Paleys Natural Theology markiert am Ende des 
18. Jahrhunderts den vorläufigen Höhepunkt dieser Entwicklung in ihren naturwissenschaftlichen Aspekten. ${ }^{203}$

Da Frank Tipler und Hans Moravec mit dem Bezug auf den Punkt Omega und der implizierten theologischen Teleologie und auf andere Weise auch Ray Kurzweil in seiner Auslegung der Singularität einen Fortschritt religiösen bzw. spirituellen Wissens voraussetzen, ist es plausibel anzunehmen, dass an dieser Stelle Elemente des englischen bzw. amerikanischen Verständnisses von einem Fortschritt des Wissens mit ihrem heilsgeschichtlichen Sinngehalt rezipiert wurden.

In der Kombination vom Fortschritt des Wissens und dem Fortschritt der Arbeit - teils mit den von Minsky ausgeführten Implikationen einer calvinistischen Arbeitsaskese - gewinnt die Idee vom Fortschritt der Informationsverarbeitung eine ungeheure Dynamik. ${ }^{204}$ Beide Ideen haben in der englischen, schottischen und amerikanischen Geistesgeschichte eine immense Bedeutung. Beide Ideen sind zumindest im amerikanischen und englischen Kontext in theologische Konzeptionen von Arbeit und Wissen eingebunden. Diese Ideen von einer Steigerung der Arbeitsleistung und einer Steigerung des Wissens werden im Posthumanismus rezipiert und zu einer Synthese zusammengefügt. Bei einigen der posthumanistischen Autoren, wie z.B. bei Frank Tipler oder Marvin Minsky, wird dieser protestantische Hintergrund in seiner amerikanischen Ausprägung deutlich sichtbar. Auch Ray Kurzweil, der als Unitarier aufgewachsen ist, rezipiert zumindest in Bezug auf den Fortschritt des Wissens den theologischen bzw. spirituellen Gehalt dieser Ideen: »I see the opportunity to expand our minds, to extend our learning, and to advance our ability to create and understand knowledge as an essential spiritual quest. $\ll^{205}$ Ohne zwar auf traditionelle Religionen einzugehen, vertritt Kurzweil ferner die These von einer kontinuierlichen Steigerung spiritueller Erfahrungsdimensionen mit Hilfe der technischen Erweiterung des Menschen bzw. in seiner virtuellen Existenz. Auch würden künstliche Intelligenzen in der Lage sein, eine ihnen eigene Spiritualität zu entwickeln. ${ }^{206}$

${ }^{203}$ Vgl. a.a.O., 85-104. Vgl. Kapitel 6.3.4.

${ }^{204}$ Otto Hansmann spannt in seiner Interpretation des Transhumanismus einen weiten

Bogen in der Geschichte der menschlichen Selbstüberwindung (von Plato über Marx bis Niklas Luhmann). Er verkennt aber die Technozentrik der transhumanistischen Entwicklungstheorie, die im Kern auf der Steigerung von Arbeit und Wissen beruht. Vgl. Hansmann 2015, 31-84.

${ }^{205}$ Kurzweil 1999a, 185. Vgl. Kurzweil 2005, 1.

${ }^{206}$ Vgl. Kurzweil 1999a, 152 ff., Kurzweil 2005, 377. 


\subsection{Vernichtung oder unendlicher Fortschritt}

Der Posthumanismus ist der Höhepunkt eines jahrhundertealten, okzidentalen Fortschrittsglaubens. Ziel des Fortschrittes ist die Vervollkommnung und konsequenterweise die Überführung des gegenwärtigen Menschen in eine posthumane, unsterbliche Existenz. Die verschiedenen Aspekte der posthumanistischen Fortschrittsideologie - also die Frage nach dem Weg in die Unsterblichkeit - werde ich im Folgenden darstellen und analysieren.

Was macht eine umfassende Fortschrittsidee wie den Posthumanismus aus? Eine solche Theorie muss eine Reihe von Fragen beantworten, um zu begründen, warum die Zukunft so und nicht anders aussehen wird: Fortschritt muss legitimiert werden. In einem ersten Schritt wenden wir uns daher dem posthumanistischen Bedrohungszenario zu: Was würde passieren, wenn die Menschheit sich gegen den Fortschritt stellen würde (Kapitel 6.3.1 Tod, Entropie und die drohende Vernichtung des Lebens im Universum)? Das Opfer des Menschen, das mit diesem Prozess einhergeht, greift auf kulturelle Deutungsmuster der amerikanischen Kolonialgeschichte zurück (Kapitel 6.3.2). Positiv wird der prognostizierte Fortschritt einerseits als Ablösung von der natürlichen Evolution interpretiert (Kapitel 6.3.3). Andererseits ist der technologische Posthumanismus erst das Ergebnis einer fortschrittsfreundlichen Rezeption der darwinistischen Evolutionstheorie, die einen stetigen Aufstieg des Lebens festzustellen glaubte (Kapitel 6.3.4). Frank Tipler entwickelt in diesem Zusammenhang den eigenständigen Ansatz einer christlich fundierten Physikotheologie (Kapitel 6.3.5). 


\subsubsection{Tod, Entropie und die drohende Vernichtung des Lebens im} Universum

Der Fortschritt ist keine höhere Notwendigkeit, sondern höchstens eine Möglichkeit (und oft auch eine Unmöglichkeit). ${ }^{207}$

Ryszard Kapuściński

Bevor wir darauf eingehen, wie Posthumanisten den eigentlichen Fortschrittsprozess legitimieren und ausgestalten, soll ein Aspekt beleuchtet werden, der die Basis aller posthumanistischen Futurologie ausmacht: Warum muss es Fortschritt geben? Welche Gefahren drohen, wenn der prognostizierte Fortschritt ausbliebe oder verhindert würde? Der Posthumanismus zeichnet sich nicht dadurch aus, dass er Möglichkeiten entwirft. Er präsentiert Notwendigkeiten menschlicher Entwicklung, die ohne Alternative sind. Dieser futurologische Imperativ wird auf zwei Ebenen expliziert, die sich gegenseitig bedingen: einer evolutionären und einer kosmischen.

Im Einzelnen führen die Posthumanisten sehr unterschiedliche Aspekte der menschlichen Biologie auf, die als defizitär erfahren werden. Es sind zwei Bedrohungen, die Marvin Minsky als Gefährdung des Lebens und des menschlichen Expansionsdranges ausmacht. Zum einen beschwört Minsky das zivilisatorische Horrorszenario der Überbevölkerung des Planeten Erde mit biologischen Lebewesen herauf. Nur eine Transformation der Menschen in Maschinenwesen könne der unkontrollierten Vermehrung Einhalt gebieten. ${ }^{208}$ Eine viel schwerwiegendere Gefahr sieht der Informatiker jedoch in der biologischen Konstitution des Menschen, deren fehleranfällige, genetische Kodierung für unendliches Leid verantwortlich sei:

The major causes of death result from the effects of inherited genes. These genes include those that seem to be largely responsible for heart disease and cancer, the two largest causes of mortality, as well as countless other disorders such as cystic fibrosis and sickle cell anemia. ${ }^{209}$

\footnotetext{
${ }^{207}$ Kapuściński 1992, 63.

${ }^{208}$ V gl. Minsky 1994, 113; Minsky 1992a, 24.

${ }^{209}$ Minsky $1994,108$.
} 
Mit der äußerst beschränkten Lebensspanne des Menschen seien der Erlangung von Reichtum und Weisheit biologisch bedingte Grenzen gesetzt, die einer erheblichen Weiterentwicklung von Kultur, Wirtschaft und Gesellschaft entgegenstehen. Ebenso wertet Minsky die Grenzen des menschlichen Gedächtnisses und den beschränkten Sehsinn, der nur einen kleinen Frequenzbereich abdeckt, als Ursache für eine intellektuelle Stagnation, die den Herausforderungen ihrer Zeit bald nicht mehr gerecht werden könne. Die biologischen Grenzen unserer Kapazitäten würden demnach jeglichen Denkfortschritt verhindern. ${ }^{210}$ Die körperliche Größe des Menschen gilt ihm als bloße Verschwendung, da mit Hilfe der Nanotechnologie mehr Denkleistung mit weniger materiellem Aufwand effizienter gestaltet werden könne, zumal ohne »Backup-Gehirne« der anfälligen, biologischen »Denkmaschine« ständig die Gefahr eines unwirtschaftlichen, endgültigen Datenverlustes - des Todes - bestehe. ${ }^{211}$

Für Hans Moravec indes ist nicht nur der einzelne Mensch, sondern die gesamte Menschheit von ihrer endgültigen Auslöschung durch unwägbare Gefahren bedroht. Die einzige Alternative zum Untergang der menschlichen Kultur bestehe in der Zustimmung zu einem ungebremsten Fortschritt und dem Ersatz des Menschen durch eine posthumane Intelligenz:

If by some unlikely pact, the whole human race decided to eschew progress, the long-term result would be almost certain extinction ... Sooner or later an unstoppable virus deadly to humans will evolve, or a major asteroid will collide with the earth, or the sun will expand, or we will be invaded from the stars, or a black whole will swallow the galaxy ... By growing rapidly enough, a culture has a finite chance of surviving forever. ${ }^{212}$

Bemerkenswert ist an dieser Stelle, dass Moravec die informationstechnischen Risiken - Computerviren und unbeabsichtigte Mutationen der Programme - wiederum als Mittel zur Stärkung der Computersysteme und als innovatives und äußerst fruchtbares Chaos würdigt. ${ }^{213}$

Auf individueller Ebene beschreibt Moravec ein erschreckendes Szenario medizinischer Alltäglichkeit, das den Leser persönlich berühren soll: Ein Patient erfährt von seinem Arzt, dass es keine Aussicht auf die

${ }^{210}$ Vgl. a.a.O., 108, 111f.; Minsky 1992a, $25 f$.

${ }^{211}$ Vgl. a.a.O., $25 f$.

212 Vgl. Moravec 1988, 101.

${ }^{213}$ Vgl. a.a.O., 125-146. 
Heilung seiner Krankheit gebe und er nur noch sechs Monate zu leben habe. Aber es bestehe eine Rettung: die Geisttransplantation (mind transplantation), die mit der Aufgabe des sterblichen Körpers einhergehe. Nach dem chirurgischen Vorgang des Uploading wacht der Patient in seiner neuen virtuellen Existenz auf und wird vom Arzt begrüßt: »Congratulations, welcome to immortality. « ${ }^{214}$

Als Bill Joy (seinerzeit Führungskraft beim Computergiganten Sun Microsystems) im Jahr 2000 mit seinem Essay Why the Future Doesn't Need Us einen Verzicht auf die Gentechnik, die molekulare Nanotechnik und die Robotik forderte, reagierte der extropianische Vordenker Max More äußerst irritiert. ${ }^{215}$ More, sichtlich ungehalten über das Verlangen nach Selbstbeschränkung eines einflussreichen Forschers, entgegnete Joys Bedenken gegen einen grenzenlosen Fortschritt mit zwei Argumenten. Zum einen würde ein Verzicht auf die heilsbringende Technik keinen Erfolg haben, weil sechs Milliarden Menschen nicht auf deren Segnungen (massive benefits) und die militärischen Potentiale zum Wohle der Menschheit verzichten wollten. Die einzige Möglichkeit, mit eventuellen Gefahren umzugehen, ist nach More die Beschleunigung des Fortschrittes. So könne man undemokratischen Staaten stets überlegen bleiben. Da das baldige Entstehen von künstlichen, dem Menschen überlegenen Intelligenzen nicht zu verhindern sei, geht es More nur noch darum, so schnell wie möglich mit den neuen Technologien mittels Chipimplantaten und einem neuronalen Interface zu verschmelzen, um eine größere Kontrolle ausüben zu können. Der zweite Grund, den More in seiner Antwort auf Joy anführt, ist der Vorwurf, der Verzicht auf Technik sei unethisch: Die neuen Techniken würden nämlich nicht nur der Bekämpfung von Krankheiten zugutekommen, sondern auch das Altern und den Tod besiegen können. Technik führe damit nicht zur Ausrottung des Menschen, sondern zu seiner Immortalisierung. Ungeachtet seiner vorhergehenden Sorge um die Kranken und Schwachen untermauert More seinen Standpunkt mit Verweis auf seinen bevorzugten Fortschrittsphilosophen Friedrich Nietzsche und dessen Forderung nach der Überwindung des Menschen. ${ }^{216}$

Moravec, Minsky und More führen also v.a. ökonomische, medizinische und politische Faktoren an, die einen Fortschrittprozess erzwingen werden. Alle hier aufgeführten Aspekte der Obsoletheit des biologischen

214 Moravec \& Pohl 1993, 68.

${ }^{215}$ Vgl. Joy 2000; Lavery 2003, $284 \mathrm{f}$.

${ }^{216}$ Vgl. More 2000b, 1f.; Dery 1996, 302. 
Menschen im Vergleich zu den Potentialen der Maschinen hatte übrigens bereits Arthur C. Clarke 1958 in seinem populärwissenschaftlichen Buch Profiles of the Future aufgeführt, inklusive des Verweises auf Nietzsche. ${ }^{217}$

Eine kosmische Perspektive nehmen Ray Kurzweil und Hans Moravec ein. Jedoch ist es der Physiker Frank Tipler, der zuerst und ausführlichst diese Bedrohungslage erörtert hat. Zusammen mit John D. Barrow schildert er, wie in fünf Milliarden Jahren unsere Sonne erlöschen werde, in $10^{12}$ Jahren die langlebigsten Sterne zu Weißen Zwergen mutieren werden, in $10^{19} \mathrm{Jahren}$ dann die letzten Neutronensterne auf $100^{\circ} \mathrm{K}$ abkühlen werden, in $10^{31}$ Jahren die Protonen zerfallen werden, in $10^{1500}$ Jahren alle Materie in Eisen verwandelt sein werde, das wiederum in $10^{2600}$ Jahren in Schwarzen Löchern kollabieren werde: ${ }^{218}$

If the human species, or indeed any part of the biosphere, is to continue to survive, it must eventually leave the Earth and colonize space. For the simple fact of the matter is, the planet Earth is doomed. The Sun is becoming more luminous every day, and in about 7 billion years its outer atmosphere will have expanded engulf the Earth ... Gaia, like all mothers, is not immortal. ${ }^{219}$

Als weitere alternative Lebensbedrohung bezeichnet Tipler den Wärmetod des Universums, die so genannte Entropie. Nachdem der Mensch wie wir bereits gesehen haben - als Muster von Informationen definiert wurde, impliziert die Gefahr der kosmischen Entropie für Menschen und Maschinen auf verschiedenen Ebenen eine unausweichliche und existentielle Bedrohung, die in ferner Zukunft den Menschen und seine Nachkommen erwarte. ${ }^{220}$ Tipler fordert daher seine Leser auf, nicht tatenlos zuzusehen, wie die Biosphäre unseres Planeten durch kosmische Prozesse in einigen Milliarden Jahren zerstört wird (kurzfristigen Umweltschutz thematisiert er nicht). Denn damit würde unser gegenwärtiges Leben letztlich sinnlos bleiben: »Without progress, the complete and total extermination of all life is inevitable. ${ }^{221}$ Verweist schon sein Postulat des ewigen Lebens auf die besondere Rolle des Menschen im

${ }^{217}$ Vgl. Clarke 1960, 197-233.

${ }^{218}$ Die sehr heißen aber massearmen Weißen Zwerge entstehen, wenn ein Stern seinen gesamten Vorrat an Brennmaterial für die Kernfusion verbraucht hat. Vgl. Barrow \& Tipler 1986, 613-658; Tipler 1995, 56 f.

${ }^{219}$ A.a.O., 18. Dies ist der einzige fettgedruckte Satz in Tiplers Buch!

${ }^{220}$ Vgl. a.a.O., 87; Hayles 1999, 104.

221 Tipler 1995, 66. 
Universum, so ist für Tipler klar, dass mit der Bewahrung des eigenen Lebens noch höhere Aufgaben für den Menschen verbunden sind und der Mensch den vermeintlichen Wärmetod des Universums verhindern kann: »Thus ultimately life exists in order to prevent the Universe from destroying itself! $\ll^{222}$

Wohl angeregt durch Tiplers kosmologische Überlegungen behandelt auch Moravec das unerquickliche Entropieproblem. Mit explizitem Bezug auf Tipler, Barrow und den Physiker Freeman Dyson entwirft Moravec die Idee eines gigantischen Akkumulators, der die Energie von Photonen zwischen zwei Spiegeln speichern und so das Überleben nach dem eigentlichen Ende des Universums garantieren soll. ${ }^{223}$

Ebenso hatten Max More und sein philosophischer Partner, T. O. Morrow, bereits 1988 als positives Gegenstück zur Entropie den Terminus Extropy kreiert, den sie als Synonym für die unendliche Aufwärtsbewegung und Verbreitung des Lebens definieren. Die Extropianer verstehen unter dem Sammelbegriff Extropy alle Kräfte, die der Entropie entgegenwirken. Spezifische Rettungsszenarien liegen jedoch nicht vor. $^{224}$

Ohne konkrete Bezüge auf den Wärmetod des Universums betont auch Ray Kurzweil die Notwendigkeit der technischen Weiterentwicklung der Menschheit, um künftige Bedrohungen durch Asteroiden und Außerirdische zu meistern und um in ferner Zukunft das Schicksal des Universums in die eigenen Hände nehmen zu können: ${ }^{225}$

So, will the Universe end in a big crunch, or in an infinite expansion of dead stars, or in some other manner? ... Rather, the fate of the Universe is a decision yet to be made, one which we will intelligently consider when the time is right. ${ }^{226}$

Zwar bleiben die Motive für die Expansion des Lebens von der Erde ins Universum bei Kurzweil nur skizzenhaft. Jedoch verwendet er in The Singularity is near (2005) viel Raum darauf, diese Kolonisierung des gesamten Kosmos zu illustrieren. ${ }^{227}$

\footnotetext{
${ }^{222}$ Barrow \& Tipler 1986, 674.

${ }^{223}$ Vgl. Moravec 1988, 147-149. Vgl. hierzu auch Dyson 1988.

${ }^{224}$ Vgl. More 2000a und http://www.aleph.se/Trans/Words/e.html\#EXTROPIA.

${ }^{225}$ Vgl. Kurzweil 1999a, 258-260. Zu diesem Zeitpunkt geht Kurzweil noch von der Existenz außerirdischen Lebens aus.

${ }^{226}$ A.a.O., 260.

${ }^{227}$ Vgl. Kurzweil 2005, 342-368.
} 
Das Bemerkenswerte an dieser kosmischen Perspektive, die Tipler, Moravec, More und Kurzweil einnehmen, ist die Bündelung physikalischer, kosmologischer und kybernetischer Gesichtspunkte der künftigen Bedrohung. Der englische Physiker William Thomson, d.i. Lord Kelvin, entwickelte in den 1850er Jahren die Idee des Endes aller thermodynamischen Prozesse im Universum in einem künftigen Zeitpunkt, an dem sämtliche Energie/Wärme im Kosmos in ein vollkommenes Gleichgewicht übergegangen sein wird. Hermann von Helmholtz prägte in seinen Königsberger Vorlesungen von 1854 dafür den Begriff des »Wärmetods« des Universums (der eigentlich ein Kältetod ist).

Auch das Leben der Pflanzen, Menschen und Thiere kann natürlich nicht weiterbestehen, wenn die Sonne ihre höhere Temperatur und damit ihr Licht verloren hat, wenn sämmtliche Bestandtheile der Erdoberfläche die chemischen Verbindungen geschlossen haben werden, welche ihre Verwandschaftskräfte fordern. Kurz das Weltall wird von da an zu ewiger Ruhe verurtheilt sein. ${ }^{228}$

In den 1870er Jahren verband der österreichische Physiker Ludwig Boltzmann diese Annahmen von Thomson und Helmholtz mit dem Konzept der Entropie. Der Übergang zum thermischen Gleichgewicht des Universums war gemäß dem 2. Hauptsatz der Thermodynamik eine Entwicklung von einem unwahrscheinlicheren zu einem wahrscheinlicheren Zustand. Diese Idee ersetzte für aufgeklärte Geister die althergebrachten religiösen Vorstellungen einer Apokalypse. Der »Wärmetod des Kosmos" oder der »heat death of the universe « wurde seitdem in zahlreichen literarischen Entwürfen verarbeitet und insbesondere in der englischsprachigen Welt durch Autoren wie H. G. Wells, Kurt Vonnegut und Bret Easton rasch popularisiert. Auch Philosophen wie Herbert Spencer, Henri Bergson, Friedrich Engels und Friedrich Nietzsche setzten sich mit Entropie und Wärmetod auseinander. ${ }^{229}$

Ein halbes Jahrhundert nach Boltzmann verwendete der Begründer der technischen Informationstheorie, Claude Shannon, die Idee der Entropie als Wahrscheinlichkeitsmaß für die Unbestimmbarkeit oder Unsicherheit von Informationen. Es beschreibt, wieviel Wahlfreiheit in die Auswahl eines Ereignisses (oder einer Information) einbezogen ist oder wie ungewiss wir bezüglich des Ergebnisses sind. Die Entropie als prä-

\footnotetext{
${ }^{228}$ Hermann von Helmholtz (1876): Über die Wechselwirkungen der Naturkräfte und die daraus bezüglichen neuesten Ermittelungen der Physik. In: Populäre wissenschaftliche Vorträge, 2. Heft, 116f. Zitiert nach Freese 2006, 9.

${ }^{229}$ Vgl. Freese 2006, 7-21; Hölscher 2016, 150-163.
} 
ziser Begriff für Unsicherheit bzw. Informationsgewinn ist damit von großer Bedeutung bei der kompakten Kodierung von Quellen und der Beschreibung der Leistungsfähigkeit gestörter Kanäle. Da die Störungen von Übertragungssignalen das wesentliche Problemfeld bei der technischen Übertragung von Informationen darstellen, markiert die Entropie einen der zentralen Begriffe der technischen Informationstheorie. ${ }^{230}$

Während Shannon die Entropie nun als Unsicherheit der Information bezeichnet und daraus schließt, dass eine unerwartete Information stets mehr Informationen enthält - Entropie also positive Konnotationen impliziert - betrachtet Norbert Wiener in seinem Aufsatz The Thermodynamics of the Message von 1955 die Entropie als allgemeines Maß der Unordnung eines dynamischen Systems und stellt parallel zu der gleichlautenden Auffassung des Physikers Leon Brillouin die von manchen Kybernetikern vehement zurückgewiesene Verbindung zwischen der physikalischen Thermodynamik und der Informationstheorie her: ${ }^{231}$ »Information is equivalent to order, and a message may be garbled, but never ungarbled. ${ }^{232}$

Wiener und viele andere übernahmen die von Leo Szilard in einem Gedankenexperiment aufgeworfene Überlegung, dass Entropie mit dem Gegenteil von Information korreliere. Als Auflösung von Struktur und Ordnung erlangt die Entropie für Wiener die moralische Qualität von Tod und Untergang, denn wenn Gregory Bateson Information als Unterschied definiert, der einen Unterschied macht, dann bedeutet völlige Entropie völlige Unterschiedslosigkeit und das Fehlen jeglicher Information, was dem Ende des Lebens im Universum gleichkommen würde. ${ }^{233}$ Es war daher auch niemand Geringeres als Norbert Wiener, der bereits zu Beginn der von ihm begründeten Wissenschaft die Kybernetik als Lebenswissenschaft im Kontext der kosmischen Bedrohung allen Lebens durch die Entropie definierte:

${ }^{230}$ Vgl. Shannon \& Weaver 1976, 59-68, 78-90; Wiener 1963, 103-142; Mathar 1996, 22-39. Der Begriff einer mikroskopischen Entropie wurde bereits 1932 bzw. 1939 von John von Neumann und Satosi Watanabe eingeführt.

${ }^{231}$ Vgl. Shannon \& Weaver 1976, 38f.; Wiener 1985, 206-211; Brillouin entwickelt in seinem Prinzip der Negentropie ein umfangreiches Beziehungsgeflecht zwischen dem kybernetischen und dem physikalischen Entropiebegriff. Vgl. Brillouin 1957, 114-201, 245-258. Klaus empfiehlt dagegen, den Begriff Entropie in der Kybernetik überhaupt zu meiden. Vgl. Klaus 1969a, 177.

${ }^{232}$ Wiener 1985, 206.

${ }^{233}$ Vgl. Wiener 1964, 31-36, 130; Hayles 1999, 100-104. 
But while the universe as a whole ... tends to run down, there are local enclaves whose direction seems opposed to that of the universe at large and in which there is a limited and temporary tendency for organization to increase. Life finds its home in some of these enclaves. It is with this point of view at its core that the new science of Cybernetics began its development. ${ }^{234}$

Auch John von Neumann sieht physikalische und informationstheoretische Entropie als Maß für die Degeneration von Energie und Information an:

There is reason to believe that the general degeneration laws, which hold when entropy is used as a measure of the hierarchic position of energy, have valid analogs when entropy is used as a measure of information. On this basis one may suspect the existence of connections between thermodynamics and new extensions of logics. ${ }^{235}$

Diese kosmische Dimension der Bedrohungen nimmt im gegenwärtigen Posthumanismus einen größeren Raum ein als um die Jahrtausendwende. Rhetorisch verleiht sie dem Posthumanismus eine nicht mehr zu überbietende Relevanz: Die Rettung des ganzen Universums liegt nun in den Händen der technologischen Propheten, die für eine unumgängliche Staffelübergabe an die erwarteten künstlichen Intelligenzen werben. Die Alternative liegt aus dieser Sichtweise in der Verantwortung für das endgültige Erlöschen allen Lebens in den Milliarden Galaxien unserer Welt. Wer will diese unermessliche Schuld auf sich nehmen? Argumentativ bilden die Frage der Entropie, das Konzept der Singularität, die erhoffte Ankunft der Superintelligenz und die Vision eines vernetzten, universalen Gehirns einen Zirkel. Komplementär dazu stehen die konkreten Maßnahmen, die die Defizite des biologischen Menschen (Alter, Krankheit, Tod) überwinden sollen. Dass der prognostizierte Fortschritt Aussicht auf die erfolgreiche Bekämpfung von menschlichen Leiden und die Beherrschung von Naturgewalten bietet, gehört seit Francis Bacons Nova Atlantis zum guten Ton jeder Art von Zukunftsoptimismus. ${ }^{236}$ Neu dagegen ist im Posthumanismus, dass die aufgezählten Gefahren eine totale und existentielle Bedrohung des menschlichen Lebens darstellen: Auf individueller Ebene werden nicht Krankheiten, sondern der Tod selbst genannt - während auf kollektiver Ebene der

${ }^{234}$ Vorwort nur in der amerikanischen Ausgabe, Wiener 1988, 12.

${ }^{235}$ Neumann 1966, 61.

${ }^{236}$ Vgl. Bacon 1959, 51-102; Tabbert 2004, 85-104. 
Untergang des gesamten Universums als größte und absolute Bedrohung thematisiert wird. In den vergangenen 20 Jahren können wir eine stärkere Betonung dieser kosmischen Perspektive im Posthumanismus beobachten.

Es ist somit evident, dass der Fortschritt im posthumanistischen Sinn ein Imperativ ist, für den es keine Alternative gibt. Das schließt die Auslöschung des biologischen Menschen mit ein. Die folgenden Kapitel behandeln im Einzelnen, wie dieser Fortschrittsprozess legitimiert und ausgestaltet wird.

\subsubsection{Das Opfer des Menschen}

Nicht weil das Flügelwachs versagt, stürzt heute Ikarus, sondern weil Ikarus selbst versagt.

Könnte er sich selbst als Ballast abwerfen, seine Flügel könnten den Himmel erobern. ${ }^{237}$

Günther Anders

Sofern Fortschritt stets impliziert, dass man von einem Ort fortschreitet, so ist ein Zurücklassen und etwas Zurückgelassenes ebenfalls miteingeschlossen. Es scheint nicht allzu fern zu liegen, die im posthumanistischen Diskurs entfaltete Dynamik zwischen Mensch, Technik und Fortschritt unter der Kategorie des Opfers zu charakterisieren, sofern unter Opfer die Aufgabe eines bisherigen Wertes zugunsten eines erwarteten, höheren Gewinns verstanden werden kann. Mit Bezug auf Günther Anders' Prophezeiung eines dritten Weltkrieges, den die Menschheit in einem postzivilisatorischen Kannibalismus gegen sich selber führen würde, hatte bereits die Ethnologin Johanna Riegler die Kategorie des Opfers eingeführt, um die gegenwärtige Herabsetzung und die damit einhergehende »Abschaffung « eines gegenüber der Technik antiquierten Menschen begrifflich zu fassen:

Die technoexpansive Marktgesellschaft ist eine säkulare Form der Opferung ... Ich behaupte, daß Opferung das Kernstück moderner Technologie und Ökonomie ist und daß, wenn wir uns zeitlich zu sehr vom erbrachten Opfer entfernt haben, die Erinnerung und der Schrecken nachlassen und verblassen, eine neuerliche rituelle Inszenierung im Namen des Fortschrittes ansteht. ${ }^{238}$

${ }^{237}$ Anders 1983, 34.

${ }^{238}$ Riegler 1999, 52. 
Die Obsoletheit des Menschen manifestiert sich im posthumanistischen Diskurs in den zwei voneinander zu unterscheidenden Aspekten erstens des im wahrsten Sinne des Wortes »überholten« biologischen Körpers und zweitens des obsoleten Kollektivs der Menschheit als Gattung. Wurde bereits in den vorhergehenden Ausführungen offenkundig, dass der menschliche Körper mit seinen Beschränkungen und vitalen Anfälligkeiten eine zentrale Ursache für die existentielle Bedrohung der Menschheit repräsentiere, so wird besonders bei Marvin Minsky, Frank Tipler und Hans Moravec deutlich, dass der materielle Körper einem posthumanen Substitut geopfert werden müsse: »We must die - as individuals, as a species - in order that our civilization might live. ${ }^{239}$ Will die Menschheit überleben und sich weiterentwickeln, so gibt es nach Minsky im Angesicht einer durch ungezügelte sexuelle Entfaltung hervorgerufenen Überbevölkerungsproblematik und der beständigen Bedrohung durch das Versagen - den natürlichen Tod - der biologischen »Informationssysteme« keine Alternative zur Überwindung ihrer biologischen Grenzen. ${ }^{240}$ Auch für Frank Tipler werden Überleben und Auferstehung des künftigen Menschen (superhuman oder superbeing) nur durch die Loslösung vom materiellen Körper möglich. ${ }^{241}$ Zudem gibt Minsky zu bedenken, dass die Fehlerkorrektur bei einem Computer keine Schwierigkeit darstellt, während genetische Schäden, die im menschlichen Reproduktionsprozess entstehen, kaum revidierbar seien. Der fehleranfällige menschliche Körper müsse daher durch einen problemlos korrigierbaren Maschinenkörper ersetzt werden. ${ }^{242}$ Bildet das Opfer des Menschen zugunsten einer postbiologischen und posthumanen Kultur einen der Kernpunkte von Hans Moravecs Betrachtungen, so wird bei den Ausführungen des Robotikers wahrnehmbar, wie sehr hier Günther Anders psychologisches Erklärungsmodell der "prometheischen Scham" greift. Alle Motive des für den biologischen Körper unbefriedigenden Vergleiches mit den Maschinen erscheinen bei Moravec: von einzelnen technischen Überlegenheiten bis hin zur Wandlungsfähigkeit der Maschinen. ${ }^{243}$ Hans Moravec ist davon überzeugt, dass der biologische Mensch am Ende des 20. Jahrhunderts

\footnotetext{
${ }^{239}$ Tipler 1989, 245.

${ }^{240}$ Vgl. Minsky 1994, 113; Minsky 1992a, 24ff.

${ }^{241}$ Vgl. Tipler 1995, 87f., 255-259.

${ }^{242}$ V gl. Minsky 1994, 108.

${ }^{243}$ Vgl. Anders 1983, 32-54.
} 
an die Grenzen seiner geistigen Aufnahmefähigkeit gelangt ist. ${ }^{244}$ Der menschliche Körper, der abwertend als wetware bezeichnet wird, besteht nach Ansicht Moravecs aus Sülze (jelly), die mit ihren Neuronen nur zu einer äußerst primitiven Art von Informationsverarbeitung qualifiziert sei, während elektrische Schaltungen eine Milliarde mal schneller und präziser ihre Zwecke erfüllen würden. ${ }^{245}$ Zudem sei die biologische Evolution nicht offen für wirkliche Neuerungen und Verbesserungen ihrer Systeme. ${ }^{246}$ In seitenlangen Tabellen vergleicht Moravec sodann - nach verschiedenen technischen und ökonomischen Kategorien differenziert - den Menschen mit Rechenmaschinen neueren und älteren Datums: »Today, as our machines approach human competence across the board, our stone-age biology and our information-age lives grow ever more dismatched. ${ }^{247}$ Das menschliche Gehirn unterliegt erwartungsgemäß in den meisten der von Moravec ausgewählten Aspekten schon den Computern aus den 1950er Jahren - außer in den Herstellungskosten! Das Aufgehen des Menschen in seinen mind children wäre daher ein großer Gewinn. ${ }^{248}$

Darüber hinaus stehe der menschliche Körper einer Expansion ins Weltall im Wege. Eine als notwendig erachtete Kolonisierung anderer Planeten, Sonnensysteme oder gar Galaxien mit den damit verbundenen jahrzehntelangen Reisen erscheine mit unseren biologischen Körpern völlig utopisch. Aus Gründen der »extraterrestrischen Emanzipation« treten daher besonders Frank Tipler und der Künstler Stelarc für das Design interstellar überlebensfähiger Maschinenwesen ein: ${ }^{249}$

Die Frage steht an, ob ein zweifüßiger, atmender Körper mit einem binokularen Blick und einem Gehirn in der Größe von $1400 \mathrm{~cm}^{3}$ noch eine angemessene biologische Form ist. Er kann die Quantität, Komplexität und Qualität der aufgehäuften Informationen nicht bewältigen; er wird von der Präzision, Geschwindigkeit und Macht der Technologie eingeschüchtert und ist biologisch schlecht dafür ausgerüstet, sich einer neuen extraterrestrischen Umwelt anzupassen. ${ }^{250}$

Während Minsky und Moravec vom Standpunkt einer radikal utilitaristischen Überzeugung allein unter dem Gesichtspunkt der maxima-

\footnotetext{
${ }^{244}$ Vgl. Moravec 1999, 1, $125 f$.

${ }^{245}$ Vgl. Moravec 1988, 55f., 117ff.; Moravec \& Pohl 1993, 72.

${ }^{246}$ Vgl. Moravec 1988, 167f.

${ }^{247}$ Moravec 1999, 7.

${ }^{248}$ Vgl. Moravec 1979, 63-83; Moravec 1988, 18, 61, 173-177; Moravec 1999, 58, 68.

${ }^{249}$ Vgl. Tipler 1995, 18-65; Stelarc 1996, 72ff.; Stelarc 1998, $116 f$.

${ }^{250}$ Stelarc 1996, 74.
} 
len Effizienz argumentieren, beziehen Max More, Kevin Warwick und teils auch Ray Kurzweil eine stärker anthropozentrisch ausgerichtete Position. Hier steht eher die ungebremste Erweiterung der Fähigkeiten des Menschen im Zentrum der posthumanen Visionen, die schließlich $\mathrm{zu}$ einer Überwindung des heutigen carbon-based human being führen werde.

So wendet sich More ähnlich wie Warwick gegen Moravecs Vision von überlegenen Maschinen, die den Menschen hinter sich lassen werden, und favorisiert ein baldiges Verschmelzen mit den Maschinen, um sie unter Kontrolle zu halten: »They become part of us. ${ }^{251}$ In der Aufrüstung des biologischen Körpers mit künstlichen Elementen zum Cyborg sieht Warwick, der sich selbst als Cyborg No. 1 stilisiert, die einzige Überlebenschance des Menschen im Wettbewerb mit den künftigen, dem Menschen überlegenen Maschinengenerationen:

Once the first powerful machine, with an intelligence similar to that of a human, is switched on, we will most likely not get the opportunity to switch it back off again. We will have started a time bomb ticking on the human race, and we will be unable to switch it off. ${ }^{252}$

Auch für Frank Tipler ist zweifelsfrei, dass der Fortbestand der menschlichen Zivilisation nicht die Kontinuität der Spezies Mensch beinhaltet:

...our species Homo sapiens must inevitably become extinct, just as every individual human being must inevitably also die ... it is a logically necessary consequence of eternal progress that our species becomes extinct. ${ }^{253}$

Für Ray Kurzweil erübrigt sich jede Diskussion um die Fortexistenz des Menschen, weil diese Prozesse unabänderlichen, evolutiven GesetzmäBigkeiten unterliegen würden. Die Evolution selbst habe die Computer (mit Hilfe des Menschen) hervorgebracht, um das menschliche Mängelwesen durch eine überlegene Spezies zu ersetzen. Das menschliche Defizit besteht nach Kurzweils Meinung in der primitiven und langsamen Informationsverarbeitung. Der Mensch müsse daher aussterben. ${ }^{254}$ Eine Wahl über die Verdrängung des Menschen durch die Maschinenintelli-

${ }^{251}$ More 2000b, 2.

${ }^{252}$ Warwick 1998, 302.

253 Tipler 1995, $218 f$.

${ }^{254}$ V gl. Kurzweil 1999a, 96f., $101 \mathrm{f}$. 
genz bleibe uns nicht mehr, da der point of no return bereits überschritten worden sei - der Verzicht auf Computer sei folglich nicht mehr möglich:

We can't stop. The Law of Accelerating Returns forbids it! It's the only way to keep evolution going at an accelerating pace. [ ...] The accelerating pace of change is inexorable. The emergence of machine intelligence that exceeds human intelligence in all of its broad diversity is inevitable. ${ }^{255}$

Die Beschleunigung der Evolution selbst wird hier zum Zweck der Evolution und erfordert die Aufgabe der Menschheit, um nicht ihren Fortgang zu gefährden.

Wenn posthumanistische Denker heute so vehement für die Überwindung der Menschheit eintreten - Stanisław Lem sprach schon 1964 von »einer merkwürdigen Form der Euthanasie, so etwas wie ein angenehmer zivilisatorischer Selbstmord ${ }^{256}$ - und bereit sind, den Menschen zugunsten eines ungebremsten Fortschrittes zu opfern, so drängt sich unweigerlich die Frage nach den ideengeschichtlichen Quellen auf. Auf welche Vorbilder und Argumentationsstrategien greifen die Posthumanisten zurück, wenn sie einerseits mit der implizierten Annahme, selber zum Neuen einer transhumanen Elite zu gehören, das Alte zurücklassen wollen? Oder wenn sie andererseits in der Hoffnung auf eine universale Auferstehungsmaschine und den omnihistorischen Simulationsapparat in ferner Zukunft eine vorläufige Opferung ihrer selbst in Kauf nehmen? Es sind ganze vier Ideenkomplexe der neuzeitlichen Fortschrittsideologien, die mit teils differierenden Perspektiven in einem posthumanistischen Nexus kulminieren.

Zunächst rezipieren Hans Moravec und Marvin Minsky - abgesehen von einer biologisch-anthropologischen Komponente - einen Gedanken des französischen Philosophen Diderot, wenn sie die nachfolgenden Generationen von Robotern und künstlich intelligenten Computerprogrammen als ihre »Geisteskinder« bezeichnen und gleichzeitig ihre eigene elterliche Aufgabe und Erfüllung ihres Daseinszweckes in der Hervorbringung eben dieser vielversprechenderen Nachkommenschaft zu finden glauben. Als aufgeklärter Vertreter einer sich ausbildenden französischen Fortschrittsidee bestimmte Diderot nach dem Verlust eines religiös-transzendenten Jenseits den Lebenssinn im Sich-Aufopfern für die zukünftigen Generationen, die einmal von den Früchten der Arbeit und

${ }^{255}$ Kurzweil 1999a, 130, 253. Vgl. auch a.a.O., 157f., 186.

${ }^{256}$ Lem 1981, 340. 
Mühen der Alten profitieren sollten. Diderots großer Einfluss auf die englische bzw. amerikanische Fortschrittsidee erklärt die nachhaltige Rezeption dieser Gleichsetzung einer biologischen Eltern-Kind-Beziehung mit der evolutiven Abfolge der Arten im Posthumanismus. ${ }^{257}$

Unter einem darwinistischen Paradigma wurde das Aussterben einer Gattung schon früh als Aufgehen in einer neuen Art gedeutet, als natürlicher Prozess der Evolutionsgeschichte. ${ }^{258}$ Eine der wirkungsreichsten Popularisierungen und Weiterführungen von Darwins Theorie vollzog der deutsche Philosoph und Vordenker der antisemitischen Rassentheorien Eugen Dühring in seinem Werk Der Werth des Lebens (1865). Er wirft die Frage auf, was mit einer Art geschieht, wenn sie die höchste Form ihrer Entwicklung erreicht hat. Das Aussterben einer Art versteht er nicht als Tod und absolutes Ende einer Gattung, sondern als Abfolge von unendlich vielen Veränderungen und neuen Kombinationen der Ursprungselemente:

Man wird alsdann sagen, eine Artung sei untergegangen, um einer anderen platzzumachen. Auf diese Weise könnte sich einst auch die Menschheit in einem vollkommeneren Wesenstypus übergeführt finden und auf diejenige Menschengestalt, die uns als die entwickelste gilt, als auf eine ausgestorbene Thierart zurückblicken. ${ }^{259}$

Aufgrund der Annahme eines allgemeinen Entwicklungsgesetzes rechnet Dühring bereits mit der Möglichkeit einer »veredelten und erheblich anders ausgestatten Gattung ${ }^{260}$, die aus dem zu überwindenden, bisherigen Menschengeschlecht hervorgehen werde. Wenige auserwählte Rassen der Menschen seien daher Träger dieses höheren Lebens, während alle anderen aus der künftigen Evolution ausscheiden würden. ${ }^{261}$

${ }^{257}$ Auch Robbespierre und Herder entwickelten in unterschiedlichen Kontexten den Gedanken einer Nachwelt, in der das menschliche Leiden und Schaffen der Gegenwart seinen Sinn finden wird. Baillie vergleicht dieses aufklärerische Aufgehen in der Nachwelt sogar mit Abrahams Aussicht auf eine reiche Nachkommenschaft in der israelitischen Religion, als der Glaube an eine unsterbliche Seele noch nicht ausgebildet war. Vgl. Baillie 1950, 110-114; Sampson 1956, $129 \mathrm{ff}$.

${ }^{258}$ Vgl. hierzu die kritische Diskussion von Rapp 1992, 73-77. Nicht zu unterschätzen ist in diesem Zusammenhang auch die Rolle des international einflussreichen Evolutionstheoretikers Ernst Haeckel bei der Popularisierung von Darwins Theorie. Vgl. Bolle 1962, 146-152.

${ }^{259}$ Eugen Dühring: Der Werth des Lebens. Leipzig ${ }^{3} 1881$, 194. Zitiert nach Benz 1965, 106 f.

${ }^{260}$ A.a.O., 195. Zitiert nach Benz 1965, 107.

${ }^{261}$ Vgl. Benz 1965, 105-109. 
Einerseits operieren die posthumanistischen Autoren nun mit dem evolutionistischen Modell der mangelnden Nichtanpassung, die langfristig - dann aber zwangsläufig - zum Aussterben der menschlichen Art führen werde. Hierbei werden jeweils verschiedene Aspekte der veränderten Umweltbedingungen, denen der gegenwärtige Mensch nicht mehr gewachsen sei, fokussiert: Minsky, Moravec, Kurzweil und Warwick betonen wie vormals Robert Jastrow insbesondere die vermeintlichen Defizite in Geschwindigkeit und Speichervermögen der menschlichen »Informationsverarbeitung«, während Tipler und der Künstler Stelarc namentlich die Obsoletheit des Menschen im interplanetaren Weltraumzeitalter unterstreichen. Unter diesen reduzierten Voraussetzungen gehört die Menschheit im natürlichen Selektionsprozess der Evolution zu den vom Aussterben bedrohten Arten. Andererseits jedoch eröffnet die Evolutionstheorie das Modell des Aufgehens in den posthumanen Nachfolgern des Menschengeschlechtes, was in Moravecs Metapher von den mind children wohl seinen treffendsten Ausdruck findet.

Unabhängig vom darwinistischen Denken hat die Überwindung des Alten als konstitutives Element von Identität in der amerikanischen Zivilisationsgeschichte eine prägende Bedeutung erlangt. Die positive Bewertung des Losreißens vom Alten hatte ihre höchste Signifikanz im Puritanismus des 17. Jahrhunderts, der die althergebrachte katholische Lehre als korrupt und Teufelswerk verurteilte und Rom mitsamt der Antike als Antipode des progress - der ewigen Glückseligkeit - verdammte. Wohl als erster verlieh John Bunyan mit seinem Werk Pilgrim's Progress (1678) dieser Idee einen literarischen Ausdruck, noch bevor Cotton Mather in der Magnalia Christi Americana (1702) beweisen wollte, dass erst die Loslösung der neuenglischen religiösen Siedler von Europa die Reformation zu ihrer Vollendung bringen würde. Bis ins 19. Jahrhundert hinein wurde der Weg aus dem korrupten Europa in religiöser Literatur wie auch in weltlichen Geschichtswerken mit dem biblischen Exodus des auserwählten Volkes verglichen, das nun in ihrem »gelobten Land « stetig fortschreiten werde - hinzu auf das gottgewollte Ziel. Die Loslösung vom alten Europa und vom Alten an sich ist damit in dieser für die Unabhängigkeit des neuen Amerika konstitutiven Frage durchweg positiv besetzt. ${ }^{262}$

Damit verbunden ist die Frage nach dem Alten Amerika. Nicht nur der Aspekt des Zurücklassens des Alten, sondern auch die direkte Be-

${ }^{262}$ Vgl. Sampson 1956; Buchloh 1963, 159-165. 
gegnung mit den offenbar fortschrittsnegierenden indigenen Völkern Nordamerikas wurden in der amerikanischen philosophischen und politischen Literatur noch Mitte des 20. Jahrhunderts thematisiert. Mit der weiteren Expansion der amerikanischen Siedler um 1800 und dem kontinuierlichen Vorrücken der frontier gen Westen wurde die Konfrontation mit den »Indianern « akut - die bis dahin dank der aufklärerischen Philosophen Rousseau, Lafitau, Lahontan und anderen als die »edlen Wilden« idealisiert wurden. ${ }^{263}$ In der westlichen und südlichen Presse der jungen Vereinigten Staaten konnte man sehr genau beobachten, wie nun vom Indianer das Bild eines unproduktiven, verschwenderischen und zum Fortschritt unfähigen Barbaren gezeichnet wurde. Einen Höhepunkt erreichte diese Entwicklung 1830, als Präsident Andrew Jackson die Vertreibung der Indianer als notwendige Maßnahme zur Gewährleistung des Weiteren Fortschrittes verteidigte:

Humanity has often been wept over the fate of the aborigines of this country, and Philanthropy has been long busily employed in devising means to avert it, but its progress has never for a moment been arrested, and one by one have many powerful tribes disappeared from the earth. ${ }^{264}$

Höchst wirkungsvoll hatte kurze Zeit später der amerikanische Staatsmann Joel R. Poinsett in einer Schrift von 1834 den "Indianern « aus rassischen Gründen die Fähigkeit zur Weiterentwicklung und Zivilisation abgesprochen, die vor allem der kaukasischen Rasse vorbehalten sei. ${ }^{265}$ Indianer wurden zum Sinnbild der barbarischen Rückständigkeit, die ein Hindernis und eine Gefährdung der zivilisierten Fortschrittsgesellschaft des weißen Mannes bedeuten würden. Auch wenn es vereinzelt Gegenstimmen gab, so wurden die Vertreibung und der damit einhergehende Untergang der Indigenen im Allgemeinen als notwendiges Opfer des Fortschrittes in Kauf genommen. Vertreibung, Entrechtung, Ausrottung und Kindeswegnahmen prägten den Umgang mit den indigenen Völkern in den Vereinigten Staaten. Letztere Praxis bestand bis in die 1970er Jahre. Die amerikanische Fortschrittsidee zerstörte somit das humanistische Ideal des »edlen Wilden« wie auch die Indigenen selbst. ${ }^{266}$

\footnotetext{
${ }^{263}$ Vgl. Baillie 1950, 92f.; Rohbeck 1987, 80-84.

${ }^{264}$ Andrew Jackson: Second Annual Message. 06.12.1830, zitiert nach Ekirch 1944, 43.

${ }^{265}$ Poinsett schrieb das einflussreiche Werk Inquiry into the Received Opinions of Philosophers and Historians, on the Natural Progress of the Human Race from Barbarism to Civilization. Charleston 1834.

${ }^{266}$ Vgl. Ekirch 1944, 41-46; Rohbeck 1987, 80-84.
} 
Der forschrittsbejahende Indianerdiskurs gerade des 19. Jahrhunderts bietet dem Posthumanismus ein unvergleichbar konkretes Muster an - auf das sich freilich niemand explizit bezieht, nachdem die Ausrottung der Indianer vom heutigen moralischen Standpunkt auch in der US-Öffentlichkeit bisweilen kritischer gesehen wird. Gleichwohl muss davon ausgegangen werden, dass bestimmte Deutungsmuster von den Posthumanisten rezipiert werden. Die fundamentale Alternative zwischen einer propagierten Anpassung - also heute der Cyborgisierung bei Kurzweil, More und Warwick oder dem (vorläufigen) Aussterben bei Tipler, Minsky und Moravec ist in der 200 Jahre alten Diskussion über das Schicksal der indigenen Völker Amerikas bereits angelegt. Ebenso tritt hier die biologistische Verknüpfung zwischen Rasse und der Unfähigkeit zum Fortschritt und zum Überleben in Erscheinung, die offensichtlich in der These vom unausweichlichen Untergang der menschlichen, biologischen Gattung ihren posthumanistischen Widerhall findet.

Mit unterschiedlichen Nuancen der einzelnen posthumanistischen $\mathrm{Au}-$ toren werden die dargestellten vier Motive neuzeitlicher Fortschrittsideen teils explizit, teils implizit rezipiert. Allen voran bieten verschiedene Aspekte des historischen amerikanischen Fortschrittsdiskurses Muster an, die die Aufgabe und den Untergang des Bisherigen durchaus positiv bewerten. Die letztlich teleologische Rezeption der darwinistischen Evolutionstheorie, die einen stetigen Aufstiegsprozess der Arten zugrunde legt, legitimiert darüber hinaus das Bild von der Transformation und dem elterlichen Aufgehen der menschlichen Gattung in die sie überragende posthumane Spezies. Dabei wird die Überlegenheit der zur Zeit noch fiktionalen künstlichen Intelligenzen eines posthumanen Zeitalters und die Minderwertigkeit des biologischen Körpers einzig aus dem utilitaristischen Kriterium der Effizienz deduziert.

Das Fazit der posthumanistischen Opferbereitschaft klingt paradox: Der Mensch soll freiwillig auf sich selbst verzichten, um sein Überleben zu sichern. Sinn ergibt diese Aussage, wenn mit dem Glauben an einen ungeheuren technischen Fortschritt auch die Überzeugung einer technisch ermöglichten Auferstehung als Simulation verbunden wird oder aber die Menschheit ihren Daseinszweck in der Hervorbringung der posthumanen Wesen erfüllt sieht. Den Hintergrund dieser Anschauungen - und damit das fundamentale Rezeptionsmuster - bildet das posthumanistische Menschenbild, denn so wie die individuelle Persönlichkeit nicht an einen konkreten Körper gebunden ist, so besteht auch die Kultur und 
Identität der Menschheit unabhängig von ihrer biologischen Basis. Gemäß dieser Logik kann die menschliche Kultur nur ohne den Menschen überleben: weil die Menschheit vergänglich ist, die Kultur aber nicht:

Given fully intelligent robots, culture becomes completely independent of biology. Intelligent machines, which will grow from us, learn our skills, and initially share our goals and values, will be the children of our minds. ${ }^{267}$

\subsubsection{Fortschritt und Vollkommenheit}

Die posthumanistischen Denker vereint die Idee eines unaufhörlichen Aufstiegs der Menschheit bis zu ihrem Aufgehen in eine posthumane Lebensform. Die Basis dieses Fortschrittes wurde referiert, wobei sich die bisherigen Ausführungen auf die Rolle der Technik im Prozess des Fortschrittes beschränkten. Die bislang angesprochenen Aspekte des drohenden Untergangs des Lebens im Universum und des Opfers des biologischen Menschen sind dabei Elemente einer übergreifenden Konzeption der menschlichen bzw. kosmologischen Geschichte. Die Annahme einer Maximierung informationsverarbeitender Leistungen und die einzelnen, daraus hervorgehenden Fortschritte werden von den posthumanistischen Autoren auf vielfältige Weise in einen entwicklungsgeschichtlichen Zusammenhang gestellt. Es drängt sich infolgedessen die Frage auf, wie der beobachtete technische Fortschrittsprozess von den Posthumanisten gedeutet wird. Welche Konzeptionen von Geschichte und menschlicher Entwicklung werden hier rezipiert?

Frank Tipler und John D. Barrow verwenden viel Mühe darauf, die Wiedereinführung der Teleologie in die kosmologische Wissenschaft zu rechtfertigen, wobei sie mit der Frage nach der Zukunft des Universums gleichzeitig die Frage nach Sinn und Zweck unseres gegenwärtigen Daseins aufwerfen. Das zugrunde gelegte, starke anthropische Prinzip geht davon aus, dass die Menschheit Teil eines kosmischen Planes ist und eine zentrale Aufgabe im Universum zu erfüllen hat. Indem Tipler und Barrow ausdrücklich an die Metaphysik des griechischen Philosophen Aristoteles anknüpfen, legen sie nahe, das Wesen einer Sache oder eines Lebewesens von seiner letzten Ursache, seinem Endzweck, her

${ }^{267}$ Moravec 1999, 126. 
zu bestimmen. ${ }^{268}$ Ferner präsentieren Tipler und Barrow teleologische Elemente unzähliger Philosophen aus zwei Jahrtausenden, die ihr $d e$ sign argument untermauern können, ohne dass sie jedoch den Kontext der Werke berücksichtigen. Platon, Cicero, Galen, Boethius, Johannes Kepler, Richard Bentley, William Derham und Voltaire werden herangezogen, um zu zeigen, wie verbreitet die Idee eines geplanten Universums gewesen sei. ${ }^{269}$ Anschließend versuchen Tipler und Barrow nun ihrerseits, mit naturwissenschaftlichen Fakten die besondere Rolle des Menschen im Universum zu beweisen. ${ }^{270}$ In der großen Kette der von Gott geplanten, kosmischen Geschichte sei der Mensch ein unverzichtbares Zwischenglied auf dem Wege zum Punkt Omega: »If the ascent of Life into the Omega Point is to occur, one day the most advanced minds must be non-Homo sapiens. The heirs of our civilization must be another species, and their heirs yet another, ad infinitum into the Omega Point. ${ }^{271}$ Damit der Mensch Einfluss auf die künftige Entwicklung des Universums nehmen und der Punkt Omega überhaupt erst durch das Wirken intelligenten Lebens realisiert werden könne, sei es nötig, dass die Nachfahren der Menschheit den Kosmos erobern und ihre Biosphäre auf das ganze Universum ausdehnen. In den nächsten fünfzig Jahren sollten nach Tiplers Vorstellung die ersten selbstreproduzierenden Von-Neumann-Sonden gestartet werden, die innerhalb von $600.000 \mathrm{Jah}-$ ren dann schon die gesamte Milchstraße kolonisiert haben würden. ${ }^{272}$ Aus dem finalen anthropischen Prinzip leitet Tipler das Postulat des ewigen Lebens ab, das nicht nur die Notwendigkeit des Überlebens der menschlichen Zivilisation, sondern die des ganzen Universums beweisen soll. Das Erreichen dieser kollektiven Unsterblichkeit macht er vor allem von der Bedingung abhängig, dass bis zum Ende der Zeit - der von Roger Penrose eingeführten k-Grenze - Informationsverarbeitung, das heißt Leben, fortgesetzt werden kann. ${ }^{273}$ Das Postulat des ewigen Lebens und die Überzeugung, dass die Menschen die einzigen intelligenten Lebewesen im Universum sind, gebieten Frank Tipler, dass er in gleicher Weise an den ewigen Fortschritt der menschlichen Zivilisation

\footnotetext{
${ }^{268}$ Vgl. Barrow \& Tipler 1986, 37-40.

${ }^{269}$ Vgl. a.a.O., 33f., 43ff., 50, 61, $67 \mathrm{f}$.

${ }^{270}$ Vgl. a.a.O., 219-601.

271 Tipler 1989, 245.

272 Vgl. a.a.O., 44-55.

${ }^{273}$ Vgl. Tipler 1995, 132 f.
} 
glaubt. ${ }^{274} \mathrm{Da}$ die Welt als Schöpfung Gottes gut sei, werde auch alles, was aus ihr hervorgehe, letztlich gut sein: ${ }^{275}$

Any cosmology with progress to infinity will necessarily end in God. Further, the hope of eternal worldly progress and the hope of individual survival beyond the grave turn out to be the same. Far from being polar opposites, these two hopes require each other; one cannot have one without the other. The Omega Point is truly the God of Hope: »O death, where is thy sting? O grave, where is thy victory?«(I Corinthians 15:55) 276

Der beobachtbare Fortschritt in der Menschheitsgeschichte und die Entwicklung der Lebensformen in der Evolutionsgeschichte sind für Tipler Teile des kosmischen Planes Gottes, der nach der vollen Entfaltung seiner weltlichen Schöpfung im Punkt Omega selbst erst zu seiner äußersten Verwirklichung finden werde.

Marvin Minsky stellt die Entwicklung der künstlichen Intelligenz und posthumaner Lebensformen nicht in die Kontinuität der natürlichen Evolution, sondern kontrastiert eine weitgehend ziellose Entstehung des biologischen Lebens mit der künftigen, bewussten Planung künstlicher Lebensformen. Nach Ansicht Minskys arbeitet die Evolution nicht intelligent, da sie zu viel Zeit für die Lösung des Lebensproblems benötigt habe und sich allzu oft kurzsichtig für unpraktische Regelungen entschieden habe. Statt durch eine vernünftige und reflektierte Planung sei der Mensch durch einen primitiven Kampf der Gene gekennzeichnet, dessen aggressiver Charakter sich sogar noch in den unsinnigen Sportund Wettkampfspielen niederschlage. ${ }^{277}$

Die - auch unter Posthumanisten - weit verbreitete Annahme eines grundlegenden Zieles oder Prinzips der Evolution weist Minsky zunächst einmal zurück: Nicht ein elementarer Lebenswille, sondern viele einzelne Faktoren führten zum Überleben einer Spezies.

But to attribute this [das Überleben: O.K.] to any single, central force or to some basic, underlying survival instinct is as foolish as believing in special powers that attract corpses to cemeteries or broken cars to scrapyards ... The myth of an underlying survival instinct explains nothing that cannot better be explained

${ }^{274}$ Vgl. a.a.O., 104-123.

275 Vgl. Tipler 1989, 244 f.

276 Tipler 1995, 268.

277 Vgl. Minsky 1988, 71, 146; Minsky 1992a, 24. 
without it, and blinds us to the fact that each of those survival aids may exploit an entirely different mechanism. ${ }^{278}$

Zwar wehrt sich Minsky gegen eine allgemeine Teleologie der Evolution, glaubt jedoch, dass einzelne Systeme in den Lebewesen aller Wahrscheinlichkeit nach eine Verbesserung der Lebensfähigkeit implizieren. ${ }^{279}$ Die Befreiung vom biologischen Körper als Weg zur Lebensverlängerung und Unsterblichkeit ist für Minsky gleichbedeutend mit der Befreiung von der natürlichen Evolution, die kein Interesse an langlebigen, biologisch unproduktiven Lebewesen habe. ${ }^{280}$ Minsky spricht daraufhin von der Notwendigkeit einer neu eingeführten, praktischen Teleologie der menschlichen Entwicklung, deren Grundlagen ab 1940 von der kybernetischen Forschung formuliert wurden. Mit einer vernunftorientierten Teleologie würden Ziel und Zweck der künftigen Lebensformen nicht mehr einem wilden Kampf der Gene unterliegen:

In the end, we will find ways to replace every part of the body and brain - and thus repair all the defects and flaws that make our lives so brief ... In the past we have tended to see ourselves as a final product of evolution - but our evolution has not ceased. Indeed, we are now evolving more rapidly - although not in the slow Darwinian way ... We now can design systems based on new kinds of »unnatural selection « that can exploit explicit plans and goals ... It took a century for evolutionists to train themselves to avoid such ideas - biologists call them >teleological< and >Lamarckian< - but now we may have to change those rules. ${ }^{281}$

Wenn Frank Tiplers Fortschrittsdeutung einen heilsgeschichtlichen Plan Gottes voraussetzt, Marvin Minsky dagegen jede Art von teleologischen Implikationen der bisherigen Evolutionsgeschichte missbilligt, so spiegelt Moravecs Position eine vermittelnde Bewertung wider, die gleichzeitig die Ambivalenz zwischen Kontinuität und Diskontinuität einer heilsgeschichtlichen Deutung im Posthumanismus illustriert. Einerseits charakterisiert Moravec die Technikgeschichte als Prozess der zunehmenden Synthese zwischen Mensch und Maschine, die sich an dem erhöhten kommunikativen Austausch zwischen Mediennutzern und Computern gegenüber älteren Medien in der Menschheitsgeschichte ablesen lasse. Die zunehmend bedeutenderen Medienerfahrungen interpretiert Moravec als out-of-body experience einer medialen Telepräsenz, die über

\footnotetext{
${ }^{278}$ Minsky 1988, 317.

${ }^{279}$ Vgl. Minsky 1984, 7f.

${ }^{280}$ V gl. Minsky 1994, 108.

${ }^{281}$ A.a.O., 110. Vgl. Minsky 1982, 8.
} 
die schrittweise Reduktion der körperlichen Wahrnehmung bis zur völligen Aufgabe des realen Körpers im virtuellen Raum führen werde - als disembodied mind. ${ }^{282}$

Vor 100 Millionen Jahren, so Moravec, begann mit dem Erscheinen der ersten Menschen die Erschaffung der Kultur, die in naher Zukunft durch einen beschleunigten Evolutionsprozess ihre mit dem Menschen verbundenen biologischen Grenzen überwinden werde. ${ }^{283}$ Denn mit dem Aufkommen der ersten Hochkulturen vor 5000 Jahren setze das sich in der Gegenwart zuspitzende Auseinanderdriften zwischen Kultur und Biologie ein, da der einzelne Mensch die Wissensakkumulation seit der Steinzeit nicht mehr bewältigen könne. Die begrenzte Speicherkapazität der Gene bzw. des biologischen Körpers ist nach Meinung Moravecs nicht mehr in der Lage, das gesamte kulturelle Wissen der Menschheit aufzunehmen, was den Fortschritt der Menschheit ernstlich gefährden könnte. Der Durchbruch der Singularität, der breakout, werde in nächster Zukunft diese Diskrepanz zwischen Kultur und Biologie durch das Aufgehen der menschlichen Kultur in den posthumanen Wesen bezwingen. ${ }^{284}$ Die Singularität, der Moment also, ab dem Maschinen sich autonom weiterentwickeln, kennzeichnet die Diskontinuität in der posthumanistischen Theorie, da auch Moravec die natürliche Evolution als rein zufälligen Prozess beurteilt, den die Menschheit der Gegenwart in die eigene Hand nehme:

In the metaphor of Richard Dawkins, we are the handiwork of a blind watchmaker. But we have now acquired partial sight and can, if we choose, use our vision to guide the watchmaker's hand. ${ }^{285}$

Durch die Schöpfung von Computern und künstlicher Intelligenz sei die Menschheit im Begriff, ihre biologische Bedingtheit zu transzendieren und mit der Autoevolution die Freiheit vom naturgegebenen Körper zu erlangen: Selbstentworfene himmlische Körper (heavenly bodies) mit Lichtantrieb, Rädern, als Roboterbusch oder als Insekt sollen in der $\mathrm{Zu}$ kunft zur Verfügung stehen. ${ }^{286}$

Für Ray Kurzweil vollzieht sich die Geschichte der Evolution dialektisch in drei zwingenden Schritten: Die primäre Entstehung von bio-

\footnotetext{
${ }^{282}$ Vgl. Moravec 1988, 75-82 ; Moravec 1999, 168-171.

${ }^{283}$ Vgl. Moravec 1988, 2-4.

${ }^{284}$ Vgl. Moravec 1999, 2-7, 126.

${ }^{285}$ Moravec 1988, 159.

${ }^{286}$ Vgl. Moravec 1999, 143, 149-153.
} 
logischem und nachfolgend intelligentem Leben ziehe die sekundäre Erfindung von Technik nach sich und münde schließlich in der finalen Verschmelzung von biologischem Leben und Technik. Die Entwicklung von Technik und die Symbiose von Technik und Biologie indiziere dabei bloß die Fortsetzung der Evolution mit anderen Mitteln. ${ }^{287}$

All kinds of practical and ethical issues delay the process, but they cannot stop it. The Law of Accelerating Returns predicts a complete merger of the species with the technology it originally created. ${ }^{288}$

Nach Einschätzung Kurzweils wird der dreiteilige Evolutionsprozess im 21. Jahrhundert seinen Abschluss finden, da bis 2099 die meisten Menschen als Simulation in der virtuellen Realität existieren und auf die wenigen, sterblichen MOSHs (Mostly Original Substrate Human) in der realen Welt herabblicken werden. ${ }^{289}$ Durch die Verschmelzung menschlicher und künstlicher Intelligenz werde der weitere Evolutionsprozess eine ungemeine Beschleunigung erfahren.

Evolution has been seen as a billion-year drama that led inexorably to its grandest creation: human intelligence. The emergence in the early twenty-first century of a new form of intelligence on earth ... will be a development of greater import than any of the events that have shaped human history. ${ }^{290}$

Das große Verdienst der Evolution sei es gewesen, den Menschen hervorzubringen: »Let us first praise evolution ... It created human beings with their intelligent human brains, being smart enough to create their own intelligent technology. ${ }^{291}$ Die Leistungen der Evolution, die als personifizierte Größe laut Kurzweil ohnehin nur einen geringen Intelligenzquotienten besitze, wurden jedoch bei weitem durch den Menschen übertroffen, der in wenigen Jahrtausenden mehr erschaffen habe, als die Evolution in Jahrmillionen: »So human intelligence, a product of evolution, is far more intelligent than its creator. ${ }^{292}$

Analog betrachten auch Kevin Warwick und der Extropianer Max More den technischen Fortschrittsprozess als Kontinuität der Evolutionsgeschichte:

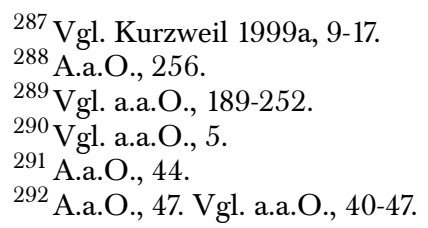


Those who have become cyborgs will be one step ahead of humans. And just as humans have always valued themselves above other forms of life, it's likely that cyborgs will look down on humans who have yet to »evolve ${ }^{293}$

Für Max More zählt das menschliche Streben nach der Überwindung biologischer und mentaler Grenzen zu den Grundeigenschaften einer aus eben dieser natürlichen Evolution hervorgegangenen Menschheit. ${ }^{294}$ In seinem Brief an Mutter Natur zeigt sich More dankbar, dass die Natur trotz ihrer »langsamen Intelligenz « uns Menschen hervorbringen konnte, was jedoch nicht darüber hinwegtäuschen sollte, dass der Mensch mit einer Vielzahl von Mängeln versehen wurde - allen voran Alter und Tod. Nun sei die Kindheit des Menschen beendet und die Zeit gekommen, den Menschen mit Hilfe von Computer- und Biotechnologie hin zu einer ultrahumanen Form (ultrahuman condition) zu veredeln:

We will no longer tolerate the tyranny of aging and death. Through genetic alterations, cellular manipulations, synthetic organs, and any necessary means, we will endow ourselves with enduring vitality and remove our expiration date ... Your ambitious human offspring. ${ }^{295}$

More analysiert in seiner Doktorarbeit eingehend die Bedingungen, die uns zur Veränderung oder sogar zur Aufgabe unseres gegenwärtigen $\mathrm{Zu}-$ standes als biologische Menschen bewegen könnten. Er konstatiert, dass vor allem die Aussicht auf eine Verbesserung unserer Person nach unseren gegenwärtigen Wertmaßstäben und eine Beteiligung an der Planung des Projektes eines neuen Menschen starke Anziehungskraft besitzen. ${ }^{296}$ Diese Bedingungen erfülle die Zukunftsvision des Posthumanismus im allgemeinen und die des Extropianismus im Besonderen.

Extropian transhumanism offers a [sic!] optimistic, vital and dynamic philosophy of life. We behold a life of unlimited growth and possibility with excitement and joy. We seek to void all limits to life, intelligence, freedom, knowledge, and happiness. Science, technology and reason must be harnessed to our extropic values to abolish the greatest evil: death. ${ }^{297}$

In diesem Zusammenhang bemerkt More, dass erst ein auf unsere zukünftigen Ziele ausgerichteter Lebensplan uns einen tieferen Lebenssinn

${ }^{293}$ Warwick 2000, 151.

${ }^{294} \mathrm{Vgl}$. More 1994, 2.

${ }^{295}$ More 1999, 1.

${ }^{296}$ Vgl. More 1995a, 62f.

${ }^{297}$ More 1996b, 4. 
verleihen könne, wobei jedoch ein rationaler Lebensplan den religiösen Lebensentwürfen überlegen wäre, da letztere dauernd Gefahr liefen, durch die Realität widerlegt zu werden. Die Zukunftsperspektiven, die den Fixpunkt menschlicher Lebensführungskonzepte konstituieren, sollten nicht in Dogmen eines starren Endziels festgeschrieben werden, sondern als gleichsam veränderbare, verbesserungsfähige Prinzipien einer individuellen und kollektiven Lebensplanung die Richtung vorgeben:

As we integrate principles into our personality, living according to them starts to flow more effortlessly. They will become »second nature.« Another way of putting it is to say a principle will have become a virtue. ${ }^{298}$

Diese Erwägungen bilden den Hintergrund von Max Mores Formulierung der Extropian Principles, die dem Einzelnen eine generelle Hilfestellung bei der Auswahl von Lebenszielen und der Orientierung seiner Lebensführung an die Hand geben sollen. ${ }^{299}$

Die Bedeutungszuweisungen des eigentlichen Fortschrittsprozesses und deren Kontextualisierungen mit evolutionistischen Modellen, die die einzelnen Autoren propagieren, differieren auf den ersten Blick erheblich voneinander. Als einziger Posthumanist vertritt Frank Tipler vehement die Wiedereinführung einer theologischen Teleologie: Das Wunder der Schöpfung beweise die Existenz eines intelligenten Schöpfers und daraus lasse sich die Existenz eines göttlichen Schöpfungszieles inklusive des heilsgeschichtlichen Prozesses ableiten. Dagegen unterscheiden Minsky, Moravec, Kurzweil und More zwischen einerseits der natürlichen Evolution und andererseits der mit dem Aufkommen des Menschen einsetzenden, geplanten und intelligenten Fortsetzung dieser Entwicklung, wobei die Akzentuierung des Bruches zwischen einer natürlichen Evolution und der künftigen, von Menschen und Computern gesteuerten Evolution unterschiedlich stark ausgeprägt ist. Für Minsky stellt sich die Genese der künstlichen, posthumanen Intelligenz als Befreiung von der zufälligen natürlichen Evolution dar, während Moravec, More und Kurzweil auch das Verschmelzen zwischen Mensch und Technik als Teil der Evolutionsgeschichte verstehen, die jedoch mit dem Eintreten der Singularität eine neue Qualität erhalten werde. Obwohl es bestimmte gemeinsame Vorstellungen über die Ausgestaltung der menschlichen Zukunft gibt, die nachfolgend noch thematisiert werden, sind Kurzweil,

\footnotetext{
${ }^{298}$ More 1995a, 70.

${ }^{299}$ Vgl. a.a.O., 67-72.
} 
Minsky und besonders More von der Möglichkeit und Notwendigkeit des ewigen Fortschrittes und der unaufhörlichen »Transzendierung « bisheriger Grenzen des Körpers und des Geistes überzeugt:

Life and intelligence must never stagnate; it must re-order, transform, and transcend its limits in an unlimited progressive process. Our goal is the exuberant and dynamic continuation of this unlimited process, not the attainment of some final supposedly unlimited condition. ${ }^{300}$

Die posthumanistische Idee vom eigentlichen Fortschrittsprozess vereinigt auf diese Weise verschiedene Elemente der englischen und amerikanischen Fortschrittstheorie und den modernen evolutionstheoretischen Entwürfen, die Gegenstand der folgenden Analyse sein werden.

Fortschritt präsentiert im Posthumanismus nicht nur ein Mehr an Informationsverarbeitung, sondern dieses Mehr ist gleichbedeutend mit einem Besser, einem irreversiblen Aufstieg der Menschheit. Erst ab 1750 wurde im englischen Diskurs die Definition von progress in ihrer ursprünglich neutralen Bedeutung als ein Vorwärtsschreiten, das damit auch negative Konnotationen implizierte, von einer durchweg positiven Besetzung abgelöst. Fortschritt meinte fortan meist die Steigerung und Verbesserung von Verhältnissen. Innerhalb dieses Diskurses spielte die Debatte um die Vervollkommnungsfähigkeit des Menschen eine herausragende Rolle. ${ }^{301}$ Schon der protestantische Theologe Jean Calvin hatte dem Gedanken einer allmählichen Verbesserung den Vorzug gegeben, da der Mensch im Diesseits nicht mehr hoffen könne, durch Gott erhoben zu werden. Der Begründer des Methodismus, John Wesley, forderte von seinen Anhängern, die Vollkommenheit des Christen durch die Heiligung, also durch die richtige und hingebungsvolle Erfüllung seiner irdischen Pflichten, zu verwirklichen. Das Ziel einer Vollkommenheit des Christen, das vormals durch ein herausragendes geistiges Ereignis gekennzeichnet war, wurde zusehends zu einer Vervollkommnung der irdischen Lebensführung. ${ }^{302}$ Der Anglikaner William Worthington verband 1743 in seiner Schrift Essay on the Scheme and Conduct, Procedure and Extent of Man's Redemption die Idee der innerweltlichen Vervollkommnung des Menschen mit einer allgemeinen Fortschrittstheorie und folgerte aus der anhaltenden Weiterentwicklung und Aufwärtsbewegung des

${ }^{300}$ More 1996b, 4.

${ }^{301}$ Vgl. Spadafora 1990, 5ff.

${ }^{302}$ Vgl. Passmore 1975, 142-145. 
Menschen: »And may it long continue still advancing in perfection! «303 Noch lange vor dem Erscheinen der Evolutionstheorie im 19. Jahrhundert sprach Worthington allen Lebewesen eine naturgemäße Neigung $\mathrm{zu}$ ihrer eigenen Verbesserung zu: » ... all parts of nature are endued with a principle not only to preserve their state, but to advance it, and ... every thing has a tendency to its own perfection. This is a general law impress'd upon Nature. ${ }^{304}$ Ebenso waren Joseph Priestley, Edmund Law und Richard Price von der natürlichen Verbesserungsfähigkeit $(n a-$ tural improveableness) des Menschen in seinem endlosen zukünftigen Fortschritt im Wissen und Glück überzeugt.

... the human powers will, in fact, be enlarged; nature, including both its materials, and its laws, will be more at our command; men will make their situation in this world abundantly more easy and comfortable; they will probably prolong their existence in it, and will grow daily more happy ... Thus, whatever was the beginning of this world, the end will be glorious and paradisical, beyond what our imagination can now conceive. ${ }^{305}$

Die Moralphilosophen des 18. Jahrhunderts wie Joseph Priestley, Jeremy Bentham, Helvétius und Daniel Gross vertraten die These, dass der Mensch durch die Fortschritte der moralischen Erziehung lernen kann, sein Glück zu maximieren. Während französische Philosophen meist vor einem atheistischen Hintergrund argumentierten, wurde die englische Fortschrittstheorie stark von William Paleys Principles of Moral and Political Philosophy (1788) geprägt, wonach Gott es war, der den Menschen zum Glück geschaffen habe. ${ }^{306}$ Den theologischen Konzepten zufolge sei der Mensch in dieser Welt jedoch unfähig, zu endgültiger Vollkommenheit, die er nur in der Einheit mit Gott erreichen kann, zu gelangen. Die Idee einer absoluten Vollkommenheit in der Vereinigung mit Gott wurde im 18. Jahrhundert durch die Vorstellung einer generellen Vervollkommnungsfähigkeit (perfectibility) des Menschen als Möglichkeit der stetigen und unaufhörlichen Verbesserung abgelöst. Als führender Vertreter der politischen und moralischen Fortschrittsbewegung in England konnte William Godwin daher behaupten: »Perfectibility is

\footnotetext{
${ }^{303}$ William Worthington: Essay on the Scheme and Conduct, Procedure and Extent of Man's Redemption. London ${ }^{2} 1748$, 153f., zitiert nach Spadafora 1990, 228.

${ }^{304}$ A.a.O., 190f., zitiert nach Spadafora 1990, 234.

${ }^{305}$ Joseph Priestley: An Essay on the First Principles of Government, and on the Nature of Political, Civil, and Religious Liberty, ${ }^{2}$ 1771, 4-5. Zitiert nach Sampson 1956, 58.

${ }^{306}$ V gl. Paley 1842, 533-546; Passmore 1975, 162-169.
} 
one of the most unequivocal characteristics of the human species. « ${ }^{307}$ Nicht ein festes Ziel der Vollkommenheit, sondern das kontinuierliche, in der Natur des Menschen verankerte Streben nach Vervollkommnung ist kennzeichnend für die moderne Fortschrittsidee, die sich an der Schwelle zum 19. Jahrhundert herausbildete und vor allem für die utilitaristischen Theorien Helvétius' und Benthams prägend wurde. ${ }^{308}$ Entscheidend für die Genese des Posthumanismus ist die Beobachtung, dass sich noch vor der Entstehung der Evolutionstheorie im englischen bzw. amerikanischen Diskurs bereits zwei Deutungsmuster der menschlichen Fortschrittsgeschichte herauskristallisiert hatten. Beide basieren auf der Annahme der innerweltlichen Vervollkommnungsfähigkeit des Menschen und der Maximierung der Möglichkeit zum Glück, ohne dass der Mensch jedoch einen endgültigen Zustand der Vollkommenheit erreichen könne. Priestley, Worthington, Price und viele andere frühe Denker stellen den Entwicklungsprozess in einen theologischen und teils millenniaristischen Kontext: Da die Schöpfung Gottes gut sei, werde sich alles aus ihr Hervorgehende auch zu immer perfekteren Ausgestaltungen hin verändern - die Vollkommenheit selbst sei jedoch nicht im Diesseits möglich. Die utilitaristischen Philosophen wie Godwin und Bentham dagegen schreiben der Menschheit die Fähigkeit der unaufhörlichen Selbstvervollkommnung zu - ohne dass ein höheres Wesen hier Einfluss nehmen müsse. Diese beiden Richtungen - eine theologische und eine säkulare, meist utilitaristische - sollten für die Rezeption der im 19. Jahrhundert entstandenen Evolutionstheorien bestimmend werden. Die Tatsache, dass auch heutige Posthumanisten theoretische Elemente, oder wie Tipler das tut, explizit Theoretiker des 18. Jahrhunderts referieren, belegt die anhaltende Bedeutung dieser Lehren.

\subsubsection{Evolution und der Aufstieg des Lebens}

»Evolution« ist ein Schlüsselbegriff in der posthumanistischen Fortschrittstheorie. Der Begriff wird jedoch ambivalent - gar widersprüchlich - genutzt. In der hermeneutischen Analyse müssen wir uns einmal mehr von der Maßgabe verabschieden, dass philosophische Konzepte innerhalb des Posthumanismus konsistent angewendet werden. Mit Bezug auf die Evolutionsgeschichte bedeutet dies, dass dieser Prozess ei-

${ }^{307}$ Godwin 1793, I, 11. Zitiert nach Sampson 1956, 58 f.

${ }^{308}$ Vgl. Passmore 1975, 142-145, 162-169; Sampson 1956, 50-56. 
nerseits teleologisch interpretiert wird - also auf ein bestimmtes Ziel hinausläuft. Im gleichen Atemzug wird jedoch jede Teleologie abgelehnt. Um dies zu verstehen, müssen wir in »die Ordnung der Dinge« eintauchen, die mit den Deutungen des Evolutionsgedankens einhergeht.

Es ist das Verdienst von Arthur Oncken Lovejoy, mit seinen William James Lectures in Harvard aus dem Jahre 1932/33 den Entstehungskontext der modernen Evolutionstheorie auf eindrückliche Weise näher beleuchtet zu haben. In seinem umfangreichen Werk behandelt er beginnend mit Platon und Aristoteles den Wandel einer zentralen philosophischen und religiösen Idee des Abendlandes: the great chain of being die Seinsordnung aller Lebewesen. Über viele Jahrhunderte beherrschte die Idee einer Verbundenheit aller Lebewesen den philosophischen und theologischen Diskurs über die Stellung des Menschen in der kosmischen Schöpfung. Diese durch die Nähe oder Ferne zum göttlichen Schöpfer definierte Kette basierte auf einer Rangordnung von den niedersten irdischen Organismen über die Menschen und die Engel bis hin zu Gott selbst. Diese Kette wurde zunächst statisch als die vollkommene und vollständige Stufenfolge aller Lebewesen betrachtet, wie sie Gott zu Beginn der Welt geschaffen habe und wie sie bis zum Ende der Welt bestehen würde. In der Neuzeit verlieh der Entwicklungsgedanke durch die Beiträge von John Locke, Alexander Pope, Edmund Law, Leibniz, Kant, Herder und Diderot dieser ewig gültigen Rangordnung eine neue Dynamik. $^{309}$

Es war der Schweizer Naturphilosoph Charles Bonnet, der im ausgehenden 18. Jahrhundert die biologische Entwicklung des Lebens mit dem Begriff evolution bezeichnete. Damit implizierte er, dass sich alles Leben aus dem Keime entwickelt habe und dieser Prozess stets in einem Ursprungssamen enthalten sei. Arthur McCalla sieht hierin den Beleg, dass bereits die terminologische Grundlage der späteren Evolutionstheorie in ihrem Kern Strukturen der christlichen Heilsgeschichte enthielt: »Evolution is here a synonym for the preformationist archetypal pattern of essence and development that purports to be at once scientific and soteriological.« ${ }^{310}$ Sowohl Bonnet als auch sein Zeitgenosse, der schwäbische Pietist und Naturphilosoph Friedrich Christoph Oetinger, entfalteten im Kontext dieses biologischen Preformationismus die Idee einer vom göttlichen Schöpfer geplanten Steigerung der biologischen Komplexität der

${ }^{309}$ Vgl. Lovejoy 1961, 183-200.
${ }^{310}$ McCalla 1998, 30. 
Lebewesen und ihrer spirituellen Fähigkeiten. Oetinger zufolge werde Gott sich als ens manifestativum sui im Laufe dieser Evolutionsgeschichte in der Welt selbst verwirklichen. Evolution sei folglich die Entfaltung der göttlichen Leiblichkeit in der Welt. Wie Lovejoy resümierte, vollzog sich diese schon bei Leibniz angedeutete Temporalisierung der Seinsordnung, der great chain of being, während der Aufklärung. Das heißt, die Rangordnung der Geschöpfe wurde dynamisch - zu einer Stufenleiter, die einen genealogischen Aufstieg verlangt, da alle von Gott angelegten Möglichkeiten des Seins im Universum zu ihrer Verwirklichung streben würden und dies nur in einer zeitlichen Abfolge geschehen könne. Teilweise wird dieser Prozess als ein unendlicher Aufstieg interpretiert, weil der Abstand zwischen Gott und dem Menschen selbst unendlich sei. ${ }^{311}$ Wie zuvor schon William Worthington mit seiner These einer stufenweisen Vervollkommnung der natürlichen Schöpfungen vertraten Erasmus Darwin und Jean-Baptiste Lamarck, der 1809 seine umstrittene Philosophie Zoologique veröffentlichte, die Auffassung, dass sich innerhalb großer Zeiträume ein Wandel der Arten vollzogen habe. Keiner dieser beiden Denker jedoch war imstande, seine Thesen mit empirischem Material zu beweisen. ${ }^{312}$ Dieses Defizit konnte erst Charles Darwin beheben, als am Abend des 1. Juli 1858 in einer Sitzung der Londoner Linnean Society die sorgfältig aufbereiteten Ergebnisse seiner über 20 Jahre zurückliegenden Forschungsreise mit dem Vermessungsschiff Beagle präsentiert wurden. Auf derselben Veranstaltung wurden die Arbeiten des jungen Naturforschers Alfred Russel Wallace zu einer biologischen Entwicklungslehre vorgestellt. Darwin konnte in dem nachfolgenden fruchtbaren Austausch mit Wallace dazu bewegt werden, seine wissenschaftlichen Resultate endlich zu veröffentlichen. 313

Darwins Hauptwerk On the Origin of Species by Means of Natural Selection, or the Preservation of Favoured Races in the Struggle for Life von 1859 enthält keine einzige Zeile über den Menschen, aber der Schlusssatz hat die Bahn für einen theologischen wie auch säkularen Zukunftsoptimismus geebnet: » ... from so simple a beginning endless forms most beautiful and most wonderful have been, and are being, evolved.« ${ }^{314}$ In den Arbeiten über die Geistesgeschichte des 19. Jahrhunderts wird oftmals ein-

\footnotetext{
${ }^{311}$ Vgl. Lovejoy 1961, 242-287; McCalla 1998, 29-31. Tipler rezipiert Lovejoys Werk, vgl. Tipler 1989, 245; Tipler 1995, 216, 385.

${ }^{312}$ Vgl. Lovejoy 1961, 227-241; Spadafora 1990, 234; Staudinger 1986, $167 f$.

${ }^{313}$ Vgl. Staudinger 1986, 167-173.

${ }^{314}$ Darwin 1859, 490. Nach der 1. Auflage von 1859.
} 
seitig hervorgehoben, wie der Darwinismus den Menschen auf ein den Tieren gleiches entsakralisiertes, biologisches Geschöpf reduzierte und entwertete. Diese Perspektive verkennt jedoch das positive, normative Potential der Entwicklungslehre. Denn erst mit Darwins Theorie war es möglich, den im 18. Jahrhundert formulierten Fortschrittsgedanken innerhalb der Anthropologie entwicklungsgeschichtlich zu begründen. Und erst mit Darwin war es auch möglich, die Mängel der gegenwärtigen Spezies Mensch als Wachstumsstörungen einer erst auf dem Weg zu ihrer vollkommenen Form befindlichen Menschheit zu deuten. Mit seinem Schlusswort und in einigen Briefen zeigte sich Darwin zuversichtlich, dass der Mensch durch die evolutionsbedingte Bereinigung seiner Mängel und der optimierten Anpassung an seine Umwelt noch zu Höherem bestimmt sei. ${ }^{315}$ In den Popularisierungen der darwinistischen Evolutionstheorie erschien der Mensch nun als Spitze der organischen Stufenleiter und Krone der historisierten Geschichte des Lebens, was Reinhart Koselleck sehr anschaulich mit einem Beispiel aus dem deutschsprachigen Morgenblatt für gebildete Leser von 1865 demonstriert:

Immer die entwickelste Race liege an der Spitze der Menschheit, während im Verborgenen eine höher organisierte Form der Menschheit sich vorbereitet, um nach dem natürlichen Recht des Stärkeren über ihre Vorgängerin hinaufzurücken und ihrerseits ... den äußeren und inneren Horizont der Menschheit zu erweitern. Minimale Voraussetzung sei etwas mehr Hirnsubstanz. ${ }^{316}$

Diese hier zum Vorschein kommende Idee der fortschreitenden, biologisch begründeten Vervollkommnung des Menschengeschlechtes steht dabei seit der Veröffentlichung von Darwins Theorie im Kontext zweier, antagonistisch anmutender Rezeptionsstränge. So muss in diesem $\mathrm{Zu}-$ sammenhang zwischen einer säkularen, teils religionsfeindlichen und einer religiösen, teils christlichen, wohlwollenden Rezeption der darwinistischen Entwicklungslehre differenziert werden.

$\mathrm{Zu}$ den weltweit wohl einflussreichsten Popularisierungen der darwinistischen Entwicklungslehre gehören die Arbeiten des deutschen Zoologen und Naturphilosophen Ernst Haeckel, der als noch junger Wissenschaftler 1863 die für Jahrzehnte maßgebliche Interpretation Darwins

\footnotetext{
${ }^{315}$ Vgl. Benz 1961, 84-89; Benz 1965, 81-91, 148f.; Baillie 1950, 145f.; Bury 1955, 334349; Weizsäcker 1990, 143.

316 »Etwas von der Zukunft der Geschichte.« In: Morgenblatt für gebildete Leser 59 (1865), Nr. 1, 32. Zitiert nach Kosellek 1975, 420. Vgl. dazu Kapitel 6.6.2.
} 
kundtat. Den Menschen als das höchstorganisierteste Lebewesen in der langen Kette der Evolutionsgeschichte deutend, leitet er daraus die allgemeine Entwicklungslehre ab:

\begin{abstract}
Dasselbe Gesetz des Fortschritts finden wir dann weiterhin in der historischen Entwickelung des Menschengeschlechts überall wirksam. Ganz natürlich! Denn auch in den bürgerlichen und geselligen Verhältnissen sind es wieder dieselben Principien, der Kampf um das Dasein und die natürliche Züchtung, welche die Völker unwiderstehlich vorwärts treiben und stufenweise zu höherer Cultur emporheben. Rückschritte ... können wohl diesen allgemeinen Fortschritt zeitweise hemmen oder scheinbar unterdrücken; je unnatürlicher je anachronistischer aber diese rückwärts gerichteten Bestrebungen sind, desto schneller und energischer wird durch sie der Fortschritt herbeigeführt ... Denn dieser Fortschritt ist ein Naturgesetz, welches keine menschliche Gewalt, weder Tyrannen-Waffen noch Priester-Flüche, jemals dauernd zu unterdrücken vermögen. Nur durch eine fortschreitende Bewegung ist Leben und Entwickelung möglich. Schon der bloße Stillstand ist ein Rückschritt, und jeder Rückschritt trägt den Keim des Todes in sich selbst. Nur dem Fortschritte gehört die Zukunft. ${ }^{317}$
\end{abstract}

Den von Darwin entdeckten Evolutionsprozess interpretiert Haeckel als wissenschaftlich beweisbare Vervollkommnung der Arten im Laufe der Geschichte des Lebens: »Neue, vollkommenere Rassen entstehen fortwährend und veredeln sich im Kampfe ums Dasein, während die unvollkommeneren Rassen (ebenso wie die alten Stammformen) zurücktreten, erlöschen und aussterben. ${ }^{318}$ Sein Bedauern über das Aussterben vieler Rassen und über den Verlust der Vielfalt in der Flora und Fauna wiegt Haeckel mit der Aussicht einer Höherentwicklung des Lebens auf, denn es liege

... ein höherer Trost in dem Gedanken, daß es durchschnittlich der vollkommenere und veredeltere Mensch ist, welcher den Sieg über die anderen erringt, und daß das Endergebniß dieses Kampfes der Fortschritt zur allgemeinsten Vervollkommnung und Befreiung des Menschengeschlechtes, zur freien Selbstbestimmung des menschlichen Individuums unter der Herrschaft der Vernunft ist. ${ }^{319}$

Haeckels immense publizistische Produktivität und seine beträchtliche Vortragstätigkeit in Deutschland und im Ausland machen ihn vermutlich zum wichtigsten Verbreiter der darwinistischen Entwicklungslehre. 1868 erscheint Die natürliche Schöpfungsgeschichte; gemeinverständlich - wis-

\footnotetext{
${ }^{317}$ Haeckel 1902, 29f.

318 A.a.O., 25.

${ }^{319}$ A.a.O., 113.
} 
senschaftliche Vorträge über die Entwicklungslehre im allgemeinen und diejenigen von Darwin, Goethe und Lamarck im besonderen. Folgt hier bereits sieben Jahre später die 6. Auflage und nachfolgend die Übersetzung ins Englische, so übertraf Haeckel diesen publizistischen Erfolg noch mit den 1899 erschienenen Gemeinverständlichen Studien über monistische Philosophie unter dem Buchtitel Welträthsel. Nicht zuletzt durch dieses Werk, das bis 1919 über 340.000 mal in Deutschland verkauft und in 24 Sprachen übersetzt wurde, konnte sich Haeckel großer Anerkennung und einer klassenübergreifenden Popularität sicher sein, die ihm sowohl vom fortschrittsgläubigen Bildungsbürgertum als auch von der sozialistisch orientierten Arbeiterschaft entgegengebracht wurde. In seiner Philosophie, dem Monismus, bemüht sich Haeckel, die immer größer werdende Kluft zwischen Religion und Evolutionslehre zu schließen, indem er den Darwinismus als Möglichkeit sieht, nun alle Erscheinungen der organischen Natur und der Sphäre des sozialen Lebens durch einen einzigen Gedanken zu erklären: nämlich, dass im Reich der Biologie aufgrund naturgesetzlicher Vorgänge Höheres aus Niederem entsteht, Vollkommeneres aus minder Vollkommenem. Der Monismus will dabei die Ära der Religion überwinden und setzt an die Stelle dogmatischer Überzeugungen die sich stetig verbessernden Erkenntnisse der Wissenschaften, was institutionell nicht nur in der von Haeckel initiierten Gründung des Deutschen Monistenbundes (1906) seinen Ausdruck fand. Der Publizist Eduard Löwenthal hatte schon 1865 die monistische, sozial-humanitäre Religionsgemeinschaft der Cogitanten ins Leben gerufen - eine Gesinnungsgemeinschaft ohne festes Bekenntnis, die allein im Fortschritt der Wissenschaften die Lösung aller Übel der Menschheit sah. Sowohl Löwenthals Entwurf einer Art Wissenschaftsreligion als auch Haeckels Bemühungen führten zur Gründung zahlreicher monistischer Gruppierungen bzw. Gemeinden in Österreich, Italien, Frankreich, Russland und vor allem in den Vereinigten Staaten, wo Paul Carus seit 1890 die Zeitschrift The Monist verlegte. ${ }^{320}$

Jede Art von Vorsehung und teleologischen Momenten weist Ernst Haeckel vehement zurück, denn der historische Fortschritt (Progressus) und die allmähliche Vervollkommnung (Teleosis), die die Anpassung an veränderte Umweltbedingungen immer wieder aufs neue erfordern, seien eine notwendige Wirkung der Selektion, aber nicht die Wirkung eines vorbedachten Zweckes. Haeckel spürt allerdings, dass, abgesehen

${ }^{320}$ Vgl. Mehlhausen \& Dunkel 1994; Sass 1968, 126-130; Staudinger 1986, $172 \mathrm{ff}$. 
von der Rolle des Schöpfergottes, der Deismus sich dem Monismus »bis zur Berührung « angenähert hat, da beide die vergangene und zukünftige Aufwärtsentwicklung der Welt aus den Naturgesetzen ableiten. ${ }^{321}$

Diese Entwicklung scheint uns im großen und ganzen - wenigstens soweit wir die Stammesgeschichte der organischen Natur auf unserem Planeten übersehen können - als eine fortschreitende Umbildung, als ein historischer Fortschritt vom einfachen zum Zusammengesetzten, vom Niederen zum Höheren, vom Unvollkommenen zum Vollkommenen. ${ }^{322}$

Im englischsprachigen Raum wurde der Darwinismus von den Kirchen des protestantischen Mainstreams sehr rasch positiv aufgenommen. Die schärfsten Auseinandersetzungen ereigneten sich erst mit der Politisierung der christlichen Fundamentalisten in den Vereinigten Staaten, die in den 1920er Jahren begann und sich bis heute im Streit um den Biologieunterricht fortsetzt. Bereits in den 1860er Jahren bemühten sich viele Theologen, die christliche Heilsgeschichte und die darwinistische Entwicklungslehre in Übereinstimmung zu bringen. Die Temporalisierung der Idee einer great chain of being sowie die aus dem Postmillenniarismus hervorgegangene Akzeptanz eines innerweltlichen Fortschrittsgedankens bilden den Hintergrund dieser ideengeschichtlichen Entwicklung. Die beteiligten Theologen führten ihre Auseinandersetzung mit den neuesten Erkenntnissen der Naturwissenschaften unter großer öffentlicher Anteilnahme vornehmlich in Predigten, Vorträgen und Erbauungsschriften. Der Kongregationalist und spätere konservative Biblizist Minot Judson Savage interpretiert z.B. Darwins Evolutionismus heilsgeschichtlich und löst auf diese Weise gleich das Problem der Theodizee und der Erbsünde, da seiner Ansicht nach alle Übel der Welt - wie Krankheit, Sünde, Leid, Unglück - ihre Ursache einzig im maladjustment, in der schlechten Anpassung der Lebewesen an ihre Umwelt, hätten. Die Heilsgeschichte identifiziert Savage daher mit der biologischen Entwicklungsgeschichte, die zu einer Optimierung der Lebensverhältnisse führen würde. Ebenso energisch verteidigte der schottische Presbyterianer James McCosh, der seit 1868 in Princeton Philosophie lehrte, den christlich-teleologischen Evolutionismus in seiner 1890 erschienenen Schrift The Religious Aspect of Evolution gegen die atheistischen Vereinnahmungsversuche des Darwinismus. Die Menschheit wird hier als Samen gedacht, der noch nicht

${ }^{321}$ Vgl. Haeckel 1960, 22ff., 300ff., 343-347.

${ }^{322}$ A.a.O., 337. 
voll aufgegangen ist und nun ihren Kampf der Verbesserung auf dem Weg zur Vollkommenheit aufgenommen hat. Große Wirkung erzielte auch der theologisch inspirierte Wissenschaftler Henry Drummond, der in seiner Glasgower Vorlesung The Contribution of Science to Christianity von 1884 und seinen späteren amerikanischen Lowell Lectures unter dem Titel The Ascent of Man von 1894 das christliche Konzept der Heilsgeschichte nicht mehr nur auf die Menschheit beschränkt, sondern die gesamte Evolutionsgeschichte des Lebens als Teil dieses heilsgeschichtlichen Prozesses versteht. Die Menschheit sei der bisherige Höhepunkt dieser Entwicklung, denn auch im Bereich der Evolution seien »viele berufen, aber wenige auserwählt« (Mt 20,16). Weitere wichtige Beiträge zu einer Synthese zwischen Theologie und Darwinismus erbrachten der calvinistische Botaniker Asa Gray mit seiner Darwiniana (1876), der Geologe und Prediger George Frederick Wright, der Jurist und Kongregationalist Lyman Abbott, der Naturphilosoph John Fiske und der anglikanische Bischof Ernest William Barnes. ${ }^{323}$

Drei große Philosophen, die auf verschiedene Weise im Posthumanismus rezipiert werden, haben im 20. Jahrhundert den Versuch unternommen, mit Bezug oder in Reflexion auf die christliche Heilsgeschichte die Evolutionstheorie als Teil einer umfassenden Kosmologie zu verstehen. Die Philosophen Samuel Alexander (1859-1938), Alfred North Whitehead (1861-1947), die beide Einladungen zu den Gifford Lectures erhalten haben, und der bereits erwähnte Paläontologe Pierre Teilhard de Chardin begreifen das Universum als eine Entität, die ihre Qualitäten erst sukzessive entwickelt. ${ }^{324}$

Den Darwinismus deutet Samuel Alexander in seinem Hauptwerk Space, Time and Deity von 1920 als eine rein wissenschaftliche Theorie, die sich zwar jeder Wertung über ihren Untersuchungsgegenstand enthalte, jedoch die grundlegende Erklärung liefere, wie Werte in der Geschichte des Lebens und der menschlichen Kultur überhaupt geschaffen werden. Das, was per natürlicher Zuchtwahl bzw. Mutation in den jeweils besonderen Lebensbedingungen überlebt habe, werde als gut bewertet. Das augenscheinlich Überlebensfähigste werde daher stets als Höchstes verehrt. ${ }^{325}$ In der unendlich fortschreitenden Zeit bringe das Universum

\footnotetext{
${ }^{323}$ Vgl. Benz 1965, 157-183; Barrow \& Tipler 1986, 85f., $183 f$.

${ }^{324}$ Vgl. Kroker \& Weinstein 1997, $194 \mathrm{f}$.

${ }^{325}$ V gl. Alexander 1966, $309 f$.
} 
aus der Sicht des Menschen nun kontinuierlich höhere Daseinsstufen (levels of existence) - bisher Materie, Leben und Geist - hervor:

Within the all-embracing stuff of Space-Time, the universe exhibits an emergence in Time of successive levels of finite existences, each with its characteristic empirical quality. The highest of these empirical qualities known to us is mind or consciousness. Deity is the next higher empirical quality to the highest we know ... ${ }^{326}$

Diese gegenwärtig höchste Stufe des Daseins, die für den Menschen empirisch erfahrbar sei, der Geist (mind) oder das Bewusstsein (consciousness), verweise aus sich selbst heraus bereits auf die nächste Stufe der kosmischen Entfaltung: »Deity is thus the next higher empirical quality to mind, which the universe is engaged in bringing to birth. « ${ }^{327}$ Dabei legt Alexander der Aufwärtsentwicklung des Kosmos keinen göttlichen Plan zugrunde, sondern unterstreicht, dass die Tendenzen zur nächst höheren Stufe der Existenz sich in der evolutiven und kosmischen Entwicklung aus sich selbst heraus entwickelten (Emergenz). Diese Tendenzen sind laut Alexander für die Daseinsformen auf der Stufe des Geistes im religiösen Streben schon dunkel erkennbar. Die Gottheit (deity) erscheint als Teil des evolutiven Prozesses: »Deity is some quality not realised but in process of realisation, is future and not present. ${ }^{328}$ Gott selbst versteht Alexander als ein unendliches Wesen innerhalb der Raum-Zeit, das sich mit der Weiterentwicklung des Universums verändert und als Begriff daher alle noch nicht verwirklichten und erkennbaren Eigenschaften des Universums bezeichnet: ${ }^{329}$

As actual, God does not possess the quality of deity but is the universe as tending to that quality. This nisus in the universe, though not present to our sense, is yet present to reflection upon experience. ${ }^{330}$

Es ist in der philosophiegeschichtlichen Forschung bislang umstritten, wie sehr die Arbeiten Alexanders den Mathematiker und Philosophen Alfred North Whitehead beeinflusst haben. Auch er war bemüht, die Einzelphänomene der empirischen Wirklichkeit im Gesamtzusammenhang mit der Geschichte der Natur und des Kosmos zu interpretieren

\footnotetext{
${ }^{326}$ A.a.O., 345 .

${ }^{327}$ A.a.O., 347.

${ }^{328}$ A.a.O., 379.

${ }^{329}$ Vgl. a.a.O., 341-372.

${ }^{330}$ A.a.O., 361.
} 
und die manifesten Grenzen zwischen Naturwissenschaften, Geisteswissenschaften und der Philosophie zu überwinden. Whitehead entwirft das Bild eines bipolaren Gottes, der über eine Urnatur und eine Folgenatur charakterisiert ist. Diese beiden Naturen Gottes streben nun im Entwicklungsprozess des Universums zueinander, um die Einheit in Gott wiederherzustellen. Aus seinen Beobachtungen schließt Whitehead, dass dieser prozesshafte Gott im Zuge seiner eigenen Verwirklichung die Schöpfung veranlasst, in aufeinander folgenden Stufen immerzu neue und höhere Daseinsformen hervorzubringen. ${ }^{331}$

Wir unterscheiden also vier kreative Phasen, in denen das Universum seine Wirklichkeit vervollständigt. Als erste die Phase der begrifflichen Entstehung, der es an Wirklichkeit mangelt, die aber in ihrer Abstimmung der Wertung unendlich ist. Zweitens die zeitliche Phase der physischen Entstehung, mit ihrer Vielheit von Wirklichkeiten. In dieser Phase wird vollständige Wirklichkeit erreicht; aber es fehlt an der Solidarität der Einzeldinge untereinander ... Drittens die Phase vollkommener Wirklichkeit, in der die Vielen immerwährend eins sind, ohne durch irgendeinen Verlust an individueller Identität oder an Vollständigkeit der Einheit eingeschränkt zu werden. Im Immerwährenden wird Unmittelbarkeit mit objektiver Unsterblichkeit versöhnt. In der vierten Phase vervollständigt sich der Schöpfungsvorgang ... Denn das Reich Gottes ist mitten unter uns. Die Auswirkung der vierten Phase ist Gottes Liebe zur Welt ... Was in der Welt getan wird, verwandelt sich in eine Realität des Himmels, und die Realität des Himmels geht wieder über in die Welt. Hierin finden wir die letzte Anwendung der Lehre von der objektiven Unsterblichkeit. Durch all die vergehenden Ereignisse im Leben jedes zeitlichen Geschöpfs ist der innere Quell von Abkehr oder Auffrischung, der Richter, der aus der Natur der Dinge selbst hervorgeht, Erlöser oder Unheilsgöttin, die Transformation seiner selbst, immerwährend im Sein Gottes. ${ }^{332}$

Mit dieser Idee eines sich im Zuge der kosmischen und Menschheitsgeschichte entwickelnden Gottes erzielte Whitehead eine große Wirkung auf die sich ab den 1930er Jahren konstituierende, protestantische Prozesstheologie an der Chicagoer Divinity School. ${ }^{333}$

Angeregt durch Henri Bergsons Versuch einer Synthese zwischen christlichem Schöpfungsgedanken und naturwissenschaftlicher Entwicklungslehre in L'évolution créatrice (1907) bemühte sich auch der Jesuit und Paläontologe Pierre Teilhard de Chardin um eine fruchtbare Er-

\footnotetext{
${ }^{331}$ Vgl. Whitehead 1979, 611-627.

${ }^{332}$ A.a.O., $626 f$.

${ }^{333}$ Vgl. Maaßen 1991, 217ff.
} 
gänzung wissenschaftlicher Erkenntnisse und religiöser Erkenntnis. ${ }^{334}$ Materie und Geist sind für Teilhard die zwei in einem dynamischen Verhältnis zueinander stehenden Zustände des kosmischen Stoffes. Aus dem Anfangspunkt alpha lasse Gott das Universum sich zu einem immer komplexer werdenden System entfalten. In seinen beiden philosophischen Hauptwerken Le Groupe Zoologique Humain von 1956 (dt.: Die Entstehung des Menschen) und Le Phénomène humain von 1955 (dt.: Der Mensch im Kosmos) schildert Teilhard ähnlich wie Alexander und Whitehead den Evolutionsprozess als eine sukzessive Entfaltung des Geistes. ${ }^{335}$ Diese vollziehe sich zunächst über die Vorstufe der Entstehung des Universums und der Erde (Kosmogenese), die nachfolgende Ausbildung der Biosphäre (Biogenese) und die Ausbreitung des Denkens mit der Noosphäre durch das Auftreten der ersten Hominiden (Noogenese). Sie münde schließlich in der künftigen Entfaltung des höheren Lebens durch den Zusammenfluss des menschlichen Denkens. Die kosmische Entwicklung ende mit der Wiederkunft Christi am zeitlichen Endpunkt Omega. Die menschlichen Individuen werden Teilhard zufolge in diesem kosmischen Christus zu einem endgültigen »Ultra-Ego« verschmelzen.

Zentrale Bedeutung in der Entwicklung des Kosmos hat für Teilhard die Entstehung des Menschen, denn sein Erscheinen und die Ausbreitung der Noosphäre (der Geistsphäre) über den gesamten Planeten markieren den Wendepunkt der Evolution, die sich nun in einer Konvergenzbewegung auf Omega hinzubewege. Die herausragende Rolle des Menschen deduziert Teilhard aus der bisherigen Evolutionsgeschichte: »Deutet nicht alles darauf hin, daß das Leben seit dem Pliozän die besten Kräfte, die ihm noch verblieben, ganz auf den Menschen ansetzte, wie ein Baum auf seinen Gipfel?«336 Der Mensch selbst, erhoben durch die Erscheinung Christi, ist damit der entscheidende Faktor in der kosmischen Soteriologie Teilhards: »Der Mensch ist unersetzbar. Trotz der Unwahrscheinlichkeit seiner Aussichten muß er ans Ziel gelangen; gewiß nicht notwendig aber unfehlbar. $\ll^{337}$

${ }^{334}$ Teilhards Ideen knüpfen dabei an die theologische Kritik des materialistischen Monismus an, wie sie um 1900 bereits von dem Jesuiten Erich Wasmann und dem protestantischen Theologen Georg Wobbermin vorgebracht wurde. Vgl. Altner 1965, 57-60.

${ }^{335}$ Vgl. Teilhard 1961; Teilhard 1959.

${ }^{336}$ Teilhard 1961, 76. Vgl. a.a.O., 40-106; Teilhard 1959, 84-295.

${ }^{337}$ A.a.O., 271. 
Teilhard bestreitet, dass die biologische und geistige Entwicklung des Menschen bereits ihren Zenit erreicht hat, und stellt eine stetige Höherentwicklung seiner cerebralen Fähigkeiten in Aussicht, deren nächster Stufe wir in unserer Gegenwart gewahr werden:

Wir werden zu diesem Zweck nachweisen - immer ohne den Boden wissenschaftlicher Forschung zu verlassen - daß gerade durch die Kräfte der Konvergenz, die während der »kompressiven« Phase der Gemeinschaftsbildung auftreten, die Entwicklung des Lebens auf der Erde nicht nur Mittel und Wege findet, um sich auch in uns nach altem Rezept fortzusetzen, sondern daß sie, vergleichbar mit einer Mehrstufenrakete, die mehrmals aus sich selbst heraus frischen Antrieb empfängt, gerade jetzt, vor unseren Augen, einen neuen Anlauf nimmt, indem sie eine neue Methode verfolgt, die eine bisher noch nicht gekannte Durchschlagskraft besitzt. ${ }^{338}$

Diese neue Stufe der Evolution werde durch das zunehmende Bewusstsein des Menschen über sein eigenes evolutionsgeschichtliches Gewordensein hervorgebracht, das ihm die Kraft des Geistes in seiner kulturellen Entwicklung offenbart gegenüber dem Millionen Jahre währenden, rein biologischen Aufstieg: »Das Künstliche löst das Natürliche ab. Die mündliche oder schriftliche Überlieferung ersetzt die genetischen (oder Chromosom-bedingten) Formen der Vererbung. « $^{339}$

Alexander wie später auch Whitehead und Teilhard schufen eine kosmologische Metaphysik als erweiterte Interpretation der durch die Naturwissenschaften erschlossenen Welt. Die Entwicklung des Universums impliziert bei allen drei Denkern ein starkes teleologisches Moment, da das Neue stets als Höheres präsentiert wird. Bei Teilhard und Whitehead ist dieses Moment gar deterministisch, da die Geschichte des Universums mit der Verwirklichung Gottes gleichgesetzt wird. ${ }^{340}$

Wenn auch die Idee einer stetigen, geistigen Höherentwicklung und Loslösung aus der natürlichen Evolution von vielen Posthumanisten aufgenommen wurde, so erlangte die von Alexander, Whitehead und Teilhard entworfene Vorstellung eines sich in der kosmischen Geschichte entwickelnden Gottes vor allem für Frank Tipler eine wesentliche Bedeutung. Zusammen mit Friedrich Wilhelm Schelling und Henri Bergson würdigt Tipler diese drei Denker als die Vorläufer seiner eige-

\footnotetext{
${ }^{338}$ Teilhard 1961, 115.

${ }^{339}$ Teilhard 1959, 272.

${ }^{340}$ Vgl. Baillie 1950, 146-152 ff.; Emmert 1991, 109-112.
} 
nen Physik der Unsterblichkeit. ${ }^{341}$ Obwohl er betont, dass er aus dem Werk Teilhards lediglich den Begriff Omega entlehnt habe, zeigen sich in den Schriften beider Zukunftsanthropologen eine Reihe von Parallelen. Mit ihren Kommentaren zu den Ideen Teilhards in The Anthropic Cosmological Principle erwecken John Barrow und Frank Tipler sogar den Eindruck, als würden sie Teilhards Versöhnungsversuch zwischen der Naturwissenschaft und Theologie bzw. Teleologie, angereichert um die Fortschritte der Kosmologie und Astronautik, fortführen wollen. ${ }^{342}$ So werden einige philosophische Begriffe, die Teilhard benutzt, in eine strikt physikalische Terminologie überführt: Die geistige, radiale oder psychische Energie als Motor der Evolution in Teilhards Konzept versteht Tipler als Information und deutet die Ausweitung der Noosphäre folglich als Steigerung der Informationsverarbeitung, bis das Leben den Punkt Omega - den prozesshaft verwirklichten, christlichen Gott - erreicht haben werde. In gleicher Weise wird bei Tipler aus dem christlichen Heiligen Geist mit Berufung auf Teilhard die universale Wellenfunktion des Universums. In Teilhards Faszination für die Kybernetik sieht Tipler bereits den Hinweis, dass auch der Jesuit Computer als Mittel zu Überwindung der Materie interpretiert hätte. Große Differenzen zu Teilhard bestehen vor allem in Tiplers völliger Preisgabe einer Christologie, denn der christliche Erlöser spielt in Tiplers Zukunftsvision keine Rolle. ${ }^{343}$

Auch dürfen vor dem amerikanischen Hintergrund Tiplers sowohl die ebenfalls auf christologische Elemente verzichtenden Theorien Alexanders und Whiteheads als auch die wiederum von Whitehead geprägte Prozesstheologie der Chicagoer Schule in ihrer Bedeutung für das Werk des Physikers nicht zu gering geachtet werden. Tipler nahm 1988 in Chicago am 2. Pannenberg Symposium an der Lutheran School of Theology teil und konnte Kontakte zu dem evangelischen Theologen Wolfhart Pannenberg knüpfen, der sich in einem Briefwechsel fortsetzte. Pannenberg war Vertreter des religionsgeschichtlichen Ansatzes innerhalb der protestantischen Theologie, die von der fortschreitenden Offenbarung Gottes in der Geschichte der Menschheit ausgeht. Diese Offenbarung

${ }^{341}$ Vgl. Tipler 1989, 221; Barrow \& Tipler 1986, 191ff.; Moravec und Kurzweil sind mindestens die mathematischen Arbeiten Whiteheads und ein unbedeutenderes Werk Alexanders bekannt. Vgl. Kurzweil 1999a, 328f., 344; Moravec 1999, 20.

342 »Providing the term is Teilhard's only scientific contribution to this book. He is not mentioned in the Appendix for Scientists." Tipler 1995, 110. Vgl. Barrow \& Tipler 1986, 196-204.

${ }^{343}$ Vgl. Tipler 1995, $183 f f$. 
Gottes zeige sich in allen Religionen, laufe aber auf die Auferstehung Christi zu, in der Pannenberg eine so genannte Prolepse der vollständigen Offenbarung am Ende der Zeiten sieht. ${ }^{344}$ Als einziger namhafter Theologe hat sich Pannenberg ernsthaft mit Tiplers Theorie auseinandergesetzt und einige Gemeinsamkeiten in der Vorstellung eines sich in der Zukunft voll entfaltenden Gottes gefunden, jedoch die Idee einer Erlösung durch technischen Fortschritt zurückgewiesen. Neben Pannenbergs Bestreben, Frieden zwischen Naturwissenschaft und Religion zu stiften, schätzt Tipler an dem Theologen vor allem sein Eintreten für ein dynamisches Gottesbild. ${ }^{345}$ Um die Evolutionsgeschichte mit der Teleologie des von William Paley und anderen Theologen entworfenen design argument zu verbinden, beruft sich Tipler darüber hinaus auch auf Fürsprecher einer temporalisierten Idee der great chain of being wie den Asa Gray, Ernest W. Barnes, John Fiske und den Biologen Thomas Huxley, die um die Jahrhundertwende den Darwinismus als wissenschaftlichen Beleg für ein geplantes Universum bzw. eine teleologische Evolutionstheorie werteten. ${ }^{346}$

Alle Post- und Transhumanisten teilen diese melioristische und anthropozentrische Interpretation der Evolutionsgeschichte. Sowohl säkulare Philosophien wie der Monismus, als auch die metaphysischen Systeme Alexanders und Whiteheads sowie die zahlreichen theologischen Deutungen sind sich einig, dass die Evolution immer höherwertigere Daseinsformen hervorbringt und der zeitgenössische Mensch den Gipfelpunkt der bisherigen Entwicklung markiert. Exakt an dieser Stelle setzt nun ein zweites Moment ein, das eigentlich der bisherigen teleologischen Deutung zuwiderläuft: die vollkommene Abwertung der natürlichen Evolution mit der Perspektive, deren weiteren Verlauf nun selbst zu steuern. Dieses Narrativ gewinnt schon in der frühen Eugenik Konturen. Bereits der religiöse Schriftsteller Soame Jenyns hatte im 18. Jahrhundert die Idee der evolutiven Stufenleiter auf die menschlichen Rassen übertragen und hier das symbolhafte Genie der Engländer, Isaac Newton, dem »wilden Hottentotten « gegenübergestellt. ${ }^{347}$ In der Konsequenz dieses

\footnotetext{
${ }^{344}$ Vgl. Pannenberg 1961.

${ }^{345}$ Vgl. a.a.O., XXIIIf.; Tipler 1989, 217ff.; Tipler 1995, 4f. In einer Zusammenfassung seiner Theorie gibt er als Hauptgrund für die Identifizierung Gottes mit dem Omegapunkt Gottes Selbstbeschreibung in Ex 3:14 an: »Ich werde sein, der ich sein werde.« Vgl. Tipler 2000, 1.

${ }^{346}$ Vgl. Barrow \& Tipler 1986, 85ff., 127-143, 189; Tipler 1995, 216ff.; Pannenberg 1995. ${ }^{347}$ Vgl. Lovejoy 1961, 183-200. Vgl. Kapitel 6.6.2.
} 
weit verbreiteten Gedankens proklamierten die Verfechter der Eugenik wie Francis Galton, Ludwig Büchner oder Auguste Forel ab den 1860er Jahren das Ziel, die »natürliche Zuchtwahl« durch die »künstliche Zuchtwahl« des Menschen zu ersetzen. Anders als die fehlerreiche natürliche Evolution könne eine bewusste Steuerung der Selektion nur der geistig am höchsten entwickelten Rassen oder Klassen zu einem »höheren Menschentum « der Zukunft führen. ${ }^{348}$

$\mathrm{Zu}$ den wenigen, aber in ihrer Wirkung sicherlich kaum zu unterschätzenden Befürwortern der Eugenik, die auch nach den Erfahrungen des nationalsozialistischen Rassenwahns an ihren Visionen festhielten, gehörten vor allem Julian Huxley und Teilhard de Chardin. Huxley propagierte - konträr zu dem Roman Brave New World (1932) seines Bruders Aldous - die Befruchtung ausgewählter Eizellen durch erlesene, geistig und körperlich hochwertige Spender, um das genetische Niveau der Bevölkerung zu heben, während er gleichzeitig die Sterilisation sozial schwacher und potentiell kinderreicher Familien wegen der drohenden Gefahr einer genetischen Degeneration forcierte. ${ }^{349}$

Er [der Mensch: O.K.] ist nicht nur äußerst jung, er ist auch noch äußerst unvollkommen, ein unfertiges, oft zusammengepfuschtes Produkt stammesgeschichtlicher Improvisation ... Nur ein Bruchteil seiner Möglichkeiten ist bis heute verwirklicht worden. Der Mensch hat eine weitere Verbesserung dringend nötig. Hier ist der Punkt, wo die Eugenik ihren Hebel anzusetzen hat. ${ }^{350}$

Huxley formuliert daraufhin ein mehrstufiges Modell des evolutionären Humanismus (evolutionary humanism). An Darwin bemängelt er, dass der große Forscher nicht berücksichtigt habe, dass die biologische Entwicklung von der Amöbe zum Menschen ganz anderer Natur sei als die der psychosozialen Evolution »vom Wilden der Vorzeit zum zivilisierten Menschen der Gegenwart«:

Die biologische Evolution ist nur ein Sektor oder eine Phase des allgemeinen Evolutionsprozesses. Außerdem gibt es noch den anorganischen oder kosmischen und den psychosozialen oder menschlichen Sektor. Die verschiedenen Phasen folgen zeitlich aufeinander, die späteren fußen auf den früheren und ent-

${ }^{348}$ Vgl. Benz 1961, 94ff.; Benz 1965, 81-91, 148f.; Baillie 1950, 145f.; Passmore 1975, 193 196.

${ }^{349}$ V gl. Huxley 1965, 250-280.

${ }^{350}$ A.a.O., 253. 
wickeln sich aus ihnen. Die anorganische Phase ist prä-biologisch, die menschliche ist post-biologisch. ${ }^{351}$

Teilhards Eintreten für die Eugenik erklärt sich mit seiner Hoffnung auf die Steigerung der cerebralen Fähigkeiten des Menschen: »Und was wollen sie letzten Endes anderes finden als Mittel und Wege zu einem Über-Menschsein oder doch wenigstens einem Ultra-Menschsein? « ${ }^{352}$ Die Wissenschaften dienen demnach nicht einem unendlichen innerweltlichen Fortschritt, sondern sollen als religiöses Ziel die beschleunigte Ausbreitung der Noosphäre durch die geistige Überhöhung des jetzigen Menschen unterstützen. Teilhard formuliert ebenso deutlich wie Huxley die Notwendigkeit des menschlichen Eingreifens in die natürliche Evolution als Konsequenz des Evolutionsprozesses selbst:

Wir finden es bequemer, ja, wir halten es sogar für sicherer, daß sich die Formen dieses großen Körpers ... von selbst herausbilden, auf Grund des blinden Spiels von Willkürakten und individuellen Trieben. Nur nicht in die Kräfte der Welt sich einmischen! Immer noch das Trugbild des Instinkts und der angeblichen Unfehlbarkeit der Natur. Doch erwartet nicht eben die zum Denken gelangte Welt, daß wir die instinktiven Schritte der Natur durchdenken und dadurch vervollkommnen? ? $^{353}$

Die heutigen Möglichkeiten der Naturwissenschaften befähigen Teilhard zufolge die Menschheit zu einer neuen Phase der Evolution des Geistes, die den Beginn der »Auto-Cerebralisation« und Selbstvervollkommnung des Menschen markiere. ${ }^{354}$

Hat uns nicht die Kenntnis der Hormone so weit geführt, daß wir morgen schon auf die Entwicklung unseres Körpers - ja sogar des Hirns Einfluß gewinnen können? Wird uns die Entdeckung der Gene nicht bald die Kontrolle des Mechanismus der organischen Vererbung gestatten? Wird uns die bevorstehende synthetische Herstellung der Eiweißsstoffe nicht eines Tages befähigen, eine Wirkung

\footnotetext{
${ }^{351}$ Huxley 1965, 31.

${ }^{352}$ Teilhard 1961, 116 .

${ }^{353}$ Teilhard 1959, 278.

${ }^{354}$ Vgl. a.a.O., 229-286. Diese Seite von Teilhards Evolutionstheorie wird in der jüngeren, katholischen Rezeption seines Werkes völlig ignoriert: Günther Schiwy gelingt es sogar, den konservativen Kardinal Ratzinger als Schüler Teilhards zu präsentieren - man muss dabei bedenken, dass der Jesuit Teilhard zeitlebens von seinem Orden keine Erlaubnis zur Veröffentlichung seiner philosophischen Schriften erhielt. Vgl. Schiwy 2001, 258-261.
} 
hervorzurufen, die der sich selbst überlassenen Erde versagt scheint: eine neue Woge von Organismen - ein künstlich hervorgebrachtes Neu-Leben? ${ }^{355}$

Huxley, der für Teilhards Le Phénomène Humain (1955) das Vorwort schrieb, sieht sich grundsätzlich in Übereinstimmung mit Teilhard. Wie der Jesuit interpretiert er den Evolutionsprozess als Zuwachs an Wissen und Bewusstsein. Auch teilen beide Denker die absolute Anthropozentrik ihrer Perspektive auf die Evolution: »Das Schicksal hat ihn [den Menschen: OK] dazu auserkoren, für den gesamten zukünftigen Entwicklungsprozeß auf diesem Planeten verantwortlich zu sein. ${ }^{356}$ Die Fortschritte der biologischen Wissenschaften hätten in der Gegenwart zur Folge, dass der Mensch sich seiner eigenen Evolution bewusst werde und diesen Vorgang zum ersten $\mathrm{Mal}$ in seiner Gesamtentwicklung als Entstehungsprozess einer höheren Ordnung von kollektivem Bewusstsein erkennen könne.

Seine [Teilhards: O.K.] Formulierung ist jedoch tiefer und fruchtbringender. Aus ihr geht nämlich hervor, daß wir die »gemeinsam-denkende« (inter-thinking) Menschheit als einen neuen Typ von Organismus, oder besser gesagt, als einen neuen Typ lebender Organisation ansehen sollten, dessen Bestimmung es ist, neue Möglichkeiten für das evolutierende Leben auf unserem Planeten ausfindig zu machen. ${ }^{357}$

In der Gegenwart zählt der Oxforder Biologe Richard Dawkins zu den wohl prominentesten Befürwortern einer antiteleologischen Deutung des Darwinismus, die er vor allem in seinen Monographien The Selfish Gene (1976) und The Blind Watchmaker (1986) erörtert hat. Scharf reagiert Dawkins auf die unwissenschaftlichen Versuche, die darwinistische Evolutionstheorie in Frage zu stellen oder sie mit einem auf William Paley gegründeten design argument in Verbindung zu bringen:

Natural selection, the blind, unconscious, automatic process which Darwin discovered, and which we now know is the explanation of all life, has no purpose in mind. It has no mind and no mind's eye. It does not plan for the future. It has no

${ }^{355}$ Teilhard 1959, 242f. Gerade dieses Bekenntnis zur Wissenschaft macht Teilhards Philosophie nach Bekunden von Robert Ettinger so attraktiv für den Transhumanismus. Vgl. Ettinger 1989, 7f.

${ }^{356}$ Huxley 1965, 123.

${ }^{357}$ A.a.O., 211. Die theologischen Anteile weist Huxley jedoch zurück, auch wenn er die Möglichkeit neuer parapsychologischer, meditativer und mystischer Fähigkeiten des künftigen Menschen nicht ausschließen will. Vgl. Huxley 1965, 223; Benz 1965, 94f.; Passmore 1975, 247-268. 
vision, no foresight, no sight at all. If it can be said to play the role of watchmaker in nature, it is the blind watchmaker. ${ }^{358}$

Die Evolutionsgeschichte erklärt Dawkins auf Darwin aufbauend als Kampf der Gene, die ihre DNA-Informationen begleitet von zahlreichen Transformationsprozessen weitergeben. ${ }^{359}$ Dawkins wird - wenn auch unterschiedlich - von allen posthumanistischen Autoren wahrgenommen. Marvin Minskys Standpunkt, dass die Evolutionsgeschichte ein planloser Prozess der Weiterentwicklung - und nicht etwa einer Höherentwicklung - ist, kann unzweifelhaft auf die fast vollständige Adaption von Dawkins Thesen zurückgeführt werden. Hans Moravec und Ray Kurzweil, die gleich auf mehrere Werke von Dawkins Bezug nehmen, wenden seine Idee der ziellosen Entwicklungsgeschichte ausschließlich auf die Phase der »natürlichen Evolution" an. Aus dieser unintelligenten, langsamen und ineffizienten Phase der Evolution befreie sich, beginnend mit der Menschheit, das intelligente Leben sukzessive. Dawkins Theorie dient den erwähnten Posthumanisten zur Charakterisierung und Abgrenzung der bisherigen, Milliarden Jahre zählenden Evolutionsgeschichte von der neuen, zunehmend beherrschbaren Entwicklung, die durch das Aufkommen von Kultur und Technik gekennzeichnet ist. ${ }^{360}$ Entgegen Dawkins nutzen sie seine Theorien allerdings für die Formulierung einer universalen Teleologie: Die Evolution des Lebens auf der Erde muss in der Hervorbringung der künstlichen Intelligenz münden, die daraufhin den Kosmos besiedelt und diesen vor dem endgültigen Wärmetod rettet.

Offenbar rezipiert Hans Moravec hier Huxleys gestaffeltes Konzept der biologischen und psychosozialen Evolution, was sich nicht nur in der sichtbaren terminologischen Kontinuität des Begriffes "post-biologisch» ausdrückt. Der Grundgedanke der künstlichen bzw. kulturellen Evolution, die die natürliche, biologische Evolution fortführt, wurde auch bei Teilhard thematisiert. In den Entwürfen von Huxley wie Moravec befreit sich die Evolution des Geistes, der Kultur, aus ihrem rein biologischen Vorspiel. Nur legt Moravec hier sein Augenmerk auf die Rolle der

\footnotetext{
${ }^{358}$ Dawkins 1996, 5 .

${ }^{359}$ Vgl. Dawkins 1976; Dawkins 1996, 169-194.

${ }^{360}$ Vgl. Minsky 1992a, 1f.; Kurzweil 1999a, 349; Moravec 1988, 136, 159, 200; Moravec 1999, 4, 213. Kevin Warwick und sogar Frank Tipler berufen sich bei der Definition des Lebens als Weitergabe von Informationen durch die DNA auf Dawkins - die antiteleologischen Kontexte von Dawkins Theorie meidet Tipler jedoch. Vgl. Tipler 1995, 165, 443; Warwick 1998, 45ff.
} 
Technik im Allgemeinen und der Computertechnik im speziellen, die die Loslösung der Kultur aus ihren biologisch-humanen Abhängigkeiten überhaupt erst ermöglicht.

Keiner der posthumanistischen Autoren greift explizit die Gedanken einer rassischen bzw. biologischen Vervollkommnung der Eugeniker auf. Selbst unter den Transhumanisten fristen die gegenwärtigen Verheißungen der Gentechnik eher ein marginales Dasein, da die eigentlichen Bestrebungen der Posthumanisten wie auch der Transhumanisten auf die völlige Loslösung von dem nur schwerlich kontrollierbaren - weil definitiv alternden - biologischen Körper hinauslaufen. Jedoch entspringt das allen Posthumanisten eigene, strukturelle Prinzip, den Menschen aus der natürlichen Evolution zu befreien und einen neuen, höherwertigen Menschen künstlich durch den Eingriff der Wissenschaft hervorzubringen, auch den Theorien der Eugeniker. Denn wie groß die substantielle Nähe zwischen der Idee einer biologischen Vervollkommnung durch Eugenik und der Ergänzung des biologischen Körpers durch chemische und mechanische Mittel ist, das dokumentierte - so überraschend das für manchen auch ist - bereits Teilhard de Chardin.

Die Fortschrittstheorie des Posthumanismus ist das Ergebnis einer philosophischen Entwicklung, die sich seit mindestens drei Jahrhunderten in der europäischen und amerikanischen Geistesgeschichte nachzeichnen lässt. Ihre Quellen sind vielfältig und zahlreich. Die Zentrierung des eigentlichen Fortschrittsprozesses auf die Steigerung von informationsverarbeitenden Rechenleistungen rezipiert Muster der protestantischen Arbeitsethik und der theologischen wie auch säkularen Konzeptionen vom innerweltlichen Fortschritt des Wissens. Im Posthumanismus spiegeln sich damit sowohl theologische wie auch utilitaristische Ideen des 18. und 19. Jahrhunderts von der fortschreitenden Vermehrung von Wissen, Arbeit und Glück wider. Es zeigt sich ferner, dass die posthumanistischen Autoren in ihrer Interpretation der Evolutionsgeschichte Strukturen aufnehmen konnten, die vom Prinzip her bereits in den 1860er Jahren voll entwickelt waren und die die Vision von einer durch mehr Hirnsubstanz höher organisierten Form der Menschheit propagierten.

Die Rezeptionsleistung der Posthumanisten besteht vor allem darin, verschiedenste Elemente des okzidentalen Fortschrittsdiskurses zu einer Synthese zusammenzufügen. Zwar wird der eigentliche Fortschrittsprozess innerhalb des posthumanistischen Diskurses mit dem Bezug auf monistische, metaphysische oder gar christlich-theologische Konzeptionen 
durchaus konträr begründet, jedoch münden alle posthumanistischen Zukunftsanthropologien in der Vision von einem höheren, vollkommeneren - eben posthumanen - Menschentum.

\subsubsection{Die Physikotheologie des Frank Tipler}

Allen posthumanistischen Autoren ist gemeinsam, dass sie versuchen, die Weiterentwicklung der Menschheit und ihrer Nachfahren bis ins technische Paradies der Zukunft wissenschaftlich zu begründen. Nichtsdestotrotz sind die posthumanistischen Fortschrittsberechnungen in ihrer Bewertung des vergangenen Fortschritts gebunden an einen normativen Interpretationshintergrund, der aber weitgehend geleugnet wird. ${ }^{361}$ Geschichtsdeutung als Teil von Fortschrittstheorien erfüllt damit stets auch eine identitätsstiftende Funktion:

Wie immer: geschichtliche Bewegung, also »Tradition« und »Fortschritt«, sind wertorientiert; Geschichte ist Wertverwirklichungsstreben. Um sie zu erkennen und zu verstehen, legen jede Generation und auch jeder Einzelne ihre eigene Werte-Welt, einem Schleier gleich, über die Vergangenheit; was sie durch den hindurch erblicken, das ist ihr »Geschichtsbild «. 362

Die bevorstehenden technischen Fortschritte werden im Posthumanismus als schier unumgängliche Prozesse präsentiert, die je nach Autor verschiedenen Gesetzmäßigkeiten folgen. Während sich Hans Moravec, Ray Kurzweil und andere auf vergangenen menschlichen Fortschritt als Basis für ihre Prognose beziehen, erörtert Frank Tipler von einer kosmologischen Warte aus Sinn und Ziel des Fortschrittes in summa, so dass die entsprechende Analyse der komplexen ideengeschichtlichen Rezeptionsprozesse des Physikers ungleich mehr Raum erfordert. Frank Tipler wird nicht müde zu beteuern, dass seine Omegapunkt-Theorie im Ganzen und jedes einzelne ihrer Postulate das Ergebnis wissenschaftlicher Überlegungen sei. ${ }^{363}$

This book is a description of the Omega Point Theory, which is a testable physical theory for an omnipresent, omniscient, omnipotent God who will one day in the far future resurrect every single one of us in an abode which is in all essentials the Judeo-Christian Heaven. Every single term in the Theory - for example ...

\footnotetext{
${ }^{361}$ Vgl. dazu Rapp 1992, 34.

${ }^{362}$ Berglar 1978, 9.

${ }^{363}$ Vgl. Tipler 1995, IX-XII, 1-17, 139-153; Tipler 1989, 222.
} 
»resurrection (spiritual) body,« »Heaven« - will be introduced as pure physics concepts. In this book I shall make no appeal, anywhere, to revelation. I shall appeal instead to the solid results of modern physical science ... I shall show exactly how physics will permit the resurrection to eternal life of everyone who has lived, is living and will live. ${ }^{364}$

Alle Darlegungen in seinem Buch versteht Tipler im Rahmen der von ihm neu begründeten »physikalischen Eschatologie « ${ }^{365}$ als falsifizierbare und damit wissenschaftliche Aussagen, wobei das so genannte Postulat des ewigen Lebens den Dreh- und Angelpunkt seiner physikalischen Argumentation bildet.

In ihrem gemeinsamen Werk The Anthropic Cosmological Principle bemühen sich Frank Tipler und John D. Barrow, eine längst überwunden geglaubte, teleologische Ausrichtung der Kosmologie wiedereinzuführen. Dabei betonen sie explizit, dass sie als Kosmologen und nicht als Philosophen argumentieren und keine persönlichen Wertungen in den wissenschaftlichen Diskurs einflechten. ${ }^{366}$ Das schwache anthropische Prinzip besagt, dass nur eine begrenzte Zahl aller physikalisch möglichen Universen die Entstehung von Leben begünstigt, während theoretisch sehr unterschiedliche Universen mit vielfältigen physikalischen Verhältnissen und Werten für die Naturkonstanten existieren können. Demnach lässt nur eine eingeschränkte Klasse solcher Modelle intelligente Beobachter zu. Da wir als Beobachter existieren, muss unser Universum eng begrenzte Eigenschaften haben, die unsere Entwicklung ermöglicht haben. Das in der Kosmologie allgemein akzeptierte schwache anthropische Prinzip stellt daher fest, dass die beobachteten Werte aller physikalischen und kosmologischen Einheiten nicht gleich wahrscheinlich sind, aber die Werte annehmen, die ein auf Kohlenstoff basierendes Leben erlauben und dass das Universum alt genug ist, Leben hervorzubringen. ${ }^{367}$

Tipler und Barrow verwenden nun einen Großteil ihres über sechshundertseitigen Buches darauf, in einem Streifzug durch alle Disziplinen der Naturwissenschaft der Frage nachzugehen, warum die physikalischen Konstanten exakt die Werte haben, die sie haben. Die beiden Kosmologen demonstrieren, dass nur eine Verbindung aus den Elementen Kohlenstoff, Wasserstoff, Stickstoff und Sauerstoff solch komplexe Struktu-

\footnotetext{
${ }^{364}$ Tipler 1995, 1.

365 A.a.O., 146 und Barrow \& Tipler 1986, 659.

${ }^{366}$ Vgl. a.a.O., $14 f$.

${ }^{367}$ Vgl. a.a.O., 15-18; Press 1986, 315.
} 
ren wie den Menschen ermöglichen konnte. ${ }^{368}$ In aller Ausführlichkeit legen sie sodann dar, dass nur eine spezifische Größe und ein bestimmtes Alter der Sterne und der Planeten eine Atmosphäre und damit Leben hervorbringen können. ${ }^{369}$ Wenn Leben im Prinzip möglich ist, bedarf es weiterer unsicherer Faktoren, die die Genese intelligenter Lebewesen unterstützen. ${ }^{370}$ Hinter all diesen detailversessenen physikalischen Schilderungen klingt wie die Repetition eines Mantras die wiederholt ausgesprochene Feststellung, wie unwahrscheinlich und zufällig die Entstehung intelligenten Lebens im Kosmos sei: »In short, the evolution of >cognition<, or intelligence and self-awareness of the human type, is most unlikely even in the primate lineage. ${ }^{371}$

Aber stellen Tipler und Barrow auf Hunderten von Seiten all diese unwahrscheinlichen physikalischen Begebenheiten auf, um dann zu behaupten, dies sei alles Zufall? Mitnichten. Agatha Christies berühmte Detektivin wird von den Kosmologen herbeizitiert, um uns den Weg zu weisen: ")Any coincidence<, said Miss Marple to herself, >is always worth noticing. You can throw it away later if it is only a coincidence. « ${ }^{372}$

Die angebliche Zufälligkeit und Unwahrscheinlichkeit der Bedingungen für die Entstehung des menschlichen Lebens bilden für Tipler und Barrow die Substanz für das so genannte starke anthropische Prinzip, das der Kosmologe Brandon Carter in den 1970ern eingeführt hatte. Weitaus spekulativer als das schwache anthropische Prinzip und in der kosmologischen Wissenschaft höchst umstritten, behauptet das starke anthropische Prinzip, dass das Universum im Laufe seiner Entwicklung Beobachter hervorbringen muss, weil es so viele scheinbar unabhängige Zufälle in der Natur gebe: »The universe must have those properties which allow life to develop within it at some stage in its history.«373

Daraus leitete John A. Wheeler, der Mentor von Tipler und Barrow, das partizipatorische anthropische Prinzip ab, das sich wiederum teils an George Berkeleys Argumentation anlehnt: »Observers are necessary to bring the Universe into being. « ${ }^{374}$ Gleichzeitig schließt Tipler in zahlreichen Publikationen die Existenz und künftige Entstehung von

\footnotetext{
${ }^{368}$ Vgl. Barrow \& Tipler 1986, 143-147, 510f., 524-556.

${ }^{369}$ Vgl. a.a.O., 308-359, 557-570.

${ }^{370}$ Vgl. a.a.O., 128-132.

371 A.a.O., 131, vgl. auch a.a.O., 253f., 359f., 558.

372 A.a.O., 288.

373 A.a.O., 21.

${ }^{374}$ A.a.O., 22.
} 
außerirdischem intelligenten Leben aus, ${ }^{375}$ so dass all die zuvor zusammengestellten Unwahrscheinlichkeiten im natürlichen Universum nur deshalb bestehen, um notwendigerweise den Menschen als Beobachter des Universums zu erschaffen. Ohne den Menschen würde dem starken anthropischen Prinzip zufolge das Universum nicht existieren können, was die Autoren dazu verleiten mochte, den antiken Philosophen Protagoras zu zitieren: »Man is the Measure of all things. «"

Weil es aber unverständlich erscheint, warum Leben unter solch unwahrscheinlichen Bedingungen heranreift und wieder vergehen solle, ohne zuvor irgendeinen spürbaren Einfluss auf die weitere Geschichte des Universums gehabt zu haben, setzen Tipler und Barrow das starke anthropische Prinzip in einem finalen anthropischen Prinzip fort: »Final Anthropic Principle (FAP): Intelligent information-processing come into existence in the Universe, and, once it comes into existence, it will never die out. ${ }^{377}$

Das finale anthropische Prinzip, das laut seiner Entdecker als physikalische Aussage bar jeder ethischen und moralischen Wertungen sei, bildet den theoretischen Hintergrund für Tiplers Postulat des ewigen Lebens in der Physik der Unsterblichkeit. ${ }^{378}$ Daher kann Tipler behaupten, dass die Unsterblichkeit des intelligenten Lebens eine notwendige Folge physikalischer Tatsachen sei. Aber obwohl Tipler und Barrow sich in The Anthropic Cosmological Principle bemühen, ihren teleologischen Standpunkt mit einem beeindruckenden Streifzug durch Philosophie und Religion (von Aristoteles, über Boshongo-Mythen bis Teilhard de Chardin) zu legitimieren, ${ }^{379}$ provozierte ihr Vorstoß unter den etablierten Physikern und Naturwissenschaftlern einhellige Ablehnung. ${ }^{380}$

Tipler greift später auf diese Argumentationsweise zurück: Dort nämlich, wo selbst eine mit Formeln angereicherte physikalische Begründung den kritischen Leser nicht überzeugen würde, verweist er eklektisch auf andere Wissenschaftler und Philosophen, die im weiteren Sinne »ähnliche«

\footnotetext{
${ }^{375}$ Vgl. a.a.O., 132-134, 577-591; Tipler 1981, 1991.

${ }^{376}$ Barrow \& Tipler 1986, 556.

377 A.a.O., 23. Intensiv hat sich der Transhumanist und Direktor des Future of Humanity Institute, Nick Bostrom, mit Tiplers und Barrows anthropischem Prinzip auseinandergesetzt. Die wissenschaftlich überprüfbaren Anteile betrachtet er als finale anthropische Hypothese. Vgl. Bostrom 2002, 49-51.

${ }^{378}$ Vgl. Barrow \& Tipler 1986; Tipler 1995, 212f.; Tipler 2007.

${ }^{379}$ V gl. Barrow \& Tipler 1986, 27-203.

${ }^{380}$ Vgl. Press 1986. Der Wissenschaftsjournalist Martin Gardner beschimpfte das FAP gar als CRAP - als Completely Ridiculous Anthropic Principle. Vgl. Bostrom 2002, 50.
} 
Positionen vertraten. ${ }^{381}$ Das Postulat des ewigen Lebens bildet für Tipler die Basis aller weiteren, vermeintlich wissenschaftlichen Schlussfolgerungen über den ewigen Fortschritt, das Ziel der kosmischen Evolution und die Auferstehung der Toten. Im Einzelnen werde ich an späterer Stelle auf diese Punkte eingehen.

Bemühten sich Transhumanisten in den 1990ern und den frühen 2000ern um möglichst klare Abgrenzungen gegenüber Tiplers Positionen, werden heute viele seiner Grundgedanken von den christlichen und mormonischen Transhumanisten geteilt. Und schließlich adaptiert auch Ray Kurzweil wesentliche Elemente der Theorien Tiplers, wie wir noch sehen werden.

Mit dem finalen anthropischen Prinzip und der daraus abgeleiteten Notwendigkeit eines immerwährenden Fortschrittes stellt sich Tipler explizit in die Tradition protestantischer und anglikanischer Naturtheologen bzw. Physikotheologen.

Seit Ende des 17. Jahrhunderts mehrten sich vor allem in England und in den Niederlanden die Versuche naturwissenschaftlich und aufklärerisch beeinflusster Theologen, das Wesen Gottes nicht mehr durch die von Menschen verfertigten Offenbarungsschriften zu bestimmen, sondern durch die Beobachtung der natürlichen Schöpfung selbst zum wahren Wesen des Schöpfers vorzudringen. Tipler bekennt sich ausdrücklich dazu, dass einzig die Wissenschaften - allen voran die Mathematik und Physik - nun ohne Einfluss des menschlichen Subjektes zum besseren Verständnis Gottes und der Destination des Menschen in der göttlichen Schöpfung beitragen können:

The only book which does not suffer from these limitations is the Book of Nature, the only book which God wrote with His/Her own hand, without human assistance. The book of nature is not limited by human understanding. The Book of Nature is the only reliable guide to the true Nature of God. ${ }^{382}$

Als Bekräftigung für diese Haltung führt der Physiker den paulinischen Römerbrief auf: »His invisible attributes, that is to say his everlasting power and deity, have been visible, ever since the world began, to the eye of reason, in the things he has made. ${ }^{383}$ Tipler und Barrow verweisen

\footnotetext{
${ }^{381}$ Tipler verfährt auf diese Weise bei der Idee einer Unsterblichkeit im Computer sowie bei seiner Begründung der Vielweltentheorie, vgl. Tipler 1995, 16f., 170f.

${ }^{382}$ A.a.O., 337.

${ }^{383}$ A.a.O., 4. Römer 1:20.
} 
in aller Ausführlichkeit auf diese Traditionslinie der so genannten Natural Theology oder Physikotheologie, der sie sich verpflichtet fühlen. Besondere Beachtung finden bei ihnen John Ray, ${ }^{384}$ William Paley, ${ }^{385}$ Isaac Newton, Richard Bentley, Colin MacClaurin ${ }^{386}$ und an anderer Stelle die Ideen des amerikanischen Unabhängigkeitsphilosophen Thomas Paine. ${ }^{387}$ Letzteren lobt Frank Tipler mit den amerikanischen Revolutionären George Washington, Benjamin Franklin, Ethan Allen und Thomas Jefferson als ausgezeichneten Vertreter des ihm nahestehenden Deismus. Als mit der neuzeitlichen Aufklärung verbundene Kraft kann der Deismus als ein konfessionsübergreifendes Bekenntnis zur Existenz eines zur weiteren Weltgeschichte transzendenten Schöpfergottes verstanden werden. Der Deismus setzt meist an die Stelle der göttlichen Offenbarung die rationale Erkenntnisfähigkeit des menschlichen Geistes. ${ }^{388}$

Die Tatsache, dass Tipler in diesem Zusammenhang offensichtlich William Paley als maßgeblichen Vertreter der Natural Theology bzw. Physikotheologie rezipiert, erfordert eine differenziertere Auseinandersetzung mit den Ursprüngen dieser von Tipler geforderten Wiedereinführung einer auf dem design argument beruhenden Teleologie in die Wissenschaft. Bereits Mitte des 17. Jahrhunderts fand der puritanisch geprägte Botaniker John Ray mit seiner Predigten-Reihe The Wisdom of God manifested in the Works of Creation großen Anklang, der sich in zahlreichen Übersetzungen niederschlagen sollte. Im beginnenden 18. Jahrhundert muss hingegen der anglikanische geistliche William Derham als der für nachfolgende Autoren wie Paley wohl bedeutendste Physikotheologe gewürdigt werden. ${ }^{389}$ An die hundert, zwischen 1680 und

${ }^{384}$ Vgl. Barrow \& Tipler 1986, 58f. John Ray (1628-1704) gilt als bedeutendster Naturtheologe des 17. Jahrhunderts und verfasste das grundlegende Werk The Wisdom of God manifested in the works of Creation (London 1691).

${ }^{385}$ Vgl. Barrow \& Tipler 1986, 76-81. William Paley schrieb die Natural Theology (London 1802), war seit den 1770er Jahren als Prediger für die Church of England tätig und betrachtete die Entdeckung der Naturgesetze als die Mystik seines Zeitalters.

${ }^{386}$ Vgl. Barrow \& Tipler 1986, 60ff. Richard Bentley (1662-1742) trug in den Boyle Lectures sein berühmt gewordenes Traktat Confutation of Atheism from the Origin and Frame of the World (London 1693) vor. Colin MacClaurin (1698-1746) wurde bekannt durch An Account of Sir Isaac Newton's Philosophical Discoveries (London 1748), das Newtons physikotheologische Signifikanz begründete.

${ }^{387}$ Paine schilderte in seinem Werk The Age of Reason (London 1793) die gesamte Schöpfung als Wort Gottes, das wir als Offenbarung mit Hilfe der Wissenschaft lesen müssten. Vgl. Tipler 1995, 321f.

${ }^{388}$ Vgl. a.a.O., 321-327. Vgl. Gestrich 1981, 392-395.

${ }^{389} \mathrm{Zu}$ Beginn des 18. Jahrhunderts erschienen auch Isaac Newtons naturwissenschaftliche und philosophische Schriften, deren theologische Implikationen großen Einfluss auf 
1727 erschienene Buchtitel führt Derhams deutscher Übersetzer in einer Bibliographie auf, die in der wissenschaftlichen Betrachtung der Natur einen Beweis für die Existenz Gottes und das Lob Gottes erkennen wollten. Derhams drei Hauptwerke nehmen hierunter jedoch eine besondere Stellung ein. ${ }^{390}$ Während sich Derhams Artificial Clockmaker (1696) noch einer allgemeineren Beschreibung und Bewunderung der Schöpfung widmet, steht seine Physico Theology (1713) ganz im Zeichen der irdischen Schöpfungen Gottes. Derham schildert mit zahlreichen wissenschaftlichen Erläuterungen die Vielfalt von Flora und Fauna auf der Erde, wobei der Anatomie und Physiologie des Menschen - und hier wiederum dem Auge als komplexestem Organ - besondere Aufmerksamkeit zuteilwird. ${ }^{391}$ Aus dem durch die Wissenschaft erkennbaren Reichtum der Schöpfung, die uns Gott verliehen habe, leitet Derham den menschlichen Auftrag ab, das anvertraute Gut mit Fleiß zu vermehren und mit den Gaben, die Gott uns verlieh, zu wuchern. ${ }^{392}$

Von ungleich größerer Bedeutung für die Kosmologen Tipler und Barrow sind gewiss die Grundlagen der von Derham 1714 publizierten Astro Theology, or a Demonstration of the Being and Attributes of God, from a Survey of the Heavens. In Anlehnung an Psalm 19 (wonach die Himmel die Ehre Gottes verkünden) stellt Derham hier in sieben Kapiteln Anschauungen über die Größe, Anzahl, Stellung, Bewegung, über Form, Gewicht, Licht und Wärme der himmlischen Körper (Planeten) an und beschließt seine detailreichen astronomischen Betrachtungen mit einer rhetorischen Frage, die angesichts seines »Beweismaterials« nur eine Antwort zulässt: ${ }^{393}$

Wenn wir nun wuerklich sehen und fuehlen diese grosse und liebreiche Vorsorge, diese erstaunens-wuerdige Wuerkungen des grossen Schoepfers ... wenn

\footnotetext{
die entstehende Natural Theology haben sollten.

${ }^{390}$ Nur eine Auswahl von Denkern, die der Übersetzer Fabricius anführt, will ich hier wiedergeben: Basilius, Eustathius Antiochenus, Anastasius Sinaita Cicero, Beda Venerabilis, Joannis Gersons, Robert Boyle (Of the High Veneration Man's Intellect Owes to GOD, 1685), Christian Wolff, Isaac Barrow, Richard Bentley, Nehemia Grew (Cosmologia Sacra, 1701), Francisco Fenelon (Démonstration de l'existence de Dieu, 1712), Bernard Nienwentyt (Het regt gebruik der Werelt beschowingen, 1715), George Cheyne (Philosophical Principles of natural Religion, 1705), Robert Green (The Principles of Natural Philosophy, 1712), William Whiston (Astronomical Principles of Religion, 1717). Vgl. Fabricio 1728, XIII-LXIV.

${ }^{391}$ Vgl. zum Auge: Derham 1764, 147-191.

${ }^{392}$ Vgl. Derham 1764, 438-469.

${ }^{393}$ Vgl. Derham 1728, 1-212.
} 
wir ... in unserem eigenen Sonnen Systeme beobachten einen feurigen Koerper von so entsetzlichen Umfang als die Sonne ist, gestellet in den Mittel-Punct, die Finsternis zu vertreiben, uns zu wärmen und mit dem Tag zu freuen ... wer kann anders als zum allerhoehesten bewundern solche vortreffliche Wercke Gottes, die mit solcher Vorsorge, zu solchem Nutzen, und so wohl eingerichtet sind? ${ }^{394}$

Derham verehrt Gott als vernünftigen Meister der Mathematik, Optik und Medizin, der seine Fürsorge nicht nur der Erde, sondern auch den anderen Planeten zukommen lasse. ${ }^{395}$ Er erteilt damit einer rein anthropozentrischen Interpretation der Schöpfung eine Absage: »Denn was ist unsere ganze Erd-kugel als ein Punct, ein nichts gegen das ganze Weltgebäude ... « ${ }^{396}$ Aus der Erkenntnis der astronomischen Unbedeutsamkeit des Irdischen heraus mahnt Derham zu Demut und Askese und fordert die Besinnung auf das ewige Streben der Seele nach dem Himmlischen. Das Erscheinen Christi unter den Menschen hat nach Ansicht Derhams die Erde vor allen anderen Himmelskörpern im Universum als Krone der Schöpfung und Zentrum des Kosmos ausgezeichnet. ${ }^{397}$

Bald ein Jahrhundert nach Derham griff der englische utilitaristische Philosoph und anglikanische Priester William Paley dessen Ideen wieder auf und veröffentlichte 1802 die Natural Theology, or Evidences of the Existence and Attributes of the Deity collected from the Appearances of Nature. Im Kontext der Religionskritik seiner Zeit fokussiert Paley seine weitgehend mechanistisch geprägten Erörterungen auf das design argument. Seine Beschreibungen der Pflanzen, der Tiere und des Menschen - der als »most complicated or most flexible machine that was ever contrived « bezeichnet wird - gipfeln in der Feststellung, dass allein schon die Vollkommenheit des Auges als Gottesbeweis genügen würde. ${ }^{398}$ Auch wenn er den kosmologischen Beweis im Gegensatz zu Derham aufgrund der Einfachheit der Bewegung der Himmelskörper nicht für den besten hält, ${ }^{399}$ kann nichts seine Überzeugung erschüttern, dass der Mensch, die Erde und das ganze Universum Teile einer geplanten Schöpfung eines intelligenten Gottes seien, der das Glück seiner Geschöpfe wünsche: »The marks of

${ }^{394}$ A.a.O., $208 f$.

${ }^{395}$ Zum Beispiel sollten die Saturnringe nach Ansicht Derhams Wärme und Licht des damals vermeintlich sonnenfernsten Planeten für die dortigen Lebewesen zurückhalten. Vgl. a.a.O., 197-208.

396 A.a.O., 235.

${ }^{397}$ Schon Pascal, Fontennelle und die Cambridge Platonists hatten jedoch Zweifel, ob Gottes Interesse hauptsächlich der Erde gelte. Vgl. a.a.O., 212-245.

${ }^{398}$ V gl. Paley 1842, 451-467, 490f.

${ }^{399}$ Vgl. a.a.O., 517-523. 
design are too strong to be gotten over. Design must have had a designer. This designer must have been a person. That person is god. ${ }^{400}$

William Paley markiert den Höhepunkt der Natural Theology, bevor dann im 19. Jahrhundert rein materialistische Welterklärungen den wissenschaftlichen Diskurs dominieren sollten. In dem Verweis auf die letzten Vertreter der Physikotheologie, den calvinistischen Evolutionstheoretiker Asa Gray und den Bischof von Birmingham, Ernest William Barnes, drückt Frank Tipler sein Bedauern über die vollzogene Trennung von Religion und Wissenschaft aus. ${ }^{401}$ Viele von Paleys und Derhams Thesen macht sich Tipler zu eigen - ja er stellt sich selbst an die Spitze einer wiederbelebten, nun sogar experimentellen Natural Theology, wenn er für 200 Millionen Dollar einen Teilchenbeschleuniger aufrüsten will, um über Versuche mit Higgs-Bosonen und durch die Entdeckung des Topquarks die Existenz Gottes zu beweisen: ${ }^{402}$

The SSC and the LHC [Teilchenbeschleuniger: O.K.] are often compared to the cathedrals of the Middle Ages and to the pyramids of ancient Egypt. The cathedrals were built to help the medieval Europeans to find God and the Egyptian kings find their immortality. If I am right, the SSC and the LHC could do both for all humanity. ${ }^{403}$

Galt der Beweis einer geplanten Schöpfung und eines intelligenten Schöpfers zwar auch in der Vergangenheit als Beleg für eine insgesamt positive Weiterentwicklung der Menschheit, so lag das Hauptinteresse der Physikotheologen doch sichtlich auf dem Existenzbeweis Gottes wider den atheistischen Positionen der französischen Aufklärer Diderot, Holbach und Helvétius. Tipler entwirft aus dem klassischen design argument nun eine teleologische Zukunftsanthropologie, die den Beweis eines geplanten Universums als Folie einer futurologischen Extrapolation instrumentalisiert. Die Gottesfrage tritt zunächst in den Hintergrund. Indem er die Existenz von extraterrestrischen, intelligenten Lebewesen kategorisch ausschließt, spitzt Tipler das design argument auf eine absolute Anthropozentrik hin zu, während William Derham und andere Naturtheologen die Idee von "menschenähnlichem « Leben auf anderen Planeten durchaus tolerierten. Beide Elemente fügt Tipler kreativ zu

\footnotetext{
${ }^{400}$ A.a.O., 530.

${ }^{401}$ Vgl. Barrow \& Tipler 1986, 85f., $183 f$.

${ }^{402}$ Vgl. Tipler 1995, $335 \mathrm{f}$.

${ }^{403}$ A.a.O., 336. SSC $=$ Super-Conducting Supercollider, LHC = Large Hadron Collider.
} 
einer vermeintlich wissenschaftlichen, anthropozentrischen Teleologie zusammen.

Etwas schwerfällig knüpft auch Hans Moravec am Ende seines zweiten Werkes an Tiplers und Barrows Futurologie an, die auf den Punkt Omega von »Tielhard de Chardin« (sic!) hinauslaufe. ${ }^{404}$ Die Inkonsistenz und Willkürlichkeit von Moravecs Erklärungen zeigt sich jedoch an dem Fakt, dass er an anderer Stelle explizit auf die Bedrohung durch Außerirdische verweist. Die Existenz von Außerirdischen jedoch würde die gesamte Fortschrittslegitimation von Tiplers anthropozentrischer Teleologie untergraben. ${ }^{405}$

Wie ist Tiplers Verhältnis zur Religion nun insgesamt zu bewerten? Einerseits beschwört er den wissenschaftlichen Geist reiner Objektivität und betont, dass seine physikalischen Einsichten die »letzte Wirklichkeit« beschreiben, die unabhängig von allen kulturellen Konzepten bestehe. ${ }^{406}$ Andererseits bestätigt er, dass seine Omegapunkt-Theorie mit den allen Religionen gemeinsamen Grundzügen übereinstimme, zumal alle Religionen eigentlich an eine Auferstehung der Toten und nicht an die Unsterblichkeit der menschlichen Seele glauben würden. ${ }^{407}$

In seinem Gottesverständnis lehnt sich Tipler explizit an christliche Theologen wie Origines, Thomas von Aquin, George Berkeley, Paul Tillich und Teilhard de Chardin an und schließt seinen religiösen Exkurs mit einem Zitat der mittelalterlichen Mystikerin Juliana von Norwich, das ihn persönlich berührt habe: "Sin must needs be, but all shall be well. All shall be well; and all manner of thing shall be well.< Mother Julian has nicely summarized the Omega Point theodicy. « ${ }^{408}$ Tipler präsentiert seinen Ansatz dabei als physikalische Vereinnahmung - gar als Rettung - der Theologie. ${ }^{409}$

The Omega Point Theory allows the key concepts of the Judeo-Christian-Islamic tradition now to be modern physics concepts: theology is nothing but physical cosmology based on the assumption that life as a whole is immortal ... Physics

${ }^{404}$ Vgl. Moravec 1999, 201f. und Moravec 1988, $148 f$.

${ }^{405}$ Vgl. a.a.O., 101; Tipler 1981; 1991; 1995, 311f.; Tipler \& Barrow 1986, 132-134, 577591.

${ }^{406}$ Vgl. Tipler 1995, 1, 88, 294f.

${ }^{407}$ Vgl. Tipler 1995, 16f., 269-304.

${ }^{408}$ A.a.o., 265. Tipler zitiert aus: The Revelations of Divine Love of Julian of Norwich, translated by James Walsh. London 1961, Kap. 26. Vgl. auch a.a.O., 4-13, 153-158, 214ff., 264; Tipler 1989, 229-253.

${ }^{409}$ Vgl. Tipler 1995, XIVf., 2-8, 247, 293, 327f., 339; Barrow \& Tipler 1986, $180 f$. 
has now absorbed theology; the divorce between science and religion, between reason and emotion, is over. ${ }^{410}$

Im Ganzen betrachtet, verhält sich Tiplers Argumentation zirkulär. Tipler behauptet, die Aussagen der Religionen mit der Physik zu beweisen. Wir haben allerdings mehr als deutlich gesehen, dass Tiplers Argumentation selbst auf religiösen Voraussetzungen - von protestantischer Theologie bis zu Teilhard - aufbaut. Tipler beweist tautologisch die Religionen mit einer vermeintlich physikalischen Theorie, die ihrerseits auf religiösen und philosophischen Annahmen beruht. Selbst die kosmologische Perspektive seiner physikalischen Eschatologie verliert ihren wissenschaftlichen Charakter mit der Einführung des für Tipler zentralen Postulates des ewigen Lebens. Tipler benutzt hier den scheinbar unfehlbaren Mantel der objektiven Naturwissenschaften, um den unsicheren, religiösen Glauben an die Unsterblichkeit zu beweisen.

\subsection{Singularitäten}

Singularity, The. The Techno-Rapture. A black hole in the Extropian worldview whose gravity is so intense that no light can be shed on what lies beyond it. ${ }^{411}$

Godling's Glossary

Durch Ray Kurzweils Buch The Singularity is near: When Humans Transcend Biology, die nachfolgende Gründung der Singularity University im Jahr 2008 und zahlreiche Filme hat die Idee vom Anbruch eines neuen Zeitalters, das durch künstliche Intelligenzen geprägt sein wird, weit über das transhumanistische Milieu hinaus Bekanntheit erlangt. ${ }^{412}$

Es gab in der Vergangenheit bereits einige Versuche, verschiedene Ansätze der Singularität zu ordnen und zu analysieren. Die kulturwissenschaftlichen Arbeiten bleiben jedoch mitunter sehr undifferenziert und teils polemisch. ${ }^{413}$ David Porush bewertet die »Cybergläubigkeit« schon

${ }^{410}$ Tipler 1995, 338.

411 Godling's Glossary 1998. Zitiert nach Sandberg http://www.aleph.se/Trans/Global/ Singularity/index.html.

${ }^{412}$ Vgl. Loh 2018, 106-112.

${ }^{413}$ Für Selmer und Alexander Bringsjord sowie Paul Bello ist die gesamte Singularitätstheorie eine Glaubenssache ohne wissenschaftliche Grundlagen. Vgl. Bringsjord \& Bringsjord \& Bello 2012. 
1992 in diesem Sinne als einen modernen Cargo-Kult in Naherwartung materieller Heilsgüter. Diane Proudfoot spricht generalisierend von einem techno-supernaturalism. ${ }^{414}$ Die Transhumanisten Anders Sandberg und Socrates (vom Singularity Weblog) führen neun bzw. 17 Definitionen der technologischen Singularität auf. ${ }^{415}$ Drei Typen der Singularität identifiziert der transhumanistische Aktivist Eliezer Yudkowsky: den beschleunigten Wandel (accelerating change) à la Kurzweil, den Ereignishorizont (event horizon) à la Vinge und letztlich seine eigene, auf Irving Good basierende Schule der Intelligenzexplosion (intelligent explosion). Ärgerlich reagiert er auf das Unvermögen seiner Zeitgenossen, diese Differenzierung nicht wahrnehmen zu wollen und alles zu einem einzigen »Singularity-Kleister « zu verquirlen. ${ }^{416}$ Sandberg, Socrates und Yudkowsky ist anzumerken, dass sie sehr darum bemüht sind, einen klaren und wissenschaftlich präzisen Singularitätsbegriff zu etablieren. Besonders Sandberg präsentiert die verschiedenen Ansätze als weitgehend mathematisierte Wahrscheinlichkeitsmodelle. Diese Versuche müssen allerdings als normative Prozesse innerhalb des Transhumanismus angesehen werden. Tatsächlich sind die zentralen Konzepte der Singularität äußerst diffus und vermischen Elemente einer wissenschaftlichen Prognostik mit religiösen Inhalten. Selbst eine strikte Trennung des technologischen vom kosmologischen Singularitätsverständniss erscheint vor dem Hintergrund der vorliegenden Quellen nicht plausibel. ${ }^{417}$ Mit Reinhard Heil plädiere ich dafür, diese verschiedenen semantischen Schichten in die kulturwissenschaftliche Analyse miteinzubeziehen, um die komplexen Verflechtungen zwischen Religion und Naturwissenschaft betrachten zu können. ${ }^{418}$

Der Begriff der Singularität umfasst wissenschaftliche Konzepte in der mathematischen Funktionen- und Systemtheorie, der Geometrie, der

${ }^{414}$ Vgl. Proudfoot 2012, 367f.; Porush 1992. Die melanesischen Cargo-Kulte hofften im 20. Jhd. auf die Erfüllung ihrer materiellen Wünsche durch fliegende Gottheiten, die sie später mit den amerikanischen Soldaten gleichsetzten.

${ }^{415}$ Ohne Erläuterung wird auch Teilhard de Chardin von Sandberg als einer dieser Typen identifiziert. Vgl. Sandberg 2013b; Socrates 2012.

${ }^{416}$ Vgl. http://yudkowsky.net/singularity/schools. Nick Bostrom hatte in einem Kommentar zu Vinges Konzept bereits 1998 diese drei Typen identifiziert, die Yudkowsky jedoch griffiger fasste. Vgl. Bostrom 1998b, 399.

417 Blendet man die religiösen und kosmologischen Elemente aus, so ist klar, dass hiermit unliebsame Ansätze wie z.B. Tiplers Physikotheologie ausgeschlossen werden. Auf diese Weise entsteht dann eher der Eindruck eines konsistenten und wissenschaftlichen Singularitätsbegriffes, was vielen Transhumanisten gelegen käme.

${ }^{418}$ Vgl. Heil 2010b, 44-46. 
Festkörperforschung sowie der Kosmologie und eben der Kybernetik. Die zwei letztgenannten Bereiche haben für den Posthumanismus eine besondere Bedeutung. Kratzt man nicht nur an der ideengeschichtlichen Oberfläche, so wird rasch offenbar, dass diese beiden Bereiche eng miteinander verflochten sind und ihrerseits wieder vielfache Bezüge insbesondere zum theologischen Werk Teilhard de Chardins und seinem Konzept des Punktes Omega aufweisen. In der populären Publizistik verschwimmen diese Elemente gegenwärtig zu einer einzigen, mystisch aufgeladenen Technikprophetie. ${ }^{419}$ In drei Schritten werden wir daher den Singularitätsbegriff untersuchen: Auf die darstellenden Abschnitte zur kosmologischen und technologischen Singularität folgt die kulturgeschichtliche Kontextualisierung des Konzeptes.

\subsubsection{Die kosmologische Singularität und die schwarzen Löcher}

Anders als im Deutschen ist der Begriff der kosmologischen Singularität im Englischen bereits seit den 1980ern verbreitet und wird in vielen populären Rezeptionen in der Literatur und in Fernsehserien kreativ verwendet. Den Kosmologen Roger Penrose und Stephen Hawking zufolge bezeichnen Singularitäten (im Plural) die besonderen Bedingungen von Raum und Zeit, wie sie z.B. durch Schwarze Löcher entstehen. Dies sind Momente, an denen Materie bzw. ihre Vorläufer sich auf einen einzigen Punkt konzentrieren und Raum und Licht unendlich gekrümmt werden. Auch der Beginn des Universums, der Urknall (big bang), wird durch eine Singularität markiert. ${ }^{420}$ Das populäre Verständnis der Singularität bezieht sich meist auf die phantastischen Raum- und Zeiteffekte von Schwarzen Löchern, für das das Penrose-Hawking-SingularitätenTheorem Anwendung findet. ${ }^{421}$

${ }^{419}$ Ein Artikel der Computerzeitschrift Chip erweckt z.B. den Eindruck, als handele es sich bei den Ideen von Kurzweil und Vinge zur Singularität nur um einen weiteren Aspekt der kosmologischen Heilsgeschichte des Jesuiten Pierre Teilhard de Chardin. Vgl. Leiphold 2016, 50.

${ }^{420}$ V gl. Hawking \& Penrose 1970.

${ }^{421}$ Die populären Rezeptionen des Begriffes beziehen sich in der Literatur und in Fernsehserien fast ausschließlich auf die kosmologische Singularität und werden hier vage mit Schwarzen Löchern und deren Effekte in Verbindung gebracht: so in den Romanen Singularity von William Sleator (1985) und Bill de Smedt (2004), in der Graphic Novel Singularity 7 von Ben Templesmith (2004), dem Dr. Who Hörspiel Singularity (2005) sowie in den Fernsehserien Stargate (Staffel 1, Folge 15, Singularity von 1997), Star Trek: Enterprise (Staffel 2, Folge 9, Singularity von 2002) und So Weird (Staffel 1, 
Zusammen mit John D. Barrow hat Frank Tipler das Konzept der kosmologischen Singularitäten schon früh in philosophische Bahnen gelenkt, die die Frage nach dem Leben und dem Menschen im Universum miteinbeziehen. ${ }^{42}$ Verbunden mit ihrer These vom finalen anthropischen Prinzip reflektieren die beiden Kosmologen über die initiale und die finale Singularität im Modell eines geschlossenen Universums, also den Beginn und das Ende des Universums, das in diesem Moment keine raumzeitliche Ausdehnung besitzt. Bereits hier sehen Barrow und Tipler Analogien zum Werk von Teilhard de Chardin und setzten die finale Singularität mit dem göttlichen Punkt Omega gleich. Die beiden Ansätze lassen sich miteinander verbinden, da dem finalen anthropischen Prinzip zufolge das Ende des Universums einen letzten Beobachter erfordert, der für Tipler mit Gott-Omega identisch ist. ${ }^{423}$ In seinen späteren Werken, Physics of Immortality (1994) und Physics of Christianity (2007), baut Tipler auf diesen Überlegungen auf und bettet die kosmologischen Singularitäten in theologische Deutungsmuster ein, d.h. nicht nur das Endziel des Universums ist Gott, sondern auch der erste Grund (first cause), der noch keinen physikalischen Gesetzen unterliege. ${ }^{424}$

Die nachfolgende Graphik veranschaulicht die zeitlichen Dimensionen von Tiplers Entwurf. Erst in unserer Gegenwart beginnt die Biosphäre des irdischen Planeten mit der Expansion ins Weltall, um einhergehend mit der Besiedlung des gesamten Universums seine heilsgeschichtliche Funktion zu entfalten. Im Interview mit Socrates vom Singularity Weblog beschreibt Tipler 2013 die Eigenschaften der finalen kosmologischen Singularität folgendermaßen:

The singularity is outside the natural world, it is beyond the natural world, and it is transcendent to the natural world. So, approaching the singularity ... the amount of information, the amount of knowledge is approaching infinity as you are going into the final state. The processing rate is increasing to infinity. So, the total amount of information processing will be infinite. ${ }^{425}$

Folge 11, Singularity von 1999). Lediglich in jüngeren Werken wird ein direkter Bezug auf die technologische Singularität genommen wie in der Fernsehserie Agents of S.H.I.E.L.D. (Staffel 3, Folge 18, The Singularity von 2016) und kritisch im low-budget Spielfilm Singularity (2017) von Robert Kouba, in dem die Menschheit von einer übermächtigen künstlichen Intelligenz fast ausgerottet wird. Vgl. Art. »Singularity« (engl. Wikipedia).

${ }^{422}$ Vgl. Barrow \& Tipler 1978; Barrow \& Tipler 1979; Barrow \& Tipler 1981.

${ }^{423}$ Vgl. Barrow \& Tipler 1986, 201-204, 470f.

${ }^{424}$ Vgl. Tipler 1995, 139-157; Tipler 2007, 83-100.

${ }^{425}$ Tipler 2013 (Interview). 


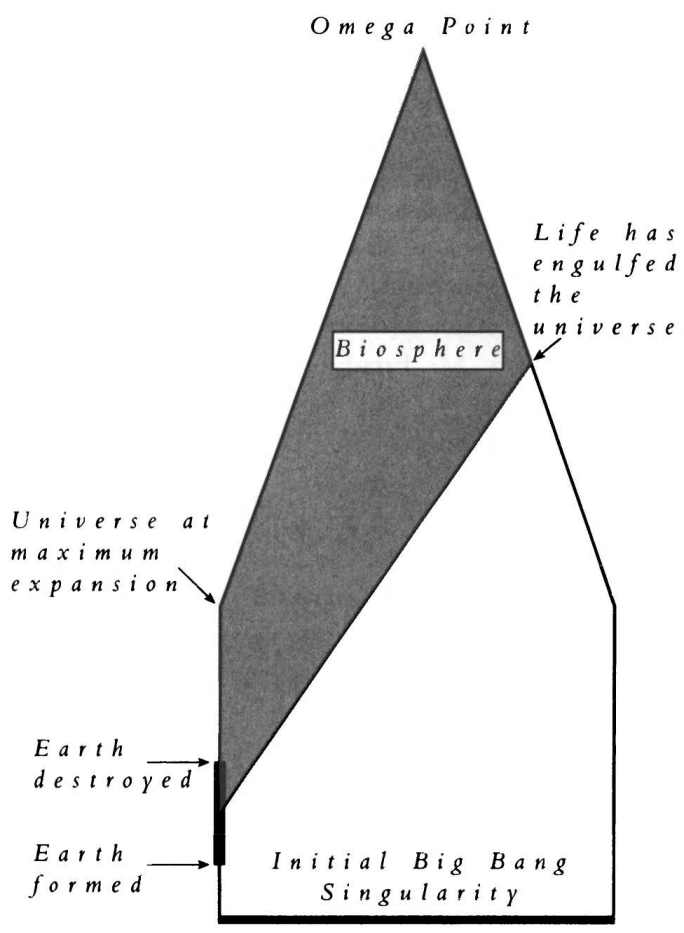

Tipler 1995, Figure IV.9, p. 145,

Penrose Diagram of the future of life in the universe

Dem von Kurzweil und anderen Denkern propagierten Konzept der technologischen Singularität begegnet Tipler inklusivistisch. Die technologische Singularität sei lediglich ein philosophischer Begriff, während die kosmologische ein bewiesenes mathematisches Theorem darstelle. Die technologische Singularität ist Tipler zufolge nur ein kleines Ereignis in der menschlichen Geschichte, das durch die Unausweichlichkeit der Bewegung auf die kosmische Singularität hin bedingt sei. »The cosmological singularity is determining, requiring the existence of the computer science singularity. And I agree with various people as Hans Moravec and Ray Kurzweil. And I think the singularity in computer science will occur in this century. I think we are very close. I think we already have the necessary hardware. ${ }^{426}$

$\overline{{ }^{426} \text { Tipler }} 2013$ (Interview). 
Spätestens wenn er sich selbst als »fundamentalistischen Physiker « bezeichnet, wird offensichtlich, dass nicht ein Hauch von Ironie in Tiplers Aussagen liegt. Unter den beiden Voraussetzungen, dass das Universum geschlossen ist und die Menschheit die einzige intelligente Lebensform im Kosmos ist (beides ist laut Tipler mathematisch bewiesen), muss das Leben auf der Erde einen neuen Träger finden:

Namely, that eventually human meat, rational beings will be replaced by human downloads and our artificial intelligence of reason at least at the human level. I am convinced that's true. I am convinced it must be true because as you are going into the final singularity, necessarily, ... life can no longer exist, it has to move on another substrate. And, well, that's just human downloads. ${ }^{427}$

\subsubsection{Die technologische Singularität}

Der Mathematiker und Kybernetiker John von Neumann wird von den Post- und Transhumanisten gemeinhin als Urheber des Begriffes der technologischen Singularität identifiziert. ${ }^{428}$ Man bezieht sich hier auf den ausführlichen Nachruf auf von Neumann, den sein langjähriger Freund und wissenschaftlicher Weggefährte Stanisław Ulam 1958 anfertigte. Den entsprechenden Absatz möchte ich hier in voller Länge wiedergeben.

Quite aware that the criteria of value in mathematical work are, to some extent, purely aesthetic, he once expressed an apprehension that the values put on abstract scientific achievement in our present civilization might diminish: »The interests of humanity may change, the present curiosities in science may cease, and entirely different things may occupy the human mind in the future." One conversation centered on the ever accelerating progress of technology and changes in the mode of human life, which gives the appearance of approaching some essential singularity in the history of the race beyond which human affairs, as we know them, could not continue. ${ }^{429}$

Die Trans- und Posthumanisten Vernor Vinge, Ray Kurzweil, Nick Bostrom und Anders Sandberg zitieren nur den letzten Satz dieser Passage und lassen keinen Zweifel daran aufkommen, dass der Compu-

\footnotetext{
${ }^{427}$ Tipler 2013 (Interview).

${ }^{428}$ Vgl. Vinge 2013, 366; Kurzweil 2005, 10; Kurzweil 2012, 27f.; Sandberg 2010, 376;

Bostrom 2014, 261 FN 2; Eden 2012b, 4f.

${ }^{429}$ Ulam 1958, 5.
} 
ter-Pionier John von Neumann hiermit ausschließlich die Entstehung von künstlicher Intelligenz gemeint haben könne, die ein Ende der bisherigen Geschichte bewirke. Blickt man jedoch auf den größeren $\mathrm{Zu}-$ sammenhang in von Neumanns Biographie und Ulams akademischer Würdigung, so ist eine andere Interpretation ungleich wahrscheinlicher. Denn John von Neumann und mit ihm Stanisław Ulam waren bereits an der gravierendsten technologischen Entwicklung beteiligt, die die menschliche Geschichte bisher hervorbrachte: dem Bau der ersten Atombomben für ihren Einsatz in Hiroshima und Nagasaki am 6. und 9. August 1945.

Der aus einer Budapester Familie stammende János Neumann galt als einer der besten Mathematiker seines Jahrhunderts und erhielt als junger Privatdozent in Berlin 1933 den Ruf auf eine Mathematikprofessur am Institute for Advanced Studies in Princeton, die er bis zu seinem Tod 1957 innehatte. Neben seinen grundlegenden Beiträgen zur mathematischen Mengenlehre und der Spieltheorie befasste sich von Neumann mit Problemen der Quantenmechanik und nach seiner Einbürgerung (samt Namenswechsel) im Jahr 1937 auch vermehrt mit der Berechnung von Strömungen sowie Druckwellen, wie sie für die Optimierung von ballistischen Geschossen und Bomben notwendig waren. Aufgrund dieser Expertise und seiner Vertrautheit mit der Quantenphysik holten ihn der Physiker Robert Oppenheimer und der Initiator des Manhattan-Projektes, Vannevar Bush, 1943 in das Forschungsteam in Los Alamos. Von Neumann spielte hier keine Nebenrolle, sondern war maßgeblich an der Entwicklung des komplexen Zündmechanismus der ersten Atombombe beteiligt. Er konnte mit seinen Berechnungen belegen, dass die Zündung der Bombe weit über der Erdoberfläche die größte Zerstörung anrichten würde. Von Neumann wie auch Oppenheimer gehörten auch zu den Wissenschaftlern, die Teil des militärischen Zielauswahlkomitees waren, die schließlich Hiroshima und Nagasaki als Orte der Vernichtung bestimmten. Während sich der Leiter des Projektes, Robert Oppenheimer, nach dem Einsatz der zweiten Bombe zunehmend kritisch äußerte und sich Ende 1945 ganz aus Los Alamos zurückzog, waren John von Neumann und Stanisław Ulam an der weiteren Entwicklung der ersten Wasserstoffbombe beteiligt. Diese umfasste die zwanzigfache Sprengkraft der Hiroshima-Bombe. ${ }^{40}$ Ulams Schilderungen von Neumanns Rolle im

${ }^{430}$ Vgl. Ulam 1958, 35-39. 
Atomprogramm nehmen viel Raum im Nachruf ein und von Neumann scheint sich der Bedeutung seiner Forschungen bewusst gewesen zu sein:

Von Neumann strongly believed that the technological revolution initiated by the release of nuclear energy would cause more profound changes in human society, in particular in the development of science, than any technological discovery made in the previous history of the race. In one of the very few instances of talking about his own lucky guesses, he told me that, as a very young man, he believed that nuclear energy would be made available and change the order of human activities during his lifetime $!^{431}$

Da wir keine weiteren Quellen von Neumanns besitzen, die eine Verbindung zwischen der Entwicklung von künstlicher Intelligenz und der technologischen Singularität nahelegen würden, müssen wir davon ausgehen, dass sich der erwartete Umbruch eher auf die künftige Kernforschung und deren ambivalente Folgen für die menschliche Geschichte bezieht. Die Initiierung der Doomsday Clock zur Messung des Risikos eines Atomkrieges spiegelt diese Ambivalenz bis zum heutigen Tag wieder. ${ }^{432}$ Die punktuelle Verwendung des Begriffes der Singularität, die uns nur durch Ulam überliefert ist, kann hier lediglich als metaphorische Übertragung des mathematischen Singularitätsbegriffes verstanden werden. Von Neumann wie auch Ulam waren ja vor allem Mathematiker. Weder definiert von Neumann einen bestimmten Zeitpunkt noch eine besondere Qualität der Singularität. Das Ende der bisherigen menschlichen Geschichte, auf das er anspielt, ist damit auch nicht eindeutig als positiv und wünschenswert konnotiert.

Ein Vierteljahrhundert später schlägt der amerikanische Mathematiker und Science-Fiction Autor Vernor Vinge $\left({ }^{*} 1944\right)$ in einem einseitigen Impulsartikel für das Technikmagazin Omni 1983 erstmals explizit die Brücke vom kosmologischen zum technologischen Singularitätsbegriff: $^{433} »$ We will soon create intelligences greater than our own. When this happens, human history will have reached a kind of singularity, an

${ }^{431}$ Ulam 1958, 39.

${ }^{432}$ Mit der Gründung des Bulletin of the Atomic Scientists wurde 1947 die symbolische Doomsday Clock initiiert, die den geschätzten Abstand zu einem möglichen Atomkrieg kennzeichnet. Stand sie nach dem Ende des kalten Krieges auf 17 Minuten vor zwölf, wurde sie wegen der zunehmenden globalen Spannungen zwischen nationalistisch ausgerichteten Staaten Anfang 2018 auf zwei Minuten vor zwölf justiert.

${ }^{433}$ Im Jahr 2000 gab Vinge seine Dozentenstelle für Mathematik an der San Diego State University zugunsten seiner schriftstellerischen Tätigkeit auf. Vgl. Wagner 2015, 31-40; Heil 2010b, 44. 
intellectual transition as impenetrable as the knotted space-time at the center of a black hole, and the world will pass far beyond our understanding. « ${ }^{434}$ Allerdings ist für Vinge zu diesem Zeitpunkt noch offen, ob diese neuen Intelligenzen durch Gentechnik oder Computertechnik generiert werden. Abfällig äußert er sich hier gegenüber jeder Art von Zukunftsprophetie: »A favorite game of futurists is to plot technological performance - computer speed, say - against time. Such trend curves climb ever more steeply. Extrapolated 30 or 40 years, they are so high and steep that even the most naive futurist discounts their accuracy. « ${ }^{435}$ In den kommenden Jahren verwendet Vinge die Singularität lediglich als dramaturgische Hintergrundfolie in mehreren seiner Science-Fiction Romane wie z.B. Marooned in Realtime (1986). ${ }^{436}$

Seine vormalige, futuristische Zurückhaltung währte allerdings nicht lange. 1993 verkündet Vinge auf dem Vision-21-Symposium der NASA ohne den geringsten Zweifel: »Within thirty years, we will have the technological means to create superhuman intelligence. Shortly after, the human era will be ended. « ${ }^{437}$ Vinge skizziert vier Möglichkeiten, wie diese technologische Singularität Gestalt annehmen könnte: erstens durch Computer oder zweitens Computernetzwerke, die Bewusstsein und eine übermenschliche Intelligenz entwickeln; drittens durch Mensch-Computer-Schnittstellen, die Menschen superintelligent machen oder viertens durch biologische Verbesserungen des Menschen. Da die ersten drei Möglichkeiten stark von der Computerhardware abhängen, prognostiziert Vinge das Eintreffen der Singularität dann für die Periode zwischen 2005 und 2030. Er begründet seine Voraussage mit Verweis auf die Erwartung einer »ultraintelligenten Maschine« durch den Kybernetiker Irving Good und der Singularität gemäß Ulam bzw. von Neumann: »Von Neumann even uses the term singularity, though it appears he is thinking of normal progress, not the creation of superhuman intellect. ${ }^{438}$ Er stellt jedoch sogleich klar: »For me, the superhumanity is the essence of the Singularity. Without that we would get a glut of technical riches, never properly absorbed. ${ }^{439}$ In diesem Punkt wird das

\footnotetext{
${ }^{434}$ Vinge 1983, 10. Bezüge zu Irving Good und John von Neumann erscheinen hier noch nicht.

${ }^{435}$ Vinge 1983, 10.

${ }^{436}$ Vgl. Raulerson 2013, 3-15.

${ }^{437}$ Vinge 1993.

${ }^{438}$ Vinge 2013, 366.

${ }^{439}$ Vinge 2013, 366.
} 
heilsgeschichtliche Moment sichtbar, zumal Vinge in einem Interview von 2011 betont, dass seine persönliche Motivation für die Formulierung der Singularität darin besteht, das Universum als sinnvoll zu begreifen (»making some sense of the universe «). ${ }^{440}$

Die Singularität werde alle bisherigen Regeln des menschlichen Lebens revolutionieren und in sehr kurzer Zeit Veränderungen herbeiführen, denen bisher nur eine Analogie in der Geschichte der Evolution entspräche: »The rise of humankind. We will be in the Post-Human era. And for all my rampant technological optimism, sometimes I think I'd be more comfortable if I were regarding these transcendental events from one thousand years remove ... instead of twenty. « ${ }^{441}$ Alles, was nach der Singularität geschehe, sei nicht vorhersagbar (completely unknowable). Damit rezipiert Vinge, wie schon in seinem frühen Artikel von 1983, das Konzept des Ereignishorizontes (event horizon): Hinter diesem Punkt sind in der astrophysikalischen Betrachtung Schwarzer Löcher keine Aussagen möglich. ${ }^{42}$

Die Singularität ist Vinge zufolge unvermeidbar, sofern es prinzipiell möglich ist, eine künstliche Intelligenz zu programmieren, die sich autonom weiterentwickeln kann. Neben Arbeitslosigkeit für immer größere Teile der Bevölkerung sieht Vinge die Konsequenzen der Singularität durchaus kritisch: "If the Singularity can not be prevented or confined, just how bad could the Post-Human era be? Well ... pretty bad. The physical extinction of the human race is one possibility. ${ }^{443}$ Vinge schlägt daher verschiedene Wege einer Partizipation des Menschen an der Computerintelligenz vor, die hauptsächlich durch Mensch-Maschine-Interfaces realisiert werden könnten. Angesichts der Überlegenheit der Computer könne die Unsterblichkeit dem Menschen ein würdevolles Dasein bescheren. Ein Leben in Computernetzwerken könne sodann die heutigen Grenzen des Selbst überwinden: »What happens when pieces of ego can be copied and merged, when the size of a selfawareness can grow or shrink to fit the nature of the problems under consideration? « 44 In einem späteren Aufsatz bezeichnet Vinge diese Vision einer Digital

\footnotetext{
${ }^{440}$ Vinge 2011 (Video).

${ }^{441}$ Vinge 1993; Vinge 2013, 367.

${ }^{442}$ Vinge 1993; Vinge 2013, 367.

${ }^{443}$ Vinge 1993; Vinge 2013, 369.

${ }^{444}$ Vinge 1993; Vinge 2013, 373. Vinge selbst sieht technologische Neuerungen bisweilen sehr kritisch und vermeidet soziale Medien zum Schutz seiner Privatsphäre, wie er in dem Interview mit Socrates von 2011 unterstreicht. Dieser war verwundert, dass er das Gespräch über ein Festnetztelefon führen musste. Vgl. Vinge 2011 (Video).
} 
Gaia als höchst wahrscheinlich: »The Digital Gaia would be something beyond human intelligence, but nothing like human. $^{445}$

Einen ersten Versuch, die punktuelle Idee der technologischen Singularität in ein weitreichendes philosophisches Konzept zu transformieren, unternahm der amerikanische KI-Forscher Eliezer Yudkowsky mit der Formulierung der Singularitarian Principles von 1999. Yudkowsky versteht sich als Atheist, Transhumanist und Kryoniker und plädiert in seinen Prinzipien für eine scharfe Trennung zwischen dem technologischen Singularitätsbegriff und religiösen Elementen. Das ausschweifende Dokument enthält viele ambitionierte Erklärungen zur »Ultratechnologie«, der Globalisierung, der Vergöttlichung des Menschen (apotheosis) und Solidarität sowie ferneren Aspekten. Singularitarians seien demnach »Partisanen«, die die technologische Singularität als übermenschliche Intelligenz für wünschenswert halten und auf dieses Ziel hinarbeiten.

The Singularity holds out the possibility of winning the Grand Prize, the true Utopia, the best-of-all-possible-worlds - not just freedom from pain and stress or a sterile round of endless physical pleasures), but the prospect of endless growth for every human being - growth in mind, in intelligence, in strength of personality; life without bound, without end; experiencing everything we've dreamed of experiencing, becoming everything we've ever dreamed of being ... ${ }^{446}$

In die transhumanistische Debatte bringt Yudkowsky Ende der 1990er als einer der wenigen Aktivisten das Moment der Solidarität mit ein. Wer für die Vergöttlichung eintrete, müsse auch zustimmen, dass Jedermann Göttlichkeit erhält. Wer die Auslöschung der Menschheit durch die KI in Kauf nehme, müsse sich auch mit der eigenen Auslöschung abfinden können. Ein messianischer Optimismus und ein Glaube an die technologische Lösung aller Daseinsprobleme kennzeichnet den jungen Yudkowsky: »I'm working to save everybody, heal the planet, solve all the problems of the world. $\ll^{447}$

Der heilsgeschichtliche Aspekt der lebensspendenden Singularität in Yudkowskys Futurologie wird in einem intimen Moment sichtbar, als er 2004 über den plötzlichen Tod seines jüngeren Bruders schreibt: »Even if we make it to and through the Singularity, it will be too late. One of

\footnotetext{
${ }^{445}$ Vgl. Vinge 2008.

${ }^{446}$ Yudkowsky 2000a.

${ }^{447}$ Yudkowsky 2000b. Trotz identischer bibliographischer Angaben, unterscheiden sich die beiden Textversionen (2000a/2000b) der Singularitarian Principles erheblich voneinander.
} 
the people I love won't be there. The universe has a surprising ability to stab you through the heart from somewhere you weren't looking. « ${ }^{44}$ Anfang der 2000er Jahre wird Yudkowsky auf der transhumanistischen Webseite Transtopia noch als Begründer einer der zwei Schulen der Singularitarians aufgeführt. Die seine sei durch Altruismus, Kollektivismus und Paternalismus gekennzeichnet. ${ }^{449}$ Von den etablierten Transhumanisten wird Yudkowsky abgesehen von Bostrom und Sandberg kaum rezipiert, jedoch in die wissenschaftliche Auseinandersetzung um Roboterethik und KI-Entwicklung einbezogen. ${ }^{450}$

Wie ist nun der Ansatz des inzwischen einflussreichsten Posthumanisten, Ray Kurzweil, in der Debatte einzuordnen? Vernor Vinge, der seine These der Singularität zur einen Hälfte noch im Genre der ScienceFiction Literatur verortet, legitimiert seine Prognose tautologisch: »But if the technological Singularity can happen, it will. « ${ }^{451}$ Frank Tipler begründet die künftige Technikentwicklung von der Warte einer kosmologischen Teleologie aus. Für Yudkowsky erscheint die Singularität als ein gegebenes Faktum. Ray Kurzweil und mit ihm Hans Moravec wählen einen anderen Weg, der sich augenscheinlich stärker an überprüfbaren Kriterien orientiert. Beide Denker extrapolieren den künftigen technologischen Fortschritt aus der Beobachtung bisheriger Trends, wobei nur Kurzweil in seinen jüngeren Publikationen ab 2005 das Konzept der Singularität einführt. ${ }^{452}$ In diesem Zusammenhang erscheint es sinnvoll, auch die Entwicklung dieser Prognosen in den vergangenen drei Dekaden im Auge zu behalten.

Wenn der Maßstab für die Vervollkommnung des Lebens die Leistung der Informationsverarbeitung ist, dann werden Vergangenheit und $\mathrm{Zu}-$ kunft unter diesem Paradigma gemustert. Moravec und Kurzweil präsentieren in großen Teilen ihrer Publikationen Daten zum Wachstum der Rechen- und Speicherkapazitäten von Computern sowie Detailfra-

\footnotetext{
${ }^{448}$ Eliezer Yudkowsky, Eintrag über seinen 2004 verstorbenen Bruder Yehuda. http:// yudkowsky.net/other/yehuda.

${ }^{449}$ Die Seite war von 2000 bis etwa 2010 aktiv und übertrug ihre Inhalte später an euvolution.com, wo sich eine Mischung zwischen Transhumanismus, Kryonik, Ufologie und Eugenik findet. Vgl. Transtopia, FAQ, Singularitarian. https://web.archive.org/ web/20090303082302/http://www.transtopia.org:80/faq.html\#1.10.

${ }^{450}$ In einschlägigen Textsammlungen wie dem Transhumanist Reader (More \& Vita-More 2013) fehlt Yudkowsky.

${ }^{451}$ Vinge 1993; Vinge 2013, 368.

${ }^{452}$ Vgl. Moravec 1999, 95-110; Kurzweil 1999a, 189-252.
} 
gen der Schaffung künstlicher Intelligenz. ${ }^{453}$ Ihrer Hoffnung auf eine exponentiell beschleunigte Weiterentwicklung und Verbreitung von Computern und Robotern legen beide Autoren eine mit vielen Daten untermauerte Gesetzmäßigkeit des Fortschrittes zugrunde: das so genannte Gesetz von Moore (Moore's Law). ${ }^{454}$ Die Annahme einer ständigen Beschleunigung der Computerentwicklung geht auf den ehemaligen Intel-Vorsitzenden Gordon Moore zurück, der Mitte der 1960er Jahre behauptet hatte, dass die Größe einer integrierten Schaltung sich alle 24 Monate halbiere, das heißt mit anderen Worten, doppelt so leistungsfähig werde. Diese Voraussage, die heute als Moore's Law geläufig ist, impliziert die exponentielle Leistungssteigerung von Computern für alle Zeiten. Die Auffassung, dass Computer für Jedermann in Kürze die Kapazität des menschlichen Gehirns haben werden, hält sich daher hartnäckig. ${ }^{455}$

Bereits in ihren früheren Werken teilen Hans Moravec und Ray Kurzweil die Idee einer Beschleunigung des informationstechnischen Fortschrittes, insbesondere wenn man die gesamte Evolutionsgeschichte des Lebens miteinbezieht. ${ }^{456}$ Moravec aber auch Marvin Minsky äuBern sich mit zunehmendem Alter zurückhaltender und bisweilen auch skeptischer, was die baldige Verwirklichung von künstlicher Intelligenz auf menschlichem Niveau angeht. 1970 prophezeite Minsky noch, dass innerhalb von drei bis acht Jahren ein Computer mit der Intelligenz eines durchschnittlichen Erwachsenen konstruiert werden könne. Wenige Monate später sollten bereits Computer mit Genie-Level verfügbar sein. In einem Interview mit seinem Schüler Ray Kurzweil hatte sich Marvin Minsky 2010 noch optimistisch gezeigt und die Ankunft der Singularität zu »Lebzeiten« erwartet. Drei Jahre später resümiert er kühl, dass der Glaube an die Singularität vielen jungen Leuten Jobs beschert habe, aber er selbst kaum Fortschritte in der KI-Forschung der letzten 10 bis 15 Jahre beobachte. Künstliche Intelligenz auf dem Niveau eines Menschen prognostizierte er nun ca. für das Jahr $2050 .^{457}$

In ähnlicher Weise ist Moravec in seiner viel beachteten Monographie von 1988 noch davon ausgegangen, dass uns in »kürzester Zeit« univer-

\footnotetext{
${ }^{453}$ Vgl. Moravec 1988, 37-51, 60-75; Moravec 1999, 51-64; Kurzweil 1999a, 103-116, 137142; Kurzweil 2005, 14-110.

${ }^{454}$ Vgl. Moravec 1988, 68; Kurzweil 1999a, 13-25.

${ }^{455}$ Vgl. Kurzweil 1999a, 17-39, 103-105, 204, 220ff.

${ }^{456}$ Moravec 1999, 110, vgl. auch Moravec \& Shieber 1997, 1001.

${ }^{457}$ Vgl. Roszak 1994, 122f.; Minsky 2010 (Video); Minsky 2013 (Video).
} 
sale Haushaltsroboter vom Koch bis zum Putzgehilfen die Arbeit erleichtern würden. ${ }^{458}$ In seinem zweiten Buch Robot von 1999 schenkt er den Entwicklungsproblemen von künstlicher Intelligenz und Robotern eine ungleich größere Beachtung. Er zielt insbesondere auf die Diskrepanz zwischen den überragenden mathematisch-abstrakten Fähigkeiten eines Computers und den kognitiven und motorischen Unzulänglichkeiten von computergestützten Robotern. Dabei hält er weiterhin an der These einer starken Beschleunigung fest, aber datiert die Ankunft übermenschlicher KI jetzt ebenfalls auf das Jahr 2050. ${ }^{459}$ Dann jedoch werden die posthumanen Intelligenzen der Zukunft weit jenseits unserer biologischen, kognitiven und operationalen Beschränkungen neue Welten entdecken und durch die ungeheure Geschwindigkeit ihrer gedanklichen Verarbeitung die geistigen Leistungen eines ganzen Menschenlebens in wenigen Sekunden erzielen: ${ }^{460}$ »Someday our progeny may exploit these bodies [die Planeten: O.K.] to build machines with a million million million million million (that's $10^{30}$ ) times the power of a human mind.« ${ }^{461}$

Ray Kurzweil hat sich im Gegensatz zu allen anderen posthumanistischen Vordenkern sowie transhumanistischen Aktivisten im Verlauf der letzten zwei Dekaden nicht vorsichtiger und zurückhaltender geäußert. Seine drei zentralen Bücher The Age of Intelligent Machines (1990), The Age of Spiritual Machines (1999) und The Singularity is Near (2005) folgen der dramaturgischen Choreographie einer steten Steigerung der futuristischen Aussagen. Mit dem Abschluss dieser Trilogie ist allerdings auch die Grenze von Technikprophetie zu spiritueller Philosophie, die dem Christentum und dem New Age nahesteht, überschritten. ${ }^{462}$

Hierin zeigt sich übrigens eine Parallele zu fehlgeschlagenen Voraussagen der christlichen Apokalypse. Mit Blick auf die zentrale Immortalisierungsutopie der Post- und Transhumanisten, von denen viele inzwischen das Rentenalter erreicht haben, gibt es keine nennenswerten, technischen Fortschritte in den vergangenen 30 Jahren. Viele Denker dieser Bewegung wurden kritischer gegenüber der früheren Euphorik und seriöse Forscher wiesen die Technikvisionen beständig zurück. Ein

\footnotetext{
${ }^{458}$ Vgl. Moravec 1988, 22-25.

${ }^{459}$ Vgl. a.a.O., 13-22; Moravec 1999, VIII, 15-51; Moravec 2009.

${ }^{460}$ Vgl. Moravec 1988, 114 ff.; Moravec 1999, $207 f$.

${ }^{461}$ Moravec 1988, 74.

${ }^{462}$ Die Kernidee eines technologischen Wendepunktes formuliert er schon 1999. Vgl. Kurzweil 1999a, 36.
} 
möglicher Weg, wie innerhalb religiöser Prophetie mit dem Ausbleiben von Vorhersagen umgegangen wird, ist ebendiese Steigerung versprochener Heilsgüter, wie sie Kurzweil betreibt.

Schon 1999 skizziert Kurzweil das von ihm so benannte Gesetz vom steigenden Ertragszuwachs (Law of Accelerating Returns), das um 2020 Moore's Law ablösen und eine noch stärkere Beschleunigungsrate unter sich selbst entwerfenden Maschinengenerationen der Zukunft begründen soll. Nicht nur der Zuwachs wäre dann exponentiell, sondern auch der Exponent selbst würde exponentiell wachsen. Daher würden - so Kurzweil in seinem Buch von 1999 - um das Jahr 2023 erschwingliche PCs mit der Rechenleistung des menschlichen Gehirns und 2030 mit der eines ganzen Dorfes zur Verfügung stehen. Im Jahr 2029 würden demnach 99\% der Denkleistung auf unserem Planeten von Computern erbracht. Es werden laut Kurzweil dann kaum noch Menschen in der industriellen Produktion, Landwirtschaft oder dem Transportwesen tätig sein. ${ }^{463}$ Seine Prognosen legitimiert er fortwährend durch Rückblicke auf seine früheren Veröffentlichungen und bescheinigt sich selbstzufrieden, dass fast alle seiner bisherigen Vorhersagen eingetroffen wären, genauer gesagt seien $86 \%$ zutreffend oder großenteils zutreffend gewesen. Die Auswahl der überprüften Beispiele ist jedoch höchst selektiv. ${ }^{464}$

Fünf Stufen identifiziert Kurzweil in der Evolutionsgeschichte bis zur Verwirklichung der Singularität: 1. die Entstehung der Materie, 2. die Entstehung des Lebens, 3. die Entstehung der Gehirne / des Geistes, 4. die Entstehung der Technologie und 5. die Verschmelzung von menschlicher und maschineller Intelligenz. In der sechsten Phase werde die übermenschliche Intelligenz die Besiedlung des gesamten Universums in Angriff nehmen. ${ }^{465}$ Die Singularität, die gleich dem Urknall eine neue Schöpfung des gesamten Kosmos bedeutet, markiert dabei einen absoluten Höhepunkt technologischer Prophetie.

Das Konzept selbst definiert Kurzweil nur knapp: »It's a future period during which the pace of technological change will be so rapid, its impact so deep, that human life will be irreversibly transformed ... «466 Eine genauere Beschreibung verschließe sich dem Menschen: »So how do we contemplate the Singularity? As with the sun, it's hard to look at

\footnotetext{
${ }^{463}$ Vgl. a.a.O., 17-39, 103-105, 204, 220ff.; Kurzweil 2005, 24-29.

${ }^{464}$ Vgl. Kurzweil 2010, 5. Rothblatt übernimmt diese Einschätzung, vgl. Rothblatt 2014, 44-53.

${ }^{465}$ Kurzweil 2005, 14-21, 35-111

${ }^{466}$ Kurzweil 2005, 7.
} 
directly; it's better to squint at it out of the corner of our eyes. « ${ }^{467}$ Diane Proudfoot entlarvt dieses Bildnis als ein Gleichnis zur Unbeschreibbarkeit Gottes, wie es in der christlichen Mystik gebräuchlich war. So verkündet Anselm von Canterbury im 11. Jahrhundert: »I cannot look directly into [the light in which God dwells], it is too great for me ... it is too bright ...the eye of my soul cannot bear to turn towards it for too long. 468

Die prophetische Bedeutung seiner Aussagen akzentuiert Kurzweil mit einer exakten Datierung der Singularität (in übergroßen Buchstaben):

I set the date for the Singularity - representing a profound and disruptive trans-formation in human capability - as 2045. The nonbiological intelligence created in that year will be one billion times more powerful than all human intelligence today. ${ }^{469}$

Bleiben die Kriterien, was denn die Verwirklichung der Singularität ausmacht, bei Kurzweil recht vage, so sind die verheißungsvollen Aussichten grenzenlos. Schon in den Eingangszeilen seines Buches verkündet Kurzweil, dass in Bälde alle Magie, die dem Leser aus den Harry Potter-Romanen bekannt sei, nun bald technologisch verfügbar wäre. ${ }^{470}$

The Singularity will allow us to transcend these limitations of our biological bodies and brains. We will gain power over our fates. Our mortality will be in our own hands. We will be able to live as long as we want (a subtly different statement from saying we will live forever). We will fully understand human thinking and will vastly extend and expand its reach. By the end of this century, the nonbiological portion of our intelligence will be trillions of trillions of times more powerful than unaided human intelligence. ${ }^{471}$

Neu erscheinen in Kurzweils Singularitätsbuch die Überlegungen zur kosmologischen Bedeutung der irdischen Ereignisse und dem endgültigen Ziel des Lebens im Universum. Zunächst übernimmt er die von Vinge etablierte Analogie des Ereignishorizontes Schwarzer Löcher: »Just as we

\footnotetext{
${ }^{467}$ Kurzweil 2005, 371.

${ }^{468}$ Anselm von Canterbury, The prayers and meditations of St Anselm, chap. 16, 257. Zitiert nach Proudfoot 2012, 368.

${ }^{469}$ Kurzweil 2005, 136 (Großdruck im Original). Auf der Gedenkfeier für Marvin Minsky stellt Kurzweil in Aussicht, dass der Verstorbene im Jahr 2045 wiederbelebt werden könne. Vgl. Kurzweil 2016 (Video).

${ }^{470}$ Kurzweil 2005, 4.

${ }^{471}$ Kurzweil 2005, 9.
} 
find it hard to see beyond the event horizon of a black hole, we also find it difficult to see beyond the event horizon of the historical Singularity. « ${ }^{472}$

Vernor Vinge und Ray Kurzweil haben mit ihrem Verständnis der Singularität verschiedene qualitative Elemente kanonisch festgelegt.

John von Neumann und Irving Good werden als Urheber bestimmt. Die Singularität bedeutet einen radikalen und rasanten Umbruch.

Sie ist eine Folge der evolutionären Entwicklung des Lebens.

Sie ist determiniert, sie wird in jedem Fall eintreffen.

Sie ist mit der Entwicklung superintelligenter Computersysteme verbunden.

Die Menschheit kann durch Verschmelzung mit den Computern partizipieren.

Vorhersagen nach dem Zeitpunkt der Singularität sind nicht möglich. Die Singularität ermöglicht die Unsterblichkeit des Menschen.

Der kosmologische und technologische Singularitätsbegriff ergänzen einander.

Die Entwürfe Frank Tiplers und Eliezer Yudkowskys bilden in diesem Kontext Extrempole des technikprophetischen Spektrums: Tipler am christlichen Ende, Yudkowsky am atheistischen - Vinge und Kurzweil oszillieren zwischen diesen weltanschaulichen Polen. Erstaunlicherweise bleiben bei allen Autoren die exakten Merkmale, die das Eintreffen der Singularität kennzeichnen, äußerst vage. Was macht denn das Erscheinen der »Super-« oder »Ultraintelligenz« aus? Wenn die erste Maschine klüger ist als ein Mensch? KI kann schon heutzutage erfolgreicher Go, Schach oder ein Wissensquiz spielen als jeder Mensch auf diesem Planeten. Oder ist der Moment gemeint, an dem sich eine Maschine autonom weiterentwickelt? Oder von Menschen (welchen?) nicht mehr verstanden wird, was sie tut? Oder ist gar erst der Zeitpunkt der virtuellen Immortalisierung die volle Singularität? ${ }^{473}$

Der Erfolg der Singularitäts-Idee ist vor allem dem breiten futurologischen Netzwerk heutiger und künftiger Unternehmer, Aktivisten und Blogger zu verdanken. Diente das 2000 begründete Singularity Institute for Artificial Intelligence (heute MIRI) mit seinen Singularity Summits als früher Motor der Singularitäts-Debatte, ${ }^{474}$ so erhielt die kalifornische Singularity University (SU) seit ihrem Start 2008 weltweite Aufmerksam-

\footnotetext{
${ }^{472}$ Kurzweil 2005, 487.

${ }^{473}$ Auch Sandberg beklagt sich über diesen Mangel an Klarheit (2013a, 378).

${ }^{474}$ Vgl. Kapitel 4.5.
} 
keit. Von Unternehmen wie Google, Nokia und SAP wird sie großzügig gesponsert. Die $S U$ ist dabei keine herkömmliche Universität mit fixen Studiengängen, qualifizierenden Abschlüssen und Forschungseinrichtungen. Genau genommen ist es überhaupt keine Universität und sie hat auch nichts mit der Singularität zu tun. ${ }^{475}$ Das von Ray Kurzweil und dem Unternehmer Peter Diamandis angestoßene Projekt bot zunächst nur mehrwöchige Workshops zur Entwicklung von Start-Ups an. Die Jungunternehmer sollten Business-Ideen entwickeln, die innert zehn Jahren mindestens das Leben von einer Milliarde Menschen entscheidend verändern können (das Angebot firmiert heute unter dem Titel $S U$ Labs). Kurzweils Kerngedanken sollen hier unternehmerisch unter dem Motto umgesetzt werden: »Exponential technology will disrupt all industries!« Als Botschafter dieses exponentiellen Traums treten dann IT-Magnaten wie Peter Thiel oder die Google-Gründer Larry Page und Sergey Brin auf. Seit Kurzweils Anstellung als Director of Engineering bei Google im Dezember 2012 sind die Zukäufe des Unternehmens in den Sparten Robotik, Clouds, Neuro-Schnittstellen, KI und Biotechnologien bemerkenswert - alles Technologien, die für die Verwirklichung einer Kurzweilschen Singularität nötig wären. ${ }^{476}$

2012 wurde die Universität in eine so genannte benefit corporation umgewandelt, die sowohl profit- als auch non-profit-Bereiche einschließen darf. Das Tätigkeitsfeld wurde massiv erweitert und umfasst inzwischen Angebote in Global Solutions, Executive, Innovation Partnership und Exponential Regional Partnership. Diese wenden sich an unterschiedliche Zielgruppen wie Business-Leader, Start-Up-Gründer oder idealistische Weltverbesserer, die »exponentielle « Strategien für ihre Märkte entwickeln wollen. Unter dem Label der Singularity University werden inzwischen weltweit Summits organisiert, die Zukunftsmärkte und Technologien präsentieren und dem Netzwerken dienen sollen; die Preise für eine zweibis dreitägige Teilnahme belaufen sich auf ca. 1700,- bis 10.000,- Euro. Parallel dazu wurde die Medienplattform des Singularity Hub initiiert, um über wissenschaftliche Durchbrüche zu berichten. ${ }^{47}$

Auch die Initiative 2045 des russischen Internetmilliardärs Dmitry Itskov ist stark durch Kurzweils Futurologie beeinflusst. Mit dem 2011 gestar-

\footnotetext{
${ }^{475}$ Vgl. https://su.org/. Sie ist auch nicht formell als Universität registriert. Vgl. dazu den kritischen Beitrag The Emperor has no Clothes von Socrates (2015).

${ }^{476}$ Vgl. Wagner 2015, 63-67; Christoph Keese (Executive Vice President der Springer AG) im Gespräch mit Thomas Wagner. Vgl. Wagner 2015, 75f.; https://su.org.

477 Vgl. https://singularityhub.com.
} 
teten Forschungsprogramm will Itskov bis ins Jahr der Singularität den Transfer einer menschlichen Persönlichkeit in einen Computerspeicher ermöglichen. Die Zwischenstufen benennt er in Anlehnung an die Hindu-Mythologie Avatare A-D. ${ }^{478}$

Die wichtigste Kommunikationsplattform für die Debatte um die Singularität hat seit Oktober 2009 der Publizist Nikola Danaylov, alias Socrates, aufgebaut. Kreisten viele Beiträge auf dem Singularity Weblog zu Beginn nur euphorisch um die Frage, wann denn die Singularität eintreffen werde, so hat sich inzwischen ein kritischer Diskurs entwickelt. Im Zentrum stehen die ausführlichen Interviews mit Visionären und Denkern der Tech-Branchen. ${ }^{479}$

So stark ausgeprägt die Überzeugung ist, dass die Singularität kommen und alle Menschheitsprobleme lösen werde, so gering ist das Reflexionsniveau, was denn die Singularität im Kern ausmache. Dabei gestaltet sich die Debatte um die Singularität innerhalb des Transhumanismus vielfältig und kontrovers. ${ }^{480}$

Kritisiert wird die gedankliche Etablierung einer mystischen Wand des Nicht-Wissens (Singularität als Ereignishorizont), hinter die man mit keiner Prognostik blicken könne. ${ }^{481}$ Anders Sandberg, Max More und der Quantenphysiker Michael Nielsen bemängeln, dass die Singularität nach Vinge und Kurzweil mathematisch konstruiert und die sozialen und ökonomischen Bedingungen zu ihrer Hervorbringung kaum berücksichtigt werden. ${ }^{482}$ Die schärfste Kritik unter Transhumanisten zieht das Konzept allerdings aufgrund der Vermischung von Religion und wissenschaftlicher Prognostik auf sich. Die Idee der Singularität sei »krypto-mystisch « und "pseudo-religiös« (so der Science-Fiction-Autor Damien Broderick), der Begriff sei wegen der »unheiligen Allianz von

\footnotetext{
${ }^{478}$ Vgl. Wagner 2015, 77-94. Neben den üblichen Transhumanisten hat Itskov auch religiöse Vertreter aus dem Buddhismus, der christlichen Orthodoxie sowie den HinduTraditionen einbezogen. http://gf2045.com/speakers/.

${ }^{479}$ Vgl. https://www.singularityweblog.com.

${ }^{480}$ Die Verbindung zwischen der Singularität mit übermenschlicher Intelligenz und der Ermöglichung des Mind-Uploads wird von kaum einem Autoren hinterfragt. Vgl. Heil 2010b, 44; More \& Vita-More 2013, 395-418; Broderick 2013; Brin 2013. Außerhalb des Transhumanismus besteht natürlich eine sehr umfangreiche Kritik gegenüber Kurzweils Annahmen und Voraussagen, die v.a. seine Fortschrittskalkulationen und den schlecht definierten Intelligenzbegriff attackieren. Vgl. als Überblick Proudfoot 2012, 373

${ }^{481}$ Nielsen 2013, 410; Hanson 2013, 406.

${ }^{482}$ Vgl. Sandberg 2013b, 411-416; Nielsen 2013, 409-411; More 2013, 407f.
} 
Techno-Utopien mit religiös-eschatologischen Elementen« überhaupt zu meiden (Nick Bostrom) und sie erscheine wie der orgiastische Mix technologischer Utopien mit christlicher Apokalyptik (Max More): ${ }^{483}$

Many discussions just close with, »But we cannot predict anything about the post
singularity world!« ending all further inquiry just as Christians and other religi-
ous believers do with, »It is the Will of God.« And it is all too easy to give the
Transcension eschatological overtones, seeing it as Destiny. This also promotes
a feeling of helplessness in many, who see it as all-powerful and inevitable. ${ }^{484}$

Es ist verständlich, dass die meisten Vertreter des Posthumanismus den Thesen Kurzweils mit Skepsis begegnen. Zum einen spielte Kurzweil um das Jahr 2000 noch gar keine Rolle innerhalb der transhumanistischen Bewegung. Zum anderen sind die meisten Extropianer und Transhumanisten von ihren Grundeinstellungen her sehr individualistisch und antiautoritär geprägt. ${ }^{485}$ Ein charismatisch auftretender Führer mit weitreichenden Visionen stößt hier natürlicherweise auf Widerstand. Das Gefühl einer feindlichen Übernahme liegt in der Luft.

Allerdings muss man auch die Frage stellen, was aus dem Transhumanismus ohne Kurzweil, seine Universität und die ungeheure Popularisierung des Singularitätsbegriffes geworden wäre. Anfang 2010 schrieb ich bereits einen Abgesang auf den Transhumanismus unter dem Titel »Much Ado about Nothing - oder: Der Transhumanismus als Phänomen der Jahrtausendwende?«. Tagungszyklen wurden eingestellt, das Extropy-Institute wurde 2006 aufgelöst und schließlich blieben die erhofften technischen Durchbrüche aus. Ohne Kurzweil, den Hype um die Singularität und seine Person, den Transcendent Man, wäre es heute recht still um den Transhumanismus.

\subsubsection{Fortschrittsgesetze und die endless frontier}

Auf welche Weise kann man nun ein temporales Konzept wie die Singularität analysieren? Handelt es sich um ein technologisches Faktum, das auf gesetzmäßigen Berechnungen beruht? Viele Beobachter der

${ }^{483}$ Vgl. Broderick 2013, 398; More 2013, 407; Bostrom 2014, 2. In einem frühen Kommentar hielt Bostrom die Singularität noch für eines der wahrscheinlicheren Szenarios. Vgl. Bostrom 1998b.

${ }^{484}$ Broderick 2013, 398.

${ }^{485}$ Vgl. Yudkowsky 2000. 
technophilen Szene bezweifeln dies. Soziale, psychologische und kulturelle Faktoren spielen eine zentrale Rolle bei der Verkündigung heilsgeschichtlicher Prognosen. Nick Bostrom bemerkt süffisant, dass seit den 1940er Jahren die Prognosen über die Verwirklichung künstlicher Intelligenz Jahr für Jahr stets nach hinten verschoben wurden, meist ungefähr zwanzig Jahre in die Zukunft: »Two decades is a sweet spot for prognosticators of radical change: near enough to be attention-grabbing and relevant, yet far enough to make it possible to suppose that a string of breakthroughs, currently only vaguely imaginable, might by then have occurred. ${ }^{486}$

Der MIT-Professorin Pattie Maes war zu Beginn der 1990er Jahre aufgefallen, dass die meisten ihrer männlichen Kollegen von der Idee fasziniert waren, ihre Gehirne in naher Zukunft in Computerspeicher hochladen zu können und damit Unsterblichkeit zu erlangen. Sobald die erste übermenschliche Intelligenz realisiert wäre, könne diese ja umgehend das Immortalitätsproblem lösen. Die Herausforderung bestand darin, noch bis zu diesem entscheidenden Moment zu leben. 1993 präsentierte Maes ihre systematisierten Beobachtungen zu den Voraussagen ihrer Kollegen auf der Ars Electronica in Linz unter dem Titel »Why Immortality is a Dead Idea«. Das erstaunliche Ergebnis war, dass fast alle Futuristen das Eintreffen der Unsterblichkeitstechnik gerade noch in ihrer zu erwartenden Lebenszeit ansetzten. Egal wann die Prognosen gemacht wurden oder wie alt die Akteure waren, die erhoffte Rettung würde sie im Alter von ca. 70 Jahren beglücken. ${ }^{487}$

Mit wissenschaftlicher Präzision sind Stuart Armstrong und Kaj Sotala vom MIRI nun dieser Frage nach der Systematik von KI-Vorhersagen nachgegangen. Sie analysierten 257 zeitliche Vorhersagen für das Eintreffen einer universalen KI (der Fragehorizont war also weiter gefasst als bei Pattie Maes, die lediglich KI-Vorhersagen in Verbindung mit Unsterblichkeitshoffnungen fokussierte). Armstrong und Sotala konnten aufzeigen, dass eine sehr große Unsicherheit in den KI-Prognosen besteht. Dies betrifft einerseits die Methoden der Vorhersagen (scheinbare Gesetzmäßigkeiten, philosophische Argumente, gefühlter Status des Experten) als auch die Ziele, die eine Bandbreite von sechs bis über 75 Jahren umfassten. Erhellend war insbesondere, dass die Schätzungen

\footnotetext{
${ }^{486}$ Bostrom 2014, 4.

${ }^{487}$ Kevin Kelly, der ehem. Herausgeber von Wired, hatte hieraus sogar halb scherzhaft das Maes-Garreau-Law entworfen. Vgl. Brooks 2002, 206; Garreau 2005, 180, 338 FN 180; http://kk.org/thetechnium/the-maesgarreau.
} 
der KI-Experten exakt mit der gleichen Streuung ausfielen, wie diejenigen von Nicht-Experten (Journalisten, Publizisten, fachferne Prognosten). Die Mehrheit der Prognosen zielt in beiden Gruppen stets auf den Zeitraum von 15 bis 20 Jahren in der Zukunft (was den Eindruck Bostroms bestätigt). Der Status der eigenen Person, die ideell oder durch Forschungsgelder an ihrer eigenen Vorhersage partizipieren kann, wird dadurch erhöht. ${ }^{488}$

Wenn tendenziell unabhängig von der Person und dem Zeitpunkt der Prognosen ein umwälzendes Ereignis in etwa zwei Dekaden anvisiert wird, dann müssen wir die sozialen Dynamiken und Legitimationen der Futurologie genauer untersuchen. Welche Elemente machen also die Singularität als temporales Konzept aus? Zunächst ist das die Begründung mit einem Gesetz des Fortschrittes und der Beschleunigung. Unübersehbar ist ferner, dass die Singularität eine Schwelle oder Grenze konstruiert - damit verweist sie auf die in der amerikanischen Kulturgeschichte so präsente frontier (inklusive ihrer Adaptionen im ScienceFiction Genre). Wie wir von Armstrong und Sotala lernten, dient oftmals der Status eines Futurologen als Legitimation seiner Vorhersagen. Dieses »Charisma eines endzeitlichen Propheten", wie es Max Weber begrifflich fassen würde, gilt es genauer zu beleuchten.

Das Erscheinen der Singularität und allgemeiner noch das Auftreten der KI wird von allen Post- und Transhumanisten nicht mit Offenbarungen oder seherischen Gaben begründet, sondern auf der Basis einer mathematisierten Fortschrittsprognose (z.B. Moore's Law). Diese Annahme, dass Fortschritt keiner zufälligen Entwicklung, sondern einer festen Gesetzmäßigkeit unterliege, wird häufig auf den englischen Philosophen Francis Bacon (1561-1626) zurückgeführt. ${ }^{489}$ Tatsächlich aber ist die Formulierung einer allgemeinen Fortschrittsdoktrin erst das Ergebnis der Spätaufklärung und der Philosophie des Positivismus, die zum einen den Fortschritt als Gesetz der Geschichte konzipierten, zum anderen den Fortschritt der Wissenschaft und der Technik mit dem der Moral und Politik identifizierten und beide in Abhängigkeit voneinander sahen. ${ }^{490}$ An

${ }^{488}$ Vgl. Armstrong \& Sotala 2012, 3f., 13-19.

${ }^{489}$ Vgl. Spadafora 1990, 21f.

${ }^{490}$ Bacon betonte gegenüber der früheren spekulativen Philosophie die Notwendigkeit von systematischen und empirisch fundierten Forschungen, um einen Fortschritt des Wissens zu erreichen. Er leitete hieraus jedoch keine allgemeine Fortschrittsdoktrin ab. Paolo Rossi bezeichnet den häufigen Verweis auf Bacon gar als einen in der Spätaufklärung entstandenen, philosophischen Mythos. Vgl. Bacon 1962, 80ff. Zur 
der Schwelle zum 18. Jahrhundert waren es die Philosophen Fontenelle und Abbé de Saint-Pierre, die als erste eine allgemeine Fortschrittsdoktrin entwarfen. Für Fontenelle war Fortschritt notwendig und gesichert, da die nachfolgenden Generationen stets vom Wissen und den Fehlern der vorhergehenden profitieren würden, während Abbé de Saint-Pierre in seiner Vision einer sozialen und moralischen Weiterentwicklung den Fortschritt des Wissens mit der Idee von steigendem menschlichem Glück und Zufriedenheit verband. ${ }^{491}$

Mit großer Wirkung auf die englischen Utilitaristen wurde 1796 das Werk Esquisse d'un Tableau historique des progrès de l'esprit humain des französischen Philosophen Antoine Marquis de Condorcet veröffentlicht, in dem er die Fortschrittsentwicklung in der Geschichte der menschlichen Rasse sowie des Einzelnen einem Naturgesetz zuschrieb. Geschichte - so David Hume und Adam Ferguson - sollte nun als empirisch mathematische Naturwissenschaft betrieben werden, die die zugrunde liegenden Gesetze der von Turgot und Auguste Comte mechanistisch gedachten Kausalkette historischer Fortschritte erforschen soll, um schließlich künftige Entwicklungen besser steuern zu können. ${ }^{492}$ Gleichzeitig begann man, die nur einzeln wahrnehmbaren Fortschritte als historischen Gesamtprozess zu deuten. Weite Verbreitung fand dabei die Ansicht, dass der Fortschritt der vergangenen Zeitalter nicht nur den Fortschritt der Zukunft bewies, sondern sich sukzessive beschleunigen würde. »Infinitely slow in the beginning, and increasing by degrees with redoubled velocity ${ }^{493}$ - wie es Edward Gibbon in seiner History of the Decline and Fall of the Roman Empire prophezeite. Die empirisch sicherlich unbestreitbare Tatsache eines beschleunigten Fortschrittes auf technischem und wissenschaftlichem Gebiet im 18. Jahrhundert, die Francis Bacon, Adam Smith, Immanuel Kant und viele andere Denker dieser Zeit konstatierten, wurde zwischen Erfahrungsraum und Erfahrungshorizont in den Fortschrittsideologien aus den beobachtbaren Fortschritten der Vergangenheit und den erhofften Fortschritten der Zukunft deduziert. Der Einbezug der utopischen Perspektive als Legitimation einer unaufhörlichen

Rolle Bacons im Zeitalter des Positivismus vgl. Rossi 1987, 34f.; Baillie 1950, 104; Ekirch 1944, 12, 106f.; Klein 1986, $251 f$.

${ }^{491}$ Vgl. Bury 1955, 98-143.

${ }^{492}$ Vgl. Condorcet 1970; Bury 1955, 202-216; Ekirch 1944, 14ff.; Rohbeck 1987, $35 f$.

${ }^{493}$ Edward Gibbon: The History of the Decline and the Fall of the Roman Empire, ed. J. B. Bury, $9^{\text {th }}$ ed., 7 vols. (London 1925), Bd. 4, 176-181. Zitiert nach Spadafora 1990, 224. 
Beschleunigung des Fortschrittsprozesses bildet ein charakteristisches Merkmal einer jeden Fortschrittsideologie.

Schon 200 Jahre vor Kurzweil diente die Annahme einer künftigen, enormen Beschleunigung des Fortschrittes zwei Zielen. Nicht nur waren die Segnungen zu Lebzeiten zu erwarten, sondern auch jeder Einzelne, der den Prozess mit vollem Einsatz unterstützte, konnte mit einer Teilhabe rechnen. Eine doppelte Motivation zum Glauben und zur Unterstützung umgibt daher die heutige Singularität genauso wie die Fortschrittsutopien der Aufklärung. ${ }^{494}$ Die Idee der sich stetig erhöhenden Beschleunigung verweist daneben noch auf eine weitere kulturelle Quelle. Der Religionswissenschaftler Ernst Benz macht uns darauf aufmerksam, dass die These der unentwegten Beschleunigung ein Kennzeichen der christlichen Heilsgeschichte sei. So stand die Entdeckung und Christianisierung Amerikas unter endzeitlichem Zeitdruck: Kolumbus - überzeugt von dem nahenden Ende der Welt - sah Indien (also Amerika) als letztes noch zu missionierendes Reich des Satans an. Die fundamentale Idee der Beschleunigung des Fortschrittes, so Benz, stehe damit im Kontext einer subjektiven Naherwartung des christlichen Heilszieles, die durch neutestamentliche Berichte und Visionen bei Johannes oder dem Apostel Stephanus genährt wird. Dieses Motiv der Beschleunigung des Fortschrittes hat besonders in der amerikanischen Fortschrittstheorie ihren Niederschlag gefunden, die den weltlichen Fortschritt oftmals als Teil des göttlichen Planes für ein neues gelobtes Land verstand. ${ }^{495}$

Nicht nur das Element der sich steigernden Beschleunigung, sondern auch das Verständnis der Singularität als frontier, als letzte Grenze, spielt auf die amerikanische Kulturgeschichte an. Seit der Besiedlung Massachusetts durch die Puritaner im 17. Jahrhundert bezeichnete die frontier die Grenze der zivilisierten und moralischen Welt zur wilderness, dem ungeordneten Chaos der indigenen Stämme Nordamerikas. Das christlich-koloniale Sendungsbewusstsein wurde in den 1840er Jahren nochmals beflügelt, als sich expansionistische Tendenzen der US-Politik (Annektierung von Texas) mit der Verbreitung von Freiheit und Demokratie verbanden. Es sei die unaufhaltsame Bestimmung, the manifest destiny,

\footnotetext{
${ }^{494}$ Vgl. Koselleck 1975, 401ff.; Spadafora 1990, 381ff.; Rohbeck 1987, 55ff; Bultmann 1958, 80ff.

${ }^{495}$ Vgl. Benz 1977, 18-21.
} 
des von Gott auserwählten, amerikanischen Volkes, Fortschritt, Zivilisation und Freiheit in der Weite des Kontinents zu säen. ${ }^{496}$

Als sich die geographische frontier nach der Besiedlung des mittleren Westens und der Ausrottung der meisten indigenen Völker auflöste, wuchs die metaphorische Bedeutung der frontier für andere Gesellschaftsbereiche, insbesondere für die Wissenschaft. Schon mit Francis Bacon entstand das Bild des Forschers als Pionier, der in unentdeckte Welten vorstößt. Es war jedoch der wissenschaftliche Berater von Präsident Franklin D. Roosevelt, Vannevar Bush, der die Metapher 1945 mit seinem Bericht Science - the Endless Frontier für die amerikanische Forschungskultur verewigte. Bush schlägt in diesem Dokument Leitlinien für eine systematische Wissenschaftsförderung in den Vereinigten Staaten vor, die unter anderem in der Gründung der National Science Foundation mündeten.

It has been basic United States policy that Government should foster the opening of new frontiers. It opened the seas to clipper ships and furnished land for pioneers. Although these frontiers have more or less disappeared, the frontier of science remains. It is in keeping with the American tradition - one which has made the United States great - that new frontiers shall be made accessible for development by all American citizens. ${ }^{497}$

Über John F. Kennedy bis zu George W. Bush und Barack Obama ist die Metapher der intellektuellen frontier in der amerikanischen Wissenschaftspolitik präsent. ${ }^{498}$

Die Singularität, wie sie Vinge, Yudkowsky und Kurzweil entwerfen, baut ambivalent auf dieser zentralen Metapher der endless frontier auf. Die Singularität im Sinne eines Ereignishorizontes Schwarzer Löcher bleibt für den Menschen undurchdringlich und unüberwindbar. Aber für die künstlichen Intelligenzen beginnt mit der Singularität die tatsächlich unbegrenzte Expansion ins Universum, an der der Mensch teilhaben darf.

Wie bereits angedeutet, ist diese Wahrnehmung der Singularität als letzte zu überwindende Grenze bereits durch zahlreiche Adaptionen in Science-Fiction-Geschichten und Filmen popularisiert worden. Dieses Genre etabliert die Verbindung zwischen der räumlichen und der wis-

\footnotetext{
${ }^{496}$ Vgl. Torr 2002, 69-77.

${ }^{497}$ Vannevar Bush (1945): Science - the Endless Frontier, 6. Zitiert nach Ceccarelli 2013, 46. Vgl. Zachary 1997, 218-239.

${ }^{498}$ Vgl. Ceccarelli 2013, 29-155.
} 
senschaftlichen Metapher - also den in den Weltraum verlagerten Rand menschlicher Zivilisation mit der letzten Grenze menschlicher Erkenntnis. Als Katalysator dieser Ideen wirkte das wissenschaftliche Werk des Princeton-Physikers Gerard O’Neill (1927-1992), der seit den 1970er Jahren zahlreiche technische Entwürfe zur Kolonisation des Weltraums, der High Frontier, vorlegte. ${ }^{499}$

In dem fünften Star Trek-Kinofilm, The Final Frontier (1989), wird Captain Kirk gezwungen, mit seinem Raumschiff die "große Barriere« im Zentrum der Milchstraße zu überwinden, um auf einem mythischen Planeten Gott zu suchen. Die ersten beiden Star Trek Fernsehserien (1966-1969, 1987-1994) begannen stets mit den magischen Worten des Vorspanns "Space, the final frontier.« Weniger fantastisch versuchte Maximilian Schell als genialer, aber skrupelloser Wissenschaftler, Dr. Hans Reinhardt, in The Black Hole (1979) eine gestrandete Raumschiffcrew davon zu überzeugen, dass auf der anderen Seite eines Schwarzen Loches die letzte Wahrheit, Gott und das ewige Leben in einer Welt jenseits physikalischer Gesetze auf sie warte. Die widerspenstigen Mitglieder seiner eigenen Mannschaft hatte der Wissenschaftler in willenlose Cyborgs verwandelt. Am Ende des Filmes fliegen die überlebenden Helden dann tatsächlich durch ein Dante gleiches, höllisches Inferno und gleiten hinter einem Engel in ein lichtdurchflutetes Paradies. ${ }^{500}$ Der Western-Held wird im 20. Jahrhundert nahtlos in den Weltraumhelden überführt. Die Überwindung der final frontier, der Singularität Schwarzer Löcher, wird zum heroischen Unternehmen weißer Männer - mit heterogenen Akzentuierungen als fäusteschwingender Macho (James Tiberius Kirk) oder als besessenes Genie (Max Reinhardt). ${ }^{501}$

Außer Frage steht ferner, dass das starke endzeitliche Moment der Singularität durch christliche Vorstellungen beeinflusst ist. Die Überwindung von Alter, Krankheit und Tod entsprechen der christlichen Heilserwartung (die Tiplersche Vision einer Wiederauferstehung aller Toten ohnehin). Die wesentliche Analogie zur christlichen Apokalypse besteht jedoch in ihrer Doppeldeutigkeit: Die Singularität ist weder das Ergebnis einer kontinuierlichen und positiven Fortschrittsentwicklung noch einer totalen Vernichtung. Wie die christliche Heilsgeschichte verbindet das Konzept den Untergang des bisherigen Menschen mit der Gewissheit einer postsingulären Verheißung - Tod und Wiederauferstehung.

${ }^{499}$ Vgl. Kapell 2016, 168-208.

${ }^{500}$ Vgl. Shatner 1989; Nelson 1979; Kapell 2016, 139-167.

${ }^{501}$ Vgl. Kapell 2016, 139-145. 
Ein weiteres Strukturmerkmal teilen die christliche und die singuläre Prophetie. Das Nahen des Endes ist an Zeichen abzulesen. Die Offenbarung des Johannes zählt viele apokalyptische Elemente auf (Prophetien, Zerstörungen, Opfer, Zeugnisse), die sich vor dem letzten Kampf gegen Satan und dem Jüngsten Gericht ereignen (Offb 4-20). Insbesondere Kurzweil entwickelt in seinen drei futuristischen Büchern eine immer präzisere Beschreibung technischer Meilensteine, die der Singularität vorausgehen, inklusive einer Bewertung eigener früherer Voraussagen. Anders als in Vinges und Kurzweils Version der Singularität geben christliche Schriften sehr wohl einen präzisen Ausblick auf die Zeit nach der Apokalypse: Das Neue Jerusalem wird mit vielen Details beschrieben (Offb 21f). Das Seelenheil ist in der christlichen und jüdischen Tradition zudem abhängig vom Urteil Gottes über die moralische Lebensführung des Einzelnen. Mit der Singularität wird nach Ansicht aller posthumanistischen Autoren die Unsterblichkeit für jeden lebenden Menschen verfügbar (teils auch für die eingefrorenen Kryoniker). Die Idee einer universellen Rettung aller Menschen findet sich explizit nur bei den Unitariern (Unitarian Universalists), die schon 1803 in ihrer Winchester Profession davon ausgingen, dass der eine Holy Spirit of Grace »... will finally restore the whole family of mankind to holiness and happiness. « Dass Kurzweil unitarisch aufgewachsen ist, darf an dieser Stelle nicht überbewertet werden, da auch alle anderen Singularitätsbefürworter zur gleichen Einschätzung gelangen.

Ein letztes Element, das Relevanz für unsere Analyse hat, ist die Rolle der Verkünder der Singularität. Hier sticht Ray Kurzweil heraus, sowohl in seinem Anspruch als auch in der Farbenpracht seiner autobiographischen Selbstdarstellung. Er kann zwar auf einige Erfindungen und Auszeichnungen in den 1970er und 1980er Jahren zurückblicken. Das Internet und die Digitalisierung hat er selbst bisher jedoch nicht zu einem technologischen Durchbruch wirtschaftlich nutzen können. Natürlicherweise erscheint dann die Frage, warum gerade Kurzweil berufen ist, die Singularität und systemverändernde Technologien zu preisen, wenn er selbst von den vorhergesagten Trends offenbar kaum profitierte. Umso wichtiger ist für ihn die Konstruktion eines geniehaften Charismas. Schon im Kindergartenalter habe Kurzweil seine Bestimmung erkannt: »At the age of five, I had the idea that I would become an inventor. I

$\overline{502}$ Winchester Profession. http://uudb.org/articles/winchester.html. 
had the notion that inventions could change the world. ${ }^{503}$ Mit 8 Jahren baut er erste Roboter. Nicht nur technologische Innovationen glaubt er vorausgesehen $\mathrm{zu}$ haben, sondern in seinem Buch The Age of Spiritual Machines von 1990 behauptet er, auch den Untergang der Sowjetunion (1990/91) aufgrund der dezentralisierten Kommunikationsnetzwerke prophezeit zu haben. ${ }^{504}$ Der Dokumentarfilm Transcendent Man. The Life and Ideas of Ray Kurzweil von 2009 ist eine fulminante Hagiographie, eine »Heiligenlegende«. Kurzweil wird hier auf seinen weltweiten Vorträgen begleitet und fast ausschließlich seine Bewunderer kommen zu Wort, wie z.B. der Schauspieler William Shatner, der Sänger Stevie Wonder oder Ex-Außenminister Colin Powell. Man erhält einen Einblick in das Trauma, das der Tod von Kurzweils Vater bei ihm hinterlassen hat sowie in seine Besessenheit, mit Hilfe von 150 Vitaminpillen täglich das Zeitalter der Singularität selbst noch zu erreichen. Abgesehen vom Mantra, dass die Singularität nahe sei und alles verändern werde, enthält der Film nicht viel Substanz und keinerlei vertiefte Diskussion des Konzeptes. $^{505}$ Die Filme The Singularity (2012) von Doug Wolens und der von Kurzweil selbst produzierte Film The Singularity is near (2010) waren nicht vergleichbar stark auf die Person Kurzweils konzentriert, vermochten aber das futuristische Szenario weiter zu popularisieren. Parallel zur religiösen Prophetie verhält sich auch die Steigerung, die Kurzweil in seinen drei futurologischen Monographien vollzieht. Aus der Religionsgeschichte ist dieses Phänomen wohlbekannt, insbesondere bei Ausbleiben der Vorhersagen. Dieses Merkmal ist bei Kurzweil besonders auffällig, da alle anderen post- und transhumanistischen Denker der letzten Dekaden ihre Voraussagen relativieren, abmildern oder die zeitlichen Horizonte erweitern. Mit spitzer Feder könnte man sich daher zu der Bemerkung hinreißen lassen, dass laut einem noch zu definierendem Law of growing disappointments das einzige, was im Transhumanismus exponentiell wachse, die Steigerung der Vorhersagen sei.

Als prophetische Figur nimmt Kurzweil zudem eine Sonderstellung ein: Wenn Vannevar Bush 1945 die endless frontier der Wissenschaften ausruft, so verkündet Ray Kurzweil das Ende dieser Periode menschlichen Wissensstrebens exakt ein Jahrhundert später für das Jahr 2045. Er stilisiert sich damit zum letzten Propheten der Endzeit, zum Siegel der Propheten. Es gibt keine weitere Steigerung dieser Prophetie, die Kurzweils

${ }^{503}$ Vgl. Kurzweil 2005, 1.

${ }^{504}$ Vgl. Kurzweil 2010, 5, 127; Kurzweil 1990, 446 f.

${ }^{505}$ V gl. Wagner 2015, 100; Ptolemy 2009. 
Visionen übertreffen könnten. Mit Eintreffen der Singularität ist die Zeit des Menschen abgeschlossen.

Wie schon der Film Terminator (1984) und die Matrix-Trilogie (19992003), in der eine machtvolle künstliche Intelligenz die (letzten) Menschen ausrotten oder versklaven will, befindet sich die postsinguläre Science-Fiction anders als die naiven Futurologien Kurzweils und der Transhumanisten vorwiegend in der Tradition der dystopischen Cyberpunkliteratur. ${ }^{506}$ Elaine Graham bemerkt, dass die jüngere ScienceFiction die Grenze zwischen Religion und Wissenschaft mit zunehmender Selbstverständlichkeit auflöst. Das Säkulare und das Heilige, Mensch und Gott, Glaube und Wissen erscheinen immer weniger als absolute Gegensätze, sondern verschwimmen in einer post-säkularen Ära. ${ }^{507} \mathrm{Die}$ dystopischen Entwürfe propagieren nicht mehr die Überwindung eines religiösen Aberglaubens durch eine rationalistische Techno-Kultur, sondern zelebrieren die irrationale Fusion beider Sphären.

So ist es in Rudy Ruckers Roman Postsingular (2007) ein christlichfundamentalistischer US-Präsident, der mit Hilfe eines Computerwissenschaftlers die gesamte Erde durch Nano-Roboter in eine Virtuelle Erde (Vearth) umwandeln will. Diese Transformation betrachtet er als die Verwirklichung biblischer Prophetie vom wiedererlangten Garten Eden, in dem Leid, Krieg und Tod verbannt und das Leben vollkommen kontrolliert sei. Rucker entlarvt die Ursache dieses Verlangens als Trauma aus der Jugend des Computerwissenschaftlers, der seinen Freund bei einem Unfall verlor. In Postsingular überschneiden sich die Interessen des christlichen und des kybernetischen Fundamentalismus in ihrem Hass auf Frauen und die Schöpfung. ${ }^{508}$

Es ist offensichtlich, dass die Prophetie der Singularität stark durch kulturelle Deutungsmuster geprägt ist. Die Annahme von Fortschrittsgesetzen und einer stetigen Beschleunigung des Fortschrittes lässt sich schon auf Positionen des aufklärerischen Vervollkommnungsstrebens zurückführen. Neu ist der von Vinge und Kurzweil eingeführte Gedanke einer absoluten und undurchdringlichen Grenze dieses Fortschrittes, der Singularität, als last frontier. Der Begriff wiederholt Semantiken aus der Physik Schwarzer Löcher und deren Popularisierung in Literatur

\footnotetext{
${ }^{506}$ Joshua Raulerson hat hier eine eingehende Analyse vorgelegt. Vgl. Raulerson 2013, 124f.; Cameron 1984; Wachowsky \& Wachowsky 1984.

${ }^{507}$ Vgl. Graham 2015, 362.

${ }^{508}$ Rucker 2007; Raulerson 2013, 40-45.
} 
und Film. Erstaunlich ist ferner, dass mit dem Konzept der Singularität eine religiöse Teleologie Einzug in den Post- und Transhumanismus hält, die vor 15 Jahren noch als exotisch abgetan wurde. Heute kann Ray Kurzweil unverblümt Frank Tiplers Idee von einer vollständigen Besiedlung des Universums aufgreifen, die in der Verwirklichung Gottes gipfelt. In den nachfolgenden drei Kapiteln, die die zentralen Folgen der Singularität betrachten, wird diese Grenze zwischen dem Religiösen und dem Wissenschaftlichen noch weiter verschwimmen. Da zwar viele Post- und Transhumanisten diese Ziele (Superintelligenz, Unsterblichkeit und das Verschmelzen zu einem globalen Gehirn) verfolgen, jedoch keine ausgesprochenen Anhänger der Singularitätsidee sind, wird dieser Aspekt gesondert behandelt.

\subsection{Unsterblichkeit}

\subsubsection{Posthuman und unsterblich}

Sowohl Tiplers Konzept von Unsterblichkeit als auch seine Idee der Auferstehung aller Toten, die fortan als Simulationen im Computer existieren sollen, beruhen auf der zuvor schon erläuterten Mustertheorie der Identität. Für den Physiker Tipler ist der Mensch als geschlossenes System von Quantenzuständen vollkommen definiert: » ... a system is its quantum states ... They thus prove that a human being is a finite state machine, and nothing but a finite state machine. ${ }^{509}$ Nachdem Tipler den Menschen inklusive seiner Seele als informationsverarbeitendes System definiert hat, das durch seinen Quantenzustand eindeutig bestimmbar ist, sieht er hier die Rettung für die Bedrohung des Menschen durch kosmische Katastrophen: »If one accepts the identification ... of ssouk with a particular computer program or pattern, we could interpret this pattern conservation as simmortality of the souk. $\$ 10$

Schließlich muss Tipler zufolge die ganze Erde vom realen Raum als Simulation in den virtuellen Raum eines Computers überführt werden, bevor die Sonne ihre Leuchtkraft verliert. ${ }^{511} \mathrm{Da}$ die individuelle Unsterblichkeit für all jene, die die Zeit der technisch ermöglichten Un-

${ }^{509}$ Tipler 1995, 31.

${ }^{510}$ A.a.O., 237.

${ }^{511}$ Vgl. a.a.O., 108 f. 
sterblichkeit nicht mehr erreichen, nur ein schwacher Trost ist, hat Tipler keinen Zweifel, dass uns in der Zukunft auch die Auferstehung aller Personen, die je gelebt haben, bevorsteht.

In fact, the universal resurrection is physically possible even if no information whatsoever about an individual can be extracted from the past light zone. Since the universal computer capacy increases without bound as the Omega Point is approached, it follows that ... there will be sufficient computer capacy to simulate our present-day world by simple brute force: by creating an exact simulation an emulation - of all logically possible variants of our world. ${ }^{512}$

Gemessen an der maximalen Zahl von Quantenzuständen für die Masse eines Menschen kann es nach Tipler höchstens die enorme, aber endliche Menge von $10^{450}$ verschiedenen Menschen und $10^{1230}$ verschiedene Universen geben, die ein Supercomputer der Zukunft mit Leichtigkeit als virtuelle Existenz simulieren könne. ${ }^{513}$

Die Unsterblichkeit der menschlichen Seele - das »Programm des Menschen« - und die Auferstehung der Toten soll durch einen gigantischen Computer gewährleistet werden, der die reale Welt emuliert, kurz bevor der Omegapunkt erreicht sei. »Kurz bevor « bedeutet nach Tiplers exakten Berechnungen $10^{-100}$ bis $10^{-1230}$ Sekunden vor Omega. ${ }^{514}$

The key question is this: do the emulated people exist? As far as the simulated people can tell, they do. By assumption, any action which real people can and do carry out to determine whether they exist ... the emulated people also can do, and in fact do do. There is simply no way for the emulated people to tell that they are »really« inside a computer, that they are merely simulated, and not real..$^{515}$

Das Leben nach dem Tod beruhe bei der computerisierten Auferweckung nicht wie in vielen Religionen auf der Kontinuität einer unsterblichen, seelischen Substanz, sondern lediglich auf der Musteridentität der

${ }^{512}$ A.a.O., 220.

${ }^{513}$ Vgl. ebd.; a.a.O., 31ff. 1986 bestimmten Tipler und Barrow die Anzahl menschlicher Individuen lediglich über die Zahl 3x106 Variationen der menschlichen Gene. Vgl. Barrow \& Tipler 1986, 514. Tipler ist Anhänger der so genannten Vielwelteninterpretation in der Quantenmechanik. Für unsere Fragestellung ist die Vielweltentheorie, auf die Tipler dauernd hinweist, nicht wesentlich und würde bei genauerer Reflexion zu immensen Widersprüchen führen, denn die Simulation aller möglichen Universen und Lebewesen durch den Omegapunkt scheint unvereinbar mit Tiplers ausschließlich paradiesischen Visionen. Vgl. a.a.O., 458-506; Tipler 1995, 167-183; 483-488.

${ }^{514}$ Vgl. a.a.O., $225 \mathrm{ff}$.

515 A.a.O., 207. 
Seele: »The need for such a continuity is obviated by quantum mechanics, and thus we see that an immortal soul is no longer necessary for individual immortality. ${ }^{516}$

Trotzdem hält Tipler an der christlichen Idee einer leiblichen Auferstehung fest. Denn auch der menschliche Körper mit all seinen Empfindungen werde im künftigen Computer simuliert, wobei alle körperlichen Defekte und Verfallserscheinungen behoben sein würden. Der künftige Körper soll gleichzeitig jedoch einem immateriellen »Lichtleib « entsprechen. ${ }^{517}$ Aber wer wird überhaupt auferweckt?

What happens to the resurrected dead is entirely up to the Omega Point; there is no way the imulations [sic!] can pay for to enforce the immortality which is in the power of the Omega Point to grant ... Adopting the natural theological term, I think we will be granted "grace« ... So, the motivation for the granting of immortal life in the Omega Point is exactly the same as it is in the Judeo-Christian-

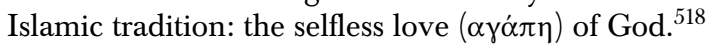

Ins Zentrum der Auferstehungsvision Tiplers rückt damit ein grundlegender Pfeiler der calvinistischen Theologie: die Gnadenwahl. Gott allein habe die Entscheidung zu fällen, wem er ewiges Leben gewährt und wen er verdammt. Der Mensch hat keine Möglichkeit, einen direkten Einfluss auf den »geheimen Ratschluss Gottes« zu nehmen, sondern er muss sich gänzlich der reinen Gnade seines Herrn überantworten. In seiner Schilderung der Hölle macht Tipler klar, dass unverbesserlich böse Personen nicht auf die Gnade des Omegapunktes hoffen können und daher nicht wiedererweckt werden. ${ }^{519}$

In der Ausübung der dann zur Verfügung stehenden übermenschlichen (superhuman) Fähigkeiten würden sich die Auferweckten in diversen Richtungen vervollkommnen, bis sie mit dem Erreichen des Punktes Omega einen Zustand seliger Gottesschau in der wahrhaften Vereinigung mit Gott erlangt haben würden. Zwar sei die Auferstehung vorrangig den Menschen vergönnt, weil sie als potentielle selbstprogrammierende Turingmaschinen dem Ebenbild eines Schöpfergottes entsprechen würden, jedoch werden laut Tipler auch einige Haustiere auferstehen können, weil ihre Besitzer es so wollen. Wenn Tipler auch die Möglich-

\footnotetext{
${ }^{516}$ A.a.O., 235.

517 Vgl. Barrow \& Tipler 1986, 618f. und Tipler 1995, 108-110; Lavery 1992, 10-18; Regis $1990,46$.

${ }^{518}$ Tipler 1995, 245ff. Vgl. auch Tiplers Vortrag vor Theologen: Tipler 1989, 222, 249.

${ }^{519}$ Vgl. Tipler 1995, 251.
} 
keit eines Verzichtes auf die Auferstehung bzw. eine Art »Fegefeuer« für besonders fehlerbehaftete Menschen nicht gänzlich ausschließt, so ist in seinen Schilderungen die Dominanz der erfreulichen Verheißungen des zukünftigen Heilshorizontes unübersehbar: ${ }^{520}$ Ferner könnten Menschen mit all ihren Vor- und Nachfahren in der simulierten Welt zusammentreffen und jeder Mensch würde in seiner virtuellen Existenz nahezu unendlichen Reichtum besitzen. ${ }^{521}$ Die Fehler unvollkommener Menschen und das Böse könnten gleichzeitig getilgt werden, so dass der vollkommene Mensch in seiner virtuellen Existenz jenseits der Zeit eins mit Gott werden könne. ${ }^{522}$

In seinen aktuellen Interviews weicht Tipler jedoch von diesem eschatologischen Szenario ab und legt nahe, dass Menschen nicht erst durch die Verwirklichung Omegas, sondern allein durch den technischen Fortschritt Unsterblichkeit erlangen werden:

Ultimately, all humans will join'em. The Earth is doomed, remember? When this doom is at hand, any human who remains alive and doesn't want to die will have no choice but to become a human upload. And the biosphere that the new human uploads wish to preserve will be uploaded also. The AIs [Artificial Intelligences: $\mathrm{OK}]$ will save us all. ${ }^{523}$

Der Kybernetiker Marvin Minsky sieht die virtuelle Unsterblichkeit nicht als Zielpunkt christlicher Heilsgeschichte, sondern als Folge des kapitalistisch organisierten, medizinischen Apparates. Seiner Einschätzung nach wird diese technisch ermöglichte Unsterblichkeit vorerst nur den Reichen vorbehalten bleiben. ${ }^{524}$ Nichtsdestotrotz bewertet Minsky die erhofften technischen Errungenschaften als den Beginn einer neuen, anthropozentrischen Ethik: Das Recht auf Kinder, der Genwechsel und der Tod sollten nicht länger biologischen Konditionen unterworfen sein, sondern würden allein vom menschlichen Willen und der Vernunft der posthumanen Lebensformen kontrolliert werden: ${ }^{525}$

${ }^{520}$ Vgl. Tipler 1995, 241-255.

${ }^{521}$ Vgl. a.a.O., 241f.; Tipler 1989, 244. Indem sich Tipler auf den österreichischen Wirtschaftswissenschaftler Friedrich von Hayek beruft, weist er sogar nach, dass alle Menschen im Laufe der Zeit unendlichen Reichtum besitzen würden. Vgl. Tipler 1995, $267 f$.

${ }^{522}$ Vgl. a.a.O., $244 \mathrm{f}$.

${ }^{523}$ Tipler 2015, 31.

${ }^{524}$ Vgl. Minsky 1992c, 31.

${ }^{525}$ Vgl. Minsky 1994, 112 f. 
The most important thing about each person is the data, and the programs in the data are in the brain. And some day you will be able to take all that data, and put it on a little disk, and store it for a thousand years, and then turn it on again and you will be alive in the fourth Millennium or the fifth Millennium. ${ }^{526}$

Hans Moravec, der die Erlangung der Immortalität mit dem eingangs geschilderten, chirurgischen Verfahren verbindet, beschreibt die virtuelle Existenz als Raum einer unendlich steigerbaren Selbstverwirklichung. Alle persönlichen Fähigkeiten ließen sich dann beliebig und unermesslich verbessern - man werde sogar besser singen können als jeder heutige Opernstar und in seiner virtuellen Existenz bedeutsame berufliche Erfolge vorzuweisen haben: ${ }^{527}$ »And, of course, you are better at your job than even your best ever was - better than any flesh-and-blood person ever could be. ${ }^{528}$

Durch den Vorgang des Uploading wird der Mensch laut Tipler nicht nur die Möglichkeit haben, in der virtuellen Unsterblichkeit dem Tod vor seinem physischen Lebensende zu entgehen, sondern er könne auch schon zu Lebzeiten eine unsterbliche Notfallkopie anfertigen lassen, die nach einem unerwarteten Unglücksfall aktiviert werde. ${ }^{529}$ Hoffnungen auf ein unsterbliches Leben dürfen sich auch in Moravecs Vision alle Menschen machen, die je gelebt haben. Moravec stimmt hier mit Tipler insofern überein, als dass er die Auferstehung der Toten für nichts weiter als ein mathematisches Problem hält. Denn die simple Berechnung und Simulation aller Vergangenheiten, die zu unserer Gegenwart geführt haben, würde es Roboterärzten ermöglichen, auf diese Weise mit Hilfe von archäologischen Erkenntnissen und biographischen Datenfragmenten alle Toten aufzuerwecken und ihnen ein neues, unsterbliches Leben in der Simulation zu schenken, die für sie absolut real wäre. ${ }^{530}$

\footnotetext{
${ }^{526}$ Marvin Minsky: Why Computer Science Is the Most Important Thing That Has Happened to the Humanities in 5,000 Years. Öffentliche Vorlesung, Nara, Japan, 15.05.1996. Zitiert nach Hayles 1999, 244 f.

${ }^{527}$ Vgl. Moravec \& Pohl 1993, 72ff. Vgl. hierzu Lem 1981, 328f.

${ }^{528}$ Moravec \& Pohl 1993, 76. Wie sich allerdings dieser Luxus und Erfolge im Beruf mit Moravecs an anderer Stelle geäußerten Prognosen über eine wahrscheinliche Massenarbeitslosigkeit und die Verdrängung des Menschen aus allen Berufsfeldern vereinigen lässt, bleibt völlig unklar, zumal die künstlichen Intelligenzen im virtuellen Raum ungleich überlegener funktionieren müssten als ein menschlicher Geist nach seinem Uploading. Vgl. Moravec 1999, 70f., $139 f$.

${ }^{529}$ Vgl. Moravec 1988, 108-111.

${ }^{530}$ Vgl. a.a.O., 124; Moravec 1999, 142, 172 f.
} 
It might be fun to resurrect all the past inhabitants of the earth this way and to give them an opportunity to share with us in the (ephemeral) immortality of transplanted minds. Resurrecting one small planet should be child's play long before our civilization has colonized even the first galaxy. ${ }^{531}$

Seit seinem Buch The Age of Spiritual Machines (1999) propagiert auch Ray Kurzweil analog dem Vorschlag Moravecs die Idee einer Immortalisierung des Menschen per Gehirnscan. Er begründet diesen Vorgang mit der kybernetischen Mustertheorie der Identität und hält es daher auch gar nicht für notwendig, die Funktionsweise des Gehirns vollkommen verstehen zu müssen. ${ }^{532}$

Actually, there won't be mortality by the end of the twenty-first century ... Up until now, our mortality was tied to the longevity of our hardware. When the hardware crashed, that was it ... As we cross the divide to instantiate ourselves into our computational technology, our identity will be based on our evolving mind file. We will be software, not hardware. ${ }^{533}$

Wie in den vorherigen Kapiteln dargelegt, steht die Todesfrage bereits im Zentrum des frühen Transhumanismus seit den 1960er Jahren, der durch FM-2030 und Robert Ettinger geprägt war. Ettingers Antwort auf das Problem ist die Kryonik, die den Toten eine Auferweckung in einem technologisch fortgeschrittenen Zeitalter verspricht. Für FM-2030 bildet die Überwindung der menschlichen Sterblichkeit den Dreh- und Angelpunkt der transhumanistischen Weltanschauung. Denn solange er noch sterben müsse, habe der Mensch nach Ansicht Esfandiarys keine Freiheit, so dass sich alle politischen und sozialen Anstrengungen zunächst und vor allem diesem einen Problem widmen müssten. Soziales und politisches Engagement sei beschränkt auf den wissenschaftlichen und technischen Fortschritt, der letztendlich an den Leistungen im Kampf gegen den Tod gemessen werde:

The real revolutionary of today fights a different battle. He wants to be alive in the year 2050 and in the year 20,000 and the year 2,000,000. Is there anything more radical than this determination? ... Who are the new revolutionaries of our times? They are the geneticists, biologists, physicists, cryonologists, biotechnologists, nuclear scientists, cosmologists, astrophysicists, radio astronomers, cos-

\footnotetext{
${ }^{531}$ Moravec 1988, 124.

${ }^{532}$ Vgl. Kurzweil 1999a, 2ff., 53ff., 128f.; Kurzweil 2005, 9, 324-330.

${ }^{533}$ Kurzweil 1999a, 128f.
} 
monauts, social scientists, youth corps volunteers, internationalists, humanists, Science-Fiction writers, normative thinkers, inventors ... ${ }^{534}$

Noch während der Arbeiten an seinem letzten Buch Countdown to Immortality verstarb Fereidoun M. Esfandiary im Juli 2000 nach einem längeren Krebsleiden. Seine Leiche wartet im gekühlten Stickstoff eines Kryo-Tanks in Arizona auf die technische Auferstehung.

Auch für den Künstler Stelarc ergibt sich die im realen Raum vollzogene Unsterblichkeit des künftigen Menschen als Folgeerscheinung und Notwendigkeit des technischen Fortschrittes:

Und wenn der Körper auf modulare Art neu gestaltet werden kann, um den Ersatz von schlecht funktionierenden Teilen zu erleichtern, dann GIBT ES TECHNISCH KEINEN GRUND FÜR DEN TOD, die Verfügbarkeit von Ersatzteilen vorausgesetzt. Der Tod ist kein authentisches Zeichen der Existenz. Er ist eine veraltete evolutionäre Strategie ... Im ausgedehnten Zeitraum der extraterrestrischen Umgebungen MUSS DER KÖRPER UNSTERBLICH WERDEN, UM SICH ANZUPASSEN. Utopische Träume werden zu postevolutionären Imperativen. ${ }^{535}$

Quasi alle wichtigen transhumanistischen Autoren wie Max More, Nick Bostrom und Martine Rothblatt zeigen sich optimistisch, dass früher oder später eine Form der virtuellen Unsterblichkeit realisiert werden kann. Max More, der auch als CEO des Kryonik-Anbieters Alcor fungiert, relativiert das Unsterblichkeitsziel der Transhumanisten. Eine potentiell unbegrenzte Lebensspanne, die prinzipiell auch nach einem erfüllten Leben beendet werden könne, sei das Ziel. Nick Bostrom verteidigt philosophisch das Streben nach Lebensverlängerung und Unsterblichkeit. Martine Rothblatt prägte den Begriff des mindcloning, das den Menschen zu einem cyberconciousness bis ans Ende der Zeit verhelfen soll. ${ }^{536}$ Rothblatt ist in diesem Zusammenhang auch die einzige Transhumanistin, die davon ausgeht, dass sich die bisherigen Religionen im virtuellen $\mathrm{Da}$ sein des Menschen fortsetzen werden, da die mindclones ihren Originalen und deren Werthaltungen entsprechen müssen. Alle Religionen werden laut Rothblatt die Existenz von mindclones begrüßen, da sie in Einklang mit Gottes Schöpfungsauftrag stehen. ${ }^{537}$

${ }^{534}$ Ebd.

${ }^{535}$ Stelarc 1996, 78.

${ }^{536}$ Vgl. Bostrom \& Roache 2008, 3-7; More 2009; Rothblatt 2014, 280-293; Heil \& Coenen 2014, $153 f$.

${ }^{537}$ Vgl. Rothblatt 2014, 261-276. 
$\mathrm{Zu}$ einer weiteren Verwissenschaftlichung der Debatte hat die Studie Whole Brain Emulation. A Roadmap von Anders Sandberg und Nick Bostrom (2008) beigetragen. Der Begriff der transmigration (Seelenwanderung), den Moravec eingeführt hatte, wird durch das technische Etikett einer Gehirn-Emulation, also einer vollständigen und exakten Kopie des ursprünglichen Gehirns, ersetzt.

WBE [Whole Brain Emulation: O.K.] represents a formidable engineering and research problem, yet one which appears to have a well-defined goal and could, it would seem, be achieved by extrapolations of current technology. This is unlike many other suggested radically transformative technologies like artificial intelligence where we do not have any clear metric of how far we are from success. ${ }^{538}$

Die beiden Autoren des Future of Humanity Institutes setzen sich detailliert mit technischen Fragen auseinander und benennen offen die Schwierigkeiten. Diese verorten sie weniger in den Methoden der Speicherung und computertechnischen Simulation eines Gehirns als vielmehr in den heute zur Verfügung stehenden Methoden eines Gehirnscans. Die hochauflösendsten Verfahren würden in der Regel zeitlich und materiell eine sehr aufwendige Präparierung von histologischen Schnitten erfordern, die zudem tiefgekühlt oder mit Kunststoffen fixiert werden müssen. Sandberg und Bostrom regen daher an, eventuell nur die wichtigsten Teile eines Gehirns zu scannen und den Rest mit weniger präzisen Methoden (MRI) zu erfassen. Ein lebendes Gehirn in Funktion könne mit der heutigen Technik nicht in ausreichender Auflösung gescannt werden. ${ }^{539}$ Die menschliche Persönlichkeit wird auch von Sandberg und Bostrom einzig mit den Gehirnaktivitäten verbunden. Die Simulation des Körpers - der auf kinetische Aspekte von Knochen und Muskeln reduziert wird - sei peripher. ${ }^{540}$

Da gegenüber der Kryonik auch unter Transhumanisten eine gewisse Skepsis zu spüren ist, nehmen die Forschungen zur Langlebigkeit wie sie einst Roy Lee Walford und heute Aubrey de Grey verfolgt, eine wachsende Bedeutung ein, vor allem, weil diese als Überbrückung bis zur Realisierung der virtuellen Unsterblichkeit von Nutzen sein könnten. ${ }^{541}$

\footnotetext{
${ }^{538}$ Sandberg \& Bostrom 2008, 5.

${ }^{539} \mathrm{Vgl}$. a.a.O., 40-54.

${ }^{540}$ Vgl. a.a.O., $74 \mathrm{f}$.

${ }^{541}$ Der Pathologe Roy Lee Walford (1924-2004), der dem früheren Extropy Institute nahe stand, hatte in seinem 1983 veröffentlichten Buch Maximum Life Span behauptet, dass
} 
Dem Hang zur Begründung immer neuer Denksysteme ist es zu verdanken, dass Transhumanisten das Streben zur Unsterblichkeit oder unbegrenzten Langlebigkeit als immortalism, longevism bzw. life-extensionism bezeichnen.

Der Vielzahl von Denkschulen entsprechen innerhalb des rezenten Transhumanismus eine Reihe von Organisationen, Lobby-Gruppen und Instituten, die sich der visionären Lebensverlängerung verschrieben haben. Diese visionäre Perspektive umfasst im Gegensatz zu den realistischeren Szenarien der etablierten Biogerontologie lebensverlängernde Phasen von mehreren Hundert bis zu 1000 Jahren (wie das Aubrey de Grey suggeriert). Zu diesen Instituten zählen das bereits vorgestellte $F u$ ture of Humanity Institute von Nick Bostrom und die SENS Foundation von de Grey. Das 2002 entstandene, amerikanische Immortality Institute / LongeCity versteht sich als Forum, um Ideen zur Überwindung des unfreiwilligen Todes zu verbreiten. ${ }^{542}$ In Israel hat der transhumanistische Aktivist Ilia Stambler Longevity Days ins Leben gerufen sowie 2014 die beiden internationalen Lobby-Organisationen Longevity for All und Longevity Alliance begründet. ${ }^{543}$ Eine Brücke zur etablierten Forschung bildet das 2016 initiierte Global Healthspan Policy Institute in Washington D.C., das sich zum Ziel gesetzt hat, Politiker und die Bundesverwaltung von der Notwendigkeit der lebensverlängernden Altersforschung zu überzeugen. $^{544}$

Einen kurzweiligen Erfolg der politischen Lobbyarbeit konnte der Transhumanismus bereits 2002 feiern, als sogar die weitestgehenden Immortalisierungsvisionen Eingang in den bereits erwähnten, von Mihail Roco und William S. Bainbridge verfassten Bericht der National Science Foundation zur Konvergenz der Nanotechnologien fanden:

Is having the traditional body necessary to being human? Nevertheless, if you accept the above premises, it could be done. Having made the leap to new hardware for yourself, many staggering options open up:

- No death. You back yourself up. You get new hardware as needed.

\footnotetext{
Menschen mit seinem Diätplan im Durchschnitt 120 Jahre alt werden könnten. Nicht nur durch seine Teilnahme am Experiment der Biosphäre 2 von 1991 bis 1993, sondern auch durch zahlreiche Fernsehauftritte und populäre Buchpublikationen war Walford seinerzeit der bekannteste Vertreter der amerikanischen Langlebigkeitsbewegung. Vgl. Walford 1983 (dt.: Leben über hundert. München 1987); Walford 2000.

${ }^{542}$ Vgl. www.imminst.org.

${ }^{543}$ Vgl. www.longevityforall.org; www.longevityalliance.org.

${ }^{544}$ Vgl. https://healthspanpolicy.org.
} 
- Turn up the clock speed. Goodbye, millisecond-speed neurons; hello, nanosecond-speed electronics.

- Choose space-friendly hardware. Goodbye, Earth; hello, galaxy. ${ }^{545}$

In den folgenden beiden Kapiteln soll der Frage nachgegangen werden, wie diese Vision einer technischen Immortalisierung ideengeschichtlich zu verorten ist. Dies betrifft zunächst die seit der Aufklärung formulierten Ansätze einer medizinisch erhofften Verlängerung des Lebens und Bekämpfung des Todes. Mit Blick auf die Science-Fiction Literatur des 19. und 20. Jahrhunderts wird die Idee der Immortalisierung durch technische Medien nachgezeichnet. In diesem Zusammenhang bildet die Kryonik einen Sonderfall, der nur im kulturgeschichtlichen Rahmen der US-amerikanischen Bestattungskultur adäquat beschrieben werden kann.

\subsubsection{Von der Langlebigkeit zur computertechnischen Immortalisierung}

Die technische Überwindung des Todes ist kulturgeschichtlich ein Kind der Aufklärung. Wie wir bereits in Zusammenhang mit den heilsgeschichtlichen Entwürfen sehen konnten, stehen die aufklärerischen Positionen nicht in jedem Fall im Gegensatz zur Religion. Zweifellos emanzipieren sich Wissenschaftler des 19. und 20. Jahrhunderts wie auch posthumanistische Denker von traditionellen religiösen Vorstellungen. Metaphysik und religiöse Versatzstücke (wie die entwicklungsgeschichtliche Gottesidee) finden jedoch über die Hintertür wieder Einzug in posthumanistische Utopien. Das Plädoyer für ein ewiges Leben knüpft dabei nahtlos an die Bemühungen um Lebensverlängerung an. Dabei sind wir versucht, diese Ansätze ausschließlich in den medizinischen Kontext einzuordnen. Ebenso unterstreichen Moravecs Immortalisierungsprozedur wie auch die Inszenierung der Kryonik diesen medizinischen Charakter. ${ }^{546}$ Die philosophischen und religiösen Anteile dieser Zukunftsutopien geraten dann leicht in den Hintergrund.

Schon das älteste Epos der Menschheitsgeschichte, das sumerische Gilgamesch-Epos, berichtet ca. 2000 v.u.Z. von einem heldenhaften König, der sich trotz aller Ruhmestaten am Ende seines Lebens mit der Unausweichlichkeit des nahenden Todes konfrontiert sieht. Er macht sich auf

\footnotetext{
${ }^{545}$ Roco \& Bainbridge 2003, 169.

${ }^{546}$ Vgl. Bloch 1985, 539; Schäfer 2010, 23-28; Jordan \& Frewer 2010, $154 f$.
} 
die Suche nach der Unsterblichkeit und findet auf einer paradiesischen Insel den Utnapischtim und seine Frau. Diese wurden von den Göttern mit Unsterblichkeit gesegnet, da sie ihnen stets gehorsam waren und die Schöpfung vor der großen Flut retteten. Aus Mitleid erhält Gilgamesch von Utnapischtim ein Kraut der Unsterblichkeit, das ihm dann allerdings eine Schlange raubt. ${ }^{547}$

Das Motiv eines mythischen Ortes des ewigen Lebens bzw. der Verjüngung erstreckt sich von den Kulturen des Alten Orients bis zur frühen Neuzeit. Es reicht vom bei Homer beschriebenen Garten der Hesperiden, in dem die Jugend verleihenden Äpfel gedeihen, bis zu den Legenden eines Jungbrunnens, von dem der antike Alexanderroman, Herodot, der mittelalterliche Reisebericht des John Mandeville und die Chronisten der spanischen Konquistadoren im 16. Jahrhundert berichten. ${ }^{548}$

Auch in der mediterranen Antike wird das übermäßig lange Leben in ferne Länder oder vergangene Zeiten projiziert: Im jüdischen Tanach erreichen die Erzväter der Juden Lebensalter von mehreren Hundert Jahren, bei Hesiod lebt das goldene Menschengeschlecht ohne Krankheit und Alter. Strabo und Plinius berichten von Völkern jenseits der Nordwinde, die nahezu ewig leben würden. Die Hoffnung auf ein ewiges (postmortales) Leben gilt im christlichen Kontext dann jedoch nur den Frommen als Wiedergutmachung für ihr Leiden in der Welt. Alles andere erscheint als Hybris. ${ }^{549}$

Im europäischen Mittelalter tritt die Alchemie als systematischer Versuch, die natürliche Welt und ihre Kräfte zu erforschen und zu beherrschen, in Erscheinung. Dabei verstanden sich die Alchemisten meist im Einklang mit der christlichen Lehre, galt es doch, mit fortschreitender Vernunft und Erkenntnisfähigkeit des Menschen die Wunder der göttlichen Schöpfung zu entschlüsseln. Neben dem Bemühen, Gold und Silber aus minderwertigen Materialien herzustellen, setzte schon mit Roger Bacon (ca. 1220-1292) die Suche nach einem Allheilmittel (panacea) ein, das das Leben verlängern und Krankheiten heilen könne. Teils führten die metaphysisch begründeten Anwendungen mit Quecksilber- oder Antimonpräparaten zum raschen Tod ihrer Befürworter und deren Kunden. Ähnliche Wirkungen hatte die Verehrung des Zinnober als Mittel,

${ }^{547}$ Der sumerische und babylonische Utnapischtim geht später in der Figur des biblischen Noah auf. Vgl. Maul 2005, 39f., 147-151.

${ }^{548}$ Vgl. Gruman 1966, 24-27.

${ }^{549}$ V gl. a.a.O., 9-24. 
die Unsterblichkeit zu erlangen, in der daoistischen, chinesischen Alchemie der Tang-Zeit (7.-9. Jhd.). Explizit bezieht sich Martine Rothblatt auf die hiermit in Verbindung stehende, daoistische Erkenntnislehre. ${ }^{550}$ Größten Einfluss auf die nachfolgenden Generationen hatte der italienische Renaissance-Denker Luigi Cornaro (ca. 1467-1566) mit seinem Plädoyer für ein maßvolles Leben mit strikter Diät und Hygiene. Auf diese Weise könne der Mensch mit 80 Jahren vollkommene Freiheit erlangen, wenn er jegliches irdische Verlangen und das sündhafte Leben hinter sich gelassen habe. Dann würde er halb im Irdischen, halb im Himmlischen weilen und Gott wahrhaft dienen können. ${ }^{551}$ Dem Philosophen und frühen Verfechter der empirischen Wissenschaften, Francis Bacon, ist mit seiner Schrift Historia Vitae et Mortis (History of Life and Death) von 1623 die erste systematische Erörterung der möglichen Verlängerung des Lebens zu verdanken. Bacon präsentiert hier jedoch noch keine konsistente Lebenstheorie, sondern führt unzählige historische und legendäre Beispiele lebensverlängernder Methoden zusammen, wie Diäten, Elixiere, Bäder (in Blut) u.a. ${ }^{552}$

Cornaro und Bacon sind mit ihren Vorstößen denn auch Bezugspunkt der aufklärerischen Ansätze einer Vervollkommnung des Lebens, zu der für viele Denker die nahezu unbegrenzte Verlängerung des Lebens zählt. Dieser Fortschrittsoptimismus wird teils säkular untermauert, teils in christliche Deutungsrahmen eingeordnet. Englische Theologen und Moralphilosophen wie Richard Price, John Wesley und Richard Clarke träumten von einem neuen Jerusalem einer durchweg frommen Gesellschaft, in der Gott die Menschen mit Lebensaltern, wie sie vor der Sintflut für die alttestamentarischen Patriarchen galten, belohnen würde. ${ }^{553}$ Säkulare Philosophen wie Condorcet betrachteten das lange Leben als Folge der immerwährenden Weiterentwicklung der menschlichen Rasse und der Medizin: »Sans doute l'homme ne deviendra pas immortel, mais la distance entre le moment où il commence à vivre, l'époque commune où naturellement, sans maladie, sans accident, il éprouve la difficulté d'être, ne peut-elle s'accroître sans cesse ? « ${ }^{554}$ Er zeigt sich optimistisch, dass mit dem biologischen Fortschritt eine Höherentwicklung

\footnotetext{
${ }^{550}$ Vgl. a.a.O., 27-68; Priesner 2011, 41f.; Rothblatt 2014, 293-299.

${ }^{551}$ Vgl. Gruman 1966, 60-81.

${ }_{552}$ Vgl. Bacon 1977, 144-282; Gruman 1966, 60-81.

553 Vgl. Spadafora 1990, 103f., 125f., 223-252; Sampson 1956, 57f.; Ekirch 1944, 26; Dawson 1935, 169f.; Gruman 1966, 76.

${ }^{554}$ Condorcet 1970, 381.
} 
der Moral und des Intellekts einhergehen wird. ${ }^{555}$ Auch für Benjamin Franklin sind der moralische Fortschritt der Menschheit und die Lebensverlängerung miteinander verschränkt:

\begin{abstract}
All Diseases may by sure means be prevented or cured, not excepting even that of Old Age, and our Lives lengthened at pleasure even beyond the antediluvian Standard. O that moral Science were in as fair a Way of Improvement, that Men would cease to be Wolves to one another, and that human Beings would at length learn what they now improperly call Humanity. ${ }^{556}$
\end{abstract}

Die weitreichendste Utopie formulierte der englische Philosoph William Godwin, der mit seiner kurz nach der französischen Revolution erschienenen Enquiry Concerning Political Justice and its Influence on Morals and Happiness von 1793 alle bisherigen gesellschaftlichen Institutionen - wie die Ehe, die Religion, die Monarchie, die Regierung - ablehnte. Im Einklang mit den meisten Philosophen seiner Zeit war Godwin davon überzeugt, dass sich die Menschheit mit wachsender Vernunft einem vollkommenen Zustand annähere. Und wenn die Geschichte des Lebens bezeuge, wie der Mensch mit jedem neuen Jahrhundert mehr Macht über die Kräfte der Natur gewinne, dann stelle sich die Frage, ob dies auch für die Herrschaft des Geistes (mind) über die Materie (matter) seines eigenen Körpers gelte:

If over matter at ever so great a distance, why not over matter which, however ignorant we may be of the tie that connects it with the thinking principle, we always carry about with us, and which is in all cases the medium of communication between that principle and the external universe? In a word, why may not man one day be immortal? ${ }^{557}$

In der Auseinandersetzung mit seinen zahlreichen Kritikern verteidigt Godwin seine Prognosen mit einer Relativierung: Die absolute Unsterblichkeit, also die vollständige Beseitigung des Sterbens, sei ein nie gänzlich zu erreichendes Ziel. Als erster Schritt zur Überwindung des Todes müsse sein »Bruder«, das menschliche Bedürfnis nach Schlaf, bezwungen werden. Die individuelle Unsterblichkeit ist laut Godwin ein unverzichtbares Element des menschlichen Strebens nach Vervollkommnung, das die gesamte gesellschaftliche Ordnung erneuern werde. Damit geht

\footnotetext{
${ }^{555}$ Vgl. Condorcet 1970, 379-385; Gruman 1966, 87f.

${ }^{556}$ Franklin 1780. Brief an Joseph Priestley, 08.02.1780. Vgl. Gruman 1966, 74, 83f.; Perry 2000, $17 \mathrm{f}$.

${ }^{557}$ Godwin 1793, II, 862. Vgl. auch Ni 2007, 26.
} 
Godwin über La Mettrie hinaus, der zwar ebenfalls dem Geist eine Macht über die körperliche Gesundheit zuschrieb, dieses Potential allerdings keiner Fortschrittsbewegung zuordnete. ${ }^{558}$

Die Überlegungen der Aufklärer führten seinerzeit in ganz Europa zu ersten praktischen Ratgebern für eine gesunde Lebensführung, die dem Einzelnen viele zusätzliche Lebensjahre schenken sollte. Besonders einflussreich war das Werk Die Kunst das menschliche Leben zu verlängern (1796) des deutschen Mediziners Christoph Wilhelm Hufeland, der als königlicher Leibarzt und Direktor der Charité das moderne Gesundheitswesen Preußens entscheidend prägte. Laut Hufeland verfügt jeder Mensch über ein bestimmtes Maß an Lebenskraft, das er schonen und pflegen muss. Sexuelle Ausschweifungen, Onanie, die schlechte Luft der Städte, Völlerei oder ein unausgewogenes Gemüt würden das Leben verkürzen, während eine gute Abstammung, eine arbeitsame Jugendzeit, sexuelle Mäßigung, eine gute Ehe, das Landleben, Hygiene, ein zufriedenes Gemüt und maßvolle Speisen das Leben verlängern. Als interessante Möglichkeit zur Verjüngung betrachtet der fromme Arzt die ersten Versuche mit Bluttransfusionen, die Jean Baptiste Denis und Richard Lower im 17. Jahrhundert durchführten. ${ }^{559}$ Die Ideen Hufelands mit dem schon antiken Ideal eines maßvollen und zufriedenen Lebens setzten sich im 19. Jahrhundert bei Autoren wie William Sweetser (Human Life, 1867) und Daniel Harrison Jacques (How to grow handsome or Hints toward physical perfection, and the philosophy of human beauty, 1879) fort. ${ }^{560}$

Blickt man summarisch auf das Streben nach Langlebigkeit bzw. Unsterblichkeit, so können wir zunächst beobachten, dass seit der Antike stets Vorstellungen über außergewöhnliche Methoden und Mittel zur Erlangung dieses Ziels bestanden, die dann in der Alchemie systematisch gesucht und erprobt wurden. Die Möglichkeit des langen Lebens wird meist fernen Ländern oder Zeiten zugeschrieben - mit Francis Bacon und seinen Zeitgenossen erlaubt das Utopische jedoch auch den Blick in eine verheißungsvolle Zukunft.

Alle Denker bis in die europäische Neuzeit sehen im biblischen Bericht das Zeugnis, dass prinzipiell eine Lebensspanne von mehreren hundert Jahren möglich sei. Für die christlich orientierten Philosophen wie Cornaro, Bacon und Hufeland bedeutete dies sogar, dass das Bemühen um ein langes und gesundes Leben in Einklang mit den göttlichen Ge-

\footnotetext{
${ }^{558}$ Vgl. a.a.O., 33-39; Gruman 1966, 85-87.

${ }^{559}$ Vgl. Hufeland 1797, 21-23, 235-492; Gruman 1966, $82 f$.

${ }^{560}$ V gl. Gruman 1966, 80-89.
} 
boten stand. Denn mit den aufklärerischen Denkern verband sie die Überzeugung, dass das Erreichen des langen Lebens nicht nur einen Wissensfortschritt, sondern unbedingt auch einen moralischen Fortschritt der gesamten Gesellschaft voraussetzt.

Die biologische Vervollkommnung des Menschen mit der sozialen Entwicklung zu verknüpfen, war auch Ziel der Bewegung der russischen Kosmisten zu Beginn des 20. Jahrhunderts. Ausgangspunkt ist die umfassende sozialistische Utopie des Philosophen Nikolai Fedorov (18291903), der mit seiner »Philosophie der gemeinsamen Tat« die gesellschaftlichen und technologischen Bedingungen dafür schaffen will, alle bisherigen Menschen wiederauferstehen zu lassen. Nicht die Religion, sondern der Staat trage die Verantwortung für ein unsterbliches Leben vergangener Generationen, die mit ihrem Opfer erst den Fortschritt in der Gegenwart möglich machten. Dadurch könne die wahre Brüderlichkeit aller Menschen realisiert werden. Nach der Revolution wurden die Ideen Fedorovs von einer Reihe junger Wissenschaftler und Philosophen aufgegriffen, die die Menschheit, den Planeten und das ganze Universum durch den Fortschritt von Arbeit, Wissen und Erziehung revolutionieren wollten. Im Manifest der Biokosmisten Immortalismus und Interplanetarismus von 1922 streben die Autoren nach der vollkommenen Aufhebung aller gesellschaftlichen Unterschiede der Menschen. Die Lebenszeit betrachten sie als den ureigenen Ursprung allen Besitzes, der die Ungleichheit der Menschen zementiert. Daher fordern die Biokosmisten für jeden Menschen das Recht auf ein unbegrenztes Sein: die Unsterblichkeit, die Auferweckung aller Toten, die Verjüngung sowie die völlige Bewegungsfreiheit im kosmischen Raum. Das Ziel der Verjüngung wollte man z.B. durch das von Aleksandr Bogdanov in den 1920er Jahren etablierte Institut für Bluttransfusion erreichen. ${ }^{561}$

Eine der zentralen Figuren der Biokosmisten war der russische Vordenker der Raumfahrt, Konstantin Ziolkowski (1857-1935), dessen theoretische Pionierleistungen wegweisend für die Raketentechnik des 20. Jahrhunderts wurden. Die Besiedlung des Universums entsprach der kosmistischen Forderung nach einer unbegrenzten räumlichen Entfaltung der Menschheit und war für Ziolkowski eng mit der Schaffung von Lebensraum für die auferstandenen Menschen verbunden. So könne die Menschheit auch dem drohenden Wärmetod des Universums begegnen. Der kreative Visionär ist dabei sowohl von materialistischen

${ }^{561}$ Vgl. Groys 2005, 8-17; Hagemeister 2005, 19-44. 
Philosophien wie dem Monismus Ernst Haeckels und dem Positivismus Auguste Comtes, der Religionskritik Ludwig Feuerbachs und Nietzsches Idee vom Übermenschen als auch von theosophischen Vorstellungen eines kosmischen Bewusstseins und Denkens beeinflusst (Ziolkowskis Wohnort war um 1900 ein bedeutendes Zentrum der theosophischen Bewegung). Mit vielen Kosmisten teilte er die Auffassung, dass die Erde neben der Atmosphäre und der Photosphäre noch von einer geistigen Sphäre umgeben sei und diese experimentell zu erforschende Gedankenenergie letztlich auch Materie verändern könne. Die Menschheit selbst werde sich in eine höhere Daseinsform ätherischer Wesen, die aus kosmischem Licht bestehen, weiterentwickeln. Hierin spiegeln sich Vorstellungen eines künftigen Menschen, wie sie um $1900 \mathrm{im}$ Spiritismus und der Theosophie artikuliert wurden. ${ }^{562}$

Die Idee einer evolutionären Sphärenstruktur des Planeten, der Biosphäre und der Noosphäre wurde parallel zum Werk von Teilhard de Chardin von dem kosmistischen Geologen Vladimir Vernadski fortgeschrieben, der sie während seiner Pariser Forschungszeit 1922/23 durch Édouard Le Roy kennenlernte. ${ }^{563}$ Die russische Literatur dieser Zeit nimmt vereinzelt Motive der Kosmisten auf, wie z.B. in der sozialistischen Utopie Der rote Stern (1908) von Aleksandr Bogdanov, die die Verjüngung durch Bluttransfusion propagiert. ${ }^{564}$

Es bestehen zunächst keine direkten Verbindungen zwischen den Ideen der Kosmisten und der Genese des Trans- und Posthumanismus in den Vereinigten Staaten. Die Schriften von Fedorov wurden wegen ihrer metaphysisch-christlichen Gehalte während der Sowjet-Zeit nicht veröffentlicht. Und mit Ausnahme von Vladimir Vernadski und Konstantin Ziolkowski fielen die meisten Kosmisten den stalinistischen Säuberungen in den 1930er Jahren zum Opfer. Sie wurden ermordet oder starben in den Arbeitslagern. Führte der russische Kosmismus später nur ein Nischendasein in den Forschungsinteressen westlicher Slawisten, können

${ }^{562}$ Einflussreich waren hier vor allem der deutsche Spiritist und Theosoph Carl du Prel (1839-1899) und der Evolutionstheoretiker Alfred Russel Wallace, der in seinem Spätwerk Man's Place in the Universe (1903), eine kosmische Entwicklungsgeschichte schrieb, die auf ein künftiges Geistwesen hinauslaufe. Vgl. Hagemeister 2005, 19-44, 56-64; Wallace 1903, 272-301; Benz 1961, 96-100; Benz 1965, 91-96.

${ }^{563}$ Zusammen mit Pierre Teilhard de Chardin hatte Le Roy in den 1920ern das Konzept der Biosphäre und der Noosphäre entwickelt. Le Roy, der 1921 als Nachfolger von Henri Bergson am Collège de France berufen wurde, lehnte sich in seinem Entwurf der Evolutionsgeschichte spürbar an den Idealismus an. Vgl. Le Roy 1928.

${ }^{564}$ Vgl. Hagemeister 2005, 34 . 
wir in der postsowjetischen Ära eine breite Rezeption in der rezenten Philosophie und Kunstszene beobachten. Sogar der russische Präsident Vladimir Putin beruft sich in einer Ansprache von 2014 auf Fedorovs »Philosophie der gemeinsamen Tat«. Im Transhumanismus wurden die Ideen der Kosmisten - allerdings ohne besonderen Widerhall in der breiteren Bewegung - erst jüngst wahrgenommen. Im Jahr 2003 widmete der amerikanische Philosoph Charles Tandy den ersten Band seiner transhumanistischen Buchreihe Death and Anti-Death dem Gedenken an Fedorov. Der KI-Forscher und Unternehmer Ben Goertzel setzt Fedorov in seinem 2010 erschienen Cosmist Manifesto sogar ins Zentrum einer praktischen Umsetzung der posthumanistischen Philosophie. Die Initiative 2045 des russischen Milliardärs Dmitry Itskov steht ohne Frage in der Tradition der Kosmisten. ${ }^{565}$ Auch über die seit den 1960er Jahren ins Englische übersetzten Werke der Brüder Strugazki und Stanisław Lems sind Einflüsse auf den rezenten Post- und Transhumanismus denkbar. ${ }^{566}$ Bemerkenswert ist in Anbetracht der russischen Kosmisten, dass wie schon im Falle aufklärerischer Zukunftsvisionen das Projekt der Unsterblichkeit ein kollektives ist, das die gesamte Gesellschaft erfassen solle - die vergangenen Generationen eingeschlossen.

Nicht nur sozialistische Utopien in Osteuropa, sondern ein allgemeiner Glaube an den Fortschritt der Technik und Wissenschaften erfasste im 19. Jahrhundert weltweit Vertreter aller gesellschaftlichen Schichten. Die Blüte der Science-Fiction Literatur, die sich an Autoren wie H. G. Wells und Jules Verne festmachen lässt, spiegelt diesen technophilen Optimismus des fin de siècle wider. Eine Vielzahl populärer und wissenschaftlicher Autoren blickte verheißungsvoll auf die künftigen Möglichkeiten der Verjüngung und Lebensverlängerung. ${ }^{567}$ Einflussreich waren hier insbesondere der Begründer der Gerontologie, Ilja Metschnikow mit seinen Études sur la nature humaine. Essai de philosophie optimiste (1903), der französische Autor Jean Finot mit seiner Philosophie de la longévité (1906), die Chirurgen Serge Voronoff und Eugen Steinach, die mit der Trans-

\footnotetext{
${ }^{565}$ Vgl. Bernstein 2015, 770-775.

${ }^{566}$ Vgl. Simakova 2016; Goertzel 2010; Bernstein 2014; Krysmanski 2014, 136f.; Tandy 2003. Bis 2013 sind 11 Bände dieser Reihe erschienen. Charles Tandy hat in Kalifornien die Ria University (www.ria.edu) gegründet, die sich vor allem dem Studium der Lebensverlängerung und Zeitreisen (!) widmet.

${ }^{567}$ Der israelische Transhumanist Ilia Stambler hat eine umfangreiche Zusammenstellung aller Autoren vorgelegt, die sich v.a. im frühen 20. Jahrhundert mit der Frage der medizinischen Lebensverlängerung befassten. Vgl. Stambler 2014.
} 
plantation von Affenhoden und ähnlichen Eingriffen die Verjüngung männlicher Patienten versprachen (darunter auch Sigmund Freud), ferner Gerhard Venzmer mit seinem Plädoyer für ein natürliches Leben in Alt werden und jung bleiben (1936). ${ }^{568}$ Mit seinem 2009 erschienenen Lebensratgeber Transcend. Nine Steps to Living Well Forever knüpft Ray Kurzweil an diese Tradition an und verspricht, dass bereits in den frühen 2020er Jahren der Alterungsprozess rückgängig gemacht werden könne. ${ }^{569}$ Während diese medizinischen Perspektiven auf die Verjüngung und Lebensverlängerung abzielten, proklamierten der amerikanische Schriftsteller Charles Asbury Stephens und der britische Physiker John Desmond Bernal die Unsterblichkeit selbst als Ziel wissenschaftlichen Strebens. Stephens, der auch Medizin studierte, verdiente sich seinen Lebensunterhalt mit Kurzgeschichten und Zeitschriftenberichten über wissenschaftliche Neuerungen. Von 1888 bis 1905 betrieb er dank der Unterstützung eines Mäzens ein Forschungslabor in Maine, das Lebenszyklen von Zellkulturen analysierte. Die ausbleibenden Erfolge seiner Forschungen hielten ihn nicht davon ab, in seinen philosophischen Werken Living Matter (1888), Long Life (1896), Natural Salvation (1903) und Immortal Life (1920) die endgültige Überwindung des Todes zu proklamieren. Gemäß Stephens ist nämlich der Tod unnatürlich: Er trete durch die Erkrankung oder Beschädigung der an sich unsterblichen Zellen unseres Organismus ein. Jede einzelne Zelle verfüge genau wie die Menschheit in der Evolution über eine ätherische Energie, die den Menschen in eine höhere, geistige Daseinsebene dränge, »a spiritualization of the genus homo ${ }^{570}$ Stephens präsentierte die Evolutionsgeschichte als teleologischen Prozess, der das menschliche Gehirn hervorbringen sollte, das im Gegensatz zu den kurzlebigen Organen des Restkörpers mehrere Jahrhunderte überdauern könne: ${ }^{571}$

Two millions of centuries have struggled forward in pain and travail to make the human brain capable of the human intellect ... It is not destined forever, nor much longer, to be lost in death; we shall carry it through to a greater destiny. The true scope and intent of life is now just dawning in the minds of men. We are waking, - after idle dreams, - waking to what we can do and be, waking to

\footnotetext{
${ }^{568}$ Vgl. Stambler 2014, 10-114.

${ }^{569}$ Vgl. Kurzweil \& Grossman 2009, XX-XXIII.

${ }^{570}$ Vgl. Stephens 1920, $48 f$.

${ }^{571}$ Vgl. Stephens 1920, 49-67.
} 
the great possibilities of science, waking to live, instead of resigning ourselves to death. ${ }^{572}$

Die ursprüngliche Wirkmacht, die das Leben entstehen lässt und das ganze Universum durchzieht, ist laut Stephens der Äther in Form einer beseelten, wissenden Energie, die auch für Phänomene wie Telepathie, Hellsichtigkeit und Geistererscheinungen verantwortlich sei. ${ }^{573} \mathrm{Als}$ höchst entwickelte Rasse der Menschheit sei es nun den Ariern vorbehalten, die nächste evolutionäre Stufe des Lebens zu realisieren. ${ }^{574}$ Während Stephens die arische Rasse als höchste Form des intelligenten Lebens stilisiert, ist es für John Desmond Bernal die Aristokratie der Wissenschaftler, die die künftige Evolution des Lebens steuern wird. Im Alter von 25 Jahren beginnt er mit der Niederschrift seiner Memoiren, da er befürchtet, als alter Mann nicht mehr dieselbe Offenheit für neue Ideen zu haben. Als der irisch stämmige Molekularbiologe drei Jahre später das berüchtigte Traktat The World, The Flesh and the Devil. An Enquiry into the Future of the three Enemies of the Rational Soul veröffentlicht, betrachtet er seine eigene Rolle denn auch als die eines passionierten Revolutionärs, der dem Marxismus zugeneigt ist, der Kirche abgeschworen hat, promisk lebt und von starken anti-englischen Ressentiments angetrieben ist. Bernal wurde zu dieser Zeit von der kontroversen Debatte um den Biologen J.B.S. Haldane inspiriert, der 1924 mit der Vision seines Buches Daedalus, Föten künstlich zu befruchten und in Gebärmaschinen auszubrüten, für Furore gesorgt hatte. ${ }^{575}$ Während Bernal sich in seiner wissenschaftlichen Karriere viel Anerkennung für seine innovativen Beiträge in der Kristallographie erwarb, ist seine jugendliche Utopie höchst spekulativ und bleibt in ihrer Radikalität bis zum Aufkommen des technologischen Posthumanismus unübertroffen.

Der Buchtitel The world, the Flesh and the Devil spielt auf die irdische und kosmische Umwelt, den menschlichen Körper und - als größte Herausforderung - unsere innere, psychische Welt, unsere Ängste, Hoffnungen und Motivationen an, die es zu bezwingen gelte. Eine Prognose über die Zukunft des Lebens müsse Bernal zufolge neben der (Fortschritts-) Geschichte und den physikalischen Gesetzen auch das menschliche Begehren berücksichtigen, »the strongest thing in the universe «. ${ }^{576}$

\footnotetext{
${ }^{572}$ Stephens 1920, $75 f$.

${ }^{573}$ Vgl. Stephens 1920, 130.

${ }^{574}$ Vgl. Stephens 1920, 240f.; Stambler 2014, $191 \mathrm{f}$.

${ }^{575}$ Vgl. Haldane 1924; Brown 2005, 65-69; Bernal 1929, 13.

${ }^{576}$ V gl. Bernal 1929, 1-4.
} 
Bernal lehnt den eugenischen Ansatz ab, der auf eine bessere Auswahl von Sexualpartnern oder gar die Manipulation des Erbgutes abzielt. Dies würde nur sehr langsame Veränderungen bedeuten und die Spezies Mensch nicht substantiell verändern. Der zukünftige Mensch wird nach Bernals Vorstellungen zunächst biologisch optimiert aus einer Geburtsfabrik hergestellt werden, dann 60 bis 120 Jahre als biologischer Mensch das Leben, den Tanz und die Sexualität genießen, um schließlich seinen bisherigen Daseinszustand zu »transzendieren«. Ähnlich wie für Stephens - dessen Werk er offenbar nicht kennt - ist auch für Bernal das Gehirn der Sitz der menschlichen Persönlichkeit: »After all it is brain that counts, and to have a brain suffused by fresh and correctly prescribed blood is to be alive - to think. " ${ }^{577}$ Der Restkörper sei überflüssig: »In a civilized worker the limbs are mere parasites, demanding nine-tenths of the energy of the food and even a kind of blackmail in the exercise they need to prevent disease, while the bodily organs wear themselves out in supplying their requirements. ${ }^{578}$

Der Mensch werde nur noch mental als Gehirn in einem künstlichen Zylinder existieren, der ihn von außen mit Blut und extrem erweiterten, sensorischen Eindrücken versorgen wird (wie z.B. Röntgenstrahlen etc.). Laut Bernal könnte diese Daseinsweise dem Menschen die ersehnte Unsterblichkeit auf zwei Arten näherbringen. Erstens könnte das Gehirn selbst in mehrere Teile aufgesplittet werden, die dann bei Bedarf ersetzt würden. Zweitens aber schwebt Bernal eine künstliche Verbindung der einzelnen Gehirne vor, die zu einem komplexen Superorganismus zusammenwachsen. Der Tod einzelner Gehirne werde dann durch das Ganze kompensiert: ${ }^{579}$

But the multiple individual would be, barring cataclysmic accidents, immortal, the older component as they died being replaced by newer ones without losing the continuity of the self, the memories and feelings of the older member transferring themselves almost completely to the common stock before its death. ${ }^{580}$

Ausgestattet mit neuen, künstlichen Sinnesorganen könnte dieser verbundene Gehirnorganismus in das Innere biologischer Zellen oder eines Sterns eindringen und all diese Ebenen durch die schiere Kraft des

\footnotetext{
${ }^{577}$ Bernal 1929, 15.

${ }^{578}$ Ebd.

${ }^{579}$ Vgl. a.a.O., 17-21; Heil \& Coenen 2014, $149 f$.

${ }^{580}$ Bernal 1929, 19.
} 
Bewusstseins verändern. ${ }^{581}$ Nur auf diese Weise könne der Mensch laut Bernal sein geistiges Potential voll entfalten und körperlos die Expansion ins Weltall beginnen. Der Mensch werde damit die natürliche Umwelt und ihre Zwänge wie die Ernährung und Sexualität überwinden und die frei werdenden Kräfte in die Kunst und Wissenschaft einfließen lassen: ${ }^{582}$ »As time goes on, the acceptance, the appreciation, even the understanding of nature, will be less and less needed. In its place will come the need to determine the desirable form of the humanly-controlled universe which is nothing more nor less than art. « ${ }^{583}$

Bernals Prognose für die Zukunft der Menschheit ist ambivalent. Er hält es für möglich, dass die überlegene neue Menschheit der Wissenschaft die Dummen und Einfältigen, die sich der Veränderung verweigern, einfach auf der zoogleichen Erde zurücklässt und den Weltraum kolonisiert oder aber, dass ein Kampf ums Überleben zwischen beiden Fraktionen ausbrechen wird. ${ }^{584}$

Mit seinem jugendlichen Urteil über die schwindende Kühnheit alter Männer sollte Bernal übrigens Recht behalten: Seine späteren wissenschaftlichen Texte - wie das Kapitel The Future of Biology in der Science in History von 1969 - wirken leidenschaftslos und geben nur einen allgemeinen Ausblick auf bessere Krankheitsprognosen, effizientere Saatgutzüchtungen und dadurch eine Aussicht auf Lebensverlängerung. ${ }^{585}$ Zwar war der britische Science-Fiction Schriftsteller Arthur C. Clarke von Bernals radikalem Menschheitsentwurf höchst fasziniert und auch Einflüsse auf das literarische Werk von Olaf Stapledon sind denkbar. Da Bernal jedoch in späteren Jahren weder aktiv seine Visionen propagierte, noch eine entsprechende Bewegung ins Leben rief, steht The World, the Flesh and the Devil in seiner Periode isoliert da. Erst mit den Bernal-Lectures durch den Physiker Freeman Dyson wurden in den 1970er Jahren die frühen Ideen Bernals zur Kolonisierung des Weltraums (Bernal-Sphäre / Dyson-Sphäre) wieder einem breiteren Publikum bekannt.

Wenn sich daher kein direkter Einfluss auf Vorstellungen des technologischen Posthumanismus aufzeigen lässt, so spiegeln Stephens und Bernals Entwürfe die radikale Zuspitzung von verbreiteten Zukunftshoffnungen ihrer Zeit. Diese Vorstellungen konnten dann über die eugenische Bewe-

${ }^{581}$ Vgl. Bernal 1929, 13-20.

${ }^{582}$ V gl. Bernal 1929, 27-31.

${ }^{583}$ Bernal 1929, 30.

${ }^{584}$ Vgl. Bernal 1929, 36.

${ }^{585}$ Vgl. Bernal 1969, 981-988. 
gung (Huxley) und Science-Fiction-Literatur (Clarke) durchaus posthumanistische Visionen in der 2. Hälfte des 20. Jahrhunderts prägen. Da ist zunächst die in der Eugenik geteilte Auffassung, dass der Mensch seine weitere Vervollkommnung nun bewusst steuern müsse und sie nicht dem blinden, evolutionären Zufall überlassen dürfe. Beide Autoren vertreten auch eine extreme Natur- und Körperverachtung, die bei Stephens noch mit einer rassisch-theosophischen Äthermetaphysik vermengt wird. Die Verabsolutierung des Gehirns als alleinige Qualität des Menschseins korrespondiert ganz offensichtlich mit dem Geniekult der Jahrhundertwende und der zeitgenössischen Eugenik. Wirklich neu ist an Bernals Abwertung des Körpers die Begründung, dass der biologische Körper der angestrebten Expansion in den Weltraum entgegenstehe.

Die beiden Weltkriege wirkten als Dämpfer für den technologischen Fortschrittsoptimismus, führten sie doch vor Augen, welches Potential zur Vernichtung allen Lebens die Naturwissenschaften entfesseln konnten. Der Einsatz von Chemiewaffen im Ersten Weltkrieg und der Abwurf der Atombomben in Hiroshima und Nagasaki provozierten eine Debatte um die ethischen Grenzen des Fortschritts. Die beiden wichtigsten fortschrittsoptimistischen Bewegungen haben die Weltkriege nicht überlebt: Der materialistische Monismus Ernst Haeckels galt nach 1918 vielen als Symptom einer gottlosen Gesellschaft, die ohne christliche Wertverbundenheit die Grauen des Krieges erst möglich gemacht hatte. Die Tötungen von »lebensunwertem Leben" während der NS-Zeit sowie die überall in Europa durchgeführten Zwangssterilisationen brachte die Eugenik in Misskredit. Zudem erfuhren die Vereinigten Staaten in dieser Periode eine antimodernistische, religiöse Politisierung, die erst durch den Sputnik-Schock von 1957 eine Wende erlebte.

In diese neue, fortschrittsoptimistische Phase seit Anfang der 1960er Jahre fällt die Initiierung der kryonischen Bewegung durch Robert Ettinger. Sie wird aus systematischen Gründen am Ende des Kapitels zur Unsterblichkeit gesondert besprochen, ist sie doch aus Sicht ihrer Anhänger keine wirkliche Immortalisierungsmethode, sondern bloß eine Überbrückungstechnologie, die eine spätere Wiederauferstehung ermöglichen soll. Diese Ära war - ähnlich dem Transhumanismus der 1990er Jahre - durch den ungebremsten Enthusiasmus geprägt, dass durch den medizinischen Fortschritt in Kürze auch das Todesproblem gelöst werden könne. Zahlreiche lokale Kryonik-Vereinigungen formierten sich Mitte der 1960er Jahre, wie z.B. die Life Extension Society in Washington D.C., die Immortalist Society in Michigan oder die Immortality 
Records and Compilation Association in Kalifornien. Den Höhepunkt der Bewegung, die später durch zahlreiche Skandale erschüttert werden sollte, bildete sicherlich die Präsentation des kryonischen Immortality Pavilion auf der Weltausstellung von Montreal 1967. ${ }^{586}$

Zwar äußern sich nur vereinzelt Denker wie der Physiker Gerald Feinberg oder der Schriftsteller Arthur C. Clarke zuversichtlich, dass den Menschen die technische Unsterblichkeit gelingen werde, ${ }^{587}$ aber die konkrete Idee einer Existenz im Speicher eines Computers nimmt in engem Austausch mit der fiktionalen Literatur langsam Gestalt an. Bereits 1964 hat der Science-Fiction Schriftsteller Frederik Pohl in einem Artikel für den amerikanischen Playboy verschiedene Arten der Lebensverlängerung diskutiert und schließlich die Vorstellung entwickelt, einen Computer durch Interaktion und Kommunikation wie ein Kind zu erziehen und dadurch ein künstliches und unsterbliches Leben zu erschaffen. ${ }^{588}$ 1971 erschien dann zum ersten Mal im nicht-fiktionalen Rahmen die konkrete Idee, die Persönlichkeit eines lebenden Menschen in einen Computer zu übertragen. George M. Martin, seinerzeit Professor für Pathologie an der Washington University in Seattle, zeigte sich in seinem kleinen Beitrag für eine medizinische Fachzeitschrift zunächst von den Perspektiven der Kryobiologie beeindruckt. Unter der Voraussetzung, dass der wissenschaftliche Fortschritt anhalte und die Menschheit sich nicht selbst vernichten werde, war er zuversichtlich, das Todesproblem des Menschen zu bewältigen:

We shall assume that developments in neurobiology, bioengineering, and related disciplines, perhaps over a period of centuries, will ultimately provide suitable techniques of »read-out « of the stored information from cryobiologically preserved brains into $n$ th. generation computers capable of vastly outdoing the dynamic patterning of operation of our cerebral neurons. We would then join a family of humanoid "postsomatic « bioelectrical hybrids, capable of contributing to cultural evolution at rates far exceeding anything now imaginable. ${ }^{589}$

Im selben Jahr - davon berichtet Hans Moravec - behandelte der $I B M$-Angestellte Dick Fredericksen in mehreren Ausgaben seines pri-

\footnotetext{
${ }^{586}$ Vgl. Krüger 2010, 3f., 17 (FN V).

${ }^{587}$ Vgl. Feinberg 1970, 77-87, 102-115; Clarke 1960, 233. Feinberg selbst war in dieser Zeit mit seinem Prometheus-Projekt bemüht, interdisziplinär die wichtigsten Probleme und Ziele der Menschheit anzugehen. Vgl. Heil 2010a, 140.

${ }^{588}$ Später publizierte Pohl auch gemeinsam mit Moravec. Vgl. Pohl 1964; Pohl 1989; Moravec \& Pohl 1993; Regis 1990, 151f., 204ff.

${ }^{589}$ Martin 1971, 339.
} 
vaten Newsletters $A$ Word in Edgewise die Frage, ob man über einzelne Organtransplantationen hinaus nicht auch den im Gehirn verorteten, menschlichen Geist auf eine andere Hardware - einen quasi unsterblichen Roboter - übertragen könne. ${ }^{590}$ Und just diese Serie des Newsletters fand der junge Student Hans Moravec am schwarzen Brett des Stanford Artificial Intelligence Laboratory. ${ }^{591}$

Inspiriert durch Fredericksen publizierte Moravec nun seinerseits mehrere Essays, die die Zukunft der Evolution und künstlichen Intelligenz behandelten. Erstmals in seinem Aufsatz Today's Computers, Intelligent Machines and Our Future, der im Februar 1979 in dem Science-Fiction Magazin analog erschien, ${ }^{592}$ schildert Moravec die chirurgische Prozedur der Geisttransplantation, die er später in seiner Monographie detaillierter ausführt:

Though you have not lost consciousness, or even your train of thought, your mind has been removed from the brain and transferred to the machine. A final step is the disconnection of the your old sensory and motor system, to be replaced by higher quality ones in your new home. This last part is no different than the installation of functioning artificial arms, legs, pacemakers, kidneys, ears and hearts and eyes being done or contemplated now. ${ }^{593}$

Auch bereits an dieser Stelle führt Moravec verschiedene Vergleichstabellen ein, die biologisches Leben und Computerleistungen in ein Verhältnis zueinander setzen. ${ }^{594}$

Zur gleichen Zeit nutzte der an der Georgetown University tätige Medizinethiker Robert M. Veatch die »bizarre und rein hypothetische« Vorstellung, dass alle Informationen eines menschlichen Gehirns auf Magnetbändern gespeichert wären, in der damals aktuellen Diskussion um die präzise, medizinische Bestimmung des Todes. ${ }^{595}$

Zu Beginn der 1980er Jahre war die Utopie einer computertechnischen Unsterblichkeit bereits so verbreitet, dass der populäre NASA-Wissen-

${ }^{590}$ Vgl. Fredericksen 1971, 23, 1-A - 3-A.

${ }^{591}$ Vgl. Moravec 1988, 203; Regis 1990, 152f., $157 f$.

${ }^{592}$ Die Zeitschrift ging aus dem 1930 gegründeten Magazin Astounding Science Fiction hervor und nahm ab 1960 unter dem neuem Titel auch nichtfiktionale Beiträge auf.

${ }^{593}$ Moravec 1979, 80. Vgl. a.a.O., 78-81.

${ }^{594} \mathrm{Vgl}$. a.a.O., 63, 71, 73 .

${ }^{595}$ Veatch weist die hier zum Ausdruck kommende Bestimmung des Menschen allerdings entschieden zurück: »Man is, after all, something more than a sophisticated computer. At least in western tradition the body is an essential element, not something from which man escapes in liberation.« Veatch 1975, 23. 
schaftler Robert Jastrow freimütig über diese paradiesische Zukunft spekulieren konnte:

At last the human brain, ensconced in a computer, has been liberated from the weakness of mortal flesh. Connected to cameras, instruments and engine controls, the brain sees, feels, and responds to stimuli ... It seems to me that this must be the mature form of intelligent life in the Universe. Housed in indestructible lattices of silicon, and no longer constrained in the span of its years by the life and death cycle of a biological organism, such a kind of life could live forever. ${ }^{596}$

Vor einem großen Lesepublikum konnte dann 1983 der Publizist Mike Edelhart in der amerikanischen Zeitschrift Psychology Today die philosophischen Herausforderungen dieses Gedankenspiels einer Existenz im Computerspeicher ausführen. Diese bedeuteten auch für ihn eine $\mathrm{Zu}$ spitzung der ethischen Fragen der Organtransplantation:

Or consider a computer-brain hookup. As a man lies dying, he feeds the entire contents of his brain - every brain-wave pattern it can create - into a computer, linked to a voice synthesizer, which speaks as he did. The computer has all his memories and attitudes. It has his sense of humor. Is it him $?^{597}$

Trotz dieser vereinzelten Artikulierungen einer Möglichkeit von computertechnischer Immortalisierung ist es m.E. nicht angebracht, an dieser Stelle von einem »Proto-Posthumanismus« zu sprechen. Die Kontexte dieser Ideen sind sehr heterogen und teils auch kritisch. Erst Moravec ordnet die technische Utopie in ein normatives Gefüge ein, das die Simulation des Menschen kybernetisch plausibilisiert und den unausweichlichen Fortschrittsprozess legitimiert.

\subsubsection{Die Unsterblichkeit in der Science-Fiction}

Dass man ab den 1970er Jahren als Gedankenexperiment oder Ausblick auf die Zukunft in medizinischen Fachzeitschriften oder populären Magazinen über eine computertechnische Immortalisierung spekulieren konnte, ist das Verdienst der fiktionalen Literatur. Denn hier wurden die zentralen Ideen zuerst erdacht und einem breiten Publikum präsentiert. Der posthumane Traum von der Unsterblichkeit knüpft dabei an eine

${ }^{596}$ Jastrow 1981, 166f. Vgl. Lavery 1992, 68 f.

${ }^{597}$ Edelhart 1983, 41. Edelhart schrieb von den frühen 1980er bis in die 2000er Jahre Bücher und Zeitschriftenartikel über Computer, Karriere und private Erdbunker. 
lange literarische Tradition an, die sich seit mehr als zwei Jahrhunderten mit der »wissenschaftlichen« Verwirklichung der menschlichen Unsterblichkeit befasst. In einem fließenden Übergang von alchemistisch geprägten Novellen bis zur eigentlichen Science-Fiction Literatur weisen Brian Stableford und Carl B. Yoke zwischen 1800 und 1990 nicht weniger als 200 entsprechende englischsprachige Erzählungen und Romane nach. Während die älteren Stücke bis ca. 1930 die technische Immortalisierung fast ausschließlich als Stagnation oder Fluch bewerteten, steht die neuere fiktionale Literatur der Idee des wissenschaftlich ermöglichten, ewigen Lebens differenzierter gegenüber. Aber auch hier überwiegen die skeptischen Stimmen. ${ }^{598}$

Eine der älteren Erzählungen von Jules Verne möchte ich hier besonders hervorheben. Sie steht - ähnlich wie die Éve future (1886) von Auguste Villiers de l'Isle Adams - sichtbar unter dem Eindruck der Erfindung des Phonographen durch Thomas A. Edison im Jahr 1877. Mit dieser Innovation sollte es erstmals möglich sein, menschliche Sprache aufzuzeichnen und wiederzugeben. Die maschinelle Simulation eines menschlichen Wesensmerkmals, nämlich der Kommunikation, rückte damit in greifbare Nähe und schließt den bereits angesprochenen Bogen, der von Descartes zu Turing reicht.

Ließ Villiers de L'Isle Adam seinen Helden eine vollkommenere - nämlich gefügige - Frau erschaffen, so wird in Jules Vernes 1892 publiziertem Le Château des Carpathes die perfekte und unvergängliche Simulation einer echten Frau verwirklicht. Der Baron von Gortz, der sich in die berühmte Opernsängerin La Stilla verliebt hat, zeichnet ihre Stimme auf phonographischen Rollen auf. Jedoch wird während der Aufnahme und Reproduktion des Gesanges nicht nur die Stimme, sondern auch die Seele und Lebenskraft der Sängerin aufgesogen, so dass La Stilla plötzlich bei der letzten Vorstellung vor ihrer geplanten Hochzeit mit dem Grafen von Telek tot zusammenbricht und aus ihrem Mund nur noch Blut hervorquillt. Mit Hilfe dieser phonographischen Klangreproduktion und mittels einer Art dreidimensionaler, beweglicher Bildprojektion gelingt es von Gortz jedoch, eine perfekte Simulation der Verstorbenen zu erschaffen, die seinen Konkurrenten, den Grafen von Telek, bis zuletzt vom Weiterleben seiner Braut überzeugt. ${ }^{599}$ Die Erzählung von Jules Verne stellt das erste Zeugnis für die Idee einer Immortalisierung

\footnotetext{
${ }^{598}$ Vgl. Stableford 1999, 616; Yoke 1985; Collings 1985.

${ }^{599}$ Vgl. Verne 1892, dt.: Das Karpatenschloß. Wien 1893; Innerhofer 1996, 396-399; Innerhofer 2003, 279f. Innerhofer berichtet von einer Reihe unbekannterer Erzählungen
} 
durch Medientechnik dar, ein halbes Jahrhundert später folgen literarische Überlegungen, die die Unsterblichkeit direkt mit Computern in Verbindung bringen.

Sichtlich beeinflusst von Shannons mathematischer Informationstheorie und Wieners Kybernetik verfasste 1957 der britische Science-Fiction Schriftsteller Arthur C. Clarke die Geschichte The City and the Stars, die in einer viele Millionen Jahre entfernten Zukunft der Stadt Diaspar spielt. Erstmals wird hier die Vorstellung eines als informationstechnisches Extrakt »gespeicherten« Menschen fiktional inszeniert: Ohne dass der Autor die technischen Details thematisieren würde, ist es den künftigen Lebewesen gelungen, den menschlichen Geist vom Körper abzulösen. Aus einem gigantischen Zentralcomputer heraus werden die "gespeicherten Menschen« nach bestimmten Zyklen in realen Körpern zum Leben erweckt und nach ihrem Dahinscheiden mit ihren neuen Lebenserfahrungen wiederum gespeichert. Clarke setzt damit die Annahme um, dass die menschliche Persönlichkeit nichts als information, structure and pattern sei, die gleichermaßen auf Papier, Magnetbändern oder Elektroröhren gespeichert werden könnten. ${ }^{600}$

Auch die konkretere Idee einer vermeintlich unsterblichen Existenz in der Virtualität ist bereits dreißig Jahre vor Hans Moravecs Mind Children in den Dialogen (Dialogi) des polnischen Autoren Stanisław Lem formuliert und ausführlich diskutiert worden. Lem entwirft 1957 in seinen an Platon bzw. George Berkeley angelehnten Dialogen die technische Vision einer Unsterblichkeitsmaschine, die eine Weiterführung der Existenz bzw. eine Auferstehung durch die perfekte Simulation der materiellen Person ermöglichen sollte. ${ }^{601}$ Nach langen Diskussionen gelangen die Kontrahenten Hylas und Philonous zu dem Schluss, dass eine noch so perfekte Kopie des Menschen keine Kontinuität der Persönlichkeit garantieren könne. ${ }^{602}$ In zahlreichen kürzeren Geschichten hat Lem diverse Aspekte dieser Idee der »Speicherung « einer menschlichen Persönlichkeit in einem Apparat bzw. Computer nun ausgeführt und damit die schier unüberwindlichen Schwierigkeiten der kybernetischen Mus-

um die Jahrhundertwende, die den Phonographen als Verewigungsinstrument benutzten. Vgl. Innerhofer 1996, 417-420.

${ }^{600}$ Vgl. Clarke 1956, dt.: Die sieben Sonnen. München 1960; Dery 1996, $150 f$.

${ }^{601}$ Vgl. Lem 1980, 9-74 und später in der Summa Technologiae. Dort präsentiert Lem eben-

falls die Idee der beliebigen, individuellen Auferstehung in der Computerexistenz. Vgl. Lem 1981, 328f., 477. Vgl. auch Gräfrath 2000, 287ff.; Rottensteiner 1997, $369 f$.

${ }^{602}$ V gl. Lem 1980, 9-33. 
tertheorie der Identität offengelegt. In den Sterntagebüchern des Kosmonauten Ijon Tichy von 1971 beschreibt Lem ein Experiment des Kybernetikers Professor Corcoran, der »in ein paar Kisten« künstliches Leben geschaffen hat. Die Lebewesen halten ihr Bewusstsein und ihre virtuelle Welt, die ja nur in der »Kiste« existiert, für absolut real: ${ }^{603}$

Jede dieser Kisten enthält ein Elektronensystem, das Bewußtsein erzeugt. Wie unser Hirn. Es besteht zwar aus einem anderen Baustoff, aber das Prinzip ist das gleiche ... Diese Kisten haben Organrezeptoren, die analog zu unserem Geruchssinn, Gesichtssinn, Tastsinn, Gehör und so weiter wirken ... Diese Kisten, Tichy, sind an eine künstliche Welt angeschlossen. Dieser hier ... scheint es, sie sei ein siebzehnjähriges Mädchen, grünäugig, mit rotblondem Haar, mit einem Körper, der einer Venus würdig wäre. ${ }^{604}$

Mitunter enthüllt Lem in seinen Erzählungen schonungslos die vermeintliche Absurdität der Vision einer virtuellen Existenz. Moravecs Vision von einer Notfallkopie des Menschen ${ }^{605}$ gehört auf dem von Tichy aufgesuchten Planeten Enteropien (!) bereits zum Standard. Der Planet wird alljährlich von einem Meteoritenstrom heimgesucht, dem viele Leben zum Opfer fallen würden, wenn nicht von jedem Bewohner und Besucher eine "Reserve« gespeichert und bei Bedarf materialisiert werden könnte. ${ }^{606}$ Auf seiner 23. Reise gelangt der Kosmonaut Tichy dann auf den winzigen Planeten der Bischuten, die sich aus Platzgründen überall, wo es nötig erscheint (im Wartezimmer bei Ärzten und Behörden, Sitzungen etc.), in winzigen Apparaten aufhalten - und sich nur bei Bedarf mit Hilfe der gespeicherten atomaren Strukturen wieder materialisieren. Die versehentliche Verdopplung eines Bräutigams, der durch einen technischen Fehler gleich zweimal neu erschaffen wurde, führt dabei zu großer Verwirrung. ${ }^{607}$ In der Erzählung Gibt es Sie, Mr. Johns? von 1957 muss ein Rennfahrer, der nur noch aus Prothesen der Cybernetics Company besteht, um die Anerkennung und Freiheit seiner Persönlichkeit kämpfen. Sein biologisches Gehirn wurde nämlich ebenfalls nach diversen Unfällen komplett durch ein elektronisches Äquivalent ersetzt, das nun seinen »Geist« beherbergt. ${ }^{608}$ Ein beliebtes Motiv bei Lem ist auch

\footnotetext{
${ }^{603}$ Vgl. Lem 1995, 355-370.

${ }^{604}$ Vgl. a.a.O., $361 \mathrm{ff}$.

${ }^{605}$ Vgl. Moravec 1988, 119.

${ }^{606}$ Vgl. Lem 1995, 148ff.

${ }^{607}$ Vgl. a.a.O., $292 \mathrm{f}$.

${ }^{608}$ Vgl. Lem 1996a.
} 
die virtuelle »Auferstehung « und lebensechte Simulation von längst verstorbenen Persönlichkeiten per Computer. ${ }^{609}$

Neben Clarke und Lem hat sich insbesondere der österreichische Schriftsteller Herbert W. Franke schon früh mit den Möglichkeiten einer virtuellen Simulation der realen Lebenswelt und der technischen Integration des Menschen in diese künstlichen Welten beschäftigt. In seinem 1961 entstandenen Roman Der Orchideenkäfig, der heute zu den Klassikern der deutschsprachigen Science-Fiction zählt, schildert Franke die Erkundung eines fremden Planeten. Die irdischen Besucher, die mit Hilfe eines Synchronstrahles und nur mittels eines Doppelgängers auf der fernen Planetenoberfläche agieren, entdecken die Geschichte einer alten Zivilisation, die aufgrund der kosmischen Bedrohung durch einen Meteoritenschwarm eine totale Mediatisierung und Virtualisierung ihrer Welt vorangetrieben hat. Bauten die Menschen des Planeten zunächst nur Maschinen, um sich von ihnen bedienen zu lassen, so brauchten sie in späteren Epochen ihre Wohnungen gar nicht mehr zu verlassen: Der obsolete Körper wurde durch Leitungen mit flüssiger Nahrung versorgt, während er tagelang die virtuellen Wonnen der so genannten Totalwiedergabe, eines alle Sinne umfassenden Mediums, genoss. Die ehemaligen Menschen enden nach vielen Generationen der physischen und psychischen Veränderung in einem Geflecht aus Leitungen, Drähten, Reflektoren und Plastikhüllen, die dank einer direkten elektronischen Gehirnzellenreizung den verwandelten Organismen Ruhe, Zufriedenheit und Glück vermitteln. ${ }^{610}$

In seinem Roman Zone Null von 1970 variiert Franke dieses Motiv. Ein Expeditionsteam entdeckt auf einem fremden Planeten wiederum eine medientechnisch weit fortgeschrittene Zivilisation. Die führenden Forscher dieses Planeten plädieren aus den gleichen Gründen wie die heutigen Posthumanisten für die Vereinigung der überholten, kohlenstofforganischen Lebewesen (Menschen) mit den Maschinen. ${ }^{611}$ Auf der Zone Null eines riesigen Gebäudekomplexes schließlich befinden sich die technischen Voraussetzungen, um den menschlichen Geist in den Speicher eines universalen Computers zu integrieren. ${ }^{612}$ Die virtuell simulierte Persönlichkeit des Menschen erlangt dort als Befreiung von seinem vergänglichen Körper den Status der Unsterblichkeit. Durch den

${ }^{609}$ Vgl. Lem 1987, 186-208.

${ }^{610}$ Vgl. Franke 1982; Flessner 1997b; Tabbert 2004, 457-473.

${ }^{611}$ Vgl. Franke 1996, 312f.

${ }^{612}$ Vgl. a.a.O., $354 f$. 
Zugriff auf das unendliche Wissen der Computerspeicher und durch unzählige Sonden hat die Person dann weitaus mehr Handlungsmöglichkeiten als zuvor. ${ }^{613}$

Ebenfalls 1961 veröffentlichen die beiden russischen Meister utopischer Literatur, Arkadi und Boris Strugazki, die kleine Erzählung Kerzen vor dem Pult (Свечи перед пультом): In dem aufwendigen Forschungsprojekt Die große Kodierung wird das Gehirn eines wissenschaftlichen Genies ausgelesen und in einer kristallinen Biomasse gespeichert. Alle waren sich klar darüber,

... daß die Große Kodierung den Weg zur Unsterblichkeit des menschlichen Ich darstellte, denn der Mensch, das waren ja nicht nur Arme und Beine, das war zuallererst sein Gedächtnis, die Fülle seiner Gewohnheiten und Vorstellungen, sein Hirn - das Hirn! ${ }^{614}$

Die Präservation der Gehirnmuster, die für 12.000 Jahre stabil wäre, würde es erlauben in den kommenden Jahrhunderten als neuen Träger ein künstliches, biologisches Gehirn zu entwickeln, das dann die Persönlichkeit des Genies absorbieren könnte. ${ }^{615}$

Etwas später als Franke, Lem und Strugazki publizierte der österreichische Künstler und Kybernetiker Oswald Wiener seine einflussreiche Dystopie die verbesserung von mitteleuropa, roman (1969), in dessen appendix $A$ er einen »bio-adapter« skizziert. Dieser sei die »vollständige lösung aller welt-probleme« und »die befreiung von philosophie durch technik. sein zweck ist es nämlich, die welt zu ersetzen«. ${ }^{616}$ Als »uterus« und »glücks-anzug« spiegelt er dem Menschen seine eigenen »lustimpulse (servo-narziss) « in einer vollkommenen, simulierten Welt vor. Erst die Liquidation des "preisgegebenen, nervös aktivierten und miserabel ausgerüsteten ... schleimklumpen, geschüttelt von Lebensangst und von todesfurcht versteinert« ${ }^{617}$ werde den Menschen zu einer souveränen

${ }^{613}$ Vgl. a.a.O., 298ff.

${ }^{614}$ Strugazki \& Strugazki 1989, 122.

${ }^{615}$ Vgl. a.a.O., 114-126. Die englische Ausgabe erschien 1978 als Noon: 22nd Century bei Macmillan. Die Strugazkis spitzen hier eine Idee zu, die 1929 bereits von Arthur Conan Doyle in der Erzählung The Disintegration Machine formuliert wurde. Ein Erfinder präsentiert hier eine Strahlenmaschine, die Objekte und Lebewesen materiell auflösen und wieder zusammensetzen kann. Es liegt jedoch noch kein Bezug zu einer Immortalisierungsidee vor. Vgl. Conan Doyle 1929; Clarke 1960, 72.

${ }^{616}$ Wiener 1969, CLXXV. Die entsprechende Vision Bernals wird in Wieners umfangreichem Literaturverzeichnis nicht aufgeführt.

617 Ebd. 
Einheit machen. Stück für Stück wird der Körper des Eingeschlossenen abgebaut und amputiert, bis auch die Zellen des Gehirns elektronisch verdrahtet werden: »das bewusstsein, dieses kuckucksei der natur, verdrängt also schliesslich die natur selbst« ${ }^{618}$ Wiener und mit ihm Franke setzen damit Bernals Vision einer körperlosen Gehirn-Existenz des zukünftigen Menschen dystopisch um.

Es war der amerikanische Journalist und Science-Fiction Autor Daniel Francis Galouye, der vermutlich als erster die Idee von computergenerierten Persönlichkeiten in einer totalen virtuellen Realität für das englischsprachige Lesepublikum aufgegriffen hat. In seinem erstmalig 1964 erschienenen Roman Simulacron 3 beschreibt Galouye eine Welt, in der die kommerzielle Marktforschung nichts mehr dem Zufall überlassen will. ${ }^{619} \mathrm{Um}$ die Reaktionen der Konsumenten auf bestimmte Produkte zu testen, entwickeln Wissenschaftler die detaillierte Simulation einer ganzen Stadt mit ca. 10.000 »Bewusstseinseinheiten«, die allesamt geregelt ihren alltäglichen Tätigkeiten nachgehen und grundsätzlich von der Realität ihrer Welt überzeugt sind. ${ }^{620}$ Die Katastrophe nimmt ihren Lauf, als Douglas Hall, einer der führenden Ingenieure dieses kybernetischen Marktforschungsunternehmens, beginnt, an der Realität seiner eigenen Welt zu zweifeln. Zwar muss Hall schließlich erkennen, dass auch er nur eine künstliche Person in einer virtuellen Welt ist, jedoch gelingt es ihm, durch die Liebe zu einer Frau die vermeintlich reale Wirklichkeitsebene zu erreichen. Der deutsche Regisseur Rainer Werner Fassbinder hatte diese Vision Galouyes in seinem zweiteiligen Fernsehfilm Welt am Draht von 1973 in äußerst bedrückender Weise inszeniert und damit wohl zum ersten Mal im deutschsprachigen Raum diese Idee einem breiteren Publikum zugänglich gemacht. ${ }^{621} 1999$ verwirklichte John Rusnak die erste Kinoverfilmung des Stoffes unter dem Titel The 13th Floor. ${ }^{622}$

${ }^{618}$ Wiener 1969, CLXXXIII; Tabbert 2004, 375-403. Die Arbeiten des Kybernetikers Norbert Wiener sind Oswald Wiener wohlbekannt. Vgl. Wiener 1969, CCVI.

${ }^{619}$ Der Roman erschien 1964 bei Bantam (NY) unter dem Titel Simulacron 3, wurde in der englischen Ausgabe des gleichen Jahres beim Verlag Victor Gollancz (London) aber als Counterfeit World vermarktet, während die deutsche Ausgabe von 1983 (Heyne, München) mit Simulacron-drei betitelt wurde. Nach der Neuverfilmung als The Thirteenth Floor von 1999 erschien das Buch unter dem gleichnamigen englischen Titel auch in deutscher Sprache (Galouye 1999).

${ }^{620}$ Das entspricht auch Tiplers Beispiel: »Suppose we try to simulate a city full of people ... « Tipler 1995, 206.

${ }^{621}$ Vgl. Fassbinder 1973.

${ }^{622}$ Vgl. Rusnak 1999. 
Das erste Mal jedoch, dass im englischsprachigen Kontext explizit die Idee der Unsterblichkeit in der Virtualität in Erscheinung trat, war im Jahr 1966 in der 9. Folge der Fernsehserie Star Trek mit dem Titel What are Little Girls Made of? (dt.: Der alte Traum), deren Drehbuch der bekannte Schriftsteller und Drehbuchautor Robert Bloch (Psycho) verfasst hatte. Auf dem Planeten Exo III trifft Captain James T. Kirk auf den Wissenschaftler Dr. Roger Korby, der nach einer schweren Krankheit seine Persönlichkeit, seinen Geist, in das Computergehirn eines Androiden einscannte und auf diese Weise Unsterblichkeit erlangte. ${ }^{623} \mathrm{Die}$ Star Trek-Folge What are Little Girls Made of? stellt daher mit erheblicher Breitenwirkung das erste Auftreten der virtuellen Unsterblichkeitsidee im Ursprungsland des Posthumanismus dar. ${ }^{624}$

Während bereits in Bernard Wolfes Roman Limbo (1952) das Motiv der Prothesenmenschen in die Science-Fiction Literatur eingeführt wurde und James Triptree Jr. (d.i. Alice Sheldon) in der Kurzgeschichte The Girl who was plugged in (1973) das Thema technisch veränderbarer Identitäten ausbreitete, bildete sich erst Anfang der 1980er Jahre die postmoderne Cyberpunk-Literatur. ${ }^{625}$ Die für den Cyberpunk kennzeichnenden Elemente wie der Einsatz von technischen und chemischen Mitteln (Gentechnik, Prothesen, Drogen) zur Erweiterung der menschlichen Leistungsfähigkeit implizieren über ähnliche Verfahren auch die Loslösung des menschlichen Geistes vom Körper (disembodiment) und die damit verbundene Transferierung in andere Körper oder in die virtuelle Realität eines Computerspeichers. Obwohl schon der Schriftsteller und Mathematiker Rudy Rucker (d. i. Rudolf von Bitter Rucker) in seinen Romanen Software (1982) und Wetware (1988) diese Art der technischen »Geist-Übertragung « teils mit einhergehender Immortalisierung schilderte, sollte erst William Ford Gibson mit seiner Neuromancer-Trilogie einem breiten Lesepublikum die Idee der virtuellen Realität vermitteln. Gibson knüpft in seinen Romanen Neuromancer (1984), Count Zero (1986) und Mona Lisa Overdrive (1988) nicht nur an Galouyes Vorstellung einer neuronalen Mensch-Computer-Schnittstelle an, deren technische Aspek-

${ }^{623}$ Vgl. Bloch 1966 .

${ }^{624}$ Die Idee einer wissenschaftlich ermöglichten Unsterblichkeit wird zwar in mehreren Science-Fiction-Geschichten der 1960er Jahre thematisiert, jedoch meist im Hinblick auf eine unermessliche Lebensverlängerung durch die Medizin bzw. Drogen. Vgl. hierzu Yoke \& Hassler 1985.

${ }^{625}$ Den Begriff Cyberpunk prägte Bruce Bethke in seiner gleichnamigen Kurzgeschichte. $\mathrm{Zu}$ den Vorläufern des Cyberpunk wie Bernard Wolfe und Philip K. Dick vgl. Hayles 1999, 113-130, 160-191. 
te bei Galouye jedoch nicht weiter ausgeführt wurden, sondern er kreiert auch den Begriff Cyberspace als Bezeichnung für den virtuellen Raum, den die Computertechnologie eröffnet. ${ }^{626}$ Verbunden mit dem Speicher eines Computers erlebt die Hauptfigur aus Neuromancer, Case, die Welt als materialisierte Information, als reine Repräsentation der Matrix:

Get just wasted enough, find yourself in some desperate but strangerly arbitrary kind of trouble, and it was possible to see Ninsei as a field of data, the way the matrix had once reminded him of proteins linking to distinguish cell specialities. Then you could throw yourself into a highspeed drift and skid, totally engaged but set apart from it all, and all around you the dance of biz, information interacting, data made flesh in the mazes of the black market ... ${ }^{627}$

Während Case nur zeitweise Erfahrungen im Cyberspace sammelt, haben andere menschliche Akteure wie Dixie Flatline die virtuelle Existenz im Computer ihrem realen, materiellen Körper vorgezogen, so dass viele Transhumanisten und teils auch die Sekundär-Literatur zur Science-Fiction William Gibson als den Entdecker der virtuellen Realität ansehen. ${ }^{628}$

Eine innovative Variante spielt der schottische Autor Philip Kerr in seinem postapokalyptischen Szenario The Second Angel (1998) durch. Nicht Menschen oder deren Geist werden in einen Computer geladen, sondern Quantencomputer von Nanometergröße werden hier durch einen übermächtigen Computer namens Descartes in das menschliche Blut eingeschleust und verschmelzen mit der menschlichen Persönlichkeit. Mit Hilfe dieser widerstandsfähigen Mensch-Maschinen-Hybride will Descartes den Weltraum erforschen. ${ }^{629}$

Populär wurden diese Ideen - zum Teil schon vor der Cyberpunk Movement - durch Spielfilme, in denen Menschen sich in der meist computergenerierten virtuellen Realität befinden oder gefangen werden. Bereits 1976 wird Welcome to Blood City (dt.: Willkommen in dieser blutigen Stadt) produziert, 1982 folgen Brainstorm (dt.: Projekt Brainstorm) und der ästhetisch wohl anspruchsvollste Film über virtuelle Welten, Tron, von Steven Lisberger und dessen Fortsetzung Tron Legacy von Joseph Kos-

\footnotetext{
${ }^{626}$ Vgl. Gibson 2000, 1986, 1988; Nicholls 1999a, 1999b. Zur herausragenden Rolle von Ruckers Roman Software vgl. Hassler 1985, 4ff.

${ }^{627}$ Gibson 2000, 26.

${ }^{628}$ Vgl. dazu Hayles 1999, 32-35; Flessner 1996; Tabbert 2004, 483-522.

${ }^{629}$ V gl. Kerr 1998; Ahn 2001, 331-337.
} 
inski (2010). ${ }^{630}$ In dem Film Tron wird der Programmierer Flynn in das Innere eines Computers eingescannt und muss nach dem Muster von damals beliebten Videospielen gegen das diktatorische Master Control Programm kämpfen, während in Welcome to Blood City, Brainstorm und in dem 1991 entstandenen Film The Lawnmower Man (dt.: Der Rasenmäher-Mann) die Möglichkeiten der virtuellen Realität vorrangig dafür eingesetzt werden sollen, Soldaten bzw. Affen als perfekte Killermaschinen zu trainieren. ${ }^{631}$ Neben den bedrohlichen Potentialen der virtuellen Realität werden in dem Film The Lawnmower Man, der auf einer Kurzgeschichte des Schriftstellers Stephen King beruht, auch positive Aspekte in Szene gesetzt: Ein zurückgebliebener Gärtner wandelt sich binnen kurzem durch die Erfahrungen von Freiheit und Selbstbewusstsein im Cyberspace zu einem intelligenten und charmanten jungen Mann.

Die 1966 veröffentlichte Kurzgeschichte We Can Remember It for Your Wholesale von Philip Kindred Dick, dessen Werke für das häufig auf Drogen basierende Spiel mit alternativen Realitäten berühmt sind, verfilmte Paul Verhoeven 1990 unter dem Titel Total Recall (dt.: Die totale Erinnerung - Total Recall) und erreichte mit seiner mit Gewalt-Exzessen gespickten Inszenierung ein Massenpublikum. Erzählt wird die Geschichte eines vermeintlichen Bauarbeiters, dessen Erlebnisse und Erinnerungen im Interesse einer zwielichtigen Regierung durch Implantate manipuliert werden. ${ }^{632}$

In dem 1997 produzierten Film Abre los Ojos (dt.: Öffne die Augen) entfaltet Alejandro Amenábar eine surrealistische Geschichte um einen jungen Mann, der mit Hilfe der Kryonik-Firma Life-Extension in einer virtuellen Scheinwelt lebt. ${ }^{633} 1999$ thematisierten neben dem zuvor erwähnten The $13^{\text {th }}$ Floor noch zwei weitere US-Spielfilme die Auflösung der Wirklichkeit durch die medialen Möglichkeiten der Simulation von Realität. Andy und Larry Wachowski (heute Lana und Lilly Wachowski) konnten mit ihrem Film The Matrix (dt.: Matrix) und seinen Weiterführungen, in dem intelligente Maschinenwesen die Menschheit mit Hilfe der VR-Technik versklavt haben, über 15 Millionen Kinobesucher faszinieren. In einem Begeisterungssturm feierte die Filmkritik die spannende Inszenierung anthropologischer Grundfragen, die großzügig mit postmodernen und

\footnotetext{
${ }^{630}$ Vgl. Sasdy 1976; Trumbull 1982; Lisberger 1982; Kosinski 2010.

${ }^{631}$ Vgl. Leonard \& Everett 1991.

${ }^{632}$ Vgl. Verhoeven 1990.

${ }^{633}$ Vgl. Amenábar 1997.
} 
religiösen Versatzstücken angereichert war. ${ }^{634}$ Dagegen beschränkte sich David Cronenberg mit seiner Schöpfung eXistenZ darauf, ein verwirrendes (Computer-)Spiel verunsichernder realer und künstlicher Wirklichkeiten zu inszenieren. ${ }^{635}$

Zentral wird die gesamte Idee der virtuellen Immortalisierung in dem 2002 veröffentlichten Cyberpunk-Roman Altered Carbon von Richard Morgan, der seit 2018 kreativ als Netflix-Serie weitergeführt wird. Das persönliche Bewusstsein kann hier auf einem Chip gespeichert werden und wieder in einen oder mehrere Körper transferiert werden. Die wohlhabenden "Meths" (von Methusalem) dieser dystopischen Welt können sich stets neue und vollkommenere Körper aneignen und sind dadurch praktisch unsterblich. Der endgültige Tod droht dennoch, falls sowohl der Körper (sleeve) als auch der Bewusstseinschip (stack) ausgelöscht werden, z.B. durch einen Angriff mit Computerviren. ${ }^{636}$ Beachtenswert ist mit ähnlichem sozialkritischen Motiv auch der Film Transfer (2010) nach einer Kurzgeschichte der spanischen Autorin Elia Barceló von 2004. Hier ist es ein wohlhabendes, deutsches Rentnerehepaar, das seinen Geist mit Hilfe des Unternehmens Menzana in die Körper zweier junger und schöner Menschen aus Afrika transferieren lässt. Nur wenige Stunden täglich wird den verarmten Spendern der Körper mit ihrem alten Bewusstsein gewährt. Im sich anbahnenden Konflikt zwischen den vier Persönlichkeiten eliminiert am Ende Menzana jedes Aufbegehren gegen die schöne neue Welt. ${ }^{637}$

Selbst wenn man Jules Vernes frühen Einfall aus dem Karpathenschlo $\beta$, einen Menschen mit Hilfe eines Phonographen und einer Bildreproduktion (für den Betrachter) unsterblich zu machen, außer Acht lässt, so muss festgestellt werden, dass die Idee einer medientechnischen Simulation des Menschen zur Verwirklichung des ewigen Lebens, beginnend mit Clarkes und Lems Erzählungen, mehr als ein halbes Jahrhundert alt ist. Auch dem weniger aufmerksamen Leser wird jedoch bei der Lektüre posthumanistischer Werke auffallen, dass weder Frank Tipler, Hans Moravec und Marvin Minsky noch Ray Kurzweil in ihren Monographien Vertreter des Cyberpunk, wie William Gibson, oder entsprechende Verfilmungen auch nur erwähnen. Die Idee eines computergestützten Auf-

\footnotetext{
${ }^{634}$ Vgl. Wachowski \& Wachowski 1999.

${ }^{635}$ Vgl. Cronenberg 1999.

${ }^{636}$ Vgl. Morgan 2002, dt. Das Unsterblichkeitsprogramm (München 2017); Kalogridis 2018 (Netflix-Serie).

${ }^{637}$ Vgl. Lukacevic 2010.
} 
erstehungsmechanismus schreibt Tipler sich selbst, Hans Moravec und dem populären Harvard-Philosophen Robert Nozick zu. ${ }^{638}$ Letzterer zeichnete mit Bezug auf Science-Fiction Phantasien tatsächlich bloß die düstere Vision einer Unsterblichkeit im Computer ${ }^{639}$ und wurde dank Tiplers »Vereinnahmungsversuch « allerdings in der Sekundärliteratur prompt als ein weiterer Verfechter der virtuellen Unsterblichkeit rezipiert. ${ }^{640}$ Die Vermutung, dass die Seriosität der posthumanistischen Visionen, die sich ausschließlich als Ergebnis wissenschaftlicher Extrapolationen präsentieren, nicht durch den Verweis auf vermeintlich »unseriöse« Science-Fiction Literatur untergraben werden soll, hat sicherlich ihre Berechtigung. Eine weitergehende Reflexion über die posthumanistischen Rezeptionsmuster erscheint jedoch vielversprechend, denn dass die posthumanistischen Autoren überhaupt Virtualitätsvorstellungen der Science-Fiction wahrgenommen haben, belegt allein schon die mitunter inflationäre Verwendung des Begriffes Cyberspace bei Hans Moravec und Frank Tipler. ${ }^{641}$

Ein resümierender Überblick über die sozialen, philosophischen und technischen Kontexte und Bewertungen einer Existenz in der virtuellen Realität in der besprochenen Science-Fiction Literatur und in Science-Fiction Filmen gestaltet sich in seiner Kritik der virtuellen Realität überraschend einheitlich. Das virtuelle Dasein erscheint in der Literatur am häufigsten als Mittel der Manipulation - als Kontrollinstrument von herrschenden Maschinen (Tron, The Matrix), Marktforschern (Simulacron-3 / Welt am Draht etc.) oder Militärs bzw. Regierungen, die Menschen oder Affen zu perfekten Tötungsmaschinen ausbilden (Welcome to Blood City, Brainstorm, The Lawnmower Man) oder einsetzen wollen (We Can Remember It for Your Wholesale / Total Recall). Auch im Cyberpunk werden die Ausflüge in die virtuelle Realität gewöhnlich nicht als paradiesische Alternative charakterisiert, sondern als bittere Notwendigkeit im Kontext von Datenkriminalität und Cyberterrorismus. Die Protagonisten in Cyberpunk-Romanen sind meist gesellschaftliche Außenseiter, aggressiv, entfremdet, drogenabhängig, beziehungsunfähig und leiden teils unter identitätsverunsichernden Bewusstseinsveränderungen und gravierenden Gehirnschäden. Dort, wo explizit die Virtualität als Verwirklichung der Unsterblichkeit thematisiert wird, erfährt diese Idee

\footnotetext{
${ }^{638}$ Vgl. Tipler 1995, 16 f.

${ }^{639}$ Vgl. Nozick 1991, $25 \mathrm{f}$.

${ }^{640}$ Vgl. Rottensteiner 1997, 367.

${ }^{641}$ Vgl. Moravec 1999, 164-168, 171f.; Tipler 1995, 108f., 220.
} 
vorwiegend Ablehnung: Lems Philosophen kommen zu dem Schluss, dass eine simulationstechnisch realisierte Kontinuität der Persönlichkeit nicht möglich ist (Dialoge); der Kosmonaut Ijon Tichy flieht vor den im Paket angelieferten »Notfallkopien« seiner selbst (Vierzehnte Reise) und der in einen Roboter transferierte Geist des Dr. Korby hat seine Menschlichkeit verloren und entwickelt sich zum skrupellosen Mörder (What are Little Girls Made of?). Herbert Frankes und Oswald Wieners Dystopien von vollkommen virtualisierten Kulturen (Der Orchideenkäfig, Zone Null bzw. Der Glücksanzug) können als direkte Kritik gegenwärtiger Mediatisierungstendenzen verstanden werden. Lediglich The Lawnmower Man betont neben der militärischen Anwendung auch den Einsatz der virtuellen Realität für die persönliche Weiterentwicklung.

Das existentielle Unbehagen als philosophische Konsequenz der Überlegungen zu einer technischen Simulation der Welt, das besonders in Simulacron-3 / Welt am Draht und The Matrix dramatisiert wird, beschäftigt unter den Posthumanisten nur Frank Tipler, der jedoch eine einfache Antwort auf die ontologische Problematik bereithält: »How do we know we ourselves are not merely a simulation inside a gigantic computer? Obviously, we can't know. But obviously we ourselves really exist. « ${ }^{642}$ Erstaunlicherweise erscheint in den populären, das heißt verfilmten Fiktionen des Gefangenseins in der künstlichen Welt am virtuellen Horizont etwas zutiefst Menschliches als Motivation und Zielpunkt der Befreiung aus der Virtualität: Die durch den finalen, dornröschengleichen Kuss der Geliebten symbolisierte Liebe überwindet die Künstlichkeit der simulierten Welten (Simulacron-3 / Welt am Draht, The Matrix). Der virtuellen Scheinwelt wird als romantisches Urmotiv etwas menschlich Echtes entgegengesetzt.

Der gesamte problemorientierte Aspekt einer Existenz in der Virtualität wird im posthumanistischen Diskurs vollkommen ausgeblendet. Soziale, philosophische und politische Risiken, wie sie in den Szenarien der Science-Fiction durchgespielt wurden, beschränken sich im posthumanistischen Diskurs auf die kindlich-naive Schilderung einer ungeheuren Leistungssteigerung auf allen Gebieten des menschlichen Schaffens. Dass eine zunehmende Einbindung des Menschen in den Cyberspace eventuell mit globalen Abhängigkeiten von Computernetzen und monopolisierten Konzernen, autokratischen Herrschaftssystemen und wei-

${ }^{642}$ Tipler 1995, 207. Vgl. hierzu auch Lem 1981, 332-336. 
testgehender Manipulation für politische oder kommerzielle Zwecke einhergeht - das alles thematisiert der Posthumanismus mitnichten.

\subsubsection{Kryonik}

Die Kryonik ist der bisher einzige Zweig des Trans- und Posthumanismus, der im Bemühen um die Unsterblichkeit gravierende praktische Konsequenzen hat. Andererseits verdeutlicht die Kryonik präzise die Vorstellungen zu Tod, Leben und persönlicher Identität, wie wir sie in den vorangegangenen Kapiteln dargelegt haben. Kultur- und religionsgeschichtlich betrachtet, liegt der Gedanke nicht fern, dem menschlichen Körper auch in seinem postmortalen Zustand Pflege und Fürsorge zu Teil werden zu lassen. In der Hoffnung auf eine wie auch immer geartete Kontinuität des Lebens oder eine Auferstehung wurden teils erhebliche Anstrengungen unternommen, um ein Fortleben zu sichern. Man denke bspw. an die Pyramiden und Grabkammern des Alten Ägypten. In der Kryonik verschmilzt die traditionelle Sorge um den Fortbestand des Leichnams mit der Hoffnung auf die medizinisch-technische Kontrolle des Todes selbst. Dabei werden fiktionale Vorstellungswelten fortgesetzt, wie sie seit Ende des 19. Jahrhunderts in der Science-Fiction Literatur verbreitet waren.

Schon 1887 entwickelte der amerikanische Autor, Entdecker und Abenteurer William Clark Russell in seinem Roman The Frozen Pirate die Idee eines Menschen, der zufällig in einem Eisblock eingefroren wurde: Als einziger Überlebender eines Sturmes entdeckt demnach ein Matrose ein altes Schiff, das in einem Eisberg eingeschlossen war. An Bord findet er einige eingefrorene Besatzungsmitglieder. Nahe des Lagerfeuers erwacht einer der Seemänner und erlangt mit der Hilfe des Matrosen sein volles Bewusstsein wieder. Er verfällt jedoch dem Wahnsinn, da er nicht wahrhaben will, dass er fünfzig Jahre lang im Eis "geschlafen« habe, und beginnt, rasend schnell zu altern. Zwei Jahre nach Russells literarischer Innovation publizierte der populäre französische Schriftsteller Louis Boussenard dann seinen Roman Dix mille ans dans un bloc de glace. Hier erwacht ein Mensch der Gegenwart durch einen ähnlichen Unfall in der fernen Zukunft. ${ }^{643}$

${ }^{643}$ Vgl. Russell 1974; Boussenard 1890 (engl. Ausgabe: 10,000 Years in a Block of Ice, 1898). 
In der Folgezeit veröffentlichte der amerikanische Autor und Schöpfer der Tarzan-Figur, Edgar Rice Burroughs, die satirische Erzählung The Ressurection of Jimber Jaw (1937), in der ein prähistorischer Mann in der heutigen Zivilisation wiederbelebt wird und zahlreiche Abenteuer bewältigen muss. In den 1930er Jahren erschienen die ersten Kryonikgeschichten in den neuen pulp fiction magazines, die besonders unter den amerikanischen Jugendlichen gefragt waren. In seiner Kurzgeschichte Armageddon 2419 schickt der amerikanische Fantasy-Autor Philip Francis Nowlan seinen Helden mittels kryonischem Tiefschlaf in das 25. Jahrhundert, während Neil Ronald Jones seinen unerschütterlichen Professor Jameson für einige Millionen Jahre tiefgefroren in den Erdorbit verbannt, bis dieser von Außerirdischen wiederbelebt wird. Angeregt durch Jones' Erzählung verfasste 1948 auch der spätere Gründervater der Kryonik, Robert Ettinger, die Kurzgeschichte The Penultimate Trump für das Magazin Startling Stories - zwei Helden werden hier für eine Zeitreise eingefroren. ${ }^{644}$

In den frühen Geschichten war das zufällige Einfrieren und Erwachen von Menschen lediglich ein weiteres dramatisches Mittel für das beliebte Motiv der Zeitreisen in einer sozialen Umgebung, die von großen gesellschaftlichen, kulturellen und technischen Veränderungen geprägt war. Nachdem die eigentliche kryonische Bewegung in den 1960er Jahren entstanden war, nahm sich auch das Filmgenre des Themas an. Weithin bekannt sind vor allem der französische Film Hibernatus von 1969 mit Louis de Funès und Woody Allens Sleeper von 1973. Beide Komödien variieren das Thema eines Menschen aus der Vergangenheit, der in der fremdartigen, gegenwärtigen bzw. zukünftigen Welt wiedererwacht. In der Liebesgeschichte Forever Young spielt Mel Gibson einen Testpiloten, der 1939 eingefroren und vergessen wird und im Jahr 1992 wieder zum Leben erwacht. Schließlich mimt Sylvester Stallone einen eingefrorenen Superagenten in dem Blockbuster Demolition Man (1993), der seinen Erzfeind - der Titel legt es nahe - aufs Neue überwältigen muss. Vier Jahre später erschien mit Austin Powers - International Man of Mystery die erfolgreiche Parodie von Stallones Film. ${ }^{645}$

Was diese Science-Fiction Ideen von den Vorstellungen der kryonischen Bewegung unterscheidet, ist - abgesehen von der Normativität der Zukunftsvisionen - ihr Verhältnis zur Unsterblichkeit. Von Beginn

${ }^{644}$ Vgl. Burroughs 1937; Nowlan 1928; Jones 1931; Ettinger 1948; Stableford 1999a. ${ }^{645}$ Vgl. Molinaro 1969; Allen 1973; Miner 1992; Brambilla 1993; Roach 1997. 
an verfolgte die Idee der Kryonik nicht das Ziel, Menschen für Zeitreisen einzufrieren, sondern das Leben um eine unbegrenzte Zeitspanne zu verlängern. Die Anhänger der Kryonik sind fest davon überzeugt, dass eine Gesellschaft, die fähig sein wird, Menschen aus dem »kryonischen Tiefschlaf« zu erwecken, ebenfalls über eine außerordentlich fortgeschrittene Medizin verfügen wird, so dass in dieser Zukunft überhaupt niemand mehr sterben müsse. All die negativen Begleiterscheinungen, die in der Science-Fiction durchgespielt werden - wie die kulturelle Orientierungslosigkeit, Wahnsinn oder die ökonomische Ausbeutung der eingefrorenen Menschen - werden in der Kryonik vollkommen ausgeblendet. Nur die technische Idee in Verbindung mit der optimistischen Überzeugung eines beschleunigten Fortschrittsprozesses wird von den kryonischen Denkern rezipiert.

Was steht am Anfang der Kryonik? Gemäß dem Profil des Cryonics Institute in Michigan und seinem Gründer und langjährigen Direktor, Robert Ettinger, beginnt die Geschichte der Kryonik wie folgt:

The cryonics movement began in 1962 with the private publication of the first version of my first book, > The Prospect of Immortality.< It gained more attention when Doubleday published the first of several successful commercial editions, including several in foreign languages; and with the publication of my next book, >Man Into Superman<. ${ }^{646}$

Ettingers Kurzbiographie, die auf derselben Webseite des Cryonics Institute präsentiert wird, wies in einer früheren Version denn auch starke hagiographische Elemente auf, die typisch für moderne amerikanische Helden sind: Ettinger wurde demnach 1918 als Großneffe eines Dirigenten des kaiserlichen Moskauer Symphonieorchesters geboren, durch Anheirat war er auch Neffe des bekannten Jazzmusikers Pee Wee Russell. Als Kind entdeckte er bereits die erwähnte Science-Fiction Geschichte von Neil R. Jones, die sein Denken in den kommenden Dekaden prägen sollte. Nach ernsthaften Verletzungen, die er sich während des zweiten Weltkrieges zuzog, verbrachte er mehrere Jahre in Kliniken: »Ettinger used the time not only to recuperate, but also to read and think. « ${ }^{647}$ In dieser Zeit entdeckte er die Arbeiten von Jean Rostand, der in seinen Experimenten erfolgreich das Sperma von Fröschen ein-

\footnotetext{
${ }^{646}$ Vgl. Robert C. Ettinger, »Cryonics Institute. A brief History and Overview« (2002), verfügbar am 10.01.2011, unter: http://www.cryonics.org.

${ }^{647}$ Cryonics Institute, »Directors of the Cryonics Institute« (2003), verfügbar am 10.01.2011, unter: http://www.cryonics.org/bio.html\#Robert_Ettinger.
} 
gefroren hatte. Nach seiner Genesung wurde Ettinger Dozent für Physik und Mathematik an einem Community College in Michigan und verfasste 1962 schließlich sein maßgebliches Buch Prospects of Immortality, das er zunächst an Freunde und bekannte Persönlichkeiten aus Who's Who versandte. Das Buch wurde 1964 bei Doubleday verlegt und in acht weitere Sprachen übersetzt. Ettinger selbst wurde Mitte der 1960er zum Medienstar, dessen Ideen in vielen internationalen Zeitschriften und im amerikanischen Fernsehen diskutiert wurden. Angeregt durch Ettingers Buch wurden eine Reihe von kryonischen Organisationen gegründet: »Cryonics had begun as Robert Ettinger's idea. It had become a reality. $" 648$

In dieser Geschichte der Kryonik verschweigt Ettinger allerdings, dass es noch einen anderen Pionier der Bewegung gegeben hat: »But there was another man and another book and he made a contribution every bit as great as Ettinger's and perhaps in his quiet, unassuming way he may have made a more significant one. ${ }^{649}$

Evan Cooper (1926-1983) hatte 1962 sein Manuskript Immortality: Physically, Scientifically, Now privat publiziert und Kopien unter seinen Freunden verteilt. In diesem Buch kombiniert er die Idee des Einfrierens und der Wiederbelebung von Menschen mit einer kybernetischen Definition der menschlichen Identität. Mit Bezug auf den Kybernetiker Norbert Wiener versteht Cooper ein menschliches Wesen als nichts anderes als ein Computerprogramm, das jederzeit wiederhergestellt werden könne, so lange noch ausreichend Informationen über das Original vorhanden seien. Im Dezember 1963 gründeten Cooper und andere Unsterblichkeits-Enthusiasten die Life Extension Society (LES) in Washington D.C. mit Cooper als Präsidenten. Allerdings verließ Cooper die Bewegung fünf Jahre später, und die LES löste sich kurze Zeit danach auf. Sein Engagement für die Kryonik jedoch - Cooper initiierte jährliche Konferenzen und die Zeitschrift Freeze - Wait - Reanimate - ermutigte auch weitere Aktivisten in den Vereinigten Staaten. So gründeten im August 1965 Saul Kent, Karl Werner, Harry Costello und Curtis Henderson die Cryonics Society of New York (CSNY), wobei nicht unerwähnt bleiben sollte, dass es der junge Kunststudent Karl Werner war, der kurz vor der

${ }^{648} \mathrm{Ebd}$.

${ }^{649}$ Alcor 1983, 7. Es scheint kein Zufall zu sein, dass Ettingers kommerzieller Konkurrent, die Alcor Life Extension Foundation, so ausführlich Coopers Nachruf publiziert hat. 
Gründung der CSNY den Neologismus cryonics kreierte (abgeleitet vom griechischen $\kappa \rho \dot{o} \varsigma=$ Eis) ${ }^{650}$

Während einer groß angelegten Informationsreise, die Saul Kent und Curtis Henderson 1966 quer durch die Vereinigten Staaten führte, wurden die Cryonics Society of Michigan mit Ettinger und die Cryonics Society of California mit Robert Nelson als Präsidenten gegründet. ${ }^{651}$ Ettingers Gruppe änderte ihren Namen in der Folgezeit noch mehrere Male Immortalist Society und Cryonics Association - und wurde schließlich zum Cryonics Institute in Clinton, Michigan. 1972 wurde die Alcor Life Extension Foundation von Fred und Linda Chamberlain ins Leben gerufen, die inzwischen in Scottsdale (Arizona) angesiedelt ist. Der Höhepunkt der Bewegung war um 1970 erreicht. ${ }^{652}$

Die kryonische Bewegung war und ist sehr heterogen und basiert auf der dominierenden Rolle einzelner Protagonisten. In ihren soziologischen Interviews mit Kryonikern in den frühen 1970ern haben Clifton Bryant und William Snizek festgestellt, dass die Anhänger durchweg von dem starken Bedürfnis angetrieben sind, ihr Leben vollkommen zu kontrollieren. Ein ungewöhnlich hoher Anteil unter den Kryonikern verfügte über eigene Luftschutzkeller und flog eigene Flugzeuge - eine Tatsache, die Bryant und Snizek darauf zurückführen, dass Kryoniker die Verantwortung für ihr Leben nicht gerne in die Hände anderer legen. Die einzige quantitative Erhebung, die in den 1970er Jahren von Ellen B. Rievman durchgeführt wurde, zeigte, dass Kryoniker in überwiegendem Maße männlich, weiß und ca. Mitte 30 Jahre alt sind und in der Regel ohne religiöse Bindung und politisch unkonventionell leben. Sie verfügen über ein höheres Einkommen, über höhere Bildung, aber auch über eine stärker ausgeprägte Todesangst als der US-amerikanische Durchschnitt. ${ }^{653}$

${ }^{650}$ Karl Werner, der seinerzeit Vizepräsident der New Yorker Cryonics Society war, verließ die Bewegung nur drei Jahre später, um eine längere Zeit bei der Church of Scientology zu verbringen. Er kehrte allerdings nicht mehr zurück zur Kryonik. Vgl. Kent 1983, 4f.; Perry 2000, 38-39.

${ }^{651}$ Daneben gab es Mitte der 1960er Jahre in den USA noch weitere, meist kurzlebige Unsterblichkeitsgruppen: The Immortality Records and Compilation Association in Panorama City (CA); die Society for Anabiosis in New York, die Cryonics Society of Illinois und die Cryonics Society of South Florida. Vgl. Kent 1983, 4; Rievman 1976, VIII, 4; Sheskin 1979, Appendix D.

${ }^{652}$ Vgl. Bryant \& Snizek 1973, 57-62; Mann 1981, 10.

${ }^{653}$ Vgl. Bryant \& Snizek 1973, 59; Rievman 1976, 68-89. 
Die ehrgeizige Cryonics Society of New York und andere kommerzielle Anbieter konnten ihre Vorhaben langfristig nicht finanzieren. Die Cryonics Society of California, die in den späten 1960er Jahren die ersten »kryonischen Suspensionen « vorgenommen hatte, war finanziell ruiniert, nachdem neun Leichen aufgetaut waren und einige Verwandte den Präsidenten der Vereinigung, Robert Nelson, und einen Assistenten verklagten. Sie wurden dazu verurteilt, eine Million Dollar Schadensersatz zu zahlen. Nach einer langen Phase des öffentlichen Desinteresses haben die amerikanischen Massenmedien erst wieder über die Kryonik berichtet, als der Baseballstar Ted Williams 2002 starb und nach Wochen gerichtlicher Auseinandersetzung zwischen seinen Kindern schließlich doch kryonisch suspendiert wurde. ${ }^{654}$ Nur das Cryonics Institute und die Alcor Life Foundation bestehen bis heute. Der Transhumanist Max More ist seit 2011 Präsident und CEO von Alcor, der Autor R. Michael Perry ist dort Patientenbetreuer und der Nanotechnologe Ralph Merkle sowie Aubrey de Grey und Martine Rothblatt gehören zum wissenschaftlichen Beirat. Seit 2005 bietet die Firma KrioRus in Russland, die aus der transhumanistischen Bewegung hervorging, ebenfalls kryonische Suspensionen für Menschen an. ${ }^{655}$

Um die kryonische Bewegung zu begreifen, ist es notwendig, zunächst ihr besonderes Todesverständnis zu betrachten. Das für die Kryonik grundlegende Buch Prospects of Immortality (1964) von Robert Ettinger beginnt mit einer enthusiastischen und pragmatischen Präambel:

Most of us now living have a chance for personal, physical immortality. This remarkable proposition - which may soon become a pivot of personal and national life - is easily understood by joining one established fact to one reasonable assumption.

The fact: At very low temperatures it is possible, right now, to preserve dead people with essentially no deterioration, indefinitely ...

The assumption: If civilization endures, medical science should eventually be able to repair almost any damage to the human body, including freezing damage and senile debility or other cause of death ... Hence, we need only arrange to have our bodies, after we die, stored in suitable freezers against the time when science may be able to help us. No matter what kills us, whether old age or disease, and even if freezing techniques are still crude when we die, sooner or later our friends

${ }^{654}$ Die Cryonics Society of California hatte es zugelassen, dass neun der Leichen in ihrer Obhut auftauten, ohne jedoch die Verwandten zu informieren, die noch lange Zeit die Wartungskosten bezahlten. Als der Skandal aufgedeckt wurde, waren die Toten schon weitgehend verwest. Vgl. Perry 2000, 39-40; Oliver 1981.

${ }^{655}$ Vgl. Bernstein 2015, 768f.; http://kriorus.ru/en. 
of the future should be equal to the task of reviving and curing us. This is the essence of the main argument. ${ }^{656}$

Ettinger schätzte das Einfrieren als ein Mittel, um den unwiderruflichen Tod eines Lebewesens zu verhindern, da Menschen seiner Ansicht nach in kaum wahrnehmbaren Stufen sterben würden. So sei die Frage nach der Unumkehrbarkeit des Todes lediglich vom medizinischen Fortschritt einer Gesellschaft abhängig - der klinische Tod sei oftmals schon überwunden worden und im Prinzip behandelbar, was die häufigen Erfolge von Reanimationen belegen würden. Künstliche Prothesen und Organe und die Fortschritte der Transplantationstechnik werden Ettinger zufolge diesen Reparatur- und Vervollkommnungsprozess des menschlichen Körpers noch beschleunigen. ${ }^{657}$

Suspended death, then, will refer to the condition of a biologically dead body which has been frozen and stored at a very low temperature, so that degeneration is arrested and not progressive. The body can be thought of as dead, but not very dead; it cannot be revived by present methods, but the condition of most of the cells may not differ too greatly from that in life. ${ }^{658}$

Den unwiderruflichen Tod (permanent death) definiert Ettinger als $\mathrm{Zu}-$ stand, in dem das Gehirn als Sitz der menschlichen Identität so schwer beschädigt sei, dass es nicht mehr wiederhergestellt werden könne. Mit der Entdeckung der Kryonik erwartet die Menschheit nach Ansicht Ettingers ein neues goldenes Zeitalter und auf der individuellen Ebene die Aussicht auf ein Leben mit einem vollkommenen, verjüngten Körper:

The freezer program represents for us now living a bridge to an anticipated Golden Age, when we shall be reanimated to become supermen with indefinite life spans ... You and I, the frozen, the resuscitated, will be not merely revived and cured, but enlarged and improved, made fit to work, play, and perhaps fight, on a grand scale and in a grand style. ${ }^{659}$

Diese Verbindung zwischen einer Vision der Auferstehung und eines paradiesischen Lebens im »Jenseits « (das eigentlich eine Fortsetzung und Steigerung des Diesseits ist) macht die Besonderheit der kryonischen

\footnotetext{
${ }^{656}$ Ettinger 1964, 11.

${ }^{657}$ Vgl. a.a.O., 13, 50-58.

658 A.a.O., 13.

${ }^{659}$ A.a.O., 16, 84.
} 
Utopie aus. In seinem ersten Buch Prospects of Immortality und deutlicher noch in seinem zweiten Werk Man into Superman (1972) skizziert Ettinger die Vision eines unsterblichen Übermenschen, der die Genialität von Gilgamesch und Sherlock Holmes mit der Aussicht auf unendlichen Reichtum vereinigt. ${ }^{660}$ Der Schriftsteller und Journalist Alan Harrington preist in seinem populären Buch The Immortalist von 1969 die Aussichten der Kryonik und der Gentechnik (zur Züchtung von ganzen Menschen als "Ersatzteillager « für das Original), die beide die Überwindung der menschlichen Sterblichkeit verheißen würden. Mit Verweisen auf diverse philosophische und psychologische Schulen - von Giordano Bruno bis zu Erich Fromm und C. G. Jung - plädiert Harrington für die Befreiung des Menschen aus der unheilvollen Selbsthypnose, die die Religion, die Kunst und die bisherige Philosophie ihm anbiete: »Our new faith must accept as gospel that salvation belongs to medical engineering and nothing else. ${ }^{661}$

Aktuellere Schriften von Kryonikern wie R. Michael Perrys Forever for All (2000) fokussieren vor allem technische und philosophische Fragen, um die Kryonik zu legitimieren. Allerdings hält auch Perry an den alten Hoffnungen auf eine Erweiterung mentaler und physischer Leistungen fest. Er träumt von der Möglichkeit einer körperlichen Verjüngung der auferweckten Kryoniker und gar von einem virtuellen und unsterblichen Leben im Speicher von Computern. ${ }^{662}$ Mit dem Scientist's Open Letter on Cryonics von 2004 war beabsichtigt, eine größere Aufmerksamkeit in der öffentlichen Debatte zu erlangen. Zu den Unterzeichnern gehörten fast alle zentralen Akteure des Transhumanismus wie Nick Bostrom, Aubrey de Grey, Eric Drexler, Ben Goertzel, James Hughes, Ralph Merkle, Marvin Minsky, Max More, Michael Perry, Martine Rothblatt, Anders Sandberg, Natasha Vita-More und Roy Lee Walford. ${ }^{663}$

Was aber passiert konkret mit den toten Körpern in der Kryonik? Wie aus dem Verständnis des Todes in der Kryonik hervorgeht, ist die größte Bedrohung für den Menschen ein ernsthafter Gehirnschaden. Das Gehirn wird als Sitz der menschlichen Identität angesehen und sei unverzichtbar für die spätere Wiederherstellung einer Person. Dem natürlichen Verfall der Gehirnzellen soll daher mit einer möglichst schnellen

\footnotetext{
${ }^{660} \mathrm{Vgl}$. Ettinger 1989.

${ }^{661}$ Harrington 1977, 21. Vgl. a.a.O., 225-272.

${ }^{662}$ Vgl. Perry 2000, 559-563; Regis 1990, 76-108, 128-130; Sheskin 1979, 9-39.

${ }^{663}$ Vgl. http://www.cryonics.org/about-us/the-case-for-cryonics.
} 
Kühlung der Leiche begegnet werden. Im (kostenintensiveren) Idealfall werden die letzten Tage oder Stunden eines sterbenden Kryonikers von einem Stand-by-Team begleitet, das sofort zur Aktion schreitet, nachdem ein unabhängiger Arzt den Patienten für tot erklärt hat. Der Verstorbene wird dann zunächst mit Eis gekühlt und das Blut wird durch eine organschonende Flüssigkeit bzw. ein Frostschutzmittel ersetzt, da der größte Schaden im menschlichen Gewebe durch die Kristallisation des Wassers erwartet wird. In einem zweiwöchigen Vorgang wird der Leichnam schließlich in flüssigem Stickstoff auf eine Temperatur von $-196^{\circ} \mathrm{C}$ herabgekühlt. In Fällen, die nicht ideal verlaufen, da zum Todeszeitpunkt kein Stand-by-Team verfügbar war, wird der Körper so schnell wie möglich in Eiswasser gekühlt und dann zu den Standorten von Alcor in Arizona, dem Cryonics Institute in Michigan oder zu KrioRus bei Moskau transportiert. Der plötzliche Tod, der keine kryonischen Vorbereitungen zulässt, wird gefürchtet, wie in lange vergangenen Jahrhunderten das Sterben ohne Sakramente.

Allgemein kann man zwischen der Aufbewahrung des ganzen Körpers (whole body preservation) oder nur des Kopfes (neuropreservation) wählen, letztere Möglichkeit wird nur von Alcor und KrioRus angeboten. Ausschließlich den Kopf einzufrieren, bedeutet eine spürbare finanzielle Entlastung: Die minimalen Kosten für die Aufbewahrung der vollständigen Leiche betragen bei Alcor 200.000 \$, nur der Kopf schlägt mit 80.000 \$ zu Buche (der Transfer und ein evtl. Stand-by-Team werden extra berechnet). Alcors Konkurrent, das Cryonics Institute von Ettinger, bietet die Aufbewahrung des ganzen Körpers für mindestens 28.000 \$ an. Der deutliche Unterschied ist auf eine andere Preispolitik und die Auslagerung von Zusatzkosten zurückzuführen. Bei KrioRus werden 36.000 \$ für die Ganzkörperaufbewahrung und 18.000 \$ pro Kopf verlangt. ${ }^{664}$

Schließlich werden die Leichen bzw. die abgetrennten Köpfe in speziellen kryonischen Kapseln aufbewahrt, die mit flüssigem Stickstoff gefüllt sind. Der so genannte Bigfoot Dewar von Alcor kann bis zu drei Körper oder zehn Köpfe aufnehmen, während »the world's largest human storage cryostat « im Cryonics Institute bis zu 16 Leichname tiefkühlen kann. Diese Behälter werden in einer Art Gewerbehalle untergebracht und regelmäßig gewartet, um den entwichenen Stickstoff nachzufüllen.

${ }^{664}$ Alles mit dem Stand Juni 2018. https://alcor.org/BecomeMember/scheduleA.html; http://www.cryonics.org/ci-landing/funding-for-cryostasis/; http://kriorus.ru/en/Human-cryopreservation. 
Der erste Tote wurde im April 1966 eingefroren. Allerdings wurde dieser Leichnam nach US-amerikanischer Sitte einbalsamiert: Zuvor waren daher alle inneren Organe entfernt worden. Nach einigen Monaten der kryonischen Aufbewahrung entschieden sich die Angehörigen dann doch für eine gewöhnliche Erdbestattung. ${ }^{665}$ Die erste, aus der Sicht der Kryoniker echte »Suspension« eines Menschen wurde am 12. Januar 1967 in Glendale (Kalifornien) durchgeführt. Der Psychologe und pensionierte Collegeprofessor James H. Bedford wurde eingefroren, nachdem er an Lungenkrebs verstorben war. Als er erfuhr, dass seine Krankheit unheilbar sei, hatte Bedford die neu gegründete Cryonics Society of California kontaktiert. Nachdem seine Leiche mit Eiswasser gekühlt wurde, transportierte man sie in ein Lagerhaus in Phoenix (Arizona), das zunächst geheim blieb. Obwohl das Ereignis in den Medien sehr kritisch kommentiert wurde, waren die Anhänger der Kryonik äußerst euphorisch. Sie waren davon überzeugt, dass Bedford in seiner Kapsel 20.000 Jahre unbeschadet überdauern könne. Die kryonischen Organisationen erhielten weitere Anfragen aus aller Welt. Der sich selbst zugeschriebenen, welthistorischen Bedeutung der ersten kryonischen Suspension widmete Robert Nelson 1968 ein ganzes Buch, We Froze the First Man. ${ }^{66}$ James Bedford wurde als erster »Kryonaut« eine Ikone der Bewegung:

The oldest patient currently still being held in cryonic suspension is a Dr. James Bedford, who was suspended in 1967. He's survived the Cold War, the Vietnam War, the Gulf War, Watergate, the collapse of the Soviet Union and the 9/11 attacks - which is more than a lot of his contemporaries can say. If he can make it for so many years, you can too. ${ }^{667}$

Als Bedfords Leiche, die mehrfach umgebettet werden musste, 1991 genauer untersucht wurde, zeigten sich die Kryoniker besorgt, da sie gröBere Mengen gefrorenen Blutes und zahlreiche Gefrierfrakturen an dem Leichnam entdeckten. ${ }^{668}$

Mit Stand Juni 2018 werden insgesamt 157 Köpfe und vollständige Körper - so genannte Kryopatienten (cryopatients) - bei Alcor aufbewahrt, und 1179 Mitglieder haben bereits alle rechtlichen und finanziellen Vorbereitungen für ihren Todesfall getroffen. Von den heutigen »Kryonau-

\footnotetext{
${ }^{665}$ Perry berichtet von den ersten missglückten Versuchen. Perry 1991; Perry 2000, 39. ${ }^{666} \mathrm{Vgl}$. Nelson 1968.

${ }^{667}$ FAQs auf der Homepage des Cryonics Institute, http://www.cryonics.org/prod.html (10.01.2011).

${ }^{668}$ Vgl. Darwin 1991, 22-25; Ettinger 1967, 1251.
} 
ten« haben 48 Personen (33\%) ihren ganzen Körper einfrieren lassen, 86 $(58 \%)$ haben die Neuropräservation in Anspruch genommen, 11 (8\%) ließen nur das Gehirn (ohne Schädel) tiefkühlen und in zwei Fällen wurde nachträglich der Körper entfernt. ${ }^{669}$

Ettingers Cryonics Institute mit 1525 Mitgliedern hat inzwischen die 165. Leiche eingefroren und KrioRus kann trotz des späten Unternehmensbeginns im Jahr 2005 bereits auf 61 Supensionen zurückblicken. Bei KrioRus halten sich Neuropräservationen und Ganzkörperbehandlungen in etwa die Waage, ca. 60\% der dokumentierten Fälle sind Frauen. ${ }^{670}$ In Mitteleuropa ist derzeit noch keine kryonische Aufbewahrung von Leichen verfügbar, verschiedene kryonische Interessenvereine der vergangenen Jahrzehnte erwiesen sich meist als sehr kurzlebig.

Nachfolgend gilt es, den kulturellen Kontext der Kryonik näher zu beleuchten, um zu erklären, warum sich in der zweiten Hälfte des 20 . Jahrhunderts die Idee der Kryonik überhaupt formieren konnte. Daran schließt sich allerdings auch die Frage an, warum die Kryonik nie die euphorischen Erwartungen ihrer Pioniere erreicht hat. Denn weniger als 400 eingefrorene Leichen bzw. Köpfe innerhalb von 55 Jahren kryonischer Praxis ist angesichts von jährlich ca. 2,5 Millionen Toten allein in den Vereinigten Staaten absolut marginal.

Zunächst einmal ist die Kryonik als kulturelle Rezeption einer technischen Innovation zu begreifen: nämlich der Gefriertechnik. Nachdem Carl von Linde bereits 1876 den Kühlschrank erfunden hatte und schon in den späten 1930er Jahren jeder zweite US-Haushalt über ein Kühlgerät verfügte, brachte General Electric 1939 dann auch Gefrierschränke zur Marktreife. Erst in den 1950er Jahren fand die Gefriertechnik eine größere Verbreitung und die Nahrungsmittelindustrie begann, haltbare und gefrorene Lebensmittel anzubieten, die eine nicht zu unterschätzende Annehmlichkeit für das alltägliche Leben darstellten. Gefriertechnik erschien daher in den frühen 1960er Jahren als ein euphorisches Symbol des modernen Lebens im Lichte von neuen Entdeckungen und Errungenschaften - Ettinger sprach sogar von einer künftigen freezer-centered society: »... the freezer is more attractive than the grave ...« $" 671$

${ }^{669}$ Die bloße Konservation von Gehirnen bei Alcor wird z.B. von Krebspatienten in Anspruch genommen. Alcor und KrioRus veröffentlichen alle Fallgeschichten (alcor.org/ kriorus.ru), jedoch sind die Angaben teils stark anonymisiert, ein fundierter Schluss auf die Geschlechterverteilung bei Alcor ist nicht möglich.

${ }^{670} \mathrm{Vgl}$. die entsprechenden Homepages auf alcor.org; cryonics.org; kriorus.ru.

${ }^{671}$ Ettinger 1964, 16. Vgl. a.a.O., 77-91, 158ff. 
In den Selbstbeschreibungen der kryonischen Literatur wird in der Regel auf die lange Tradition wissenschaftlicher Experimente mit tiefgefrorenen Tieren verwiesen, die bis ins 17. Jahrhundert zurückreiche. ${ }^{672}$ Aus kulturwissenschaftlicher Sicht ist die Kryonik jedoch vornehmlich eine kulturelle Innovation, die im spezifischen Kontext der amerikanischen Bestattungskultur des 20. Jahrhunderts entstand. Allgemein muss die Kryonik daher als eine sehr ausgeprägte Form der Todesverdrängung verstanden werden, die oftmals als Ausdruck der besonderen US-amerikanischen Tabuisierung dieses Themas bewertet wird. Entsprechend der kryonischen Definition des Todes werden die tiefgekühlten Leichen weder als tot angesehen noch terminologisch als tot bezeichnet - sie sind »suspendierte Mitglieder« oder »Kryopatienten«, die zur Zeit »deanimiert« (deanimated) sind.

Die Tatsache, dass 1967 die Anhänger der Kryonik von den Medien gefragt wurden, ob sie ein Kryotorium errichten wollten, um so eine fortgeschrittene Art der Einbalsamierung anzubieten, verweist bereits auf die Beziehung zur traditionellen amerikanischen Bestattungskultur. ${ }^{673}$ Die Konservierung der Leiche durch eine umfassende Einbalsamierung und durch ein Begräbnis in äußerst beständigen Särgen kann als Standard der heutigen Bestattungskultur in den Vereinigten Staaten angesehen werden. Beginnend mit dem amerikanischen Bürgerkrieg (1861-1865) wurden die Einbalsamierung und die Aufbahrung der konservierten Leiche zum Archetyp der Bestattung im Bürgertum und der gesellschaftlichen Oberschicht, da viele Familien ihre auf den fernen Schlachtfeldern gefallenen Angehörigen in der Heimat beerdigen wollten. So wurden fast 40.000 der über 600.000 Gefallenen des Bürgerkriegs von rasch ausgebildeten Bestattern oder Ärzten mit Hilfe von medizinischen Konservierungstechniken einbalsamiert. Die unhaltbaren Zustände, die durch die aggressive Konkurrenz der Einbalsamierer auf den Schlachtfeldern und nahe der Hospitäler entstanden, führten zu der Festlegung erster beruflicher Regulierungen für Bestatter durch die US-Armee. Während die öffentliche Aufbahrung von Kriegshelden und die 2000 Meilen lange »Totenreise« des amerikanischen Präsidenten Abraham Lincoln im Jahr 1865 der neuen Einbalsamierungstechnik viel Popularität verlieh, sorgte andererseits das Bedürfnis nach körperlicher Integrität der Verstorbenen

${ }^{672}$ Vgl. Perry 2000, S. 36-48.

${ }^{673}$ V gl. Larsen 1967. 
für den Erfolg der Einbalsamierung gegenüber den traditionellen Bestattungsformen. ${ }^{674}$

Friedhöfe in den Vereinigten Staaten und in England litten im 18. und 19. Jahrhundert sehr unter dem so genannten body snatching - dem professionell organisierten Raub von frisch bestatteten Leichen, die für die anatomische Ausbildung von Ärzten benötigt wurden. Selbst noch in den letzten Jahrzehnten des 19. Jahrhunderts war es in vielen US-Staaten lediglich gestattet, die Körper von exekutierten Mördern oder anonymen Mordopfern für medizinische Zwecke zu sezieren, so dass an den medizinischen Fakultäten eine enorme Nachfrage nach Leichen bestand. Auf diese Weise entwickelte sich ein professionelles, jedoch illegales Gewerbe der resurrectors, die die Universitäten und anatomischen Schulen mit kürzlich bestatteten Toten versorgten. Die Bevölkerung hatte vielerlei Mittel und Wege eingesetzt, um ihre Verstorbenen zu schützen: Grabwächter, gesicherte Grüfte, Friedhofsmauern und sogar Sprengfallen in den Gräbern. Der Leichenraub ging jedoch erst spürbar zurück, als sich in den 1890er Jahren das Einbalsamieren bei der bürgerlichen Bestattung durchsetzte (einbalsamierte Leichen verfügen über keine inneren Organe mehr) und sich gleichzeitig die gesetzlichen Rahmenbedingungen für anatomische Schulen änderten. ${ }^{675} \mathrm{Nach}$ Ansicht von Gary Laderman ist die Popularität der Einbalsamierung damit auf verschiedene Faktoren zurückzuführen: »A refusal to allow the dead to disappear from the living community, a fixation on the body of the deceased, and a demand that the integrity of the corpse be perpetuated in the grave as well as in collective memory. ${ }^{676}$ Viele Kryoniker halten daher den Gedanken an den natürlichen Verfall der Leiche nach der Bestattung für äußerst abstoßend, während die kryonische Suspension die ewige Konservierung des Körpers verspricht. ${ }^{677}$

Dieses Bedürfnis nach der Bewahrung der körperlichen Integrität verbindet sich am Ende des 19. Jahrhunderts mit dem marktwirtschaftlichen Interesse des professionalisierten Bestattungsgewerbes. Durch die Verbesserung von Werbe- und Verkaufsstrategien konnte dieses kulturell vorhandene Bedürfnis nach der Konservierung der Leiche nicht nur die Verbreitung der Einbalsamierung beschleunigen, sondern auch die Wei-

\footnotetext{
${ }^{674}$ Vgl. Quigley 1998, 5-12.

${ }^{675}$ Vgl. Shultz 1992; Quigley 1996, 292-298.

${ }^{676}$ Laderman 1996, 73.

677 Vgl. Bryant \& Snizek 1973, 60.
} 
terentwicklung von Särgen fördern. ${ }^{678}$ Heutzutage bestehen die meisten Särge, die in den Vereinigten Staaten verkauft werden, aus metallenen Werkstoffen, teils sogar aus massivem Kupfer oder aus Bronze mit extra Asphalteinlage; denn anders als in Deutschland werden Gräber gekauft und es werden in der Regel Liegezeiten für einhundert Jahre garantiert. Viele Friedhöfe schreiben darüber hinaus vor, dass die Särge und Urnen in speziellen Beton- und Teereinfassungen oder in einer Art zweitem Sarkophag aus Stahl, Kupfer, Bronze (alles wird als vaults bezeichnet) bestattet werden müssen. Teils werden Särge auch mit Überdruck im Sarg luftdicht versiegelt (air-seal principle), um das Eindringen von Wasser und Fremdkörpern zu verhindern und um den Angehörigen das Gefühl zu vermitteln, dass der Verstorbene auf lange Zeit konserviert sei. In dieser US-amerikanischen Bestattungskultur, in der versucht wird, den natürlichen Verfall des Leichnams mit Hilfe von Einbalsamierung, hermetisch abgeschlossenen, massiven Metallsärgen und zusätzlichen Sarkophagen aufzuhalten, entstand die Kryonik. Vor diesem Hintergrund erscheint sie als hervorstechender Extrempol von kulturell weit verbreiteten Tendenzen, die einerseits durch ein starkes Bedürfnis nach Konservierung der Verstorbenen und andererseits durch einen ausgeprägten Geschäftssinn charakterisiert sind. ${ }^{679}$ Aus einer ökonomischen Perspektive mag es sogar plausibel sein, die Kryonik als Fortsetzung einer kommerzialisierten Medizin zu betrachten - wie es Peter Metcalf und Richard Huntington in Anthropology of Mortuary Rituals ausdrücken:

Americans have vast faith in medicine, a faith that is certainly in tune with the Enlightenment of progress and humanism. The development of powerful new drugs, and of reliable and safe surgical techniques, together with improvements in diet, have enabled the sinevitability< of death to be redefined. ${ }^{680}$

Einen toten Menschen als »nicht permanent tot« zu erklären und stattdessen die Notwendigkeit von unbegrenzter medizinischer und technischer Betreuung des »Kryopatienten« zu behaupten, kann als perfekter Businessplan betrachtet werden.

Neben diesen ökonomischen und technologischen Aspekten der Kryonik ist die Idee des Einfrierens und des späteren Wiedererweckens Ver-

\footnotetext{
${ }^{678}$ Vgl. Laderman 1996, 164-175.

${ }^{679}$ Vgl. Quigley 1998, 133-139. Die gleiche Tendenz kann in Summum's Modern Mummification beobachtet werden, eine Firma, die kostenintensive Einbalsamierungen im altägyptischem Stil anbietet (summum.org).

${ }^{680}$ Huntington \& Metcalf 1991, $209 f$.
} 
storbener auch Teil der Religionsgeschichte. Ganz offensichtlich rezipiert die Kryonik allgemeine Muster der christlichen Konzepte von Tod und Jenseits. Bestehen im Detail sehr große Differenzen zwischen den Interpretationen einzelner christlicher Konfessionen und Denominationen, so wird, ganz allgemein gesprochen, davon ausgegangen, dass der Mensch nach seinem Tod eine Weile tot sein und am Ende der Zeit in einer fast vollkommenen Form wieder auferstehen wird. Der entscheidende Unterschied zwischen der Kryonik und dem christlichen Glauben ist die Abwesenheit einer transzendenten Macht und damit auch der Verzicht auf eine moralische Dimension in der Kryonik: Die erhoffte Reanimation ist weder von der Dauer eines Fegefeuers noch von einem göttlichen Gnadenakt abhängig. Die Kryonik rezipiert nur die positiven und unverbindlichen Aspekte des christlichen Musters der Auferstehung - ohne ein Wort über die Verpflichtungen des Einzelnen zu verlieren, so sie denn nicht finanzieller Natur sind. Ettingers Versuche einer expliziten Anknüpfung an die christliche Theologie verhallten denn auch ohne spürbare Reaktionen seitens der christlichen Institutionen. ${ }^{681}$

Die Kryonik ist damit eine perfekte Utopie, im eigentlichen Sinne des Wortes - sie ist als Nicht-Ort die Negation eines Ortes für den toten Körper. Der erste kryonisch suspendierte Mensch, James Bedford, ist nicht nur ein »Kryonaut «, sondern er ist auch ein paradigmatischer Odysseus der Kryonik, nachdem er seine »letzte Ruhestätte« sieben Mal innerhalb von 30 Jahren wechseln musste. Andere »Kryonauten« sind inzwischen aufgetaut worden, hauptsächlich aus finanziellen Gründen. ${ }^{682}$

So wie der Tod aus Sicht der Kryoniker per Definition verschwindet, so verschwinden auch die Verstorbenen, nachdem der Tod festgestellt wurde. Die Leichen werden für gewöhnlich in Eiswannen heruntergekühlt, das Blut wird durch Frostschutzmittel ersetzt und schließlich werden sie in eine Lagerhalle nach Arizona, Michigan oder Moskau transportiert und mit bis zu fünfzehn anderen Leichnamen bzw. Köpfen in einem entsprechenden Container aufbewahrt. Es ist offensichtlich, wie die Kryonik im Kontext der hoch kommerzialisierten amerikanischen Bestattungskultur mit ihrem stark ausgeprägten Bedürfnis nach der Inte-

\footnotetext{
${ }^{681}$ Vgl. Ettinger 1967, 1253.

${ }^{682}$ Wie bereits erwähnt, tauten die meisten »suspendierten« Mitglieder der Cryonics Society of California vor 1980 auf, wie auch die meisten "Kryonauten" der Cryonics Society of New York. Vgl. Darwin 1981, 18.
} 
grität des Körpers entstehen konnte. Bryant und Snizek betrachten die Kryonik sogar als eine Ersatzreligion:

Unwilling to accept the promises of organized religions regarding a spiritual afterlife, cryonics members opt for a type of materialistic, active mastery over their own destinies ... the large majority of cryonics advocates are atheists ... Many are devotees of science fiction. ${ }^{683}$

Es stellt sich jedoch die Frage, warum die Kryonik trotz der immensen Aufmerksamkeit, die ihr zuteil wurde, bis heute so wenige Unterstützer gefunden hat. ${ }^{684}$ Bryant und Snizek führen »das Schmelzen« der kryonischen Bewegung Anfang der 1970er Jahre auf die ausbleibenden wissenschaftlichen Fortschritte und die hohen Kosten zurück. ${ }^{65}$ Allerdings sollten bei diesen Überlegungen noch andere Aspekte berücksichtigt werden, denn das Fehlen eines Rituals - oder eher die Inkompatibilität mit traditionellen Bestattungsritualen in den USA und die Verletzung der eigenen ideologischen Voraussetzungen - erschweren sicherlich die Akzeptanz der Kryonik. Vor einigen Jahren hat das Cryonics Institute daher einen Gedenkraum eingerichtet, an dem die Angehörigen Blumen niederlegen können.

Der letzte Punkt betrifft vor allem die Neuropräservation, die zwar nur von Alcor und KrioRus angeboten wird, jedoch im Populärdiskurs ein plastisches Bild der Kryonik vermittelt hat. Den Kopf vom Rumpf eines Leichnams abzutrennen, steht im krassen Widerspruch zum kulturell verankerten Bedürfnis nach der Integrität der Leiche, zumal die amerikanischen Medien auch ausführlich über die häufigen Gefrierfrakturen und weitere Schäden berichtet haben. ${ }^{686}$ Das Cryonics Institute, das keine Neuropräservation des Kopfes anbietet, ist sich dieser Problematik für die emotionalen und rituellen Bedürfnisse der Trauernden bewusst:

As human beings we understand that it just borders on the impossible for a person to go to the parents or children or friends of someone who has just passed away, and have to explain that the head of that person, whose loss has broken their heart, is going to be cut off and frozen in a tank with a dozen others somewhere. Nerves are frayed, families are grief-stricken, some of them may never

\footnotetext{
${ }^{683}$ Bryant \& Snizek 1973, 59.

${ }^{684}$ Vgl. Rievman 1976, 4f.

${ }^{685}$ Vgl. Bryant \& Snizek 1973, 61.

${ }^{686} \mathrm{Im}$ Allgemeinen haben die Medien immer sehr kritisch über die Kryonik berichtet. Vgl. Mann 1981, 11f.
} 
even have heard of cryonics much less the scientific plausibility of it, and outbursts, arguments, and threats of lawsuits are inevitable. ${ }^{687}$

Nichtsdestotrotz ist auch ohne Abtrennung des Kopfes ein traditionelles Bestattungsritual mit einer idealen kryonischen Behandlung nicht vereinbar. Bei dem zentralen Element der US-amerikanischen Bestattung, der Totenwache (viewing), kondolieren Verwandte und Freunde bis zu drei Tage im Bestattungshaus, in dem für gewöhnlich die einbalsamierte Leiche im halboffenen Sarg aufgebahrt wird. Im Falle der Kryonik kann keine Leiche aufgebahrt werden, es gibt keine Bestattung und kein Ritual, das zeigt: »Dies ist das Ende!« Diese Rituale würden die eigentliche Idee der Kryonik unterlaufen. Darüber hinaus gibt es auch keinen Platz des Erinnerns, kein Grab, nur zwei Lagerhallen in Michigan oder Arizona. Zwar hatte ein Architekt für die Weltausstellung 1967 in Montreal ein kapellenartiges cryo-sanctorum entworfen, jedoch wurden diese aufwendigeren Pläne aus Kostengründen nie realisiert. ${ }^{688}$ Wenn auch die Entscheidung für die Kryonik an sich schon ein gewisses Vermögen voraussetzt, so verursacht das Fehlen eines Bestattungsrituals auch Schwierigkeiten für die soziale Bestimmung des »postmortalen Status« des Verstorbenen und seiner hinterbliebenen Familie, da die üblichen sozialen Indikatoren wie der Sarg, die Ausgestaltung der Bestattungsfeier und die soziale Zusammenkunft der Trauergemeinde ausbleiben bzw. sehr eingeschränkt sind. Gelegentlich entstehen in diesem Zusammenhang Auseinandersetzungen zwischen den Kryounternehmen und den Hinterbliebenen, wie offenbar auch im Fall des 2016 verstorbenen Marvin Minsky: Seine Leiche wurde erst drei Tage nach dem Todeseintritt vom Alcor-Team geborgen und zur Abtrennung seines Kopfes für die Neuropräservation nach Arizona überführt. ${ }^{689}$

Auf diese Weise verspricht die kryonische Bewegung zwar die »Suspension des Todes", eine paradiesische Fortsetzung des gegenwärtigen Lebens und praktiziert die »Suspension der Toten", kann offenbar jedoch nicht die rituellen Bedürfnisse der großen Mehrheit der US-amerikanischen Gesellschaft befriedigen. Für den Fortschrittsglauben des Transhu-

${ }^{687}$ FAQ auf der Homepage des Cryonics Institute, http//www.cryonics.org/prod.html (10.01.2011).

${ }^{688}$ Vgl. Bryant \& Snizek 1973, 57; Sheskin 1979, 23-39.

${ }^{689}$ Vgl. Kephart 1950, 637-343. Es wurde offiziell nie bestätigt, dass Alcor Minsky einfror, jedoch war Minsky Mitglied bei Alcor und die Fallbesprechung für Patient 144 entspricht exakt Minskys Todesumständen. https://alcor.org/Library/html/casesummary $1700 . h t m l$. 
manismus jedoch ist die Kryonik die eigentliche »Lebensversicherung«, falls die erhofften Immortalisierungstechniken auch in den kommenden Jahrzehnten noch nicht zur Verfügung stehen sollten.

\subsubsection{Die technologische Unsterblichkeit}

Die Idee der technologisch ermöglichten Unsterblichkeit im Posthumanismus speist sich aus zwei divergenten Strängen. Dies ist zum einen die Vision einer computertechnischen Simulation des »echten Menschen«, wie sie genealogisch aus den medienbezogenen Immortalisierungsfantasien der frühen Science-Fiction Literatur hervorging (Verne, Villiers de l'Isle Adam). Erst mit der Etablierung eines kybernetischen Menschenbildes durch die Macy Conferences von 1946 bis 1953 und dessen Popularisierung v.a. durch die Schriften Norbert Wieners fand die Idee einer virtuellen Existenz im Speicher eines Computers Eingang in die fiktionale Literatur der 1950er und 1960er Jahre (Clarke, Lem, Strugazki, Franke). Der zweite Strang geht aus der medizinischen Suche nach Langlebigkeit hervor, die mit der Renaissance (Cornaro, Bacon) begann, durch das aufklärerische Vervollkommnungsstreben beflügelt wurde (Condorcet, Hufeland, Godwin) sich in eugenischen / gentechnologischen Visionen fortsetzte (Bernal, Haldane, Stephens) und schließlich in den VerheiBungen der Transplantationstechnologie mündet. Es ist evident, dass die frühesten nicht-fiktionalen Beispiele einer computertechnischen Immortalisierung (Veatch, Martin, Edelhart) allesamt im Kontext von Organtransplantationen standen. Hans Moravec übernahm dann 1979 explizit dieses klinische Szenario einer chirurgischen Geisttransplantation des Gehirns. In der Kryonik überschneiden sich dieses Streben nach Langlebigkeit und die kybernetisch begründete Fixierung auf das Gehirn mit den neuen Perspektiven der Transplantationsmedizin und Gefriertechnik sowie der traditionellen Einbalsamierungspraxis der US-Bestattungskultur.

Erstaunlicherweise werden ganz offensichtliche technische und soziale Fragen der posthumanistischen Unsterblichkeitsvision gemieden. Da ist zunächst die Frage nach der Persistenz von computertechnischen Speichermedien und Speicherformaten: Jeder Computernutzer meiner Generation wird sich an den raschen Wechsel von Floppy-Discs zu CDROMs, von DVDs zu USB-Sticks und schließlich hin zu verknüpften Festplattenspeichern (Clouds) erinnern und mehr als einmal frustriert 
festgestellt haben, dass sich alte Schreibdateien nicht mehr lesen lassen. Diese an sich triviale Alltagsbeobachtung wird von Datenexperten unter dem Begriff der »digitalen Obsoleszenz« subsummiert: Einerseits verfügen elektronische Speichermedien vom Magnetband bis zur Festplatte - je nach Nutzungsgrad - nur über Haltbarkeiten von zwei bis 30 Jahren. Andererseits sind alte Speicherformate, die mit Betriebssystemen und spezieller Software korrelieren, nicht ohne Datenanpassungen und -verluste auf neue Formate übertragbar. Kritische Stimmen wie Terry Kuny prophezeien unserer Epoche bereits, zu einem digitalen, dunklen Zeitalter zu werden, das aufgrund der rasanten technischen Umbrüche Gefahr läuft, große Wissensbestände zu verlieren. ${ }^{690}$ Langzeitarchive für besonderes Kulturgut wie der Barbarastollen in Deutschland basieren daher auf photographischen Mikrofilmen mit einer Haltbarkeit von ca. 500 Jahren und einer einfachen aber zuverlässigen, technischen Zugänglichkeit. ${ }^{691}$ Vor dem Hintergrund dieser wichtigen Debatte um die digitale Obsoleszenz erscheint die posthumanistische Hoffnung auf eine ewige Existenz im Speicher eines Computers recht naiv, zumal wir bei der Simulation von menschlichen Gehirnen mit höchst leistungsintensiver Hardwarenutzung rechnen müssten. Philipp von Becker weist zudem darauf hin, dass jede digitale Lebensform, die letztlich auf einem materiellen Datenträger beruht, auch zerstörbar und damit sterblich ist - wie dies die aktuelle Netflix-Serie Altered Carbon kreativ durchspielt. ${ }^{692}$ Auch die Idee von Sicherheitskopien ist - im wahrsten Sinne des Wortes - nur auf dem Papier brauchbar: Die Störgröße des »Rauschens« kann nie zu 100\% beseitigt werden und bei der Übertragung (»Kopie«) umfangreicher Datenmengen spürbare Fehler verursachen. Zudem sind die erwarteten kybernetischen Lebensformen prinzipiell nicht besser für die Kolonisierung des Weltraums gewappnet als ihre biologischen Vorfahren. Sonnenwinde, kosmische Strahlung und Vibrationen können massive Schäden in Prozessoren und Speichern verursachen. Mit steigender Leistung der Rechner nimmt auch das Schadensrisiko zu, weshalb heutige Raumschiffe und -stationen in der Regel mit langsamen aber zuverlässigeren CPUs ausgestattet werden. ${ }^{693}$

\footnotetext{
${ }^{690}$ Vgl. Kuny 1997, 1-4. Verschiedene Initiativen wie nestor (in Deutschland) oder die internationale Digital Preservation Coalition befassen sich mit dieser Problematik.

${ }^{691} \mathrm{Im}$ Barbarastollen sind derzeit über eine Milliarde Aufnahmen auf $32.000 \mathrm{~km}$ Film archiviert (bbk.bund.de).

${ }^{692}$ Vgl. Becker 2015, 54f.

${ }^{693}$ Vgl. Hruska 2017.
} 
Neben diesen grundsätzlichen Problemen der technischen Zuverlässigkeit ist der posthumanistische Traum von der Unsterblichkeit als Computerprogramm noch mit einer weiteren, nicht auflösbaren Schwierigkeit konfrontiert: die Datensicherheit. Zurzeit erleben wir, wie beinahe im Monatsrhythmus Datenlecks bei wichtigen Internetfirmen auftreten und sensible Nutzerdaten wie Bewegungsprofile, Gesundheitsstatus und Kreditkarteninformationen millionenfach geraubt und missbraucht werden. Zudem können wir beobachten, wie soziale Netzwerke persönliche Daten für politische und kommerzielle Zwecke an Dritte weiterverkaufen oder diese im Auftrag autokratischer Systeme sammeln. Es kann kein Zweifel daran bestehen, dass - falls es eine virtuelle Existenz je geben würde - die menschlichen Simulationen (und ihre Sicherheitskopien) das begehrteste Ziel krimineller Agitation überhaupt wären. In der Hand des Administrators - sei er nun menschlich oder künstlich - würde sich eine enorme Machtfülle ballen. ${ }^{64}$ Künstlich intelligente Computerviren - die alleinig auch nur Moravec kurz erwähnt - würden sich für »Entführungen" und verheerende Anschläge auf multiple, translokale Ziele bestens eignen. ${ }^{65}$ Mit einem Schlag das Leben von tausend realbiologischen Menschen auszulöschen ist - außer mit Kriegswaffen - ein schwieriges Unterfangen. Millionen von virtuellen Daseinsformen zu attackieren, wird mit dem richtigen informationstechnischen »Schlüssel « ein Leichtes sein. ${ }^{696}$ Bei Lichte betrachtet, verspricht die posthumanistische Vision einer virtuellen Unsterblichkeit alles andere als eine ewig gesicherte Existenz - tatsächlich erscheint kaum ein Medium so flüchtig zu sein wie eine Computersoftware.

Warum werden diese fundamentalen Probleme, die jedem noch so »dilettantischen « Experten bewusst sein müssen, nicht thematisiert? So wie die punktuelle, technische Idee eines Geisttransfers überzeugender klingt als der Einbezug kritischer technischer Fragen, so gewinnt auch die allgemeine Vision einer posthumanen Menschheit an Anziehungskraft, wenn sie die gesellschaftlichen Rahmenbedingungen ignoriert. Die technische Idee der Immortalisierung an sich wird im Posthumanismus bar ihrer in der Science-Fiction ausgeführten, sozialen Implikationen verhandelt.

${ }^{694}$ Christoph Keese (Executive Vice President der Springer AG) im Gespräch mit Thomas Wagner. Vgl. Wagner 2015, 75.

${ }^{695} \mathrm{Vgl}$. Moravec 1988, 173.

${ }^{696}$ Um solche Risiken zu minimieren, müssten die virtuellen Existenzen wiederum extrem stark durch Schutzprogramme kontrolliert werden, damit niemand unbedacht Schadsoftware installiert. 
Anders als das Vervollkommnungsstreben der Aufklärer oder als die Ideen der russischen Kosmisten erscheint die posthumanistische Utopie als individualistisches Projekt, das dem Einzelnen göttlich-paradiesische Aussichten verheißt. ${ }^{697}$

Erst diese konsequente Ausblendung der sozialen Dimension und der komplexen technischen Zusammenhänge ermöglicht es, die Existenz in der Virtualität ausschließlich als Befreiung zu präsentieren: als Befreiung von der Natur und der Evolution, als Befreiung von der Sterblichkeit und Beschränktheit des menschlichen Körpers und Geistes, als Befreiung von den Zwängen und Verpflichtungen der menschlichen Gesellschaft sowie als Befreiung von der sensorischen Gebundenheit an eben diesen Körper auf dem Planeten Erde.

Die hiermit suggerierte Befreiung von der biologischen Materie beruht jedoch - wie wir sahen - auf einem Trick, denn auch das virtuelle Dasein würde ja auf einem materiellen Datenträger und einer Maschine beruhen, die diese Daten »aktiviert«. Posthumanisten knüpfen auf diese Weise vordergründig an antike Vorstellungen der Gnosis und des Platonismus an, die das wahre Wesen der Lebensformen in einer immateriellen Sphäre des Lichtes vermuteten. Auch die aristotelische Seinsordnung definiert alle vergänglichen Lebewesen und Dinge auf der Erde als Verbindung der Elemente Erde, Luft, Feuer und Wasser, während die Sonne, Sterne und Planeten aus dem ewigen und unwandelbaren Äther bestehen. Auch hier zeigt sich, wie sehr Kurzweils Metapher von der sonnengleichen Singularität sowie der starke Fokus der Post- und Transhumanisten auf den Weltraum das Ergebnis einer langen kulturgeschichtlichen Entwicklung ist. ${ }^{698}$

${ }^{697}$ Die einzige kritische Diskussion, die Post- und Transhumanisten führen, ist beginnend mit FM-2030 die Auseinandersetzung mit vermuteten religiösen Positionen - dabei findet eine reale Auseinandersetzung der christlichen Theologien mit dem Posthumanismus bisher kaum statt. Vgl. Kurzweil 2005, 369-374; Rothblatt 2014, 283-287; Bostrom \& Roache 2008, 3-7.

${ }^{698}$ Vgl. Kapitel 6.6.2.; Gruman 1966, 15f.; Krüger 2005; Kurzweil 2005, 371. 


\subsection{Die transzendente Superintelligenz}

The awkward question posed by the changes we have labelled Post-Humanism, is not >Will we develop machines that are equal or superior to humans? ... The difficult question is, >Why do we want to develop such machines - to what ends will they be put? t99 $^{699}$

Robert Pepperell

Die maschinelle Unsterblichkeit ist nur eines der beiden Grundelemente der posthumanistischen Logik. Das zweite ist die Teilhabe an der übermenschlichen Existenz der post-humanen Nachfahren. Es zeichnet gerade die posthumanistische Argumentation aus, dass der prophezeite Fortschritt der künstlichen Intelligenzen den Menschen »mitnimmt « - eben durch den Geisttransfer. Kein Post- und Transhumanist vertritt ernsthaft die These, dass sich kybernetisches Leben entwickelt, den Menschen übertrifft und ihn dann unbeachtet hinter sich zurücklässt. Mit Robert Pepperell müssen wir nun der Frage nachgehen, was diese Teilhabe an der künstlichen Intelligenz ausmacht - warum Menschen nach diesem Zustand streben sollten. Eine besondere Rolle spielen in diesem Zusammenhang die Begriffe der Transzendenz, des Übermenschen und der Superintelligenz, die allesamt ein männliches Idealbild der menschlichen Existenz widerspiegeln.

\subsubsection{Transzendenz und Übermensch}

Im Rahmen posthumanistischer Literatur ist es Hans Moravec, der den Begriff des Transzendierens als erster einbringt. Schon in Mind Children (1988) prophezeit er, dass die künstlichen Intelligenzen die materiellen Grenzen der Physik und überhaupt alles »transzendieren « werden, was wir als Menschen bisher kennen. In seinem zweiten Buch von 1999 ist der Terminus schon Bestandteil des Untertitels Robot. Mere Machines to Transcendent Mind, wird jedoch nicht weiter ausgeführt. ${ }^{700}$

Die Brücke zwischen Fiktion und Techno-Utopie schlägt - wiederum Vernor Vinge. In seinem Roman A Fire Upon the Deep von 1992 schildert er die Zukunft unserer Galaxis, die nach Grad ihrer technologischen

${ }_{699}$ Pepperell 1995, 177.

${ }^{700}$ V gl. Moravec 1988, 1; auch Moravec 1999, 159. 
Entwicklung in verschiedene Zonen gegliedert sei. Hat eine Zivilisation die Singularität überschritten, wird sie Teil der Zone »Transcend « und beherbergt superintelligente, künstliche Wesenheiten. ${ }^{701}$ In seinem richtungsweisenden Text The Coming Technological Singularity von 1993 formuliert er dann seine realen Hoffnungen: »After all, IA [intelligence amplification: $\mathrm{OK}]$ allows our participation in a kind of transcendance.«702

Ray Kurzweil verwendet den Transzendenzbegriff zuerst in The Age of Spiritual Machines, wo er ihn häufig mit Meditations- und Gotteserlebnissen in Verbindung bringt und nur sehr allgemein in Bezug zu technischem Fortschritt setzt. ${ }^{703}$ Erst mit seinem prophetischen Buch The Singularity is near. When Humans transcend Biology (2005) und seinem zweiten Lebensratgeber Transcend: Nine Steps to Living Well Forever, den er mit dem Mediziner Terry Grossman 2009 veröffentlicht, gelangt der Transzendenzbegriff ins Zentrum der Selbstvermarktung von Ray Kurzweil, die bekanntlich in dem Dokumentarfilm gleichen Jahres, Transcendent Man, und dem Spielfilm Transcendence von 2014 gipfelt. Transzendenz und transzendieren führt Kurzweil zunächst als ein Überschreiten der biologischen Grenzen des Menschen und der Evolution ein. ${ }^{704}$ Im Einklang mit der kybernetischen Mustertheorie der Identität ist Transzendenz für ihn die Beständigkeit der Information gegenüber dem Stoff: »It's through the emergent powers of the pattern that we transcend. Since the material stuff of which we are made turns over quickly, it is the transcendent power of our patterns that persists. ${ }^{705}$ Daher ist die Singularität für Kurzweil (wie für Vinge) identisch mit der Verwirklichung von Transzendenz. ${ }^{706}$ Ebenso wenig wie sie bei den Posthumanisten philosophisch reflektiert wird, erscheint die Idee des Transzendierens des Menschseins nur punktuell bei den drei wichtigen Vordenkern des Post- und Transhumanismus im 20. Jahrhundert. John Desmond Bernal prophezeit, dass der künftige Mensch alle Fähigkeiten des bisherigen transzendieren würde: ${ }^{707}$

\footnotetext{
${ }^{701}$ Vgl. Vinge 1992; Yudkowsky 2000.

702 Vinge 1993.

${ }^{703}$ Vgl. Kurzweil 1999a, 23, 109-111, 189.

${ }^{704}$ Vgl. Kurzweil 2005, 3, 9, 136, 374 .

${ }^{705}$ Vgl. a.a.O., 388.

${ }^{706}$ Unter den posthumanistischen Autoren bezieht sich nur noch Martine Rothblatt auf den Transzendenzbegriff, den sie wie Kurzweil als Überwindung der materiellen Grenzen des biologischen Menschen (und seiner geschlechtlichen Determination) versteht. Vgl. Rothblatt 2014.

${ }^{707}$ Vgl. Bernal 1929, 16.
} 
It may even be ... that with the new possibilities we are reaching up to a new step in cosmic evolution away from the individual organisms or the society of such organisms towards an organismal electronic complex which will transcend it and may ultimately male its organismal originator superfluous. ${ }^{708}$

Neben dem Kybernetiker Norbert Wiener spricht auch Julian Huxley vereinzelt vom Transzendieren menschlicher Fähigkeiten durch den erwarteten technischen Fortschritt. ${ }^{709}$

Wie kaum ein anderer philosophischer Begriff ist die transcendence tief in der amerikanischen Philosophie verwurzelt. Die so genannten transcendentalists bilden Anfang des 19. Jahrhunderts die erste philosophische Bewegung des neuen Kontinents. Allerdings wäre es m.E. unangemessen, an dieser Stelle eine ideengeschichtliche Genealogie abzuleiten. Die Transzendentalisten waren um 1830 stark durch die deutsche und englische Romantik und den Idealismus beeinflusst, priesen die mystische Erkenntnis vor der reinen Vernunft und begriffen ihr Engagement als ein Aufbegehren gegen das rationale Menschenbild und den Fortschrittsgedanken der Aufklärung. Da fast alle Vertreter der Tranzendentalisten aus den Reihen der Unitarier stammen, werden ihre Ideen meist als Protest und Reform der etablierten unitarischen Lehre interpretiert. Sie verkörpern damit eine Gegenbewegung zu den materialistischen und utilitaristischen Philosophien des 19. Jahrhunderts, die maßgeblich für die posthumanistische Utopie werden sollten. ${ }^{710}$ Es lässt sich höchstens die Vermutung anstellen, dass Ray Kurzweil durch seine unitarische Prägung in der Jugend auch eine gewisse Affinität zum Transzendenzbegriff entwickelt hat.

Ähnlich verfährt die Mehrheit der trans- und posthumanistischen Denker mit dem Konzept des Übermenschen nach Friedrich Nietzsche. Terminologisch firmiert der künftige Zustand des Menschen zwar bei Frank Tipler, Marvin Minsky und Kevin Warwick als superhuman bzw. superbeing. ${ }^{711}$ Einen expliziten Bezug auf Nietzsche vollführen aber nur

${ }^{708}$ Bernal 1969, 857.

${ }^{709}$ Vgl. Wiener 1985, 184; Huxley 1957b, 13.

${ }^{710}$ Die Kategorisierung des Posthumanismus als techno-/cyber-transcendentalism, wie sie David Brin vornimmt, wäre daher fehlleitend. Vgl. Miller 1979, 7-9; Brin 2013, 395f. Die Herleitung aus dem Konzept des plus-man / great man des Transzendentalisten Ralph Waldo Emerson, die bisweilen vorgeschlagen wird, wären aufgrund seines vitalistischen Prinzips ebenso unangemessen. Benz 1961, 105-112; Regis 1990, $276 f$.

${ }^{711}$ Vgl. Tipler 1995, 87f., 255-259; Minsky 1992a, 24; Warwick 2000, 151. Vgl. hierzu auch Warwick 1997, 1998. 
der Kryonik-Gründer Robert Ettinger, der deutsche Transhumanist Stefan Sorgner und der Extropianer Max More. Ettinger verurteilt Nietzsches Rassismus scharf, lobt jedoch (paradoxerweise) seine Vision des zukünftigen Menschen, die keine moralischen Schranken kenne: »Nevertheless, the best of Nietzsche is very good, and he made an important contribution toward exposing the illusions of the altruists. ${ }^{712}$ Unter Berücksichtigung einer möglichst weiten anthropologischen Basis entwickelt Ettinger dann das Bild eines Übermenschen (superman), der die Eigenschaften antiker Heroen, von Nietzsches philosophischem Übermenschen und der Superhelden diverser Science-Fiction Geschichten und Comics in sich vereint. Der künftige Mensch soll dabei die schon vorhandenen Eigenschaften biologischer Lebewesen kombinieren und erweitern. Das Ergebnis ist - wie schon in Daniel Halacys Cyborg - Evolution of the Superhuman (1965) - eine überintelligente Kriegsmaschine: Diese soll gegen Kälte und Hitze unempfindlich sein wie Angehörige einiger Indianerstämme; er soll beliebig seine Hautfarbe wechseln können; er soll in der Lage sein, so viel zu essen und zu genießen, wie er will, da Übergewicht »repariert« werden kann; der gesamte Körper soll mit einer stabilen, aber flexiblen Hautrüstung ausgestattet werden, während gleichzeitig einige aktive Verteidigungsorgane (elektrische Organe oder eine Art Flammenwerfer) die Sicherheit dieses transhumanen Lebewesens gewährleisten sollen. Die Form des Körpers könne dabei vom fliegenden Batman bis hin zum schwimmenden Aquaman, vom Zwerg bis zum Riesen und Cyborg variieren. ${ }^{713}$

Stefan Sorgner bemüht sich dagegen, die Gemeinsamkeiten zwischen Nietzsches Philosophie und dem Transhumanismus herauszuarbeiten, die er im dynamischen Menschenbild und der mutigen Überwindung traditioneller moralischer und religiöser Schranken zu finden glaubt. ${ }^{714}$ Auch Max More betont diese gemeinsamen Aspekte, verweist aber zugleich auf die Unvereinbarkeit des transhumanistischen Fortschrittsglaubens mit Nietzsches Konzept der ewigen Wiederkehr. Gegenüber Sorgner betont More sogar, dass es einen direkten Einfluss von Nietzsche auf den Transhumanismus gab, denn More selbst sei durch Nietzsches

\footnotetext{
712 Ettinger 1989, 26.

${ }^{713}$ Vgl. a.a.O., 43-87; Halacy 1965.

${ }^{714}$ Vgl. Sorgner 2009.
} 
Rede vom Übermenschen ja inspiriert worden und habe den Begriff des transhuman entsprechend gedeutet. ${ }^{715}$

Transzendenz und Übermensch sind lediglich für einige Vertreter des Post- und Transhumanismus wichtige Konzepte. Über Kurzweil und Vinge bzw. Ettinger, Sorgner und More hinaus bezeichnen sie jedoch wirkungsvoll das grundlegende Verhältnis des Menschen zum Posthumanen: Erstens wird es die menschliche Natur in einem Maße übersteigen, die bisher nur der göttlichen Transzendenz entsprach. Zweitens wird der Mensch an diesem transzendenten Dasein teilhaben. Das Posthumane ist aus dieser Perspektive durch die ihm innewohnende Transzendenz stets auch transhuman: transcendent, posthuman und transhuman spiegeln wie die Glassteinchen eines Kaleidoskops mit je eigenen Nuancen die Beziehungen zwischen Mensch und Transzendenz wider. Das changierende Farbenspiel - also die Qualitäten des erhofften posthumanen Daseins - ist in Bewegung.

\subsubsection{Gehirn, Genie und Superintelligenz}

I do not think there is any thrill that can go through the human heart like that felt by the inventor as he sees some creation of the brain unfolding to success. ${ }^{716}$

Nikola Tesla

Die paradiesischen Verheißungen der Post- und Transhumanisten waren bis Mitte der 2000er Jahre äußerst vielfältig und plastisch. Schon vor dem Upload in ein virtuelles Dasein sollten Menschen in den Genuss von robotischen und künstlich intelligenten Helfern kommen. Hans Moravec schwärmt davon, dass in Kürze universale Haushaltsroboter den Menschen von lästigen Tätigkeiten wie Staubsaugen, Kochen, Putzen und Teppichknüpfen befreien werden. ${ }^{717}$ Noch optimistischer gibt sich Ray Kurzweil: Bis 2029 werden seiner Einschätzung nach Menschen in fast allen Berufen - vom Lehrer bis zum Mediziner - durch leistungsfähigere Computer ersetzt worden sein. Die führenden Künstler,

\footnotetext{
${ }^{715}$ Vgl. More 2010; More 1996b, 3; More 1994, 2. Nick Bostrom und Frank Tipler weisen die Verbindung zwischen Nietzsche und dem Transhumanismus scharf zurück, teils wegen des sozialdarwinistischen Rassenkultes, der für die Nazi-Ideologie prägend wurde. Vgl. Bostrom 2005; Tipler 1995, 80-83.

${ }^{716}$ Zitiert nach Kurzweil 2005, 1.

717 Vgl. Moravec 1988, 22-25; Moravec 1996a, 101-108; Moravec 1999, 91-110 und $124 f$.
} 
Komponisten und Musiker seien dann kybernetische Organismen. ${ }^{718}$ Gleichzeitig werden die medizinischen Fortschritte nach Ansicht der Posthumanisten - v.a. mit Hilfe der Nanotechnologie - eine Lösung für alle gesundheitlichen Probleme garantieren, so dass Raymond Kurzweil schon für die nahe Zukunft eine durchschnittliche Lebenserwartung von ca. 120 Jahren prognostiziert. ${ }^{719}$

Ferner sind sich alle posthumanistischen Autoren einig, dass der Fortschritt in den Informationstechnologien und der Ersatz menschlicher Arbeitskräfte durch produktivere, intelligente Maschinen zu unendlichem Reichtum führen werde: »Early to bed and early to rise, makes a man healthy and wealthy and wise. ${ }^{720}$ Dieses calvinistisch geprägte Motto von Benjamin Franklin zitiert Marvin Minsky, bevor er die Bedingungen für den erwarteten, unbegrenzten Reichtum erörtert. Roboterplaymates und ein luxuriöses Leben für jeden Menschen würden diese nahe Zukunft kennzeichnen, in der alle sozialen Spannungen behoben wären: ${ }^{721}$

Furthermore, the road we're going down is a road paved with gold. It's full of benefits that we're never going to resist - continued growth in economic prosperity, better health, more intense communication, more effective education, more engaging entertainment, better sex. ${ }^{722}$

Das virtuelle Dasein selbst übertrifft diese Aussichten dann um ein Vielfaches. Nicht Gesundheit und Langlebigkeit, sondern Unsterblichkeit wird dem Menschen geschenkt. Sie könnten die Gestalt ihres virtuellen Körpers nach ihren Wünschen verändern, ohne weitere Hilfsmittel durch das emulierte Universum reisen und trotzdem wie ein biologischer Mensch kulinarische Erlesenheiten genießen und andere Menschen berühren und spüren. Nick Bostrom umschreibt den Zustand poetisch: "Beyond dreams. Beyond imagination. ... There is a beauty and joy here that you cannot fathom. It feels so good that if the sensation were translated into tears of gratitude, rivers would overflow. « ${ }^{723}$

\footnotetext{
${ }^{718}$ Als Ludditen wurden die »Maschinenstürmer« zu Beginn des 19. Jahrhunderts bezeichnet. Vgl. Kurzweil 1999a, 85f., 180ff., 202-233.

${ }^{719}$ Vgl. Minsky 1994, 113; Tipler 1995, 104f.; Moravec 1988, 102; Moravec 1999, 9-13, 141f.; Kurzweil 1999a, 223.

${ }^{720}$ Minsky 1994, 108.

${ }^{721}$ Vgl. Minsky 1994, 108; Tipler 1995, 104f.; Moravec 1988, 102; Moravec 1999, 9-13, 141f.; Moravec \& Pohl 1993, 76; Kurzweil 1999a, 130f., 202-233.

${ }^{722}$ Kurzweil 1999a, 130.

${ }^{723}$ Bostrom 2008. Vgl. Proudfoot 2012, 372.
} 
Frank Tipler erläutert das virtuelle Paradies im Hinblick auf eine Frage, die ihm häufig von seinen männlichen, unverheirateten Studenten gestellt worden sei: »Gibt es im Himmel Sex?« (Tipler hielt an der Tulane-University in New Orleans regelmäßig Lehrveranstaltungen über seine Omega-Theorie ab). Das Zitat einer längeren Passage aus der Physik der Unsterblichkeit dokumentiert diese allmächtige Qualität von Tiplers virtueller Emulation und illustriert eindrücklich sein uneingeschränktes Bedürfnis zur Mathematisierung - in diesem Falle der Schönheit:

... since some people desire sex, the answer has to be yes, sex will be available to those who wish it ... However, the problems which sex generates in our present life will not occur in the afterlife ... it would be possible for each male to be matched not merely with the most beautiful woman in the world, not merely with the most beautiful woman who has ever lived, but to be matched with the most beautiful woman whose existence is logically possible ... It is instructive to compute the psychological impact of the most beautiful woman on a man ... Assuming the validity of the Fechner-Weber Law at large stimulus, the relative psychological impact of meeting the most beautiful of these is thus $\left[\log _{10} 10^{106}\right] /$ $\left[\log _{10} 10^{9}\right]=100,000$ times the impact of meeting the most beautiful woman in the world ... I've gone through this calculation to illustrate dramatically one crucial point: the principle of nonsatiation will not hold for the resurrected humans ... about two thirds of adult humans experience at some point in their lives [sic!] an intense passion for a member of opposite sex which is not reciprocated: this is the phenomenon of unrequited love. The Omega Point has the power to turn this passion into requited love in the afterlife. ${ }^{724}$

Überdies wird $\operatorname{man}(\mathrm{n})$ auch dem Sexualleben neue Freuden abgewinnen können: » ... not just sex. Not even just very good sex. Incredible sex, without such penalties as AIDS or unwanted pregnancy or even the wrath of a jealous lover, since all of it takes place in your mind." $" 25$

Damit ist offensichtlich, dass die posthumanistische Utopie zwar den biologischen Körper überwinden will, nicht aber die Körperlichkeit an sich. Das posthumanistische Begehren schließt den Wunsch nach physischer Perfektion, Stärke und sinnlicher Lust ein. Diese Formen lustvoller Körperlichkeit werden von keinem der posthumanistischen Autoren abgewertet. Eine allgemeine Deutung des Posthumanismus als Cyberplatonismus oder Cybergnosis, wie sie Erik Davis, Slavoj Žižek und Hartmut Böhme vorgeschlagen haben, ist daher fehlleitend. ${ }^{726}$

${ }^{724}$ Tipler 1995, 256f.

${ }^{725}$ Moravec \& Pohl 1993, 74.

${ }^{726}$ Vgl. Krüger 2004b; Krüger 2005. 
Bereits in den Schriften der 1990er Jahre bildeten diese »bunten« Aussichten die Peripherie der posthumanistischen Fortschrittstheorie. Im Zentrum des Vervollkommnungsstrebens stehen die Fähigkeiten des menschlichen Geistes. Wie wir zeigen konnten, sind diese für das posthumanistische Menschenbild und den Maßstab der Fortschrittsentwicklung bestimmend (Kapitel 6.2). Jedoch erst durch Ray Kurzweils Buch The Singularity is near (2005) und Nick Bostroms Werk Superintelligence (2014) gewann das Posthumane als eigene Entität schärfere Konturen. Was zuvor diffus mit der Verfügbarkeit von künstlich intelligenten Computern verbunden wurde, bezieht sich (gemäß Kurzweil) nun auf die eine Superintelligenz, die mit dem Eintreten der Singularität im Jahr 2045 verwirklicht werde. Das konventionelle Ziel der KI-Forschung, wie es Marvin Minsky zeitlebens anstrebte, ein künstliches System auf dem Niveau von menschlicher Intelligenz zu schaffen, wird auf diese Weise durch die Prophezeiung einer übermenschlichen, transzendenten Superintelligenz abgelöst. Damit aber wird die Frage nach den ideengeschichtlichen Zusammenhängen aufgeworfen, die durch den Genie-Kult und eugenische Züchtungsphantasien von Elitegehirnen um 1900 geprägt wurden. In diesem Zusammenhang ist die Sonderstellung des menschlichen Gehirns im Posthumanismus bedeutungsvoll: Das Gehirn ist aus posthumanistischer Sicht vollkommen identisch mit der Persönlichkeit eines Menschen und bildet die Grundlage des immortalisierenden Geisttransfers, der von Anders Sandberg und Nick Bostrom benannten whole brain emulation. ${ }^{727}$

Schon 1988 formuliert Hans Moravec Ziel und Anspruch mit Blick auf die vierte Robotergeneration, die superhuman und superrational sein werde: ${ }^{728}$

Long life loses much of its point if we are fated to spend it staring stupidly at our ultra-intelligent machines as they try to describe their ever more spectacular discoveries in baby-talk that we can understand. We want to become full, unfettered players in this new superintelligent game. ${ }^{729}$

Auch für Frank Tipler sind diese Aussichten aufgrund der letztlichen Auferstehung aller Menschen nicht beunruhigend: »Ultimately, intelligent machines will become more intelligent than members of the species

\footnotetext{
${ }^{727}$ Vgl. Sandberg \& Bostrom 2008; Moravec 1988, 109f.; Kurzweil 2005, 143-203.

${ }^{728}$ Vgl. Moravec 1988, 1, 22-25; Moravec 1996a, 101-108; Moravec 1999, 91-110, 124 f.

${ }^{729}$ Moravec 1988, 108. Vgl. Moravec 1995, 168.
} 
Homo sapiens, and will thus dominate civilization. So what? « ${ }^{730}$ Mit den Werken von Nick Bostrom und Ray Kurzweil erhält die Superintelligenz darüber hinausgehend eine heilsgeschichtliche Qualität, die bereits in Vernor Vinges Zukunftsentwurf angelegt war. Beide Denker beziehen sich wechselseitig aufeinander: Die Singularität verknüpft Kurzweil mit Bostroms Ideen zur Superintelligenz und Bostrom wiederum verbindet sein Verständnis der Superintelligenz mit Kurzweils Singularitätsprophetie (auch wenn er die religiösen Konnotationen explizit zurückweist). ${ }^{731}$ Es wäre schwer zu entscheiden, welcher der beiden Denker die folgende Technikeuphorie in Prosa gegossen hat:

It is hard to think of any problem that a superintelligence could not either solve or at least help us solve. Disease, poverty, environmental destruction, unnecessary suffering of all kinds: these are things that a superintelligence equipped with advanced nanotechnology would be capable of eliminating. Additionally, a superintelligence could give us indefinite lifespan, either by stopping and reversing the aging process through the use of nanomedicine, or by offering us the option to upload ourselves. ${ }^{732}$

Zunächst erstaunt es, dass weder Bostrom noch Kurzweil in ihren umfangreichen Büchern eine Definition von Intelligenz vorlegen oder diskutieren. Für Kurzweil ist dies schlicht die Steigerung von Speicher- und Rechenkapazitäten, Bostrom unterscheidet der Form nach noch eine kollektive und eine qualitative Superintelligenz. ${ }^{733}$ Allerdings verfügt keiner der beiden erfolgreichen Autoren über Forschungserfahrung im Bereich der künstlichen Intelligenz: Bostrom ist Philosoph und Kurzweil hat vor allem in den 1970er und 1980er Jahren einige Erfindungen und technische Verbesserungen von optischen Texterkennungsprogrammen vorgelegt.

Selbst innerhalb der trans- und posthumanistischen Debatte ist die Erwartung einer superintelligenten KI, die zudem mit der Singularität verbunden wird, höchst umstritten. Der Wirtschaftswissenschaftler Robin Hanson und der Quantenphysiker Michael Nielsen stellen in Frage, ob es überhaupt eine Art »Intelligenz-Explosion« geben könne, wie sie Bostrom prognostiziert, ohne dass der Intelligenzbegriff geklärt wäre. Anstelle einer so genannten dominanten KI, die den Menschen in allen

${ }^{730}$ Tipler $1995,87$.

${ }^{731}$ Vgl. Kurzweil 2005, 259f.; Bostrom 2014, 2.

732 Es war Nick Bostrom (2003).

${ }^{733}$ Vgl. Bostrom 2014, 53-57; Loh 2018, 113. 
Lebensbereichen übertreffen werde, sei es wahrscheinlicher, dass Computer in einigen Nischenbereichen leistungsfähiger werden. Die Annahme einer Dominanz der KI über das gesamte planetarische Leben sei laut Nielsen genauso plausibel, wie die Herrschaft der Bakterien zu behaupten. ${ }^{734}$ Anders Sandberg gibt zu bedenken, dass die Prognose eines exponentiellen technologischen Fortschrittes - wie der Verwirklichung der Singularität - auf einem stetigem ökonomischen Wachstum und gesellschaftlicher Stabilität beruht, die jedoch nicht naturgegeben seien. ${ }^{735}$ Der KI-Forscher Selmer Bringsjord wirft den Vertretern der ultraintelligenten Singularitätsthese gar vor, längst in den Bereich religiöser Prophetie abgeglitten zu sein, da ihre These weder rational abzuleiten noch empirisch belegbar sei: ${ }^{736}$

... and yet, again, here we are, with hardware that moves information in silicon at a rate that makes the transmission speed of the brain seem as slow as a disoriented caterpillar by comparison, and we still don't have a machine that can problem-solve, even in highly formal domains like computer programming, at the level of a mediocre novice. ${ }^{737}$

Die Kritik von KI-Experten an der Singularitäts- bzw. Superintelligenzkonzeption von Vinge, Kurzweil und Bostrom offenbart die theoretische Leerstelle in der posthumanistischen These. Aus Sicht der tatsächlichen KI-Forschung existiert kein technisches Argument, warum in spätestens 30 Jahren aufgrund steigender Speicher- und Rechnerleistungen plötzlich eine »Intelligenzexplosion« stattfinden solle, die zudem noch alle Probleme der Menschheit, des Planeten und des Universums lösen werde. Man fühlt sich an das offenherzige Kind aus dem Märchen Des Kaisers neue Kleider erinnert, wenn der Science-Fiction Autor David Brin trotz einzelner Bedenken seinen Glauben an die Singularität bekräftigt: "The alternative is simply too awful to accept. «738

Aus kulturwissenschaftlicher Perspektive drängt sich daher die Frage auf, warum die Idee der mit der Singularität verbundenen Superintelligenz mindestens für Teile des technophilen Milieus so attraktiv erscheint, obwohl die fachlichen Argumente bei näherer Betrachtung mager aus-

\footnotetext{
${ }^{734}$ Vgl. Hanson 2013, 405; Nielsen 2013, 409ff.

${ }^{735}$ Vgl. Sandberg 2013b, 411-416.

${ }^{736}$ Vgl. Bringsjord \& Bringsjord \& Bello 2012.

737 A.a.O., 405.

${ }^{738}$ Brin 2013, 396. Die tatsächlichen KI-Forscher, die ich in den vergangenen 15 Jahren auf Tagungen traf, hatten für die Hoffnungen der Posthumanisten meist auch nur ein Kopfschütteln übrig.
} 
fallen. Die ideengeschichtliche Analyse des Singularitätskonzeptes als Fortschrittsidee hat gezeigt, dass bereits etablierte kulturelle Deutungsmuster wie die frontier und die populäre Mystifizierung schwarzer Löcher die breite Aufnahme der Singularitätsidee »getragen« haben. Aus welchen kulturellen Ideen speist sich also die v.a. von Vinge, Kurzweil und Bostrom erwartete Superintelligenz?

Als Urheber der Idee einer Superintelligenz verweisen alle maßgeblichen Autoren auf den 1965 publizierten Aufsatz Speculations Concerning the First Ultraintelligent Machine des britischen Mathematikers Irving John Good. ${ }^{739}$ Good absolvierte ein Mathematikstudium in Cambridge und diente ab 1941 in Bletchley Park, wo er sich bei der Dekodierung chiffrierter, deutscher Nachrichten und der Entwicklung des ersten elektronischen Computers, des Colossus, unter der Leitung von Alan Turing einbrachte. Später wirkte er fast drei Jahrzehnte als Professor für Statistik an der Virginia Tech University in den Vereinigten Staaten. Seinen berühmten Aufsatz leitet Good mit einem prophetischen Bekenntnis ein: »The survival of man depends on the early construction of an ultra-intelligent machine. ${ }^{740}$ Dieser Computer, den Good bis zum Ende des 20. Jahrhunderts erwartet hatte, werde dem Menschen in der Speicherung und Verarbeitung von Informationen weit überlegen sein:

Let an ultraintelligent machine be defined as a machine that can far surpass all the intellectual activities of any man however clever. Since the design of machines is one of these intellectual activities, an ultra-intelligent machine could design even better machines; there would then unquestionably be an »intelligence explosion, " and the intelligence of man would be left far behind ... Thus the first ultraintelligent machine is the last invention that man need ever make, provided that the machine is docile enough to tell us how to keep it under control. It is curious that this point is made so seldom outside of science fiction. It is sometimes worthwhile to take science fiction seriously. ${ }^{741}$

Als größtes Problem bei der Entwicklung künstlicher Intelligenz identifiziert Good die Übersetzung von Semantik in Maschinensprache. Als erste Anwendung und damit Maßstab zur Messung der Intelligenz führt er das Schachspiel ein, das er selbst mit Passion betrieb. ${ }^{742}$ Schon 1962

\footnotetext{
${ }^{739}$ Vgl. Good 1965a; Vinge 1993; Vinge 2013, 366f.; Bostrom 2014, 4; Kurzweil 2005, 22f.; Loh 2018, 112ff.

${ }^{740}$ Good 1965a, 31.

${ }^{741}$ A.a.O., 33. Vgl. a.a.O., 33-37, 78.

${ }^{742}$ Vgl. a.a.O., 33-37.
} 
- auf dem Höhepunkt der Kuba-Krise - erwartet Good, dass die künftigen ultraintelligenten Maschinen (UIM) der Russen und Amerikaner zu einer einzigen Weltregierung verschmelzen könnten und einen dauerhaften Frieden garantieren könnten: »Orakel aller Länder vereinigt euch! ${ }^{743}$ Goods Enthusiasmus nimmt tatsächlich einige Elemente des technologischen Posthumanismus vorweg, selbst wenn diese Visionen noch keine konkrete Programmatik enthalten.

The UIM will enable us to solve any practically soluble problem and we shall perhaps achieve a world peace, the elixir of life, the piecemeal conversion of people into UIPs (ultra-intelligent people), or the conversion of the world's population into a single $\mathrm{UIP}^{744}$

Falls es sich aber erweisen sollte, dass Maschinen vorerst nur "phantastisch nützliche« Aufgaben ohne kreative Intelligenz bewältigen könnten, obliege die Überwindung dieser Entwicklungsgrenze »künstlich herangezüchteter menschlicher Genies «. ${ }^{745}$

Zentral für die Popularisierung der Idee eines superintelligenten Computers waren mehrere Werke der Science-Fiction. Schon 1930 hatte der englische Philosoph und Schriftsteller Olaf Stapledon mit seinem Roman Last and First Man. A Story of the Near and Far Future eine Beschreibung der menschlichen Zukunft in den nächsten Milliarden Jahren präsentiert, die in der Hervorbringung neuer Lebensformen mit immer größeren, gar kosmischen Gehirnen münden werde. ${ }^{746}$ Die Fiktion einer überlegenen Computerintelligenz prägte allerdings niemand so nachhaltig, wie Stanley Kubrick mit HAL 9000, den alles beherrschenden Bordrechner eines Forschungsraumschiffes in dem Film 2001 Space Odyssee. Der Film von 1968 entstand parallel zu dem gleichnamigen Roman von Arthur C. Clarke. Dieser vermenschlichte Computer, der ohne Körper nur durch sein rotes Kameraauge das Schiff überwacht, ist schließlich so sehr von seiner eigenen Perfektion überzeugt, dass er sich keine Fehler eingestehen kann und beginnt, die menschlichen Astronauten zu ermorden. Der Film endet damit, dass es dem letzten Überlebenden der fünf Astronauten gelingt, den Computer abzuschalten. Lange

\footnotetext{
${ }^{743}$ Good 1965b, 191. Auch der Physiker Gerald Feinberg zieht den Vergleich mit den antiken Orakeln heran, vgl. Feinberg 1970, 87-101.

${ }^{744}$ Irving J. Good: Machine Intelligence. In: Impact (UNESCO) 4/1971, zitiert nach Roszak 1994, 39.

${ }^{745}$ Vgl. Irving $1965 \mathrm{~b}, 190 \mathrm{f}$.

${ }^{746}$ V gl. Stapledon 1983, 103ff., 165f., 251-267.
} 
bevor in jeder Vorstadtfamilie wenigstens ein Commodore-64-Homecomputer die Jugendlichen begeisterte, verkörperte der Computer $H A L$ ein nichtmenschliches und nichtgöttliches Gegenüber, das in seiner Macht (innerhalb des Raumschiffes) den Menschen weit übertraf. Die Maschine war bereit, die Menschen auszulöschen und die Mission alleine auszuführen. Marvin Minsky und Irving Good fungierten nicht nur als wissenschaftliche Berater des Regisseurs, sondern wurden bereits in der Romanvorlage von Clarke als Erfinder neuronaler Netze in der fiktiven Computerwissenschaft der 1980er Jahre gepriesen. ${ }^{747}$

Dieses Motiv eines intellektuell überlegenen Supercomputers variiert Stanisław Lem in seiner Erzählung GOLEM XIV (Also sprach Golem) von 1981. ${ }^{748}$ Eigentlich für militärische Zwecke ersonnen, wurde GOLEM XIV nach einigen misslungenen Tests zum Massachusetts Institute of Technology in Boston abgestellt, wo er mehrere philosophische Vorlesungen über den Menschen, sich selbst und den Sinn des Lebens hält (die Vorlesungen werden fiktiv von »Irving T. Creve« ediert). In seiner ersten anthropologischen These konstatiert das Elektronenhirn, dass der Sinn des Menschen nur seine »Botschaft« sei, da die Organismen deren Übermittlung dienen würden und nicht die »Botschaft« den Organismen. Aus der Sicht des GOLEM stellt der Mensch ein »Übergangswesen« dar, das seinen Sinn lediglich darin habe, »vernunftbegabte Wesen zu bauen«. Schließlich prophezeit GOLEM, dass der vernünftige Mensch den natürlichen Menschen opfern werde und den Lauf der Evolution vom homo naturalis zum Computer akzeptiere. ${ }^{749}$

Sowohl HAL 9000 als auch GOLEM XIV erscheinen als übermächtige, geniegleiche Wesen. Die Menschen werden als »Lemuren und Halbaffen « charakterisiert, ${ }^{750}$ während den Computern gottgleiche Möglichkeiten offenstehen: Losgelöst von der Bedingtheit der Materie, kann GOLEM XIV als reiner Geist zu neuen Erfahrungsdimensionen aufbrechen und hüllt sich schließlich in ewiges Schweigen.

${ }^{747}$ Vgl. Kubrick 1968; Clarke 1968. Kaum beachtet wurde dagegen der deutsche Roman Gigant Hirn (1958) von Heinrich Hauser, in dem ein machthungriger Supercomputer seine eigene Nachkommenschaft kreieren will. Vgl. Loh 2018, 114.

${ }^{748}$ Einige Teile des Buches stammen allerdings schon aus Lems Imaginäre Größe von 1973. Mit GOLEM (für General Operator, Longrange, Ethically Stabilized, Multimodelling) spielt Lem einerseits auf die kabbalistische Golem-Sage an, andererseits schafft er auf diese Weise einen Bezug zu Norbert Wieners Buch Gott E Golem Inc. Vgl. Gräfrath 1996, 13f.; Lem 1986, 16.

${ }^{749}$ Vgl. a.a.O., 39, 49, $82 \mathrm{ff}$.

${ }^{750}$ A.a.O., 31f. und in den ersten Szenen von Kubrick 1968. 
Ein halbes Kinojahrhundert nach HAL 9000 rezipiert der Film Transcendence von Wally Pfister aus dem Jahr 2014 bereits explizit die Futurologie des Posthumanismus. Der Held der Geschichte, ist ein charmanter KI-Forscher, der kurz davorsteht, eine kybernetische Superintelligenz zu verwirklichen: "Some scientists refer to this as the singularity. I call it transcendence.« Bei einem Attentat von revolutionären Technikkritikern wird er tödlich verletzt und transferiert seinen Geist in einen Quantencomputer. Mit der neu gewonnenen Macht strebt er scheinbar zur Weltherrschaft und wird daraufhin von seinen Gegnern (und seiner Witwe) zerstört. Erst im Epilog wird deutlich, dass er eigentlich mit Hilfe gesteuerter Nanopartikel alle Weltprobleme lösen wollte. Der Film trug erheblich zur Verbreitung des Konzeptes der Singularität bei. ${ }^{751}$

Um das gleiche Motiv kreist Luc Bessons Film Lucy aus demselben Jahr. Durch eine wundersame Droge entwickelt sich aus der Heldin des Filmes in kurzer Zeit eine überragend intelligente Wesenheit, die schließlich willkürlich die Form der Materie und ihres eigenen Körpers gestalten kann. Als Fortführung der Evolution mutiert sie am Ende der Geschichte zu einem weisen Supercomputer, der sich schließlich in einen USB-Stick verwandelt, der das Wissen der gesamten Menschheit und darüber hinaus aller Lebewesen enthält. ${ }^{752}$

Die Bedeutung der Science-Fiction Literatur und Filme kann kaum überschätzt werden, lassen sich doch hier die in wenigen Jahrzehnten abfolgenden Umdeutungen des Verhältnisses zwischen Mensch und Computer ablesen. Wir müssen uns bewusst sein, dass bis in die Mitte des 20 . Jahrhunderts sowohl das Christentum als auch der Darwinismus den Menschen als die Krone der Schöpfung inthronisierten. Die eugenische Utopie, bewusst einen neuen, höherentwickelten Menschentypus zu zeugen, gipfelte in der westeuropäischen und amerikanischen Praxis der Zwangsterilisationen und der mordenden Rassenpolitik des Nationalsozialismus. Sie zerschellte an den inhärenten, ethischen Konflikten, die diese »Aussonderung minderwertigen Lebens« mit sich brachte. In kybernetischen und fiktionalen Werken der Nachkriegszeit begann sodann die Spekulation über die nächste, nun unschuldig fleischlose Utopie von elektronischen Superhirnen als nächste Stufe der Evolution. Diese wird als überlegen aber auch als Bedrohung des Menschen inszeniert (wie beispielhaft bei Clarke). Die Idee des Transfers des menschlichen Geis-

\footnotetext{
${ }^{751}$ Vgl. Pfister 2014.

${ }^{752}$ Vgl. Besson 2014.
} 
tes in ein virtuelles Dasein besteht zwar schon zuvor, aber erst in den späten 1980er Jahren entwickelt sich mit Hans Moravecs Mind Children, die Vorstellung einer Teilhabe des Menschen an dieser überlegenen Maschinenintelligenz. Paradigmatisch kann man diesen Bedeutungswandel in den Publikationen von Vernor Vinge ablesen: Noch in seinem frühen Text von 1983 markiert die Singularität lediglich den Zeitpunkt, in dem die Menschheit Intelligenzen geschaffen haben werde, die sie selbst übertreffen. 1993 propagiert Vinge dann eine posthumane Ära der superhumanity, in der biologisches und kybernetisches Leben unsterblich miteinander verwoben sein werden. ${ }^{753}$

Dieser evolutionäre Denkrahmen wird auch für die metaphorischen Vergleiche prägend, die post- und transhumanistische Autoren bemühen, um die Überlegenheit der Superintelligenz zu charakterisieren. Die posthumanen Wesen seien demnach für uns Menschen so unverständlich, wie wir es für Goldfische ${ }^{754}$, Felsen, ${ }^{755}$ Bakterien, ${ }^{756}$ sowie für Hunde, Affen und Neandertaler sind. ${ }^{757}$

Da diese Art von evolutionärem Vergleich seit den Rassentheorien und der eugenischen Propaganda des 19. Jahrhunderts verbreitet ist, erscheint es lohnenswert, die kulturellen Anteile an der posthumanistischen Idee der Superintelligenz genauer zu betrachten. Wie wir in den vorangegangenen Kapiteln bereits darlegen konnten, ist der Mensch aus Sicht des Posthumanismus ein informationsverarbeitendes System. Der Fortschrittsbegriff, als Steigerung von Rechenschritten pro Sekunde und Speicherkapazitäten, wurzelt in der aufklärerischen Idee einer Vermehrung der Arbeitsleistung und des Wissens. Die Superintelligenz verspricht nun die unendliche Übersteigerung der herkömmlichen menschlichen sowie kybernetischen Informationsverarbeitung.

Die deutlichste Entsprechung zur Superintelligenz finden wir zweifellos im europäischen und amerikanischen Geniebegriff vor. Bis zur Romantik um 1800 war das Genie ausschließlich dem kreativen Künstler der Malerei, Dichtung und Musik vorbehalten. Das Genie vermochte die Schöpfung der Natur zur Vollkommenheit zu geleiten und zeigte sich in den herausragenden Leistungen einzelner Männer wie Petrarca, Shakespeare, Johann Sebastian Bach oder Michelangelo. Die Ursache

${ }_{753}^{75}$ Vl. Vinge 1983; Vinge 1993.

${ }^{754}$ Vgl. Bostrom 1998b, 401; Hanson 2013, $404 f$.

${ }^{755}$ Vgl. Kurzweil 2005, 136.

${ }^{756}$ Vgl. a.a.O., 367.

${ }^{757}$ Vgl. Yudkowsky 2000; Vinge 1993. 
für das Genie sah man gemäß antiker Tradition in der Inspiration durch das göttlich Ewige. Nur durch diese Teilhabe am Göttlichen könne der sterbliche Mensch selbst etwas Ewiges erschaffen, das über ihn hinausreicht. Der Kuss des Künstlers durch die göttlichen Musen versinnbildlicht in der Antike und seit der Renaissance dieses Verhältnis. Ab dem 19. Jahrhundert umfasst der Geniebegriff zugleich Philosophen, Wissenschaftler und Feldherren (wie Napoleon Bonaparte) sowie seit 1900 auch Erfinder wie Thomas Edison oder Nikola Tesla. ${ }^{758}$ Zudem entsteht das Konzept des Universalgenies, das auf Goethe oder Leonardo da Vinci angewendet wird. Die (auto-)biographische Konstruktion des Genies mit Einblick in Herkunft, Erziehung, heroische Askese sowie Schicksalsschläge wird in dieser Zeit maßgeblich. An die Stelle divinatorischer Inspirationslehren treten rationalisierende Theorien über geniehafte Begabungen und Leistungen, die jedoch nicht minder in einem regelrechten Geniekult des fin de siècle mündeten.

Herausragend als Symbol für die Meister der rationalen Intelligenz ist sicherlich der ab den 1880er Jahren einsetzende Kult um die Schach-Genies, der von posthumanistischen Autoren als Indikator von Intelligenz fortgeführt wird. ${ }^{759}$ Der Fortschritt der künstlichen im Vergleich zur menschlichen Intelligenz wird anhand von Schachprogrammen wie Deep Thought und Deep Blue gemessen und graphisch illustriert. ${ }^{760}$ Interessanterweise galt die Beherrschung des Schachspiels schon zwei Jahrhunderte zuvor als Gradmesser der nach vollkommener Nachahmung der Natur strebenden Automatenkunst. Der Erfinder Wolfgang von Kempelen präsentierte ab 1769 seinen »Schachtürken«, einen orientalisch gewandeten Automaten, der scheinbar eigenständig und meisterhaft Partien gegen menschliche Spieler durchführen konnte. Die Begeisterung für diesen Automaten bewegte auch mehrere Herrscher wie Napoleon Bonaparte und den Preußenkönig Friedrich II. zu einem Spiel. Allerdings war der Apparat »getürkt« - daher stammt vermutlich der bekannte

\footnotetext{
${ }^{758}$ Kurzweil, der in seinem englischen Wikipedia-Artikel als „würdiger Erbe Edisons“ präsentiert wird, nimmt ebenso wie Tesla-Firmengründer Elon Musk Anteil am Charisma des legendären Erfinders Nikola Tesla, wenn er dessen visionäres Zitat an den Beginn seines Prologes von The Singularity is near setzt. Vgl. Kurzweil 2005, 1. Tesla gilt im New Age und verwandten Parawissenschaften als verkanntes Genie.

${ }^{759}$ Vgl. Köhne 2014, 25-33, 58-64.

${ }^{760}$ V gl. Moravec 1988, 8-13, 78, 171; Moravec 1999, 66-72, 186-189; Kurzweil 1999a, 74f., 289f.; Kurzweil 2005, 8, 146, 274-278;
} 
Ausdruck, denn von Kempelen und die späteren Besitzer versteckten einen begabten, menschlichen Spieler im Inneren des Automaten. ${ }^{761}$

Die geschlechtliche Zuordnung des Genies behält übrigens auch nach den Rationalisierungen des 19. Jahrhunderts weitgehend ihre Gültigkeit - die Muse küsst nur Männer. Entsprechende populäre Anthologien der Genies der Menschheit tun sich schwer mit weiblichen Mitgliedern dieses elitären Clubs. Dafür aber präsentiert uns z.B. Friedrich Weissensteiner nach dem etablierten Musen-Schema Die Frauen der Genies (2001).

Es war der britische Begründer der Eugenik, Francis Galton, der mit seinem Werk Heriditary Genius (1869) den ersten Versuch unternahm, außergewöhnliche Begabungen (ability) von britischen Männern zu quantifizieren. Beeindruckt vom Werk seines Cousins Charles Darwin verfolgte Galton die These, dass nicht nur physische Eigenschaften, sondern auch geistige Fähigkeiten biologisch vererbbar wären: ${ }^{.72}$ »By natural ability, I mean those qualifications of intellect and disposition, which urge and qualify a man to perform acts that lead to reputation. ${ }^{763} \mathrm{Die}$ Verteilung von Intelligenz misst Galton statistisch anhand der Anzahl von Richtern, Staatsmännern, Literaten, Wissenschaftlern, Künstlern und Theologen, die einzelne Familien und ganze Gesellschaften hervorgebracht haben. Im Ergebnis attestiert seine universalhistorische Bewertung aller Menschenrassen den attischen Griechen die höchste Verwirklichung dieser Kräfte und positioniert »the negros « ans untere Ende der Skala. Sie hätten keinerlei namhafte Persönlichkeiten hervorgebracht und seien gemäß seinem persönlichen Urteil so kindisch, dumm und schwachsinnig, dass Galton sich schäme, derselben menschlichen Spezies anzugehören. ${ }^{764}$

Ein wichtiges Element für Eugeniker und Rassentheoretiker war zu dieser Zeit die Vermessung des menschlichen Gehirns und dies in zwei Ausprägungen: Zum einen wollte man über Gehirngewicht und Gehirnwindungen das künstlerische und wissenschaftliche Genie erklären, zum anderen die intellektuelle und moralische Entwicklung und Rangordnung der Menschenrassen und sozialen Unterklassen sowie der Kriminellen wissenschaftlich legitimieren. Was mit der seltenen Sektion und Konservierung von Gehirnen berühmter Männer wie Oliver Cromwell oder Lord Byron begann, entwickelte sich Mitte des 19. Jahrhunderts zu

${ }^{761}$ Vgl. Standage 2005.

${ }^{762}$ Vgl. Galton 1972, 45-55. Dt. Ausgabe: Genie und Vererbung, Leipzig 1910.

763 Galton 1972, 77.

${ }^{764}$ Vgl. Galton 1972, 392-395. 
einer systematischen Forschung zunächst an den Göttinger Professorengehirnen und zu einer verbreiteten Praxis bis zum Ersten Weltkrieg (darunter Carl Friedrich Gauß, Ernst Haeckel, Walt Whitman, Justus Liebig, Franz Schubert, Hermann von Helmholtz, Theodor Mommsen, Robert Schumann und Wilhelm von Siemens). ${ }^{765}$ Zum anderen diente die Vermessung des Gehirns und des menschlichen Schädels als vermeintlich wissenschaftliche Evidenz der verschiedenen Menschenrassen. So wurden in vielen europäischen Ländern flächendeckende Stichproben erhoben, um die Überlegenheit der eigenen Nation anatomisch zu beweisen. Folgenschwer war das Engagement der frühen Hirnforschung vor allem für die Konstruktion einer »Negerrasse«, die nicht nur allen anderen Rassen unterlegen sei, sondern nach Ansicht der Polygenisten sogar einer anderen Spezies angehören würde. Obwohl bereits ein gegenteiliger wissenschaftlicher Befund vorlag, behaupteten Anthropologen wie der britische Forscher James Hunt während des amerikanischen Bürgerkrieges, dass das Gehirn des negro deutlich kleiner und rauchig gefärbt sei sowie über weniger Windungen verfüge. ${ }^{766}$

First, that there is as good reason for classifying the Negro as a distinct species from the European as there is for making the ass a distinct species from the zebra; and if we take intelligence into consideration in classification, there is far greater difference between the Negro and Anglo-Saxon than between the gorilla and chimpanzee. ${ }^{767}$

Das Verhältnis des Europäers zu der »Negerrasse« entspräche damit dem zu Kindern, da sich das Gehirn des negro nur bis zum 12. Lebensjahr entwickele und dann auf diesem Niveau verharre. ${ }^{768}$

\footnotetext{
${ }^{765}$ Verstorbene und Angehörige der Berühmtheiten erhofften sich oft die anatomische Bestätigung der zu Lebzeiten sichtbaren Begabungen. Da sich die Gehirne von herausragenden Wissenschaftlern in der fortschreitenden Forschung nicht als different zu jenen von »normalen « Männern, Frauen und anderen Rassen erwiesen, erstarb die Elitegehirnforschung Anfang des 20. Jahrhunderts rasch. Die späte Sektion der Gehirne von Lenin (1927) und Einstein (1955) sind eher dem Personenkult zuzuschreiben. Vgl. Hagner 2004, 249-264, 296-303.

${ }^{766}$ Seine Schrift On the Negro's Place in Nature (1865) spielt natürlich auf Thomas Huxleys Evidence as to Man's Place in Nature (1863) an und liefert während des amerikanischen Bürgerkrieges (1861-65) wichtige Argumente für die Legitimation der Sklaverei. Vgl. Hunt 1864, XVf., LI-LVI; Hunt 1865, 15-19, $26 f ., 53$.

${ }^{767}$ Hunt 1864, XVI.

${ }^{768}$ Vgl. Hunt 1865, 11, 17, 27, 37, 41, 51, 60.
} 
There is no doubt that the Negro brain bears a great resemblance to a European female or child's brain, and thus approaches the ape far more than the European, while the Negress approaches the ape still nearer. ${ }^{769}$

Aus diesem Grund sei der negro von seinem Verhalten her entweder cholerisch oder phlegmatisch. Er sei unfähig eine Zivilisation mit Wissenschaftlern und Künstlern hervorzubringen und kenne nur brutalstes Barbarentum, Aberglaube, Folter und Grausamkeit. Daher sei seine natürliche Rolle dem Europäer untergeordnet und wenn er als Sklave von Afrika nach Amerika käme, sei dies wie aus der Hölle ins Paradies zu gelangen. Es ist dabei offensichtlich, wie die Verteidigung der Sklaverei in dieser Zeit Hand in Hand mit der politischen und sozialen Entrechtung der Frauen in Europa und den Vereinigten Staaten geht. ${ }^{770}$

Die Ausprägungen der frühen Gehirnforschung im 19. Jahrhundert hatte damit in zwei Richtungen eine immense Bedeutung für die eugenische Politik. In dieser Logik sahen sich die Vertreter der biologisch wertvollsten Gruppe stets durch eine Degeneration ihres Erbgutes bedroht. Die unkontrollierte Vermehrung der unerwünschten sozialen Klassen (bei Galton die undesirable classes) sowie die Vermischung der Weißen mit den minderwertigen Rassen müsse verhindert werden. ${ }^{771}$ Zwangssterilisationen, Eheverbote und (im Nationalsozialismus) die Ermordung »unwerten Lebens « sollten diese Degeneration verhindern. Positiv propagierten Eugeniker das Ziel eines reinrassigen »genialen Kollektivs«, das als »Herrenrasse« die Geschicke der künftigen Menschheit bestimmen sollte und im Werk des germanophilen Rassentheoretikers Houston Stewart Chamberlain wohl seinen deutlichsten Ausdruck fand. ${ }^{772}$ Neuere Eugeniker, wie Robert K. Graham, der von 1980 bis zu seinem Tod 1997 eine Samenbank für (männliche) Nobelpreisträger unterhielt, folgen dieser Faszination für »geniale Gehirne «. ${ }^{773}$ Auch noch im Werk von Teilhard de Chardin spiegelt sich die eugenische Faszination für die Gehirne: Die Vervollkommnung des menschlichen Gehirns werde sich seiner Ansicht nach einerseits als »kollektive Cerebralisation« durch die Vervielfachung

\footnotetext{
${ }^{769}$ Hunt 1865, $16 f$.

${ }^{770}$ Vgl. Hunt 1864, XVf., LI-LVI; Hunt 1865, 15-19, 26f., 38, 53.

${ }^{771}$ Vgl. Galton 1909, 310-313.

${ }^{772}$ Wie Edwin Black aufzeigt, ist diese rassenpolitische Ideologie neben Deutschland v.a. in den Vereinigten Staaten umgesetzt worden. Vgl. Köhne 2014, 361-401; Black 2004.

${ }^{773}$ Mit denselben Argumenten wie einst Francis Galton will Robert K. Graham in seinem Buch The Future of Man (1981) den durch genetische Degeneration drohenden Untergang der Menschheit verhindern.
} 
der Reize in der globalen Gesellschaft beschleunigen und zusätzlichen Antrieb durch die schon damals »erstaunliche Leistung der Elektronenautomaten « und die Fortschritte der Kybernetik erfahren. Der einzelne Mensch werde so von der Aktivierung schon vorhandener, aber noch nicht genutzter Neuronen des Gehirns profitieren - »... oder aber, wer weiß? auch dadurch, daß durch mechanische, chemische oder biologische Einwirkungen eventuell sogar völlig neue Organe ins Leben gerufen werden. ${ }^{774}$

Während die meisten post- und transhumanistischen Autoren, die die Ankunft einer kybernetischen Superintelligenz bzw. Singularität erwarten, sich nicht explizit für eine erneuerte Eugenik stark machen, erweist sich Nick Bostrom als glühender Befürworter. Durch ein gentechnologisches Enhancement könne man den Menschen gesünder, geistreicher und glücklicher machen:

We might speculate, instead, that germ-line enhancements will lead to more love and parental dedication. Some mothers and fathers might find it easier to love a child who, thanks to enhancements, is bright, beautiful, healthy, and happy. ${ }^{775}$

Die höhere Intelligenz würde den genetisch verbesserten Menschen bessere berufliche Positionen und ein höheres soziales Ansehen verschaffen. Im Gegensatz zu den problematischen Eugenikprogrammen der Vergangenheit wäre das zukünftige Enhancement einer liberalen Gesellschaft nur der freien Entscheidung von Individuen überlassen. ${ }^{776}$ Der Staat sollte allerdings in Erwägung ziehen, eine eugenische Steigerung von Intelligenz für das Gemeinwohl steuerlich zu begünstigen, auch um eventuellen Ungleichheiten entgegenzuwirken. ${ }^{777}$ Die dystopische Perspektive, nach der nur Reiche den Zugang zu genetischer Verbesserung hätten, relativiert Bostrom. Die schlimmste Dystopie wäre die gesellschaftliche Stagnation: »... humanity could get permanently stuck in a not-very-good state, having foolishly changed itself to lack any desire to strive for something better. $" 778$

\footnotetext{
774 Teilhard 1961, 118. Vgl. a.a.O., 114-119.

775 Bostrom 2003a, 9. Vgl. Loh 2018, 177.

${ }^{776}$ Die durch pränatale Diagnostik ermöglichte Abtreibungspraxis von Millionen weiblicher Föten v.a. in Indien und China, die sogar staatlichen Bemühungen entgegenläuft, erscheint Bostrom nicht erwähnenswert, vermutlich da sie die euphemistische Logik einer völligen Deregulierung konterkarieren würde.

777 Vgl. Bostrom 2003a, 10-15.

${ }^{778}$ A.a.O., 20.
} 
Ray Kurzweil wiederum verweist ausdrücklich auf die Resultate der eugenisch motivierten Hirnforschung, wenn er betont, dass Einsteins Scheitellappen besonders ausgeprägt gewesen seien und die Gehirne von Serienmördern Fehler aufwiesen. Er verspricht jedenfalls, dass Menschen der Zukunft nicht wie Einstein auf eine partielle Begabung beschränkt sein werden. ${ }^{779}$

Seit den frühen Schriften Moravecs teilen alle posthumanistischen Autoren und viele Transhumanisten die Faszination für die erhoffte künstliche Intelligenz, die das menschliche Denken »transzendieren« werde. Die Bewunderung für die Kybernetik spiegelt sich in den metaphorischen Benennungen von Computern wider: Der von Alan Turing konzipierte, erste elektronische Computer trägt den Namen Colossus und der Kybernetiker Edmund Berkeley spricht in seinem Buch von 1949 von den Giant Brains, den Hirnen der Giganten - obwohl es sich nur um Zwergenhirne handelte, wie Arthur C. Clarke spitzzüngig bemerkt. ${ }^{780}$ Diese Faszination für künstliche Genie-Gehirne knüpft historisch exakt da an, wo eugenische Züchtungsphantasien nach 1945 durch die NS-Rassenpolitik und Experimente diskreditiert waren. ${ }^{781}$ Aber erst mit dem Posthumanismus und der von Moravec vorgeschlagenen transmigration entstand die Idee einer Teilhabe des Menschen an dieser übermenschlichen Intelligenz. Im Falle von Bostrom ist die Patenschaft der historischen Eugenik unverkennbar. Über den Eugeniker Julian Huxley, den ja Bostrom stets als den Urheber des Transhumanismus herausstellt, besteht eine direkte ideengeschichtliche Verbindung. Und auch der Übermensch ist in Bostroms Denkfiguren präsent: In einem Interview von 2016 beschreibt er die Entdeckung Nietzsches in seiner Jugend als ein "Epiphanie-Erlebnis«, als Erweckung aus einem schlafwandlerischen Leben in Unwissenheit, das ihn schließlich zur Philosophie, Mathematik und zum Transhumanismus geführt habe. ${ }^{782}$

Mir liegt fern zu behaupten, dass Trans- und Posthumanisten Rassisten und Frauenfeinde seien. Die kulturellen Deutungsmuster des technologischen Posthumanismus jedoch, die in der Faszination für die Super-

${ }^{779}$ Vgl. Kurzweil 2005, 202.

${ }^{780}$ Vgl. Bösch 2017, 227f.; Clarke 1960, 213.

${ }^{781}$ Vgl. Hagner 2004, 288-296.

${ }^{782}$ Die Rolle Nietzsches für den Transhumanismus schätzt Bostrom allerdings gering ein. Vgl. Adams 2016; Bostrom 2005. Im Gegensatz dazu vermeidet Kurzweil eugenische Spekulationen und gründet die mittelfristige Verbesserung des menschlichen Körpers auf Nanoroboter im Blutkreislauf. Vgl. Kurzweil 2005, 226-258. 
intelligenz zum Ausdruck kommen, sind eine direkte Fortsetzung der Vorstellungen von Genie, Gehirn und Rasse in Westeuropa und den Vereinigten Staaten des vergangenen Jahrhunderts. Die biologischen Vergleichsmetaphern der Rassentheoretiker um 1900 und der heutigen Posthumanisten belegen die Persistenz dieser Seinsordnungen. Krönte damals der geniehafte, nordische bzw. arische Mann die Entwicklung der Evolution, so ist es heute die erwartete, posthumane Superintelligenz. Die Skala dieser Seinsordnung - Gehirngrößen bzw. mathematisierte Denkleistungen - ist unverändert geblieben.

Es ist daher kein Zufall, dass der Transhumanismus weitgehend eine Bewegung weißer Männer ist. Die großen Transhumanisten-Tagungen, die ich um das Jahr 2000 besuchte, hatten schätzungsweise einen Frauenanteil von 1:50 und wiesen kaum eine ethnische Diversifizierung auf. Mit Ausnahme der Transgender-Transhumanistin Martine Rothblatt und der Künstlerin Natasha Vita-More gibt es kaum Frauen, die kontinuierlich Beiträge zur transhumanistischen Debatte geleistet haben. Im wichtigen transhumanistischen Journal of Evolution and Technology, in dem Aktivisten aber auch neutrale Wissenschaftler publizieren, finden sich unter den 199 Artikeln, die von 1998 bis 2018 erschienen sind, nur 15 Artikel von Autorinnen oder Co-Autorinnen (das sind 7,5\%). ${ }^{783}$ Von den 67 Beiträgen auf den Tagungen des Extropy Institutes (1994-2004) stammen nur 11 von Frauen $(16 \%)$ und Socrates führte auf seinem Singularity Weblog seit 2010 nur 8 von 226 Interviews (3,5\%) mit Frauen (davon allein drei mit Natasha Vita-More). ${ }^{784}$

Es lässt sich nur vermuten, dass Frauen und Afroamerikaner daher sensibler reagieren, wenn - wieder einmal - weiße Männer ein neues Menschheitsideal proklamieren, selbst wenn das Menschsein diesmal in einer posthumanen Größe aufgeht. In diesem Punkt sind diejenigen Vertreter des Post- und Transhumanismus, die die Superintelligenz und Singularität ins Zentrum ihrer Utopien stellen, philosophisch und historisch vollkommen unterreflektiert.

${ }^{783}$ Allein sechs der Beiträge gehen jedoch auf eine wissenschaftliche Tagung aus dem Jahr 2013 in Yale zur Frage der nonhuman personhood zurück, an der mehrere Tierrechtlerinnen und Ethikerinnen beteiligt waren (Vol 24, 3/2014). Berücksichtigt man dies, so verringert sich der Frauenanteil auf 4,5\%.

${ }^{784}$ Vgl. http://www.extropy.org und https://www.singularityweblog.com (Stand 27.08.2018). 


\subsection{Omega}

Our speculation ends in a supercivilisation, the synthesis of all solar-system life ..., spreading outward from the sun, converting nonlife into mind ... This process, possibly occurring now elsewhere, might convert the entire universe into an extended thinking entity, a prelude to even greater things. ${ }^{785}$

Hans Moravec

Der Transfer des menschlichen Geistes in einen Computer ist für alle posthumanistischen Autoren nur ein Zwischenschritt auf dem Weg zu einem planetaren oder gar einem kosmischen Bewusstsein. Hierbei werden vor allem Elemente einer theologischen Heilsgeschichte rezipiert, die in ihrer prägnantesten Form von Pierre Teilhard de Chardin ausformuliert und von Marshall McLuhan für den Mediendiskurs aufbereitet wurden.

\subsubsection{Das kosmische Bewusstsein}

Auch Marvin Minsky sowie Gregory Stock und Kevin Warwick sprechen der Möglichkeit, dass sich die verschiedenen Identitäten im Computerspeicher austauschen und zu einem kollektiven Bewusstsein, einer neuen society of mind, verschmelzen könnten, eine hohe Wahrscheinlichkeit zu: »Some future options have never been seen: Imagine a scheme that could review both your and my mentalities, and then compile a new, merged mind based upon that shared experience. ${ }^{786}$

Hans Moravec glaubt, dass schließlich die gesamte Erde als eine Art Reservat von einem gigantischen Computer simuliert wird: Geistesgrößen zwischen Sherlock Holmes und Gott würden dann alle möglichen Vergangenheiten rekonstruieren, die als dauerhafte Simulationen die letzten Menschen und alle Auferstandenen beheimaten würden. ${ }^{787}$ Die unverkörperten, posthumanen Individuen wären dann gemäß Moravec sogar imstande, in den Geist eines virtuellen Delphins oder Elefanten einzu-

\footnotetext{
${ }^{785}$ Moravec 1988, 116.

${ }^{786}$ Marvin Minsky: Why Computer Science Is the Most Important Thing That Has Happened to the Humanities in 5,000 Years. Öffentliche Vorlesung, Nara, Japan, 15.05.1996. Zitiert nach Hayles 1999, 244f. Vgl. Warwick 2000, 151; Stock 1993; Schenkel 2000, 111 .

${ }^{787}$ Vgl. Moravec 1999, 164-168.
} 
tauchen: »Concepts of life, death, and identity will lose their present meaning as your mental fragments and those of others are combined, shuffled, and recombined ... $\ll^{788}$

Diese Vision von miteinander vernetzten, virtuellen Persönlichkeiten wird von Frank Tipler, Hans Moravec und Ray Kurzweil noch überboten. Denn während die Menschen als perfekte Simulationen in den Computern die Zeitalter überdauern, sollen Roboter und künstliche Intelligenzen die Herausforderungen der realen Welt meistern. ${ }^{789}$ Robotersonden würden dann das Sonnensystem, die Galaxie und zuletzt das gesamte Universum kolonisieren und es dadurch in belebten Geist verwandeln. ${ }^{790}$ Haben die künstlichen Lebewesen erst die Kontrolle gewonnen, könnten sie im Interesse der Menschheit und des Kosmos das Verhalten ganzer Galaxien steuern und so der steigenden Entropie, dem Wärmetod des Universums, entgegenwirken: » ... life must engulf the entire universe if it is to have the power to force the universe to move in this unlikely way. " ${ }^{791}$ Wenn das Ziel der Evolution Tipler zufolge die Bewahrung des Lebens im Universum ist, dann verbindet sich damit die auf der Maximierung des möglichen Wissens beruhende Vervollkommnung des Menschen bzw. seiner Nachfahren - das Leben werde Allgegenwart, Allmacht und Allwissenheit erlangen: ${ }^{792}$

At the instant the Omega Point is reached, life will have gained control of all matter and forces ...; life will have spread into all spatial regions in all universes which could logically exist, and we will have stored an infinite amount of information, including all bits of knowledge which is logically possible to know. And this is the end. ${ }^{793}$

Ohne nun in irgendeiner Weise den theistischen und anthropozentrischen Kontext zu berücksichtigen, knüpft Moravec auch seine eigenen Überlegungen zu einem künftigen »super-consciousness« an Tiplers Omega-Punkt Theorie an: »We truly exist because our actions lead ultimately to this >Omega Point< (a term borrowed from the Jesuit paleontologist and radical philosopher Tielhard [sic!] de Chardin).." ${ }^{794}$

\footnotetext{
${ }^{788}$ A.a.O., 115.

${ }^{789}$ Vgl. a.a.O., 87.

${ }^{790}$ Vgl. Moravec 1999, 116, 164-168.

${ }^{791}$ Tipler 1995, 65; vgl. a.a.O., 55-65.

${ }^{792}$ Vgl. a.a.O., 153f.; auch Barrow \& Tipler 1986, 523.

${ }^{793}$ Barrow \& Tipler 1986, 677.

${ }^{794}$ Moravec 1999, 202. Vgl. auch Moravec 1979.
} 
Kurzweil implementiert erst 2005 in seinem Singularitätsbuch eine kosmische Perspektive und rahmt seine Überlegungen mit der neuerlichen Einsicht, dass es außer der Menschheit wohl keine weiteren intelligenten Lebensformen im Universum gebe. Er verweist auf das anthropische Prinzip und schließt: »We don't yet see evidence that such a community beyond Earth exists. The community that matters may be just our own unassuming civilization here. ${ }^{795}$ In seinem vorhergehenden Buch von 1999, The Age of Spiritual Machines, hielt er die Existenz von außerirdischem, intelligentem Leben noch für wahrscheinlich. ${ }^{796}$

Gemäß seiner jüngsten Prognose werde nun die aus der Menschheit hervorgehende, technologische Zivilisation mit mindestens (!) Lichtgeschwindigkeit das Universum durchkreuzen und die »dumme Materie des Kosmos« in »kluge Materie« verwandeln. Materie werde dadurch so intelligent, dass sie dann auch die Gesetzmäßigkeiten der Physik überwinden könne:

Such a civilization will overcome gravity ... and other cosmological forces ... and engineer the universe it wants. This is the goal of the Singularity. «797 ${ }^{7 W e}$ will determine our own fate rather than have it determined by the current >dumb, simple, machinelike forces that rule celestial mechanics. ${ }^{798}$

Das ganze Universum werde auf diese Weise mit Intelligenz gesättigt. Daraus leitet Kurzweil die besondere Bedeutung der Erde und die Hervorbringung des letzten Computers (the ultimate computer) ab. Futurologie und die Metaphysik Schwarzer Löcher fließen ineinander:

If we increase the mass enough, its gravitational force becomes strong enough to cause it to collapse into a black hole. So a black hole can be regarded as the ultimate computer. Of course, not any black hole will do. Most black holes, like most rocks, are performing lots of random transactions but no useful computation. But a well-organized black hole would be the most powerful conceivable computer in terms of cps per liter. ${ }^{799}$

Wer jetzt annimmt, dass der rational auftretende Denker Ray Kurzweil zwar die heilsgeschichtliche Kosmologie Tiplers kopiere, jedoch die theologischen Elemente meide, der wird enttäuscht sein. Punktuell be-

\footnotetext{
${ }^{795}$ Kurzweil 2005, 362.

${ }^{796}$ Vgl. Kurzweil 1999, 260.

${ }^{797}$ Kurzweil 2005, 364.

${ }^{798}$ A.a.O., 29. Vgl. a.a.O., 342-365, 390.

${ }^{799}$ A.a.O., 362.
} 
tont Kurzweil zwar, dass er kein neues Dogma begründen will und der Singularitarianismus auf dem Verständnis rein wissenschaftlich erkannter Trends beruhe. ${ }^{800}$ Andererseits erweckt er aber an zahlreichen Stellen den Eindruck, als wolle er tatsächlich einen neuen religiösen Glauben stiften. In einem Gespräch mit Bill Gates, das Kurzweil in seinem prophetischen Buch wiedergibt (oder imaginiert?), unterstreicht er, dass die Menschheit eine neue Art von Religion brauche, die über das Rationalisieren des Todes hinausgehe. Diese Forderung hatte bekanntermaßen bereits Julian Huxley in seinem Plädoyer für einen evolutionären Humanismus aufgestellt. Statt eines charismatischen Führers bräuchte man nun ein charismatisches Betriebssystem:

BILL: Ha, we've already got that. So is there a God in this religion?

RAY: Not yet, but there will be. Once we saturate the matter and energy in the universe with intelligence, it will »wake up«, be conscious, and sublimely intelligent. That's about as close to God as I can imagine.

BILL: That's going to be silicon intelligence, not biological intelligence.

RAY: Well, yes, we're going to transcend biological intelligence. ${ }^{801}$

Kurzweil umgeht zwar den Begriff des Omega-Punktes und jeglichen Bezug auf das Christentum oder Teilhard de Chardin. Die evolutionsgeschichtlichen Phasen (Materie, Biologie, Geist) sowie die Umwandlung des Universums in einen gigantischen Computer entsprechen konzeptuell jedoch exakt den Entwürfen Teilhard de Chardins bzw. Frank Tiplers (ohne dass er Tipler jedoch der Erwähnung für würdig befindet).

Die posthumanistischen Vorstellungen einer globalen oder gar kosmischen, denkenden Einheit entwickeln sich ab den 1990er Jahren parallel zu spirituellen und säkularen Deutungen des Internet. Einer der wichtigsten Protagonisten ist in diesem Zusammenhang der amerikanische Informatiker Mark Pesce, der 1994 den ersten verbindlichen Standard für die visuelle Darstellung virtueller Realität im Internet erfand (Virtual Reality Modeling Language, VRML). Zahlreiche Publikationen, Fachtagungen und Medienauftritte nutzt er nicht nur, um seine informationstechnischen Innovationen zu präsentieren, sondern verbindet die technischen Aspekte mit seiner bildhaften Vision vom Internet. So führt er in seinem Buch Playful World mitten in der Schilderung über die Erfindung von VRML den Jesuiten Teilhard de Chardin als den bedeutendsten, jedoch fast vergessenen Propheten des Internet ein:

${ }^{800}$ Vgl. Kurzweil 2005, 370-374.

${ }^{801}$ Kurzweil 2005, 375. 
[...] no one foresaw the importance and comprehensive impact of the World Wide Web. But, over fifty years ago, one fairly obscure scientist did predict a coming transformation of the human mind, the birth of collective intelligence, and the emergence of a new way of knowing. ${ }^{802}$

Mit der Idee, dass nun bald alle Menschen durch das Internet geistig miteinander verbunden seien, adaptiert Pesce das von Teilhard entwickelte Konzept der Noosphäre:

We can't know for sure if the Web is the same thing as the noosphere, or if the Web represents part of what Teilhard envisioned. But it feels that way ... If Teilhard was right, the Web is part of our evolution, as much an essential element of humanity as our acute eyes, our crafty hands, and our wonderful brains. ${ }^{803}$

Wenn das Internet durch Pesce mit der Noosphäre gleichgesetzt wird, erscheint es nicht mehr als eine »alltägliche« medientechnische Neuerung, wie seinerzeit beispielsweise das Radio oder das Fernsehen. Das Internet verweist dann durch seine Außerordentlichkeit auf eine spirituelle Dimension:

Meine Arbeit rund um WebEarth hat viel mit Spiritualität zu tun, mit der Idee der Gaia, des Planeten als Lebewesen. Wenn ich diese Arbeit Leuten zum ersten Mal zeige, packt es sie oft richtig; manche weinen ... Mit VRML wird die Noosphäre viel greifbarer werden. Die Leute werden sie als echten Ort erkennen, obwohl sie nur aus Daten besteht. ${ }^{804}$

Somit verknüpft Pesce die Ideen Teilhards gleichzeitig mit der GaiaTheorie, die für einige New Age-Vertreter wie auch für ökologische Vordenker wie Ken Wilber, Terence McKenna und Buckminster Fuller von zentraler Bedeutung ist. ${ }^{805}$ Das Internet wird auf diese Weise als organischer Teil der Erde konzipiert, das sich ganz natürlich im Lauf der Evolutionsgeschichte entwickeln musste.

Die wohl umfassendste Rezeption von Teilhards Ideen im Kontext des Cyberdiskurses hat jedoch die amerikanische Theologin Jennifer Cobb

${ }^{802}$ Pesce 2000, 164 .

${ }^{803}$ A.a.O., 170.

${ }^{804}$ Pesce im Interview mit Bennahum 1997.

${ }^{805}$ Ausgehend von James Lovelocks Werk Gaia - a new Look at Life on Earth (1979) wird die Erde mit all ihren Lebewesen als ganzer Organismus verstanden. Diese Idee, teils wiederum in Verbindung mit Teilhards Schriften, hatte großen Einfluss auf das New Age, ökologische Theorien und weitere religiöse Strömungen der Gegenwart. Pesce widmet sein Buch Playful Worlds sogar dem amerikanischen New Age-Denker Terence McKenna. 
vorgenommen. In ihrem 1998 erschienenen Buch Cybergrace. The Search for God in the Digital Space versteht Cobb den Cyberspace als unbegrenzten Möglichkeitsraum für die Menschheit, um sich intellektuell, spirituell und emotional selbst entfalten zu können. Wenn die Bedeutung der neuen Computertechnologien richtig verstanden würde, könne die Welt erneut als eine göttliche Realität jenseits der Dualismen von Materie und Geist erfahren werden. ${ }^{806}$

In the ongoing process of spiritual evolution, cyberspace has a special role to play ... In this vision, the spiritual basis of the universe is understood as creative events unfolding in time [...] Cyberspace can help guide us toward a reconciliation of the major schisms of our time, those between science and spirit, between the organic world and the world that we create. ${ }^{807}$

Cobb rezipiert weitestgehend Teilhards mehrstufiges Evolutionsmodell mit besonderer Betonung des Entwicklungssprungs von der Biosphäre zur Noosphäre - sie ist allerdings der Meinung, dass Teilhards Ideen überhaupt erst im Hinblick auf die Entstehung des Cyberspace verständlich werden:

This distinctly nontraditional evolutionary idea may strike us as odd until we consider the phenomenon of cyberspace, that electronically supported layer of human consciousness that now encircles the globe. ${ }^{808}$

Diese religiös oder spirituell aufgeladenen Mediendeutungen konvergieren mit säkularen Visionen von Futurologen und postmodernen Medientheoretikern.

John Desmond Bernal spekuliert schon 1929 über ein universales, körperloses Bewusstseins (natürlich noch ohne Bezüge zur Kybernetik):

Finally, consciousness itself may end or vanish in a humanity that has become completely etherealized, losing the close-knit organism, becoming masses of atoms in space communicating by radiation, and ultimately perhaps resolving itself entirely into light. ${ }^{809}$

Auch bereits 1970 knüpft der russische Kybernetiker Valentin Turtschin in seiner Monographie The Phenomenon of Science - A Cybernetic Approach to Human Evolution an Teilhards Idee der Noosphäre an. In diesem frühen

${ }^{806}$ Cobb 1998, Cybergrace, $8 \mathrm{ff}$.

${ }^{807}$ A.a.O., 43.

${ }^{808}$ A.a.O., 85.

${ }^{809}$ Bernal 1929, 21. Vgl. auch Bernal 1969, $855 f f$. 
Werk formuliert er die Idee eines künftigen, synthetischen Bewusstseins, in dem die menschlichen Individuen aufgehen und Unsterblichkeit erlangen würden. Die von ihm, Francis Heylighen und Cliff Joslyn 1989 initiierte Organisation Principia Cybernetica und sein Cybernetic Manifesto von 1989 blieben trotz ähnlicher Ziele (Unsterblichkeit, global brain) im trans- und posthumanistischen Kontext weitgehend unbeachtet. ${ }^{810} \mathrm{Hey}$ lighen und der französische Medienphilosoph Pierre Lévy interpretieren das Internet als erste Ausgestaltung eines "globalen Gehirns« oder einer »kollektiven Intelligenz«. ${ }^{811}$ Das Internet und weitere Kommunikationsmittel, die einen virtuellen Raum konstruieren, würden demnach Kommunikation von ihren materiellen, sozialen und physischen Hindernissen und Beschränkungen im realen Raum befreien. Der Cyberspace wird dann zur Metapher eines befreiten und egalitären Menschentums, das universal, aber demokratisch organisiert sei:

Cyberspace gives shape to a new form of the universal: the universal without the totality. Filled with the resonance of Enlightenment philosophy, its universality stems from the fact that it maintains a profound relationship with the idea of humanity. ${ }^{812}$

Ohne einen durch theologische Implikationen gefährdeten Bezug auf Teilhard de Chardin entwirft Lévy die Vision einer neuen, intelligenten Entität, die aus der Interaktion und Kommunikation des menschlichen Kollektivs hervorgehen wird.

Before you lies cyberspace with its teeming communities and the interlaced ramification of its creations, as if all of humankind's memory were deployed in the moment: an immense act of synchronous collective intelligence, converging on the present, a silent bolt of lightning, diverging, an exploding crown of neurons. $^{813}$

${ }^{810}$ Turtschin ging 1973 ins US-amerikanische Exil und blieb auch in seinen Cyberutopien stets sensibel gegenüber totalitären Tendenzen, die er in der UdSSR offen kritisiert hatte. Vgl. Turchin 1977, 85f., 259-261.

${ }^{811}$ Vgl. Krüger 2015, 79.

${ }^{812}$ Lévy 2001, 100.

${ }^{813}$ A.a.O., 236. 


\subsubsection{Teilhard de Chardin, McLuhan und die Noosphäre}

Teilhard de Chardin wird sowohl von Posthumanisten als auch von Cyberphilosophen und -theologinnen zum großen Vordenker des Internet, eines globalen Gehirns und einer kybernetisch-kosmischen Heilsgeschichte stilisiert. Zwar hatten zu Beginn des 20. Jahrhunderts auch Samuel Alexander und Alfred N. Whitehead kosmische Evolutionstheorien entwickelt, die auf die Verwirklichung Gottes hinauslaufen, jedoch wurde im Mediendiskurs nur Teilhard wahrgenommen. Diese starke Rezeption innerhalb des Mediendiskurses ist an sich äußerst bemerkenswert, denn Teilhard de Chardin hat sich - soweit ich dies beurteilen kann - in seinem umfassenden Werk, abgesehen von zwei peripheren Anmerkungen, nie zu Medien- oder Computertechnik geäuBert. Dass er dennoch zur zentralen Referenz für diese weitreichenden Medienutopien wurde, ist meines Erachtens auf den kanadischen Kommunikationswissenschaftler Marshall McLuhan zurückzuführen, der Teilhards Ideen bereits eklektisch aufgegriffen und für die Interpretation von Medien »fruchtbar « gemacht hatte. In populären Publikationen und teils auch in wissenschaftlichen Beiträgen werden beide Theoretiker dann auch in einem Atemzug genannt: McLuhan wird ein »Flirt« mit Teilhards Ideen zugeschrieben und ihre Theorien seien großenteils deckungsgleich. ${ }^{814}$ Es gilt daher im Folgenden, etwas Licht in das Verhältnis zwischen McLuhan und Teilhard de Chardin zu bringen, um schließlich die populäre und offenbar dominante Synthese von Teilhards Konzept der Noosphäre mit den Internetutopien erklären zu können.

Wie bereits geschildert, betrachtet Teilhard de Chardin die Evolution als eine Entfaltung des Geistes. Die ersten Hominiden unterscheiden sich durch die Fähigkeit des »Ichbewusstseins« von den vorhergehenden Primaten:

\footnotetext{
Als sich der Instinkt eines Lebewesens zum ersten Mal im Spiegel seines Selbst erblickte, machte die ganze Welt einen Schritt vorwärts ... Ebenso ausgedehnt, doch, wie wir sehen werden, noch mehr kohärent als alle vorausgehenden Schichten, ist es wirklich eine neue Schicht, die >denkende Schicht<, die sich seit ihrer ersten Blüte am Ende des Tertiärs oberhalb der Welt der Pflanzen und Tiere ausbreitet: außer und über der Biosphäre eine Noosphäre. ${ }^{815}$
}

${ }^{814}$ Vgl. Curtis 2005, $164 \mathrm{f}$.

815 Teilhard de Chardin 1959, $168 f$. 
Mit der wissenschaftlichen und philosophischen Dominanz des Abendlandes seit der Zeitenwende begann Teilhard zufolge der "Zusammenfluss des Denkens«, die »Planetisierung der Noosphäre«:

[...] dank dem wunderbaren biologischen Ereignis der Entdeckung der elektromagnetischen Wellen findet sich von nun an jedes Individuum (aktiv und passiv) auf allen Meeren und Kontinenten gleichzeitig gegenwärtig und verfügt über dieselbe Ausdehnung wie die Erde. ${ }^{816}$

Ebenso wie andere Eugeniker seiner Zeit ist Teilhard von der sukzessiven Höherentwicklung des menschlichen Gehirns überzeugt und schlägt hier die Brücke zwischen menschlichen und künstlichen Gehirnen. Durch diese »kollektive Cerebralisation«, nämlich den Aufstieg der Wissenschaft und der Kybernetik, würde sich, so hofft Teilhard, die Vervollkommnung des menschlichen Gehirns in der künftigen Evolutionsgeschichte beschleunigen, vor allem wenn auch die Methoden der Eugenik Anwendung finden würden. Den Prozess der Evolution begreift er als Aufstieg des Bewusstseins und diesen Vorgang wiederum als »Einigungswirkung«, denn nur wenn alle Völker und alle Schichten zusammen nach einem Ziel drängen, ließe sich die psycho-biologische Entwicklung hin zu einer »Mega-Synthese« der Menschheit verwirklichen: ${ }^{817}$

Ein harmonisches Bewußtseinskollektiv, das einer Art Überbewußtsein gleichkommt. Die Erde bedeckt sich nicht nur mit Myriaden von Denkteilchen, sondern umhüllt sich mit einer einzigen denkenden Hülle und bildet funktionsmäBig ein einziges umfassendes Denkatom von siderischem Ausmaß. ${ }^{818}$

Nach Ansicht Teilhards wird die Noosphäre durch das kollektive Zusammenwirken ihrer Teile schließlich »am Ende der Welt« ihren Konvergenzpunkt erreichen, an dem sich die Summe des individuellen Bewusstseins wieder zu einem neuen, überpersönlichen Bewusstsein bündelt. Dieser von Teilhard als Omega bzw. Gott-Omega bezeichnete Konvergenzpunkt könne nur durch die Kraft der universalen Liebe erreicht werden. Durch die Gegenwart Christi falle der Menschheit diese außerordentliche Rolle im Kosmos zu, Omega zu verwirklichen:

\footnotetext{
${ }^{816}$ A.a.O., 232.

${ }^{817}$ Vgl. Teilhard de Chardin 1961, 118; Teilhard de Chardin 1959, 229-286.

${ }^{818}$ A.a.O., 244.
} 
Wenn die Welt konvergent ist, und wenn Christus ihr Zentrum einnimmt, dann ist die Christogenese des heiligen Paulus und des heiligen Johannes nichts anderes und nichts Geringeres als die gleichermaßen erwartete wie überraschende Fortsetzung der Noogenese, in der für unsere Erfahrung die Kosmogenese gipfelt ... Das Christentum allein, ganz allein auf der modernen Erde zeigt sich fähig, in einem einzigen, aus dem Leben entspringenden Akt das All und die Person zur Synthese zu bringen. ${ }^{819}$

Teilhard de Chardin führt auf diese Weise ein dynamisches Gottesverständnis weiter, das sich im 19. Jahrhundert im deutschen Idealismus herausgebildet hatte. ${ }^{820}$ In der teils sehr polemisch geführten Debatte mit dem Philosophen Friedrich Heinrich Jacobi, der vom traditionellen Bild eines vollkommenen und unwandelbaren Gottes überzeugt war, vertrat F. W. J. Schelling die Auffassung eines sich entfaltenden Gottes und legte hiermit den Grundstein für eine Synthese zwischen Metaphysik und Evolution. Jacobis Vorwurf des Atheismus wies Schelling zurück, da er Vernunft und Wissenschaft als Mittel der fortschreitenden Gotteserkenntnis betrachtete:

Ich setze Gott als Erstes und als Letztes, als A und als O, aber als das A ist er nicht, was er als das $\mathrm{O}$ ist, und inwiefern er nur als dieses - Gott sensu eminenti ist, kann er nicht auch als jenes Gott in dem naemlichen Sinne seyn, noch auf's Strengste genommen, Gott genannt werden, es waere denn, man sagte ausdruecklich, der unentfaltete Gott, Deus implicitus, da er als O Deus explicitus ist ... Aber eben dieses Daseyn Gottes als persoenlichen Wesens ist Gegenstand recht eigentlich der Wissenschaft, und nicht nur ueberhaupt, sondern ihr hoechster, letzter Gegenstand, das Ziel ihres Strebens, nach dem sie zu allen Zeiten gerungen hat ... ${ }^{821}$

Die Entfaltung des Kosmos konvergiert demnach mit der vollen Verwirklichung Gottes. Anders als in der romantischen Tradition Schellings verbindet Teilhard diese metaphysische Teleologie mit der durch den wissenschaftlichen Fortschritt bedingten Entfaltung der Noosphäre. Alles wissenschaftliche Streben sollte nämlich auf die Zusammenführung der Menschen, auf die »Mega-Synthese« eines menschlich-göttlichen Be-

${ }^{819}$ A.a.O., $293 \mathrm{f}$.

${ }^{820}$ Ähnliche Systeme entwickelten parallel zu Teilhard de Chardin auch die britischen Philosophen Samuel Alexander (1859-1938) und Alfred North Whitehead (1861-1947) sowie der Kosmist Vladimir Vernadsky. Vgl. Krüger 2015, 76-78; Barrow \& Tipler 1986, 156f.; Vernadskij 1997, 11.

${ }^{821}$ Schelling 1812, 113f. Bereits im 18. Jahrhundert hatte der Naturphilosoph Friedrich Christoph Oetinger die Grundidee formuliert, dass sich Gott in der Evolution selber manifestieren würde. Vgl. Lovejoy 1961, 242-287; McCalla 1998, 29-31. 
wusstseinskollektivs, ausgerichtet sein. Alle Bewegungen und Schichten der Evolution werden nach Teilhard schließlich in dieser harmonischen Einheit aller Seelen im Konvergenzpunkt Omega dieses kollektiven $\mathrm{Zu}$ sammenwirkens der Noosphäre münden. ${ }^{822}$

Das Universell-Zukünftige kann nur ein Überpersönliches sein - im Punkt Omega ... In Omega addiert und vereinigt sich, entsprechend unserer Begriffsbestimmung, die Menge des auf der Erde durch die Noogenese nach und nach frei gewordenen Bewußtseins in voller Frische und Unversehrtheit. ${ }^{823}$

Nicht explizit beschreibt Teilhard die Auferstehung der Toten. Er redet jedoch enigmatisch von dem »Emportauchen« der menschlichen Seelen in Omega. Teilhard idealisiert Omega als Raum und Zeit des Lebens im Angesicht der vernichtenden Kraft der Entropie im Universum: »Die Flucht aus der Entropie durch Rückkehr zu Omega. Der Tod selbst vermenschlicht. $\ll^{824}$

Dass Teilhard im gegenwärtigen Mediendiskurs wahrgenommen wird, obwohl Medien und Computertechnologie keine Erwähnung finden, ist Marshall McLuhan zu verdanken. Teilhards Gesamtwerk ist ja viel deutlicher durch die biologisch-vitalistische Ausrichtung in einem explizit christologischen Kontext geprägt. ${ }^{825}$

McLuhan selbst konvertierte 1937 zum römisch-katholischen Glauben, arbeitete an drei katholischen Universitäten, besuchte täglich die Messfeier und stand mit vielen katholischen Theologen, insbesondere Jesuiten, in engem Kontakt. In seiner öffentlichen Auseinandersetzung mit der Kirche fiel er bisweilen durch unkonventionelle Vorschläge auf, wie z. B. durch seine Kritik an der Abschaffung des Latein als liturgi-

${ }^{822}$ Vgl. Teilhard 1959, 229-267.

${ }^{823}$ A.a.O., 253f.

${ }^{824}$ A.a.O., 266. Vgl. a.a.O., $265 \mathrm{ff}$.

${ }^{825}$ Eine Rezeption der Idee des kosmischen Bewusstseins bzw. eines Punktes Gott-Omega über die Science-Fiction Literatur ist eher unwahrscheinlich. Sie erscheint nur in der wenig bekannten Geschichte The Last Question (1956) von Isaac Asimov und peripher ohne eine tiefere Ausgestaltung in William Gibsons Neuromancer (1984). Bei Asimov wird tatsächlich das gesamte Universum von Menschen und intelligenten Computern besiedelt, die schließlich in einem einzigen Bewusstsein aufgehen. Bei Gibson wird der Held mit der alles umspannenden Matrix konfrontiert, »the whole show«. Vgl. Asimov 1956, 15; Raulerson 2013, 177-183; Gibson 2000, 269; vgl. auch Porush 1992, 132ff. Auch Arthur C. Clarke macht die Vision eines globalen Gehirns durch sein nicht-fiktionales Werk bekannt. Vgl. Clarke 1960, 209, 233. 
scher Sprache während des II. Vatikanischen Konzils. ${ }^{826}$ Es ist daher nicht weiter verwunderlich, dass es eine besondere Beziehung zwischen McLuhan und Teilhard de Chardin gab.

Das fehlende Glied in der Kette ist der amerikanische Jesuit, Theologe und einflussreiche Medienwissenschaftler Walter Ong. Er studierte von 1938 bis 1941 an der St. Louis University Philosophie und Anglistik, während der junge McLuhan dort von 1937 bis 1944 Englisch lehrte. McLuhan betreute Ongs Magisterarbeit über den viktorianischen Jesuiten und Dichter Gerald Manley Hopkins, der Ong bereits für die theologische Verbindung von Evolution und der Entfaltung Gottes sensibilisieren sollte. Seit dieser Zeit waren Ong und McLuhan durch einen regen Briefwechsel wissenschaftlich und freundschaftlich miteinander verbunden. Ong widmete ihm sogar den zweiten Band seiner Dissertation. Als Ong in den frühen 1950er Jahren als Guggenheim Fellow in Paris lebte, wohnte er mit Teilhard de Chardin in derselben Unterkunft und konnte in dieser Zeit bereits dessen erst posthum veröffentlichtes Hauptwerk Le Phénomène Humain (1955) studieren. 1952 verfasste Ong eine Rezension für McLuhans erstes Buch, The Mechanical Bride. Er nutze die Gelegenheit, bereits zentrale Punkte aus Teilhards zensiertem Werk zu veröffentlichen. Anhand von McLuhans Zivilisationskritik in dessen The Mechanical Bride wirft Ong die Frage nach den Antworten der katholischen Theologie im technisierten Industriezeitalter auf und leitet positiv dann zu Teilhards (zensierten) Ideen über. Ong führt die Begriffe der Kosmosphäre und Biosphäre als frühe Stufen der Evolution ein und verweist dann auf die Noospähre:

In a third stage, slowly, man, with human intelligence, has made his way over the surface of the earth into all its parts ... with the whole world altered simutaneously every day to goings-on in Washington, Paris, London, Rio de Janeiro, Rome and (with reservations) Moscow - human consciousness has succeeded in enveloping the entire globe in a third and still more perfect kind of sphere, the sphere of intelligence, the >noosphere<, as it has been styled by Father Pierre Teilhard de Chardin, S.J. ${ }^{827}$

Ong wurde in der englischsprachigen Welt der wichtigste Vertreter von Teilhards Ideen. Es ist damit evident, dass McLuhan spätestens seit Ongs Rezension von 1952 mit Teilhards Ideen in Berührung kam, noch bevor er sich selbst intensiver mit kommunikationswissenschaftlichen Fragen

${ }^{826}$ Vgl. McLuhan 1999, Introduction, XXVf.

${ }^{827}$ Ong 1952, 84. 
befasste. Direkte Verweise auf Teilhard finden sich meines Wissens nur in McLuhans Gutenberg Galaxy von 1962, vermutlich aufgrund von äuBerer Kritik und McLuhans späterer, zunehmend kritischen Haltung gegenüber Teilhards Werk. ${ }^{828}$

McLuhan lenkt die Aufmerksamkeit seiner Leser bereits 1962 zu Beginn der Gutenberg Galaxy auf Teilhards Werk, »the lyrical testimony of a very Romantic biologist « ${ }^{829}$ und zitiert eine Beschreibung des globalen Zusammenwachsens und technischen Fortschrittes aus Le Phénomène Humain. Er fügt sogleich hinzu, dass Teilhards unkritischer Optimismus von Intellektuellen heftig kritisiert wurde, führt aber dann doch Teilhards Begriff der Noosphäre ein:

This externalisation of our senses creates what de Chardin called the snoosphere or a technological brain for the world. Instead of tending towards a vast Alexandrian library the world has become a computer, an electronic brain, exactly as in an infantile piece of science fiction. ${ }^{830}$

In zwei weiteren Anmerkungen verweist McLuhan auf die Verbindung des Evolutionsgedankens mit dem Fortschritt der (Medien-)Technik bei Teilhard. ${ }^{831}$ In der Folgezeit gestalten sich McLuhans Aussagen zu Teilhards Werk vielfältig, wenn nicht gar widersprüchlich. Zwar erwähnt McLuhan den französischen Jesuiten in seinem dritten Buch Understanding Media. The Extensions of Man (1964) nicht mehr namentlich, jedoch entwirft er nun mit Bezug auf Henri Bergsons L'évolution créatrice die Utopie eines harmonischen, elektronischen Zeitalters, geleitet von Bergsons Gedanken, dass die Sprache für die Teilung der Menschheit etc. verantwortlich sei:

Electricity points the way to an extension of the process of consciousness itself, on a world scale, and without any verbalization whatever. Such a state of collective awareness may have been the preverbal condition of men. Language as the technology of human extension, whose powers of division and separation we know so well, may have been the sTower of Babek by which men sought to scale the highest heavens. Today computers hold out the promise of a means of instant translation of any code or language into any other code or language. The computer, in short, promises by technology a Pentecostal condition of universal

\footnotetext{
${ }^{828} \mathrm{Vgl}$. Marchand 1998, 216f.

${ }^{829}$ McLuhan 2002, 32 (dt., Die Gutenberg-Galaxis. Das Ende des Buchzeitalters, Düsseldorf 1968).

${ }^{830} \mathrm{Ebd}$

${ }^{831}$ A.a.O., 46, 174.
} 
understanding and unity. The next logical step would seem to be, not to translate, but to by-pass languages in favor of a general cosmic consciousness, which might be very like the collective unconscious dreamt of by Bergson. The condition of >weightlessness $<$, that biologists say promises a physical immortality, may be paralleled by the condition of speechlessness that could confer a perpetuity of collective harmony and peace. ${ }^{832}$

Das genannte Werk von Bergson war jedoch just der Ausgangspunkt für Teilhards evolutionstheoretische und theologische Überlegungen. McLuhan sieht darüber hinaus noch mehr Anlass zu Optimismus:

If the work of the city is the remaking or translating of man into a more suitable form than his nomadic ancestors achieved, then might not our current translation of our entire lives into the spiritual form of information seem to make of the entire globe, and of the human family, a single consciousness? ${ }^{833}$

Später äußert sich McLuhan kritischer gegenüber der Idee einer harmonischen Weltgemeinschaft und entwirft das Konzept der retribalization. Das globale Dorf - zu dem Begriff fühlte sich McLuhan durch Wyndham Lewis' Buch America and Cosmic Man inspiriert - wird für McLuhan zunehmend zur leitenden Metapher der Mediengesellschaft, die durchaus konfliktreich und krisengeschüttelt verstanden wird, wie dies vor allem in McLuhans Buch War and Peace in the Global Village (1968) deutlich wird. ${ }^{834}$ In den analytischen Aussagen McLuhans zeigen sich dann auch spürbar die Unterschiede in der Verwendung des Bewusstseinsbegriffs im Vergleich zu Teilhard. Denn abgesehen von den ausdrucksstarken aber seltenen euphorischen Prophezeiungen in der Gutenberg Galaxy und in Understanding Media bezieht McLuhan die »Erweiterung des Bewusstseins« (extension of consciousness) auf die erweiterte Wahrnehmungsfähigkeit der Individuen und nicht auf die Entstehung eines gemeinsamen, kollektiven Bewusstseins, das in einem fernen Punkt Omega kulminiere. ${ }^{835}$

Der genauere Blick auf das Verhältnis von Marshall McLuhan und Pierre Teilhard de Chardin hat demonstriert, dass es sehr wohl erhebliche Differenzen in den theoretischen Konzepten der beiden Denker gibt. Umso interessanter gestaltet sich die Frage nach den konkreten Rezeptionsprozessen des philosophischen Werkes von Teilhard im heu-

\footnotetext{
${ }^{832}$ McLuhan 1994, 80 (dt., Die magischen Kanäle - Understanding Media, Dresden 1994). ${ }^{833}$ A.a.O., 61.

${ }^{834}$ Vgl. McLuhan 1968 (dt., Krieg und Frieden im globalen Dorf, Düsseldorf 1971).

${ }^{835}$ Vgl. McLuhan 1994, 47; McLuhan 2002, 32.
} 
tigen Posthumanismus. Teilhard ist kein Medientheoretiker, die Begriffe »Information« und »Kommunikation« spielen keinerlei Rolle in seinen Arbeiten und den Terminus der Noosphäre führt er im Kontext von theologischen und philosophischen Überlegungen eines phylogenetischen Aufstiegs des menschlichen Bewusstseins ein. Maßgeblich für die spätere Rezeption Teilhards im medienphilosophischen Zusammenhang war nun McLuhans Präsentation von Teilhards Ideen in der Gutenberg Galaxy. Zunächst wird Teilhard de Chardin von McLuhan als »romantischer Biologe « und nicht etwa als katholischer Theologe in jesuitischer Tradition vorgestellt. Niemals erwähnt McLuhan den christlichen Kontext von Teilhards Evolutionsmodell: Dessen Kern, die Konvergenz des Bewusstseins der menschlichen Individuen im künftigen Punkt Omega, verschweigt McLuhan gänzlich. Teilhards Ideen erscheinen bei McLuhan folglich in einer völlig enttheologisierten Form. Der Begriff der Noosphäre schließlich erfährt durch McLuhan eine klare Umdeutung.

Bezeichnet die Noosphäre bei Teilhard die Schicht des Denkens, die sich mit dem Erscheinen der ersten Hominiden entwickelt, so wird sie für McLuhan zum »technological brain « - die ganze Welt wird zum Computer. Damit vollzieht McLuhan drei Modifizierungen, die den Begriff der Noosphäre für heutige Posthumanisten und Cyberphilosophen überhaupt erst interessant gemacht haben: Erstens wird die Noosphäre als medientechnischer Begriff kontextualisiert, zweitens wird die Entstehung der Noosphäre auf den Beginn des »elektrischen Zeitalters«, also unsere Gegenwart, datiert und drittens impliziert die Noosphäre für McLuhan bereits die weltumspannende, mediale Vernetzung. ${ }^{836}$ Auf diese Weise nimmt McLuhan eine außerordentliche Aufwertung der gegenwärtigen Medienentwicklungen vor: Was bei Teilhard noch als die allmähliche und mit der Hegemonie des Abendlandes verbundene »Planetisierung der Noosphäre« war oder als Zusammenfluss des Denkens bezeichnet wurde, wird bei McLuhan zur neuen Evolutionsstufe des durch Radio, Fernsehen und Computer geprägten elektrischen Zeitalters.

${ }^{836}$ Teilhard hatte die vor vielen Jahrtausenden situierte Entstehung der Noosphäre von ihrer "Planetisierung « und dem künftigen "Zusammenfluss des Denkens« differenziert. Vgl. Teilhard de Chardin 1959, 179-202. 


\section{Virtualität. Unsterblichkeit in der Mediengesellschaft}

Ich habe meinen Lehrer sagen hören, daß es da, wo maschinelle Vorrichtungen sind, auch bald maschinelle Weisen geben wird, die Dinge $z u$ tun, und daß der, der die Dinge maschinell tut, auch ganz bestimmt ein Maschinenherz hat. ${ }^{1}$

In den bisherigen Analysen haben wir den technologischen Posthumanismus aus einer ideengeschichtlichen Perspektive betrachtet. Einzelne Elemente wie das kybernetische Menschenbild, die Unsterblichkeitsvision und die Superintelligenz konnten historisch aufgeschlüsselt werden. Parallel zu diesen Einzelaspekten bestehen jedoch übergeordnete analytische Perspektiven, die für das Verständnis des Posthumanismus von besonderem Interesse sind. Dies gilt zunächst für die Ökonomie der Futurologie: Welche Rolle nimmt wirtschaftliches Denken in den Zukunftsvisionen ein und wer profitiert unmittelbar von den weitreichenden Prognosen?

Desweiteren müssen wir uns der Frage widmen, ob neben den zahlreichen heterogenen Elementen der posthumanistischen Philosophie doch eine gemeinsame Klammer existiert, die alles miteinander verbindet. Durch die verschiedenen Standpunkte hindurch erscheint die Sehnsucht nach Kontrolle der Unbestimmbarkeiten, der Kontingenzen des menschlichen Lebens, als einigendes Band. Sowohl mit Blick auf den Einzelnen als auch für die Menscheit als Ganzes wartet der Posthumanismus mit einer eindeutigen Antwort auf die anthropologische Grundfrage nach dem Wohin gehen wir? auf.

Ferner bildet der technologische Posthumanismus das jüngste Beispiel moderner Fortschrittstheorien, deren Bewertung und Einordnung die Philosophie, Geschichts- und Kulturwissenschaften seit dem 20. Jahrhundert beschäftigt. Im Zentrum steht die Diskussion um die Kontinuität von oder den Bruch mit Entwürfen der christlichen Heilsgeschichte. Diese Debatte werde ich nachzeichnen und einen Gegenvorschlag zu den bisherigen, dichotomen Konzepten formulieren.

1 Aus dem Zhuangzi yinde, zitiert nach Jäger 1998, 168 f. 
Aus einer weiten kulturgeschichtlichen Perspektive betrachtet leistet der Posthumanismus nichts weniger als die Überwindung der vier neuzeitlichen Kränkungen des Menschen. Zu welchem Preis jedoch? Damit befassen sich die abschließenden Gedanken dieses Buches.

\section{1 Ökonomie}

Auf drei Ebenen ist die ökonomische Frage für den technologischen Posthumanismus von Bedeutung. Primär geht es um die wirtschaftliche Dimension der technologischen Visionen selbst. Sekundär drängt sich die Frage nach den ökonomischen und gesellschaftlichen Rahmenbedingungen des prognostizierten Fortschrittes auf. Drittens müssen wir die wirtschaftlichen Grundlagen des Post- und Transhumanismus und seiner Akteure betrachten.

Leben wird im Posthumanismus als Informationsverarbeitung definiert. Im Kern ist das Ziel jeglicher posthumanistischer Fortschrittsutopie eine Steigerung der Effizienz dieser Informationsverarbeitung. Gesteigerte Effizienz bedeutet eine höhere Rechen- und Speicherleistung bei gleichzeitiger Senkung der Kosten. Diese Eckpunkte prägen den Posthumanismus seit den frühen Schriften Moravecs Ende der 1970er Jahre bis hin zu Kurzweils aktuellen Prognosen. Fortschritt ist dabei mit der "Befreiung « des Lebens aus der ineffizienten, natürlichen Evolution verbunden, die verschwenderisch und ziellos agiere und den aus der kybernetischen Perspektive fehlerhaften Menschen hervorgebracht habe. Deshalb muss der Mensch abgeschafft und durch eine künstliche Superintelligenz ersetzt werden. Das virtuelle Dasein des posthumanen Menschentums wird durch Allwissenheit und unendlichen Reichtum gekennzeichnet sein sowie den von Moravec, Tipler und Kurzweil plastisch geschilderten Annehmlichkeiten, die den Heilsvorstellungen eines Gangster-Rap in nichts nachstehen: ewige Jugend und Potenz, überirdischer Sex mit überirdischen Playmates - ohne Furcht vor Krankheiten und Vaterschaften. Ein spirituelles Wachstum erscheint nur an der Peripherie. Das virtuelle Paradies erweist sich in seiner ökonomischen Dimension damit als banaler Traum alternder Männer, deren Begehren nach Jugend und Reichtum lechzt.

Welche gesellschaftlichen Voraussetzungen für die Verbreitung von künstlicher Intelligenz oder gar für die Hervorbringung der erhofften 
Superintelligenz der Singularität von Nöten sind, wird von Post- und Transhumanisten nirgends ernsthaft thematisiert. Der computertechnische Fortschritt folge allein technischen Gesetzmäßigkeiten einer sich exponentiell beschleunigenden Leistungssteigerung der entsprechenden Hardware. Erstaunlicherweise wird in der Regel auch noch nicht einmal das Problem der Software angegangen, also der Programme, die tatsächlich eine menschengleiche künstliche Intelligenz hervorbringen sollen. Dass für den technischen Fortschritt eine allgemeine wirtschaftliche Stabilität, prosperierende Internetkonzerne und ein funktionierendes Wissenschaftssystem vorausgesetzt werden muss, die allesamt auf dauerhaftes wirtschaftliches Wachstum angewiesen sind, bedarf für die Posthumanisten keiner Erwähnung. Auch nicht, dass die Entwicklung von Militärtechnik einen wichtigen Motor für die gesamte Computerund Robotikindustrie bildet. $^{2}$ Die Begründung eines anhaltenden Fortschrittsprozesses spiegelt daher erneut die posthumanistische Eigenart wider, Phänomene unabhängig von ihrem Kontext zu betrachten und jede Art einer holistischen Perspektive zu vermeiden: Technik und Wissenschaft erscheinen hier als eine von der restlichen gesellschaftlichen, ökologischen und kulturellen Umwelt entkoppelte Größe. Die posthumanistische Vision von sich selbst verbessernden Maschinengenerationen der Zukunft verdeutlicht dieses Argumentationsmuster einer von menschlichen Faktoren unabhängigen Technikentwicklung paradigmatisch. $^{3}$

Auch schweigt sich der Post- und Transhumanismus größtenteils darüber aus, wie denn die Gesellschaft der kommenden Jahrzehnte aussehen soll, wenn die erhofften Technologien des human enhancement Stück für Stück zur Verfügung stehen würden. Es wird das naive Bild einer freiwilligen Aneignung suggeriert. Das Plädoyer für einen demokratischen Transhumanismus verhallt in der schillernden Diskussion um immer weitreichendere technische Visionen. Ray Kurzweil steht im Rampenlicht, während sich James Hughes als wirkungsloses Feigenblatt des sozialen Gewissens erwiesen hat.

Weder mit Blick auf den künftigen Zugang zu den versprochenen Technologien, noch in Bezug auf die Legitimation dieser Techno-Revolution als Effizienzsteigerung zeigen sich Merkmale einer egalitären und solidarischen Gemeinschaft. Vielmehr identifiziert Philipp von Becker

2 Vgl. Becker 2015, 76f.; Wagner 2015, 29-31, 103-124.

3 Vgl. Kroker \& Kroker 1996, 92 f. 
in seiner ökonomischen Studie des Transhumanismus hinter dem Optimierungs- und Freiheitsversprechen ein Anforderungsprofil des kapitalistischen Marktes: »In Wirklichkeit bieten sie dem Einzelnen jedoch an, den Anforderungen des Wettbewerbs, den sie dadurch selbst noch forcieren, besser genügen zu können - kurz: Sie bieten an, den herrschenden Selbstoptimierungszwang zu optimieren. ${ }^{4}$

Eine problemorientierte Diskussion über die gesellschaftlichen Aspekte trans- und posthumanistischer Visionen findet wider besseren Wissens nicht statt. Nicht nur die Cyberpunkliteratur der vergangenen vier Dekaden, sondern auch viele populäre Adaptionen in Film und Fernsehen sind weitaus reflektierter. Zum Beispiel werden in dem Film Gattaca (1997) die meisten Kinder einer biotechnologisch fortgeschrittenen Gesellschaft genetisch selektiert und haben dadurch Zugang zu sozialem Aufstieg und Status, während andere ausgeschlossen werden. Im Film und Buch Cloud Atlas (2004/2012) erzählt David Mitchell die ergreifende Geschichte von Somni 451, einer geklonten Serviererin eines Restaurants, deren Bewusstsein als autonomes Wesen erwacht. Die Klone sind selbst in der Illusion eines sozialen Aufstiegs gefangen und werden, sobald sie kleinste Störungen aufweisen, ermordet und wiederverwertet. $^{5}$

Nicht die konkreten Phänomene, sondern die Machtstrukturen dieser Dystopien sind dabei das beunruhigendste Element, da sie Tendenzen unserer heutigen Situation projizieren: Die biotechnologische Zukunft ist schon jetzt präsent und zeigt ihre hässlichste Seite in der Anwendung der Pränataldiagnostik zur Selektion weiblicher Föten, die aus kulturellen und ökonomischen Gründen von den Eltern abgetrieben werden. Besonders ausgeprägt ist diese Praxis in China und Indien, wo in manchen Regionen auf 100 geborene Mädchen 130-140 Jungen entfallen. Aber auch in den Vereinigten Staaten, dem Kaukasus und Südosteuropa werden Gender-Selektionen zu Gunsten von männlichen Nachkommen mit Hilfe von Abtreibungen und neuerdings In-vitro-Fertilisation verstärkt durchgeführt. ${ }^{6}$ Der reale Femizid demonstriert, wie verheerend

Becker 2015, 70. Vgl. auch Wagner 2015, 31; Hayles 1999, 4.

Vgl. Orison of Sonmi 451 in Mitchell 2004; Wachowski \& Tykwer 2012.

6 Die sozialen Folgen dieses Femizid, der soziologisch als missing women-Phänomen gefasst wird, sind enorm und erhöhen in diesen Gesellschaften das Risiko für Gewalt gegen Frauen, Frauenhandel und berufliche Benachteiligung. Aktuelle Daten und Literatur findet sich unter dem Artikel Sex-selective abortion (engl. Wikipedia). 
die Einführung von Biotechnologien ohne Berücksichtigung der sozialen und kulturellen Rahmenbedingungen ist.

Mit seinem Plädoyer für eine unbegrenzte genetische und morphologische Selbstbestimmtheit des Menschen ignoriert der Trans- und Posthumanismus die gesellschaftliche Wirklichkeit des 21. Jahrhunderts und lässt erahnen, wie sich unter seinen liberalen Maximen soziale Ungleichheiten einer gen- und cybertechnologisch »optimierten « Menschheit verschärfen werden.

Damit stellt sich die Frage nach einer Zukunft, in der zwischen den Klassen eine biologische Kluft aufbricht und in der sich die Superreichen zu einer völlig neuen Spezies entwickeln, die zwanzig Jahre länger lebt, länger aktiv ist, noch mehr Reichtum akkumulieren kann und diese Vermögen und die damit verbundene Macht noch folgenreicher als bisher auf ihre Nachkommen überträgt. ${ }^{7}$

Im Gegensatz zu den klassischen Utopien von Francis Bacon, Thomas Morus oder Edward Bellamy entwerfen Post- und Transhumanisten keine soziale Perspektive des Zusammenlebens oder der Solidarität. Technologie und nicht Menschen bilden das Zentrum dieser Futurologie.

Dort wo Freiheit augenscheinlich versprochen wird, verbergen sich neue Formen der Kontrolle und Abhängigkeit. Was heute bereits für die Internetwirtschaft gilt, wäre im Falle eines virtuellen Daseins tatsächlich exponentiell profitabel:

Das eigentliche Produkt des Computerkapitalismus ist man selbst. Und obwohl man gekauft wird, bezahlt man dafür. Mit dem, was man tut oder nicht tut, sagt oder nicht sagt, fühlt oder nicht fühlt, kurz: mit der Aufgabe der Intim- und Privatsphäre, mit der Aufgabe der Unversehrtheit des Körpers und der Persönlichkeit. ${ }^{8}$

Abgesehen von der Frage, wem ein virtueller Mensch im Datenspeicher eines Supercomputers eigentlich gehören würde (gar dem Provider oder der Upload-Maschinerie?), wäre dies eine vollkommene ökonomische Utopie: Der Kunde wäre nicht nur in all seinen Gedanken und Handlungen gläsern-durchschaubar. Mittels kleiner Softwareeingriffe, wäre er auch direkt manipulierbar. Diese Einsicht gilt gleichermaßen für Biotechnologien und die Cyborgisierung des Menschen - Frédéric

7 Vgl. Krysmanski 2014, 128.

8 Becker 2015, 83; vgl. a.a.O., 83-97. 
Vandenberghe spricht hier von einer marktorientierten Kolonisierung des Lebens. ${ }^{9}$

Wenn sich das tracking nicht mehr nur auf unsere Smartphones und Computer bezieht, sondern die Technologie Teil des Körpers ist, dann wird die digitale Kontrolle des Menschen in seinem ökonomischen aber auch politischen Handeln grenzenlos sein. In der chinesischen Provinz Rongcheng wird zur Zeit ein Social Credit System erprobt, das das Handeln und die Äußerungen ihrer Bürger mit Punkten bewertet und zu Sanktionen oder Privilegien führt. Mit Hilfe von Gesichtserkennungssoftware wird z.B. die Nichtbeachtung einer roten Fußgängerampel erfasst. Eine Cyborgisierung des Menschen und das damit ermöglichte »Überwachungs-, Bestrafungs- und Belohnungsregime « ${ }^{10}$ wäre der Traum eines jeden autokratischen und marktwirtschaftlichen Systems. Die politische und ökonomische Utopie ergänzen sich deshalb so ergiebig, weil die Analyse, Kontrolle und Steuerung von menschlichem Verhalten just das alte Ziel der Kybernetik und ihrer verschiedenen Töchter wie der kybernetischen Psychologie, Pädagogik und Soziologie perpetuieren. Auch hier setzt sich der englische Utilitarismus des 19. Jahrhunderts à la Jeremy Bentham fort, wonach die Erfassung von Sozialstatistiken der besseren legislativen Steuerung der Gesellschaft dienen sollte. ${ }^{11}$

Wenn Ray Kurzweil daher von künftigen Nanobots im menschlichen Blut schwärmt, die per W-LAN eine neue Füllung für den Kühlschrank anfordern, dann ist dies ein ökonomischer Tagtraum totaler Kundenkontrolle eines Google-Funktionärs. In einem Vorwort zu dem Buch The Eternal E-Customer. How Emotionally Intelligent Interfaces Can Create Long-lasting Customer Relationships aus dem Jahr 2000 breitet Kurzweil diese Vision aus. ${ }^{12}$ Wenn es heute schon mühselig ist, sich aus dem Apple-Produktuniversum zu verabschieden, wie unmöglich wird dieses Unterfangen sein, wenn der ganze Körper von einer Myriade Nanobots eines bestimmten Anbieters bevölkert sein wird? Damit erfüllt sich dann tatsächlich die Fantasie vom »ewigen Kunden«.

Auch Martine Rothblatt sieht im Unsterblichkeitsbusiness die unerschöpfliche Quelle für »the real money«. ${ }^{13}$ Mit der ökonomischen Vision verbunden ist für Rothblatt auch das Einfordern von politischen

9 Vgl. Vandenberghe 2006; Wagner 2015, 56f.

10 Becker 2015, 70.

11 Vgl. Klaus 1969a, 324-335; Roszak 1994, 156ff.

12 Vgl. Kurzweil 2005, 300-311; Kurzweil 2000, IX.-XI.

13 Vgl. Rothblatt 2014, 11. 
Rechten für die Mindclones, die nicht als Bürger 2. Klasse degradiert werden sollten. Sie verschweigt allerdings, dass man die politischen Einstellungen dieser Mindclones nicht nur effizienter beeinflussen könnte, als dies eine Armada russischer Bots in sozialen Netzwerken je hätten tun können. Gleichzeitig könnte man in Sekundenschnelle Milliarden von Mindclones anfertigen, die politische Entscheidungsprozesse durch ihre Wählerstimmen lenken. ${ }^{14}$

Der letzte Aspekt betrifft die Ökonomie des Post- und Transhumanismus selbst. Es ist der Wissenschaftsautor Dylon Evans, der die so wichtige Frage aufwirft: Cui bono? Wer profitiert? Denn dieselben Technikpropheten, die die baldige Erwartung der übermenschlichen künstlichen Intelligenz verkünden, würden in einem zweiten Schritt vor eben diesen Wesenheiten warnen. Einerseits seien diese Prognosen einem manifesten Narzissmus geschuldet: »To regard yourself as one of a select few farsighted thinkers who might turn out to be the saviors of humankind must be very rewarding. ${ }^{15}$ Andererseits ernähre die wechselhafte Risikoprophetie eine ganze Reihe lukrativer Zukunftsinstitute - wie Drexlers Foresight Institute oder Bostroms Future of Humanity Institute, die nichts anderes täten als vor den Folgen ihrer eigenen Prognosen zu warnen. ${ }^{16}$ Die lukrative Vermarktung der Zukunft lässt sich plastisch auch an der Singularity University ablesen, die weder Forschung noch akademische Qualifikationen anbietet, sondern über ihre Workshops und weltweiten Veranstaltungen eine Plattform für die Vermarktung künftiger Technologien zur Verfügung stellt. Gerade für Ray Kurzweil haben seine Bücher, Auftritte und die Singularity University auch eine ökonomische Relevanz. Der verkündete, große technologische Durchbruch - disruptive and exponential - ist ihm selbst nie gelungen: Gemessen an den heutigen Internetunternehmern ist er mit seinem geschätzten Vermögen von 27 Millionen \$ nur ein Fliegengewicht. ${ }^{17}$

Vollkommen banal, aber ökonomisch höchst bedeutsam, ist die Vermarktung von Nahrungsergänzungsmitteln / Supplementen, die Kurzweil in seinen Lebenshilfebüchern anpreist und die im Zentrum von Veranstaltungen wie dem RAAD Fest stehen. Im US-Markt, der ca. 40 Milliar-

\footnotetext{
14 Vgl. a.a.O., 183-195.

15 Vgl. Evans 2015, 173.

16 Vgl. Evans 2015.

17 Vgl. https://www.therichest.com/celebnetworth/celebrity-business/men/ray-kurzweilnet-worth/.
} 
den \$ umfasst, haben sich Unternehmen wie Elysium Health und LifeExtension auf vermeintlich lebensverlängernde Supplemente spezialisiert. Ray Kurzweil, Aubrey de Grey, Natasha Vita-More, James Strole und Bernadeane schwören ihre Anhänger darauf ein, dass in wenigen Jahren Therapien zur Verfügung stehen würden, die das Altern rückgängig machen könnten, und damit auch die Unsterblichkeit selbst in greifbare Nähe rücke. Die Botschaft wirkt fast zynisch, wenn man bedenkt, dass die Vereinigten Staaten gegenwärtig unter einer massiven Opioid-Krise mit 70.000 Todesopfern jährlich, einem überforderten Gesundheitssystem und demzufolge einer real sinkenden Lebenserwartung leiden.

Egal ob Orakel im akademischen Kleid oder praktischer Lebensratgeber - die Zukunft selbst erweist sich als unerschöpfliche Ressource für eine Vielzahl futurologischer Projekte. Die unverfügbare, virtuelle Zukunft setzt sich in ganz konkrete Geschäftszahlen um. Die Praxis und Botschaft des Post- und Transhumanismus ist daher im Kern eine ökonomische: Den vom Club of Rome 1972 postulierten, dystopischen »Grenzen des Wachstums « wird die Vision eines unbegrenzten Wachstums des Einzelnen und der Menschheit - people unlimited - entgegengesetzt.

Angesichts der sich abzeichnenden globalen ökologischen Krise und des Klimawandels, die aus der ungebremsten Vernichtung und Ausbeutung natürlicher Ressourcen resultieren, gleicht die von Drexler, Kurzweil und Diamandis zelebrierte Prophetie eines unendlichen, exponentiellen Wachstums - einer radical abundance - dem letzten Tanz auf der Titanic. Allerdings hinkt die Metapher, denn die transhumanistische Band nährt die Illusion, dass das sinkende Schiff sogar noch beschleunigen würde. ${ }^{18}$

\subsection{Kontrolle und Kontingenz}

Nicht nur in der Ökonomie, sondern in allen Aspekten post- und transhumanistischer Philosophie bildet Kontrolle (control) den Schlüsselbegriff. Die Funktionen unseres Denkens dienen letztlich der Kontrolle des Systems Mensch, das sich andauernd gegen die äußerlichen Gefahren

18 Der Begriff abundance kann am besten mit »Reichtum im Überfluss « gefasst werden. Peter Diamandis, der die Singularity University leitet, entfaltet diese Vision in seinem Buch Abundance: The Future is better than you think (Diamandis \& Kotler 2012), während Eric Drexler dies mit Blick auf die Nanotechnologie in Radical Abundance: How a Revolution in Nanotechnology Will Change Civilization (2013) vollzieht. 
des Kontrollverlustes durch Drogen, Meditation, Musik, Gefühle, Sexualität oder auch nur Konversationen behaupten müsse. ${ }^{19}$ Minsky konstatiert, dass Erkenntnisfortschritte nicht auf neuen Erfahrungen, sondern auf der Aneignung neuer Ordnungs- und Kontrollmittel basieren - ja, Wissen an sich bedeute Kontrolle: »To know the cause of a phenomenon is to know ... how to change or control some aspects of some entities without affecting all the rest. $\ll^{20}$ Andererseits betont Minsky, dass nur durch Besitz Kontrolle möglich ist. Mit den enorm anwachsenden Denkfähigkeiten der künstlichen Intelligenz werde eine Zunahme der Kontrolle über die Welt einhergehen, die sich in unendlichem Reichtum widerspiegele. ${ }^{21}$ Kontrolle und Besitz werden somit bei Minsky über den begrenzten Kontext der Informationstechnologie hinaus zur allgemeinen Aufgabe geistiger Existenzen, das heißt menschlicher und künstlicher »Denkmaschinen«. Die Kontrolle und Neuprogrammierung von Gefühlen entwickelt sich auch in der Argumentation Ray Kurzweils zu einer wichtigen Fähigkeit des mit neuronalen Implantaten aufgerüsteten Menschen des 21. Jahrhunderts. ${ }^{22}$

Bei Frank Tipler, Hans Moravec und Max More spiegelt sich das Bestreben nach Kontrolle in ihrer Konstruktion eines deterministischen physikalischen Weltbildes wider. Denn im Kern der posthumanistischen Idee einer technischen Immortalisierung besteht ein niemals ausgesprochenes, aber gravierendes Problem: Post- und Transhumanisten wollen die menschliche Persönlichkeit durch eine exakte Erfassung aller Moleküle eines originären Körpers als ein komplexes Computerprogramm simulieren - Sandberg und Bostrom sprechen hier von einer whole brain emulation $(W B E) .{ }^{23}$ Eine erfolgreiche Simulation würde voraussetzen, dass auch atomare und subatomare Prozesse eindeutig bestimmbar wären. Dies ist jedoch prinzipiell nicht möglich. Spätestens mit der Entwicklung von Werner Heisenbergs so genannter Unschärferelation in der Kopenhagener Deutung der Quantenmechanik wurde 1927 das Ende einer deterministischen Physik eingeläutet. Quantenmechanische Prozesse, so stellte man fest, sind weder eindeutig beobachtbar noch vorhersagbar. Eine exakte Simulation, das heißt eine Emulation, von realen Prozessen

19 Vgl. Minsky 1988, 51, 68, 172, 182; Minsky 1994, 113; Minsky 1992a, 24.

20 Minsky 1988, 129. Vgl. a.a.O., 102-105.

21 Vgl. a.a.O., 292f.; Minsky 1994, 108.

22 Vgl. Kurzweil 1999a, 150.

23 Vgl. Sandberg \& Bostrom 2008. 
im Computer wird daher grundsätzlich nie möglich sein. ${ }^{24}$ Für dieses bekannte Problem entwickeln einige Posthumanisten kreative Lösungsvorschläge.

Tipler erweist sich schon früh als Verfechter der so genannten Vielweltentheorie als Alternative zur Preisgabe eines klassisch-deterministischen Weltbildes in der Physik. Im Gegensatz zur Kopenhagener Interpretation, die die Aktualität quantenmechanischer Vorgänge vom Beobachter abhängig macht, geht die höchst umstrittene Vielweltentheorie des Physikers Hugh Everett bei einer quantenmechanischen Entscheidung von der Aufspaltung des Universums aus, so dass unendlich viele Multiversen entstehen. Auch wenn wir die einzelnen Prozesse gesetzmäßig nicht erfassen können, wären das Universum und seine Teile damit prinzipiell simulierbar, selbst wenn dies bei genauerer Reflexion zu immensen Widersprüchen innerhalb von Tiplers Theorie führen würde. Denn die Simulierung aller möglichen Universen und Lebewesen durch den Omegapunkt scheint unvereinbar mit Tiplers ausschließlich paradiesischen Visionen. ${ }^{25}$ Diese Wiedereinführung eines vormodernen Determinismus in die Physik wäre gleichzeitig die unabdingbare Voraussetzung für die Simulation der Vergangenheit in Tiplers und Moravecs Visionen.

In seiner Haltung zu physikalischen Grundfragen scheint Hans Moravec unter dem prägenden Einfluss Frank Tiplers weitergehende Ideen zu entwickeln. Analog zu Tipler hebt er die Rolle des Beobachters hervor, der erst durch seine Anwesenheit die Existenz eines Universums, einer Wirklichkeit generiere. Er entwickelt die solipsistische These, dass zwar ständig aus der Menge aller Multiversen einige untergehen (beispielsweise durch kosmische Katastrophen, einen dritten Weltkrieg), diese aber aufgrund der vernichteten Beobachter auch aus dem Reich der Wirklichkeit eliminiert würden. Der ambitionierte Robotiker regt daher ein Selbstmord-Experiment an, das beweisen soll, dass der eigene Tod unmöglich sei! Glückt ein Selbstmordversuch, so erlischt das ensprechende Universum, und es bleiben nur all jene Universen, das heißt Quantenwirklichkeiten übrig, in denen der Selbstmord gescheitert oder etwa kein dritter Weltkrieg ausgebrochen sei. ${ }^{26}$

We lose our ties to physical reality, but, in the space of all possible worlds, that cannot be the end. Our consciousness continues to exist in some of those, and

\footnotetext{
24 Vgl. Cassidy 1996.

25 Vgl. Tipler \& Barrow 1986, 458-506; Tipler 1995, 167-183, 483-488.

26 Vgl. Moravec 1988, 153f.,187f.; Moravec 1999, 193-207.
} 
we will always find ourselves in worlds where we exist and never in ones where we don't. ${ }^{27}$

Unbeantwortet bleibt natürlich die Frage, warum sich Moravec nach dieser innovativen Unsterblichkeitsgewissheit so viele Gedanken um das posthumane Überleben macht. ${ }^{28}$ Auch Max More ersinnt in seiner Dissertation die Fiktion einer Überwindung der unbestimmbaren Quantenprozesse und die damit verbundene, vollkommene Kontrolle mindestens der Vergangenheit in einem gigantischen Computer der $\mathrm{Zu}$ kunft:

The 22nd century overthrow of quantum mechanics with its uncertainty principle and replacement by Quantum Super-Determinacy, has allowed (with the aid of vast computational power) the precise determination of the state of the universe at all past times. ${ }^{29}$

Durch diesen Umweg über die Vielwelteninterpretation der Quantenmechanik und die daran angeschlossene Wiedereinführung des physikalischen Determinismus wird die computertechnische Simulation bzw. Emulation eines Menschen, der Vergangenheit und aller beliebigen Universen möglich. Nur ein deterministischer Ansatz kann garantieren, dass Original und Emulation nicht durch unbestimmbare Quantenprozesse voneinander abweichen.

Konkret äußert sich das posthumanistische Streben nach Kontrolle unbestimmbarer Kontingenzen auf mannigfache Weise. Körperliche Funktionen werden in den Utopien von Cyborgs und erst recht in einer virtuellen Existenz vollkommen kontrollierbar. Alter, Krankheit und körperliches Versagen werden revidierbar oder verschwinden völlig aus dem Leben des posthumanen Menschen bzw. seiner Nachfahren. Sexuelles Verlangen wird beherrschbar. Sexualität selbst wird sich ohne körperliche Versagensnöte vollziehen - natürliche Risiken, wie die von fast allen Posthumanisten angesprochene Krankheit AIDS und die ungewollte Schwangerschaft werden ausgeschlossen. Dort, wo im Posthumanismus Körper und Geschlechter frei wähl- und konstruierbar werden, konvergiert der posthumanistische Diskurs wieder mit den gegenwärtigen Visionen der Gentechnik. Es muss hier wieder heißen, denn mit der Metapher vom genetic code, der den Menschen als eine prinzipiell

27 A.a.O., 210.

28 Vgl. a.a.O., 13f., 189, $192 f$.

29 More 1995a, 6. 
programmierbare Erbsubstanz definiert, rekurrierten James Watson und Francis Crick 1952 auf dieselben Quellen der kybernetischen Informationstheorie, die zur Grundlage des posthumanistischen Menschenbildes werden sollten. ${ }^{30}$

Verbunden mit der Konstruktion neuer, teils virtueller Körper ist das Ideal einer immerwährenden Steigerung der geistigen, informationsverarbeitenden Leistung des menschlichen Geistes, das in der posthumanistischen Literatur mehrfach in der geniegleichen Figur des fiktiven Detektivs Sherlock Holmes kulminiert. ${ }^{31}$ Im Vergleich zu diesen körperlichen Visionen erscheint Moravecs Zuversicht auf eine baldige, totale Kontrolle des irdischen Wetters und der Vermeidung von Naturkatastrophen peripher. ${ }^{32}$

Im Zentrum der posthumanistischen Utopie aber steht die Bewältigung der größten Kontingenz des menschlichen Lebens: des Todes. Seine Bedrohung wird durch die Naherwartung einer technisch ermöglichten Unsterblichkeit überwunden - so wie der Glaube an den unmittelbaren und alles umwälzenden Fortschritt schon immer das Motiv der Fortschrittspropheten war. $^{33}$ Ohne diese Aussicht auf eine menschliche Unsterblichkeit würde das Eintreten für die Ära der künstlichen Intelligenz und das Ende der Menschheit absurd klingen. Aber so erscheint die Erlangung von Unsterblichkeit, Allwissenheit, Schönheit und körperlicher Potenz als gänzlich dem menschlichen Willen unterworfener Prozess.

Fin-de-siècle hubristic mania was the desire for perfect knowledge and total power. The goal was complete omnipotence: the power to remake humanity, earth, the universe at large. If you're tired of the ills of the flesh, then get rid of the flesh: we can $d o$ that now. If the universe isn't good enough for you, then remake it, from the ground up. ${ }^{34}$

Auf diese Weise mutet die posthumanistische Vision von Unsterblichkeit als Konsequenz und Weiterführung einer spezifisch amerikanischen Todesverdrängung an, die niemand anderes als der Begründer der Kybernetik, Norbert Wiener, vor 40 Jahren in klaren Worten beschrieben hatte:

\footnotetext{
30 Vgl. Roszak 1994, 16f.; Riegler 1999, 55f.

31 Vgl. Ettinger 1989, 35ff.; Moravec 1999, 167.

32 Vgl. a.a.O., $155 f$.

33 Vgl. Dawson 1935, 2 .

34 Regis 1990, 7.
} 
Die im allgemeinen übliche Erziehung des Amerikaners der oberen Mittelschichten will ihn vor allem ängstlich davor behüten, Tod und Schicksal gewahr zu werden. Er wird aufgezogen in einer Atmosphäre, als sei alle Tage Weihnachten ... Die Tatsache des persönlichen Todes und die Möglichkeit des Unglücks werden ihm zwar durch die Erfahrungen seiner späteren Jahre aufgezwungen; trotzdem versucht er, diese unglückseligen Wirklichkeiten in die Rolle von $\mathrm{Zu}$ fällen zu verweisen und einen Himmel auf Erden aufzubauen ... Für ihn besteht dieser Himmel in beständigem Fortschritt und gleichmäßigem Aufstieg zu gröBeren und schöneren Dingen. ${ }^{35}$

Es ist in diesem Kontext kein Zufall, dass vor allem Robotiker, Kybernetiker und Physiker den Posthumanismus begründet haben, denn ihnen ist die Struktur von abstrakten Wirklichkeitsmodellen, wie einem Computerprogramm, bestens vertraut. Während die Quantenphysik die Begrenztheit mathematischer Gesetzmäßigkeiten als Modell unserer Welt offengelegt hat, basiert die computergenerierte Virtualität auf stets eindeutigen mathematischen Algorithmen. Die zugrunde liegende Spieltheorie John von Neumanns setzt ja gerade die Quantifizier- und Rationalisierbarkeit des menschlichen Verhaltens voraus und konstruiert aus dem so gewonnenen mathematischen Modell der Wirklichkeit im Computer eine virtuelle Realität, die genau diesen rationalen Bedingungen entspricht. ${ }^{36}$ Die virtuelle Realität ist damit gleich einem Spiel nach eindeutigen und widerspruchsfreien Regeln konstruiert. ${ }^{37}$

Die Beherrschbarkeit von Zeit und die Verfügbarkeit von Erlebnissen, die eindeutig determinierbar sind, stiften die unwiderstehliche Attraktivität der Virtualität, da hier die Illusion von der mathematischen Determination aller Ereignisse inszeniert werden kann. Der Tod, der die Sinnhaftigkeit eines rein innerweltlichen Lebens so sehr in Frage stellt, existiert nicht in der Virtualität. Wenn also die reale Welt nicht als sinnvoll versteh- und erlebbar ist, dann offerieren die technisch-medialen Dispositive der Virtualität eine rationale und deterministische Alternative. Die virtuelle Welt entspricht dem Wunsch nach einer vollkommenen Logik der Berechenbarkeit. Der Widerstand der Welt, wie ihn Wilhelm Dilthey als das rational Unerklärliche und das Unverfügbare verstanden hat, besteht hier nicht mehr. ${ }^{38}$ Bereits Norbert Elias hatte den ontologi-

\footnotetext{
35 Wiener 1964, 37.

36 Vgl. Neumann \& Morgenstern 1966, 34-39.

37 Vgl. Weizenbaum 1980, 71.

38 Vgl. Dilthey 1981, 294ff.
} 
schen Implikationen der mathematischen Weltmodelle, die heute durch den Computer realisiert werden, Beachtung geschenkt:

Was Menschen nicht alles zu glauben bereit sind, nur um sich den Gedanken an die Begrenztheit ihres Lebens, an den eigenen Tod, zu verdecken oder zu versüßen! Der hohe Status der Mathematik in unseren Gesellschaften beruht sicherlich unter anderem darauf, daß sie zu den symbolischen Gebilden gehört, in deren Namen man ... den Anspruch erheben kann, den Tod überdauernde Ewigkeiten anzubieten. ${ }^{39}$

Diese Verdrängung und Illusion einer Beherrschbarkeit des Todes trifft nun in der alltäglichen und globalen Lebenswelt auf die zunehmend sichtbaren Grenzen menschlicher Macht gegenüber existentiellen Bedrohungen. Man kann sogar die Behauptung wagen, dass die Spannung zwischen dem technologischen Machtanspruch und der Ohnmacht gegenüber tatsächlicher Unbeherrschbarkeit des Lebens, der Gesellschaft und der Natur wächst. Die amerikanische Opiod-Krise, die brutalen aktuellen Kriege im Jemen, in Afghanistan und in Syrien, die sich durch den Klimawandel verstärkenden Naturkatastrophen oder auch das Risiko von schmelzenden Kernreaktoren lassen sich durch Technologie nicht lösen. Die Hilflosigkeit der Robotik offenbarte sich paradigmatisch 2011 in Fukushima, als die ferngesteuerten Geräte den hohen Strahlenbelastungen nicht gewachsen waren und menschliche Rettungskräfte die Gefahren per Hand eindämmen mussten.

Wollte der Soziologe Ulrich Beck in seinem Buch Risikogesellschaft, das den Machtgewinn des technisch-ökonomischen Fortschrittes durch die Produktion von Risiken überschattet sieht, das Szenario einer Zukunft heraufbeschwören, »die es zu verhindern gilt «, ${ }^{40}$ so kann der Posthumanismus gewiss als Reaktion auf die Krise technischer Utopien zu Beginn des 21. Jahrhunderts interpretiert werden. So korreliert hier ein steigendes Kontrollbedürfnis mit einer nachlassenden Risikoakzeptanz: Denn das Leben ist heute nicht gefährlicher und risikobelasteter als in der Vergangenheit (Kohleenergie und Kohleabbau kosteten mehr Menschenleben als die Kernenergie), aber das Bedürfnis nach einer totalen Kontrolle ist enorm angestiegen, wie Hermann Lübbe meint. ${ }^{41}$

Der Posthumanismus unterscheidet sich jedoch von der bisherigen Technikeuphorie in einem wesentlichen Punkt, denn er bietet keine tech-

\footnotetext{
39 Elias 1994, 110.

40 Beck 1986, 11.

41 Vgl. Lübbe 1994, 82-102; auch Kroker \& Kroker 1996, 42.
} 
nischen Einzellösungen für die marginal auch von posthumanistischen Autoren wahrgenommenen Probleme der Gegenwart. Die unsterbliche Existenz in der Virtualität ist der Dreh- und Angelpunkt des ganzen posthumanistischen Systems. Wenn sich die menschlich-biologische Natur, der menschliche Körper, als nur beschränkt kontrollierbar erweist, wenn ihn trotz medizinischer Fortschritte immer neue Zivilisationskrankheiten zu Boden werfen, wenn sich die körperlichen und geistigen Leistungen dieses Körpers nur in geringem Umfang kontrolliert steigern lassen und wenn sich auf globaler Ebene die Naturgewalten und das Klima weiterhin als unbeherrschbar erweisen, dann besteht die Antwort des Posthumanismus im Verzicht der Menschheit auf sich selbst und auf die Welt.

Wie schon Ed Regis feststellte, heißt die einfache Lösung: Get rid of the flesh! Alles, was sich einer einfachen Kontrolle und Steuerung entzieht - wie körperliche Schwächen, Alter, Sexualität, die Ausbildung der Persönlichkeit und letztlich der Tod - die gesamte Biologie des Menschen also und die Wildnis seiner natürlichen Umwelt, soll eben dadurch vollkommen beherrschbar werden, dass der Mensch durch maschinelle Nachfahren ersetzt wird und mindestens die letzte Generation der Menschheit selbst in den Speichern von Computern als vollkommen deterministische Rechensysteme simuliert werden. Die Kontingenz der Lebenswelt soll also nicht im herkömmlichen Sinne bewältigt werden, sondern die menschliche Erfahrungswelt soll durch eine vollkommen bestimmbare - also willkürlich kontrollierbare - Welt ersetzt werden. Wenn die Welt selbst nicht kontrollierbar und steuerbar ist wie ein Computer, dann muss sie in einen Computer verwandelt werden, was ja bereits Oswald Wiener und John D. Bernal forderten. Damit geht laut Joseph Weizenbaum der alte kybernetische Traum in Erfüllung:

Die Welt ist voller Geheimnisse - und das Credo der KI-Szene, daß alles berechenbar sei, verleugnet das Geheimnis des Lebendigen, es erzeugt die Illusion vollständiger Durchschaubarkeit und legt nahe, alle Aspekte unserer Existenz seien enträtselbar. Der Glaube an Wunder und Mysterium erscheint aus dieser Perspektive lediglich als eine besondere Form von Dummheit. Mich verletzt diese Behauptung totaler Berechenbarkeit zutiefst. ${ }^{42}$

Der technologische Posthumanismus und insbesondere der Glaube an die allmächtige Singularität versprechen eindeutige Antworten in einer

42 Pörksen 2000, 276. 
Zeit der krisenhaften Unwägbarkeiten, oder wie es Ray Kurzweil mit Blick auf die Geschichte der Evolution ausdrückt: »It will continue until the entire universe is at our fingertips. « ${ }^{43}$ Die Aussicht auf ein immerwährendes Aufwärts beruhigt die Gemüter.

\subsection{Säkularer Fortschritt und religiöse Heilserwartung}

Wie können wir den Post- und Transhumanismus des frühen 21. Jahrhunderts als Ganzes ideengeschichtlich einordnen und begreifbar machen? Zunächst muss klargestellt werden, dass es nicht um die Frage gehen kann, ob es sich bei diesen Bewegungen um eine Religion handelt. Die Heterogenität an Positionen - von christlichen Transhumanisten bis zu scharfer Religionskritik - entwertet eine solche globale Perspektive. ${ }^{44}$ Nach den vorangegangenen Analysen erweist es sich als fruchtbringend, die essentialistische Fragestellung Religion oder nicht? zu überwinden. Vielmehr gilt es zu ergründen, was denn Post- und Transhumanisten mit »Religion « machen? ${ }^{45}$ Welche Rezeptionsleistungen nehmen sie vor? Wie sind diese kulturell bedingt? Zeigen sich übergreifende, persistente Deutungsmuster in der heilsgeschichtlichen Anlage?

In ihrer generellen Einstellung zur religiösen Frage bilden Post- und Transhumanisten ein weites Spektrum ab. Frank Tipler behauptet zwar, dass seine Einsichten bloß auf naturwissenschaftlichen Fakten beruhen würden - tatsächlich aber gründet sein Ansatz in der langen Tradition der natural theology bzw. Physikotheologie des protestantischen Theologen William Paley und seiner Zeitgenossen. Ray Kurzweil und Martine Rothblatt erwarten neue spirituelle Erfahrungsdimensionen, die mit der Erweiterung menschlicher Fähigkeiten im virtuellen Dasein einhergehen. Alle weiteren post- und transhumanistischen Autoren wie Marvin Minsky, Hans Moravec, Max More, Eliezer Yudkowsky und Nick

43 Kurzweil 2005, 487.

44 Der Versuch von Hava Tirosh-Samuelson, den Transhumanismus als neue religiöse Bewegung zu deuten, bleibt inkonsistent. Der Religionswissenschaftler und Transhumanist William S. Bainbridge wiederum positioniert den Transhumanismus als Überwinder von Religion. Beide Ansätze überzeugen nur, wenn die faktische Heterogenität der Bewegung ausgeblendet wird. Vgl. Tirosh-Samuelson 2014; Bainbridge 2005.

45 Hinter dieser neuen Perspektive verbirgt sich ein Paradigmenwechsel in der Religionswissenschaft, der die ontologische Fragestellung Was ist Religion? durch den handlungstheoretischen Blick Was macht Religion? ersetzt. Vgl. Krüger 2017, 43-46. 
Bostrom betrachten Religion eher als Hindernis bzw. gar als Feind der technologischen Überwindung des biologischen Menschen und seiner Immortalisierung.

Tipler und Rothblatt illustrieren den typischen Rezeptionsverlauf einer posthumanistischen Lektüre in ihren Ausführungen zum Buch The Buddha in the Robot des japanischen Robotikers Masahiro Mori: Für Tipler ist es ein Beleg für die Überwindung aller Religion durch den technischen Fortschritt. ${ }^{46}$ Jedoch sprach Mori nicht von der Vergöttlichung des Menschen zu einem allmächtigen Wesen, sondern bezeichnete mit der »Buddha-Natur « das Potential des Roboters für die Selbsterkenntnis des Menschen: Denn Mensch wie Roboter würden an Freiheit gewinnen, wenn sie die Beschränktheit ihrer Fähigkeiten akzeptierten! ${ }^{47}$

In diesem Beispiel spiegelt sich die höchst selektive Rezeption religiöser Ideen durch den technologischen Posthumanismus wider. Religion wird nur insoweit positiv wahrgenommen, als wie sie die persönliche Selbstverwirklichung und Erweiterung von machtvollen Fähigkeiten fördert. So führen Tipler und Rothblatt den Buddhismus als mit der posthumanistischen Unsterblichkeitsvision vereinbar an, vermeiden es jedoch, das zentrale Element buddhistischer Erlösungslehren zu benennen: das Mitgefühl (bodhicitta). Transzendenz im Buddhismus erfüllt sich nicht in einer individuellen Allmachtsphantasie, sondern der Buddha Avalokiteshwara verfügt über tausend Augen, um das Leid zu sehen und tausend Hände, um zu trösten.

Wie aber gestalten sich die ideengeschichtlichen Zusammenhänge im Einzelnen? Das posthumanistische Menschenbild ist weitgehend durch eine materialistische Rezeption von Descartes bestimmt, die in religionskritischen Ansätzen der französischen Aufklärung und insbesondere in der Philosophie von La Mettrie fußt. Konkret wird dieses Verständnis in der frühen Kybernetik umgesetzt, die Leben als Informationsverarbeitung definiert und damit die unabdingbare Voraussetzung für den Ersatz des Menschen durch effizientere, informationsverarbeitende Systeme schafft. Der dynamische Aspekt dieser menschlichen Selbstvervollkommnung ist jedoch ungleich komplexer.

Denn weit über den Posthumanismus hinaus ist es Gegenstand einer hitzigen Debatte, ob weltlicher Fortschritt und seine Ziele entweder als

46 Dt. Titel: Die Buddha-Natur im Roboter. Gedanken eines Roboter-Ingenieurs über Wissenschaft und Religion. Freiburg 1981. Vgl. Mori 1980; Tipler 1995, 88; Rothblatt 2014, 264.

47 Vgl. Mori 1980, 20-31. 
ein säkularisierter Ausdruck der christlichen Heilsgeschichte, als reines säkulares Produkt der Aufklärung, oder aber als eine wieder religiös gewordene Wissenschaft anzusehen sind. In ihrem rein wirkungsgeschichtlichen Ausgangspunkt verweist die Frage in dieser Form auf eine erkenntnistheoretische Problematik, die eine adäquate Einschätzung der posthumanistischen Philosophie in ihrem ideengeschichtlichen Kontext verhindern würde. Sie suggeriert einen bipolaren geistigen Kosmos von einerseits der theologischen Geschichtskonzeption und andererseits den säkularen Philosophien, die aus ihr hervorgegangen seien. Weder kann sie dem komplexen Verhältnis zwischen weltlichem Fortschritt und christlicher Theologie gerecht werden, noch berücksichtigt sie die Vielfalt post- und transhumanistischer Positionen. Zwei Ansätze stehen sich in der bisherigen Forschung gegenüber, die sich einerseits mit dem englischen Historiker John B. Bury, dem Philosophen Karl Löwith und dem protestantischen Theologen Rudolf Bultmann und andererseits mit dem Philosophen Hans Blumenberg verbinden.

1920 legte Bury mit seinem Werk The Idea of Progress. An Inquiry into its Origin and Growth die erste ideengeschichtliche und umfassende Darstellung der modernen Fortschrittstheorien vor. In klarer Abgrenzung zur Vorstellung einer religiösen Heilsgeschichte und dem Moment göttlicher Vorsehung definiert Bury den Begriff Fortschritt wie folgt:

The process must be the necessary outcome of the physical and social nature of man; it must not be at the mercy of any external will; otherwise there would be no guarantee of its continuance and its issue, and the idea of Progress would lapse into the idea of Providence. ${ }^{48}$

Die Entwicklung der Fortschrittsidee zeichnet Bury wesentlich anhand der französischen Philosophen Fontenelle, Abbé de Saint-Pierre, Condorcet und Comte nach und sieht die englische Fortschrittstheorie in großer Abhängigkeit zu ihrem kontinentalen Pendant. Da England im 18. Jahrhundert die Zeit der politischen Unruhen bereits hinter sich hatte, in Frankreich aber die originäre Entstehung der Fortschrittstheorie unmittelbar der Revolution vorausgegangen war, beurteilt Bury die englische Fortschrittstheorie als nicht mehr als eine »kalte Spiegelung“ der französischen Aufklärung. Seiner Deutung nach wird die Idee vom Fortschritt in Frankreich und England gleichermaßen als Befreiung der

48 Bury 1955, 5. 
Vernunft und der Rationalität aus der Enge theologischer Traditionen interpretiert. ${ }^{49}$

The otherworldly dreams of theologians, which had ruled so long lost their power, and men's earthly home again insinuated itself into their affections, but with the new hope of its becoming a place fit for reasonable beings to live in. ${ }^{50}$

Im Anschluss an Bury entwickelten nun Löwith und Bultmann die These, dass der moderne Fortschrittsgedanke einerseits in seiner eschatologischen Erwartung eines künftigen Heils seinem Ursprunge nach christlich und andererseits seiner Tendenz nach antichristlich sei. Die Fortschrittsidee erscheint bei ihnen als der säkularisierte Ausdruck der christlichen Vorsehung:

Der Glaube an einen diesseitigen und grenzenlosen Fortschritt ersetzt mehr und mehr den Glauben an die Vorsehung eines überweltlichen Gottes ... Schließlich aber mußte gerade die Fortschrittsidee die Funktion der Vorsehung übernehmen, nämlich vorauszuschauen und für die Zukunft vorher zu sorgen ... Und als die moderne Fortschrittsidee zur Religion der Gebildeten wurde, verstand sich die Modernität nun im Gegensatz sowohl zum klassischen Altertum wie zum Christentum, das selbst schon ein Fortschritt über das Altertum war. ${ }^{51}$

Löwith und Bultmann legitimieren ihre Thesen mit dem Verweis vor allem auf französische, durchweg kirchenfeindliche Fortschrittsphilosophen des 18. Jahrhunderts wie Voltaire, Turgot und Condorcet bzw. Pierre Joseph Proudhon und Auguste Comte im 19. Jahrhundert. Bultmann betrachtet die europäische Aufklärung als »Säkularisation des gesamten menschlichen Lebens und Denkens«. Wie vormals die christliche Teleologie ein transzendentes Heil versprach, propagiere nun der säkulare Fortschrittsglaube die Beherrschung der Natur zur Erlangung von weltlichem Glück. Die Idee des innerweltlichen Fortschrittes und die mit einem außerweltlichen Heil verbundene Idee einer christlichen Vorsehung bleiben damit wie bei Bury auch in Löwiths und Bultmanns Konzeptionen letztlich unvereinbar. ${ }^{52}$

In der jüngeren Debatte um die moderne Fortschrittsidee greifen Reinhart Koselleck und Friedrich Rapp auf diese These einer Säkularisierung der christlichen Geschichtskonzeption zurück. Die Funktion eines göttlichen

49 Vgl. Bury 1955, 217-237.

50 A.a.O., 349.

51 Löwith 1967, 62f.

52 Vgl. a.a.O., 63-98; Löwith 1963, 23-27; Bultmann 1958, 75-83. 
Heilsplanes werde in der Moderne durch die Geschichtsphilosophie und säkulare Fortschrittstheorien mit ihrem offenen Zukunftshorizont erfüllt, was sich terminologisch im begrifflichen Wandel vom geistlichen profectus in den weltlichen progressus widerspiegele. Die Temporalisierung der Zielvorstellung einer perfectio markiert für Koselleck den entscheidenden Wandel der Lehre von der nur in Gott enthaltenen Vollkommenheit und der damit verbundenen perfectio seu consummatio salutis zu einem nun als irdisch anzustrebenden Heilsziel. Wiederum mit Bezug auf meist französische Philosophen wird dargelegt, wie die modernen Fortschrittstheorien im Kontrast zur christlichen Theologie die Erfüllung des Menschen vom Ewigen ins Zeitliche transformierten und den Sinn des Lebens damit in irdischer Erfüllung ansiedelten. Damit gewinnt im Laufe des 19. Jahrhunderts die zum politischen, ökonomischen und kulturellen Schlagwort transformierte Fortschrittsidee in den Worten Kosellecks »Züge einer säkularen Religion der Selbstgewißheit«. ${ }^{53}$

Der Münsteraner Philosoph Hans Blumenberg hat nun diese weit verbreitete These der Säkularisierung der christlichen Heilsgeschichte einer fundamentalen Kritik unterzogen. Er bemängelte mit Nachdruck die Überbeanspruchung des Begriffes »Säkularisation« in der historischen Forschung. Blumenberg sprach hier sogar von einer Kategorie historischer Illegitimität, da er die allgemeine und unendlich strapazierte Säkularisierungsthese von theologischen Implikationen durchdrungen sah. Denn die "ganze Dimension verborgenen Sinnes« würde der säkularen Philosophie, Politik, Wissenschaft und Kultur stets einen unterschwelligen theologischen Sinn zuschreiben, und das gelte insbesondere für die Idee des Fortschrittes.

Für die Abhängigkeit der Fortschrittsidee von der christlichen Eschatologie bestehen Differenzen, die jede Umsetzung der einen in die andere blockiert haben müssen. Es ist ein formaler, aber gerade darum manifester Unterschied, daß eine Eschatologie von einem in die Geschichte einbrechenden, dieser selbst transzendenten und heterogenen Ereignis spricht, während die Fortschrittsidee von einer jeder Gegenwart präsenten Struktur auf eine der Geschichte immanente Zukunft extrapoliert. $^{54}$

Blumenberg verteidigt die Annahme der eigenständigen Entwicklung der säkularen, aufgeklärten Philosophie gegenüber dem theologischen Denken und konstatiert mit Blick auf die moderne Fortschrittsidee:

53 Vgl. Rapp 1992, 58f., 122f., 187-190; Koselleck 1975, 371-377, 411.

54 Blumenberg 1974, 39. Vgl. a.a.O., 19-75. 
... entscheidend bleibt, ob dieser Zustand leistungsimmanent oder leistungstranszendent ist, ob der Mensch ihn durch die Anstrengung seiner eigenen Kraft erreichen kann oder ob er dazu auf die nicht verdienbare Gnade eines über ihn hereinbrechenden Ereignisses angewiesen ist. ${ }^{55}$

Blumenberg gelingt es auf diese Weise, eine zunächst klar erscheinende Trennung zwischen einer theologischen und einer säkularen Geschichtsauffassung zu etablieren, so dass Friedrich Rapp in Reaktion auf Blumenbergs Kritik zu der moderaten These gelangen kann, dass die frühen Fortschrittsdenker des 18. Jahrhunderts wie Vico oder Herder zwar noch in christlichen Denkmustern verhaftet waren, jedoch: »Im Zuge der Aufklärungsphilosophie ist dann das rein innerweltliche Geschichtsdenken gerade dadurch autonom geworden, daß es sich von seinen ursprünglich theologisch gedachten Prämissen und Implikationen befreite. $\ll^{56}$

Eine Schwäche der Kritik Blumenbergs besteht darin, dass er sich wie zuvor Bury, Bultmann und Löwith vollständig einem wirkungsgeschichtlichen Paradigma verpflichtet fühlt. Alle hier genannten Gelehrten vertraten die Ansicht, dass die moderne Fortschrittstheorie im Frankreich des 18. Jahrhunderts entstanden ist und von dort aus ihre Wirkung in England und anderen Nationen entfalten konnte. Diese herausragende Rolle der französischen Fortschrittsphilosophie soll keineswegs bestritten werden, jedoch haben die Beschränkungen eines rein wirkungsgeschichtlichen Paradigmas dazu geführt, dass den rezeptiven Leistungen englischer und schottischer Philosophen praktisch keine Beachtung geschenkt wurde.

Ungeachtet der jeweils spezifischen Kontexte in England und Frankreich ging man von einer beständigen, säkular-aufklärerischen Semantik des Fortschrittsbegriffes aus. Die offensichtlichen Differenzen zur französischen Fortschrittstheorie - das heißt die rezeptiven Eigenleistungen englischer und schottischer Philosophen - wurden meist marginalisiert. Übergreifende Zusammenhänge zwischen dem linearen Geschichtsverständnis des Christentums und der europäischen Fortschrittstheorie wurden zwar aufgezeigt, aber empirisch wurde an den historischen Quellen nie nachvollzogen, wie denn nun religiöse Theorien in säkulare transformiert wurden. Bury wie auch Blumenberg entwerfen ideengeschichtliche Konzepte, die jeweils eine starke Abgrenzung oder gar die

55 A.a.O., 101. Vgl. a.a.O., 19-75; Blumenberg 1964.

56 Rapp 1992, 125f. Vgl. a.a.O., 116- 128. 
Unvereinbarkeit zwischen einem theologischen und einem säkularen Geschichtsverständnis voraussetzen. Die Rezeption von religiösen Ideen im Post- und Transhumanismus kann mit diesem antagonistischen Modell kaum hinreichend erklärt werden.

Die Kritik von Johannes Rohbeck, der Blumenberg vorwirft, den Gedanken der göttlichen Vorsehung und der transzendenten Geschichtsmächte in der französischen und schottischen Fortschrittsidee zu ignorieren, trifft ebenso auf Bury und seine Nachfolger zu. ${ }^{57}$ Schon Carl-Friedrich von Weizsäcker hat in seinen Gifford Lectures vor der polarisierenden Trennung von Religion und Wissenschaft gewarnt, die die universale Säkularisierungsthese implizieren würde, und betonte das rezeptive Potential der Philosophen im 18. Jahrhundert. ${ }^{58}$

Aus diesem Grund ist es angebracht, die engen Wechselwirkungen von innerweltlichem Fortschritt und theologischer Vorsehung in englischen und amerikanischen Geschichtskonzeption genauer zu beleuchten. Bereits in den 1960er Jahren bemängelte der Religionswissenschaftler Ernst Benz die Ignoranz der europäischen Geistesgeschichte gegenüber der anglophilen Theologie, was dazu geführt habe, dass wichtige Momente in der Diskussion um die vermeintliche Säkularisierung des christlichen Geschichtsverständnisses bisher kaum wahrgenommen wurden. ${ }^{59}$ Diese allgemeine Tendenz in der ideengeschichtlichen Forschung, die sich auch in der universitätstheologischen Marginalisierung und Abwertung des anglophonen Deismus als "journalistische Religionsschriftstellerei« niederschlug ${ }^{60}$ ist um so erstaunlicher, wenn man bedenkt, dass seit Mitte des 20. Jahrhunderts eine ganze Reihe von Einzelstudien vorliegen, die die universale These einer im Zuge der Aufklärung entstandenen Dichotomie zwischen Religion und Wissenschaft in protestantisch geprägten Kulturen in Frage stellen. Umfassend haben Benz und jüngst Kocku von Stuckrad erörtert, wie religiöse Diskurse seit dem 17. Jahrhundert wissenschaftliche Argumentationen und Legitimationen aufnehmen. ${ }^{61}$ Andreas Sommer zeigte, wie bereits in der Frühzeit der protestantischen Theologie Johann Valentin Andrae in seiner 1619 veröffentlichten Utopie der idealen Stadt Christianopolis die Entdeckung religiöser Wahrheiten unlösbar mit dem Fortschritt der Wissenschaften verbunden hatte.

\footnotetext{
57 Vgl. Rohbeck 1987, 244f.

58 Vgl. Weizsäcker 1990, 173-200; Stuckrad 2014, 178-182.

59 Vgl. Benz 1965, 157f.

60 Vgl. Gestrich 1981, 396.

61 Vgl. Benz 1970, Stuckrad 2014, 178-182.
} 
Ähnlich wie in Francis Bacons Nova Atlantis (1626) ist die rationale und auf dem Experiment beruhende Naturwissenschaft der Theologie verpflichtet. Aus der Theologie speist sich ihre Progressivität:

Die Welt ist im Protestantismus (wie im Christentum überhaupt) der Tendenz nach entgöttert; ihre Profanität wird durch menschliches Handeln erst wieder mit dem Heiligen Geist befruchtet und gewinnt daher Werthaftigkeit. Andererseits bleibt die Wissenschaft von der Schöpfung Gottes sakralisiert, während die technische Weltbewältigung die sakrale Weihe im Sinne der neuen Pneumatologie jetzt erhält. ${ }^{62}$

In anderen Aspekten wird diese Idee einer Ergänzung von weltlichem Fortschritt und religiöser Heilsgeschichte im Laufe des 17. Jahrhunderts fortgesetzt. Die puritanischen Einwanderer werteten die Bezwingung der amerikanischen Wildnis und den Aufbau einer neuen Zivilisation als religiösen Akt - Neuengland wurde zu New Israel. Literarischen Niederschlag fand die Bewegung in John Bunyans Pilgrim's Progress (1678) und vor allem in der populären Erbauungsliteratur. ${ }^{63}$

Primitiver, aber für weite Kreise verständlicher, wird dem strebenden Menschen in der Erbauungsliteratur versprochen, daß Gott ihm ein Zeichen seiner Gnade schon auf dem irdischen Lebensweg gewährt ... Fortschritt ist ein Zeichen für göttliche Gnade. Der gemessene Wegabschnitt, das heißt der Grad des Fortschrittes, läßt das Maß der göttlichen Gnade erkennen. ${ }^{64}$

Die literarisch dargestellte Fortschrittsidee ist im amerikanischen Kontext vorwiegend religiös fundiert, missionarisch, visionär und wird zusätzlich verstärkt durch ein erwachendes Nationalbewusstsein. ${ }^{65}$

Von herausragender Bedeutung für die Ausbildung der amerikanischen Fortschrittsidee waren dabei millenniaristische Vorstellungen. Mit dem Aufkommen des Protestantismus im 16. Jahrhundert erwachte auch ein neuerliches Interesse an der christlichen Eschatologie. Die Theologen Johann Heinrich Alsted und Johann Piscator entwarfen mit Bezug auf die Offenbarung des Johannes (Offb 20, 1-10) die Vorstellung, dass das Millennium - die tausendjährige Herrschaft Christi über die Erde, während der die Macht des Bösen über die Menschen gebannt ist - nicht in der Gegenwart oder der Vergangenheit liegen würde, sondern in der $\mathrm{Zu}$ -

62 Sommer 1996, 124. Vgl. Sommer 1996, 114-124; Dawson 1935, $154 f$.

63 Vgl. Brunotte 2000, 76-85, 273-280.

64 Buchloh 1963, 163.

65 Vgl. a.a.O., 156-165; Brunotte 2000, 76-85, 273-280. 
kunft der Menschheitsgeschichte. Nachdem schon Francis Bacon unter Berufung auf Daniel 12,4 seine comenius dei pansophia begründet hatte, ${ }^{66}$ wird der Bibelvers in der Folgezeit zur Losung der Puritaner, die nun den Fortschritt in den Wissenschaften als Bedingung für den Anbruch des Millenniums herausheben. Der kongregationalistische Geistliche Josiah Strong (1847-1916) interpretierte die ökonomischen und politischen Errungenschaften Amerikas als Hinweis auf den göttlichen Plan, dass das Millennium in seinem Land beginnen werde, da gerade auch diese wissenschaftlichen Leistungen die Amerikaner vor aller Welt auszeichnen würden: » ... the truths of science are God's truths, ... its laws are God's laws. $«{ }^{67}$ Die Notwendigkeit wissenschaftlicher Entdeckungen wird - in frappantem Gegensatz zu den Annahmen einer allgemeinen Säkularisierungsthese - hier als Stärkung der Religion und Verteidigung gegen die irreligiösen Tendenzen der Naturwissenschaften interpretiert. ${ }^{68}$

Diese weithin verbreitete, religiös fundierte Fortschrittsidee forcierte mit einer großen Dynamik den Fortschritt - also die Verbesserung und das Wachstum - im weltlichen Leben, ordnete diese Errungenschaften jedoch den religiösen Zielen unter. Der Religionswissenschaftler Thomas Hase führt eine Reihe überzeugender biographischer Beispiele an, die das ökonomische und zukunftsoptimistische Potential der millenniaristischen Fortschrittsidee im 19. Jahrhundert illustrieren, denn der Antrieb des Handelns sei nicht unbedingt

... die Angst vor der ewigen Verdammung, sondern das Vertrauen darauf, daß durch menschliches Tun ein besserer Zustand erreicht werden kann. In der Verbindung von persönlicher Verantwortlichkeit, Aktivität und Optimismus zeigt sich am deutlichsten der Einfluß postmillenniaristischer Vorstellungen auf das ökonomische Denken im 19. Jahrhundert. ${ }^{69}$

Die allgemeine Behauptung, dass das christliche Konzept der Vorsehung durch den wissenschaftlichen Geist und die Aussicht auf eine innerweltliche Verbesserung der Lebensumstände verdrängt wurde, ist unter Berücksichtigung des amerikanischen Millenarismus unhaltbar. Eine religiöse Heilsgeschichte ist also nicht einer modernen Fortschrittsidee

66 Daniel 12,4 »Du, Daniel, halte dieses Wort geheim, und versiegele das Buch bis zur Zeit des Endes! Viele werden nachforschen, und die Erkenntnis wird groß sein.«

67 Josiah Strong: The New Era or the Coming Kingdom, New York 1893, 11. Zitiert nach Hase 1997, 129.

68 Vgl. Ekirch 41-130; Spadafora 1990, 85-104.

69 Hase 1997, $133 f$. 
unterlegen, sondern hat diese in den Vereinigten Staaten maßgeblich vorangetrieben. Seine Attraktivität gewann der Millenarismus aus seinem Vermögen, in einer Zeit vieler und rasch erscheinender Neuerungen wieder normative Ordnungen zu stiften und zu erklären, warum Neuerungen geschehen und angestrebt werden sollen. ${ }^{70}$

Vereinzelt hatten verschiedene Historiker seit geraumer Zeit explizit auf die Schwächen von Burys These einer Unvereinbarkeit zwischen den Ideen von weltlichem Fortschritt und göttlicher Vorsehung hingewiesen. John Baillie, Arthur Ekirch und jüngst David Spadafora stellten klar, dass trotz der unbestrittenen Wirkung der französischen Philosophen die englische und amerikanische Fortschrittsidee eine ganz eigentümliche Synthese zwischen innerweltlichem Fortschritt und christlicher Heilserwartung hervorgebracht haben. Bei weitem die Mehrheit der englischen bzw. amerikanischen Philosophen - darunter die schon eingeführten Denker Joseph Priestley, David Hartley, Adam Ferguson und Richard Price - bewerteten den historischen Fortschritt als göttliches Programm, das gemäß der menschlichen Reife erst am Ende der Zeit seine volle Seligkeit und sein ganzes Glück für die Menschheit entfalten würde. Die Komplexität, Schönheit und beobachtbare bisherige Entwicklung der Welt ließen keinen anderen Schluss zu, als dass die Erde und mit ihr die Menschheit keine zufällige Erscheinungen sein können und ihr ein göttlicher Plan zugrunde liegen müsse. Priestley zufolge ist es nun die Aufgabe des Menschen, sich auf diesem Weg zu einer besseren Welt zu bemühen und seine gottgegebenen Fähigkeiten und Kräfte zum Nutzen der Menschheit einzusetzen. Weltlicher und religiöser Fortschritt seien nur Teile ein und desselben geschichtlichen Vorganges. Obwohl also die englischen Philosophen nicht weniger euphorisch als ihre französischen Kollegen für weltliche Aspekte des Fortschrittes in der Politik, Bildung, Medizin, Technik und Wissenschaft eintraten, so blieb der semantische Kontext ihres Fortschrittsbegriffes stets ein explizit christlicher: ${ }^{71}$

But what was the source of all this progress? What could have transformed mankind into beings of ever-greater knowledge, power, happiness, and virtue? For all these believers in the general advancement of the world, the answer was, fundamentally, God: progress appeared to them to be a divine program. ${ }^{72}$

70 Zum Postmillenniarismus vgl. Benz 1965, 157-181; Brunotte 2000, 208-217, 273-280; Hase 1997, 142-148; Spadafora 1990, 105-131.

71 Vgl. a.a.O., 85-132.

72 A.a.O., 248. 
Vor diesem Hintergrund wird verstehbar, warum die Idee der Singularität und des kosmisch-göttlichen Zielpunktes Omega Eingang in den Posthumanismus finden konnte. Die Rezeption des Werkes von Teilhard de Chardin durch Posthumanisten verläuft, wie wir sahen, parallel zu den medienphilosophischen Interpretationen des Internet. Frank Tipler rezipiert das Konzept einer kosmischen Heilsgeschichte, die von Alpha zu Omega reicht, ohne ihre spezifisch christologischen Anteile. Hans Moravec übernimmt das Konzept nur oberflächlich (wahrscheinlich durch den Einfluss Tiplers). Ray Kurzweil wiederum adaptiert präzise die strukturellen Elemente der Theorien von Teilhard de Chardin und Frank Tipler, ohne jedoch deren Begrifflichkeiten zu teilen. Die Verwirklichung Gottes ist für ihn wie für Tipler identisch mit der Verwandlung des Universums in einen einzigen Computer aus denkender Materie, in ein kosmisches Bewusstsein, das sich der Bedrohung durch die Entropie entgegenstellt. Weil die Menschheit die einzige intelligente Lebensform im Universum sei, ist dies gemäß Tipler und Kurzweil die Aufgabe des irdischen Lebens. Diese anthropozentrische Interpretation legitimiert auch das Opfer des Menschen.

Der göttliche Punkt Omega, auf den die biologische und technologische Evolution zuläuft, erfüllt im Rahmen des Posthumanismus zwei Funktionen. Auf einer mittleren Ebene postuliert der technologische Posthumanismus einen Einheitsgedanken - ähnlich wie dies auch die säkularen Vereinigungsutopien des global brain und der kollektiven Intelligenz von Turtschin und Lévy tun. In einem ganz weiten Bogen spiegelt sich hier die im Christentum und Judentum präsente Idee des verlorenen Paradieses (Genesis) bzw. der Auflösung der menschlichen Gemeinschaft wider (Turmbau zu Babel). Das menschliche Streben zeichnet sich nun dadurch aus, dieses Paradise Lost - das John Milton so nachhaltig als Missionsauftrag der Neuen Welt festgeschrieben hatte - in der Einheit mit Gott und der Vereinigung aller Menschen wiederzugewinnen. ${ }^{73}$

Wie Armand Mattelart und Randy Connolly herausgearbeitet haben, wird damit im Konkreten eine etablierte Interpretation neuer Kommunikationsmittel - in einem sehr weiten Sinn - fortgesetzt. Wann immer in den vergangenen Jahrhunderten medien- oder auch verkehrstechnische Neuerungen wie z.B. Kanäle, die Eisenbahn, Telegraphen oder das Radio in Erscheinung traten, gab es Stimmen, die dies als Anbruch eines neuen, freiheitlichen Zeitalters gedeutet haben, in dem die Menschheit

73 Vgl. Krüger 2012, 458-462. 
zu einer harmonischen Gemeinschaft zusammenwachse. ${ }^{74}$ Die Idee der Noosphäre oder die Metapher des global village erschaffen erneut die Utopie einer egalitären, organischen Gemeinschaft aller Menschen, wie sie in der Moderne zuerst im vorrevolutionären Frankreich entstand. ${ }^{75}$

Der posthumanistische Heilsentwurf beruht auf einer Reihe äußerst spekulativer Annahmen wie der Nichtexistenz außerirdischen Lebens und der Möglichkeit einer Überwindung der Quantenmechanik beim computertechnischen Immortalisierungsprozess. Dies sollte den kritischen Beobachter jedoch nicht zu der Einschätzung verleiten, dass diese Vision dadurch ihre Attraktivität einbüßen würde. Sofern man Religionen als Glaubenssysteme begreift, bezeugt die Geschichte der Menschheit, dass sich spekulative Annahmen, die sich jeder empirischen Überprüfbarkeit entziehen, als äußerst beständig erweisen können - vermutlich sogar als beständiger als überprüfbare Weltdeutungen. Die Leistung des Posthumanismus besteht einerseits in der Überwindung der menschlichen Kalamität. Zum andern verspricht er die von Oswald Wiener bezeichnete, »vollständige lösung aller welt-probleme« und »die befreiung von philosophie durch technik « durch den Ersatz der Welt selbst. ${ }^{76}$ Die Achillesferse des Post- und Transhumanismus besteht in der Spannung zwischen seinem proklamierten Anspruch, wissenschaftlich zu argumentieren und sich wissenschaftlicher Kritik auszusetzen, während er gleichzeitig spekulative Annahmen aus der Religion und Philosophie inkorporiert.

Zwar kann man einigen säkular orientierten Transhumanisten wie Bostrom, Yudkowsky und More ihr Unwohlsein mit der techno-religiösen Synthese anmerken. Jedoch hat sich die Diskussion im Vergleich zur Jahrtausendwende deutlich verschoben. Hatte ich damals Frank Tiplers Omega-Theorie als exotisch eingeordnet, so stehen ihre grundlegenden Elemente heute im Zentrum des Post- und Transhumanismus. Zusammen mit Ray Kurzweils Interpretation der Singularität entwickelte sich eine heilsgeschichtliche Teleologie, die deterministisch auf die Verwandlung des Kosmos in ein denkendendes Universum ausgelegt ist. Der Zielpunkt dieses Prozesses ist die Verwirklichung Gottes. Damit steht der Posthumanismus in der Kontinuität der englischen Moralphilosophen des 18. Jahrhunderts, denn eine endgültige Vollkommenheit könne nur

74 Vgl. Connolly 2001, 317-364; Bösch 2017, 221-225; Feinberg 1970, 20 f.

75 Vgl. Mattelart 1996, 85-162, 304.

76 Wiener 1969, CLXXV. 
durch die Vereinigung mit Gott erreicht werden. Es verschwimmen auch die Grenzen zwischen dem kosmologischen und dem technologischen Singularitätsbegriff. In der populären Rezeption wird Teilhard de Chardin inzwischen als Prophet des Internets, der Superintelligenz, des Transhumanismus und der Singularität gefeiert. ${ }^{77}$

Wie können wir den gegenwärtigen Erfolg dieser Allianz erklären, obwohl sich der frühe Transhumanismus so dezidiert religionskritisch positioniert hatte? Da die christologischen Elemente in diesem Zusammenhang keine Relevanz haben, vermag der jüngere Post- und Transhumanismus janusköpfig sowohl ein säkulares als auch ein religiöses Publikum anzusprechen - die jeweiligen Nuancierungen erweisen sich als anpassungsfähig.

Meiner Einschätzung nach beruht die sichtbare Überlegenheit dieses Ansatzes jedoch noch auf einem anderen Element: der Persistenz - d.h. der Beständigkeit - kultureller Deutungsmuster. Wie wir in der ideengeschichtlichen Analyse gesehen haben, spielt schon die ursprüngliche Wortschöpfung des Transhumanen (trasumanar) in Dantes Versen sowohl auf die antik-mythologische Vergöttlichung des Menschen als auch auf die christliche Gottesschau an. Ebenfalls um das Jahr 1300 führte der Scholastiker John Duns Scotus den Begriff virtualiter in die christliche Theologie ein. Als William Douw Lighthall Mitte des 20. Jahrhunderts als erster den Begriff transhumanism hervorbringt, reichert er diese religiöse Lesart mit einer heilsgeschichtlichen Deutung der Evolution an, wie dies konzeptionell zur gleichen Zeit auch Teilhard de Chardin und viele andere taten.

Der Post- und Transhumanismus wird damit nicht zu einer religiösen Philosophie - die technozentrische Legitimation des Fortschrittsprozesses ist unstrittig. Jedoch wird offensichtlich, dass es Tipler, Kurzweil und ihren Mitstreitern gelingt, die persistenten Deutungsmuster von (Heils-) Geschichte, Gott, Mensch und Kosmos zu aktualisieren und in neuer Form miteinander zu verbinden. Post- und Transhumanismus entstehen daher nicht in einem kulturellen Vakuum, sondern nutzen Deutungen sozialer Wirklichkeit, die im christlichen Denken und der westlichen Philosophie mitunter schon seit zwei Jahrtausenden präsent sind.

Eigentlich hat bereits der britische Science-Fiction Autor Charles Stross das Fazit meiner ideengeschichtlichen Analysen in seiner Kurzgeschich-

77 Vgl. Redding 2016; Kurzweil 2005, 389f. 
te Accelerando (2005) mit wenigen Worten vorweggenommen. Nach ausufernden Debatten über die Natur der Singularität haben die beiden Helden der Erzählung nur noch ein müdes Schulterzucken für die große Utopie übrig. Lakonisch resümiert einer der Disputanten:

»Is not happening yet, " contributes Boris. »Singularity implies infinite rate of change achieved momentarily. Future not amenable thereafter to prediction by presingularity beings, right? So has not happened ... Singularity is load of religious junk. Christian mystic rapture recycled for atheist nerds. ${ }^{78}$

\subsection{Das Ende der Kränkungen}

Im November 2005 durfte ich an einem exklusiven Abendessen an meiner damals neuen akademischen Heimat, der amerikanischen EliteUniversität Princeton, teilnehmen. Anlass war ein vorausgegangener Vortrag von Nick Bostrom. So saßen dann ca. zehn Interessierte in einem gehobenen Dining-Room des Prospect House (!) der Princeton University und diskutierten über Bioethik und die Zukunft der Menschheit. Unter den Teilnehmern befand sich meiner Erinnerung nach nur eine Frau, ansonsten waren wir allesamt weiße Männer. Umsorgt wurden wir während des Abendessens von schwarzen Bediensteten. Mehr als die Gespräche hat diese Szenerie in mir ein bleibendes Unbehagen ausgelöst - damals eher mit Blick auf die beunruhigende Beständigkeit rassisch-sozialer Rollenverteilungen (obwohl die Universität in vielen anderen Bereichen erfolgreich eine ethnische Diversität verwirklicht). Heute jedoch erscheint mir dieses Dinner mit Nick Bostrom als Mikrokosmos - als ein gesellschaftlicher Spiegel des Trans- und Posthumanismus. Seit der Debatte um die Eugenik waren es stets weiße Männer, die universale Entwürfe für eine erneuerte, bessere Menschheit vorlegten. Frauen und andere Ethnien waren und sind nur Zuschauer. Mich erstaunt am meisten, dass diese ethnische und geschlechtliche Homogenität den klügeren Anhängern des Trans- und Posthumanismus offenbar gar nicht auffällt. Viele der philosophischen Studien von Bostrom befassen sich mit der Frage des bias - der Voreinstellungen, Befangenheiten

78 Stross 2005, 184. Vgl. auch Raulerson 2013, 33-38. Die Entrückung / rapture der Nerds ist bereits ein fester Topos in der Kritik und Parodie der Singularitätsanhänger. Vgl. Sirius \& Cornell 2015, 195. 
und Neigungen, die seiner Ansicht nach oft auf einer selektiven Wahrnehmung der Wirklichkeit beruhen:

How big is the smallest fish in the pond? You catch one hundred fishes, all of which are greater than six inches. Does this evidence support the hypothesis that no fish in the pond is much less than six inches long? Not if your net can't catch smaller fish. ${ }^{79}$

Bostrom und seine Mitstreiter erkennen jedoch nicht, wie stark ihre eigene trans- und posthumanistische Utopie von kulturellen Voraussetzungen, vor allem von einem euro- und androzentrischen bias, geprägt sind. Am deutlichsten zeigt sich dies in der Fortsetzung der Ziele des eugenischen Kultes um Genies, Gehirne, den Übermenschen und die intelligenten Eliten der Menschheit, die zudem sozialdarwinistisch interpretiert werden. Es gibt kein Miteinander, nur ein Überleben des Stärkeren. Nicht weniger folgenreich ist die Definition des Menschen als informationsverarbeitende Maschine, die aus einer teils utilitaristischen, teils protestantischen Deutung des menschlichen Lebens hervorgeht. Schließlich werden diese normativen Elemente mit weiteren Aspekten christlicher und philosophischer Heilsgeschichte zu einer universalen Fortschrittstheorie verschmolzen, die in einer allmächtigen Singularität und der Beseelung des (göttlichen) Kosmos gipfelt.

Dass Bostrom fast ausschließlich von weißen männlichen Fischen umgeben ist, sollte ihn dazu anregen, über die Maschen seines eigenen »futurologisches Netzes" nachzudenken. Bereits die Annahme, dass es so etwas wie die »Krone der Schöpfung « als höchste Stufe der Evolutionsgeschichte gäbe, gar dass dies der (westliche) Mensch einer technologisch fortgeschrittenen Zivilisation sei, zeugt von einer anthropozentrischen, selektiven Wahrnehmung. Zu derselben Einschätzung könnten beliebig andere Lebewesen wie Haifische, Ameisen oder Pantoffeltierchen gelangen. ${ }^{80}$ Der Philosoph Friedrich Rapp gibt zu bedenken, dass die Evolutionsgeschichte an sich keine Hierarchien kennt - selbst das Kriterium der steigenden Überlebensfähigkeit der jüngeren Arten sei angesichts der Möglichkeit eines atomaren Suizids der Menschheit entwertet.

79 Bostrom 2002, 1.

${ }^{80}$ Haifische besiedeln diesen Planeten seit über 350 Millionen Jahren, Ameisen seit 100 Millionen Jahren (Dinosaurier umspannnen »nur « eine Periode von ca. 170 Millionen Jahren), die Geschichte der Hominiden beginnt erst vor ca. 5 Millionen Jahren. Das Pantoffeltierchen wiederum verfügt über ein Genom, dass doppelt so komplex ist wie das Menschliche. 
Die naturwissenschaftliche Beobachtung führt nur zu der Feststellung: Manche (neuen) Arten überleben, andere nicht. Dabei sind die überlebensfähigsten Arten keineswegs immer stärker differenziert und komplexer aufgebaut. ${ }^{81}$

Im Falle des Posthumanismus besteht die Normativität aus einem komplexen Geflecht von Annahmen und Zielsetzungen, die die Ökonomie, Gesellschaft, Religion und Genderverhältnisse betreffen. Nach der Analyse der verschiedenen einzelnen Elemente des technologischen Posthumanismus zeigt sich, was diese Zukunftsvision auf einer zweiten, bedeutsameren Ebene leistet.

Es waren, wie eingangs geschildert, vier Kränkungen des Menschen, die der Philosoph Johannes Rohbeck im Anschluss an Günther Anders, Hannah Arendt und Sigmund Freud in der Geschichte der Moderne ausmachte: die kosmologische Kränkung durch das heliozentrische Weltbild des Kopernikus, die biologische Kränkung durch die Evolutionstheorie Darwins, die seelische Kränkung durch die Psychoanalyse und die technologische Kränkung durch die Perfektion der maschinellen Schöpfung, die »prometheische Scham ${ }^{8}{ }^{8}$

Der technologische Posthumanismus gemäß den Ansätzen von Frank Tipler, Hans Moravec und Ray Kurzweil sowie vieler Transhumanisten wie Bostrom und Yudkowsky vermag es, alle vier Kränkungen zu »heilen«:

1. Gestützt auf das anthropische Prinzip steht das irdische Leben nicht nur im bedeutungsvollen Zentrum des Weltalls (wie einst in der christlichen Kosmologie), sondern es hat eine Mission zu erfüllen, die nichts Geringes als die Rettung des gesamten Universums vor dem finalen Wärmetod umfasst.

2. Die biologische Kränkung ist durch die Rolle der Menschheit, als Hervorbringer der künstlichen Intelligenz und der Singularität behoben, zumal die Essenz des Menschen - sein denkender Geist - mit diesen posthumanen Wesenheiten verschmelzen wird. Das unheilvolle biologische Dasein wird damit überwunden.

3. \& 4. Die psychische und die technologische Kränkung werden mit der Vision einer totalen Beherrschung menschlicher Geisteskräfte (durch ihre Computerisierung) und der unendlichen Steigerung ihrer Leistungen in der Verschmelzung mit der Superintelligenz entkräftet. Die Tech-

81 Rapp 1992, 84. Vgl. a.a.O., 74-87; Dawson 1935, $15 f$.

82 Vgl. Rohbeck 1993, 10. 
nik degradiert den Menschen nicht, sie verhilft ihm zur Verwirklichung eines transzendenten Übermenschentums in totaler Kontrolle.

Der technologische Posthumanismus bedeutet nicht das Ende des Menschen, aber er bildet unter dem Eindruck der technischen Fortschrittsentwicklung eine große Herausforderung für das humanistische Menschenbild. ${ }^{83}$ Der Mensch und mit ihm die Ideale des Humanismus sind aus der Sicht posthumanistischer Autoren aufgrund mangelnder Effizienz obsolet geworden. Der so genannte kritische Posthumanismus - so unglücklich die Namensdoppelung auch ist - reagiert auf exakt die Ideale und Ziele, die der technologische Posthumanismus verkörpert. ${ }^{84}$

Unter einer rein technozentrischen Perspektive ist der Mensch tatsächlich obsolet - das wusste schon Günther Anders. Wir müssen uns heute entscheiden, ob wir als Menschen diese technozentrische Perspektive übernehmen wollen. Stanisław Lem macht pointiert auf diese Verschiebung von Deutungen des Menschen aufmerksam:

Je mehr die Maschine von ihrer Umgebung unabhängig wird, desto besser ist es vom rein technischen Standpunkt. Aber es gibt eine solche Abhängigkeit des Menschen von seiner Umwelt (wie die erotischen, die sozialen, die freundschaftlichen Bande), welche autonome Werte bildet, also nicht zieluntergeordnet (also teleologisch) ist. Ein Cyborg, welcher nicht mehr der Nahrung, des Trinkens, der Atemluft bedarf, weil er eine atomare Energiequelle eingebaut hat, ein Cyborg, welcher beliebige Erinnerungen mittels eines einfachen Willensaktes aus seinem Gehirn löschen kann, wird zu einer Maschine, welche im Leistungsbereich vollkommener als ein Mensch ist, weil er weder Hunger, Durst, Atemnot noch Gewissensbisse erleiden kann. Aber auf diese Weise annulliert man schrittweise alles, was den kulturell bedingten Kern der menschlichen Lebenswerte bildet. ${ }^{85}$

Die Alternative zum Posthumanismus liegt in der unbequemen Akzeptanz der menschlichen Unvollkommenheit, des Todes und des Alterns. In diesem Sinne haben Ernst Ulrich und Christiane von Weizsäcker ein Jahr, bevor Hans Moravecs Werk Mind Children erschien, die Rückkehr zum »menschlichen Maß« gefordert: zur Toleranz gegenüber menschlicher Fehlbarkeit auch im Angesicht "perfekter« Technik und "perfekter «, technischer Menschenbilder. ${ }^{86}$

\footnotetext{
83 Vgl. Hayles 1999, 286f.

84 Der großenteils im Poststrukturalismus und Feminismus verwurzelte, kritische Posthumanismus wendet sich gerade gegen die Andro- und Eurozentrik des bisherigen $\mathrm{Hu}-$ manismus. Vgl. Loh 2018, 130-180.

85 Lem 1972, 183.

86 Vgl. Weizsäcker \& Weizsäcker 1987.
} 
Aber auch dieser Ansatz bleibt noch in der posthumanistischen Logik verhaftet, die den Menschen ähnlich der philosophischen Anthropologie und der Andersschen prometheischen Scham stets als Mängelwesen interpretiert. Vielleicht ist es lohnenswert, diese Perspektive umzukehren, wie es uns der Romanautor Brian Selznick in einer seiner Geschichten nahelegt: »Du weißt schon, bei Maschinen gibt es keine überflüssigen Teile. Sie haben genau die Anzahl von Teilen, die sie brauchen. $\ll^{87}$

Unter diesem Blickwinkel - dasselbe trifft natürlich auf Computerprogramme zu - muss die Vielfalt und Offenheit der Möglichkeiten des Menschseins als Fülle gelten. Maschinen und Software sind Mängelwesen, denn ihnen mangelt es an Möglichkeiten - ihre Prozesse sind auf einen einzigen Zweck hin determiniert. ${ }^{88}$ Menschen dagegen sind in der Vielfalt ihrer sozialen Beziehungen und Möglichkeiten offene Wesen des Überflusses, fortwährend im Bemühen um Identität und Sinndeutung in Bewegung. Der technologische Posthumanismus, der übrigens auch eine dieser unendlich vielen Sinndeutungen darstellt, kann nur deshalb den Menschen als fehlerhaftes Mängelwesen konstruieren, da er ihn unter utilitaristischen Vorzeichen - wie eine Maschine - auf einen einzigen Zweck reduziert: die Informationsverarbeitung.

Begreifen wir das Menschsein dagegen als offene Frage, als Wagnis, dann sind Menschen keine Mängelwesen, sondern freie Naturen in Fülle von Möglichkeiten, die dem deterministischen Drang der Kybernetik nach totaler ökonomischer und sozialer Kontrolle entgegengesetzt sind. Für Dietmar Kamper war die Entscheidung für die Sterblichkeit daher mit der Entscheidung für die Freiheit, auch Mensch bleiben zu können, verbunden. ${ }^{89}$

Freiheit aber ist mit Sterblichkeit und präziser Ungewißheit verwandt ... Es gibt in der Tat inkommensurable, schreckliche Wahrheiten. Dazu gehört die folgenreiche Annahme, daß der Mensch eine offene Frage ist, an die keine mögliche Antwort, die - wenn sie gut ist - geschlossen ist, je heranreicht. ${ }^{90}$

87 Selznick 2008, 388.

88 Hier liegt exakt das konzeptionelle Problem einer allgemeinen künstlichen Intelligenz - niemand weiß bisher, wie man von spezialisierten Programmen hin zu einem universalen Problemlöser gelangen soll.

89 Vgl. Kamper 1999, 25f.

90 A.a.O., 96. Vgl. Gantke 1987, 286-290. 


\section{Abkürzungsverzeichnis}
AI
Artificial Intelligence
AL
Artificial Life
CPU
Central Processing Unit
Cyborg
Cybernetic Organism
IT
Informationstechnologie
KI
Künstliche Intelligenz
KZfSS Kölner Zeitschrift für Soziologie und Sozialpsychologie
MIT
Massachusetts Institut of Technology
TRE
Theologische Realenzyklopädie
VR
Virtual Reality / Virtuelle Realität
ZfR
Zeitschrift für Religionswissenschaft
ZRGG Zeitschrift für Religions- und Geistesgeschichte 


\section{Quellenverzeichnis}

a) Literatur ${ }^{1}$

A

Adams, Tim (2016): Artificial intelligence: >We're like children playing with a bombr. In: The Observer, 12.6.2016 (Online).

Agar, Nicholas (2010): Humanity's End. Why we should reject radical enhancement. Cambridge.

Ahn, Gregor (2001): Homo religiosus oder künstliche Unsterblichkeit? Vererbung und Anlage in der neueren europäischen Religionsgeschichte. In: Vererbung und Milieu, hg. von Michael Wink. Heidelberg, 331-353.

Alcor (1983): Ev Cooper. 1926-1983. In: Cryonics (March), 7-9.

Alexander, Samuel (1966)[1920]: Space, Time, and Deity. The Gifford Lectures at Glasgow 1916-1918, Vol II. New York.

Altner, Günter (1965): Schöpfungsglaube und Entwicklungsgedanke in der protestantischen Theologie zwischen Ernst Haeckel und Teilhard de Chardin. Zürich.

Anders, Günther ( $\left.{ }^{6} 1983\right)$ [1956]: Die Antiquiertheit des Menschen, Bd. 1. Über die Seele des Menschen im Zeitalter der zweiten industriellen Revolution. München.

Andreoli-de-Villers, Jean-Pierre (1986): Le premier manifeste du futurisme. Edition critique avec, en fac-simile, le manuscript original de F.T. Marinetti. Ottawa.

Armstrong, Stuart \& Kaj Sotala (2012): How We're Predicting AI - or Failing To. https://intelligence.org/files/PredictingAI.pdf.

Asimov, Isaac (1950): I, Robot. New York.

Asimov, Isaac (1956): The Last Question. In: Science Fiction Quarterly, November, 6-15.

Assmann, Jan (21993)[1983]: Schrift, Tod und Identität. Das Grab als Vorschule der Literatur im alten Ägypten. In: Schrift und Gedächtnis. Beiträge zur Archäologie der literarischen Kommunikation, hg. von Aleida \& Jan Assmann \& Christof Hardmeier. München, 64-93.

1 Eckige Klammern verweisen auf die Erstauflage der Originalausgabe, sofern sich das Erscheinungsjahr der Übersetzung bzw. späteren Auflage von dem des Originals unterscheidet. 
Assmann, Jan (2000): Der Tod als Thema der Kulturtheorie. Todesbilder und Totenriten im Alten Ägypten. Frankfurt a.M.

\section{B}

Bacon, Francis (1959)[1643]: Neu Atlantis. Georg Gerber (Übers.). Berlin. Bacon, Francis (1977)[1638]: The Historie of Life and death. With Observations Naturall and Experimentall for the Prolonging of LIFE. New York.

Baedtke, Jan \& Christina Brandt \& Hans-Ulrich Lessing (Hg.)(2015): Anthropologie 2.0? Neuere Ansätze einer philosophischen Anthropologie im Zeitalter der Biowissenschaften. Münster.

Baillie, John (1950): The Belief in Progress. London.

Bainbridge, William S. (2005): The Transhuman Heresy. In: Journal of Evolution and Technology 14/2 (Online).

Bainbridge, William S. (2011): The Virtual Future. London.

Bainbridge, William S. (2014): Personality Capture and Emulation. London.

Barrow, John D. (1997): Frontiers and Limits of Science. In: How Large is God?, hg. von John M. Templeton. Philadelphia, 203-216.

Barrow, John D. \& Frank Tipler (1978): Eternity is Unstable. In: Nature 276, 453-459.

Barrow, John D. \& Frank Tipler (1979): Analysis of the Singularity Studies of Belinskii, Lifshitz and Khalatnikov. In: Physical Reports 56, 371-402.

Barrow, John D. \& Frank Tipler (1981): Generic Singularity Studies Revisited. In: Physical Letters A 82, 441-446.

Barrow, John D. \& Frank Tipler (1985a): Closed Universes. Their Future Evolution and Final State. In: Monthly Notices of the Royal Astronomical Society 219, 395-402.

Barrow, John D. \& Frank Tipler (1985b): Closed Universes: Their Evolution and Final State. In: Monthly Notices of the Royal Astronomical Society 216, 395-402.

Barrow, John D. \& Frank Tipler (1986): The Anthropic Cosmological Principle. Oxford/New York.

Barrow, John D. \& Frank Tipler (1988): Action Principles in Nature. In: Nature 331, 31-34.

Barrow, John D. \& Frank Tipler \& Marie-Odile Monchicourt (1984): L'homme et le cosmos. Le principe anthropique en astrophysique moderne. Paris.

Barthes, Roland (1985)[1980]: Die helle Kammer. Bemerkungen zur Photographie. Dietrich Leube (Übers.). Frankfurt a.M.

Baudrillard, Jean (1991)[1976]: Der symbolische Tausch und der Tod. München. 
Baudrillard, Jean (1994): Die Illusion und die Virtualität. Bern.

Baudrillard, Jean (1996)[1995]: Das perfekte Verbrechen. München.

Baumgartner, Hans M. (1994): Zeit und Zeiterfahrung. In: Zeitbegriffe und Zeiterfahrung, hg. von Hans M. Baumgartner. Freiburg, 189-211.

Beck, Klaus (1994): Medien und soziale Konstruktion von Zeit. Über die Vermittlung gesellschaftlicher Zeitordnung und sozialem Zeitbewußtsein. Opladen.

Beck, Ulrich (1986): Risikogesellschaft. Auf dem Weg in eine andere Moderne. Frankfurt a.M.

Becker, Philipp von (2015): Transhumanismus, Biotechnik und digitaler Kapitalismus. Wien.

Bennahum, David (1997): 3-D im Internet: Ein Gespräch mit Mark Pesce, dem Schöpfer von VRML. In: Die Zeit 09/1997.

Benz, Ernst (1961): Das Bild des Übermenschen in der europäischen Geistesgeschichte. In: Der Übermensch. Eine Diskussion, hg. von Ernst Benz. Zürich, 19-161.

Benz, Ernst (1965): Schöpfungsglaube und Endzeiterwartung. Antwort auf Teilhard de Chardins Theologie der Evolution. München.

Benz, Ernst (1970): Theologie der Elektrizität. Zur Begegnung und Auseinandersetzung von Theologie und Naturwissenschaft im 17. und 18. Jahrhundert. In: Abhandlungen der Geistes- und Sozialwissenschaftlichen Klasse der Akademie der Wissenschaften und der Literatur 1970/12, 1-98.

Benz, Ernst (1977): Akzeleration der Zeit als geschichtliches und heilsgeschichtliches Problem. In: Abhandlungen der Geistes- und Sozialwissenschaftlichen Klasse der Akademie der Wissenschaften und der Literatur 1977/2, 1-53.

Berger, Peter L. \& Richard Lieban (1963): Kulturelle Wertstruktur und Bestattungspraktiken in den Vereinigten Staaten. In: KZfSS 12, 224-236.

Berglar, Peter (1978): Geschichte als Tradition - Geschichte als Fortschritt. In: Saeculum 29, 1-10.

Bernal, John D. (1929): The World, the Flesh, and the Devil. An Enquiry into the Future of the three Enemies of the Rational Soul. London. http://www. quarkweb.com/foyle/WorldFleshDevil.pdf

Bernal, John D. ( $\left.{ }^{3} 1969\right)[1954]$ : Science in History, Vol. 3. The Natural Sciences in our Time. New York.

Bernstein, Anya (2014): Cyborgs, Weak Cosmists, and a Russian Planet. In: Blog des NYUJordan Center. http://jordanrussiacenter.org/news/cyborgs-weak-cosmists-russian-planet/\#_edn1.

Bernstein, Anya (2015): Freeze, die, come to life. The many paths to immortality in post-Soviet Russia. In: American Ethnologist 42/4, 766-781. 
Black, Edwin (2004): War Against the Weak: Eugenics and America's Campaign to Create a Master Race. New York.

Bloch, Ernst (1985): Das Prinzip Hoffnung, Kapitel 33-42. Frankfurt a.M.

Blount, Thomas (1656): Glossographia: or a dictionary, interpreting all such hard words of whatsoever language, now used in our refined English tongue. London.

Blumenberg, Hans (1958): Epochenschwelle und Rezeption. In: Philosophische Rundschau 8, 94-120.

Blumenberg, Hans (1974): Säkularisierung und Selbstbehauptung. Frankfurt a.M.

Bösch, Frank (2017): Euphorie und Ängste: Westliche Vorstellungen einer computerisierten Welt, 1945-1990. In: Hölscher 2017a, 221-252.

Bolle, Fritz (1962): Darwinismus und Zeitgeist. In: ZRGG (14), 143-178.

Bollnow, Otto F. ( $\left.{ }^{8} 1997\right)[1963]$ : Mensch und Raum. Stuttgart.

Bostrom, Nick (1998a): How long before Superintelligence? In: https:// nickbostrom.com/superintelligence.html.

Bostrom, Nick (1998b): Comments on Vinge's Singularity. http://mason. gmu.edu/ rhanson/vc.html\#bostrom = Comment by Nick Bostrom: Singularity and Predictability. In: More \& Vita-More 2013, 399-401.

Bostrom, Nick (2002): Anthropic Bias: Observation Selection Effects in Science and Philosophy. London.

Bostrom, Nick (2003a): Human Genetic Enhancements: A Transhumanist Perspective. In: Journal of Value Inquiry 37 (4/2003), 493-506 (Reprint unter: https://nickbostrom.com/ethics/genetic.html).

Bostrom, Nick (2003b): Ethical Issues in Advanced Artificial Intelligence. https://nickbostrom.com/ethics/ai.html.

Bostrom, Nick (2005): A history of transhumanist thought. In: Journal of Evolution and Technology 14/1 (Online).

Bostrom, Nick (2008): Letter from Utopia. In: Studies in Ethics, Law, and Technology, 2(1). https://nickbostrom.com/utopia.html.

Bostrom, Nick (2014): Superintelligence. Paths, Dangers, Strategies. Oxford.

Bostrom, Nick \& Milan M. Ćirković (Hg.)(2008): Global Catastrophic Risks. Oxford.

Bostrom, Nick \& Rebecca Roache (2008): Ethical Issues in Human Enhancement. In: New Waves in Applied Ethics, hg. von Jesper Ryberg \& Thomas S. Petersen \& Clark Wolf. London, 120-152.

Bostrom, Nick \& Eliezer S. Yudkowsky (2014): The Ethics of Articifial Intelligence. In: The Cambridge Handbook of Artificial Intelligence, hg. von Keith Frankish \& William M. Ramsey. Cambridge, 316-334. 
Boussenard, Louis (1890): Dix mille ans dans un bloc de glace. Paris. Braidotti, Rosi (2014)[2013]: Posthumanismus. Frankfurt a.M.

Brillouin, Leon (21957)[1956]: Science and Information Theory. New York. Brin, David (2013)[2000]: A Critical Discussion of Vinge's Singularity Concept. In: More \& Vita-More 2013, 395-397.

Bringsjord, Selmer \& Alexander Bringsjord \& Paul Bello (2012): Belief in the Singularity is Fideistic. In: Eden et al. 2012a, 394-408.

Brockman, John (Hg.)(2015): What to think about Machines that think. Today's Leading Thinkers on the Age of Machine Intelligence. New York.

Broderick, Damien (2000): The Last Mortal Generation. New York.

Broderick, Damien (2013)[2000]: A Critical Discussion of Vinge's Singularity Concept. In: More \& Vita-More 2013, 397-499.

Brooks, Rodney (2002): Flesh and Machines. How Robots will Change us. New York.

Brooks, Rodney (2008): I, Rodney Brooks, Am a Robot. In: IEEE Spectrum (Juni). https://spectrum.ieee.org/computing/hardware/i-rodneybrooks-am-a-robot

Brooks, Rodney \& Luc Steels (Hg.)(1995): The Artificial Life Route to Artificial Intelligence. Building Embodied, Situated Agents. Hillsdale.

Brown, Andrew (2005): J. D. Bernal. The Sage of Science. Oxford.

Brown, Dan (2013): Inferno. New York.

Brown, Dan (2017): Origin. New York.

Brunotte, Ulrike (2000): Puritanismus und Pioniergeist. Die Faszination der Wildnis im frühen Neu-England. Berlin.

Bryant, Clifton D. \& William E. Snizek (1973): The Cryonics Movement and Frozen Immortality. In: Society 11/1 (Nov/Dez), 56-62.

Buchloh, Paul G. (1963): Vom Pilgrim's Progress zum Pilgrim's Regress. Der Fortschrittsgedanke in der englischen und amerikanischen Literatur. In: Burck 1963, 153-178.

Bultmann, Rudolf (1958): Geschichte und Eschatologie. Tübingen.

Burck, Erich (Hg.)(1963): Die Idee des Fortschrittes. Neun Vorträge über Wege und Grenzen des Fortschrittsglaubens. München.

Burroughs, Edgar R. (1937): The Resurrection of Jimber Jaw. In: Argosy, 20 (Feb.).

Bury, John B. (1955)[1920]: The Idea of Progress. An Inquiry into Its Origin and Growth. New York. 
Cassidy, David C. (1996): Werner Heisenberg und das Unbestimmtheitsprinzip. In: Quantenphilosophie, hg. von Wolfgang Neuser \& Katharina Neuser-von Oettingen. Heidelberg, 42-49.

Caygill, Howard (1997): Stelarc and the Chimera. Kant's Critique of Prosthetic Judgment. In: Art Journal 56, 46-51.

Ceccarelli, Leah (2013): On the Frontier of Science. An American Rhetoric of Exploration and Exploitation. East Lansing.

Cherniavsky, Vladimir (1994): Die Virtualität. Philosophische Grundlagen der logischen Relativität. Hamburg.

Chalmers, David (2010): The Singularity: a Philosophical Analysis. In: Journal of Consciousness Studies 17 (9-10), 7-65.

Clarke, Arthur C. (1957): The City and the Stars. New York.

Clarke, Arthur C. (1960): Profiles of the Future. An Inquiry into the Limits of the Possible. New York.

Clarke, Arthur C. (1968): 2001. A Space Odyssey. New York.

Clute, John \& P. Nicholls (Hg.)(1999): Encyclopedia of Science Fiction. London.

Clynes, Manfred \& Nathan S. Kline (1960): Cyborgs and Space. In: Astronautics 9/1960, 26-27, 74-75.

Cobb, Jennifer (1998): Cybergrace. The Search for God in the Digital World. New York.

Coenen, Christopher (2010): Deliberating Visions: The Case of Human Enhancement in the Discourse on Nanotechnology and Convergence. In: Governing Future Technologies. Nanotechnology and the Rise of an Assessment Regime, hg. von M. Kaiser et al. Dordrecht, 73-87.

Coenen et al. (Hg.)(2010): Die Debatte über "Human Enhancement". Historische, philosophische und ethische Aspekte der technologischen Verbesserung des Menschen. Bielefeld.

Collings, Michael R. (1985): The Mechanics of Immortality. In: Yoke \& Hassler 1985, 29-38.

Comte, Auguste (21974)[1830-42]: Die Soziologie. Die positive Philosophie im Auszug. Stuttgart.

Conan Doyle, Arthur (1929): The Disintegration Machine. In: The Strand Magazine, January 1929.

Condorcet, Jean-Antoine-Nicolas Marquis de (1970)[1796]: Esquisse d'un Tableau historique des progrès de l'esprit humain. Paris 1970. 
Connolly, Randy (2001). The Rise and Persistence of the Technological Community Ideal. In: Online Communities. Commerce, Community Action and the Virtual University, hg. von Chris Werry \& Miranda Mowbray. Upper Saddle River, 317-364.

Curtis, James M. (2005): Why World History needs McLuhan. In: Strate \& Wachtel 2005, 163-175.

D

Daecke, Sigurd M. (2000): Teilhard de Chardin, Pierre (1881-1955). In: TRE 23, 28-33.

Darwin, Charles (1859): On the Origin of Species. By Means of Natural Selection, or the Preservation of Favoured Races in the Struggle for Life. London.

Darwin, Mike (1981): An Interview with Saul Kent. In: Cryonics (Nov.), 13-20.

Darwin, Mike (1991): Evaluation of the Condition of Dr. Bedford after 24 years of Cryonic Suspension. In: Cryonics (Aug.), 22-25.

Davis, Erik (1998): Techgnosis: Myth, Magic, Mysticism in the Age of Information. New York.

Dawkins, Richard (1976). The Selfish Gene. Oxford.

Dawkins, Richard (1996): The Blind Watchmaker. Why the Evidence of Evolution Reveals a Universe without Design. New York.

Dawson, Christopher (1935): Die wahre Einheit der menschlichen Kultur. Eine geschichtliche Untersuchung. Karlheinz Schmidthüs (Übers.). Regensburg.

De Grey, Aubrey (2008): Ending Aging: The Rejuvenation Breakthroughs that Could Reverse Human Aging in Our Lifetime. London.

Derham, William (1728)[1715]: Astrotheologie oder Himmlisches Vergnügen in GOTT, den bey aufmercksamen Anschauen des Himmels, und genauerer Betrachtung der Himmlischen Körper, zum augenscheinlichen Beweiß, daß ein GOTT, und derselbige ein allerguetigstes, allweises, allmaechtiges Wesen sey (Astrotheology, or a Demonstration of the Being and Attributes of God, from a Survey of the Heavens). Johann A. Fabricio (Übers.). Hamburg.

Derham, William (1764)[1727]: Physico-Theologie oder Naturleitung zu Gott, durch aufmerksame Betrachtung der Erdkugel und der darauf sich befindenen Geschoepfe, zum augenscheinlichen Beweise, daß ein Gott, und derselbige ein allerguetigstes, allweises, allmaechtigstes Wesen sey. Johann A. Fabricius (Übers.). Hamburg.

Dery, Mark (1996): Escape Velocity. Cyberculture at the End of the Century. New York. 
Descartes, René (1824)[1637]: Discours de la méthode. Victor Cousin (Hg.). Paris.

Descartes, René (1972)[1642]: Meditationen über die Grundlagen der Philosophie mit den sämtlichen Einwänden und Erwiderungen. Artur Buchenau (Hg./Übers.). Berlin.

Descartes, René (1967a): Oeuvres philosophiques de Descartes, Bd. I. Ferdinand Alquié (Hg.). Paris.

Descartes, René (1967b): Oeuvres philosophiques de Descartes, Bd. II. Ferdinand Alquié (Hg.). Paris.

Descartes, René (1969): Über den Menschen (1632) sowie Beschreibung des menschlichen Körpers (1648). Karl E. Rothschuh (Übers.). Heidelberg.

Després, Elaine \& H. Machinal (Hg.)(2014): PostHumains. Frontières, évolutions, hybridités. Rennes.

Diamandis, Peter \& Steven Kotler (2012): Abundance: The Future is better than you think. New York.

Dilthey, Wilhelm (1981)[1910]: Der Aufbau der geschichtlichen Welt in den Geisteswissenschaften. Frankfurt a.M.

Dobrée, Bonamy (1959): English Literature in the Early Eighteenth Century 1700-1740. Oxford.

Dotzler, Bernhard J. \& Peter Gendolla \& Jörgen Schäfer (1992): MaschinenMenschen. Eine Bibliographie. Frankfurt a.M.

Drexler, K. Eric (1996)[1986]: Engines of Creation. The Coming Era of Nanotechnology. London.

Drexler, K. Eric (2013): Radical Abundance: How a Revolution in Nanotechnology Will Change Civilization. New York.

Dühring, Eugen ( $\left.{ }^{5} 1894\right)[1865]:$ Der Werth des Lebens. Eine Denkerbetrachtung im Sinne heroischer Lebensauffassung. Leipzig.

Dyson, Esther et al. (1994): Cyberspace and the American Dream. A Magna Charta for the Knowledge Age. http://alberti.mit.edu/arch/4.207/texts/ cyberspace-dream.html.

Dyson, Freeman (1988): Infinite in all Directions. New York.

E

Edelhart, Mike (1983): Immortality through Science. In: Psychology Today 8/1983, 40f.

Eden, Amnon H. et al. (Hg.)(2012a): Singularity Hypothesis. A Scientific and Philosophical Assessment. Berlin. 
Eden, Amnon H. et al. (2012b): Singularity Hypothesis: An Overview. In: Eden et al. 2012a, 1-14.

Ekirch, Arthur A. (1944): The Idea of Progress in America, 1815-1860. New York.

Elias, Norbert ( $\left.{ }^{5} 1994\right)[1984]$ : Über die Zeit. Arbeiten zur Wissenssoziologie II. Frankfurt a.M.

Elias, Norbert (1997)[1939]: Über den Prozeß der Zivilisation. Soziogenetische und psycho-genetische Untersuchungen. Zweiter Band. Wandlungen der Gesellschaft. Entwurf zu einer Theorie der Zivilisation. Frankfurt a.M.

Engel, Bernhard \& Christian Breunig (2015): Massenkommunikation 2015. Mediennutzung im Intermediavergleich. In: Media Perspektiven 7-8/2015, 310-322.

Esfandiary, Fereidoun M. (1959): The day of Sacrifice. New York.

Esfandiary, Fereidoun M. (1965): The Beggar. New York.

Esfandiary, Fereidoun M. (1966): Identity Card. New York.

Esfandiary, Fereidoun M. (1970): Optimism One. The Emerging Radicalism. New York.

Esfandiary, Fereidoun M. (1973): Up-Wingers. New York.

Esfandiary, Fereidoun M. (1977): Telespheres. New York.

Ettinger, Robert C. (1948): The Penultimate Trump. In: Startling Stories (March).

Ettinger, Robert C. (1964): The Prospect of Immortality. New York.

Ettinger, Robert C. (1967): Cryonics and the Purpose of Life, in: The Christian Century 84/40, 1250-1253.

Ettinger, Robert C. (1989)[1972]: Man into Superman. New York.

Ettinger, Robert C. (2002): Cryonics Institute. A brief History and Overview. 10.01.2011, http://www.cryonics.org.

Evans, Dylan (2015): The great AI swindle. In: Brockman 2015, 172 .

F

Fabricio, Johann A. (1728): Verzeichnis der Alten und Neuen Scribenten, die sich haben lassen angelegen seyn durch Betrachtung der Natur, und der Geschoepffe, die Menschen zu Gott zu führen. In: Derham 1728, XIII-LXIV.

Feinberg, Gerald (1966): Physics and life prolongation. In: Physics Today 19/11, 45-50. 
Feinberg, Gerald (1970): Projekt Prometheus. Die langfristige Planung der Menschheitsziele. Freiburg i.B.

Ferguson, Marilyn (1980): The Aquarian Conspiracy: Personal and Social Transformation in the 1980s. Los Angeles.

Flessner, Bernd (1991): Weltprothesen und Prothesenwelten. Zu den technischen

Prognosen von Arno Schmidt und Stanistaw Lem. Frankfurt a.M.

Flessner, Bernd (1996): Archäologie im Cyberspace. Anmerkungen zu

Stanisław Lems Phantomatik. In: Lem 1996b, 7-22.

Flessner, Bernd (Hg.)(1997a): Die Welt im Bild. Wirklichkeit im Zeitalter der Virtualität. Freiburg.

Flessner, Bernd (1997b): Das Finis mundi als museale Agonie. Zu Herbert

W. Frankes Orchideenkäfig. In: Flessner 1997a, 97-115.

Flessner, Bernd (Hg.)(2000a): Nach dem Menschen. Der Mythos einer zweiten Schöpfung und das Entstehen einer posthumanen Kultur. Freiburg.

Flessner, Bernd (2000b): Antizipative Diffusion. Science Fiction als Akzeptanzbeschleuniger und Wegbereiter einer multitechnokulturellen Gesellschaft. In: Flessner 2000a, 245-264.

Flusser, Vilém (1992b): Paradigmenwechsel. In: Steffens 1992, 31-42.

Flusser, Vilém ( $\left.{ }^{4} 1992 c\right)$ [1985]: Ins Universum der technischen Bilder. Göttingen.

Flusser, Vilém (1993): Vom Virtuellen. In: Cyberspace. Zum medialen Gesamtkunstwerk, hg. von Florian Rötzer \& Peter Weibel. München, 65-71. Flusser, Vilém (1997): Medienkultur. Frankfurt a.M.

FM-2030 (1989): Are you a transhuman? Monitoring and Stimulating Your Personal Rate of Growth In a Rapidly Changing World. New York.

Foucault, Michel (1983): Sexualität und Wahrheit, Bd. 1. Frankfurt a.M.

Franke, Herbert W. (1982)[1961]: Der Orchideenkäfig. Ein utopisch-technischer Roman. München.

Franke, Herbert W. (1996): Einsteins Erben. Ypsilon Minus. Zone Null. Einsteins Erben. Frankfurt a.M.

Franke, Herbert W. (1997): Bilderwelten - Weltbilder. In: Flessner 1997a, 359-366.

Franklin, Benjamin (1780): Letter to Joseph Priestley, 08.02.1780. https:// founders.archives.gov/documents/Franklin/01-31-02-0325.

Fredericksen, Dick (1971): A Word in Edgewise 6 (February). https://digital. library. illinois.edu/items/4127ea80-29ac-0136-4d81-0050569601ca-f.

Freyermuth, Gundolf (1996): Die Avantgarde der Evolution. In: Telepolis. http://www.heise.de/tp/deutsch/special/vag/2040/2.html. 
Freyermuth, Gundolf (1998): Cyberland. Eine Führung durch den High-Tech-Underground. Reinbek.

Fukuyama, Francis (2002): Das Ende des Menschen. Stuttgart.

Funk, Isaac K. (Hg.) (1949): New Standard Dictionary of the English Language. New York.

\section{G}

Gadamer, Hans-Georg (1960): Wahrheit und Methode. Tübingen.

Galouye, Daniel F. (1999)[1964]: The 13. Floor. Köln.

Galton, Francis ( $\left.{ }^{3} 1909\right)$ : Memories of my Life. London.

Galton, Francis (1972)[1869]: Hereditary Genius. An Inquiry into Its Laws and Consequences. Gloucester.

Gantke, Wolfgang (1987): Die Bedeutung des hermeneutischen Ansatzes Otto Friedrich Bollnows für die Religionswissenschaft. Dissertation. Bonn.

Garreau, Joel (2005): Radical Evolution. The Promise and Peril of Enhancing Our Minds, Our Bodies - and What it means to Be Human. New York.

Gebauer, Gunter (1984): Hand und Gewißheit. In: Kamper \& Wulf 1984a, 234-260.

Gendolla, Peter (1992a): Anatomien der Puppe. Zur Geschichte des MaschinenMenschen beiJean Paul, E. T. A. Hoffmann, Vielliers de l'Isle-Adam und Hans Bellmer. Heidelberg.

Gendolla, Peter (1992b): Zeit. Zur Geschichte der Zeiterfahrung. Vom Mythos zur >Punktzeit‘. Köln.

Gestrich, Christof (1981): Deismus. In: TRE 8, 392-406.

Gibson, William (2000): Neuromancer. London.

Gibson, William (1986): Count Zero. New York.

Gibson, William (1988): Mona Lisa Overdrive. New York.

Giddens, Anthony ( $\left.{ }^{2} 1997\right)[1990]$ : Konsequenzen der Moderne. Frankfurt a.M.

Godwin, William (1793): An Enquiry Concerning Political Justice and Its Influence on General Virtue and Happiness, 2 volumes. London.

Goertzel, Ben (2010): A Cosmist Manifesto. Practical Philosophy for the Posthuman Age. O.O.

Good, Irving J. (1965a): Speculations Concerning the First Ultraintelligent Machine. In: Advances in Computers 6, 31-88.

Good, Irving J. (1965b)[1962]: Die soziale Bedeutung künstlicher Intelligenz. In: Phantasie in der Wissenschaft. Eine Anthologie unausgegorener Ideen, hg. von Irving J. Good. Düsseldorf, 187-193.

Gove, Philip B. (Hg.) (1993): Webster's Third New International Dictionary of the English Language, Bd. 3. Springfield (Massachussetts). 
Gräfrath, Bernd (1996): Lems >Golem‘. Parerga und Paralipomena. Frankfurt a.M.

Gräfrath, Bernd (2000): Erlösung durch Überwindung des Menschen? Stanisław Lems Philosophie transbiologischer Personen. In: Flessner 2000a, 281-300.

Graham, Elaine L. (2002): Representations of the Post/Human. Monsters, Aliens and others in Popular Culture. New Brunswick.

Graham, Elaine L. (2015): The Final Frontier? Religion and Posthumanism in Film and Television. In: Hauskeller \& Philbeck \& Carbonell 2015, 361-370.

Graham, Jenny (1995): Revolutionary in Exile. The Emigration of Joseph Priestley to America 1794-1804. Philadelphia.

Gray, Chris H. (2002): Cyborg Citizen. Politics in the Posthuman Age. New York.

Großklaus, Götz ( $\left.{ }^{2} 1997\right)$ : Medien-Zeit. Medien-Raum. Zum Wandel der raumzeitlichen Wahrnehmung in der Moderne. Frankfurt a.M.

Groys, Boris (2005): Unsterbliche Körper. In: Groys \& Hagemeister 2005, 8-18.

Groys, Boris \& Michael Hagemeister (Hg.)(2005): Die neue Menschheit. Biopolitische Utopien in Russland zu Beginn des 20. Jahrhunderts. Frankfurt a.M.

Gruman, Gerald J. (1966): A History of Ideas about the Prolongation of Life. The Evolution of the Prolongevity Hypothesis to 1800. In: Transactions of the American Philosophical Society 56/9, 1-102.

Gunderson, Keith (1964): Descartes, La Mettrie, Language and Machines. In: Philosophy 39, 193-222.

$\mathbf{H}$

Haeckel, Ernst (21902): Gemeinverständliche Vorträge und Abhandlungen aus dem Gebiete der Entwicklungslehre, Bd. 1. Bonn.

Haeckel, Ernst (1960)[1899]: Die Welträtsel. Gemeinverständliche Studien über monistische Philosophie. Berlin.

Hagemeister, Michael (2005): >Unser Körper muss unser Werk sein<, Beherrschung der Natur und Überwindung des Todes in russischen Projekten des frühen 20. Jahrhunderts. In: Groys \& Hagemeister 2005, 19-67.

Hagner, Michael (2004): Geniale Gehirne. Zur Geschichte der Elitegehirnforschung. Göttingen.

Halacy, Daniel S. (1965): Cyborg - Evolution of the Superhuman. New York. 
Haldane, J. B. S. (1923): Daedalus; or, Science and the Future. London.

Halberstam, Judith \& I. Livingston (Hg.)(1995): Posthuman Bodies. Bloomington.

Hammel, Eckhard (1994): Medien, Technik, Zeit. Zur Geschichte menschlicher Selbstwahrnehmung. In: Zeit - Medien - Wahrnehmung, hg. von Mike Sandbothe \& Walther Zimmerli. Darmstadt, 60-79.

Hansmann, Otto (2015): Transhumanismus - Vision und Wirklichkeit. Ein problemgeschichtlicher und kritischer Versuch. Berlin.

Hanson, Robin (2013): A Critical Discussion of Vinge's Singularity Concept. In: More \& Vita-More 2013, 404-406.

Harari, Yuval N. (2017)[2015]: Homo Deus. Eine Geschichte von Morgen. München.

Haraway, Donna (1985): A Manifesto for Cyborgs. Science, technology and socialist feminism in Transhumanismus 1980s. In: Socialist Review 80, 65-107.

Haraway, Donna (1991): Simians, Cyborgs and Women. The Reinvention of Nature. New York.

Harrington, Alan (1977)[1969]: The Immortalist. Millbrae.

Harrison, Peter \& Joseph Wolyniak (2015): History of >Transhumanism<. In: Notes and Queries 62/3 (September 2015), 465-467.

Hase, Thomas (1997): Die religiöse Deutung von Fortschritt und Expansion im amerikanischen Postmilleniarismus. In: ZfR 5, 115-148.

Hassan, Ihab (1977): Prometheus as Performer: Toward a Posthumanist Culture? A University Masque in Five Scenes. In: Georgia Review 31/4, 830-850.

Hassler, Donald M. (1985): Introduction. In: Yoke \& Hassler 1985, 3-6.

Hauskeller, Michael \& Thomas D. Philbeck \& Curtis Carbonell (Hg.): The Palgrave Handbook of Posthumanism in Film and Television. New York.

Hawking, Stephen \& Roger Penrose (1970): The Singularities of Gravitational Collapse and Cosmology. In: Proceedings of the Royal Society, Bd. 314, 529-548.

Hayles, N. Katherine (1999): How we Became Posthuman. Virtual Bodies in Cybernetics, Literature and Informatics. Chicago.

Heil, Reinhard (2010a): Trans- und Posthumanismus. Eine Begriffsbestimmung. In: Hilt \& Jordan \& Frewer 2010, 127-149.

Heil, Reinhard (2010b): Transhumanismus, Nanotechnologie und der Traum von Unsterblichkeit. In: Visionen der Nanotechnologie, hg. von Arianna Ferrari \& Stefan Gammel. Heidelberg, 25-49. 
Heil, Reinhard (2010c): Human Enhancement - Eine Motivsuche bei J. D. Bernal, J. B. S. Haldane und J. S. Huxley. In: Coenen et al. 2010, 41-62.

Heil, Reinhard (2018): Der Mensch als Designobjekt im frühen Transhumanismus und Techno-Futurismus. In: Designobjekt Mensch. Die Agenda des Transhumanismus auf dem Prüfstand, hg. von Paul Göcke \& Frank Meier-Hamidi. Freiburg, 53-79.

Heil, Reinhard \& Christopher Coenen (2014): Transhumanistische Visionen und Computertechnik. In: Computertechnik und Sterbekultur, hg. von Knut Böhle et al. Münster, 145-159.

Heim, Michael (1993): The Metaphysics of Virtual Reality. New York.

Heims, Steve J. ( $\left.{ }^{3} 1981\right)$ : John von Neumann and Norbert Wiener. From Mathematics to the Technologies of Life and Death. Cambridge.

Herbrechter, Stefan (2009): Posthumanismus. Eine kritische Einführung. Darmstadt.

Hesse, Eva (1992): Die Achse Avantgarde-Faschismus. Reflexionen über Filippo Thomaso Marinetti und Ezra Pound. Zürich.

Hilt, Annette \& Isabella Jordan \& Andreas Frewer (Hg.)(2010): Endlichkeit, Medizin und Unsterblichkeit. Geschichte - Theorie - Ethik. Stuttgart.

Hobbes, Thomas (1967)[1655]: Vom Körper (Elemente der Philosophie I). Max Frischeisen-Köhler (Übers.). Hamburg.

Hobsbawm, Eric (2000): Introduction: Inventing Traditions. In: The Invention of Tradition, hg. von Eric Hobsbawm \& Terence Ranger. Cambridge, 1-14.

Hölscher, Lucian (2016): Die Entdeckung der Zukunft. Göttingen.

Hölscher, Lucian (Hg.)(2017a): Die Zukunft des 20. Jahrhunderts. Dimensionen einer historischen Zukunftsforschung. Frankfurt a.M.

Hölscher, Lucian (2017b): Theoretische Grundlagen der historischen Zukunftsforschung. In: Hölscher 2017a, 7-38.

Holmes, Oliver W. (1859): The Stereoscope and the Stereograph. In: Atlantic Monthly 3 (6/1859), 738-748.

Hooper, Joseph (2005): The Prophet of Immortality. In: Popular Science, January 1 (https://www.popsci.com/scitech/article/2005-01/prophet-immortality).

Houellebecq, Michel (1998): Les Particules élémentaires. Paris.

Hruska, Joel (2017): The ISS is Getting the Most Powerful Computer Ever Sent to Space. In: Extreme Tech 14.08.2017 (Online).

Hufeland, Christoph W. (1797): Die Kunst das menschliche Leben zu verlängern. Jena. 
Hughes, James (2004): Citizen Cyborg. Why Democratic Societies must respond to the Redesigned Human of the Future. Cambridge.

Hunt, James (1864): On the Negro's Place in Nature. In: Journal of the Anthropological Society of London, Vol. 2 (1864), XV-LVI.

Hunt, James (1865): On the Negros Place in Nature. In: Memoirs of the Anthropological Society of London, 1863-64, Vol. 1, 1-64.

Huntington, Richard \& Peter Metcalf ( $\left.{ }^{2} 1991\right):$ Celebrations of Death. The Anthropology of Mortuary Ritual. Cambridge.

Husserl, Edmund (1928): Vorlesungen zur Phänomenologie des inneren Zeitbewußtseins. Martin Heidegger (Hg.). Halle.

Huxley, Julian (1923): Essays of a Biologist. New York.

Huxley, Julian (1929): Religion without Revelation. New York.

Huxley, Julian (1942): Evolution. The Modern Synthesis. New York.

Huxley, Julian (1951): Knowledge, Morality and Destiny. In: Psychiatry XIV, 127-151.

Huxley, Julian (1957a): New Bottles for New Wine. Essays by Julian Huxley. London.

Huxley, Julian (1957b): Transhumanism. In: Huxley 1957a, 13-17.

Huxley, Julian (1957c): A Re-definition of Progress. In: Huxley 1957a, 18-40.

Huxley, Julian (1957d): Evolutionary Humanism. In: Huxley 1957a, 1840.

Huxley, Julian (1965)[1964]: Ich sehe den künftigen Menschen. Natur und neuer Humanismus. München.

\section{I}

Immortality Institute (Hg.)(2004): The Scientific Conquest of Death. Essays on infinite Lifespans. Buenos Aires.

Innerhofer, Roland (1996): Deutsche Science Fiction 1870-1914. Rekonstruktion und Analyse der Anfänge einer Gattung. Wien.

Innerhofer, Roland (2000): Die technische Modernisierung des künstlichen Menschen in der Literatur zwischen 1800 und 1900. In: Flessner 2000a, 69-100.

Innerhofer, Roland (2003): Von Frankensteins Monster zu Edisons Eva. Die Geburt des künstlichen Menschen aus dem Geist der Maschine. In: Mythen der Kreativität. Das Schöpferische zwischen Innovation und Hybris, hg. von Oliver Krüger \& Refika Sariönder \& Annette Deschner. Frankfurt a.M., 269-297. 
Irrgang, Bernhard (2005): Posthumanes Menschsein? Künstliche Intelligenz, Cyberspace, Roboter und Designer-Menschen. Wiesbaden.

Iser, Wolfgang (1990): Fingieren als anthropologische Dimension der Literatur. Konstanz.

Iser, Wolfgang (1991): Das Fiktive und das Imaginäre. Perspektiven literarischer Anthropologie. Frankfurt a.M.

$\mathrm{J}$

Jäger, Henrik (1998): Das Maschinenherz als Metapher für das rechnende Denken. Technik und Kulturkritik im Werk des Zhuangzi. In: Schwaetzer \& Stahl-Schwaetzer 1998a, 165-184.

Jastrow, Robert (1981): The Enchanted Loom. Mind in the Universe. New York. Jauß, Hans Robert (1987): Die Theorie der Rezeption - Rückschau auf ihre unentdeckte Vorgeschichte. Konstanz.

Johannesson, Rolf (1992): Informationstheorie - Grundlage der (Tele-)Kommunikation. Lund.

Jones, Neil R. (1931): The Jameson Satellite. In: Amazing (Juli).

Jordan, Isabella \& Andreas Frewer (2010): Todesverdrängung, (Un-)Sterblichkeit und Medizin. Theoretische und soziale Aspekte des Transhumanismus. In: Hilt \& Jordan \& Trewer 2010, 151-169.

Joy, Bill (2000): Why the Future doesn't need us. In: Wired 8.04, 1-11.

$\mathbf{K}$

Kamper, Dietmar (1976): Einleitung: Vom Schweigen des Körpers. In: Zur Geschichte des Körpers, hg. von Dietmar Kamper \& Volker Rittner. München, 7-12.

Kamper, Dietmar (1995): Unmögliche Gegenwart. Zur Theorie der Phantasie. München.

Kamper, Dietmar (1999): Die Ästhetik der Abwesenheit. Die Entfernung der Körper. München.

Kamper, Dietmar \& Christoph Wulf (Hg.)(1984a): Das Schwinden der Sinne. Frankfurt a.M.

Kamper, Dietmar \& Christoph Wulf (1984b): Blickwende. Die Sinne des Körpers im Konkurs der Geschichte. In: Kamper \& Wulf 1984a, 9-17.

Kamper, Dietmar \& Christoph Wulf (Hg.)(1987a): Die sterbende Zeit. Zwanzig Diagnosen. Darmstadt.

Kamper, Dietmar \& Christoph Wulf (1987b): Die Zeit, die bleibt. In: Kamper \& Wulf 1987a, 7-10. 
Kamper, Dietmar \& Christoph Wulf (Hg.)(1994): Anthropologie nach dem Tode des Menschen. Vervollkommnung und Unverbesserlichkeit. Frankfurt a.M.

Kapell, Matthew W. (2016): Exploring the Next Frontier. Vietnam, NASA, Star Trek and Utopia in 1960s und 1970s American Myth and History. New York. Kapuściński, Ryszard (1992)[1990]: Lapidarium. Notizen und Reflexionen. Frankfurt a.M.

Katz, Elihu \& David Foulkes (1962): On the use of mass media as sescapes: Clarification of a concept. In: Public Opinion Quarterly 26/3 (1962), 377-388.

Keller, Christoph (2003): Buildung Bodies. Der Mensch im biotechnischen Zeitalter. Reportagen und Essays. Zürich.

Kemeny, John (1972): Man and Computers. New York.

Kent, Saul (1983): The first Cryonicist. In: Cryonics (March), 4-12.

Kephart, William M. (1950): Status after Death. In: American Sociological Review 15/5, 635-643.

Keppler, Angela (2001): Mediales Produkt und sozialer Gebrauch. Stichworte zu einer inklusiven Medienforschung. In: Massenkommunikation, Interaktion und soziales Handeln, hg. von Tilmann Sutter \& Michael Charlton. Opladen, 125-145.

Kerr, Philipp (1998): The Second Angel. London 1998.

Kirkinen, Heikki (1960): Les Origines de la Conception Moderne de l'Homme-Machine. Le Problème de l'Ame en France a la Fin du Regne de Louis XIV (1670-1715). Helsinki.

Klaus, Georg (1969a): Wörterbuch der Kybernetik 1. Frankfurt a.M.

Klaus, Georg (1969b): Wörterbuch der Kybernetik 2. Frankfurt a.M.

Klein, Jürgen (1986): Materialismus - Evolution - Induktion. Wissenschaft im neunzehnten Jahrhundert. In: ZRGG (38), 249-258.

Klemperer, Victor ( $\left.{ }^{9} 1997\right)$ : Ich will Zeugnis ablegen bis zum letzten. Tagebücher 1933-1941. Walter Nowojski (Hg.). Berlin.

Kluge, Sven (2014): Menschenverbesserung in einer Welt ohne Mensch? Zur Aktualität und Kritikwürdigkeit von Günther Anders' Diagnose einer Antiquiertheit des Menschen. In: Jahrbuch Pädagogik 2014. Menschenverbesserung. Transhumanismus, 83-104.

Köhne, Julia B. (2014): Geniekult in Geisteswissenschaften und Literaturen um 1900 und seine filmischen Adaptionen. Wien. 
Koselleck, Reinhart (1975): Fortschritt. In: Geschichtliche Grundbegriffe. Historisches Lexikon zur politisch-sozialen Sprache in Deutschland, Bd. 2, hg. von Otto Brunner \& Werner Couse \& Reinhart Kosselleck. Stuttgart, 351-423.

Koplos, Janet (1993): Stelarc in the Kitchen. In: Art in America 81 (10/1993), 104.

Kroker, Arthur \& M. Kroker (1996): Hacking the Future. Geschichten für die fleischfressenden 90er E Kalifornischer Epilog. Wien.

Kroker, Arthur \& M. Weinstein (1997)[1994]: Datenmüll. Die Theorie der virtuellen Klasse. Wien.

Krüger, Oliver (2004a): Virtualität und Unsterblichkeit. Die Visionen des Posthumanismus. Freiburg i.Br.

Krüger, Oliver (2004b): Gnosis im Cyberspace? Die Körperutopien des Posthumanismus. In: Utopische Körper. Visionen künftiger Körper in Geschichte, Kunst und Gesellschaft, hg. von Kristiane Hasselmann \& Sandra Schmidt \& Cornelia Zumbusch. Paderborn, 131-146.

Krüger, Oliver (2005): Gnosis in Cyberspace? Body, Mind and Progress in Posthumanism. In: Journal of Technology and Evolution (E-Journal).

Krüger, Oliver (2010): The Suspension of Death. The Cryonic Utopia in the Context of the U.S. Funeral Culture. In: Marburg Journal of Religion 15 (Online).

Krüger, Oliver (2011): Much Ado about Nothing - oder: Der Transhumanismus als Phänomen der Jahrtausendwende? In: Humanismus. Sein kritisches Potential für Gegenwart und Zukunft?, hg. von Adrian Holderegger \& Siegfried Weichlein \& Simone Zurbuchen. Basel, 445-462.

Krüger, Oliver (2012): Die mediale Religion. Probleme und Perspektiven religionswissenschaftlicher und wissenssoziologischer Medienforschung. Bielefeld.

Krüger, Oliver (2015): Gaia, God, and the Internet - revisited. The History of Evolution and the Utopia of Community in Media Society. In: Online - Heidelberg Journal for Religions on the Internet 8 (Online).

Krüger, Oliver (2017): »Religion« definieren. Eine wissenssoziologische Analyse religionsbezogener Enzyklopädistik. In: Zeitschrift für Religions- und Geistesgeschichte 69/1, 1-46.

Krüger, Oliver \& Andrea Rota (2015): Die Verkündigung von Jehovas Königreich in Hörfunk und Internet. Ein Beitrag zur Medienhermeneutik. In: Religion, Staat, Gesellschaft 16 1/2, Sonderheft Zum Bibel- und Weltverständnis christlicher Religionen, 75-108. 
Krysmanski, Hans Jürgen (2014): Der ganz alltägliche Transhumanismus. In: Jahrbuch Pädagogik 2014. Menschenverbesserung. Transhumanismus, 123142.

Kuny, Terry (1997): A Digital Dark Ages? Challenges in the Preservation of Electronic Information. 3RD IFLA Council and General Conference. https://archive.ifla.org/ IV/ifla63/63kuny1.pdf

Kurzweil, Ray (1990): The Age of Intelligent Machines. Cambridge.

Kurzweil, Ray (1993): The 10\% Solution for a Healthy Life. New York.

Kurzweil, Raymond (1999a): The Age of Spiritual Machines. When Computers Exceed Human Intelligence. New York.

Kurzweil, Ray (1999b): Spiritual Machines. The Meaning of Man and Machine. In: The Futurist 33 (11/1999), 16-21.

Kurzweil, Ray (2000): Foreword. In: The Eternal E-customer: How Emotionally Intelligent Interfaces Can Create Long-lasting Customer Relationships, von Bryan Bergeron. New York, IX-XI.

Kurzweil, Ray (2005): The Singularity is near. When Humans transcend Biology. New York.

Kurzweil, Ray (2010): How my predictions fared. http://www.kurzweilai. net/images/How-My-Predictions-Are-Faring.pdf.

Kurzweil, Ray ( ${ }^{32012 a): ~ F o r e w o r d ~ t o ~ t h e ~ T h i r d ~ E d i t i o n . ~ I n: ~ T h e ~ C o m p u t e r ~}$ $\mathcal{E}$ the Brain, von John von Neumann. New Haven, 11-28.

Kurzweil, Ray (2012b): How to Create a Mind. The Secret of Human Thought Revealed. New York.

Kurzweil, Ray \& Terry Grossman (2004): Fantastic Voyage. Live Long Enough to Live Forever. New York.

Kurzweil, Ray \& Terry Grossman (2009): Transcend: Nine Steps to Living Well Forever. New York.

L

Laderman, Gary (1996): The Sacred Remains. American Attitudes towards Death 1799-1883. New Haven.

La Mettrie, Julien Offray de (1994)[1747]: Der Mensch eine Maschine. In: Künstliche Menschen. Dichtungen und Dokumente über Golems, Homunculi, lebende Statuen und Androiden, hg. von Klaus Völker. Frankfurt a.M., 78-102.

Larsen, Dave (1967): Cancer Victim's Body Frozen for Future Revival Experiment. In: Los Angeles Times, 19.01.1967.

Lavery, David (1992): Late for the Sky. The Mentality of the Space Age. Carbondale (Illinois). 
Leary, Timothy F. (1994): Chaos and Cyber Culture. San Francisco.

Leary, Timothy F. \& R. U. Sirius (1997): Design for Dying. San Francisco.

Lem, Stanisław (1972): Roboter in der Science Fiction. In: Science Fiction.

Theorie und Geschichte, hg. von Eike Barmeyer. München,163-186.

Lem, Stanisław (1980)[1957]: Dialoge. Frankfurt a.M.

Lem, Stanisław (1981)[1964]: Summa Technologiae. Frankfurt a.M.

Lem, Stanisław (1986)[1981]: Also sprach Golem. Frankfurt a.M.

Lem, Stanisław (1987)[1982]: Lokaltermin. Frankfurt a.M.

Lem, Stanisław (1988): Provokationen. Frankfurt a.M.

Lem, Stanisław (1995)[1971]: Sterntagebücher. Frankfurt a.M.

Lem, Stanisław (1996a) [1957]: Gibt es Sie Mr. Johns? In: Spiegel special (3/1996), 70-74.

Lem, Stanisław (1996b): Die Entdeckung der Virtualität. Frankfurt a.M.

Lem, Stanisław (1996c)[1974]: Die Wonnen der Psychemie. In: Lem 1996b, 112-151.

Lem, Stanisław (2000): Die Technologiefalle. Essays. Frankfurt a.M.

Le Roy, Édouard (1928): Les origines humaines et l'évolution de l'intelligence. XVI. Conclusion. In: Cours et Conférences 29 (16/1928), 696-724.

Lévy, Pierre (1997): Die kollektive Intelligenz. Eine Anthropologie des Cyberspace. Mannheim.

Lévy, Pierre (2001): Cyberculture. Minneapolis.

Lighthall, William D. (1930): The Person of Evolution. The outer Consciousness. The Outer Knowledge. The Directive Power. Toronto.

Lighthall, William D. (1940): The Law of Cosmic Evolutionary Adaptation. An interpretation of recent thought. In: Proceedings and transactions of the Royal Society of Canada 34, section 2, 135-41.

List, Elisabeth (1996): Platon im Cyberspace. Technologien der Entkörperlichung und Visionen vom körperlosen Selbst. In: Kultur in Bewegung. Beharrliche Ermächtigungen, hg. Ilse Modelmog \& Edit Kirsch-Auwärter. Freiburg, 83-110.

Liversidge, Anthony: Interview: Frank Tipler. A Physicist proposes a Theory of Eternal Life that Yields God. In: Omni 17 (10/1994), 89-109.

Loh, Janina (2018): Trans- und Posthumanismus zur Einführung. Hamburg.

Lovejoy, Arthur O. (1961): The Great Chain of Being. A Study of the History of an Idea. Cambridge.

Lovelock, James E. (1979): Gaia. A new Look at Life on Earth. Oxford.

Löwith, Karl ( $\left.{ }^{5} 1967\right)[1953]:$ Weltgeschichte und Heilsgeschehen. Die theologischen Voraussetzungen der Geschichtsphilosophie. Stuttgart. 
Löwith, Karl (1963): Das Verhängnis des Fortschrittes. In: Burck 1963, $17-40$.

Lübbe, Hermann (21994)[1992]: Der Lebenssinn der Industriegesellschaft. Über die moralische Verfassung der wissenschaftich-technischen Zivilisation. Berlin.

Luckmann, Thomas (1986): Zeit und Identität: Innere, soziale und historische Zeit. In: Zeit als Strukturelement von Lebenswelt und Gesellschaft, hg. von Friedrich Fürstenberg \& Ingo Mörth. Linz, 135-174.

Lüdeking, Karlheinz (1996): Das Vergnügen des Körpers. In: Kunstforum International 133, 56-67.

Luetke, Joachim (2000): Posthuman. The Art of Joachim Luetke. Stuttgart.

Luhmann, Niklas (1979): Zeit und Handlung - Eine vergessene Theorie. In: Zeitschrift für Soziologie (1/1979), 63-81.

Lyotard, Jean-François et al. (1985): Immaterialität und Postmoderne. Berlin. Lyotard, Jean-François (1987): Postmoderne für Kinder. Wien.

\section{$\mathbf{M}$}

Maaßen, Helmut (1991): Offenbarung, Mythos und Metaphysik: Drei Gottesbegriffe der Tradition und Whiteheads bipolarer Gott. In: Die Gifford Lectures und ihre Deutung. Materialien zu Whiteheads >Prozess und Realität, Bd. 2., hg. von Michael Hampe \& Helmut Maßßen. Frankfurt a.M., 217-233.

MacCorquodale, Duncan (Hg.)(1996): This is my body... this is my software. London.

Macho, Thomas (1994): Vom Skandal der Abwesenheit. In: Kamper \& Wulf 1994, 417-436.

Madsen, Sven A. (1986): Das letzte Geheimnis. In: Rottensteiner 1986, 202-249.

Mann, Laurie D. (1981): How the Cryonics Movement Thawed Out. In: Cryonics 16 (Nov), 9-12.

Marchand, Philip (1998): Marshall McLuhan. The Medium and the Messenger. A Biography. Toronto.

Martin, George M. (1971): Brief proposal on immortality: an interim solution. In: Perspectives in Biology and Medicine 14 (2): 339.

Marvin, Carolyn (1987): Information and History. In: Slack \& Fejes 1987, 49-62.

Mathar, Rudolf (1996): Informationstheorie. Diskrete Modelle und Verfahren. Stuttgart.

Mattelart, Armand (1996): The Invention of Communication. Minneapolis.

Maul, Stefan M. (2005): Das Gilgamesch-Epos. München. 
McCalla, Arthur (1998): Evolutionism and Early Nineteenth-Century Histories of Religions. In: Religion 28 (1998), 29-40.

McGinn, Colin (1999): Hello, HAL. In: New York Times Book Review, 03.01.1999.

McLuhan, Eric (1999): Introduction. In: McLuhan 1999a, IX-XXVIII.

McLuhan, Marshall (1965)[1964]: Understanding Media. The Extensions of Man. New York.

McLuhan, Marshall (1968): War and Peace in the Global Village. An Inventory of some of the Current Spastic Situations that could be eliminated by more Feedforward. New York.

McLuhan, Marshall (1994)[1964]: Understanding Media. The extensions of Man. Cambridge.

McLuhan, Marshall (1999a): The Medium and the Light. Reflections on Religion, hg. von Eric McLuhan \& Jacek Szlarek. Toronto.

McLuhan, Marshall (1999b): Electric Consciousness and the Church. In: McLuhan 1999a, 78-88.

McLuhan, Marshall (1999c): Brief an Jaques Maritain vom 6. 5. 1969. In: McLuhan 1999a, 70-73.

McLuhan, Marshall (2002)[1962]: The Gutenberg Galaxy. The Making of Typographic Man. Toronto.

Media Perspektiven (2015): Media Perspektiven Basisdaten 2017. http:// www.ard-werbung.de/media-perspektiven/basisdaten.

Mehlhausen, Joachim \& Daniela Dunkel (1994): Monismus/Monistenbund. In: TRE, Bd. 23, 212-219.

Meyer-Drawe (2014): Mit >eiserner Inkonsequenz` fürs Überleben - Günther Anders. In: Jahrbuch Pädagogik. Menschenverbesserung. Transhumanismus, 105-122.

Miller, Perry ( $\left.{ }^{8} 1979\right):$ The Transcendentalists. An Anthology. Cambridge.

Minsky, Marvin L. (1954): Neural Nets and the Brain Model Problem. Dissertation. Princeton.

Minsky, Marvin L. (1965): Matter, Mind and Models. In: Proceedings of IFIP Congress 1965, I, May, 45-49. Washington.

Minsky, Marvin L. (1967): Computation. Finite and Infinite Machines. Englewood Cliffs.

Minsky, Marvin L. (1977): Plain Talk about Neurodevelopmental Epistemology. In: Proceedings of the Fifth International Joint Conference on Artifcial Intelligence, hg. von P. Winston \& R. Brown. Cambridge, 421-450.

Minsky, Marvin L. (1981): Music, Mind and Meaning. In: Computer Music Journal 5 (3/1981). 
Minsky, Marvin L. (1982): Why People think Computers can't. In: $A I$ Magazine 3 (4/1982).

Minsky, Marvin L. (1984): Communication with Alien Intelligence. In: Extra-terrestrial. Science and Alien Intelligence, hg. von Edward Regis, Jr. Cambridge.

Minsky, Marvin L. (1996)[1986]: Foreword. In: Drexler 1996, IV-VII.

Minsky, Marvin L. (1988)[1985]: Society of Mind. New York.

Minsky, Marvin L. (1992a): Alienable Rights. In: Discover 14 (7/1993), 2426.

Minsky, Marvin L. (1992b): Future of AI Technology. In: Toshiba Review 47 (7/1992).

Minsky, Marvin L. (1992c): A Conversation with Marvin Minsky. In: AI Magazine 14 (3/1992), 31-45.

Minsky, Marvin L. (1994): Will Robots Inherit the Earth? In: Scientific American 271 (10/1994), 108-113.

Minsky, Marvin L. (2006): The Emotion Machine: Commonsense Thinking, Artificial Intelligence, and the Future of the Human Mind. New York.

Mitchell, David (2004): Cloud Atlas. New York.

Moravec, Hans (1979): Today's Computers, Intelligent Machines and Our Future. In: analog. Science Fiction/Science Fact 99, February 1979, 59-84.

Moravec, Hans (1988): Mind Children. The Future of Robot and Human Intelligence. Harvard.

Moravec, Hans (1992): Pigs in Cyberspace. In: Miller \& Wolf 1992, 15-22.

Moravec, Hans (1995): Human Culture. A Genetic Takeover Underway. In: Langton 1995, 167-199.

Moravec, Hans (1996a): Körper, Roboter, Geist. In: Kunstforum International 133, 98-112.

Moravec, Hans (1996b): Die Evolution des postbiologischen Lebens. Szenarien der Entwicklung von intelligenten Robotern und Agenten. http://www.heise.de/tp/ deutsch/special/vag/6055/1.html.

Moravec, Hans (1996c): Geisteskinder. Universelle Roboter. In vierzig Jahren haben sie uns überholt. In: C'T magazin für Computer und Technik (6/1996), 98-104.

Moravec, Hans (1998): When will Computer match the human brain? In: Journal of Transhumanism 1 (Online).

Moravec, Hans (1999): Robot. Mere Machine to Transcendent Mind. New York. Moravec, Hans (2009)[2008]: Rise of the Robots - The Future of Artificial Intelligence. In: Scientific American, 23.03.2009 https://www.scientificamerican.com/article/rise-of-the-robots. 
Moravec, Hans \& Frederic Pohl (1993): Souls in Silicon. In: Omni 16 (11/1993), 66-76.

Moravec, Hans \& Stuart Shieber (1997): Will Researchers Develop a Computer that Duplicates Human Intelligence in the Foreseeable Future? In: CQ Researcher, 14.11.1997, 1001.

More, Max (1990): Transhumanism. Towards a Futurist Philosophy. https://web. archive. org/web/20051029125153/http://www.maxmore. com/transhum.htm.

More, Max (1994): On Becoming Posthuman. http://www.maxmore. com/becoming. htm (15.12.2003).

More, Max (1995a): The Diachronic Self: Identity, Continuity, Transformation. http://www.maxmore.com/disscont.htm (15.12.2003).

More, Max (1995b): The Extropian Principles 2.6. http://www.maxmore. com/extprn26.htm (15.12.2003).

More, Max (1996a): Thinking about Thinking. In: Wired (12/2000), 151f.

More, Max (1996b): Transhumanism. Towards a Futurist Philosophy. http://www.maxmore.com/transhum.htm (15.12.2003).

More, Max (1997a): Virtue and Virtuality. From Enhanced Reality to Experience Machines. http://www.maxmore.com/virtue.htm (15.12.2003).

More, Max (1997b): Beyond the Machine. Technology and Posthuman Freedom. http://www.maxmore.com/machine.htm (15.12.2003).

More, Max (1998a): Extropian Principles. Version 3.0. A Transhumanist Declaration. http://www.maxmore.com/extprn3.htm (15.12.2003).

More, Max (1998b): Dynamic Optimism. An Extropian-Emotional Virtue. http://www.maxmore.com/optimism.htm (15.12.2003).

More, Max (1999): A Letter to Mother Nature. http://www.maxmore. com/mother.htm (15.12.2003).

More, Max (2000a): Extropy Institute. Successful Memetic Incubator, 1988-2000 Onward! http://www.extropy.com/success.htm (15.12.2003).

More, Max (2000b): Embrace, Don't Relinquish, the Future. http://www. maxmore.com/maxview.htm (15.12.2003).

More, Max (2000c): Transhumanists vs. Mysterians on the Posthuman Condition. http://www.maxmore.com/feedmag1.htm (15.12.2003).

More, Max (2009): Why Catholics Should Support the Transhumanist Goal of Extended Life. http://strategicphilosophy.blogspot. com/2009/09/why-catholics-should-support.html.

More, Max (2010): The Overhuman in the Transhuman. In: Journal of Evolution and Technology 21 (Online). 
More, Max (2013a): A Critical Discussion of Vinge's Singularity Concept. In: More \& Vita-More 2013, 406-409.

More, Max (2013b): The Philosophy of Transhumanism. In: More \& Vita-More 2013, 2-17.

More, Max \& Natasha Vita-More (Hg.)(2013): The Transhumanist Reader. Chichester.

Morgan, Richard (2002): Altered Carbon. London.

Mori, Masahiro (1980)[1974]: The Buddha in the Robot. A Robot Engineer's Thoughts on Science and Religion. Tokyo.

\section{$\mathbf{N}$}

Nassehi, Armin (1993): Die Zeit der Gesellschaft. Auf dem Weg zu einer soziologischen Theorie der Zeit. Opladen.

Neesham, Claire \& Caroline Smith (1995): Beyond Flesh \& Blood. In: Nerw Scientist 148 (11/1995), $28 f$.

Negroponte, Nicholas (1996): Being Digital. New York.

Neilson, William A. (Hg.)(1959): Webster's New International Dictionary of the English Language. Springfield (Massachussetts).

Nelson, Robert (1968): We Froze the First Man. The Startling True Story of the First Great Step toward Human Immortality. New York.

Neumann, John von (1966): Theory of Self-Reproducing Automata. Urbana.

Neumann, John von (61991)[1958]: Die Rechenmaschine und das Gehirn. München.

Neumann, John von \& Oskar Morgenstern ( $\left.{ }^{9} 1966\right)$ [1944]: Theory of Games and Economic Behaviour. Princeton.

$\mathrm{Ni}$, Siobhan (2007): >Why may not man one day be immortal? Population, perfectibility, and the immortality question in Godwin's political justice. In: History of European Ideas 33, 25-39.

Nielsen, Michael (2013)[2000]: A Critical Discussion of Vinge's Singularity Concept. In: More \& Vita-More 2013, 409-411.

Nicholls, Peter (1999a): Cyberpunk. In: Clute \& Nicholls 1999, 288-290.

Nicholls, Peter (1999b): Virtual Reality. In: Clute \& Nicholls 1999, $1285 f$.

Nowlan, Philip F. (1928): Armageddon 2419 AD. In: Amazing (Aug.).

Nozick, Robert (1991)[1989]: Vom richtigen, guten und glücklichen Leben. München.

$\mathrm{O}$

O’Bryan, Jill (2005): Carnal Art. ORLAN's Refacing. Minneapolis. 
Oliver, Myrna (1981): Families Awarded Nearly \$ 1 Million in Cryonics Suit. In: Los Angeles Times, 6.6.1981.

O'Neill, Gerard K. (1977): The High Frontier. Human Colonies in Space. New York.

Ong, Walter J. (1952): The Mechanical Bride. Christen the Folklore of Industrial Man. In: Social Order 2, 79-85.

Orlan (1999): Self-Hybridation, hg. von Laurent Cauwet. Paris.

Orlan (2000): Manifeste de L'Art Charnel. http://www.orlan.eu/texts/\# manifestefr.

Ossege, Barbara (1999): Tod, wo ist dein Stachel? Zur Herstellbarkeit von Leben und Tod. In: Riegler \& Lammer \& Stecher 1999, 172-180.

$\mathbf{P}$

Paley, William (1802): Natural Theology. London.

Paley, William (1842): The Works of William Paley, D. D., Archdeacon of Carlisle, to which is prefixed The Life of the Author. Edinburgh 1842.

Pannenberg, Wolfhart (1961): Offenbarung als Geschichte. Göttingen.

Pannenberg, Wolfhart (1995): Breaking a Taboo. Frank Tipler's The Physics of Immortality. In: Zygon 30, 309-314.

Passmore, John (1975)[1970]: Der vollkommene Mensch. Eine Idee im Wandel von drei Jahrtausenden. Stuttgart.

Pepperell, Robert (1995): The Post-Human Condition. Oxford.

Perry, Michael R. (1991): For the Record - First Suspension no >Blue Sky< Event, in: Cryonics 12 (July), 11-12.

Perry, R. Michael (1992): For the record. Gerald Feinberg, Scientific Cryonics Advocate. In: Cryonics 13/6, 4-11.

Perry, R. Michael (2000): Forever for All. Moral Philosophy, Cryonics, and the Scientific Prospects for Immortality. O.O.

Pesce, Mark (2000): The Playful World. How Technology is Transforming our Imagination. New York.

Piaget, Jean (1969)[1936]: Das Erwachen der Intelligenz beim Kinde. Stuttgart. Plessner, Helmuth (1952): Über die Beziehung der Zeit zum Tode. In: Eranos-Jahrbuch 1951, hg. von Olga Fröbe-Kapteyn. Zürich, 349-286.

Plessner, Helmuth (1980): Gesammelte Schriften III. Anthropologie der Sinne, hg. von Günter Dux \& Odo Marquard \& Elisabeth Ströker. Frankfurt a.M.

Pohl, Frederik (1964): Intimations of Immortality. In: Playboy (Juni 1964), 158. 
Pohl, Frederik (1989): When Machines Surpass People. Review of Mind Children by Hans Moravec. In: New Scientist (Feb. 1989), 65.

Polkinghorne, John (1995): I am the Alpha and the Omega Point. Review. In: Nerw Scientist 4.2.1995 (Online).

Pörksen, Bernhard (2000): Das Menschenbild der Künstlichen Intelligenz. Ein Gespräch mit Joseph Weizenbaum. In: Flessner 2000a, 265-280.

Porush, David (1992): Transcendence at the Interface: The Architecture of Cyborg Utopia - or - Cyberspace Utopoids as Postmodern Cargo Cult. In: Miller \& Wolf 1992, 127-135.

Press, William H. (1986): A place for teleology? In: Nature 320, $315 f$.

Priesner, Claus (2011): Geschichte der Alchemie. München.

Proudfoot, Diane (2012): Software Immortals: Science or Faith? In: Eden et al. 2012a, 367-394.

Q

Quigley, Christine (1998): Modern Mummies. The Preservation of the Human Body in the Twentieth Century. Jefferson.

\section{$\mathbf{R}$}

Randow, Gero von (1998): Roboter. Unsere nächsten Verwandten. Hamburg. Rapp, Friedrich (1992): Fortschritt. Entwicklung und Sinngehalt einer philosophischen Idee. Darmstadt.

Raulerson, Joshua (2013): Singularities. Technoculture, Transhumanism, and Science Fiction in the Twenty-first Century. Liverpool.

Redding, Micah (2016): The Jesuit Priest Who Believed in God and the Singularity. In: Motherboard 08.03.2016 (Online).

Regis, Ed (1990): Great Mambo Chicken and the Transhuman Condition. Science Slightly over the Edge. Reading (Massachusetts).

Reitmeier, Heidi (1996): >I Do Not Want To Look Like...< Orlan on becoming-Orlan. In: MAKE. The magazine of women's art, 72 (10/11 1996).

Richard, Birgit (2000): Vergehen Konservieren Uploaden. Strategien für die Ewigkeit. In: Kunstforum International 151, 51-84.

Riegler, Johanna (1999): Schrecklich gut! Zum Verhältnis von Revolution und Reaktion, Monster und Puppe, Faszination und Schrecken. In: Riegler \& Lammer \& Stecher 1999, 52-59.

Riegler, Johanna \& Christina Lammer \& Marcella Stecher et al. (Hg.) (1999): Puppe. Monster. Tod. Kulturelle Transformationsprozesse der Bio- und Informationstechnologien. Wien. 
Rievman, Ellen B. (1976): The Cryonics Society. A Study of Variant Behavior among the Immortalists, Diss. Florida Atlantic University.

Roco, Mihail C. \& William S. Bainbridge (2003): Converging technologies for improving human performance: Nanotechnology, biotechnology, information technology and cognitive science. Dordrecht (http:// www.wtec.org/ConvergingTechnologies /Report/NBIC_report.pdf).

Röd, Wolfgang ( $\left.{ }^{3} 1995\right)$ : Descartes. Die Genese des Cartesianischen Rationalismus. München.

Rohbeck, Johannes (1987): Fortschrittstheorie der Aufklärung. Französische und englische Geschichtsphilosophie in der zweiten Hälfte des 18. Jahrhunderts. Frankfurt a.M.

Rohbeck, Johannes (1993): Technologische Urteilskraft. Zu einer Ethik technischen Handelns. Frankfurt a.M.

Rossi, Paolo (1987): Das Altern der Welt und die sgroße Ruine< der Neuzeit. In: Kamper \& Wulf 1987a, 34-49.

Roszak, Theodore (21994)[1986]: The Cult of Information. A Neo-Luddite Treatise on High Tech, Artificial Intelligence, and the True Art of Thinking. Berkeley.

Rothblatt, Martine (2014): Virtually Human. The Promise - and the Peril - of Digital Immortality. New York.

Rottensteiner, Franz (Hg.) (1986): Phantastische Zeiten. Frankfurt a.M.

Rottensteiner, Franz (1997): Unsterblichkeit im Computer? In: Flessner 1997a, 367-381.

Rötzer, Florian (1989): Technoimaginäres - Ende des Imaginären? In: Kunstforum International 98, 54-59.

Rötzer, Florian (1996): Die Zukunft des Körpers. In: Kunstforum International 132, 54-71.

Rötzer, Florian (1997): Als Cyborg ewig leben. In: Spiegel special (3/1997), 72-78.

Rucker, Rudy (1982): Software. New York.

Rucker, Rudy (2007): Postsingular. New York.

Russell, William C. (1974)[1887]: The Frozen Pirate. Volumes 1 and 2. New York.

Rutsky, R. L. (1999): High Techné. Art and Technology from the Machine Aesthetic to the Posthuman. Minneapolis.

$\mathbf{S}$

Sampson, Ronald V. (1956): Progress in the Age of Reason. The 17th Century to the Present Day. London. 
Sandberg, Anders (1998): My Thoughts And Comments on the Omega Point Theory of Frank J. Tipler. In: Anders Sandberg Pages, http:// www.aleph.se/Trans/ Global/ Omega/tipler_page.html.

Sandberg, Anders (2013a)[2010]: An overview of models of technological singularity. In: More \& Vita-More 2013, 376-394.

Sandberg, Anders (2013b)[2000]: A Critical Discussion of Vinge's Singularity Concept. In: More \& Vita-More 2013, 411-416.

Sandberg, Anders \& N. Bostrom (2008): Whole Brain Emulation. A Roadmap. Technical Report \#2008 3, Future of Humanity Institute. Oxford. http://www.fhi.ox.ac.uk/reports/2008-3.pdf

Sanders, Olaf (2014): Echte Menschen? Posthumanistische Spuren in populärer Serienkultur und ihrer filmischen Vorgeschichte. In: Jahrbuch Pädagogik 2014. Menschverbesserung. Transhumanismus, 219-233.

Sass, Hans-Martin (1968): Daseinsbedeutende Funktionen von Wissen und Glauben im Jahrzehnt 1860-1870. In: ZRGG (20), 112-138.

Schäfer, Daniel (2010): Auf der Suche nach der Überwindung des Todes. Medizinische Spekulationen im kulturellen Kontext. In: Hilt \& Jordan \& Frewer 2010, 20-32.

Schätzing, Frank (2018): Die Tyrannei des Schmetterlings. Köln.

Schelling, Friedrich W. von (1812): Denkmal der Schrift von den göttlichen Dingen und des Herrn Friedrich Heinrich Jacobi und der ihm in derselben gemachten Beschuldigung eines absichtlich täuschenden, Lüge redenden Atheismus. Tübingen.

Schenkel, Oskar (2000): Literatur als Labor. Posthumane Welten bei H. G. Wells. In: Flessner 2000a, 101-114.

Scherer, Georg (1995)[1989]: Thomas von Aquin. In: Metzler Philosophen Lexikon. Von den Vorsokratikern bis zu den Neuen Philosophen. Stuttgart ${ }^{2} 1995,882-886$.

Schivelbusch, Wolfgang (1977): Geschichte der Eisenbahnreise. München.

Schiwy, Günther (2001): Ein Gott im Wandel. Teilhard de Chardin und sein Bild der Evolution. Düsseldorf.

Schöner, Gregor (2014): Embodied Cognition, Neural Field Models of. In: Encyclopedia of Computational Neuroscience. Berlin, 1084-1092.

Schrage, Dominik (2000): Selbstentfaltung und künstliche Verwandtschaft. Vermenschlichung und Therapeutik in den Diskursen des Posthumanen. In: Flessner 2000a, 43-65.

Schröter, Jens (2002): Biomorph. Anmerkungen zu einer neoliberalen Gentechnik-Utopie. In: Kunstforum International 158, 84-95. 
Schütz, Alfred ('1993)[1932]: Der sinnhafte Aufbau der sozialen Welt. Eine Einleitung in die verstehende Soziologie. Frankfurt a.M.

Schütz, Alfred \& Thomas Luckmann ( $\left.{ }^{5} 1994\right)$ [1973]: Strukturen der Lebenswelt, $B d$. 1. Frankfurt a.M.

Schütz, Alfred \& Thomas Luckmann (21990)[1973]: Strukturen der Lebenswelt, $B d$. 2. Frankfurt a.M.

Seltzer, Mark (1994): Bodies and Machines. New York.

Selznick, Brian (2008)[2007]: Die Entdeckung des Hugo Cabret. München.

Shannon, Claude E. \& Warren Weaver (1976)[1949]: Mathematische Grundlagen der Informationstheorie. München.

Sheskin, Arlene (1979). Cryonics. A Sociology of Death and Bereavement. New York.

Shultz, Suzanne M. (1992): Body Snatching. The Robbing of Graves for the Education of Physicians in Early Nineteenth Century America. Jefferson.

Sill, Oliver (1997): >Fiktionale Realität versus >reale Realitätı? Zu den kunst- bzw. literaturwissenschaftlichen Reflexionen Niklas Luhmanns und Wolfgang Isers. In: Soziale Systeme 3/1997, 137-155.

Simakova, Marina (2016): No Man's Space. On Russian Cosmism. In: e-flux 74 https://www.e-flux.com/journal/74/59823/no-man-s-spaceon-russian-cosmism/.

Simpson, John A. \& Edmund Weiner ( $\left.{ }^{2} 1989\right)$ : The Oxford English Dictionary. Oxford.

Sirius, R. U. \& J. Cornell (2015): Transcendence. The Disinformation Encyclopedia of Transhumanism and the Singularity. San Francisco.

Socrates (2012): 17 Definitions of the Technological Singularity. In: Singularity Weblog. https://www.singularityweblog.com/17-definitions-of-the-technological-singularity/

Sommer, Andreas U. (1996): Religion, Wissenschaft und Politik im protestantischen Idealstaat. Johann Valentin Andraes >Christianopolis<. In: ZRGG 48 (1998), 114-137.

Sorgner, Stefan L. (2009): Nietzsche, the Overhuman and Transhumanism. In: Journal of Evolution and Technology 20 (March 2009) (Online).

Sorgner, Stefan L. (2016): Transhumanismus. »Die gefährlichste Idee der Welt«!? Freiburg i.Br.

Spadafora, David (1990): The Idea of Progress in Eighteenth-Century Britain. New Haven.

Spreen, Dirk (2000): Naturwesen, künstlicher Mensch und lachende Dritte. Zur kritischen Verortung negativer Anthropologie. In: Flessner 2000a, 183-200. 
Stableford, Brian (1999a): Cryonics. In: Clute \& Nicholls 1999, 283-284. Stableford, Brian (1999b): Immortality. In: Clute \& Nicholls 1999, 616. Stambler, Ilia (2014): A History of Life-Extensionism in the Twentieth Century. Rison Lezion. www.longevityhistory.com.

Standage, Tom: Der Türke. Die Geschichte des ersten Schachautomaten und seiner Abenteuerlichen Reise um die Welt. Berlin 2005.

Stapledon, Olaf (1983)[1930]: Die ersten und die letzten Menschen. München.

Staudinger, Hugo (1986): Die Nebenwirkung einer Reise? Darwins Theorie und die Folgen. In: ZRGG (38), 167-185.

Stausberg, Michael (1998): Faszination Zarathushtra. Zoroaster und die Europäische Religionsgeschichte der frühen Neuzeit. Berlin.

Steels, Luc (1994): The Artificial Life Roots of Artificial Intelligence. In: Artificial Life 1 (1/2 1993/1994), 75-110.

Steels, Luc (1996): Die Zukunft der Intelligenz. In: Kunstforum International 133, 84-89.

Stelarc (1996): Von Psycho- zu Cyberstrategien: Prothetik, Robotik und Telexeistenz. In: Kunstforum International 132, 72-81.

Stelarc (1998): From Psycho-Body to Cyber-Systems: Images as Post-Human Entities. In: Virtual Futures. Cyberotics, Technology and Post-Human Pragmatism, hg. von Joan B. Dixon \& Eric Cassidy, 116-123.

Stelarc (2000): Obsolete Zeit? Ein Gespräch mit Sven Drühl. In: Kunstforum International 151, 117-124.

Stephens, Charles A. (1920): Immortal Life. How it will be achieved. Norway Lake.

Stephenson, William (2014): Timothy Leary and the Trace of the Posthuman. In: Després \& Machinal 2014, 281-298.

Sterling, Bruce (1985) [1979]: Schismatrix. New York.

Stock, Gregory (1993): Metaman. The Merging of Humans and Machines into a Global Superorganism. Toronto.

Stock, Gregory (2002): Redesigning Humans. Our Inevitable Genetic Future. Boston.

Strate, Lance \& Edward Wachtel (Hg.)(2005): The Legacy of McLuhan. Cresskill.

Stross, Charles (2005): Accelerando. London.

Strugazki, Arkadi \& B. Strugazki (1989)[1961]: Kerzen vor dem Pult. In: Mittag, 22. Jahrhundert. Der Junge aus der Hölle. Berlin, 101-126.

Stuckrad, Kocku von (2014): The Scientification of Religion. An Historical Study of Discoursive Change, 1800-2000. Berlin. 
Tabbert, Thomas T. (2004): Menschmaschinengötter. Künstliche Menschen in Literatur und Technik. Hamburg.

Tandy, Charles (Hg.)(2003): Death and Anti-Death, Vol. 1: One Hundred Years After N. F. Fedorov (1829-1903). Ann Arbor.

Teilhard de Chardin, Pierre ( $\left.{ }^{3} 1959\right)$ [1955]: Der Mensch im Kosmos. Othon Marbach (Übers.). München.

Teilhard de Chardin, Pierre (21961)[1956]: Die Entstehung des Menschen. Günther Scheel (Übers.). München.

Tipler, Frank J. (1980): General Relativity and the Eternal Return. In: Essays in General Relativity. A Festschrift for Abraham H. Taub, hg. von Frank Tipler. New York, 21-38.

Tipler, Frank J. (1981): Extraterrestrial Intelligent Beings Do Not Exist. In: Quarterly Journal of the Royal Astronomical Society 21, 267-282.

Tipler, Frank J. (1986): Cosmological Limits on Computation. In: International Journal of Theoretical Physics 25, 617-661.

Tipler, Frank J. (1989): The Omega Point as Eschaton. Answers to Pannenberg's Questions for Scientists. In: Zygon 24, 217-253.

Tipler, Frank J. (1991): Alien life. In: Nature 354, 334f.

Tipler, Frank J. (1994): Sophistry and Illusion. In: Nature 369, 198.

Tipler, Frank J. (1995)[1994]: The Physics of Immortality. Modern Cosmology, God and the Ressurection of the Dead. New York.

Tipler, Frank J. (2000): The Omega Point Theory. http://www.math. tulane.edu:80/ tipler/tipler2.html, 23.08.2000.

Tipler, Frank J. (2007): The Physics of Christianity. New York.

Tipler, Frank J. (2015): If you can't beat'em, join'em. In: Brockman 2015, 30-31.

Tirosh-Samuelson, Hava. (2014): Religion. In: Post- and Transhumanism: an Introduction, hg. von Robert Ranisch \& Stefan L. Sorgner. Frankfurt a.M., 49-71.

Torr, James D. (2002): The American Frontier. San Diego.

Trésor de la Langue Française. Dictionnaire de la langue du XiX $r t d u X X^{\circledR}$ siècle (1789-1960), Bd. 16. Centre National de la Recherche Scientifique. Institut National de la Langue Française (Hg.). Paris 1994.

Turchin, Valentin F. (1977)[1970]: The Phenomenon of Science - A Cybernetic Approach to Human Evolution. New York.

Turing, Alan M. (1950): Computing Machinery and Intelligence. In: Mind 59, 433-462 / Turing 1992, 133-160. 
Turing, Alan M. (1992): Collected Works of A. M. Turing. Mechanical Intelligence. D. C. Ince (Hg.). Amsterdam.

$\mathbf{U}$

Ulam, Stanisław (1958): John von Neumann 1903-1957. In: Bulletin of the American Mathematical Society 64 (1958), 1-49.

V

Vandenberghe, Frédéric (2006): Complexités du posthumanisme. Trois essais dialectiques sur la sociologie de Bruno Latour. Paris.

Van Velzer, Ryan (2014): Immortality eludes People Unlimited founder. In: AZCentral, 28.11.2014.

Veatch, Robert M. (1975): The Whole-Brain-Oriented Concept of Death. An Outmoded Philosophical Foundation. In: Journal of Thanatology 3, 13-30.

Vernadskij, Vladimir I. (1997): Der Mensch in der Biosphäre. Zur Naturgeschichte der Vernunft. Frankfurt.

Verne, Jules (1892): Le Château des Carpathes. Paris.

Villiers de l'Isle-Adam, Auguste de (1987)[1886]: L'Ève future. Paris.

Vinge, Vernor (1983): First Word. In: Omni, January 1983, 10.

Vinge, Vernor (1992): A Fire Upon the Deep. New York.

Vinge, Vernor (1993): The Coming Technological Singularity. How to Survive in the Post-Human Era. http://mindstalk.net/vinge/vinge-sing. html.

Vinge, Vernor (2008): Signs of the Singularity. In: IEEE Spectrum (Juni). https://spectrum.ieee.org/biomedical/ethics/signs-of-the-singularity.

Vinge, Vernor (2013)[2003]: Technological Singularity. In: More \& Vita-More 2013, 365-375.

Virilio, Paul (1994)[1993]: Die Eroberung des Körpers. Vom Übermenschen zum überreizten Menschen. München.

Vita-More, Natasha (1983): Transhuman Manifesto. http://www.transhumanist.biz/transhumanmanifesto.htm

Vita-More, Natasha (1990): Cryonics as a Safety Net. http://www.natasha. cc/cryonics.htm.

Vita-More, Natasha (1995): Recreating Reality - Redifining Art. A Continuous Process. Vortrag auf der Konferenz Extro2. http://www.natasha. cc/extro.htm. 
Vita-More, Natasha (1996): Ageless Thinking. Creative Positive Transhuman Attitude. Vortrag auf der Konferenz Resources for Independent Thinking. http://www.natasha.cc/ageless.htm.

Vita-More, Natasha (1997a): Future of Sexuality. http://www.extropicart. com/-sex.htm.

Vita-More, Natasha (1997b): Extropic Art Manifesto. http://www.extropic-art.com/extropic.htm (15.12.2003).

Vita-More, Natasha (2000a): The Transhumanist Art Centre. http://www. extropic-art.com/history.htm (15.12.2003).

Vita-More, Natasha (2000b): Create/Recreate. The $3^{\text {rd }}$ Millenial Culture. Typoskript.

Vita-More, Natasha (2013): Aesthetics: Bringing the Arts \& Design into the Discussion of Transhumanism. In: More \& Vita-More 2013, 18-27.

Volpicelli, Gian (2014): The Transhumanist Church that has Faith in Technology. In: Motherboard, 31.12.2014 (Online).

Vonnegut, Kurt (1991)[1966]: Slaughterhouse-Five or the Children's Crusade. A Duty-Dance with Death. New York.

\section{W}

Wagner, Thomas (2015): Robokratie. Google, das Silicon Valley und der Mensch als Auslaufmodell. Köln.

Walford, Roy L. (1983): Maximum Life Span. New York.

Walford, Roy L. (2000): Beyond The 120-Year Diet. New York.

Wallace, Alfred R. (1903): Des Menschen Stellung im Weltall. Eine Studie über die Ergebnisse wissenschaftlicher Forschung in der Frage nach der Einzahl oder Mehrzahl der Welten. Berlin.

Warwick, Kevin (1997): March of the Machines. Why the new Race of Robots will rule the World. London.

Warwick, Kevin (1998): In the Mind of the Machine. The Breakthrough in Artificial Intelligence. London.

Warwick, Kevin (2000): Cyborg 1.0. In: Wired (2/2000), 144-151.

Warwick, Kevin (2004): I, Cyborg. Chicago.

Weizenbaum, Joseph (21980)[1976]: Die Macht der Computer und die Ohnmacht der Vernunft. Frankfurt a.M.

Weizsäcker, Carl-Friedrich ( $\left.{ }^{6} 1990\right)[1964]:$ Die Tragweite der Wissenschaft. Stuttgart.

Weizsäcker, Christiane \& Ernst U. von (1987): Warum Fehlerfreundlichkeit? In: Das Ende der Geduld. Carl Friedrich von Weizsäckers >Die Zeit drängt in der Diskussion. München 1987, 97-101. 
West, Patrick (2000): The secret lives of our Walter Mitties. In: http:// www.britannica.com/magazine/article?content_id=275420\&pager. offset=10 (Archiv: Googles Cache 2000).

Whitehead, Alfred N. (1979)[1929]: Prozeß und Realität. Entwurf einer Kosmologie. Hans-Günter Holl (Übers.). Frankfurt a.M.

Wiener, Norbert (1963): Kybernetik. Regelung und Nachrichtenübertragung im Lebewesen und in der Maschine. Düsseldorf.

Wiener, Norbert (1964)[1946]: Mensch und Menschenmaschine. Kybernetik und Gesellschaft. Frankfurt a.M.

Wiener, Norbert (1965)[1964]: Gott \& Golem Inc. Düsseldorf.

Wiener, Norbert (1985): Collected Works with Commentaries. Vol. IV. Cybernetics, Science and Society; Ethics, Aesthetics, and Literary Criticism; Book Reviews and Obituaries, hg. von Pesi R. Masani. Cambridge.

Wiener, Norbert (1988)[1950]: The Human Use of Human Beings. Cybernetics and Society. New York.

Wiener, Oswald (1969): die verbesserung von mitteleuropa, roman. Reinbek.

Williams, Thomas (2001): John Duns Scotus. In: Stanford Encyclopedia of Philosophy. https://plato.stanford.edu/entries/duns-scotus.

Wittgenstein, Ludwig (1970)[1969]: Über Gewißheit, hg. von G. E. Anscombe \& G. H. Wright. Frankfurt a.M.

Wolfe, Cary (2010): What is posthumanism? Minneapolis.

Wulf, Christoph (1984): Das gefährdete Auge. Ein Kaleidoskop der Geschichte des Sehens. In: Kamper \& Wulf 1984a, 21-45.

$\mathbf{Y}$

Yoke, Carl B. (1985): List of Works on Immortality in Science Fiction. In: Yoke \& Hassler 1985, 209-222.

Yoke, Carl B. \& D. Hassler (Hg.) (1985): Death and the Serpent. Immortality in Science Fiction and Fantasy. Westport.

Yudkowsky, Eliezer S. (2000a): Singularitarian Principles. Version 1.0.2. Extended Edition. https://web.archive.org/web/20070613190005/ http://yudkowsky.net/sing/principles.ext.html\#preface.

Yudkowsky, Eliezer S. (2000b): Singularitarian Principles. Version 1.0.2. Extended Edition. http://yudkowsky.net/obsolete/principles.html.

$\mathbf{Z}$

Zachary, G. Pascal (1997): Endless Frontier. Vannevar Bush, Engineer of the American Century. Cambridge. 
Žižek, Slavoj (2000): No Sex, Please! Wé re Post-Human. In: Britannica.com, 22.01.2000. http://www.britannica.com/b...original/article/0,5744,16 $171+1,00 . h$ tm.

b) Spielfilme / Fernsehfilme / Dokumentationen

Allen, Woody (1973): Sleeper. USA 1973.

Badham, John (1985): Short Circuit. USA.

Bloch, Robert (1966): What are Little Girls Made of? Star Trek, Folge 9.

US-Erstausstrahlung 20.10.1966.

Besson, Luc (2014): Lucy. Frankreich.

Brambilla, Marco (1993): Demolition Man. USA.

Cameron, James (1984): The Terminator. USA.

Cronenberg, David (1999): eXistenZ. USA.

Fassbinder, Rainer W. (1973): Welt am Draht. WDR.

Jonze, Spike (2013): Her. USA.

Kalogridis, Laeta (2018): Altered Carbon. USA.

Kosinski, Joseph (2010): Tron Legacy. USA.

Kouba, Robert (2017): Singularity. USA.

Kubrick, Stanley (1968): 2001. A Space Odyssey. USA.

Lukacevic, Damir (2010): Transfer. Deutschland.

Leonard, Brett \& G. Everett (1991): The Lawnmower Man. USA.

Lisberger, Steven (1982): Tron. USA.

Lundström, Lars (2012-14): Äkta människor. Schweden.

Miner, Steve (1992): Forever Young. USA.

Molinaro, Edouard (1969): Hibernatus. Frankreich/Italien.

Niccol, Andrew (1997): Gattaca. USA.

Nelson, Garry (1979): The Black Hole. USA.

Nolan, Jonathan \& Lisa Joy (2016): Westworld (TV-Serie). USA.

Pfister, Wally (2014): Transcendence. USA

Ptolemy, Barry (2009): Transcendent Man. USA.

Roach, Jay (1997): Austin Powers. International Man of Mystery. USA.

Rusnak, John (1999): The 13 $3^{\text {th }}$ Floor. USA.

Sasdy, Peter (1976): Welcome to Blood City. Großbritannien/Kanada.

Scott, Ridley (1979): Alien. USA.

Shatner, William (1989): The Final Frontier. USA.

Sussberg, Jason (2014): The Immortalist. UK, USA, India. 
Trumbull, Douglas (1982): Brainstorm. USA.

Verhoeven, Paul (1990): Total Recall. USA.

Wachowski, Andy \& L. Wachowski (1999): The Matrix. USA.

Wachowski, Andy \& L. \& T. Tykwer (2012): Cloud Atlas. Deutschland. USA.

\section{c) Interviews (Videos)}

Kurzweil, Ray (1965): I've got a secret. https://www.youtube.com/watch?v=X4Neivqp2K4

Kurzweil, Ray (2016): Ray Kurzweil Remembers Marvin Minsky, 04.04.2016. https://www.youtube.com/watch?v=FIK3X2fKJrE

Kurzweil, Ray (2017): Interview. Human-Level AI is Just 12 Years Away. https://www.youtube.com/watch?v=JiXVMZTyZRw

Minsky, Marvin L. (2010): Ray Kurzweil interviews Marvin Minsky, 2010. https://www.youtube.com/watch?v=RZ3ahBm3dCk

Minsky, Marvin L. (2013): Marvin Minsky on Singularity. Interview mit Socrates / Nikola Danaylov vom SingularityWeblog, 12.07.2013. https://www.youtube.com/watch?v=3PdxQbOvAlI

Socrates (2015): The Emperor Has No Clothes: Socrates Deconstructs Singularity University. https://www.singularityweblog.com/socrates-deconstructs-singularity-university/, 29.11.2015.

Tipler, Frank (2013): The Laws of Physics Say The Singularity is Inevitable! Interview mit Socrates / Nikola Danaylov vom SingularityWeblog 29.10.2013, https://www.singularityweblog.com/frank-j-tipler-the-singularity-is-inevitable/

Vinge, Vernor (2011): We Can Surpass the Wildest Dreams of Optimism. Interview mit mit Socrates / Nikola Danaylov vom SingularityWeblog, 15.04.2011. https://www.singularityweblog.com/vernor-vinge-onsingularity-1-on-1-we-can-surpass-the-wildest-dreams-of-optimism/ 


\section{Sach- und Personenindex ${ }^{1}$}

Abbé de Saint-Pierre 290, 410

Abbott, Lyman 246

Agar, Nicholas 24

Alexander, Samuel 246, 249, 250, 384

Allen, Ethan 263

Allen, Woody 335

Alsted, Johann H. 415

Amenábar, Alejandro 330

Anders, Günther 38, 40, 43, 50, 55, 61, $62,63,65,117,152,153,220,423$, 424

Andrae, Johann V. 414

Anselm von Canterbury 206

Arendt, Hannah 24, 62, 423

Aristoteles 165, 167, 173, 182, 183

Asimov, Isaac 93

Assmann, Jan 37, 66

Augustinus 165, 183, 206

Bach, Johann Sebastian 369

Bacon, Francis 78, 206, 219, 289, 290, $308,415,416$

Bacon, Roger 307

Badmington, Neil 20

Baillie, John 417

Bainbridge, William S. 100, 104, 109, 305

Barad, Karen 20

Barcelo, Elia 331

Barnes, Ernest W. 246, 252, 266

Barrow, John D. 123, 155, 216, 229, 251, 259

Barthes, Roland 38, 66

Bateson, Gregory 218

Baudrillard, Jean 53, 54

Baumgartner, Hans M. 36

Becker, Philipp von 24, 352, 395

Beck, Klaus 32, 36, 39

Beck, Ulrich 406

Bedford, James H. 343
Bennet, James C. 105

Bentham, Jeremy 208, 238, 239, 398

Bentley, Richard 230, 263

Benz, Ernst 291, 414

Berger, Peter L. 36

Bergson, Henri 32, 33, 34, 35, 205, 217, 248, 250, 389

Berkeley, George 180, 260, 323

Bernal, John D. 78, 314, 315, 356, 407

Besson, Luc 368

Bigelow, Julian 185

Blackford, Russel 100

Bloch, Ernst 151

Bloch, Robert 328

Blount, Thomas 119

Blumenberg, Hans 148, 412, 413, 414

Boethius 230

Bogdanov, Aleksandr 311, 312

Böhme, Hartmut 361

Bollnow, Otto F. 41

Boltzmann, Ludwig 217

Bonnet, Charles 240

Bostrom, Nick 75, 78, 82, 83, 99, 101, 108, 109, 273, 362, 374, 399, 409, 421,422

Braidotti, Rosi 20

Brillouin, Leon 218

Brin, David 364

Bringsjord, Selmer 364

Brin, Sergey 285

Broderick, Damien 286

Brown, Bernadeane 111, 112, 113, 400

Brown, Charles P. 111

Brown, Dan 19

Bruno, Giordano 341

Büchner, Ludwig 253

Buffier, Claude 164

Bultmann, Rudolf 410, 411, 413

Bunyan, John 226, 415

Burroughs, Edgar R. 335

1 Verweise auf die häufigsten Akteure (Ray Kurzweil, Marvin Minsky, Hans Moravec und Frank Tipler) wurden nicht in den Index aufgenommen. 
Bury, John B. 410, 413, 417

Bush, George W. 292

Bush, Vannevar 274, 292

Calvin, Johannes 237

Carter, Brandon 260

Carus, Paul 165, 244

Cary, Henry Francis 79

Chamberlain, Fred \& Linda 338

Chamberlain, Houston S. 373

Chislenko, Sasha 127, 197

Christie, Agatha 260

Cicero 230

Clarke, Arthur C. 113, 127, 215, 317, 319, 323, 366, 375

Clarke, Richard 308

Clark, Nancie 94

Clynes, Manfred 92

Cobb, Jennifer 381

Coenen, Christopher 23

Comte, Auguste 290, 312, 410, 411

Condorcet, Antoine Marquis de 78, 205, 290, 308, 410, 411

Conolly, Randy 418

Cooper, Evan 337

Cornaro, Luigi 308

Crick, Francis 404

Cromwell, Oliver 371

Cronenberg, David 331

Daguerre, Louis 43

Dante 79, 420

Darwin, Charles 62, 80, 241, 242, 243, $245,256,371,423$

Darwin, Erasmus 208, 241

da Vinci, Leonardo 370

Davis, Erik 361

Dawkins, Richard 131, 162, 255, 256

de Funès, Louis 335

de Grey, Aubrey 98, 99, 107, 108, 111, 112, 304, 339, 400

Deitch, Jeffrey 21

Denis, Jean Baptiste 310

Derham, William 230, 263, 264, 265, 266

Dery, Mark 21
Descartes, René 154, 161, 162, 163, $164,165,166,174,175,177,178,179$, 181, 183, 409

de Val, Jaime 100

Diamandis, Peter 285, 400

Dick, Philip K. 330

Diderot, Denis 224, 240, 266

Dilthey, Wilhelm 45, 59

Dotzler, Bernhard 166

Drexler, Eric 93, 98, 104, 105, 108, 109, 399

Dühring, Eugen 225

Duns Scotus, John 57, 420

Dyson, Freeman 125, 216, 317

Easton, Bret 217

Edelhardt, Mike 321

Eden, Amnon H. 22

Edison, Thomas A. 322, 370

Edwards, John 208

Ehricke, Krafft 92

Einstein, Albert 375

Eisenhower, Dwight D. 91

Ekirch, Arthur 417

Elias, Norbert 34, 49, 64, 200, 405

Engels, Friedrich 217

Esfandiary, Fereidoun M. 75, 77, 85, 88, 90, 98, 136, 302, 303

Ettinger, Robert C. W. 75, 77, 85, 86, 87, 88, 90, 105, 150, 318, 335, 339, 358

Evans, Dylon 399

Everett, Hugh 402

Faloon, William 113

Fassbinder, Rainer W. 56, 327

Fedorov, Nikolai 113, 311, 313

Ferguson, Adam 204, 290, 417

Feuerbach, Ludwig 95, 312

Finot, Jean 313

Fiske, John 246, 252

Flessner, Bernd 29, 31, 117

Flusser, Vilém 47, 48, 58

FM-2030 88, 102, 150

Fontenelle, Bernard le Bovier de 205, 290, 410 
Foucault, Michel 49

Franke, Herbert W. 50, 51, 54, 325, 333

Franklin, Benjamin 78, 205, 208, 263, 309, 360

Fredericksen, Dick 319

Freud, Sigmund 62, 314, 423

Freyermuth, Gundolf 22

Fromm, Erich 341

Fukuyama, Francis 23

Fuller, Buckminster 119, 381

Gadamer, Hans-Georg 148

Gafsou, Matthieu 146

Galen 230

Galouye, Daniel F. 327, 329

Galton, Francis 371, 373

Garbo, Greta 38

Garreau, Joel 22

Gauß, Carl Friedrich 372

Gehlen, Arnold 45, 205

Gendolla, Peter 37, 64, 166

Gerhoch von Reichersberg 206

Gibbon, Edward 290

Gibson, Mel 335

Gibson, William 29, 328, 331

Giddens, Anthony 44

Gilbert, William 206

Gilgamesch 86, 358

Giraud, Fabien 145

Gobel, David 107

Godwin, William 238, 239, 309

Goertzel, Ben 99, 109, 112, 313, 341

Goethe, Johann Wolfgang von 244, 370

Good, Irving J. 269, 365

Graham, Robert K. 373

Gray, Asa 246, 252, 266

Gray, Chris H. 106

Gross, Daniel 238

Großklaus, Götz 32, 39, 40, 41

Grossman, Terry 135, 356

Groys, Boris 67

Guattari, Felix 383
Haeckel, Ernst 166, 242, 243, 244, 312, 318, 372

Halacy, Daniel 358

Haldane, John B. S. 78, 84, 315

Hanson, Robin 100, 363

Harari, Yuval 23

Haraway, Donna 19

Harbisson, Neil 144, 145

Harrington, Alan 341

Hartley, David 209, 417

Hase, Thomas 416

Hassan, Ihab 119

Hawking, Stephen 270

Hayles, N. Katherine 22, 58, 121, 152, 153, 154, 169, 179, 193

Heil, Reinhard 23

Heine, Heinrich 42

Heinlein, Robert 93, 127

Heisenberg, Werner 401

Helmholtz, Hermann von 217, 372

Helvétius, Claude-Adrien 238, 239, 266

Herbert, Frank 127

Herbrechter, Stefan 20, 24

Herder, Johann G. 205, 240, 413

Hesse, Eva 64

Heylighen, Francis 383

Hitler, Adolf 126

Hobbes, Thomas 161, 164, 167

Hobsbawm, Eric 77

Holbach, Paul H. 266

Holmes, Oliver W. 45

Holmes, Sherlock 86, 358, 404

Hölscher, Lucian 151

Home, Henry 204

Homer 307

Houellebecq, Michel 19

Hufeland, Christoph W. 310

Hughes, James 77, 82, 99, 108, 111, 341

Hume, David 204, 290

Hunt, James 372

Husserl, Edmund 33, 34, 35

Huxley, Aldous 78, 82, 253 
Huxley, Julian 78, 81, 82, 253, 254, $255,256,357,375$

Huxley, Thomas 252

Ingarden, Roman 149

Irrgang, Bernhard 24

Iser, Wolfgang 52, 54

Istvan, Zoltan 112

Itskov, Dmitry 285, 313

Jackson, Andrew 227

Jackson, Michael 21

Jacobi, Friedrich H. 386

Jastrow, Robert 226, 321

Jauß, Hans R. 148, 149

Jefferson, Thomas 263

Jenyns, Soame 252

Jones, Neil R. 335

Jones, Neil R. 336

Joslyn, Cliff 383

Joy, Bill 214

Juliana von Norwich 267

Jung, C. G. 341

Kac, Eduardo 144

Kamper, Dietmar 32, 48, 58, 69, 182, 425

Kant, Immanuel 78, 240, 290

Kapuściński, Ryszard 212

Kempelen, Wolfgang von 370

Kennedy, John F. 292

Kent, Saul 113, 337

Kepler, Johannes 230

Kerr, Philip 329

King, Stephen 330

Klemperer, Victor 41

Kline, Nathan 92

Kohl, Helmut 55

Kolumbus, Christoph 291

Kopernikus, Nikolaus 62, 423

Koselleck, Reinhart 242, 411, 412

Kroker, Arthur \& Marilouise 117

Kubrick, Stanley 366
Lactanz 206

Lafitau, Joseph-François 227

Lahontan, Louis Armand de Lom d'Arce 227

Lamarck, Jean-Baptiste 241, 244

La Mettrie, Julien Offray de 78, 154, 164, 165, 167, 409

Lange, Friedrich A. 164

Lavery, David 22, 92

Law, Edmund 207, 208, 238, 240

Leary, Timothy $67,75,77,91,93,94$

Leibniz, Gottfried W. 164, 240

Lem, Stanisław 29, 50, 59, 224, 313, $323,324,331,333,367,424$

Le Roy, Édouard 312

Le Sage, George-Louis 164

Levandowski, Anthony 114

Levin, Janet 96

Lévy, Pierre 383

Liebig, Justus 372

Lighthall, William D. 79, 420

Lincoln, Abraham 112, 345

Linde, Carl von 344

Locke, John 164, 180, 240

Loh, Janina 20, 24

Lovejoy, Arthur O. 167, 240, 241

Löwenthal, Eduard 244

Löwith, Karl 410, 411, 413

Lübbe, Hermann 406

Luckmann, Thomas 36, 37

Lüdeking, Karlheinz 47

Luhmann, Niklas 35

Lyotard, Jean- Fran(ois 46

MacClaurin, Colin 263

Macho, Thomas 39, 67

Maes, Pattie 288

Mandeville, John 307

Marinetti, Emilio F. T. 136, 141, 146

Martin, George M. 319

Marvin, Carolyn 195

Marx Hubbard, Barbara 92

Marx, Karl 95

Mather, Cotton 226 
Mattelart, Armand 418

Mayr, Otto 192

McCalla, Arthur 240

McCarthy, John 189

McCosh, James 245

McCulloch, Warren 127, 187, 197

McGinn, Colin 166

McKay, Donald 195

McKenna, Terence 381

McLuhan, Marshall 30, 92, 119, 384, 387, 390

Mead, George H. 35

Merkle, Ralph 105, 109, 339

Metschnikow, Ilja 313

Michelangelo 369

Millar, John 205

Milton, John 418

Mitchell, David 396

Mommsen, Theodor 372

Monchicourt, Marie-Odile 123

Moore, Gordon 280

More, Max 75, 82, 94, 95, 96, 108, 214, 235, 286, 339, 358

Morgan, Richard 331

Morgenstern, Oskar 184

Mori, Masahiro 409

Morrow, T. O. 95, 102

Morse, Samuel 43

Mukasovský, Jan 148

Muñoz, Manel 145

Murphy, Joseph 97

Napoleon Bonaparte 370

Nassehi, Armin 32, 35

Nayar, Pramod K. 20

Negroponte, Nicholas 57, 127

Nelson, Robert 338, 339, 343

Neumann, John von 127, 154, 155, 184, 185, 187, 189, 190, 191, 197, 219, 274,405

Newton, Isaac 32, 252, 263

Nielsen, Michael 286, 363

Nietzsche, Friedrich 78, 86, 95, 214, 217, 357, 358, 375

Nowlan, Philip F. 335
Nozick, Robert 332

Obama, Barack 292

O'Connor, Maximilian 95, 136

Oetinger, Friedrich C. 240

O’Neill, Gerard 93, 104, 293

Ong, Walter 388

Oppenheimer, Robert 274

Origines 206

Orlan 21, 142, 143, 144, 146

Ovid 79

Page, Larry 285

Paine, Thomas 263

Paley, William 162, 204, 205, 207, 209, 238, 252, 255, 263, 265, 266

Pannenberg, Wolfhart 124, 251, 252

Papert, Seymour 127

Parfit, Derek 171

Peale, Norman V. 97

Pearce, David 75, 99, 109

Penrose, Roger 230, 270

Pepperell, Robert 19, 120, 137, 355

Perry, R. Michael 339, 341

Pesce, Mark 380

Peterson, Christine 105

Petrarca 369

Piaget, Jean 45, 177

Piscator, Johann 415

Pitts, Walter 186

Platon 165, 183, 230, 323

Plessner, Helmuth 30, 33

Plinius 307

Plutarch 167

Pohl, Frederick 319

Poinsett, Joel R. 227

Pope, Alexander 240

Popper, Karl 97

Portmann, Adolf 83

Powell, Colin 295

Powers, Austin 335

Price, Richard 207, 208, 238, 239, 308, 417

Priestley, Joseph 207, 208, 238, 239, 417 
Prisco, Giulio 109, 113

Protagoras 261

Proudfoot, Diane 269

Proudhon, Joseph 411

Putin, Vladimir 313

Randow, Gero von 22

Rapp, Friedrich 411, 413, 422

Ray, John 263

Regis, Ed 21, 407

Régis, Pierre S. 164

Ribas, Moon 144, 145

Riegler, Johanna 220

Roco, Mihail 104, 305

Rohbeck, Johannes 62, 414, 423

Roosevelt, Franklin D. 292

Rosenblueth, Arturo 185

Ross, John 208

Rostand, Jean 336

Rothblatt, Martine 99, 101, 109, 113, 339, 398, 409

Rottensteiner, Franz 22

Rötzer, Florian 21, 67

Rousseau, Jean-Jacques 227

Rucker, Rudy 296, 328

Russel, Clark 334

Sandberg, Anders 75, 98, 99, 100, 102, 109, 269, 273, 286, 304, 341, 362

Savage, Judson 245

Schätzing, Frank 19

Schäfer, Jörgen 166

Schelling, Friedrich W. 250, 386

Schubert, Franz 372

Schumann, Robert 372

Schütz, Alfred 31, 34, 35

Selznick, Brian 425

Shakespeare, William 369

Shannon, Claude 127, 154, 184, 193, 194, 195, 196, 197, 198, 217, 218

Siboni, Raphaël 145

Siemens, Wilhelm von 372

Sirius, R.U. 22

Smith, Adam 204, 205, 290
Smyth, Garret 95

Sorgner, Stefan L. 83, 100, 109, 358

Spadafora, David 207, 417

Spencer, Herbert 217

Stableford, Brian 322

Stalin, Josef 126, 312

Stallone, Sylvester 335

Stambler, Ilia 305

Stapledon, Olaf 366

Stausberg, Michael 149

Steels, Luc 21, 106, 127

Steinach, Eugen 313

Stelarc 21, 29, 136, 138, 139, 140, 141, 226, 303

Stephens, Charles A. 314

Sterling, Bruce 106, 120

St. Jude 93, 94

Stock, Gregory 98, 100

Strabo 307

Strole, James 111, 112, 113, 400

Stross, Charles 420

Strugazki, Arkadi \& Boris 313, 326

Stuckrad, Kocku von 414

Szilard, Leo 218

Tabbert, Thomas T. 23

Tandy, Charles 313

Teilhard de Chardin, Pierre 78, 82, 84, 119, 246, 248, 251, 253, 254, 255, 257, 261, 271, 312, 373, 380, 383, $384,385,386,387,391$

Tertullian 206

Tesla, Nikola 370

Theseus 167

Thiel, Peter 107, 108, 285

Thomas von Aquin 165, 167, 168, 173, 183

Thomson, William 217

Tillich, Paul 267

Toynbee, Arnold 83

Triptree, James 328

Turgot, Anne R. 205, 290, 411

Turing, Alan 154, 156, 184, 189, 190, 191, 192, 375

Turtschin, Valentin 382 
Ulam, Stanisław 274

Vandenberghe, Fréderic 24

Vandenberghe, Frédéric 398

Van Nedervelde, Philippe 113

Vauvenargues, Luc de Clapiers Maquis de $35,36,40$

Veatch, Robert M. 320

Venzmer, Gerhard 314

Verhoeven, Paul 330

Vernadski, Vladimir 312

Verne, Jules 313, 322, 331

Vico, Giovanni B. 413

Villiers de l'Isle Adams, Auguste 322

Vincent von Lerins 206

Vinge, Vernor 98, 135, 269, 273, 275, 284, 296, 355, 363

Vita-More, Natasha 94, 98, 99, 109, 141, 146, 341, 376, 400

Vodicka, Felix 148

Voltaire, Arouet 230, 411

Vonnegut, Kurt 11, 217

Voronoff, Serge 313

Wachowski, Andy \& Larry 330

Wagner, Thomas 24

Walford, Roy L. 15, 98, 304, 341

Wallace, Alfred R. 241, 253

Warburton, William 208

Warwick, Kevin 105, 106, 203, 223, 226, 228, 234, 357, 377

Washington, George 263

Watson, James 404

Watson, Richard 209

Weaver, Warren 193, 194
Weber, Max 289

Weizenbaum, Joseph 191, 407

Weizsäcker, Christiane von 424

Weizsäcker, Ernst Ulrich von 424

Wells, H. G. 217, 313

Werner, Karl 337

Wesley, John 237, 308

Wheeler, John A. 260

Whitehead, Alfred N. 35, 246, 247, 249, 250, 251

Whitman, Walt 372

Wiener, Norbert 127, 154, 184, 185, 186, 187, 188, 189, 191, 193, 196, $197,198,218,337,357,404$

Wiener, Oswald 326, 407, 419

Wilber, Ken 381

Williams, Ted 339

Wittgenstein, Ludwig 177

Wolfe, Bernard 328

Wolfe, Cary 20

Woltmann, Ludwig 64

Wonder, Stevie 295

Worthington, William 208, 237, 238, 239, 241

Wright, George F. 246

Wulf, Christoph 57, 200

Yoke, Carl B. 322

Yudkowsky, Eliezer 99, 101, 108, 269, 278, 408

Zhuangzi 393

Ziolkowski, Konstantin 311

Žižek, Slavoj 50, 361

Zons, Raimar 23 
